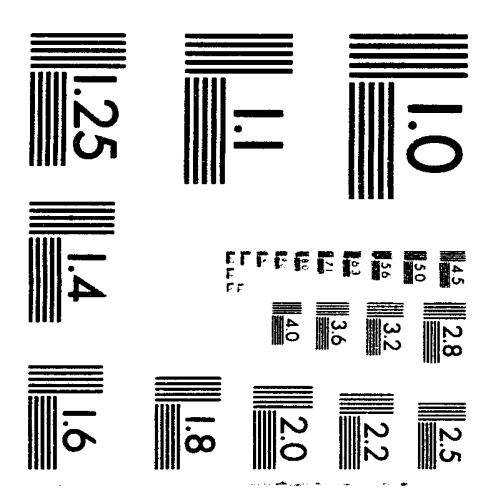



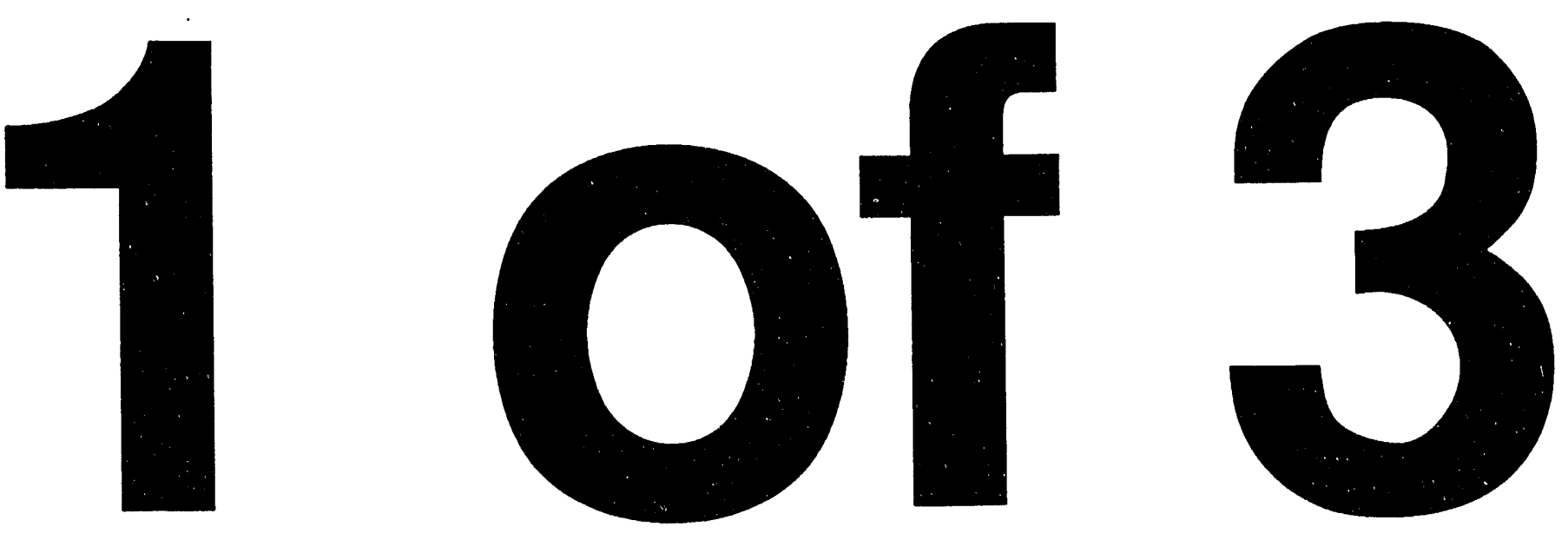


\section{ezear}

The Continuous Electron Beam Accelerator Facility

Theory Group Preprint Series

Additional copies are available from the authors.

The Southeastern Universities Research Association (SURA) operates the Continuous Electron Beam Accelerator Facility for the United States Department of Energy under contract DE-AC05-84ER40150

\section{INTERMEIIATE ENERGY SEMILEPTONIC PROBES OF 'THE HADRONIC NEUTRAL CURRENT'}

M. J. Musolfa, 'I' W. Donnelly", J. Dubach', S J Pollock",

S. Kowalskie, and E. J Br.ise'

a (enter for 'Theoretical Physics, Laboratory for Nuclear Science and I)epartment of Physics, Massachusetts lnstitute of 'Technology, (ambridge, Massachusetts 02139 USA; Department of Physics, Old Dominion Vniversity, Norfolk, Virginia 23529 USA and ('EBAA 'Theory (iroup, MS-1211, 12000 Jefferson Ave., Newport News, Virginia 23606 USA. ${ }^{2}$

b (enter for 'Theoretical Physics, Laboratory for Nuclear Sicience and Department of Physics, Massachusetts Institute of 'Technology, (ambriclge, Massachusetts 02139 USA.

c Department of Physics and Astronomy, University of Massachusetts, Amherst, Massachusetts 01003 USA

a Institute for Nuclear 'Iheory, University of Washington, IIN-12, Seattle, Washington 98195 USA; and NIKIIEF-K 'Theory, Postbus 41882, 10091)B Amsterdan, 'The Netherlands.'

- Laboratory for Nuclear Science and Department of Physics, Massachusetts Institute of 'lechnology, (ambridge, Massachusetts 02139 USA.

f W. K. Kellogg Radiation Iaboratory, (alifornia Institute of 'Technology, Pasadena, California 91125 USA; and Department of Physics, University of Maryland, College Park, Maryland 20742 USA."

DISCLAIMEH

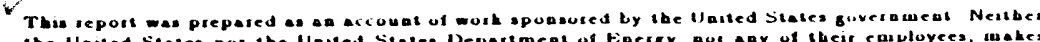

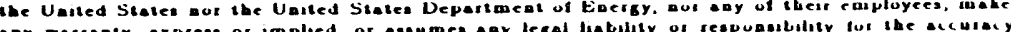

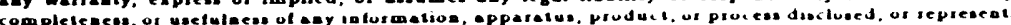

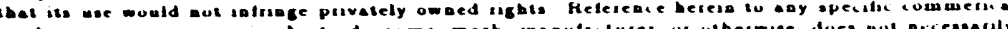

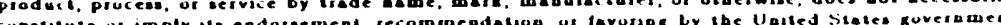

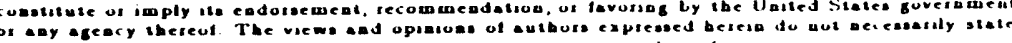

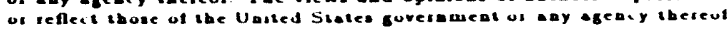

(:13AF \#'11-93-11

JuIr, 1993

This work is supported in part throught funds provided by the 11 . S. Department of lin

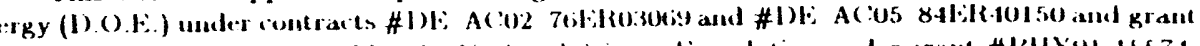

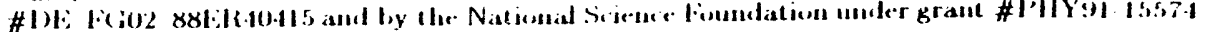

ilresent istliness. 


\title{
INTERMEDIATE-ENERGY SEMILEPTONIC PROBES OF THE HADRONIC NEUTRAL CURRENT*
}

\author{
M. J. Musolf ${ }^{1}$. T. W. Donnelly ${ }^{2}$, J. Dubach ${ }^{3}$, S. J. Pollock ${ }^{4}$, \\ S. Kowalski ${ }^{5}$, and E. J. Beise ${ }^{6}$
}

1 Center for Theoretical Physics. Laboratory for Nuclear Science and Department of Physics. Massachusetts Institute of Technology, Cambridge, Massachusetts 02139 [SA: Department of Physics, Old Dominion University, Norfolk, Virginia 23529 LS. $^{\dagger}$ and CEBAF Theory Group, .IS-12H. 12000 Jefferson Ave., . Vewport lews. Virginia 23606 CSA $^{\dagger}$.

2 Center for Theoretical Physics, Laboratory for Nuclear Science and Department of Physics. Massachusetts Institute of Technology, Cambridge, Massachusetts 02139 USA.

3 Department of Physics and Astronomy, University of Massachusetts, Amherst. Massachusetts 01003 LSA.

4 Institute for Nuclear Theory, University of Washington, HN-12, Seattle. Washington 98195 CSA: and NIKHEF-K Theory, Postbus 41882, 1009DB Amsterdam. The Netherlands ${ }^{\dagger}$.

$>$

Laboratory for .iuclear Science and Department of Physics, Massachusetts Institute of Technology, Cambridge, Massachusetts 02139 USA.

${ }^{6}$ W. K. Kellogg Radiation Laboratory, California Institute of Technology, Pasadena. California 91125 USA; and Department of Physics, University of Maryland. College Park, Maryland 20742 USA.

\footnotetext{
* This work is supported in part through funds provided by the U. S. Department of Energy (D.O.E.) under contracts \#DE-AC02-76ER03069 and \#DE-AC05-84ER40150 and grant \#DE-FG02-88ER40415 and by the National Science Foundation under grant \#PHY91-15574.

$\dagger$ Present Address
} 


\section{Table of Contents:}

I. INTRODLCTION . . . . . . . . . . . . . . . . . 3

II. PHYSICS ISSLES

II.A. Strangeness Content of the Nucleon . . . . . . . . . . . . . . . . . . . . . 6

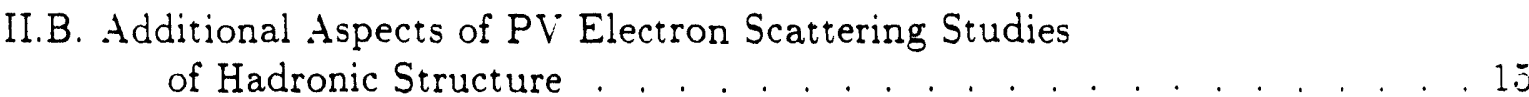

II.C. Electroweak Tests . . . . . . . . . . . . . . . . . . . . . . . . . 20

III. FORMIALIS.II . . . . . . . . . . . . . . . . . . . . . . . . 30

III.A. Currents and Couplings . . . . . . . . . . . . . . . . . . . . . . 30

III.B. Higher-order Processes and Renormalization . . . . . . . . . . . . . 36

III.C. Single-Nucleon Matrix Elements of the Electroweak Current . . . . . . 46

III.D. Nuclear Matrix Elements of the Electroweak Current _ . . . . . . . . j1

III.E. Lepton Scattering from Nucleons and Nuclei _ . . . . . . . . . . . 69

IV. SPECIFIC CASES . . . . . . . . . . . . . . . . .

IV.A. Elastic Scattering from the Proton . . . . . . . . . . . . . . 5i

IV.B. Elastic Scattering from Spin-0 Nuclei . . . . . . . . . . . . . . . .95

IV.C. Elastic Scattering from the Deuteron . . . . . . . . . . . . . . 105

IV.D. Other Discrete Nuclear Transitions . . . . . . . . . . . . . . . . 111

IV.E. Axial-Vector Hadronic Response . . . . . . . . . . . . . . . . . 118

IV.F. Quasielastic Scattering . . . . . . . . . . . . . . . . . . . . . 123

IV.G. The Nucleon-to-Delta Transition . . . . . . . . . . . . . . . . . 132

IV.H. Parity-Violating Deep Inelastic Scattering . . . . . . . . . . . . . 137

IV.I. Atomic Parity Violation . . . . . . . . . . . . . . . . . . . . . . . . 142

IV.J. . Neutrino Scattering from Nucleons and Nuclei . . . . . . . . . 147 
V. EXPERIMENTAL CONSIDERATIONS . . . . . . . . . . . 164

V.A. General Considerations . . . . . . . . . . . . . . . . . . . 164

V.B. Past Studies of Electron and Leutrino Scattering . . . . . . . . . . 172

I.C. Present and Proposed Experiments - Future Prospectives . . . . . . 187

II. SLMMARY AND CONCLLSIONS . . . . . . . . . . . 196

REFERENCES . . . . . . . . . . . . . . . . 200

\begin{abstract}
:
The present status and future prospects of intermediate-energy semileptonic neutral current studies are reviewed. Possibilities for using parity-violating electron scattering from nucleons and nuclei to study hadron structure and nuclear dynamics are emphasized, with particular attention paid to probes of strangeness content in the nucleon. Connections are drawn between such studies and tests of the electroweak gauge theory using electron or neutrino scattering. Outstanding theoretical issues in the interpretation of semileptonic neutral current measurements are highlighted and the prospects for undertaking parity-violating electron or neutrino scattering experiments in the near future are surveyed.
\end{abstract}




\section{INTRODUCTION}

Nearly a generation has passed since the minimal, $\mathrm{SU}^{*}(2)_{L} \times \mathrm{U}^{*}(1)_{Y}$ model of electroweak interactions was first proposed by Weinberg. Salam, and Glashow [Wei67. Sal68. Glai0:. During that time, a wide range of experiments, varying in energy scales from a few $\mathrm{el}^{-}$in atomic parity-riolation tests up to $\sim 100 \mathrm{GeV}$ in $e^{+} e^{-}$annihilation. have been performed with the objective of testing the tree-level predictions of the theory. Of particular interest have been experiments exploring the structure of the weak neutral current (designated in this work by $\mathrm{AC}$ ), whose form is governed by the degree of mixing between the $\mathrm{ST}^{-}(2)_{L}$ and $\mathrm{U}(1)_{Y}$ sectors:

$$
J_{\lambda}^{\mathrm{v} C}=J_{\lambda}^{W^{0}}-4 Q_{f} \sin ^{2} \theta_{w} J_{\lambda}^{E M}
$$

Here $J_{\lambda}^{W^{* 0}}$ is the neutral, weak isospin partner of the charge-changing weak currents. $Q_{f}$ is the electric charge of an elementary fermion (lepton or quark), $J_{\lambda}^{E M}$ is the electromagnetic current, and $\theta_{w}$. the Weinberg or weak-mixing angle, characterizes the degree of mixing between the gauge sectors. In the limit that $\sin ^{2} \theta_{w} \rightarrow 0$, the two sectors decouple. and the neutral current takes on the same structure as the charge-changing weak current. $J_{\lambda}^{w \pm}$. Results from the "first generation" of neutral current studies showed remarkable agreement with predictions based on Eq. (1.1), provided $\sin ^{2} \theta_{w} \approx 1 / 4$. In fact. global analyses of neutral current data from all energy scales are consistent with a common value of $\sin ^{2} \theta_{w}$ to roughly $1.5 \%$ accuracy [Ell90, Alt90, Ken90, Lan90) ${ }^{\dagger}$.

The advent of the $Z^{0}$-resonance physics at SLC and LEP, along with recent $1 \mathrm{~m}$ nrovements in both experimental and theoretical "technology" relevant to atomic parity measurements. presages a new era of higher precision neutral current tests. pushing the level of precision well beyond the $1 \%$ level. Indeed, determinations of $\sin ^{2} \theta_{w}$ from polarization asymmetries in $e^{+} e^{-}$annihilation could reduce the present uncertainty by an order of magnitude [El190, Alt90]. At this level of precision, such experiments would be sensitive to both higher-order predictions of the minimal Standard Model, thereby testing the quantum field character of the theory as well as "new physics" beyond the Standard Model. such as additional neutral vector bosons or technicolor [Mar90, Pes90, Ken90, Gol90].

In this review, we consider semileptonic neutral current studies at intermediate-energy scales (up to a few $\mathrm{GeV}$ ) as might be performed at facilities such as CEBAF, MIT/Bates. Mainz, and LAMPF. The interpretation of experiments at these energy scales involves an additional level of complexity not encountered in deep inelastic scattering or high-energy $e^{+} e^{-}$annihilation. In the latter cases, the effects of strong interactions are sufficienily small to permit a perturbative treatment of semileptonic processes in terms of quarks and gluons. Thus, in such experiments, the interpretation of results in terms of electroweak interactions ainong elementary particles is relatively straightforward and unambiguous. At the lowerenergy scales of interest here, however, QCD becomes a strong-coupling theory and such

"We follow the "mass-squared" definition of the weak mixing angle: $\sin ^{2} \theta_{w} \equiv 1-$ $V_{w}^{2} / M_{z}^{2}$; alternate definitions are discussed below. 
a perturbative treatment is no longer justified. Instead, one must work with explicitly collective hadronic degrees of freedom such as mesons, nucleons. and nuclei. Since low-and intermediate-energy properties of hadrons cannot at present be reliably calculated from first principles. the interpretation of intermediate-energy electroweak studies necessarily involves some degree of theoretical uncertainty. One then faces the question as to what limitations this uncertainty imposes on the precision with which the Standard Model may be confidently tested using intermediate-energy semileptonic probes.

On the other hand. studies of hadron structure in the strong-coupling regime are of central importance to particle and nuclear physics. The diversity of ways in which the hadronic neutral current is manifested in intermediate-energy measurements of semileptonic scattering from nucleons and nuclei makes this a potentially very fruitful direction to follow in the near future. In particular. the subject which provides the main focus of the present work. parity-violating electron scattering, presents a rich new array of hadronic quantities. such as nucleon form factors and nuclear response functions. which cannot be observed in higher-energy experiments. Indeed. since a determination of many of these observables is not possible using purely QED-type processes. intermediate-energy neutral current studies potentially offer a unique window on aspects of hadronic structure which are of fundamental interest in their own right. The realization of this potential presents both experimentalists and theorists a variety of challenges. Once some of these challenges have been met and the hadronic structure better understood, experiments in this sector could become more relevant in the search for "new" or "non-standard" physics.

In the remainder of this article, we attempt to clarify both the precision to which one may reliably test the Standard Model using intermediate-energy, semileptonic processes as well as the kind of hadronic structure information which might realistically be extracted from such experiments. We focus primarily on parity-violating (PV) polarized electron scattering and make contact with neutrino scattering as needed. Our analysis includes both theoretical as well as experimental treatments of past, present, and prospective experiments of these types. We also discuss atomic PV and deep-inelastic PV electron scattering for purposes of comparison.

Our objective is to provide both a general overview of the field as well as a "roadmap" to assist in determining what types of precision neutral current scattering measurements might be undertaken in the future. As a consequence, an important theme running through our discussion will be considerations of experimental "do-ability". Indeed. the relative importance of various hadronic quantities appearing in the observables of interest generally depends both on the choice of kinematic conditions as well as on experimental capabilities (e.g., luminosities, beam polarizations, solid angles, etc.). Since the achievable statistical precision also depends on all of these factors, issues of theoretical interpretability are correlated with considerations of experimental do-ability. In analyzing this do-ability/interpretability correlation for specific cases, we $\operatorname{tr} y$ to make reasonable assumptions for experimental conditions, based on what one expects to be achievable in the next decade at various accelerators. Hence, our projections of constraints on quantities of interest from different prospective experiments are somewhat time-dependent and should be taken more as benchmark indicators than as definitive statements. 
The remainder of this article is organized as follows. In Sect. II we review the central physics issues. including a brief summary of the status of Standard Model tests, a discussion of the hadronic form factors and response functions which might be probed with neutral current scattering, and a brief overview of the theoretical hadronic uncertainties which enter the interpretation of these experiments. In Sect. III we introduce our formalism and. in Sect. IV. give a case-by-case theoretical analysis of various classes of neutral current scattering measurements. Section $V$ contains a discussion of a variety of experimental considerations. Section VI summarizes our conclusions. particularly in regard to a prospective program of future experiments and the attendant theoretical and experimental challenges. More detailed treatments of the subjects reviewed in this paper may be found elsewhere [Mus92a. Don92]. 


\section{PHYSICS ISSUES}

The observables measured in low- and intermediate-energy neutral current processes depend on two classes of physics: (a) the structure of the underlying electroweak gauge theory and (b) hadronic matrix elements of the quark currents. For high-energy neutral current observables hadronic contributions are in general reliably calculable. whereas at the energy scales of interest here. they are more difficult to calculate but are more selectively obtained from experiment. Thus, if one considers the underlying gauge theory to be well-tested from experiments in other sectors. intermediate-energy semileptonic neutral current scattering provides an opportunity to study a variety of interesting hadronic physics issues not accessible at other energy scales. We begin this section by discussing. the strangeness content of the nucleon and then continue in Sect. II.B with a summar: of some additional aspects involved in probing hadronic structure using neutral current scattering: both subjects will be treated in more detail in the rest of the article. Following these discussions we turn in Sect. II.C to the issue of undertaking Standard Model tests using semileptonic processes.

\section{II.A. Strangeness Content of the Nucleon}

The simplest "valence-quark" picture of hadrons depicts the nucleon as consisting solely of $u$-and $d$-quarks. Many low-energy properties of the nucleon can be understood in this picture without explicitly accounting for the presence of heavier quarks in the "sea". However, there exists evidence that not all low-energy nucleon properties can be understood within this framework. In particular, analyses of $\Sigma_{\pi, v}$, the so called "sigma term" extracted from $\pi-. N$ scattering, suggests a non-negligible value for proton matrix elements of the $\bar{s} s$ operator. Defining the ratio $R$, as

$$
R_{s} \equiv \frac{\langle p|\bar{s} s| p\rangle}{\langle p|\bar{u} u+\bar{d} d+\bar{s} s| p\rangle}
$$

one has from these analyses that $R, \approx 0.1-0.2$ [Che76, Che71a. Don86a. Gas91] -- see Table 2.1. Neglect of $\bar{s} s$ pairs in the sea would, in this framework, imply a value for the nucleon mass of $\approx 600 \mathrm{MeV}$. Although the dynamical origin of this surprising result is not definitively understood, Donoghue and Nappi [Don86a] have shown that a large value of $R$, is plausible within the SU(3) Skyrme model and MIT bag model. The more recent calculation of Ref. [Mus93a] suggests that strange hadronic components of the nucleon wave function (e.g., a $K \Lambda$ intermediate state) may account for a substantial portion of the extracted value of $R_{\boldsymbol{s}}$.

These results suggest that nucleon matrix elements of other strange-quark operators could differ non-negligibly from zero. Of particular interest are s-quark vector and axialvector currents, parameterized in terms of strangeness form factors $G_{E}^{(\mathbf{s})}\left(Q^{2}\right), G_{M}^{(\mathbf{s})}\left(Q^{2}\right)$, and $G_{A}^{(s)}\left(Q^{2}\right)$ (see Eqs. (3.31), (3.41), and (3.47)). Of these three form factors, experimental constraints have been reported only for $G_{A}^{(s)}$. Its value at $Q^{2}=0$ has been derived from deep inelastic $\vec{\mu} \vec{p}$ scattering [Ash89] and from the BNL neutrino-proton scattering 


\begin{tabular}{|c|c|c|}
\hline \multicolumn{3}{|c|}{ TABLE 2.1 } \\
\hline Source & $R_{s}$ & Reference \\
\hline$\Sigma_{\approx . v}(\mathrm{~A})$ & 0.09 & {$[$ Gas91] } \\
$\Sigma_{\times, \mathrm{N}}(\mathrm{B})$ & $0.21 \pm 0.03$ & {$[$ Don86a] } \\
Skyrme & 0.23 & {$[$ Don86a] } \\
MIT Bag & $0 \rightarrow 0.29$ & {$[$ Don86a] } \\
Kaon Loops & $-0.007 \rightarrow 0.47$ & {$[$ Mus93a] } \\
\hline
\end{tabular}

Table 2.1. Strange quark scalar density of the nucleon, $R_{g}$, as defined in Eq. 2.1 . First two rows give extractions from $\pi N$ scattering data; the remaining rows give predictions from various theoretical models.

determination of the isoscalar axial-vector form factor, $\tilde{G}_{A}^{r=0}$ [Ahr87] (see Eq. (3.32b)). Results taken from these two determinations appear in Table 2.2, along with a variety of theoretical predictions. The value extracted from the EMC data assumes an $\mathrm{SC}^{-}(3)$ parameterization of the baryon octet matrix elements of the axial-vector current. Breaking of $\mathrm{SC}(3)$ symmetry could be large [Jen91, Par91], resulting in a reduction of the E.MC value for $G_{A}^{(s)}(0)$ by a factor of three or more. The BNL measurements were performed at nonzero $Q^{2}$, and the $Q^{2}$-dependence of $\tilde{G}^{T=0}$ was fit to a dipole form. The experimenters report a non-negligible correlation between $\tilde{G}_{A}^{T=0}(0)$ and the dipole mass parameter, $M_{A}^{T=0}$. The results in Table 2.2 were derived assuming that $M_{A}^{T=0}$ is the same as the mass parameter appearing in the isovector axial-vector form factor, and does not take into account the strong.$M_{A}^{T=0}$ correlation. Additional uncertainties in the BNL resuits arise from nuclear physics sources. Since $80 \%$ of the events in the experiment were generated by quasielastic (QE) scattering from protons in ${ }^{12} \mathrm{C}$ nuclei, knowledge of the ${ }^{12} \mathrm{C} Q E$ response is necessary in the interpretation of the measured cross section (see Sect. IV.J). Evidently, a more precise determination of $G_{A}^{(s)}\left(Q^{2}\right)$ is warranted.

From a theoretical point of view, elastic $\nu \mathrm{N}$ scattering offers some advantage over parity violating $\vec{e} N$ in terms of measuring $G_{A}^{(s)}$. The latter always introduces a small coefficient, $g_{v}^{e}=-\left(1-4 \sin ^{2} \theta_{w}\right)$ (see Eq. (3.2a) and Table 3.1) multiplying the nucleon axial-vector form factor, thus generically reducing the sensitivity to this term by an order of magnitude. Perhaps more significantly for theoretical interpretation, there exist large and theoretically uncertain radiative corrections arising in the $V(e) \times A(\mathrm{~N})$ amplitude which do not enter the amplitude for neutrino probes of the nucleon axial-vector current [Mus90]. The LSND experiment planned for LAMPF, intended primarily as a search for $\nu_{\mu} \rightarrow \nu_{e}$ and $\bar{\nu}_{\mu} \rightarrow \bar{\nu}_{e}$ oscillations, will also detect a significant number of recoil protons 


\begin{tabular}{|c|c|c|}
\hline \multicolumn{3}{|c|}{ TABLE 2.2} \\
\hline Source & $\tilde{G}_{A}^{r=0}(0)$ & Reference \\
\hline E.MC & $-0.194 \pm 0.055$ & [Ash89] \\
\hline BNL & $-0.15 \pm 0.09$ & [Ahr87] \\
\hline$E T+Q M$ & -0.075 & [Col78, Woli9] \\
\hline $\mathrm{ET}+\mathrm{OZI}$ & -0.046 & [Colis, Woli9] \\
\hline Kaon Loops (a) & $-0.03 i \rightarrow-0.041$ & [.Mus93a] \\
\hline Kaon Loops (b) & -0.004 & [Koe92] \\
\hline Kaon Loops (c) & \pm 0.20 & [Hol90] \\
\hline SL(3) Skyrme (B) & -0.10 & [Par91] \\
\hline
\end{tabular}

Table 2.2. Experimental results and theoretical predictions for low- $\left|Q^{2}\right|$ behavior of the isoscalar axial-vector form factor. At tree-level in the Standard Model, one has $\tilde{G}_{A}^{T=0}=G_{A}^{(s)}$. First two rows give experimental values from E.MC and BNL measurements. Statistical and systematic errors in the EMC value have been combined in quadrature. Third and fourth lines give predictions using effective theory (ET) approach of Ref. [Col78], together with quark model (QM) and SU(3)/Zweig Rule (OZI) estimates of nucleon matrix elements given in Ref. [Wol79]. Following three rows give kaon-strange baryon loop predictions under different assumptions about the meson-baryon form factor. The prediction of Ref. [Hol90] gives magnitude only. Final row gives broken (B) SU(3) Skyrme model prediction.

from elastic $\nu p$ scattering [Lou89]. The effective $\left|Q^{2}\right|$ for these scatterings is lower by approximately an order of magnitude from the BNL experiment, so that the LSND results could permit an extraction of $G_{A}^{(0)}(0)$ with significantly lower sensitivity to $M_{A}^{T=0}$. The impact of nuclear physics uncertainties in this determination remains to be analyzed. An additional source of uncertainty arises from lack of knowledge of the neutrino flux [Ahr87]. A measurement of the ratio

$$
R_{\nu}^{p n}=\frac{d \sigma\left(\nu, \nu^{\prime} p\right) / d Q^{2}}{d \sigma\left(\nu, \nu^{\prime} n\right) / d Q^{2}}
$$

could be quite sensitive to $G_{A}^{(s)}(0)$ while being independent of uncertainty in the neutrino flux [Gar92].

To date, no experimental constraints have been published for $G_{E}^{(s)}$ and $G_{M}^{(s)}$. For this 
reason. one must turn to theoretical predictions to obtain some suggestion for the scale of these form factors. While such predictions are model-dependent. there exists one rigorous theoretical constraint on $G_{E}^{(s)}$. Since the nucleon has no net strangeness, $G_{E}^{(s)}$ must vanish at $Q^{2}=0$. Consequently, it is convenient to characterize the low $-\left|Q^{2}\right|$ behavior of this form factor by a dimensionless mean square "strangeness radius". $\rho$, (see Eqs. $3.40 \mathrm{a}$ ) and $(3.41 \mathrm{a})$. The strange magnetic form factor is not constrained by any symmetry. and one characterizes its low- $\left|Q^{2}\right|$ scale (see Eqs. (3.40a) and (3.41b)) by a strange magnetic moment. $\mu_{s} \equiv G_{M}^{\left(s^{\prime}\right.}(0)$. Theoretical predictions for $\rho_{s}$ and $\mu_{s}$ are given in Table 2.3: the non-leading $Q^{2}$-dependence of $G_{E}^{(s)}$ and $G_{M}^{(s)}$ is discussed more fully in Sect. III and Ref. [Jaf89].

\begin{tabular}{|c|c|c|c|}
\hline \multicolumn{3}{|c|}{ TABLE 2.3 } \\
\hline Source & $\rho_{s}$ & $\mu_{s}$ & Reference \\
\hline Poles & $-2.12 \pm 1.0$ & $-0.31 \pm 0.009$ & {$[$ [Jaf89] } \\
Kaon Loops (a) & $0.41 \rightarrow 0.49$ & $-0.31 \rightarrow-0.40$ & {$[$ Mus93a] } \\
Kaon Loops (b) & 0.173 & -0.026 & [Koe92] \\
Kaon Loops (c) & - & \pm 0.8 & {$[$ Hol90] } \\
SL(3) Skyrme (B) & 1.65 & -0.13 & {$[$ Par91] } \\
SL(3) Skyrme (S) & 3.21 & -0.33 & {$[$ Par91] } \\
\hline
\end{tabular}

Table 2.3. Theoretical predictions for low- $\left|Q^{2}\right|$ behavior of $s$-quark vector current form factors. First row gives average of all fits in Ref. [Jaf89]. Following three rows give predictions of various loop calculations (see also Table 2.2). The estimate of Ref. [Hol90] predicts magnitude only and not sign. Final two rows are taken from Ref. [Par91] for broken (B) and symmetric (S) SU(3) Skyrme models. To set the scale, note that the dimensionless mean-square EM charge radius of the neutron is $\rho_{n} \approx 1.91$ (see Eqs. (3.35b) and (3.40a)), while the isoscalar nucleon magnetic moment is $\mu^{T=0}=0.44$.

In principle, one might hope to extract information on the strange vector current form factors by re-analyzing existing data from the BNL experiment as well as the elastic PV ${ }^{12} \mathrm{C}(\vec{e}, e)$ measurement at MIT/Bates [Sou90a] and the Mainz ${ }^{9} \mathrm{Be}\left(\vec{\epsilon}, e^{\prime}\right) \mathrm{QE}$ experiment [Hei89]. However, for reasons particular to each of these experiments, the form factor constraints extracted would be unreliable. While nonzero values of $G_{E}^{(s)}$ and $G_{M}^{(s)}$ would contribute a significant percentage to the total $\sigma_{\nu N}$ and $\sigma_{\nu N}$ in a BNL-type experiment [Bei91a], extraction of limits on the vector current strangeness form factors from the $B N L$ 
experiment would require detailed knowledge of the $\nu$ energy spectra. normalizations. correlations and other systematics. While such an analysis lies beyond the scope of the present review, one may arrive at an idea of the level of constraints possible by modeling a BNL-type experiment. Assuming measured cross sections at the BNL data points are given by their Standard Model predictions with no strangeness, and assuming only $10 \%$ statistical uncertainty in the data, one would obtain a simultaneous extraction of the vector strangeness form factors with correlated uncertainties on the order of $\delta \mu_{s} \approx \pm 0.3 . \delta \rho_{s} \approx$ \pm 5.0 ( $67 \%$ confidence). More detailed discussions of these constraints from a hypothetical B.IL-type experiment appear in Sect. IV.J.

In the case of the elastic scattering ${ }^{12} \mathrm{C}(\vec{e}, e)$ experiment, only $G_{E}^{(s)}$ contributes to $A_{L R}$ since the target is spin-0. The uncertainty in the experimental value of $\left.A_{L R}\right|^{12} C^{\prime}$, is significantly larger, at the small $\left|Q^{2}\right|$ of this experiment. than the size of the $G_{E}^{\prime \prime \prime}$ contribution one nould expect from the pole-model prediction [Jaf89] (see Table 2.31. Assuming the low- $\left|Q^{2}\right|$ behavior of $G_{E}^{(9)}$ were given by the pole-model value. neglect of this form factor would introduce roughly a three-percent theoretical error in $A_{L R}$, significantly lower than $\approx 25 \%$ experimental error. Backward-angle quasielastic electron scattering experiments of the . Mainz variety are quite insensitive to isoscalar form factors. as discussed in Sect. IV.F. Again, the scale of model predictions for the strangeness contribution to $A_{L R}\left({ }^{9} \mathrm{Be}, \mathrm{QE}\right)$ is significantly smaller than the $20 \%$ uncertainty in the measured asymmetry [Bei91a. Hei89].

In the absence of reliable experimental bounds on $G_{E}^{(s)}$ and $G_{M}^{(s)}$, the theoretical predictions of Table 2.3 take on added interest. These calculations are highly model-dependent and should be viewed more as indications of the possible overall scale and sign of the form factors than as firm and rigorous predictions. Nevertheless, the approaches followed are instructive as to the different types of physics which could give rise to strangeness contributions of varying magnitudes. In general, two pictures have been used to represent the presence of non-valence $s \bar{s}$ pairs in the nucleon: (a) a perturbative, or "high energy". picture making explicit use of quarks (Fig. 2.1), and (b) a phenomenological. or "low energy", representation employing hadronic degrees of freedom (Fig. 2.2). In the former approach, one assumes the dominant effect of heavy $q \bar{q}$ pairs is to "dress", or renormalize, operators involving the light valence quarks via gluon exchange. By integrating out the heavy quarks, one derives an effective theory involving only the lighter degrees of freedom appropriate for physics at momentum scales below the heavy-quark masses. In the case of the nucleon. for which typical internal momenta are on the order of $\Lambda_{Q C D} \sim$ few hundred $\mathrm{MeV} / c$, it is sensible to integrate out the $c, b$, and $t$ quarks, leaving the $u, d$, and $s$ quarks as effective degrees of freedom. Following this procedure, Kaplan and Manohar [Kap88] find that the heavy-quark renormalization of the light-quark vector currents is small enough to be ignored in the analysis of the $\mathrm{NC}$ observables of interest here. The induced isoscalar axial-vector current, however, is non-negligible, especially since this current vanishes at tree-level in the Standard Model. Prior to the work of Ref. [Kap88]. Collins et al. [Col78] carried the integration down through the s-quarks, and derived a somewhat larger effective isoscalar axial-vector current. While integrating out the $s$-quarks is questionable in this case, given that $m_{s} \sim \Lambda_{Q C D}$, the estimate of Ref. [Col78] gives some indication of the 
magnitude of $s$-quark effects in the axial-vector current. Nucleon matrix elements of the isoscalar axial-vector current have been estimated in two ways by Wolfenstein [Woli9]. Combining these results with the estimates of Ref. [Col78] for the coefficient of this operator leads to the two entries in Table 2.2. One observes that these estimates agree in sign but are smaller in magnitude than the E.MC and BNL results.

The second approach to modeling strangeness focuses on the low-momentum $\left(p \lesssim \Lambda_{Q C D}\right)$ part of the virtual $s \bar{s}$ loop. At these momentum scales, the heavy-quark pair interacts with the valence quarks for a sufficiently long time to permit the formation of virtual strange hadronic intermediate states. To estimate contributions of this type. one must rely on phenomenological hadronic models, since a first-principles approach involving quark degrees of freedom directly is at present intractable. The pole-model approach followed in Ref. [Jaf89] represents one estimate of this type. In this analysis, $\mu_{s}$ and $\rho_{s}$ were obtained from a three-pole $(0 . \omega$, and one higher-mass vector meson) fit to the isoscalar nucleon E.M form factors under ad hoc assumptions about the asymptotic (large- $Q^{2}$; behavior of the strangeness form factors. Alternatively, several authors have performed loop estimates [Hol90. Koe92, Mus93a], in which the virtual $s \bar{s}$ pair appear as a kaonstrange baryon intermediate state. As indicated in Table 2.3, the loop contributions to the strangeness radius are typically much smaller in magnitude than the pole contribution. whereas, depending on the cut-off procedure employed, the loop and pole contributions to $\mu_{\mathrm{s}}$ may be comparable. The extent to which these two types of contributions are either independent and ought to be added or represent the same physics is not entirely clear. Thus, one ought to take them as indicative of the magnitude and sign of $s$-quark contributions rather than as definitive predictions. One also ought to note that the calculations of Refs. [Hol90, Koe92] did not satisfy gauge invariance at the level of the Ward-Takahashi identity. A phenomenological approach in a somewhat different spirit is represented by the Skyrme model predictions, which yield values of $\rho_{\mathbf{s}}$ of roughly the same magnitude as the pole estimate. but having the opposite sign.

While it is of interest to test these model predictions experimentally, the presence of $s$-quark form factors also impacts on the feasibility of future semileptonic scattering studies as electroweak tests. For example, an uncertainty in $G_{E}^{(\mathbf{)})}$ on the order of the prediction of Ref. [Jaf89] would seriously hamper one's ability to constrain extensions of the Standard Model with PV electron scattering. Consequently, it is important not only to constrain the strangeness form factors at a level needed to distinguish among model predictions, but ultimately also to reduce the uncertainty in these constraints to a level below what is tolerable in electroweak tests. With these objectives in mind, a number of new experiments have been proposed or discussed which could provide constraints on $G_{E}^{(s)}, G_{M}^{(s)}$, and $G_{A}^{(s)}$. The so-called "SAMPLE" experiment underway at MIT/Bates [McK89] seeks to extract information on $\mu_{\mathrm{s}}$ by measuring the backward-angle asymmetry in $\overrightarrow{e p}$ scattering at an incident energy of $\epsilon \sim 200 \mathrm{MeV}$. It is expected that this measurement will constrain $\mu_{\text {s }}$ with an uncertainty of $\delta \mu_{s}= \pm 0.22$ [Bei92]. The " $G$ " experiment, conditionally approved for CEBAF, seeks to determine the $Q^{2}$-dependence of $G_{M}^{(s)}$ over the range $0.1 \leq\left|Q^{2}\right| \leq 0.5$ $(\mathrm{GeV} / c)^{2}$, with an uncertainty having the same scale as the expected SAMPLE error in $\mu_{\text {s }}$ at low- $\left|Q^{2}\right|$ and decreasing for larger $\left|Q^{2}\right|$ [Bec91]. These measurements could rule out several of the predictions listed in Table 2.3. A fundamental limitation on further tightening 
of these constraints using S.AMPLE-type experiments is imposed by hadronic uncertainties in the radiative corrections entering the axial-vector contribution to the backward-angle asymmetry. The extent to which these uncertainties would limit one's ability to determine $\mu$, from PV $\overrightarrow{e p}$ scattering is discussed in detail in Sect. IV.A. The uncertainty in the isovector component of these radiative corrections could be experimentally reduced by a measurement of the backward-angle QE PV asymmetry. Alternatively, a measurement of the backward-angle elastic $\vec{e} D$ asymmetry, which is significantly less sensitive to axialvector uncertainties than is $A_{L R}(\vec{e} p)$, might permit an improvement over the S.A.MPLE

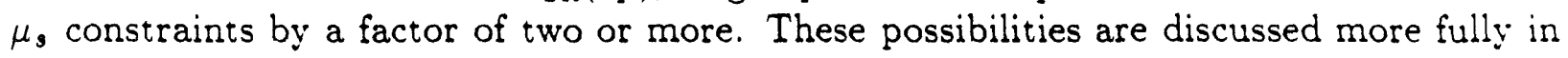
Sect. IV.

Proposals to determine $G_{E}^{(s)}$ with forward-angle PV elastic $\overrightarrow{e p}$ scattering and PY electron scattering from ${ }^{4} \mathrm{He}$ have been made at CEBAF [Fin91, Bec91. Bei91b) and have been conditionally approved. A low- $\left|Q^{2}\right|$ forward-angle measurement of $A_{L R}(\vec{\epsilon} p)$ with $10 \%$ experimental error could ultimately determine the strangeness radius to a precision of $\delta \rho_{s}= \pm 2$. A large fraction of the uncertainty actually arises from a lack of knowledge of $\mu_{\mathrm{g}}$. which also contributes to the forward-angle asymmetry. An improvement in the SA.MPLE $\mu_{s}$ bounds would correspondingly allow a tighter determination of $\rho_{s}$.

Alternatively, a determination of $G_{E}^{(s)}$ with elastic scattering from ${ }^{4} \mathrm{He}$ would be independent of uncertainties in $\mu_{\mathrm{s}}$. To the extent that $G_{E}^{(s)}$ can be extracted from the ${ }^{4} \mathrm{He}$ asymmetry in a nuclear-physics-independent manner, a series of low- and moderate- $\left|Q^{2}\right|$ measurements of $A_{L R}\left({ }^{4} \mathrm{He}\right)$ could produce constraints on $G_{E}^{(s)}$ significantly more stringent than would be attainable from a series of measurements on the proton alone (see Fig. 4.7 ). To achieve such a result, experimental uncertainties at roughly the $1 \%$ (low $\left.-\left|Q^{2}\right|\right)$ and $10 \%$ (moderate- $\left|Q^{2}\right|$ ) levels would be necessary. From the standpcint of theoretical interpretability, scattering from a $\left(J^{\pi} T\right)=\left(0^{+} 0\right)$ nucleus such as ${ }^{4} \mathrm{He}$ is an attractive case. At the simplest level. one has

$$
A_{L R}\left(0^{+} 0\right) \propto \frac{\left\langle\text { g.s. }\left\|\rho^{N C}(T=0)\right\| g . s .\right\rangle}{\left\langle\text { g.s. }\left\|\rho^{E M}(T=0)\right\| \text { g.s. }\right\rangle}
$$

where $\rho^{N C(E M)}(T=0)$ is the isoscalar weak neutral current (electromagnetic) charge operator and $\langle$ g.s. \|\| g.s. $\rangle$ is a reduced ground state matrix element (see Eqs. (4.12) and (4.13)). To the extent that transition meson-exchange currents $(e . g ., \pi-\rho)$ can be neglected, one expects the nuclear many-body contributions to these matrix elements to cancel from the ratio, leaving only a dependence on the electroweak gauge theory parameters and single nucleon charge form factors.

Corrections to this naive result arise from a number of effects. The ${ }^{4} \mathrm{He}$ ground state is not an eigenstate of strong isospin, and mixing of higher-lying $T=1$ states into the nominally $T=0$ ground state introduces a nuclear-structure-dependent correction into the asymmetry [Don89]. For sufficiently small momentum transfer, one expects this correction, and the associated theoretical uncertainty, to fall below a problematic level for a determination of $G_{E}^{(s)}$. Indeed, at low momentum transfer both the isospin-mixing correction and the term containing $G_{E}^{(s)}$ are proportional to $Q^{2}$. From the estimates of the former 
in Ref. [Don89] it appears for favorable cases such as ${ }^{4} \mathrm{He}$ and ${ }^{12} \mathrm{C}$ that the isospin-mixing correction implies an uncertainty in $\rho_{s}$ at low $-\left|Q^{2}\right|$ of approximately $\left|\Delta \rho_{g}\right| \sim 0.02$. which is conciderably smaller than the level of precision at which $G_{E}^{(s)}$ is likely to be determined in the foreseeable future. At moderate- $\left|Q^{2}\right|$ it is more difficult to be certain of the scale of the isospin-mixing corrections, although the modeling performed in Ref. [Don89] suggests that they continue to remain small compared to the uncertainties in $\rho$, that arise from other sources. such as those mentioned below, at least for the favorable cases of helium and carbon. In contrast, as discussed in Ref. [Don89], heavier nuclei (in the $s-d$ shell and beyond) are expected to have important isospin-mixing corrections and consequently are likely to prove unsuitable for $G_{E}^{(s)}$ determinations. We shall return to discuss this point in more detail in Sect. IV.B.

A potentially more serious issue in the interpretation of this asymmetry is the contribution from multi-boson-exchange "dispersion" corrections (see Fig. 3.i). Data for elastic parity-conserving $(P C)$ scattering from another spin-0, isospin-0 nucleus $-{ }^{12} \mathrm{C}-$ suggests that these corrections are significantly larger than one expects based on analyses of dispersion corrections in ep scattering [Kal89], and that at low- $\left|Q^{2}\right|$ they could enter the denominator of $A_{L R}\left(0^{+} 0\right)$ in Eq. (2.3) at a potentially problematic level. Furthermore. theoretical calculations [Fri74] of the ${ }^{12} \mathrm{C} \mathrm{PC}$ dispersion corrections are thus far in rather poor agreement with the data. It is unlikely that the PV dispersion corrections to the neutral current amplitude entering the numerator of the asymmetry will be measured directly, so that a nuclear-model-dependent estimate of these corrections will be needed. In short, a better understanding of dispersion corrections constitutes an interesting challenge for nuclear theory posed by the interpretation of the ${ }^{4} \mathrm{He}$ asymmetry.

Going beyond elastic semileptonic scattering, there exist several other possibilities for probing nucleon strangeness matrix elements with lepton probes: PV QE electron scattering, inelastic neutrino scattering and $\mathrm{PV}$ in heavy muonic atoms. In the case of $\mathrm{QE}$ electron scattering, as discussed in Sect. IV.F, contributions to the asymmetry from $G_{M}^{(s)}$ and $G_{A}^{(s)}$ are suppressed, since they enter with a multiplicative factor for the isoscalar magnetic moment. In the case of the only QE PV experiment thus far completed, a backward-angle measurement $A_{L R}\left({ }^{9} \mathrm{Be}\right)$, the largest strangeness form factor sensitivity is to $G_{E}^{(9)}$. Lnder the prediction of Ref. [Jaf89], the latter would generate a $3 \%$ contribution to the asymmetry [Bei91a] - a value significantly smaller than the $20 \%$ experimental error. A future backward-angle measurement of $A_{L_{R}}(\mathrm{QE})$ is more suited to a ditermination of $\dot{G}_{A}^{T=1}$, which enters multiplied by the isovector magnetic moment. A determination of this term, with its large and theoretically uncertain radiative correction, could reduce the associated uncertainty in a backward-angle $\vec{e} p$ determination of $G_{M}^{(s)}$. The forward-angle QE asymmetry is dominated by the transverse vector current response, since the longitudinal $P V$ response is fortuitously suppressed (see Eq. (4.61) and following). However, this asymmetry is potentially quite sensitive to $G_{E}^{(s)}$, and a series of $1 \%$ measurements of $A_{L R}(\mathrm{QE})$ at low-and moderate-momentum transfer could produce constraints on $G_{E}^{(s)}$ nearly equivalent to those ultimately attainable from a series of elastic $A_{L R}\left({ }^{4} \mathrm{He}\right)$ measurements (see Fig. 4.7). As in the case of elastic scattering, the interpretation of a forward-angle $A_{L R}(\mathrm{QE})$ measurement would require the resolution of a number of theoretical issues, including contributions 
from final-state interactions, meson-exchange currents, and nuclear correlations. In fact. the forward-angle asymmetry appears to be quite sensitive to isospin-dependent nuclear correlations at momentum transfers roughly below one $\mathrm{GeV} / c$ [Don92. Had92. Alb93a].

In principle. inelastic excitation of discrete states by PV electron scattering could be used to study specific aspects of the weak neutral current. This general approach of using nuclear transitions with carefully chosen spins and parities to "filter out" pieces of the electroweak currents was developed in the 1970's (see, for example, the review of the ideas involved in Ref. (Don79a]). For neutrino scattering we shall see that this way of proceeding is a fruitful one when we return to that subject in Sect. IV.J. Cnfortunately, as discussed in Sect. IV.D. very few practical cases of discrete-state inelastic scattering emerge in the case of PV electron scattering due to the poor figures-of-merit for such reactions which stem from the small cross sections that occur (i.e., much smaller that the coherent elastic scattering cross sections - see Figs. 3.10-3.12).

In contrast to semileptonic scattering measurements, atomic PV experiments are generally much less sensitive to nucleon form factors due to the very small effective moment um transfer associated with the interaction of an atomic electron with the nucleus. In particular. a PV ${ }^{4} \mathrm{He}(\vec{e}, e)$ would have to be carried out at $q \approx 30 \mathrm{MeV} / c$ to be as insensitive to $\rho_{s}$ as is a measurement of the weak charge in ${ }^{133} \mathrm{Cs}$ atomic PV. For this reason. atomic PV is apparently more suitable as a low-energy testing ground of the Standard Model than is semileptonic scattering, up to uncertainties associated with atomic and nuclear structure. However, in the case of muonic atoms, the muon is much more tightly bound (for a given set of orbital quantum numbers) than a corresponding atomic electron, making it sensitive to physics at hadronic length scales. In fact, a $1 \%$ measurement of the weak charge in a heavy muonic atom could constrain $\rho_{s}$ as ightly as would a forward-angle measurement of $A_{L R}(\vec{e} p)$. if the nuclear neutron distribution were also known to sufficiently high precision. 


\section{II.B. Additional Aspects of PV Electron Scattering Studies}

\section{of Hadronic Structure}

We now touch upon several additional issues where PV electron scattering may provide information on hadronic structure with the focus on issues other than the specific study of strangeness in the nucleon. Similar discussions occur for lepton scattering in general and neutrino scattering in particular (see Ref. [Don79a]) - in the present section we restrict our attention in this regard to $\mathrm{PV}$ electron scattering.

Nuclear Parity Violation

Achieving a full understanding of the strong $. \mathrm{N}-\mathrm{N}$ interaction (as well as three- and higher-body forces) has been a long-standing problem in nuclear physics. Of more recent interest have been attempts to study the weak interaction between nucleons. Since the PC part of this interaction has strength of $\mathrm{O}\left(G_{\mu} m_{v}^{2}\right) \sim 10^{-3}$ and is masked by the much stronger electromagnetic $\left[\mathrm{O}(\alpha) \sim 10^{-2}\right]$ and strong $\left[\mathrm{O}\left(\alpha_{s}\right) \sim 1\right]$ interactions. one must isolate the weak $. N-. N$ interaction by measuring $\mathrm{PV}$ observables, which provide a window on the PV part of the weak $. V-. N$ force. Direct $W^{ \pm}$- and $Z^{0}$-exchange between nucleons has a range of $\sim 0.002 \mathrm{fm}$ and is suppressed by the short-range repulsion between the nucleons. Consequently, one conventionally models a longer-range PV $\times-$. interaction as being mediated by the exchange of light pseudoscalar and vector mesons (Fig. 3.9). where the virtual meson is emitted from one nucleon through a PC strong interaction and absorbed by a second nucleon via a PV weak interaction. The resultant two-body....- . PV potential can be parameterized in terms of seven PV meson-nucleon couplings. $h_{\text {, . . . . }}$. corresponding to different exchanged mesons $(M)$ and isospin channels (see Eq. $(3.87)$ ). ${ }^{\dagger}$

Theoretical predictions, based on quark-model calculations, give a rather wide range of values for the $h_{N N M}$ [Des80]. Assuming that these couplings can be extracted from nuclear PV experiments with manageable nuclear physics uncertainties, such experiments test particle physics methods for estimating low-energy hadronic matrix elements of fourquark operators. At the nuclear level, one may treat the $h_{N N M}$ as experimentally determined parameters, and to the extent that a variety of nuclear PV measurements may be fit with the same values for these parameters, one has confirmation of this conventional picture of nuclear PV. In fact, nuclear PV experiments completed to date have placed only rather modest constraints on the $h_{N N M}$, all of which are consistent with the theoretical "reasonable ranges" of Ref. [Des80] (for a summary of these experiments and associated theoretical issues, see, e.g., Ref. [Ade85]). However, further tightening of these constraints is necessary if estimates of the four-quark hadronic matrix elements are to be tested in this way. At the nuclear level, PV experiments have produced a self-consistent set of values for the $h_{N N M}$, with one glaring exception: the value of $h_{* N N}$ extracted from the PV $\gamma$-decay of ${ }^{18} \mathrm{~F}$ is about a factor of three smaller than suggested by all other experiments

'It is conventional in the literature to denote the PV pion-nucleon coupling by $f_{\pi}$; this unfortunate choice of notation is not to be confused with the pion-decay constant, also denoted by $f_{\pi}$. 
(see Fig. 2.3). Proposed resolutions for this discrepancy include the need to account for nucleon strangeness in estimating the $h_{x, v, M}$ [Dai91] and possible contributions generated by a PV $. V . V \pi$ vertex [Kap92]. Clearly. additional experimental information would be desirable as one attempts to solve this puzzle.

Several authors have suggested the possibility of using PV electron scattering as a new probe of nuclear PV Hen 73 . Hen 79 , Ser 79. Hen82]. The contribution of nuclear PV to $A_{L R}$ would be generated by the processes illustrated in Fig. 3.9. For spin $\geq 1 / 2$ targets. the resultant, -nucleus interaction will effectively include an axial-vector component due to mixing of opposite parity nuclear levels by the PV . V-.. force (Fig. 3.9a.b) and PV" mesonexchange currents (Fig. 3.9c). For elastic scattering, this vertex induces the so-called "anapole moment" (A.1I) [Zel57, Zel60] which. unlike other electromagnetic moments. couples only to virtual photons (for a review of its properties, see Ref. Mus91. It t low- $Q^{2}$ ! the corresponding amplitude has the same form as low- $Q^{2} ! Z^{0}$-exchange with magnitude growing as $A^{2 / 3}$. Thus, for heavy nuclei the $A . I$ contribution to $A_{L R}$ can be as large as the leading axial-vector neutral current term [Fla84. Hax89. Bou91\%. In such cases, one could reasonably expect to separate the contribution of nuclear PV to $A_{L R}$ from that of the $\mathrm{NC}$. thereby providing a new means for constraining models of nuclear PY. Alternatively, Flambaum. Khriplovich. and others have observed that measurements of nuclear spin-dependent observables in atomic PV experiments would also be sensitive to the nuclear A.M [Fla80]. In fact, evidence for a large A.M term. consistent with theoretical predictions, was reported from a recent atomic PV experiment on ${ }^{133} \mathrm{Cs}$ performed by the Boulder group [Noe88].

Theoretical evaluation of the PV contribution to elastic scattering from ${ }^{13} \mathrm{C}$ has been completed by Serot [Ser 79]. who found that the effect of nuclear PV should be comparable to the $\mathrm{NC}$ contribution at low electron energies. In the same work. it was found that nuclear PV should dominate the forward-angle asymmetry for exc:tation of the $\left(J^{\pi} T\right)=11^{-1}$, level in ${ }^{12} \mathrm{C}$. Hwang, Henley, and Miller [Hwa81] considered PV electrodisintegration of the deuteron, and found comparable contributions from nuclear $\mathrm{PV}$ and from the $\mathrm{NC}$ at low incident energies. These studies are suggestive. and further analysis of PV electron scattering as a probe of nuclear PV - particularly for heavy targets - is warranted.

Determination of $G_{\varepsilon}^{n}$ and the Grouna-State Neutron Distribution

Present information on the spatial distribution of charge in the neutron and of neutrons in nuclei is much more limited than the corresponding information regarding protons. In the case of the neutron charge distribution - embodied in momentum-space form $G_{E}^{n}\left(Q^{2}\right)$ - only the mean-square radius is known to high precision [Sim80, Dum83]. A way from $Q^{2}=0$, the uncertainty in $G_{E}^{n}\left(Q^{2}\right)$ can be as much as $50 \%$. The situation is even more uncertain for $\rho_{n}(r)$, the ground-state neutron distribution in nuclei. From a theoretical perspective, the most interpretable data on the ground-state rms neutron radius. $R_{n}$, comes from moderate-energy elastic $p$-nucleus scattering. Different analyses of the data on ${ }^{208} \mathrm{~Pb}$ allow a difference between $R_{n}$ and the proton rms radius, $R_{p}$, of $\sim 0.3 \mathrm{fm}$ (see the discussion in Ref. (Don89]) and it is not certain which of $R_{n}$ and $R_{p}$ is la:ger. Knowledge of $R_{n}$ has particular importance in the interpretation of atomic PV 
experiments, where uncertainty in $R_{n}$ could limit the future use of such measurement: as Standard Model tests [For90. Pol92a]. In addition, reactions involving the scattering of hadrons other than single protons are used in extracting information on the nuclear density distributions and specifically on the neutron distribution in the nuclear ground state. Examples where nuclei far from the valley of stability can be explored include pion double charge exchange reactions and the scattering of radioactive beams. Having a few "benchmark" values of $R_{n}$ for selected nuclei might prove valuable to help calibrate those hadronic analyses.

Both the issue of the role played by the charge distribution of the neutron and that of determining the rms radius of the ground-state neutron distribution have been addressed in past work [Don88. Don89] and will be discussed further in Sect. IV.B. In summary, it was shown that it would be possible to obtain more precise information on $G_{E}^{n}\left(Q^{2}\right)$ and $\rho_{n} i r$, than presently exists by using a combination of purely electromagnetic and PV electron scattering. This possibility relies on the fact that isoscalar and isovector densities enter the electromagnetic and neutral current response functions in different linear combinations. In effect the idea relies on isospin being a good quantum number: $\mathrm{PC}$ and $\mathrm{PV}$ electron scattering in principle yield two independent responses (for given kinematics) which can be recast in terms of two form factors. one isoscalar and the other isovector. ¿ that the proton and neutron are (approximate) eigenstates of isospin with $T=1 / 2$ (see the brief discussion above in Sect. II.A and Sects. IV.A,B) the results may then be rewritten in terms of proton and neutron form factors. The particular case of elastic scattering from the proton is an important example. There only $\mathrm{PC}$ and $\mathrm{PV}$ scattering from the proton is involved and yet the charge form factor of the neutron $G_{E}^{n}$ plays an important role. For typical forward-angle scattering kinematics the effect of ignoring $G_{\Sigma}^{n}$ would amount to an error of between 10 and $25 \%$ in the asymmetry, as discussed in Ref. [Don88]. Given this rather high sensitivity to $G_{E}^{n}$ and the poor knowledge presently available about its magnitude away from $\left|Q^{2}\right| \approx 0$, it was suggested in Ref. [Don88] that PV asymmetry measurements be used together with the usual PC determinations of $G_{E}^{p}, G_{M}^{p}$ and $G_{M}^{n}$ to extract information on $G_{E}^{n}$. Specifically, in that work it was estimated that a $10 \%$ determination of $G_{E}^{n}$ at $\left|Q^{2}\right|=0.4(\mathrm{GeV} / c)^{2}$, near the maximum in the estimated figureof-merit (FOM), could be obtained in a ${ }^{1} \mathrm{H}(\vec{e}, e)^{1} \mathrm{H}$ experiment using $4 \mathrm{GeV}$ electrons at $\sim 9^{\circ}$ in 350 hours of running time. A determination of $G_{\varepsilon}^{n}$ near the beginning of the regicn where the FOM begins to fall-off $\left(\left|Q^{2}\right| \approx 1.0(\mathrm{GeV} / \mathrm{c})^{2}\right)$ would also appear to be feasible, though with less precision due to uncertainties in $G_{E}^{p}$ and $G_{M}^{n}$ which also enter the asymmetry. As discussed in more detail in Sect. IV.A, what actually enters in the asymmetry is not just $G_{E}^{n}$, but the combination $G_{E}^{n}+G_{\varepsilon}^{(s)}$. Thus, to extract values for the individual form factors will require additional measure nents such as using elastic scattering from $0^{+} 0$ nuclei to determine $G_{E}^{(s)}$ as outlined above or using PC polarized electron scattering with hadronic polarizations to determine $G_{E}^{n}$.

As for a determination of $R_{n}$, a $1 \%$ measurement for ${ }^{208} \mathrm{~Pb}$ could be obtained using PV electron scattering at $\epsilon=300 \mathrm{MeV}$ in the region below the first diffraction minimum in the charge form factor $\left(q \approx 0.6 \mathrm{fm}^{-1}\right)$, with $\sim 10$ days of running time [Don89]. A similar determination of the neutron radius for ${ }^{133} \mathrm{Cs}$ also appears feasible [Sic91]. In addition to reducing $R_{n}$ uncertainties below a problematic level for the interpretation of atomic 
PV measurements using a single isotope, such measurements would also serve to calibrate theoretical estimates of $\rho_{n}(r)$ as needed in the analysis of atomic PV measurements with a series of isotopes. Atomic theory uncertainties would be eliminated in the latter approach. and lack of knowledge of ground-state neutron distrib trions would appear to introduce the dominant theoretical error.

Isospin Decomposition of Response Functions

One of the outstanding. unsolved puzzles in electron scattering is the apparent failure of the Coulomb sum rule in $\mathrm{QE}$ scattering (see, for example. Ref. (Ber91]). Among the various explanations proffered for this failure [Alb93a]. and one which could be further explored with semileptonic scattering, is the role played by isospin-dependent nuclear correlations. In the absence of such correlations, the longitudinal PV QE response is suppressed (see Eq. (4.61)). Consequently, the sensitivity of this response function to differences between the isovector and isoscalar nuclear correlations is significant. so that a measurement of this response could provide a useful window on such correlations. A comparison of such a measurement with determinations of the $Q E$ response in purely: hadronic scattering would also be of interest [Alb88]. We return in Sect. IV.F to discuss PV QE electron scattering in more detail.

\section{The Vucleon-to-Delta Transition}

The parity-violating asymmetry in the region of the $\Delta(1232)$ is insensitive to many details of nucleon and $\Delta$ structure, and thus may provide an interesting electroweak test. This possibility is discussed in some detail in Sect. IV.G. There are also several additional contributions to $A_{L R}(N \rightarrow \Delta)$ which are of interest in themselves: (a) contributions from the axial-vector. $\mathrm{NC}$, and (b) effects of non-resonant backgrounds. Knowledge of these contributions is also relor:ant for the interpretation of $\mathrm{NC} Q E$ electron and neutrino scattering experiments. su as the $\mathrm{QE}{ }^{9} \mathrm{Be}\left(\vec{e}, e^{\prime}\right)$ measurement at Mainz mentioned above. which integrate into the "dip" region and tail of the $\Delta$ resonance. For the contribution from the axial-vector current, which is pure isovector, knowledge of $\left\langle\Delta\left|J_{\mu 3}\right| . \mathrm{V}\right\rangle$ may be obtained from charge-changing $\nu \mathrm{N}$ reactions by performing an isospin rotation, although information obtained by this means is at present rather uncertain, with model predictions varying by as much as $50 \%$ at low $-\left|Q^{2}\right|$ [Sch73). Recent experimental results [Jon89, Kit90] have been fit with a specific (highly model-dependent) form [Adl68] containing only one remaining free parameter for $\left|Q^{2}\right| \lesssim 1 \mathrm{GeV}^{2}$, with a $10 \%$ or more uncertainty in the single fit parameter. At larger- $\left|Q^{2}\right|$, the fit is even less satisfying. The low-energy limit of $A_{L R}(. V \rightarrow \Delta)$ would be the appropriate kinematic regime in which to probe $\left\langle\Delta\left|J_{\mu 3}\right| . V\right\rangle$ directly, since in this case it is suppressed only by the leptonic NC coupling $g_{v}^{e}$ and is dominated by a single multipole.

In the case of non-resonant background vector current contributions, one requires electroproduction data for bot:? proton and neutron targets in order to obtain the isospin amplitudes needed in the $\mathrm{NC}$ transition, and while such data exist for the proton, much less is known for the neutron [Vap88]. There is independent interest in further $\Delta(1232)$ electroproduction experiments at CEBAF [Bur89], as well as at medium-energy accelerators. 
and consequently it is expected that considerably better information will be available in due course. In particular, future CEBAF experiments will include measurements of nonresonant background multipoles and a complete isospin decomposition. In the absence of the latter, combining $A_{L R}(., \rightarrow \Delta)$ with existing electromagnetic data could itself be used to measure this isospin decomposition of the backgrounds - a quantitative analysis of the latter possibility is in progress [Pol93]. 


\section{II.C. Electroweak Tests}

The motivation for much of the earliest discussions of low- and intermediate-energ: semileptonic $\mathrm{NC}$ experiments was that of testing the Standard. Model of electroweak interactions Fei75. Walii. Doni9a]. Indeed. the goal of the MIT/Bates $A_{L R}\left({ }^{12} \mathrm{C}\right)$ experiment Sou90a was a determination of the isoscalar hadronic. $\mathrm{NC}$ vector coupling $;$ (defined in Table 3.2). while the . Iainz $\mathrm{QE} A_{L R}\left({ }^{9} \mathrm{Be}\right.$ ) measurement (Hei89) sought to complement the SL.AC deep-inelastic $A_{L R}\left({ }^{2} \mathrm{H}\right)$ measurement (Preis. Preigl by constraining the isovec. tor and isoscalar hadronic $\mathbb{N}^{\circ} \mathrm{C}$ axial-vector couplings, $\dot{3}$ and $\dot{\delta}$, respectively. In a similar vein. the Brookhaven $\nu_{\mu} p\left(\bar{\nu}_{\mu} p\right)$ experiment $[A$ hr87] provided constraints on the proton neutral current couplings. Since one may predict the values of these couplings using the Standard . Model. the aforementioned intermediate-energy scattering experiments could be used as tests of the standard electroweak theory. With the advent of very high precision $\epsilon^{-} \epsilon^{-}$measurements at the $Z^{0}$ pole and of precision atomic PV experiments, the role of intermediate-energy scattering in testing the Standard Model seems less clear than pre. viously thought. At present, these experiments seem more suited as probes of hadron. structure. and as such. they occupy a unique position in the broad context of $\mathrm{NC}$ studies. levertheless, it is still of interest to ask how these experiments might contribute in the search for physics beyond the Standard Model. To that end, we review briefly both the present status of electroweak tests and the issues involved in the use of semileptonic scattering for this purpose. More extensive reviews of electroweak tests may be found elsewhere [El190. Alt91. Lan90].

\section{II.C.1. STATLS OF ELECTROWEAK TESTS}

The minimal $\mathrm{SC}^{\circ}(2)_{L} \times \mathrm{C}^{*}(1)_{Y}$ Standard Model (one Higgs doublet) with three generations of fermions (including massless neutrinos) depends on 17 arbitrary parameters: the nine fermion masses. the Higgs mass.$M_{H}$, the three angles and phase which parameterize the Kobayashi-Maskawa $(\boldsymbol{K}-\mathrm{M})$ matrix and three parameters in the gauge sector. Since the $K-. . /$ parameters do not enter in tree-level neutral current amplitudes, we will not discuss them further. Their values have been determined or constrained by charge-changing processes. such as semileptonic decays and deep-inelastic neutrino scattering [RPP92]. Of the masses. only two remain largely undetermined:.$V_{H}$ and the top-quark mass. $m_{t}$. In the gauge sector. one has a choice about which three parameters to treat as independent inputs. ${ }^{\dagger}$ It is conventional to take the fine-structure constant, $\alpha$, the Fermi constant measured in muon decay, $G_{\mu}$, and the $Z^{0}$ mass,.$L_{z}$, as these three parameters, since they are the three most accurately know quantities in the gauge sector. To date, they have been determined to the psecision indicated in Table 2.4 [RPP92].

In the on-shell renormalization scheme, where the weak mixing angle is defined via

$$
\sin ^{2} \theta_{w} \equiv 1-\frac{M_{w}^{2}}{M_{2}^{2}}
$$

This point, as well as the choice of renormalization scheme, is discussed in more detail in Sect. III.

Although $G_{\mu}$ does not enter the Standard Model lagrangian directly, it is simply related to parameters which do through Eq. (3.6). 


\begin{tabular}{|c|c|}
\hline \multicolumn{2}{|c|}{ TABLE 2.4 } \\
\hline Parameter & value \\
\hline$\alpha$ & $1 / 137.0359895(61)$ \\
$G_{\mu}$ & $1.16639(2) \times 10^{-3} \mathrm{GeV}^{-2}$ \\
$M_{z}$ & $91.173 \pm 0.020 \mathrm{GeV}$ \\
\hline
\end{tabular}

Table 2.4. Gauge sector input parameters in the Standard Model.

both.$M_{w}$ and $\sin ^{2} \theta_{w}$ are determined as functions of $\left(\alpha . G_{\mu}, V_{z}\right)$ through the relation [Sir80]

$$
\sin ^{2} \theta_{w} \cos ^{2} \theta_{w}=\frac{\pi \alpha}{\sqrt{2} G_{\mu}} \frac{1}{V L_{z}^{2}}(1-\Delta r)^{-1}
$$

or

$$
\dot{M} I_{w}^{2}=\frac{1}{2} \cdot M_{2}^{2}\left[1+\sqrt{1-A_{0}}\right] \text {. }
$$

where

$$
A_{\circ}=\frac{2 \sqrt{2} \pi \alpha}{G_{\mu} M_{z}^{2}} \frac{1}{1-\Delta r}
$$

and where $\Delta r$ is a radiative correction to muon-decay [Sir80]. In this scheme. $\sin ^{2} \theta_{w}$ and .$M_{w}$ depend as well on $M_{H}$ and $m_{t}$ through the dependence of $\Delta r$ on these masses. Hence. $\sin ^{2} \theta_{w}$ cannot be determined with the same precision as $\left(\alpha, G_{\mu}, M_{z}\right)$ until both $m_{t}$ and $M_{H}$ are known.

Although $\sin ^{2} \theta_{w}$ is not rigorously an independent parameter within the context of the minimal Standard Model," one may test the theory by treating it as if it were independent and comparing experimental determinations based on this assumption with values of $\sin ^{2} \theta_{w}$ obtained from Eqs. (2.4) or (2.5a). Such an analysis, when performed without considering higher-order process (e.g., $\Delta r \rightarrow 0$ ), would test the consistency of the tree-level theory with neutral current data. However, present 1 and prospective) experiments are sensitive to $\mathrm{O}\left(\alpha G_{\mu}\right)$ effects, so one must account for second-order electroweak corrections to tree-level amplitudes when interpreting precision neutral current results. On the one hand, consideration of these radiative corrections complicates the analysis of the structure of the theory: virtual Higgs boson and top-quark loops introduce an $\left(M_{H}, m_{t}\right)$-dependence into processes not involving these particles explicitly, thereby adding ambiguity to comparisons of $\sin ^{2} \theta_{w}$ derived from Eqs. (2.4), (2.5a), and directly from experiments. ${ }^{\dagger}$ On the other

- This statement assumes that $\left(\alpha, M_{z}, G_{\mu}\right)$ are taken as inputs.

+ Some of this ambiguity may be eliminated through an alternate choice of renormalization scheme, such as $\overline{M S}$. 
hand. sensitivity to radiative corrections allows one to test the quantum field theory nature of the Standard Model. much as measurements of the anomalous magnetic moment of the electron - a quantity arising solely from higher-order processes - allow one to test QED as a quantum field theory.

Were $m_{t}$ and.$W_{H}$ known precisely, the weak mixing angle would be determined from Eq. (2.5a) to much better than $1 \%$ accuracy. For example, taking $m_{t}=100 \mathrm{GeV}$ and $\mathrm{H}_{H}=250 \mathrm{Gel}$ one finds [Lan90]

$$
\left.\sin ^{2} \theta_{w}\right|_{M_{z}}=0.2316 \pm 0.0002 \pm 0.0004
$$

where the first uncertainty is experimental, the second is theoretical. and where a somewhat older value of $W_{Z}$ has been used. The $M_{H}$-dependence of $\Delta r$ is rather weak. so that once $m_{t}$ is known. $\sin ^{2} \theta_{w}$ will be fixed by the theory to much better than $1 \%$. For comparison. the most precise determinations of $\sin ^{2} \theta_{w}$ via Eq. (2.4) are obtained from recent $p \vec{p}$ collider measurements of the ratio $M_{w} / M_{2}$ [Ali92. Abe91]. The associated uncertainty in $\sin ^{2} \theta_{w}$ is roughly $2-4 \%$. When combined with the average LEP value for $M_{z}$, these results also vield the most precise value of $M_{w}$ obtained to date, with a corresponding error in this mass of about $0.5 \%$.

Determinations of $\sin ^{2} \theta_{w}$, treated as an independent, experimentally measured quantity, come from a variety of experiments. In the purely leptonic sector, early measurements of $\sigma\left(\bar{\nu}_{e} e\right), \sigma\left(\nu_{\mu} e\right)$, and $\sigma\left(\bar{\nu}_{\mu} e\right)$, taken together with forward-backward (FB) asymmetry measurements in $e^{+} e^{-}$annihilation, confirmed predictions based on Eq. (1.1) provided $\sin ^{2} \theta_{w} \approx 0.22$ [Che84-Ch. 12]. More recent results from CHARM, BNL, and LA.MPF experiments have yielded values for $\sin ^{2} \theta_{w}$ with uncertainties ranging from $5-20 \%$ [Al190. Abe89. Dor89, Gei89, Abe87].

In the neutrino-quark sector, the first deep inelastic $\nu$-nucleon experiments with isoscalar targets showed consistency with quark neutral currents having the form in Eq. (1.1) with $\sin ^{2} \theta_{w}=0.23 \pm 0.023$ [Com83-Ch. 8]. The value of $\sin ^{2} \theta_{w}$ obtained using isoscalar targets is relatively independent of hadronic structure models [Pas73]. Determinations derived from deep inelastic $\nu(\bar{\nu})$-nucleon scattering with $T_{3} \neq 0$ targets depend more strongly on details of quark distributions in the nucleon [Com83, Che84, Ama87]. In both cases, a value of $\sin ^{2} \theta_{w}$ is determined from ratios of neutral current and charged current cross sections, such as

$$
R_{\nu(\nu)}^{D I s}=\frac{\sigma(\nu N \rightarrow \nu X)}{\sigma(\nu N \rightarrow \mu X)}
$$

The average of the latest CDHS and CHARM data [All87, Abr86, Blo89, Gei89] gives a $\nu N \rightarrow \nu X$ value for $\sin ^{2} \theta_{w}$ with roughly $3 \%$ combined experimental and theoretical error. The dominant theoretical error arises from uncertainty in the value for $m_{c}$ used in modeling the charm production threshold [Ama87]. In contrast with other direct determinations of $\sin ^{2} \theta_{w}$, those derived from deep inelastic $\nu-N$ scattering are quite insensitive to $m_{t}$, owing to an accidental cancellation in radiative corrections to $R_{\nu(D)}^{D I S}$ [Alt91]. A much less precise 
value for $\sin ^{2} \theta_{w}$ has been derived from the Brookhaven (quasi)-elastic $\nu_{\mu} p / \bar{\nu}_{\mu} p$ experiment Ahr 37 where a value of $\sin ^{2} \theta_{w}$ with roughly $14 \%$ error is reported. We discuss these results in more detail in Sect. IV.J.

Standard Model tests using charged lepton probes fall into two classes. The most precise results have been obtained from atomic PV experiments with heavy atoms. Atomic $\mathrm{PV}$ observables result from the mixing of opposite parity atomic states. induced by the PV $\triangle \mathrm{C}$ interaction of the atomic electrons with the nucleus. The quantity of interest is the so-called "weal: charge". $Q_{w}$ (see Eqs. (4.98)), which depends. at the simplest level. on the vector $: C$ couplings to the proton and neutron. Extraction of $Q_{w}$ from the experimental observatle depends on details of atomic structure. so that the uncertainty in the quoted value of $\sin ^{2} \theta_{w}$ depends on atomic theory uncertainties as well as on experimental error: and theoretical uncertainties associated with higher-order effects. Recent improvements in atomic theory techniques have reduced this error in $Q_{w}$ for ${ }^{133}$ Cs to roughly the 17 . level [Blu90]. whereas the experimental error is roughly $2 \%$ from the latest experiment by lioecker et al. [Noe88]. Additional theoretical uncertainty enters the extraction of $\sin ^{2} \theta_{w}$ from $Q_{w}$ due to the dependence of the weak charge on the ground state neutron distribution [For90. Pol92a]. A $10 \%$ uncertainty in $R_{n}$ for ${ }^{133} \mathrm{Cs}$ would result in a $1 \%$ error in $\sin ^{2} \theta_{w}$. The corresponding errors in $\sin ^{2} \theta_{w}$ from the experiment and from atomic theory are roughly $3 \%$ and $2 \%$, respectively.

Less precise Standard . Model tests have been obtained from PV electron-hadron scattering. In addition to the pioneering deep inelastic $\vec{e} D$ measurement at SLAC (Preis. Pre 79 ], which yielded a $9 \%$ determination of the weak mixing angle, determinations have also been carried out using quasielastic and elastic PV electron scattering on ${ }^{9} \mathrm{Be}$ :Hei39? and ${ }^{12} \mathrm{C}$. respectively [Sou90a]. The former yielded a $7 \%$ determination, whereas the error from the elastic ${ }^{12} \mathrm{C}$ measurement is nearly $25 \%$.

A measure of the consistency of the minimal Standard. Model with all neutral current data can be obtained by performing "global" fits to the data. Several authors have recently reported on such fits. Given the $\left(m_{t}, M_{H}\right)$-dependence of $\Delta r$ in Eq. (2.5a) as well as in the radiative corrections to other neutral current observables, such fits produce ranges for $\sin ^{2} \theta_{W}$ correlated with ranges for $m_{t}$ and $M_{H}$. Representative results from different fits are summarized in Table 2.5 .

These fits all indicate a central value for $\sin ^{2} \theta_{w}$ near 0.23 with uncertainties of approximately $1-2 \%$. Most of this uncertainty arises from uncertainty in $m_{t}$. Once $m_{t}$ is known. precision improves by an order of magnitude, signalling consistency of the Standard Model with all neutral current data at much better than the $1 \%$ level. Even the present 1-2\% level of consistency is impressive, given the wide range in energy scales which the data encompass.

With present and future NC measurements approaching the $1 \%$ level of precision or better, tests of this sort could be sensitive to physics beyond the minimal $\mathrm{SU}(2)_{L} \times \mathrm{C}(1)_{Y}$ Weinberg-Salam theory. While an in-depth discussion of this "non-standard" physics lies beyond the scope of this review and can be found elsewhere [Ama87, Alt91, Mar90, Pes90. Gol90] we highlight a few aspects relevant to intermediate-energy experiments. Deviations 


\begin{tabular}{|c|c|c|}
\hline \multicolumn{3}{|c|}{ TABLE 2.5 } \\
\hline Fit & $\sin ^{2} \theta_{w}$ & $m_{t}$ \\
\hline$[\operatorname{Lan} 90]$ & $0.2272 \pm 0.004$ & $139_{-39}^{+33} \pm 16$ \\
{$[$ Alt91] } & $0.228 \pm 0.005$ & $140 \pm 45$ \\
{$[$ El190] } & $0.2273 \pm 0.003$ & $127_{-30}^{+24}$ \\
\hline
\end{tabular}

Table 2.5. Global fits of $\sin ^{2} \theta_{w}, m_{t}$, and $\mathcal{M}_{H}$ to all electroweak data. Fit of Ref. [Alt91] includes only data from LEP, $p \bar{p}$ determination of $. M_{w} / M_{z}$, and $\nu . V^{*} \rightarrow$ $\nu . X$. The $\sin ^{2} \theta_{w}$ and $m_{t}$ values of Ref. [Ell90] assume $M_{H}=M_{Z}$. The authors of Ref. [Ell90] also performed a fit in which $M_{H}$ was allowed to vary and found $. K_{H}>1.8$ $\mathrm{GeV}$ at a $68 \%$ confidence level.

of the so-called $\rho$-parameter from its Standard Model value could indicate the presence of extra. non-doublet Higgs bosons, the presence of additional, very heavy $W^{ \pm}$and $Z^{\prime \prime}$ bosons, or heavy fermion loops associated with super-symmetric (SUSY) extensions of the Standard Model [Alt91, Ama87]. The natural parameter for discussing grand unified theories (GLT's), $\sin ^{2} \hat{\theta}_{w}$ (defined in Sect. III), seems to rule out non-SUSY GIT's such as SU(5) while providing some consistency with SUSY grand unification [Ama87].

Recently, a new framework has been introduced for discussing certain types of nonstandard physics, such as SUSY or technicolor, which would enter neutral current observables through gauge-boson self energies [Ken90, Pes90, Mar90]. While some extensions of the Standard Model - such as those associated with tree-level exchange of extra $Z^{01}$ bosons - are not described by this framework, it nonetheless constitutes a useful means of comparing different observables for purposes of electroweak tests. In one version of this parameterization, new physics is described by two parameters: $T$, which is particularly sensitive to mass splittings in boson or fermion isomultiplets, and $S$, which characterizes degenerate heavy physics contributions. In the treatment of Ref. [Mar90] which we follow here, a nonzero value for $T$ would also signal a value of $m_{t}$ different from $140 \mathrm{GeV}$. Measurements of $Z^{0}$ widths are dominantly sensitive to $T$ (at $\approx 1 \%$ level), while $Z^{0}$ asymmetries and neutrino scattering measurements are roughly equally sensitive to both $S$ and $T$. Atomic PV and elastic PV electron scattering, on the other hand, would probe primarily for nonzero values of $S$. In fact, results from the Cs atomic PV experiment [Noe88] constrain this parameter to the level $|\delta S| \approx \pm 2.3$, where the dominant error is experimental. A reduction in this uncertainty by a factor of ten would render one sensitive to contributions from either additional generations of heavy fermions or the minimal one-doublet technicolor model [Mar90]. Anticipated future improvements in the atomic PV experimental uncertainty would leave theoretical atomic structure uncertainty as the dominant source of error. It is expected that the overall atomic PV uncertainty in $S$ will decrease by a factor of three or more in the future [Lan91]. 
The completion of one or more PV electron scattering experiments with sufficient precision could complement constraints from atomic $\mathrm{PV}$ and reduce the uncertainty in $S$ to an interesting level. For purposes of illustration, we plot in Fig. 2.4 the present constraints on $S$ and $T$ from atomic PV as well as potential constraints from future. highprecision measurements of $A_{L R}$. We assume all low- and intermediate-energy experiments agree on common central values for these parameters, so that the axes give deviations from these values allowed by experimental and theoretical uncertainty. From Fig. 2.4 one observes both the rationale for exploring $A_{L R}$ measurements as electroweak tests as well as the precision needed to make such measurements relevant. Indeed, a $10 \%$ measurement of the elastic $\vec{e} p$ asymmetry or $1 \%$ measurements of either the elastic ${ }^{12} \mathrm{C}(\vec{e}, e)$ or.$V \rightarrow J$ asymmetries could nicely complement atomic PV and constrain $S$ to an interesting level.

\section{II.C.2. PRECISION TESTS WITH INTERMEDIATE-ENERGY SCATTERING}

Experimental Considerations.

From the above discussion, it should be clear that any new electroweak tests using neutrino or PV electron scattering must attain $\sim 1 \%$ precision (or $10 \%$ for $\vec{e} p$ scattering) in order to be both meaningful and competitive with tests in other sectors. This requirement presents both experimental as well as theoretical challenges. Let us begin by discussing the former. Since the typical asymmetries are very small (ranging from about $10^{-6}$ to a few times $10^{-5}$ for electrons of a few $100 \mathrm{MeV}$ to a few $\mathrm{GeV}$ ), attaining $1 \%$ precision requires a large number of scattering events with specified electron helicity. For instance, considering just the statistical precision that can be reached, this range of asymmetries implies $10^{14}$ to $10^{16} 100 \%$ polarized electron scattering events to determine the asymmetries to $1 \%$. Given that accelerators in the medium- and high-energy regime will deliver up to $100-$ $200 \mu \mathrm{A}$ (e.g., CEBAF is designed for a maximum current of $200 \mu \mathrm{A}$ ) and that there are limitations on how thick practical targets can be, as discussed in Sect. V, there is a limit to how high the attainable luminosities can be for the foreseeable future. Typically the best that can be achieved is $\mathcal{L} \sim$ few $\times 10^{38} \mathrm{~cm}^{-2} \mathrm{~s}^{-1}$. For typical asymmetries, cross sections and the resulting FOM (see Sect. III.E.2) even these extreme values of luminosity still imply experimental running times of 100 's to 1000 's of hours. It should be obvious that if luminosities on the order of a few $\times 10^{38} \mathrm{~cm}^{-2} \mathrm{~s}^{-1}$ or more cannot be reached, then most high precision PV electron scattering experiments cannot be attempted. As we shall discuss in detail for specific cases of interest (Sect. IV), for a few carefully selected hadronic transitions, the FOM is large enough to allow us to contemplate reaching the $1 \%$ precision level. However, in many cases, such as for typical inelastic excitation of discrete nuclear states, the FOM's are projected to be so small that even getting to the $100 \%$ precision level may be impractical.

In addition to these considerations, there exist additional factors which only increase the level of experimental difficulty. For example, the incident electron beams are not $100 \%$ polarized. While considerable progress has been made in recent years, and there is hope that advances will continue in the next decade, the present state-of-the-art restricts high-current (100-200 $\mu \mathrm{A}$ average) polarized electron sources to below $50 \%$ polarization. Since the experimental FOM is proportional to the square of the measured asymmetry, 
this amounts to a factor of four reduction in the effective do-ability of polarized electron experiments. Higher polarizations have been attained, but with lower average currents.

Other factors also enter into the final evaluation of the feasibility of $\mathrm{PV}$ electron scattering experiments. For instance. specific measurements may require very forwardangle scattering and the issue of limited detector solid angle must be faced. Alternately; certain measurements may require detector:; with sufficiently high energy resolution to restrict the asymmetry determination to a single specific transition. However. the available solid angle - and the corresponding achievable luminosity - is generally limited for such high-resolution detectors. Of course. if one does not require such high resolution. then rather different poor-resolution, large solid angle detectors can be employed. An example of the latter is a measurement of the elastic $\vec{e} p$ asymmetry, for which one only needs a resolution of a few hundred $\mathrm{MeV}$ in order to exclude contributions from excitation of the $\Delta(1232)$ resonance.

Beyond these considerations of statistical precision and resolution, there are issues relating to systematic errors that are discussed in some detail in Sect. V. As a distillation of past experience in performing PV electron scattering experiments at SLAC. Mainz and .MIT/Bates one arrives at the expectation that systematic errors can be controlled sufficiently well to attain the goal of $2 \%-3 \%$ asymmetry determinations. It should be realized that this is no mean feat: for an asymmetry of $10^{-6}$ this implies keeping the sum of all systematic effects below $10^{-8}$.

Theoretical Interpretability.

Even if these challenges could be surmounted, the interpretability of such high precision results would be, at this point, somewhat questionable, owing to theoretical uncertainties involved in calculating strong interaction effects at low and intermediate energies. While some of these uncertaintie: would be much less problematic for the interpretation of precision neutrino scattering measurements, experimental considerations suggest that such measurements will not approach the $1 \%$ level in the foreseeable future. The issue is more relevant, however, for PV electron scattering, where some hope does exist for attaining the requisite level of experimental precision. Over time, theoretical progress, coupled with the completion of measurements constraining some of the present sources of uncertainty in the PV asymmetry, may make electroweak tests at the level suggested in Fig. 2.4 possible with PV electron scattering. In what follows, we outline what would be required to realize this possibility.

By a suitable choice of target and/or experimental kinematics, much of the hadronic physics content of the ratio $A_{L R}$ can be eliminated, leaving only the dependence on the electroweak couplings, which would be determined from the Standard Model and its possible extensions. Two cases of particular interest are low- $\left|Q^{2}\right|$, forward-angle elastic scattering from the proton and elastic scattering from $\left(J^{\pi} T\right)=\left(0^{+} 0\right)$ nuclei. The recent ${ }^{12} \mathrm{C}(\vec{e}, e)$ experiment at Bates, from which a $25 \%$ measurement of $\sin ^{2} \theta_{w}$ was extracted, falls into the latter category. As suggested by Fig. 2.4. however, a future experiment of this type would need to achieve $\sim 1 \%$ precision in order to be competitive with atomic $\mathrm{PV}$ as a probe 
of non-standard physics. Forward-angle experiments on the proton have been discussed as possibilities for CEBAF [Nap90]. In both of these cases, the leading term in $A_{L R}$ is nominally independent of hadronic physics associated with the target (see Eqs. (4.9) and (4.11)). Target-dependent corrections to the leading terms arise from a number of sources: hadronic form factors, hadronic contributions to radiative corrections, isospin impurities in the hadronic ground state, and, in the case of an $A>1$ target, many-body currents.

In the case of forward-angle scattering from the proton. the most serious uncertainties appear to be introduced by nucleon form factors. The contribution from these form factors to $A_{L R}(\theta \rightarrow 0)$ vanish as $Q^{2} \rightarrow 0$, and naively one would expect to minimize their impact by working at sufficiently small momentum-transfer. However. the FOM for such a measurement also decreases with $\left|Q^{2}\right|$. Consequently, one cannot go to arbitrarily low $\left|Q^{2}\right|$ without sacrificing the statistical precision needed to make a meaningful electroweak test. In this regard, lack of knowledge of $G_{E}^{(s)}$ is particularly problematic. Assuming that the strangeness form factors were to be determined by a series of $A_{L R}(\vec{e} p)$ measurements alone. the remaining uncertainty in $G_{E}^{(\mathbf{s})}$ would still be larger than needed to permit one to constrain $S$ and $T$ with PV electron scattering at the level indicated in Fig. 2.4. Additional, although somewhat smaller uncertainties are introduced by lack of knowledge of other form factors. This difficulty might be overcome either by reducing the uncertainty in the strangeness form factors with an appropriate combination of $A_{L_{R}}$ measurements with $A>1$ targets, or by building a detector with sufficient solid angle at more forward angles than are contemplated for existing or planned detectors. Alternatively, if it were possible to achieve very high currents ( $\gtrsim$ few hundred $\mathrm{mA}$ ) of polarized beams at low-energy accelerators $(\epsilon \sim$ few hundred $\mathrm{MeV})$, this form factor issue could be surmounted.

For scattering from $\left(0^{+} 0\right)$ targets, the primary form factor ambiguity is also associated with a term in the asymmetry containing $G_{\varepsilon}^{(s)}$. Although this form factor vanishes at the photon point, one cannot reduce its contribution by going to arbitrarily low $\left|Q^{2}\right|$ without increasing the statistical error in $A_{L R}$ beyond the $1 \%$ level. Fortunately, one has some hope of constraining this term to the precision needed for a Standard Model test. We show in Sect. IV.B how a series of two measurements of $A_{L R}\left(0^{+} 0\right)$ would be sufficient for this purpose. In the absence of such a determination, the uncertainty in $G_{E}^{(s)}$ remaining after its determination with $\overrightarrow{e p}$ scattering would, given some model assumptions, severely restrict the kinematics at which a meaningful $\left(0^{+} 0\right)$ electroweak test would need to be performed. For purposes of comparison, we note in passing that given the very small effective momentum transfer in the interaction of an atomic electron with the nucleus, the impact of nucleon form factors in atomic PV electroweak tests can largely be ignored. Apart from the uncertainties associated with the neutron distribution and atomic structure, then, atomic PV offers a distinct advantage over semileptonic scattering as far as testing the Standard Model is concerned.

For both $A_{L R}(\vec{e} p)$ and $A_{L R}\left(0^{+} 0\right)$, additional uncertainties are generated by hadronic contributions to higher-order electroweak amplitudes. Hadronic contributions to the $Z^{0}-\gamma$ mixing tensor have been estimated using a dispersion analysis, and the associated uncertainty appears significantly below a problematic level [Mar84, Deg89]. Of more concern are the dispersion corrections discussed above in connection with strangeness measurements. 
While the scale of hadronic uncertainties in the one-body (single nucleon) dispersion amplitudes is likely to be no greater than $1 \%$ of the tree-level amplitude (Dre59, Gre69. Mar\$4: data from elastic scattering on ${ }^{12} \mathrm{C}$ [Kal89] and ${ }^{208} \mathrm{~Pb}$ [Bre90] suggest a significant manybody enhancement, at least in the case of purely electromagnetic scattering. Since the E.I and. $\mathrm{C}$ dispersion corrections are unlikely to cancel from the PV asymmetry a better understanding of this contribution appears necessary in the interpretation of $\left.A_{L R} 0^{+} 0\right)$ as an electroweak test.

One expects the effect of isospin-mixing in the nucleon to be suppressed. given the scale of mass splittings (and. hence, energy denominators) in the baryon spectrum. In particular. the first isospin-3/2 components that might isospin-mix with an isospin-1 2 "proto-nucleon" to form the physical nucleon occur at the opening of the $\pi .1$ channel. $n z$. at $140 \mathrm{MIeV}$. In the case of nuclei. however, the typical energy splitting between low-lying states is roughly an order of magnitude smaller. so that one might anticipate the presence of non-trivial isospin impurities in the nuclear ground state. In the case of $\left(0^{+} 0\right) s-p$ shell nuclei. the effect of isospin-mixing on $A_{L R}$ at low momentum transfer has been estimated to be below $1 \%$ [Don89]. This result follows from the difficulty in supporting an isovector monopole matrix element in the relevant nuclear model space. Thus. isospin-mixing is unlikely to present a problematic uncertainty in a $\left(0^{+} 0\right)$ electroweak test.

Once one goes beyond the special cases of elastic scattering from ${ }^{1} \mathrm{H}$ and $\left(0^{+} 0\right)$ targets. additional uncertainties enter the use of $A_{L R}$ measurements to test the Standard Model. In the case of $\mathrm{QE}$ scattering, for example. one encounters nuclear physics uncertainties associated with contributions from the pion-production cross section. "dip region". and radiative tail as well as from the $Q E$ peak. The present theoretical understanding of these regions is somewhat limited. particularly with regard to the dip region. and the magnitude of these uncertainties is difficult to quantify. For PV QE electron scattering. one might anticipate some cancellation of nuclear physics uncertainties from $A_{L R}(\mathrm{QE})$. since the asymmetry depends on a ratio of nuclear response functions. Investigations using a relativistic Fermi Gas model for the $Q E$ response in fact indicate that $A_{L R}(\mathrm{QE})$ is significantly less sensitive to various nuclear model parameters than are the individual response functions appearing in the PC and PV cross sections [Don92]. To the extent that future $A_{L R}(\mathrm{QE})$ measurements are carried out at kinematics for which non-QE contributions are negligible, this result gives one hope that nuclear physics uncertainties might be minimized.

Even so, uncertainties associated with single-nucleon form factors, which plague potential $\vec{e} p$ electroweak tests, also enter $A_{L R}(\mathrm{QE})$ at a non-negligible level. In the recent Mainz $\mathrm{QE}{ }^{9} \mathrm{Be}\left(\vec{e}, e^{\prime}\right)$ experiment, carried out at backward-angles, uncertainties in the radiative corrections to the axial-vector term induce a 2-3\% uncertainty in the extracted value of $\sin ^{2} \theta_{w}$ [Mus92a]. Similarly, a forward-angle determination of $\sin ^{2} \theta_{w}$ is likely to be highly sensitive to uncertainty in $G_{E}^{(s)}$. Consequently, PV QE electron scattering appears more suited to the determination of nucleon form factors than to tests of electroweak theory. As far as QE neutrino scattering is concerned, as in the BNL and LS.ND experiments, an electroweak test based on a measurement of the cross section alone incurs both form factor and nuclear physics uncertainties. It is likely that LSND will be limited by statistical accuracy: the systematic errors on the cross section are projected to be less 


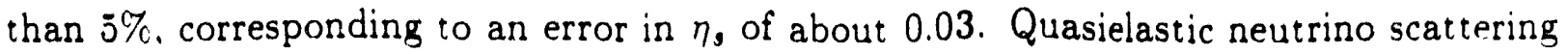
does present at least one theoretical advantage over electron scattering in that the large and uncertain radiative correction to the axial-vector response in electron scattering does not arise in processes involving neutrinos.

The inelastic $V \rightarrow \Delta(1232)$ transition, being purely isovector, provides direct information on vector and axial-vector isovector couplings. In contrast with the $\vec{e} p$ elastic cases discussed above. isoscalar nucleon structure plays a small role, and will not dominate the resulting uncertainty in extracted electroweak parameters. More problematic are contributions from the axial-vector $\mathrm{NC}$ matrix elements. As discussed above. these matrix elements are at present insufficiently well known for the purposes of $N \rightarrow \Delta$ electroweak tests at CEBAF energies or lower. The axial-vector contribution is, however, explicitly suppressed by the inverse of the incident beam energy, and thus the forward angle. high-energy limit should be the appropriate kinematic regime for such tests.

Von-resonant background contributions to $A_{L R}(. N \rightarrow \Delta)$ are also a potential source of serious uncertainty. A full isospin decomposition of $\Delta$ electroproducticn amplitudes at the same $Q^{2}$ as where a PV asymmetry measurement could be attempted ivould be sufficient to eliminate this problem, and may be undertaken at CEBAF [Bur89]. in the absence of such data, one must turn to predictions from theoretical models (see, for example. Ref. [Li82]) to estimate non-resonant isoscalar backgrounds which complicate the interpretation of the electroweak tests. Estimates of the resulting uncertainty are still needed, and some work in this direction is in progress.

From the foregoing discussion, it should be clear to the reader that semileptonic. .C scattering observables are sensitive to a variety of of physics issues (hadron structure. extensions of the Standard Model, nuclear dynamics) at potentially significant levels. At the same time, the tasks of choosing the appropriate combination of measurements and reducing the sources of theoretical uncertainty in order to draw meaningful conclusions from such measurements are non-trivial. In the near term, it appears that intermediateenergy, semileptonic . ${ }^{\circ} \mathrm{C}$ scattering is best suited as a probe of nucleon and nuclear structure - particularly nucleon strangeness. Its use to search for physics beyond the Standard Model awaits both the completion of the "first generation" of $\mathrm{NC}$ studies and progress on the theoretical issues outlined above. In the remainder of this article, we provide the rationale for these conclusions in greater detail. 


\section{FORMALISM}

The formalism for treating semileptonic electroweak interactions is straightforward and largely parallels the treatment of the electromagnetic interaction between charged leptons and hadrons based on quantum electrodynamics. Our goal here is to use this formalism in a way that emphasizes the physics issues summarized in the previous section. The first step will be to construct the relevant hadronic (both nucleon and nuclear) currents from the underlying fundamental electroweak interactions between leptons and quarks. Since the physics of interest generally requires one to consider effects at the $1 \%$ level. it will be necessary to take the theory beyond tree-level to include contributions from radiative corrections and other higher-order processes. Cpon taking nucleon matrix elements of the appropriately renormalized current operators, one encounters the interplay of strong and electroweak dynamics which complicates the interpretation of semilep nic scattering at low - and intermediate-energies. With the hadronic currents in hand. e turn to the specifics of parity-violating ( $\mathrm{PV}$ ) polarized electron scattering and neutrino scattering. We subsequently discuss the complications generated by the additional level of hadronic structure associated with nuclear $(A>1)$ targets.

\section{III.A. Currents and Couplings}

We begin with the fundamental couplings of an elementary fermion (lepton or quark) to the photon and $Z^{0}$ which we write. respectively, as

$$
i e Q_{f} \gamma_{\mu}
$$

and

$$
i \frac{g \cdot M_{Z}}{4 . M_{W}} \gamma^{\mu}\left(g_{v}^{f}+g_{A}^{f} \gamma_{s}\right) .
$$

Here $e$ and $g$ are the electromagnetic and weak coupling strengths. respectively, $Q_{f}$ is the electromagnetic charge of the fermion, and the vector and axial-vector "charges '. $g_{v}^{f}$ and $g_{A}^{f}$. respectively, are given by

$$
g_{v}^{f}=2 T_{3}^{f}-4 Q_{f} \sin ^{2} \theta_{W}
$$

and

$$
g_{A}^{f}=-2 T_{3}^{f}
$$

where $T_{3}^{f}$ is the third component of a weak isospin vector operator; acting on the weak isodoublets $\left(\begin{array}{c}\nu_{e} \\ e^{-}\end{array}\right),\left(\begin{array}{l}u \\ d\end{array}\right), \ldots, T_{3}^{f}$ gives $+\frac{1}{2}$ for the upper component and $-\frac{1}{2}$ for the lower. Thus, one obtains the values given in Table 3.1.

With these couplings, it is straightforward to compute the one-boson-exchange (photon or $Z^{0}$ amplitudes fo: scattering of a lepton from a hadronic electromagnetic (E.I) current anu weak neutral urrent (NC) associated with Figs. 3.1a and b, respectively:

$$
M^{E M}=\frac{4 \pi \alpha}{Q^{2}} Q_{\ell} \ell^{\mu} J_{\mu}^{E M}
$$




\begin{tabular}{|c|c|c|}
\hline \multicolumn{3}{|c|}{ TABLE 3.1 } \\
\hline fermion & $g_{v}^{f}$ & $g_{\lambda}^{f}$ \\
\hline$\nu_{e}, \nu_{\mu}$ & 1 & -1 \\
$e^{-} . \mu^{-}$ & $-1+4 \sin ^{2} \theta_{w}$ & 1 \\
$u, c . t$ & $1-f \sin ^{2} \theta_{w}$ & -1 \\
$d . s . b$ & $-1+\frac{f}{3} \sin ^{2} \theta_{w}$ & 1 \\
\hline
\end{tabular}

Table 3.1. Standard Model values (columns two and three) for the hadronic neutral current couplings of elementary fermions (first column). Note that the nomenclature introduced in Ref. [Don 79a] differs from that used in the present work: there the leptonic neutral current conpling were defined with respect to the quantities in the table by $a_{V}=g_{V}^{f}$ and $a_{A}=-g_{A}^{f}$.

and

$$
M^{N C}=-\frac{G_{\mu}}{2 \sqrt{2}}\left[g_{v}^{\ell} \ell^{\mu}+g_{\Lambda}^{\ell} \ell^{\mu S}\right]\left[J_{\mu}^{N C}+J_{\mu s}^{N C}\right]
$$

In Eq. (3.4) we have neglected the $Q^{2}$-dependence of the $Z^{0}$ propagator since $\left|Q^{2}\right| \ll . M_{Z}^{2}$ for all of the kinematics we consider in the present work. The PV component of the neutral current amplitude is

$$
M^{P V}=-\frac{G_{\mu}}{2 \sqrt{2}}\left[g_{V}^{\ell} \ell^{\mu} J_{\mu S}^{N C}+g_{\Lambda}^{\ell} \ell^{\mu S} J_{\mu}^{N C}\right]
$$

In these expressions, $Q_{\ell}, g_{V}^{\ell}$, and $g_{A}^{\ell}$ are the leptonic electromagnetic, vector, and axialvector charges, respectively, and $Q_{\mu} \equiv K_{\mu}-K_{\mu}^{\prime}$ is the four-momentum transfer with $K\left(K^{\prime \prime}\right)$ the initial (final) lepton four-momentum. Here, for reasons discussed in Sect. II.C.1, we have chosen to write $M^{N C}$ in terms of the Fermi constant for muon decay, $G_{\mu}$ instead of the weak coupling strength, $g$. At tree-level, these constants are related by

$$
G_{\mu}=\frac{g^{2}}{4 \sqrt{2} M_{w}^{2}}=\frac{\pi \alpha}{\sqrt{2} M_{w}^{2} \sin ^{2} \theta_{w}} .
$$

Once one works beyond tree-level, Eq. (3.6) must be modified to account for electroweak radiative corrections to muon decay, $\Delta r$ [Sir80]:

$$
G_{\mu}=\frac{\pi \alpha}{\sqrt{2} M_{w}^{2} \sin ^{2} \theta_{w}} \frac{1}{1-\Delta r} .
$$


The lepton vector and axial-vector currents. $\varepsilon^{\mu}$ and $\ell^{\mu 3}$. respectively. are just the Dirac currents

$$
\begin{gathered}
\ell^{\mu} \equiv \bar{u} \ell_{\gamma^{\mu}}^{\mu} u \ell \\
\ell^{\mu s} \equiv \bar{u}_{\ell} \gamma^{\mu} \gamma^{s} u \ell .
\end{gathered}
$$

where $u_{\ell}$ is the lepton spinor. Since the hadrons are composed of quarks. the currents $J_{\mu}^{E N} . J_{\mu}^{v c}$. and $J_{\mu 3}^{v c}$ are the hadronic matrix elements of the electromagnetic. vector. and axial-vector quark current operators:

$$
\begin{aligned}
J_{\mu}^{E M} & \equiv\left\langle H\left|\hat{J}_{\mu}^{E M}\right| H\right\rangle \\
J_{\mu}^{N C} & \equiv\left\langle H\left|\hat{J}_{\mu}^{v c}\right| H\right\rangle \\
J_{\mu 3}^{N C} & \equiv\left\langle H\left|\hat{J}_{\mu 3}^{N c}\right| H\right\rangle .
\end{aligned}
$$

where $|H\rangle$ is any hadronic state (in the present context a nucleon or a nucleus) and.

$$
\begin{gathered}
\hat{j}_{\mu}^{E M} \equiv \sum_{q} Q_{q} \bar{u}_{q} \gamma_{\mu} u_{q} \\
j_{\mu}^{N C} \equiv \sum_{q} g_{v}^{q} \bar{u}_{q} \gamma_{\mu} u_{q} \\
j_{\mu S}^{N C} \equiv \sum_{q} g_{\Lambda}^{q} \bar{u}_{q} \gamma_{\mu} \gamma_{S} u_{q},
\end{gathered}
$$

where the sums are over all quark flavors. $u, d, c, s, \ldots$

In what follows, we assume the structure of the hadronic states is dominated by the lighter quarks and limit the sums in Eqs. (3.10) to $q=u, d$, and $s$. The error introduced by neglect of the heavier quarks is expected to be of order $10^{-4}\left(10^{-2}\right)$ for the vector (axialvector) currents (see Eqs. (3.19)). With this truncation, it is convenient to decompose the current operators in terms of the $\mathrm{SU}(3)$ octet and singlet currents:

$$
\begin{gathered}
\hat{V}_{\mu}^{(a)} \equiv \bar{q} \frac{\lambda^{a}}{2} \gamma_{\mu} q \\
\hat{A}_{\mu}^{(a)} \equiv \bar{q} \frac{\lambda^{a}}{2} \gamma_{\mu} \gamma_{s} q .
\end{gathered}
$$

where $q$ represents the triplet of quarks, $q \equiv\left(\begin{array}{l}u \\ d \\ s\end{array}\right), \lambda^{0}=z 1$, and the $\lambda^{a}, a=1 \ldots \ldots 8$ are the Gell-Mann SU(3) matrices, normalized to $\operatorname{Tr}\left(\lambda^{a} \lambda^{b}\right)=2 \delta^{a b}$. (Note that no assumption of $\mathrm{SU}(3)$ syrnmetry is implied in the use of the $\mathrm{SU}(3)$ decomposition of the operators. Such an assumption enters only when SU(3) symmetry is employed to determine matrix 
elements of these operators. In the case of the electromagnetic and weak neutral currents. one requires only the six diagonal terms

$$
\begin{aligned}
& \dot{V}_{\mu}^{(0)}=\frac{1}{3}\left[\bar{u}_{\mu} u+d \gamma_{\mu} d+\bar{s}_{\gamma_{\mu}} s\right] \\
& \dot{l}_{\mu}^{(3)}=\frac{1}{2}\left[\bar{u} \gamma_{\mu} u-\dot{d} \gamma_{\mu} d\right] \\
& i_{\mu}^{\cdot(8)}=\frac{1}{2 \sqrt{3}}\left[\dot{u} \gamma_{\mu} u+d \gamma_{\mu} d-2 \bar{s} \gamma_{\mu} s\right] \\
& f_{\mu}^{(0)}=\frac{1}{3}\left[u \gamma_{\mu} \gamma_{s} u+d \gamma_{\mu} \gamma_{s} d+s \gamma_{\mu} \gamma_{s} s\right] \\
& \dot{A}_{\mu}^{(3)}=\frac{1}{2}\left[\bar{u} \gamma_{\mu} \gamma_{s} u-\bar{d} \gamma_{\mu} \gamma_{s} d\right] \\
& f_{\mu}^{(8)}=\frac{1}{2 \sqrt{3}}\left[\bar{u}_{\gamma_{\mu}} \gamma_{s} u+d \gamma_{\mu} \gamma_{s} d-2 \bar{s} \gamma_{\mu} \gamma_{s}\right] \text { ] }
\end{aligned}
$$

At the level of strong isospin. both the $0^{\text {th }}$ (singlet) and $8^{\text {th }}$ (octet) $\mathrm{SC}(3)$ components are "isoscalar" operators, while the $3^{\text {rd }}$ octet component is "isovector". The $3^{\text {th }}$ and $3^{\text {th }}$ components of the vector current are related to the isoscalar and isovector electromagnetic currents, respectively, by

$$
\begin{aligned}
& \hat{J}_{\mu}^{\Sigma M}(T=0)=\frac{1}{\sqrt{3}} \hat{V}_{\mu}^{(8)} \\
& j_{\mu}^{\Sigma M}(T=1)=\hat{V}_{\mu}^{(3)} .
\end{aligned}
$$

Since we wish to emphasize the strange-quark contribution to various processes. it is useful to note the following relationship between the two isoscalar currents:

$$
\begin{aligned}
& \hat{V}_{\mu}^{(0)}=\frac{2}{\sqrt{3}} \dot{V}_{\mu}^{(8)}+\dot{V}_{\mu}^{(0)} \\
& \dot{A}_{\mu}^{(0)}=\frac{2}{\sqrt{3}} \dot{A}_{\mu}^{(8)}+\dot{A}_{\mu}^{(0)} .
\end{aligned}
$$

where

$$
\begin{gathered}
\dot{V}_{\mu}^{(s)} \equiv \bar{s} \gamma_{\mu} s \\
\dot{A}_{\mu}^{(s)} \equiv \bar{s} \gamma_{\mu} \gamma_{s} s .
\end{gathered}
$$

With these relations we can re-write the weak neutral currents of Eqs. (3.10) in the following convenient forms:

$$
\hat{J}_{\mu}^{N C}=\xi_{V}^{T=1} \hat{J}_{\mu}^{E M}(T=1)+\sqrt{3} \xi_{V}^{T=0} \hat{J}_{\mu}^{E M}(T=0)+\xi_{V}^{(0)} \hat{V}_{\mu}^{(0)}
$$

and

$$
j_{\mu S}^{N C}=\xi_{A}^{T=1} \hat{A}_{\mu}(T=1)+\xi_{A}^{T=0} \hat{A}_{\mu}^{(8)}+\xi_{A}^{(0)} \hat{A}_{\mu}^{(0)}
$$


where

$$
\begin{gathered}
\xi_{V}^{T=1}=g_{V}^{u}-g_{v}^{d} \\
\xi_{V}^{T=0}=\sqrt{3}\left(g_{V}^{u}+g_{v}^{d}\right) \\
\xi_{V}^{(0)}=g_{V}^{u}+g_{V}^{d}+g_{V}^{d} \\
\xi_{A}^{T=1}=g_{A}^{u}-g_{A}^{d} \\
\xi_{A}^{T=0}=\sqrt{3}\left(g_{A}^{u}+g_{A}^{d}\right) \\
\xi_{A}^{(0)}=g_{A}^{u}+g_{A}^{d}+g_{A}^{d} .
\end{gathered}
$$

The extra factor of $\sqrt{3}$ appearing in Eq. (3.16) but not in Eq. (3.17) results from the use of the relation between the octet and isoscalar E.M currents in Eq. (3.13a).

IViting the hadronic neutral current in the form of Eqs. (3.16) and $(3.17$ ) delineates between the physics associated with the underlying electroweak gauge theory and the hadronic physics associated with the quark currents. The content of the former is contained in the couplings $\xi_{v . . t}^{(a)}$. whereas hadronic effects enter via matrix elements of the latter. At tree-level, the couplings $\xi_{v}^{(a)}$ are determined by the underlying electroweak gauge theory. via Eqs. (3.18). In Table 3.2 we give their values in the Standard. Model as well as their relation to other hadronic $\mathrm{NC}$ couplings defined in the literature. Fote that in earlier treatments, possible contributions from s-quarks were ignored. so that no equivalents to $\xi_{V}^{(0)}$ and $\xi_{A}^{(0)}$ are listed.

From Eqs. (3.16) and (3.17) one sees that, given a measurement of $J_{\mu}^{*} C\left(J_{\mu s}^{v C}\right)$ and independent determinations of the vector (strong) isoscalar E.M, isovector E.M . and jquark (axial-vector isoscalar, octet, and s-quark) current matrix elements, it is in principle possible to extract the $\xi_{V}^{(a)}\left(\xi_{A}^{(a)}\right)$ and thereby perform an electroweak test. As discussed in Sect. III.B below, these couplings depend on higher-order electroweak processes in the Standard Model. hadronic-structure effects, and possible contributions from extensions of the Standard Model, such as those characterized by the $S$ and $T$ parameterization discussed in Sect. II.C. The extent to which one might place constraints on the latter, however, depends on both one's ability to calculate the hadronic effects appearing in the IC couplings as well as the precision with which one may determine the E.M. octet. and strangeness matrix elements associated with the right hand side of Eqs. (3.16) and (3.1T).

Another class of hadronic contributions to the hadronic $\mathrm{NC}$ couplings arises from heavy-quark $(c, b, t)$ renormalizations of the light quark current operators. The scale of these contributions has been estimated in Ref. [Kap88] following the effective theory approach discussed in Sect. II.A (see especially Fig. 2.1). They may be included in the $R_{V, A}$ as follows:

$$
\begin{aligned}
R_{v}^{(0)} & \rightarrow R_{v}^{(0)}(\text { ewk })-\Delta_{v} \\
R_{v}^{T=0} & \rightarrow R_{v}^{T=0}(\text { ewk })-\Delta_{v} \\
R_{A}^{(0)} & \rightarrow R_{A}^{(0)}(\text { ewk })+2 \Delta_{A} \\
R_{A}^{T=0} & \rightarrow R_{A}^{T=0}(\text { ewk })+\Delta_{A},
\end{aligned}
$$




\begin{tabular}{|c|c|c|c|}
\hline \multicolumn{4}{|c|}{ TABLE 3.2} \\
\hline Coupling & $\begin{array}{l}\text { Hung and } \\
\text { Sakurai }\end{array}$ & $\begin{array}{c}\text { Donnelly and } \\
\text { Peccei }\end{array}$ & $\begin{array}{l}\text { Standard } \\
\text { Model }\end{array}$ \\
\hline $\begin{array}{c}\xi_{i}^{(0)} \\
\sqrt{3} \xi \xi_{i}^{T=0} \\
\xi T=1\end{array}$ & $\begin{array}{l}-6 \dot{\gamma} / g_{A}^{e} \\
-2 \dot{\alpha}_{/}^{\prime} g_{\Lambda}^{e}\end{array}$ & $\begin{array}{l}23_{V}^{(0)} \\
23_{V}^{(1)}\end{array}$ & $\begin{array}{c}-\left(1+R_{v}^{(0)}\right) \\
-4 \sin ^{2} \theta_{w}\left[1+R_{v}^{T=0}\right) \\
2\left(1-2 \sin ^{2} \theta_{w}\right)\left[1+R_{v}^{T=1}\right)\end{array}$ \\
\hline $\begin{array}{l}\xi_{A}^{(0)} \\
\xi_{A}^{T=0} \\
\xi_{A}^{T=1}\end{array}$ & $\begin{array}{l}2 \sqrt{3} \dot{\delta} / g_{v}^{e} \\
-2 \dot{\jmath} / g_{v}^{e}\end{array}$ & $\begin{array}{c}-2 / \sqrt{3} 3_{A}^{(0)} \\
-23_{A}^{(1)}\end{array}$ & $\begin{array}{c}1+R_{A}^{(0)} \\
\sqrt{3} R_{A}^{T=0} \\
-2\left[1+R_{A}^{T=1}\right]\end{array}$ \\
\hline
\end{tabular}

Table 3.2. Hadronic weak neutral current couplings. Columns two and three give equivalent couplings in the notation of Refs. [Hun 76) and (Don 79a), respectively. Col. umn four gives Standard Model values, including effects of higher-order contributions $\left(R_{v}\right)$. At tree-level, one has $R_{V, A}=0$.

where $\Delta_{V} \lesssim 10^{-4}$ and $\Delta_{A} \sim 10^{-2}$ according to Ref. [Kap88) and where the $R_{V}($ ewk) are contributions from higher-order electroweak processes.

Information on the matrix elements of the currents entering the right-hand side of Eqs. (3.16) and $(3.17)$ can be obtained from a variety of sources. Information on the E.II matrix elements is obtainable from parity-conserving $(P C)$ electron scattering, while a determination of the axial-vector octet matrix elemests may be performed using nuclear beta-decay and hyperon semileptonic decays. As noted in Sect. II.A, much less is presently know about the strangeness matrix elements, and their determination constitutes one of the chief goals of the experiments discussed in the present work. The strategy for doing so follows from the form of Eqs. (3.16) and (3.17). If the $\xi$ 's as well as EM and octet axialvector matrix elements are all taken as inputs from other experiments, then determinations of the NC matrix elements would allow extraction of information on the s-quark currents. Discussion of different targets and kinematic conditions that might be used for this purpose makes up the bulk of Sect. IV below. Once the s-quark matrix elements are sufficiently constrained it could be possible to perform independent determinations of the $\xi$ 's by going to the appropriate kinematic regime. As indicated by the hypothetical constraints on "non-standurd" physics of Fig. 2.4, one has reason to contemplate such a program. In what follows, we discuss in more detail how such new physics appears in the hadronic NC couplings. 


\section{III.B. Higher-order Processes and Renormalization}

As indicated by the foregoing discussion, the corrections $R_{V}$ t to the tree-level expressions for the couplings $\xi_{v+}^{(a)}$ receive a variety of contributions: electroweak radiative corrections within the framework of the Standard .lodel. corrections generated by physics beyond the Standard . Model. and hadronic physics effects of various types. We have already considered one contribution of the latter type. namely, the correction generated by neglect of $c . b$. and $t$ quarks in an effective theory approach. In this section. we discuss other higher-order contributions germane to analysis of the experiments of interest.

\section{Standard Model Radiative Currections}

Vaijvely, one might assume higher-order processes involving multiple photon and or $Z^{0}$ exchanges as in Figs. 3.2 to be suppressed by at least $\alpha / 4 \pi \approx 10^{-3}$ relative to the simple single-photon or single- $Z^{0}$ exchanges of Fig. 3.1. Thus. one might hope to be able to neglect these effects without introducing significant error in interpreting semileptonic processes. However. such an assumption breaks down in at least two ways. First. calculations of one-loop electroweak corrections may be significantly enhanced over their generic $a / 4 \pi$ scale due to the presence of large logarithms of the form $\ln \left(. M_{W} / p_{f}\right)$. where $p_{f}$ is a momentum scale (or mass) characteristic of one of the scattering leptons or quarks. In addition, there exist cases where the tree-level contribution is suppressed relative to the generic scale of tree-level $\mathrm{NC}$ amplitudes. One case is obvious from Table 3.2: $\xi_{-4}^{T=0}=0$ at tree level in the Standard Model so that the existence of such a term depends on higherorder processes. In two other cases, PV observables associated with charged-lepton probes of the $\left\langle H\left|J_{\mu s}^{\vee c}\right| H\right\rangle$ and processes which involve the neutral current charge form factor of the proton. the tree-level amplitudes are proportional to $1-4 \sin ^{2} \theta_{w} \approx 0.08$. Higher-order contributions need not carry this small factor and, thus, may be relatively more important than one naively expects.

An in-depth treatment of theoretical estimates of the higher-order terms can be found elsewhere [Sir80, Mar80, Mar81, Aok82, Mar 83, Mar84, Ros90 and references therein]: we limit ourselves to discussing briefly the main physics issues and quoting typical results. The problem of calculating electroweak radiative corrections to tree-level semileptonic amplitudes is considerably more complicated than in the case of purely leptonic scattering. In the latter instance, the scattering leptons interact only electroweakly, so that once a renormalization framework is chosen and all parameters of the electroweak model determined, the theory makes precise and unambiguous predictions for higher-order leptonic processes. Complications arise in semileptonic scattering from the interplay of the strong and electroweak interactions involving complex intermediate states characterizing the structure of the hadronic target. In high-energy processes, such as $e^{+} e^{-}$annihilation or deep inelastic scattering, one can reliably estimate strong interaction structure effects by treating hadronic quarks as quasi-free and using perturbative QCD. Such a first-principles approach breaks down, however, for medium- and low-energy processes $\left(\left|Q^{2}\right|<1(\mathrm{GeV} / \mathrm{c})^{2}\right)$ where the strong coupling $\alpha_{g}\left(Q^{2}\right)$ becomes large. In this regime, one is generally forced to rely on hadronic models in order to account for strong interaction effects. In the case 
of the vector currents, one may eliminate much of the hadronic uncertainty by employing Eq. (3.13) and experimentally determined matrix elements of the E.M currents. In other cases, however, model-dependence introduces an intrinsic theoretical uncertainty into estimates of the higher-order terms.

In order to delineate between pure Standard. Model electroweak corrections and those containing hadronic physics uncertainties, it is convenient to consider first corrections to scattering involving a single quark at a time (Fig. 3.3). Calculations of these "one-quark" amplitudes closely parallel the treatment of higher-order corrections in purely leptonic processes. The effect of the one-quark contributions is to renormalize the $\xi$ 's appearing in the current operators in a manner independent of the target's structure. However. since the quarks from which the lepton scatters are confined to a hadron (e.g. the nucleon' with a radius of about $1 \mathrm{fm}$, one ought to account for confinement where appropriate in renormalizing the quark current operators. To this end, it is reasonable to suppress contributions to loops from momenta $\lesssim 1 / R_{\text {nucleon }}$ through the use of constituent. rather than current. quark masses $\left(\mathrm{K}_{u, d} \sim 330 \mathrm{MeV}\right)$ in the quark propagators. In all other respects, the quark fields appearing in Eqs. (3.10) are those associated with the current quarks of the QCD lagrangian. The procedure for treating internal quark loops (e.g.. Fig. 3.4), in which the virtual $\vec{q} q$ pair need not be confined to the hadron interior. is somewhat different, as discussed below.

At this one-quark level, several theoretical issues should be mentioned. First is the choice of renormalization scheme. Since the use of the classical, or tree-level. theory to compute higher-order processes results in amplitudes which are infinite, the starting theory must be re-defined in such a way as to generate finite, higher-order amplitudes. The prescription followed in performing this re-definition is the "renormalization scheme". Because calculations of electroweak amplitudes are performed only to finite order in perturbation theory, estimates obtained under different renormalization schemes will, in general. differ slightly [Jeg89]. We follow the so-called "on-shell" renormalization scheme (OSR). which represents a natural extension of the prescription generally followed in renormalizing QED. In this scheme, poles in the renormalized propagators occur at physical particle masses and elementary vertices are renormalized with all particles on-shell. Moreover. $\sin ^{2} \theta_{w}$ is defined in terms of the vector boson masses (Eq. (2.4)). The independent parameters in the theory are $\left(\alpha, M_{z}, G_{\mu}\right)$ in the gauge boson sector and the fermion and Higgs masses in the remaining sectors. All other parameters (including $M_{w}$ and $\sin ^{2} \theta_{w}$ ) are determined as functions of these parameters.

Another widely used scheme is the so-called "MS-bar" ( $\overline{\mathrm{MS}})$, or modified minimal subtraction, scheme. In contrast to the OSR prescription, the $\overline{\mathrm{MS}}$ procedure defines finite inverse propagators and vertices by subtracting only the divergent parts of the loops. without placing physical requirements on the finite remainders. Moreover, the weak mixing angle in this scheme carries a dependence on a renormalization mass scale $\mu$ (typically.$_{z}$ or $M_{w}$ ) and is no longer simply related to the vector boson masses as in Eq. (2.4):

$$
\sin ^{2} \theta_{w}=1-\frac{M_{w}^{2}}{M_{z}^{2}} \longrightarrow \sin ^{2} \hat{\theta}_{w}(\mu)
$$


where $\sin ^{2} \hat{\theta}_{w}(\mu)$ defines the $\overline{\mathrm{VIS}}$ weak mixing angle. The $\overline{\mathrm{WS}}$ prescription has the attraction that $\sin ^{2} \hat{\theta}_{w}(\mu)$ is straightforwardly related to the running E.I and semi-weak couplings $e(\mu)$ and $g(\mu)$ arising in grand-unified theories [Mar81]. .Moreover. the $R_{V \cdot A}(\overline{\mathrm{VIS}})$ display a weaker dependence on the presently undetermined top-quark mass than do the $R_{V A}(\mathrm{OSR})$. On the other hand. OSR has the advantages of a simple, scale-independent definition of $\sin ^{2} \theta_{w}$ and of being a straightforward extension of the procedure conventionally followed in QED. There exist at least two other prescriptions used in the literature: the so-called "*-scheme" of Refs. [Ken89] and the scheme followed in Ref. [Con89]. In these latter schemes. the weak-mixing angle depends on the value of $Q^{2}$ at which a given process is studied. whereas in OSR, $\sin ^{2} \theta_{w}$ is independent of momentum transfer and the entire $Q^{2}$-dependence is carried by the $R_{V, A}$.

$A$ second significant issue alluded to abor is the dependence of electroweak amplitudes o: the unknown Higgs and top-quark. isses. This dependence enters the electroweak scattering amplitudes in two ways. First, $\sin ^{2} \theta_{w}$-dependent Born-level amplitudes vary with.$I_{H}$ and $m_{t}$ when $\sin ^{2} \theta_{W}$ is determined using relation (2.ja). Top-quark and Higgs loops in muon decay amplitudes induce an $\left(M_{H}, m_{t}\right)$ dependence in $\Delta r$ appearing in Eq. (2.5a) and, consequently, in $\sin ^{2} \theta_{w}$. Second, top-quark and Higgs loops in the neutral current amplitudes themselves also introduce a dependence on the unknown masses. The dependence on $m_{t}$ is stronger than the dependence on $M_{H}$; the latter enters only logarithmically, while top-quark loops introduce terms of order $\left(m_{t} / . V_{W}\right)^{2}$ in the vector boson propagators. For $m_{t}>M_{\boldsymbol{w}}$, these terms become significant.

Third, it is important to bear in mind that the electroweak corrections $R_{V \cdot A}$ (ewk) depend on both the species of lepton probe as well as the $Q^{2}$ of the process under consideration. In general, the corrections for electron scattering differ from those for neutrino processes. This distinction is particularly important in the interpretation of probes of the isoscalar. axial-vector hadronic $\mathrm{NC}$, as we discuss below. As for the dependence on momentum transfer, the $R_{V, A}$ vary rather gently with $Q^{2}$. According to Ref. [Mar80], for example. the corrections for neutrino scattering $R_{V}^{T=0}, R_{V}^{T=1}$, and $R_{V}^{n}$ (defined in Eq. 3.25b) change by $\lesssim 0.001$ over the range $0 \leq\left|Q^{2}\right| \leq 20(\mathrm{GeV} / c)^{2}$, while the variation in $R_{V}^{p}$ (defined in Eq. 3.25a) is $\sim 0.01$ over the same $Q^{2}$-range (calculated assuming $M_{H}=10 M_{z}$ and $m_{t}=18 \mathrm{GeV}$ ). For the medium-energy experiments under consideration here, corrections of this order are negligible. More significant for semileptonic experiments is the much greater $Q^{2}$-dependence of the hadronic form factors appearing in matrix elements of the quark current operators. In the case of elastic $\vec{e} p$ scattering, for example, one must know the $Q^{2}$ behavior of the neutron electromagnetic charge form factor, $G_{E}^{n}\left(Q^{2}\right)$, in order to determine the longitudinal proton neutral current response (see discussion of Sect. IV.A below). The uncertainty in $G_{\varepsilon}^{n}\left(Q^{2}\right)$ is significantly larger than any error incurred by neglecting the $Q^{2}$-dependence of the lepton-quark amplitudes and represents a much more serious issue for the interpretation of high-precision experiments.

Fourth, we note that one class of diagrams not included in the calculations cited below are those corresponding to bremsstrahlung from either of the scattering fermions. Formally, the inclusion of such diagrams is required to cancel infrared divergences in the one-loop amplitudes [Kin62, Lee64]. The finite remainders, however, depend on details of 
the experimental configuration, such as detector resolution. Hence, one requires detailed knowledge of the specific experiment before arriving at an estimate of bremsstrahlung contributions. In certain special cases, such as scattering from $\left(0^{+} 0\right)$ targets, bremsstrahlung cancels from the PV asymmetry, thereby simplifying the theoretical interpretation. We also point out that the interpretation of $\mathrm{PV}$ electron scattering asymmetries requires knowledge of QED radiative corrections as well those which renormalize the $\mathrm{NC}$ couplings of Table 3.2. The QED corrections for PC electron scattering have been worked out in detail elsewhere [.Mo69].

Fifth, in order to make contact with notation used elsewhere in the literature. the radiative corrections for atomic $\mathrm{PV}$ are sometimes written in the form (see also Eqs. (3.24)

$$
\begin{aligned}
& \xi_{V}^{p}=2 C_{1 p}=\rho_{P V}^{\prime}\left[1-4 \kappa_{P V}^{\prime}(0) \sin ^{2} \hat{\theta}_{w}\left(M_{z}^{2}\right)\right] \\
& \xi_{V}^{n}=2 C_{1 n}=\rho_{P V}^{\prime}
\end{aligned}
$$

where the $C_{1 p}\left(C_{1 n}\right)$ are the neutral vector current couplings (normalized differently than in the present work) and where the radiative corrections are contained in the parameters $\rho_{P V}^{\prime}$ and $\kappa_{P V}^{\prime}(0)$. The corrections for $Q^{2} \neq 0$ are obtained by replacing $\kappa_{P V}^{\prime}(0) \rightarrow \kappa_{P V}^{\prime}\left(Q^{2}\right)$ (the $Q^{2}$-dependence of $\rho_{P V}^{\prime}$ is negligible). This parameterization of the radiative corrections is motivated by a "factorization" of the corrections into two general classes: (a) corrections associated with the $\mathrm{SU}(2)_{L}$ components of the weak currents, and (b) those associated with the mixing of the $\mathrm{SU}(2)_{L}$ and $\mathrm{U}(1)_{Y}$ sectors. The $\rho_{P V}^{\prime}$ and $\kappa_{P V}^{\prime}$ differ from the $\rho$ and $\kappa$ terms appearing in neutrino scattering amplitudes by process-dependent terms. Writing $\rho_{P V}^{\prime}=1+\delta \rho$ and $\kappa_{P V}^{\prime}=1+\delta \kappa$, the $R_{V}$ can be written as

$$
\begin{aligned}
R_{v}^{T=0} & =\delta \rho+\delta \kappa \\
R_{v}^{T=1} & =\delta \rho-\left[\frac{2 \sin ^{2} \theta_{w}}{\left(1-2 \sin ^{2} \theta_{w}\right)}\right] \delta \kappa \\
R_{v}^{p} & =\delta \rho-\left[\frac{4 \sin ^{2} \theta_{w}}{\left(1-4 \sin ^{2} \theta_{w}\right)}\right] \delta \kappa \\
R_{v}^{n} & =\delta \rho .
\end{aligned}
$$

Our rationale for writing corrections in terms of the $R$ rather than $\rho$ and $\kappa$ is to facilitate comparison of electroweak radiative corrections with other contributions (e.g., the $\Delta_{V, A}$ of Eqs. (3.19)) which do not simply factorize into contributions associated with the two gauge groups.

With the aforementioned considerations in mind, we note the scale of typical results and refer the reader to the literature for more detail. In the OSR scheme, one has that the $R_{V}^{(a)}$ (1-quark) are on the order of $1-5 \%$ at $m_{t}=120 \mathrm{GeV}$ and $M_{H}=100 \mathrm{GeV}$ for both electron and neutrino scattering. For the axial-vector corrections, on the other hand, $R_{A}^{(a)}(1$-quark $) \sim$ few percent for neutrino scattering, significantly smaller than for electron 
scattering where $R_{A}^{(a)}(1$-quark $) \sim 25-50 \%$, due to the logarithmic enhancements and treelevel suppression factors mentioned above. This result has important consequences for the interpretation of observables containing $\left\langle H\left|J_{\mu 5}^{N C}\right| H\right\rangle$. For comparison, one has in the $\overline{\text { IIS }}$ scheme

$$
\begin{aligned}
R_{V}^{p} & =-0.054 \pm 0.033 \\
R_{v}^{n} & =-0.0143 \pm 0.0004 \\
R_{v}^{T=1} & =-0.017 \pm 0.002 \\
R_{V}^{T=0} & =-0.0113
\end{aligned}
$$

for $. W_{H}=100 \mathrm{GeV}$ and $m_{t}=140 \mathrm{GeV}$, where the values have been extracted from Ref. [Mar90] and where the uncertainties include estimated uncertainties in hadronic contributions (see below). Although the values were computed for atomic PV, they should not differ appreciably for PV electron scattering given the gentle $Q^{2}$-dependence of the $R_{V, A}$.

\section{Hadronic Contributions}

Higher-order processes which depend on both the electroweak and strong interactions generally introduce some degree of theoretical uncertainty into the $R_{V, A}$ due to the present incalculability of strong interaction effects at low-momentum scales from first principles in QCD. Such effects enter higher-order corrections in two ways: (a) via "internal" quark loops (Fig. 3.4), in which the quarks in the virtual $q \bar{q}$ pair interact strongly with each other (Fig. 3.4a) or with quarks in the target (Fig. 3.4b); and (b) via strong interactions among the valence quarks of the target, thereby introducing hadronic intermediate states into the higher-order electroweak amplitudes (Fig. 3.5). In the case of internal loops, the effects of strong interactions of Fig. 3.4a may be estimated using a dispersion theory analysis of $e^{+} e^{-}$ annihilation. Such an approach has been followed by the authors of Refs. [Mar84, Deg89]. The scale of this uncertainty, which is included in the error appearing in Eqs. (3.22), is given by these authors to be $\delta R_{V}^{(T)} \approx \pm 0.002$. Since this uncertainty is target-independent, one may associate it conceptually with the 1-quark corrections. In contrast, contributions of the type in Fig. 3.4b depend on the target, and no estimate of their scale has been performed to date.

Whereas the 1-quark corrections effectively renormalize the quark current operators of Eqs. (3.10ff), contributions involving strong interactions among the target quarks (Fig. 3.5) arise only in hadronic matrix elements of these operators. We label the latter "manyquark" hadronic corrections to distinguish them from corrections requiring no knowledge of target structure. The present intractability of a first-principles estimate of $R_{V, A}($ had) requires one to rely on phenomenological models, which necessarily introduces considerable theoretical uncertainty. While more theoretical work on this topic is needed, estimates of 


\begin{tabular}{|c|c|c|c|c|}
\hline \multicolumn{5}{|c|}{ Table 3.3 } \\
\hline Source & $\begin{array}{c}\sqrt{3} R_{A}^{T=0} \\
\text { (best) }\end{array}$ & $\begin{array}{c}R_{A}^{T=1} \\
\text { (best) }\end{array}$ & $\begin{array}{c}\sqrt{3} R_{A}^{T=0} \\
\text { (range) }\end{array}$ & $\begin{array}{c}R_{A}^{T=1} \\
\text { (range) }\end{array}$ \\
\hline $\begin{array}{c}\text { Standard Nodel } \\
\text { Hadronic }\end{array}$ & -0.43 & -0.47 & - & - \\
\hline total & -0.19 & 0.13 & $-0.43 \rightarrow 0$ & $-0.07 \rightarrow 0.31$ \\
\hline & -0.62 & -0.34 & $-0.86 \rightarrow-0.43$ & $-0.54 \rightarrow-0.16$ \\
\hline
\end{tabular}

Table 3.3. Estimates of Standard Model (one-quark) and hadronic contributions to the $R_{A}^{(T)}$ for PV electron scattering. "Best" values in hadronic contributions correspond to use of theoretical "best" estimates of Ref. [Des80] for weak meson-nucleon vertices appearing in Fig. 3.6. Ranges (columns 4 and 5) correspond to theoretical ranges in these couplings. One-quark contributions are evaluated at $m_{t}=120 \mathrm{GeV}$ and $M_{H}=100 \mathrm{GeV}$ in the OSR scheme.

$R_{A}^{(T)}$ (had) for PV electron scattering have been performed by the authors of Ref. (Mus90!. based on the loop and pole contributions of Fig. 3.6. Results are summarized in Table 3.3.

While the $R_{A}^{(T)}$ (had) receive contributions from a plethora of diagrams not considered in Ref. [Mus90], these results ought to indicate bot! the scale of hadronic effects as well as the degree of theoretical uncertainty. From Table 3.3, one observes that the resultant hadronic uncertainty may be as large as the Standard Model contributions to $R_{A}^{(T)}$ (1-quark). Note that column four gives hadronic uncertainty in the induced isoscalar axial-vector current for PV electron scattering. These results have important consequences for the interpretation of PV electron scattering experiments, as discussed in Sect. IV. Moreover, since it is unlikely that theoretical uncertainty will be significantly reduced in the foreseeable future, one would ideally like to determine these corrections experimentally. Possibilities for doing so are also discussed in Sect. IV.

Another type of many-quark diagram, the "dispersion" correction, involves multiboson exchanges between lepton and hadron as shown in Fig. 3.7. The difficulty in reliably computing this contribution derives from the lack of knowledge of the structure of the hadronic intermediate states. One does not expect this difficulty to be relevant to diagrams involving the exchange of two heavy vector bosons, since the corresponding amplitudes are dominated by large loop momenta $\left(k_{\text {loop }} \sim M_{w}\right)$. Since such diagrams generate the dominant dispersion corrections for neutrino scattering, theoretical uncertainties in neutrino dispersion corrections should be tolerably small. For amplitudes involving charged lepton probes where at least one of the exchanged bosons is a photon, however, the loop integrals no longer need be dominated by large momenta, so that contributions 
from low-lying target structure may be important. At the one-quark level. for example. the PV $e-q$ amplitudes arising form the $Z^{0}-\gamma$ box graph contain large logarithms of the form $\ln |s| / \mathcal{L}_{w}^{2}$ and $\ln |u| / \mathrm{W}_{w}^{2}$. where the Mandelstam variables $s$ and $u$ depend on the momenta of the lepton probe and target quark. Such a logarithmic dependence on widely different momentum scales indeed suggests the possible importance of low-lying target structure. In the case of two- $\gamma$ exchange, theoretical estimates of $e p$ dispersion corrections suggest that hadronic contributions do not difier significantly from the generic $\mathrm{O}(\alpha / 4 \pi)$ scale [Dre59. Gre69]. Experimental determinations of PC ep dispersion corrections are consistent with this conclusion. though the level of precision is not sufficient to constrain theoretical predictions. The situation appears rather different for scattering from nuclei. where data from recent measurements on ${ }^{12} \mathrm{C}$ suggests significant many-body enhancement of $\mathrm{PC}$ dispersion corrections over their one-body $(e p)$ scale. This situation is discussed more fully in Sect. III.D.

Dispersion corrections to $\mathrm{NC}$ e.V amplitudes have received less theoretical attention. and there exists no experimental information in this case. The authors of Ref. [Mar84] have estimated hadronic contributions to the $Z^{0}-\gamma$ box amplitude in atomic PV by including only nucleon intermediate states. The resultant corrections to the tree-level PV vector current amplitudes are $R_{v}^{p} \approx 0.013$ and $R_{v}^{n} \approx-0.0006$. The corresponding contributions to $R_{V}^{T=0}$ and $R_{v}^{T=1}$ are on the order of a few tenths of a percent. No study has been made of many-body contributions, which might result in enhancements over the one-body scale similar to those seen in $\mathrm{PC}$ scattering from carbon.

With these remarks in mind, we note several general features of dispersion corrections as they enter PV electron scattering. The relevant quantity for the experiments of interest here is tho difference

$$
R_{v, A}^{\text {disp }} \approx R_{v, A}^{\gamma Z^{0}}-R_{v}^{\gamma \gamma}
$$

where $R_{V, A}^{\gamma Z^{0}}\left(R_{V}^{\gamma \gamma}\right)$ are the dominant dispersion corrections to the $\mathrm{PV}(\mathrm{PC})$ electron scattering amplitudes. The reason one is interested in the difference is that the asymmetry measured in PV electron scattering is governed by the ratio of $\mathrm{NC}$ to E.M amplitudes (see Sect. III.D). Although one might hope for significant cancellation between these two corrections, there exist several reasons why a non-negligible difference may occur. First, electromagnetic and neutral current dispersion corrections display different $Q^{2}$-dependences at low $-Q^{2}$. The one-photon exchange diagram has a pole at $Q^{2}=0$, whereas the twophoton box-diagram cannot have such a pole, since it is one-particle irreducible. The neutral current dispersion correction, however, need not vanish at $Q^{2}=0$ since the treelevel $Z^{0}$-exchange amplitude has a pole at $Q^{2}=M_{z}^{2}$ rather than $Q^{2}=0$. Additional differences follow from the structure of the diagrams. The $Z^{0}-\gamma$ box diagrams, for example, generate a different weighting of loop momentum than occurs in the 2- $\gamma$ exchange graphs, due to the presence of a heavy vector boson propagator in the loop. Consequently, transitions to different intermediate hadronic or leptonic states are weighted differently in the two cases. Moreover, the isospin content of the neutral vector current transition matrix elements to hadronic intermediate states is not the same as for the electromagnetic current, due to the different isospin structure of the two currents. Given these features, along with experimental results for $R_{v}^{\gamma \gamma}$ for ${ }^{12} \mathrm{C}$, one has reason to invest addition theoretical effort 
in order better understand this higher-order process. Such study is especially warranted if one hopes to extract information on "new" physics from the $R_{V A}$.

Don-standard Physics

As discussed in Sect. II. there exists a variety of scenarios for extending the Standard Model beyond the minimal $\left.\mathrm{SU}^{(2}\right)_{L} \times \mathrm{U}(1)_{Y}$ Weinberg-Salam theory. While space does not permit an extensive review of different scenarios and useful discussions may be found in the literature (see, e.g. (Ama87]), we return to the $S, T$ framework to illustrate how non-standard physics could appear in the observables of interest here. to determine what level of experimental precision is needed in these observables to provide for interesting electroweak tests. and to set the corresponding scale of maximum tolerable theoretical uncertainty (e.g., from hadronic contributions) in their interpretation. To that end. we focus on the vector current corrertions $R_{v}^{(a)}$ which, owing to current conservation and one s knowledge of the $J_{\mu}^{E M}$. appear to contain smaller hadronic physics uncertainties than the $R_{A}^{(a)}$. Since we subsequently consider PV electron scattering from the proton in Sect. IV.A. we define the corresponding correction $R_{v}^{p}$ as

$$
\xi_{v}^{p}=\frac{1}{2}\left[\xi_{V}^{T=1}+\sqrt{3} \xi_{v}^{T=0}\right]=\left(1-4 \sin ^{2} \theta_{w}\right)\left[1+R_{v}^{p}\right]
$$

where $\xi_{v}^{p}$ is the $\mathrm{NC}$ coupling to the proton at $Q^{2}=0$. Likewise we have

$$
\xi_{V}^{n}=\frac{1}{2}\left[-\xi_{V}^{T=1}+\sqrt{3} \xi_{V}^{T=0}\right]=-\left[1+R_{v}^{n}\right]
$$

for neutrons. These quantities are related through the following:

$$
\begin{aligned}
& R_{v}^{p}=\frac{1}{1-4 \sin ^{2} \theta_{w}}\left[\left(1-2 \sin ^{2} \theta_{w}\right) R_{v}^{T=1}-2 \sin ^{2} \theta_{w} R_{v}^{T=0}\right] \\
& R_{v}^{n}=\left(1-2 \sin ^{2} \theta_{w}\right) R_{v}^{T=1}+2 \sin ^{2} \theta_{w} R_{v}^{T=0} .
\end{aligned}
$$

Following Ref. [Mar90], it is straightforward to determine the dependence of the $R_{V}^{(a)}$ on $S$ and $T$ [Mus93c]:

$$
\begin{aligned}
R_{v}^{T=0}(\text { new }) & \approx 0.016 S-0.003 T \\
R_{v}^{T=1}(\text { new }) & \approx-0.014 S+0.017 T \\
R_{v}^{p}(\text { new }) & \approx-0.206 S+0.152 T \\
R_{v}^{n}(\text { new }) & \approx 0.0078 T .
\end{aligned}
$$

Note that these relations are arrived at within the $\overline{\mathrm{MS}}$ renormalization scheme and that a nonzero value of $T$ would signal a top-yuark mass different from $140 \mathrm{GeV}$. It is also worth noting that the relatively larger sensitivity of $R_{V}^{p}$ to $S$ and $T$ is a consequence of the small scale of the leading-order proton coupling (see Eq. (3.24)). 
The prospective constraints on $S$ and $T$ shown in Fig. 2.4 are derived from Eq. 3.26 . assuming a $1 \%$ determination of $\xi_{v}^{T=0}$ from elastic $\mathrm{PV} C\left(\vec{e}, e^{\prime}\right)$ scattering, a $10 \%$ determination of $\xi_{V}^{p}$ from $A_{L R}(\vec{e} p)$. and a $10 \%$ extraction of $\xi_{V}^{T=1}$ from $A_{L R}(. \rightarrow \supset)$. While the first two of these prospective determinations could nicely complement results from cesium atomic $\mathrm{PV}$. roughly an order of magnitude improvement in $f_{L R}\left(. V^{*} \rightarrow \Delta\right)$ would be needed to make the latter competitive. We note that the impact of hadronic uncertainties in the radiative corrections on the prospective low-energy $S$ and $T$ constraints of Fig. 2.4 is sig. nificantly smaller than either the assumed experimental error or the uncertainty associated with poorly constrained hadronic form factors.

\section{Higher-order Contributions: Summary}

All of the higher-order 'rocesses discussed above can be folded into corrections to the couplings of Table 3.3. E..plicitly, :e have

$$
\begin{aligned}
R_{V}^{T=0} & =R_{V}^{T=0}(1 \text {-quark })+R_{V}^{T=0}(\text { had })+R_{V}^{T=0}(\text { new })-\Delta_{V} \\
R_{V}^{T=1} & =R_{V}^{T=1}(1 \text {-quark })+R_{V}^{T=1}(\text { had })+R_{V}^{T=1}(\text { new }) \\
R_{V}^{(0)} & =R_{V}^{(0)}(1 \text {-quark })+R_{V}^{(0)}(\text { had })+R_{V}^{(0)}(\text { new })-\Delta_{V} \\
R_{A}^{T=0} & =R_{A}^{T=0}(1 \text {-quark })+R_{A}^{T=0}(\text { had })+R_{A}^{T=0}(\text { new })+2 \Delta_{A} \\
R_{A}^{T=1} & =R_{A}^{T=1}(1 \text {-quark })+R_{A}^{T=1}(\text { had })+R_{A}^{T=1}(\text { new }) \\
R_{A}^{(0)} & =R_{A}^{(0)}(1 \text {-quark })+R_{A}^{(0)}(\text { had })+R_{A}^{(0)}(\text { new })+\frac{4}{3} \Delta_{A} .
\end{aligned}
$$

\section{Isoscalar Axial-Vector Current}

Before leaving this section, we note that the higher-order processes are particularly important for the isoscalar axial-vector current:

$$
J_{\mu 5}^{N C}(T=0)=\xi_{A}^{T=0} A_{\mu}^{(8)}+\xi_{A}^{(0)} A_{\mu}^{(s)}
$$

Since $\xi_{A}^{T=0}=0$ in lowest order in the Standard Model, one might naively conclude that a measurement of the isoscalar axial-vector matrix element would directly probe for strange quarks in a non-strange hadron. It should be emphasized, however, that this naïve conclusion holds only because the tree-level isoscalar Standard Model coupling vanishes and not because matrix elements of $A_{\mu}^{(8)}$, which contains the isoscalar combination of $u$ - and $d$-quark axial-vector currents, are zero. Moreover, once higher-order processes renormalize the tree-level axial-vector couplings, $\xi_{A}^{T=0}$ becomes nonzero. In this case. $\left\langle H\left|J_{\mu 5}^{N C}(T=0)\right| H\right\rangle$ does not necessarily vanish even in the absence of strange quarks. A more accurate statement is that $J_{\mu 5}^{N C}(T=0)$ is suppressed in the absence of strange-quarks. since it enters only at higher-order in electroweak couplings. In contrast, $s$-quark contributions to the isoscalar axial-vector current enter at leading order in electroweak couplings $\left(\xi_{A}^{(0)}=1\right.$ at tree-level). However, one expects matrix elements of $A_{\mu}^{(s)}$ to be suppressed for 
non-strange hadrons, so that strange-quark contributions to $\left\langle H\left|J_{\mu S}^{\mathrm{N}} \subseteq(T=0)\right| H\right\rangle$ should also be small.

A priori. one has no way of knowing whether $\xi_{A}^{T=0}\left\langle H\left|\cdot f_{\mu}^{(8)}\right| H\right\rangle$ or $\xi_{A}^{(0)}\left\langle H\left|\cdot f_{\mu}^{(3)}\right| H\right\rangle$ is larger. In fact. estimates of higher-order electroweak amplitudes and an $\mathrm{S}(\mathrm{C}(3)$ analysis of the E.IC data suggest that the two contributions could be of comparable magnitude for PV electron scattering ( see Tables 2.2 and 2.3). Furthermore. the theoretical uncertainty in $R_{A}^{T=0}($ had $)$ discussed above introduces an uncertainty into $\left\langle. V\left|J_{\mu 5}^{N C}(T=0)\right| . \dot{V}\right\rangle$ amplitudes having the same magnitude as $s$-quark contributions. The same conclusion holds for atomic PV probes of the axial-vector hadronic current. In short, the neglect of the second term in the right-hand side of $\mathrm{Eq} .(3.28)$ is potentially misleading as far as the interpretation of $P V\left(\vec{e}, e^{\prime}\right)$ measurements and atomic $P V$ is concerned. In the case of $\nu-.{ }^{*}$ scattering. on the other hand, the strange-quark term appears to dominate. while theoretical uncertainties in higher-order processes are small. This difference between neutrino and electron scattering follows from the two factors mentioned previously: (i) logarithmic enhancements appearing in one-loop amplitudes involving photon-exchange, and (ii) the fortuitous suppression of tree-level electron-hadron amplitudes by the $g_{v}^{e}=-1+4 \sin ^{2} \theta_{w}$ factor which does not arise in all of the one-loop amplitudes. 


\section{III.C. Single-Nucleon Matrix Elements of the Electroweak Current}

Single-nucleon matrix elements of the currents of Eqs. (3.13), (3.16), and $(3.17)$ are restricted by Lorentz covariance. together with parity and time-reversal invariance. to the general forms

$$
\begin{aligned}
& \stackrel{\nu}{ }\left(P^{\prime}\right) \backslash J_{\mu}^{E M}(0)|\dot{N}(P)\rangle=\bar{u}\left(P^{\prime}\right)\left[F_{1} \gamma_{\mu}+i \frac{F_{2}}{2 m_{N}} \sigma_{\mu \nu} Q^{\nu}\right] u(P) \\
& \left\langle. \vee\left(P^{\prime}\right)\left|J_{\mu}^{N C}(0)\right| \nu(P)\right\rangle=\bar{u}\left(P^{\prime}\right)\left[\check{F}_{1} \hat{\gamma}_{\mu}+i \frac{\dot{F}_{2}}{2 m_{N}} \sigma_{\mu \nu} Q^{\nu}\right] u(P) \\
& \left\langle\mathcal{N}\left(P^{\prime}\right)\left|J_{\mu \zeta}^{N C}(0)\right| N(P)\right)=\bar{u}\left(P^{\prime}\right)\left[\dot{G}_{A} \dot{*}_{\mu}+\frac{\dot{G}_{P}}{m} \eta_{\mu}\right\} ; \zeta u(P) .
\end{aligned}
$$

where $Q=P^{\prime}-P$ is the four-momentum transferred to the nucleon (see Fig. 3.1). Denoting the magnitude of the three-momentum transfer by $q=|\vec{q}|$ and the energy transfer by $\therefore$. we have $Q^{2}=\alpha^{2}-q^{2} \leq 0$. Here $F_{1(2)}$ are the usual electromagnetic Dirac and Pauli form factors of the nucleon. Throughout the remainder of this work, we usually use Sachs form factors [Sac62] defined in terms of the Dirac and Pauli vector current form factors as

$$
\begin{aligned}
& G_{\Sigma}\left(Q^{2}\right)=F_{1}\left(Q^{2}\right)-\tau F_{2}\left(Q^{2}\right) \\
& G_{M}\left(Q^{2}\right)=F_{1}\left(Q^{2}\right)+F_{2}\left(Q^{2}\right) .
\end{aligned}
$$

where $\tau \equiv\left|Q^{2}\right| / 4 m_{\mathrm{N}}^{2}$ and $m_{N}$ denotes the nucleon mass (we ignore the $n-p$ mass difference at this point).

From Eqs. (3.16) and (3.17) the form factors entering the matr elements of the neutral currents may be written

$$
\tilde{G}_{a}\left(Q^{2}\right)=\xi_{v}^{T=1} G_{a}^{T-1} \tau_{3}+\sqrt{3} \xi_{v}^{T=0} G_{a}^{T=0}+\xi_{v}^{(0)} G_{a}^{(0)}, \quad a=E, M
$$

for the vector current form factors and

$$
\tilde{G}_{a}\left(Q^{2}\right)=\xi_{A}^{T=1} G_{a}^{(3)} \tau_{3}+\xi_{A}^{T=0} G_{a}^{(8)}+\xi_{A}^{(0)} G_{a}^{(0)}, \quad a=A, P
$$

for the axial-vector form factors. Here, the $G_{a}^{r-0.1}$ denote the isoscalar and isovector combinations of the electromagnetic Sachs form factors of the nucleon and $\tau_{3}=+1(-1)$ for the proton(neutron). For use elsewhere we also define the combinations

$$
\tilde{G}_{A}^{(T=1)}\left(Q^{2}\right)=\xi^{=1} G_{A}^{(3)}
$$

and

$$
\tilde{G}_{A}^{(T=0)}\left(Q^{2}\right)=\xi_{A}^{T=0} G_{A}^{(s)}+\xi_{A}^{(0)} G_{A}^{(0)}
$$


Assuming the nucleon to be an eigenstate of isospin, one may write*

$$
\begin{aligned}
& G_{x}^{r=1}=\left\{\left(G_{k}^{p}-G_{k}^{n}\right\}\right. \\
& G_{\varepsilon}^{\tau=0}=\left\lfloor\left[G_{\varepsilon}^{p}+G_{\varepsilon}^{n}\right\rfloor\right. \\
& G_{M}^{\tau=1}=\downarrow\left|G_{M}^{p}-G_{M}^{n}\right| \\
& G_{M}^{\tau=0}=\left\lfloor\left[G_{M}^{p}+G_{M}^{n}\right\rfloor\right. \text {. }
\end{aligned}
$$

where at $Q^{2}=0$ one has

$$
\begin{gathered}
G_{E}^{r=1}(0)=G_{\Sigma}^{r=0}(0)=t \\
G_{M}^{r=1}(0)=\left(\mu_{p}-\mu_{n}\right) \equiv \mu^{T=1} \\
G_{M}^{r=0}(0)=1\left(\mu_{p}+\mu_{n}\right) \equiv \mu^{r=0} .
\end{gathered}
$$

where the magnetic mornents of the proton and neutron are $\mu_{p}=2.79$ and $\mu_{n}=-1.31$. respectively. The $Q^{2}$-dependence of these form factors is known. to varying degrees of accuracy, from unpolarized electron scatcering. The data may be summarized using the so-called "Galster parameterization" [Gal71]

$$
\begin{aligned}
G_{E}^{p} & =G_{D}^{v} \\
G_{\Sigma}^{n} & =-\mu_{n} \tau G_{D}^{v} \xi_{n} \\
G_{M}^{p} & =\mu_{p} G_{D}^{v} \\
G_{M}^{n} & =\mu_{n} G_{D}^{v},
\end{aligned}
$$

where

$$
\begin{aligned}
G_{D}^{v} & =\left(1-Q^{2} / M_{v}^{2}\right)^{-2}=\left(1+\lambda_{D}^{v} \tau\right)^{-2} \\
\xi_{n} & =\left(1+\lambda_{n} \tau\right)^{-1}
\end{aligned}
$$

with $\lambda_{0}^{v}=4.97$ and $\lambda_{n}=3.6$. This parameterization of the form factors agrees with measured proton form factors for $\left|Q^{2}\right|$ of present interest to better that $3 \%$ (the proton magnetic form factor deviates from the dipole form by about $3 \%$ at low momentum transfer (Hoh 76)). The neutron magnetic form factor is known to about $7 \%$ at low $\left|Q^{2}\right|$ (.Mar93] and is about 2 standard deviations above the dipole at $\left|Q^{2}\right|=0.1 \mathrm{GeV}^{2} / c^{2}$. The neutron electric form factor is presently constrained to the $50 \%$ level [Mad92, Pla90] below $\left|Q^{2}\right|=1.0 \mathrm{GeV}^{2} / c^{2}$. At higher momentum transfer, $G_{E}^{n}$ appears to be consistent with zero [Lun93].

Except for the value of $G_{\varepsilon}^{(0)}$ at the photon point, the vector current strangeness form factors are unknown. Since the nucleon has no net strangeness, one must have that $G_{\varepsilon}^{(0)}(0)=0=F_{1}^{(0)}(0)$. It is conventional to describe the leading, non-trivial $Q^{2}$-behavior

* In other work, such as Refs. [Don79a] and [Don89], the isoscalar and isovector nucleon form factors are defined without the factor of $1 / 2$. 
of charge form factors in terms of mean-square radius. defined. for example. in terms of $F_{1}$. Consequently, one defines an mean-square "strangeness radius" as [Jaf89]

$$
\left.\left\langle r^{2}\right\rangle^{(0)} \equiv 6 \frac{d F_{1}^{(0)}}{d Q^{2}}\right|_{Q^{2}=0}
$$

Similarly: the strangeness magnetic moment is given by the form factor at $Q^{2}=0$ :

$$
\mu_{0} \equiv F_{2}^{(0)}(0) \text {. }
$$

Working instead with the Sachs form factors and the dimensionless variable $r$. as is $\mathrm{con}$. rentional in studies of the nucleon electromagnetic form factors. one has the analogous quantities

$$
\begin{gathered}
\left.\rho_{0} \equiv \frac{d G_{\Sigma}^{(s)}}{d \tau}\right|_{r=0}=-\xi m_{.}^{2}\left\langle r^{2}\right\rangle^{(0)}-\mu, \\
\mu_{0} \equiv G_{M}^{(s)}(0) .
\end{gathered}
$$

As discussed in Sect. II. there presently exist no published experimental constraints on $\rho_{\mathrm{g}}$ and $\mu_{\mathrm{s}}$. though a careful re-analysis of the BNL $\nu p / \bar{\nu} p$ data might provide interesting bounds on the strangeness magnetic moment (see Sect. IV.J). In the absence of such experimental information, one must turn to theoretical model predictions for these form factors (see Table 2.3). In Sect. IV, we review several experimental scenarios for constraining $G_{E}^{(0)}$ and $G_{M}^{(s)}$. For the purpose of analyzing the sensitivity of prospective experiments to these form factors, it is necessary to choose some parameterization for the non-leading $Q^{2}$-dependence. To that end, we work with a natural extension of the Galster parameterization and take

$$
\begin{aligned}
& G_{\Sigma}^{(0)}=\rho_{0} \tau G_{D}^{v} \xi_{\Sigma}^{(0)} \\
& G_{M}^{(0)}=\mu_{0} G_{D}^{V} \xi_{M}^{(0)} .
\end{aligned}
$$

where $G_{D}^{v}$ is defined in Eq. (3.37a) and

$$
\xi_{x, M}^{(0)}=\left(1+\lambda_{\varepsilon, M}^{(0)} r\right)^{-1}
$$

In the remainder of this work, we set $\lambda_{M}^{(0)}=0$ and take $\lambda_{\varepsilon}^{(0)}$ as a free parameter to be determined by experiment. With this choice, the strangeness vector current form factors have the same asymptotic behavior as the neutron EM form factors: $G_{E . M}^{(0)} \sim 1 / \tau^{2}$ as $r \rightarrow \infty$. Given that these form factors arise entirely from sea quarks, it would be reasonable to assume a more rapid fall-off with $r$. Nevertheless, for purposes of comparing the constraints which different intermediatt-energy, low-to-moderate $\tau$ scattering experiments might derive on the form factors, this choice is sufficient.

In contrast to the electromagnetic and weak neutral vector currents, the neutral axialvector current is not conserved. Consequently, the $Q^{2}=0$ values of the axial-vector form factors are not constrained by any symmetry or nucleon quantum numbers. If, however. 
one assumes the nucleon to be a state of good isospin. $G_{A}^{(3)}(0)$ may be determined from Gamow-Teller 3-decay rates. If. in addition. one takes the eight lowest-lying baryons to constitute an exact $S \mathrm{C}(3)$ octet. one may also extract $G_{A}^{(8)}$ from hyperon 3-decay measurements. Inder these two assumptions, one has

$$
\begin{gathered}
G_{A}^{(3)}(0)=(D+F) \\
G_{A}^{(0)}(0)=\frac{1}{2 \sqrt{3}}(3 F-D) .
\end{gathered}
$$

with $D+F \equiv g_{A}=1.262$ and $F / D \approx 0.64$ [Gai84. Dub90. Fre90. Bou83]. Ciajuely, one might expect corrections to Eq. (3.43b) to have the same scale as that of $\mathrm{SC}(3)$-breaking in the baryon octet. namely. $\left(W_{I}-m_{v}\right) / m_{v} \approx 0.27$. Estimates using the Skyrme model Par91] and chiral perturbation theory [Jen91] suggest that the scale of these corrections could be significantly larger. with the result that the value of $G_{A}^{(8)}$ could be uncertain by a factor of two or more. The impact of this uncertainty would not be serious for the interpretation of $\nu$ scattering experiments, since $\xi_{A}^{T=0}$ is small in this case, but would introduce problematic uncertainty for the analysis of PV asymmetries beyond that associated with $R_{A}^{T=0}$ as discussed above. As for the $Q^{2}$-dependence of these form factors. data can be parameterized adequately with a dipole form

$$
\begin{aligned}
& G_{A}^{(3)}=(D+F) G_{D}^{A} \\
& G_{A}^{(8)}=\frac{1}{2 \sqrt{3}}(3 F-D) G_{D}^{A} .
\end{aligned}
$$

where

$$
G_{D}^{A}=\left(1-Q^{2} / M_{A}^{2}\right)^{-2}=\left(1+\lambda_{D}^{A} T\right)^{-2} .
$$

From charged current quasielastic neutrino reactions on isoscalar targets, one has the world average value for the axial-vector dipole mass parameter $M_{A}=1.032 \pm 0.036$ or $\lambda_{D}=3.32_{-0.22}^{+0.24}$ (Ahr87 and references therein; see also Kit90).

Values for $G_{1}^{(9)}(0)$ have been extracted from the BNL $\nu p / \bar{\nu} p$ experiment as well as from the E.MC measurement of $\int d x g_{1}(x)$. The latter determination makes use of $S(13)$ symmetry and a QCD-corrected parton model to write the $g_{1}$ sum rule for the proton as [Ash89]

$$
\int_{0}^{1} d x g_{1}^{p}(x)=\frac{1}{12}\left\{\left[1-\frac{\alpha_{0}}{\pi}\right]\left[a_{3}+\frac{1}{\sqrt{3}} a_{8}\right]+2 \sqrt{\frac{2}{3}}\left[1-\frac{33-8 N_{f}}{33-2 N_{f}} \frac{\alpha_{0}}{\pi}\right] a_{0}\right\},
$$

where $\nu_{f}$ is the number of flavors of quarks, $a_{3.8}=2 G_{A}^{(3,8)}(0)$, and $a_{0}$ may be re-written in terms of $G_{A}^{(8)}(0)$ and $G_{A}^{(0)}(0)$ in order to extract a value of the strangeness axial-vector form factor. Results of this analysis, as well as that of the BNL data, are given in Table 2.2. Several theoretical predictions agree, at least in rough order of magnitude, with these results. The presence of $\mathrm{SU}(3)$-breaking of the scale suggested by Refs. [Par91, Jen91] could reduce the E.MC value for $\left|G_{A}^{(0)}(0)\right|$ by roughly a factor of three. In the case of the BNL determination, the data were collected at sufficiently large $\left|Q^{2}\right|$ to generate a significant 
correlation between $G_{A}^{(9)}(0)$ and the axial-vector dipole mass parameter of Eq. 13.451 . It is clearly of interest to reduce the resulting uncertainty by performing a determination at much lower momentum transfer and ways for doing so are discussed in Sect. IV.J. In analyzing these proposals, we make a choice for the $Q^{2}$-dependence of $G_{A}^{(s)}$ similar to that of the vector current strangeness form factors:

$$
G_{A}^{(0)}\left(Q^{2}\right)=\eta_{0}(D+F) G_{D}^{A} \xi_{A}^{(s)} .
$$

where

$$
\begin{aligned}
\xi_{A}^{(0)} & =\left(1+\lambda_{A}^{(0)} \tau\right)^{-1} \\
\eta_{s} & =G_{A}^{(0)}(0) / g_{A} .
\end{aligned}
$$

We will generally set $\lambda_{A}^{(s)}=0$ in what follows.

Although less is known about the induced pseudoscalar form factor. $\tilde{G}_{p}$, it does not contribute to observables measured in PV electron scattering to leading order in electroweak couplings. Its contribution to elastic neutrino scattering vanishes for $m_{\nu}=0$ and is usually neglected in this case. 


\section{III.D. Nuclear Matrix Elements of the Electroweak Current}

In this section we discuss the nuclear matrix elements of the electroweak current. For crattering from nuclear targets. one must model both the nucleon and nuclear structure in obtaining the matrix elements. Accordingly. one focus in this section is to indicate how this interplay is handled in terms of a hierarchy of one-body two-body. . nuclear operators. When considering initial and final nuclear states with good angular momen. rum quantum numbers it is usually convenient to expand the hadronic currents in terms of multipole projections of the non-relativistic charge and three-current operators. This expansion leads naturally to definitions of basic vector and axial-vector form factors. as discussed in Sect. III.D.1. The dominant contributions to the nuclear matrix elements 14.1 ally arise from the one-body parts of the general current operators I see Sect. III.D.2 I with Iypirally small corrections from two-body meson-exchange currents isee Sect. III.D.3, In many instances, as discussed in more detail in Sect. IV. assuming the murlear states in be eigenstates of parity and isospin. in addition to angular momentum. and runcatitin the many-body hierarchy to one- and two-body operators is sufficient in treating the nu. clear physics contributions to observables of interest. However. the extraction of nucleon structure information from these observables often requires that we understand the residual uncertainty in the nuclear modeling, so we must also evaluate the role played by dynarnical ingredients which go beyond these basic assumptions. Two specific problems of this type are mentioned in the last two subsections: the role of isospin-mixing in the nuclear states (Sect. III.D.4) and the general character of parity-mixing in the nuclear states. including the the role of the anapole moment (Sect. III.D.5). Although these additional ingredient, generally produce negligible changes to the interpretation of the hadronic matrix element within the context of the basic set of assumptions. there exist a few cases. discussed in detail in Sect. IV. for which it may be possible to address these issues using PV electron scattering.

\section{III.D.1. ELECTROWEAK FORM FACTORS}

The multipole expansions of the E.M charge and three current are given by

$$
\begin{aligned}
& \dot{\rho}(\vec{q})=\sum_{J \geq 0} \sqrt{4 \pi(2 J+1)} i^{J} \cdot \dot{M}_{J 0}(q) \\
& \dot{J}_{\lambda}(\vec{q})=- \begin{cases}\sum_{J \geq 0} \sqrt{4 \pi(2 J+1) l} J \dot{L}_{J 0}(q), & \lambda=0 \\
\left.\sum_{J \geq 1} \sqrt{2 \pi(2 J+1) l} \dot{T}_{j \lambda}^{\mathrm{el}}(q)+\lambda \dot{T}_{J \lambda}^{\mathrm{mag}}(q)\right], & \lambda= \pm 1,\end{cases}
\end{aligned}
$$

where the Coulomb $(\hat{i})$, longitudinal $(\hat{L})$, and transverse electric and magnetic ( $\dot{T}^{\text {el, mag }}$ ) multipole operators are defined in Refs. [deF66. Don75. Wal75, Don79a]. As usual. the $z$-axis has been chosen to lie in the $\vec{q}$-direction. Let us assume an initial state with good quantum numbers $J_{1}^{\pi_{1}} T_{1}, M_{J_{1}}, M_{T_{1}}$ and a final state with good quantum numbers $J_{f}^{\pi \prime} T_{f}, M_{f_{f}}, M_{T_{f}}$. Since our main focus is $\mathrm{PC}, \mathrm{PV}^{\prime}$ electron scattering (see Sect. III.E) which involves only neutral electroweak currents. we require matrix elements between 
states in a given nucleus; hence, $M_{T_{1}}=. V_{T_{f}}=M_{T}$. The following electromagnetic form factors may then be defined:

$$
\begin{aligned}
& F_{C J}(q) \equiv \frac{1}{\sqrt{2 J_{1}+1}} \sum_{T=0,1}(-)^{T_{f}-M_{T}}\left(\begin{array}{ccc}
T_{f} & T & T_{i} \\
-M_{T} & 0 & M_{T}
\end{array}\right)<J_{f} ; T_{f}: \ddot{:} \hat{M}_{J ; T}(q): \because: J_{i}: T_{i}> \\
& \left.F_{L J}(q) \equiv \frac{1}{\sqrt{2 J_{i}+1}} \sum_{T=0,1}(-)^{T_{f}-M_{T}}\left(\begin{array}{ccc}
T_{f} & T & T_{i} \\
-M_{T} & 0 & M_{T}
\end{array}\right)<J_{f} ; T_{f}: \because \hat{L}_{J: T}(q) \ddot{\because} J_{i}: T_{i}\right\rangle \\
& F_{E J}(q) \equiv \frac{1}{\sqrt{2 J_{i}+1}} \sum_{T=0,1}(-)^{T_{f}-M_{T}}\left(\begin{array}{ccc}
T_{f} & T & T_{i} \\
-M_{T} & 0 & ._{T}
\end{array}\right)<J_{f} ; T_{f}:: \hat{T}_{J: T}^{\mathrm{el}}(q): \because: J_{i}: T_{l}> \\
& \left.F_{: I J}(q) \equiv \frac{1}{\sqrt{2 J_{\imath}+1}} \sum_{T=0,1}(-)^{T_{f}-M_{T}}\left(\begin{array}{ccc}
T_{f} & T & T_{i} \\
-M_{T} & 0 & . M_{T}
\end{array}\right)<J_{f} ; T_{f}:: i \hat{T}_{J: T}^{\operatorname{mag}}(q)::: J_{i}: T_{i}\right\rangle \text {. }
\end{aligned}
$$

where the symbols $::$ are used to denote matrix elements reduced both in angular momentum and in isospin. With the factor $i$ in the definition of the magnetic form factor it can be shown that a phase convention can be adopted in which the form factors are all real. For conserved vector currents $(\mathrm{CVC})$ such as the electromagnetic current, one may eliminate matrix elements of the longitudinal projection of the the three-vector current, which involves only the divergence of the current, using instead matrix elements of $[\hat{H} . \hat{\rho}]$ :

$$
<J_{f} ; T_{f}:: \hat{L}_{J ; T}: \because J_{i} ; T_{i}>=\frac{\omega}{q}<J_{f} ; T_{f}: \because \hat{M}_{J ; T}: \because J_{i} ; T_{i}>\text {, }
$$

where $\omega=E_{f}-E_{i}$ with $E_{i}\left(E_{f}\right)$ the energy of the initial (final) nuclear state. Thus, in considering such CVC cases, one need discuss only the Coulomb and transverse operators. The conventions used in defining the form factors in Eqs. (3.50) are those of Refs. [deF66. Don75. Don79a, Do'79b, Don80, Don84, Don89]. They may be compared with different cinvices of nnrmalization by computing the elastic $\mathrm{C} 0$ form factor in the long wavelength lim: : $(q \rightarrow 0)$ :

$$
\begin{aligned}
& <J_{i} ; T_{i}: \hat{M}_{0 ; 0}: \because J_{i} ; T_{i}>\longrightarrow \frac{1}{\sqrt{4 \pi}} \sqrt{\left(2 J_{i}+1\right)\left(2 T_{i}+1\right)} \frac{1}{2} A \\
& <J_{i} ; T_{i}: \hat{M}_{0 ; 1}: \because J_{i} ; T_{i}>\longrightarrow \frac{1}{\sqrt{4 \pi}} \sqrt{\left(2 J_{i}+1\right)\left(2 T_{i}+1\right)} \sqrt{T_{i}\left(T_{i}+1\right)},
\end{aligned}
$$

using the general arguments in Ref. [Don84]. This implies that

$$
F_{C 0} \longrightarrow Z / \sqrt{4 \pi}
$$

in the long wavelength limit.

The above definitions can easily be extended to include charge-changing weak interaction processes such as $\beta$-decay, charged lepton capture and $\nu$-reactions as well [Don79a]. 
In that case only isovector matrix elements occur and the following vector current form factors appear as natural generalizations of Eqs. (3.50):

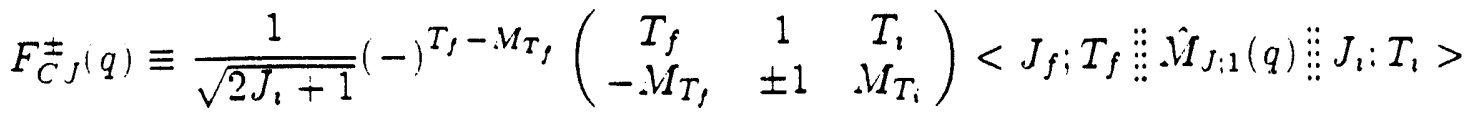

$$
\begin{aligned}
& F_{L J}^{ \pm}(q) \equiv \frac{1}{\sqrt{2 J_{2}+1}}(-)^{T_{f}-M_{T_{f}}}\left(\begin{array}{ccc}
T_{f} & 1 & T_{i} \\
-M_{T_{f}} & \pm 1 & . M_{T_{i}}
\end{array}\right)<J_{f}: T_{f}:: \hat{L}_{J: 1}(q): \because: J_{i}: T_{i}> \\
& F_{E J}^{ \pm}(q) \equiv \frac{1}{\sqrt{2 J_{i}+1}}(-)^{T_{f}-M_{T_{f}}}\left(\begin{array}{ccc}
T_{f} & 1 & T_{i} \\
-. M_{T_{f}} & \pm 1 & M_{T_{i}}
\end{array}\right)<J_{f}: T_{f}:: \hat{T}_{J: 1}^{\mathrm{el}}(q)::: J_{i}: T_{l}> \\
& F_{., I J}^{ \pm}(q) \equiv \frac{1}{\sqrt{2 J_{i}+1}}(-)^{T_{f}-M_{T_{f}}}\left(\begin{array}{ccc}
T_{f} & 1 & T_{i} \\
-M_{T_{f}} & \pm 1 & M_{T_{i}}
\end{array}\right)<J_{f} ; T_{f}:: i \hat{T}_{J_{i 1}}^{\mathrm{mag}}(q): \because: J_{l}: T_{\imath}>.
\end{aligned}
$$

where now $. M_{T_{f}}=M_{T_{i}} \pm 1$. In fact the isovector-vector reduced matrix elements appearing in all electroweak processes are the same (the "isovector triplet hypothesis") except for the electroweak couplings. 1 or $\xi_{v}^{T=1}$ (see below). The isovector form factors in Eqs. (3.j0) and (3.54) differ only in that the appropriate $3-\mathrm{j}$ symbols must be used:

$$
F^{\lambda}(T=1) \sim\left(\begin{array}{ccc}
T_{f} & 1 & T_{i} \\
-M_{T_{f}} & \lambda & M_{T_{i}}
\end{array}\right)
$$

where one has the isovector part of one of the electromagnetic form factors in Eqs. (3.50) when $\lambda=0$ and one of the charge-changing weak form factors in Eqs. (3.54) when $\lambda= \pm 1$. Of course, when discussing charge-changing weak interaction processes it is also necessar: to consider axial-vector currents as well as vector currents. The analogous operators for multipole expansions of the axial-vector currents will be denoted with a " 5 " superscript:

$$
\begin{aligned}
& F_{C J_{s}}^{ \pm}(q) \equiv \frac{1}{\sqrt{2 J_{i}+1}}(-)^{T_{f}-M_{T_{f}}}\left(\begin{array}{ccc}
T_{f} & 1 & T_{i} \\
-M_{T_{f}} & \pm 1 & M_{T_{i}}
\end{array}\right)<J_{f} ; T_{f}: \ddot{:} i \hat{M}_{J_{i 1}}^{5}(q): \because: J_{i} ; T_{i}> \\
& \left.F_{L J_{5}}^{ \pm}(q) \equiv \frac{1}{\sqrt{2 J_{i}+1}}(-)^{T_{f}-M_{T_{f}}}\left(\begin{array}{ccc}
T_{f} & 1 & T_{i} \\
-M_{T_{f}} & \pm 1 & M_{T_{i}}
\end{array}\right)<J_{f} ; \Gamma_{f}: \ddot{:} i \hat{L}_{J: 1}^{5}(q) \ddot{:} J_{i}: T_{l}\right\rangle \\
& F_{E J_{5}}^{ \pm}(q) \equiv \frac{1}{\sqrt{2 J_{i}+1}}(-)^{T_{f}-M_{T_{f}}}\left(\begin{array}{ccc}
T_{f} & 1 & T_{i} \\
-M_{T_{f}} & \pm 1 & M_{T_{i}}
\end{array}\right)<J_{f} ; T_{f}: \ddot{:} i \hat{T}_{J ; 1}^{\mathrm{els}}(q): \because: J_{i} ; T_{i}> \\
& F_{M J_{s}}^{ \pm}(q) \equiv \frac{1}{\sqrt{2 J_{i}+1}}(-)^{T_{f}-M_{T_{f}}}\left(\begin{array}{ccc}
T_{f} & 1 & T_{i} \\
-M_{T_{f}} & \pm 1 & M_{T_{i}}
\end{array}\right)<J_{f} ; T_{f}:: \hat{T}_{J_{i 1}}^{\mathrm{mag} 5}(q): \because: J_{i} ; T_{i}>.
\end{aligned}
$$

Again the factors $i$ have been included so all form factors here are real where the same phase convention mentioned above is adopted. In the case of the axial-vector current, which is not conserved, longitudinal projections cannot be re-written in terms of other multipoles and consequently all four types of form factor in Eq. (3.56) are required in general.

The final set of form factors required for a complete treatment of hadronic matrix elements of the electroweak current are those associated with the weak neutral current 
interaction in parallel with the above formalism. For extensive discussion of the interrelationships that exist within the full set of electroweak current matrix elements see Ref. [Doni9a]. Here we only summarize the developments that lead to the remaining form factors needed in the rest of the present work. Let us use Eqs. (3.31) to write the vector and axial-vector $\mathrm{NC}$ multipole operators in the form

$$
\begin{aligned}
& \left(\dot{O}_{J}\right)^{v c}=\xi_{V}^{T=1} \hat{O}_{J}^{E M}(T=1)+\sqrt{3} \xi_{V}^{T=0} \hat{O}_{J}^{E M}(T=0)-i_{v}^{(0)} \hat{O}_{J}(s) \\
& \left(\hat{O}_{J}^{5}\right)^{v c}=\xi_{A}^{T=1} \hat{O}_{J}^{5}(3)+\xi_{A}^{T=0} \hat{O}_{J}^{5}(8)+\xi_{A}^{(0)} \hat{O}_{J}^{5}(s),
\end{aligned}
$$

where $\hat{O}_{J}\left(\hat{O}_{J}^{5}\right)$ is any one of the vector (axial-vector) multipole operators introduced above. Here the notation $(s)$ refers to the multipole projections of $V_{\lambda}^{(s)}$ and $A_{\lambda}^{(s)} \cdot(T=0.1$ i denotes projections of the isoscalar and isovector E.M currents, and (3.8) correspond to projections of the octet axial-vector currents. Operationally, one may take the isoscalar matrix elements and form factors defined in Ref. [Don79a] and replace them with the corresponding quantities involving strange or octet neutral currents. For single-particle matrix elements (see Sect. III.D.2) this procedure entails making the following replacements:

$$
\begin{aligned}
& G_{E, M}^{T=0} \longrightarrow G_{E, M}^{(s)} \\
& \frac{1}{2} G_{A}^{(0)} \longrightarrow G_{A}^{(s)} \text { or } G_{A}^{(8)},
\end{aligned}
$$

where $G_{A}^{(0)}$ is the isoscalar axial-vector form factor used in Ref. [Don79a] and differs by the same factor of $1 / 2$ as do the vector form factors in Eqs. (3.33). follows:

In analogy with Eqs. (3.50) we may then define weak neutral current form factors as

$$
\begin{aligned}
& \tilde{\Sigma}_{i j j} \equiv \frac{1}{2 \sqrt{2 J_{\imath}+i}}(-)^{T_{f}-M_{T}}\left\{\left(\begin{array}{ccc}
T_{f} & 0 & T_{i} \\
-M_{T} & 0 & M_{T}
\end{array}\right)\right. \\
& \times\left[\sqrt{3} \xi_{v}^{T=0}<J_{f} ; T_{f}: \because \hat{O}_{J}(T=0): \because J_{i} ; T_{i}>+\xi_{v}^{(0)}<J_{f} ; T_{f}:: \hat{O}_{J}(s): \because: J_{i} ; T_{i}>\right] \\
& \left.+\left(\begin{array}{ccc}
T_{f} & 1 & T_{i} \\
-M_{T} & 0 & M_{T}
\end{array}\right) \xi_{v}^{T=1}<J_{f} ; T_{f}:: \dot{O}_{J}(T=1):: J_{i} ; T_{i}>\right\} \\
& \equiv \frac{1}{2}\left[\sqrt{3} \xi_{v}^{T=0} F_{X J}(T=0)+\xi_{v}^{(0)} F_{X J}(s)+\xi_{v}^{T=1} F_{X J}(T=1)\right] \\
& \tilde{F}_{X J_{s}} \equiv-\frac{1}{2 \sqrt{2 J_{i}+1}}(-)^{T_{f}-M_{T}}\left\{\left(\begin{array}{ccc}
T_{f} & 0 & T_{i} \\
-M_{T} & 0 & M_{T}
\end{array}\right)\right. \\
& \times\left[\xi_{A}^{T=0}<J_{f} ; T_{f}:: \hat{O}_{J}^{5}(8): \because: J_{i} ; T_{i}>+\xi_{A}^{(0)}<J_{f} ; T_{f}: \because \hat{O}_{J}^{5}(s): \because: J_{i} ; T_{i}>\right] \\
& \left.+\left(\begin{array}{ccc}
T_{f} & 1 & T_{i} \\
-M_{T} & 0 & M_{T}
\end{array}\right) \xi_{A}^{T=1}<J_{f} ; T_{f}: \hat{O}_{J}^{3}(T=1): \because J_{i} ; T_{i}>\right\} \\
& \equiv-\frac{1}{2}\left[\xi_{A}^{T=0} F_{X J_{s}}(8)+\xi_{A}^{(0)} F_{X J_{s}}(s)+\xi_{A}^{T=1} F_{X J_{5}}(T=1)\right] \text {, }
\end{aligned}
$$


where for clarity the $q$-dependences have been suppressed. Here $X=C$. L. E or. $\mathrm{K}$ with corresponding operators $\hat{O}_{J}\left(\dot{L}_{J} . \dot{L}_{J} . \dot{T}_{J}^{\mathrm{el}}\right.$. and $\left.i \dot{T}_{J}^{\text {mag }}\right)$ for the vector currents and $\hat{O}_{J}^{3}\left(i, \hat{Y}_{J}^{3}, i \hat{L}_{J}^{3}, i \hat{T}_{J}^{\text {els }}\right.$, and $\left.\hat{T}_{J}^{\text {mag }}\right)$ for the axial-vector currents. The factors of $\pm 1 / 2$ have been introduced to keep the normalization and sign the same as that used previously in Ref. [Doni9a] (with strangeness matrix elements set to zero). Employing Eqs. (3.j2) it is straightforward to verify that for the elastic $\mathrm{C} 0$ form factor one has the following long wavelength limit:

$$
\begin{aligned}
\tilde{F}_{C 0} & \rightarrow \frac{1}{\sqrt{4 \pi}} \times \frac{1}{2}\left[\sqrt{3} \xi_{V}^{T=0} \frac{A}{2}+\xi_{V}^{T=1} \cdot M_{T}\right] \\
& =\frac{1}{\sqrt{4 \pi}} \times \frac{1}{2}\left[Z \xi_{V}^{p}+. V \xi_{V}^{n}\right] .
\end{aligned}
$$

where we have defined the couplings

$$
\begin{aligned}
& \xi_{V}^{p}=\frac{1}{2}\left[\sqrt{3} \xi_{V}^{T=0}+\xi_{V}^{T=1}\right] \\
& \xi_{V}^{n}=\frac{1}{2}\left[\sqrt{3} \xi_{V}^{T=0}-\xi_{V}^{T=1}\right] .
\end{aligned}
$$

as in Eqs. (3.24).

We wish to emphasize that our previous comments about the isoscalar axial-vector form factor $\tilde{G}_{A}^{T=0}$ also apply to the isoscalar axial-vector multipole matrix elements (see Eq. (3.32))< $<J_{f}: T_{f}: \because \hat{O}_{J}^{S}(T=0): \because: J_{i} ; T_{i}>=\xi_{A}^{T=0}<J_{f} ; T_{f}:: \hat{O}_{J}^{S}(8): \because J_{i} ; T_{i}>+\xi_{A}^{(0)}<$ $J_{f}: T_{f}: \because \hat{O}_{J}^{5}(s): \because J_{i} ; T_{l}>$. In the case of $\nu$ scattering, $\xi_{A}^{T=0}$ is sufficiently small and theoretically certain to render $<J_{f} ; T_{f}:: \hat{O}_{J}^{5}(T=0):: J_{i} ; T_{i}>$ effectively a meter of strangeness contributions. For PV electron scattering, however, the two terms here have the same scale, with theoretical uncertainty in the first term of comparable magnitude. Moreover. many-body contributions associated with nuclear PV introduce additional theoretical uncertainties beyond those associated with the one-body (single-nucleon) .NC, as discussed in Sect. III.D.5. In fact, a measurement of the matrix element of $\hat{O}_{J}^{3}(T=0)$ with PV electron scattering could in principle serve better as a probe of nuclear $P V$ rather than of the axialvector strangeness current (see Sects. III.D.5 and IV.E). In contrast. contributions from nuclear $\mathrm{PV}$ to $\nu$-scattering are negligible, so that from a theoretical standpoint, neutrinos ought to provide a "cleaner" probe of the nuclear axial-vector strangeness current.

\section{Selection Rules}

All of the form factors defined above are matrix elements of either vector or axialvector multipole operators. For states with good parity quantum numbers $\pi_{i}$ and $\pi_{f}$, the operators must carry the total parity $\pi=\pi_{1} \pi_{f}$ (see below for discussion of the extensions which occur when parity-mixing in the nuclear states is considered). The form factors divide into two classes, viz., natural parity transitions, where $(-)^{J}=\pi$ and only the following occur:

$$
\begin{aligned}
F_{C J}, F_{L J}, F_{E J} & \text { and } \tilde{F}_{C J} \tilde{F}_{L J} \tilde{F}_{E J} \\
F_{M J_{s}} & \text { and } \tilde{F}_{M J_{s}}
\end{aligned}
$$


and non-natural parity transitions, where $(-)^{J+1}=\pi$ and only the following occur:

$$
\begin{gathered}
F_{. M J}, \text { and } \tilde{F}_{. M J} \\
F_{C J_{3}}, F_{L J_{3}}, F_{E J_{3}} \text { and } \tilde{F}_{C J_{3}} \tilde{F}_{L J_{3}} \tilde{F}_{E J_{3}} .
\end{gathered}
$$

To illustrate. consider a transition from a nuclear ground state having $J_{1}^{\pi_{1}}=\frac{3}{2}^{-}$to a final state having $J_{f}^{\pi f}=\frac{5}{2}^{+}$. implying that $1 \leq J \leq 4$ and $\pi=-$. The above parity and angular momentum selection rules then allow only $F_{C 1}, F_{L 1}, F_{E_{1}}, F_{M_{13}} ; F_{M_{2}}, F_{C_{23}}, F_{L_{23}}, F_{E_{23}}$ :

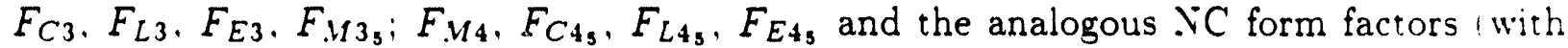
tildes).

For elastic scattering one may make use of time-reversal invariance. in addition to invoking parity and angular momentum conservation together with hermiticity. to limit the number of multipoles permitted to a smaller set [deF66. Don 76]: only the even-J vector form factors $F_{C J}$ and the odd-J vector. $F_{M J}$, and axial-vector. $F_{L J_{5}}, F_{E J_{3}}$. form factors (and their analogs with tildes) may be nonzero. Of course. from angular momentum conservation one has $0 \leq J \leq 2 J_{i}$ for elastic scattering. As discussed in Ref. [Doni6]. if. in contrast to our assumption here, the second-class axial-vector tensor current were to be nonzero, then in addition there could be odd- $J$ form factors $F_{C J_{s}}$ and $\tilde{F}_{C J_{3}}$. An example of using these general symmetry properties, discussed in more detail in Sect. IV.C. is that of elastic scattering from deuterium. There the ground state has $J_{i}=1$ and consequently the form factors permitted are $F_{C 0}, F_{C 2}, F_{M 1}, F_{L_{1}}$ and $F_{E_{1} s}$, together with the analogous quantities with tildes.

\section{III.D.2. .VCCLEAR MATRIX ELEMENTS OF ONE-BODY OPERATORS}

The electroweak currents in nuclei can usually be treated as one-body operators with corrections from two-body meson-exchange currents (see Sect. III.D.3). Any one-body operator can be written in the form [Feti1]

$$
\dot{U}_{J M_{J} ; T M_{T}}^{[1]}(q)=\sum_{\alpha \alpha^{\prime}}<\alpha^{\prime}\left|O_{J M_{J} ; T M_{T}}^{[1]}(q)\right| \alpha>c_{\alpha^{\prime}}^{\dagger} c_{\alpha},
$$

where we use $\alpha$ and $\alpha^{\prime}$ to label any complete set of single-particle wave functions. Typically $\alpha=\left\{a: m_{j_{a}} m_{t_{a}}\right\}$ with $a=\left\{n_{a}\left(l_{a} \frac{1}{2}\right) j_{a} ; t_{a}=\frac{1}{2}\right\}$. Also $-\alpha \equiv\left\{a ;-m_{j_{a}}-m_{t_{a}}\right\}$. Working with single-particle states above and below the Fermi surface, $\epsilon_{a}>\epsilon_{F}$ and $\epsilon_{a} \leq \epsilon_{F}$. respectively, we have

$$
c_{\alpha}=\theta\left(\epsilon_{a}-\epsilon_{F}\right) a_{\alpha}+\theta\left(\epsilon_{F}-\epsilon_{a}\right) S_{\alpha} b_{-\alpha}^{\dagger}
$$

where $a_{\alpha}$ is a particle destruction operator and $b_{-\alpha}^{\dagger}$ is a hole creation operator. The phase factor $S_{\alpha} \equiv(-)^{j_{a}-m_{j_{a}}}(-)^{\frac{1}{2}-m_{i_{a}}}$ is included to maintain the irreducible tensor character of $c_{\alpha}$ and its adjoint $c_{\alpha}^{\dagger}$. Thus a one-body operator can be expanded in a complete set of single-particle matrix elements $\left\langle\alpha^{\prime}\left|O_{J M_{J} ; T . M_{T}}^{(1)}(q)\right| \alpha\right\rangle$ which multiply bilinear products of creation and destruction operators - any complete set will make this expansion an 
identity. The form factors defined in the previous subsection all involve doubly-reduced matrix elements of multipole projections of the currents. For one-body operators these can be cast in the following form:

$$
<J_{f}: T_{f} \because: \hat{O}_{J: T}^{(1]}(q): \because: J_{i} ; T_{\imath}>=\sum_{a, a^{\prime}} \psi_{J: T}^{\left(f_{1}\right)}\left(a^{\prime} a\right)<a^{\prime}: \because O_{J: T}^{[1]}(q): \because: a>
$$

i.e.. as expansions over a complete set of doubly-reduced single-particle matrix elements weighted by one-body density matrix elements defined by

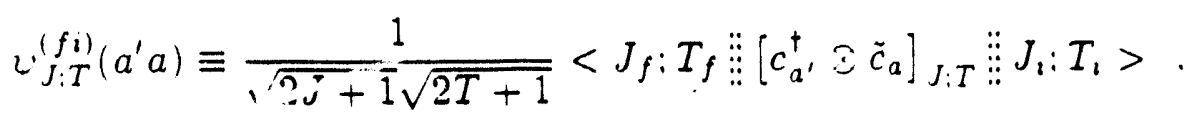

where $\tilde{c}_{\alpha} \equiv S_{\alpha} c_{-\alpha}$. Treatments of the single-particle matrix elements of the operators that occur in semi-leptonic electroweak interaction studies can be found in Refs. ¿Doni 9 b. Don80].

Equation $(3.65)$ is basic to most treatments of nuclear matrix elements of the electroweak current operators and their associated multipole projections. The many-body matrix elements (the left-hand side of the equation) is expressed in terms of sirgle-particle matrix elements and specific numbers - the one-body density matrix elements - that embody the nuclear structure physics content (the right-hand side of the equation). The former are obtained as non-relativistic reductions of the quantities discussed in Sect. III.C with allowance made for the fact that the single-particle wave functions used in Eq. (3.65) are usually to be viewed as solutions to the Hartree-Fock approximation (see. for examp.e. Ref. [Fet 71$]$ ), rather than as plane waves as is appropriate for free single nucleons. Details concerning this connection may be found in Refs. [deF66, Don79a]. Importantly in the present context is the observation that in the one-body approximation. strangeness is contained in these single-nucleon matrix elements. The nuclear many-body dynamics are represented by the one-body density matrix elements $\psi_{J_{i} T}^{(f)}\left(a^{\prime} a\right)$. Two approaches may be followed in obtaining these quantities for specific nuclear transitions: one is to obtain approximate solutions to the nuclear many-body problem (Hartree-Fock approximation. Random Phase approximation (RPA), nuclear shell model diagonalizations, etc., as discussed for example in [Fet71]); another is to use information from PC electron scattering and perhaps $\beta$-decay to constrain the density matrix and thus permit one to predict the form factors for (as yet) unmeasured electroweak processes. The latter approach is discussed in some detail in Ref. [Don79a and references therein]. Both approaches require truncating the sums in Eq. (3.65), an approximation that leads to some level of uncertainty in the nuclear modeling and consequently impacts the degree to which the single-nucleon content in the nuclear matrix elements can be extracted. In Sect. IV we return to a ess this issue in specific circumstances. 


\section{III.D.3. .IESON-EXCH.A.IGE CLRRE.NTS}

Most modern attempts to treat nuclear structure microscopically describe the nucleus in terms of the position and motion of the individual nucleons. But these treatments also include an interaction between the nucleons mediated by the exchange of mesons. Construction of the electromagnetic and weak neutral currents of the nucleus must. there. fore. include both the nucleonic and the mesonic degrees of freedom. However. since the exchanged meson is emitted by one nucleon and eventually absorbed on another. most treatme...is do not address the meson degrees of freedom explicitly but instead introduce additional multi-nucleon current operators to account for the contributions from the exchanged mesons. A few examples of diagrams contributing to these meson-exchange currents (.MEC) are shown in Fig. 3.8.

Two-body operators are developed in a way that is completely analogous to that in the previous section. Any two-body operator can be written in the form icf. Eq. 13.63 ,

$$
\dot{O}_{J . M_{J}, T . M_{T}}^{(2)}(q)=\sum_{\alpha \alpha^{\prime}} \sum_{33^{\prime}}<\alpha^{\prime} 3^{\prime}\left|O_{J . M_{J}: T . M_{T}}^{(2)}(q)\right| \alpha 3>c_{\alpha^{\prime}}^{\dagger} c_{3^{\prime}}^{\dagger} c_{3} c_{\alpha}
$$

Thus any two-body operator can be expanded in a complete set of two-particle matrix elements $\left\langle\alpha^{\prime} 3^{\prime}\left|O_{J . M_{J}: T, M_{T}}^{i 2}(q)\right| \alpha 3>\right.$ which multiply quadrilinear products of creation and destruction operators. The form factors defined in Sect. III.C all involve doubly-reduced matrix elements of multipole projections of two-body current operators of this type. as well as of the one-body operators discussed above. In analogy with Eq. (3.65) we have:

$$
\begin{aligned}
& \left\langle J_{f}: T_{f}:: \hat{O}_{J: T}^{(2 !}(q): \because: J_{i} ; T_{1}>\right. \\
& =\sum_{\left(a^{\prime}, b^{\prime}\right) J_{a^{\prime} b^{\prime}} T_{a^{\prime} b^{\prime}}} \sum_{(a, b) J_{a b} T_{a b}} \psi_{J ; T}^{(f)}\left(\left(a^{\prime}, b^{\prime}\right) J_{a^{\prime} b^{\prime}} T_{a^{\prime} b^{\prime}} ;(a, b) J_{a b} T_{a b}\right) \\
& \times<\left(a^{\prime}, b^{\prime}\right) J_{a^{\prime} b^{\prime}} T_{a^{\prime} b^{\prime}}: \because O_{J I T}^{[2]}(q): \because\left[(a . b) J_{a b} T_{a b}>.\right.
\end{aligned}
$$

where the two-body density matrix elements (Cf. Eq. (3.66)) are defined by

$$
\begin{aligned}
& \iota_{J: T}^{\left(f_{1}^{\prime}\right)}\left(\left(a^{\prime}, b^{\prime}\right) J_{a^{\prime} b^{\prime}} T_{a^{\prime} b^{\prime}} ;(a, b) J_{a b} T_{a b}\right) \equiv-\frac{1}{\sqrt{2 J+1} \sqrt{2 T+1}} \\
& \times<J_{f} ; T_{f}::\left[\left[c_{a^{\prime}}^{\dagger} \otimes c_{b^{\prime}}^{\dagger}\right]_{J_{a^{\prime} b^{\prime}} ; T_{a^{\prime} b^{\prime}}} \otimes\left[\tilde{c}_{a} \otimes \tilde{c}_{b}\right]_{J_{a b} ; T_{a b}}\right]_{J_{J} T}: \because J_{i} ; T_{1}>.
\end{aligned}
$$

These density matrix elements depend on the detailed nature of the nuclear states involved, $\mid i>$ and $\mid f>$. Given, for example, a shell-model description of the nuclear ground-state and some specific excited state in the same nucleus, it is straightforward in principle (although frequently rather more complicated in practice - see Ref. [Dubi5. Dubi6]) to evaluate the matrix elements in Eq. (3.69). To obtain the current matrix elements in Eq. (3.68) it remains to decide on specific forms for the two-particle matrix elements $<\left(a^{\prime}, b^{\prime}\right) J_{a^{\prime} b^{\prime}} T_{a^{\prime} b^{\prime}}: \because O_{J ; T}^{[2]}(q): \because \because(a, b) J_{a b} T_{a b}>$. 
Following (Dubij. Dubi6. Dub80) for completeness and to show the characteristic structure of the two-particle current operators let us record without proof the E.I pionic MEC contributions corresponding to the diagrams in Fig. 3.8. For the pair current of Fi.g 3.8a one obtains

$$
\begin{aligned}
& \left.J_{\mu}^{[2]} \mid \vec{x}_{1}, \vec{x}_{2}: q\right)_{\text {pair }}
\end{aligned}
$$

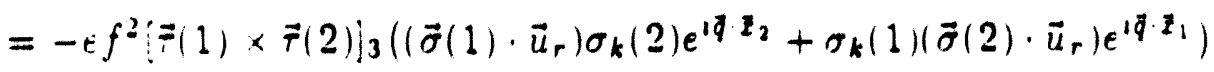

$$
\begin{aligned}
& \times\left(1+x_{\pi}\right) \frac{e^{-x}}{x_{\pi}^{2}} \quad \mu=k=1.2 .3 \\
& \approx 0 \quad \mu=0 \text {. }
\end{aligned}
$$

where $f$ is the pion-nucleon coupling, $x_{\pi} \equiv m_{\pi} r$ and $\vec{u}_{r}$ is a unit vector in the $\vec{r}$-direction. Corresponding to diagram (b) in Fig. 3.8 is the pion-in-flight current:

$$
\begin{aligned}
J_{\dot{\mu}}^{(2)}\left(\vec{x}_{1}, \vec{x}_{2} ; q\right)_{\text {pionic }} \\
=\epsilon\left(f / m_{\pi}\right)^{2}[\vec{\tau}(1) \times \vec{\tau}(2)]_{3}(\vec{\sigma}(1) \cdot \vec{\Gamma}(1))(\vec{\sigma}(2) \cdot \vec{\nabla}(2)) \\
\quad \times \int_{-1 / 2}^{+1 / 2} d v[i \vec{q} r v+\vec{x}]_{k} \frac{e^{-x}}{x} \exp i \vec{q} \cdot(\vec{R}-v \vec{r}) \quad \mu=k=1,2.3 \\
\approx 0 \quad \mu=0 .
\end{aligned}
$$

where $\vec{r} \equiv \vec{x}_{1}-\vec{x}_{2}, \vec{R} \equiv\left(\vec{x}_{1}+\vec{x}_{2}\right) / 2$, and $\vec{x} \equiv L_{\pi} \vec{r}$ with $L_{\pi} \equiv\left(m_{\pi}^{2}-q^{2}\left(v^{2}-1 / 4\right)^{1 / 2}\right.$ Finally, for the nucleon resonance term in Fig. $3.8 \mathrm{c}$ one has

$$
\begin{aligned}
& J_{\mu}^{(2)}\left(\vec{x}_{1} \cdot \vec{x}_{2}: q\right)_{N} \cdot \\
&=\frac{i m_{\pi}^{3} \mu^{T}}{6 m_{N} x_{\pi}}\left\{4 h _ { 2 } \left[\left(\vec{q} \times \vec{u}_{r}\right)_{k} \vec{u}_{r} \cdot\left(\tau_{3}(2) \vec{\sigma}(2) e^{i q \cdot z_{1}}+\tau_{3}(1) \vec{\sigma}(1) e^{i \vec{q} \cdot z_{2}}\right)\left[x_{\pi} d / d x_{\pi}-1\right]\right.\right. \\
&\left.+\tau_{3}(2)(\vec{q} \times \vec{\sigma}(2))_{k} e^{i q \cdot z_{1}}+\tau_{3}(1)(\vec{q} \times \vec{\sigma}(1))_{k} e^{i \vec{q} \cdot z_{2}}\right] \\
&-h_{1}[\vec{\tau}(1)\times \vec{\tau}(2)]_{3}\left[\vec{q} \times\left((\vec{\sigma}(1) \times \vec{\sigma}(2))\left(e^{i \vec{q} \cdot z_{1}}+e^{i q \cdot z_{2}}\right]\right.\right. \\
&+\left[\left(\vec{\sigma}(1) \times \vec{u}_{r}\right)\left(\vec{\sigma}(2) \cdot \vec{u}_{r}\right) e^{i q \cdot z_{1}}-\left(\vec{\sigma}(2) \times \vec{u}_{r}\right)\left(\vec{\sigma}(1) \cdot \vec{u}_{r}\right) e^{i \vec{q} \cdot \vec{z}_{2}}\right] \\
&\left.\left.\left.\times\left[x_{\pi} d / d x_{\pi}-1\right]\right)\right]_{k}\right\} \frac{d}{d x_{\pi}}\left(\frac{e^{-x_{*}}}{x_{\pi}}\right) \quad \mu=k=1,2,3 \\
& \approx 0 \quad \mu=0,
\end{aligned}
$$

where $h_{1}=0.074 m_{\pi}^{-3}$ and $h_{2}=0.0658 m_{\pi}^{-3}$ are the couplings used [Chei1b] to take into account both the $\Delta_{33}$ and Roper resonances. Two-particle matrix elements may then be obtained using appropriate single-particle wave functions, for instance, using plane waves when treating quasielastic scattering or harmonic oscillator wave functions when treating discrete states via the nuclear shell model.

Two considerations are critical in understanding the role of MEC in electron scattering. The first is the range of the exchanged meson. Since MEC involve (at least) two 
nucleons and since the repulsive hard core of the nucleon-nucleon interaction tends to keep nucleons apart, one can on general grounds expect the longer-ranged meson exchanges to be the most important. In addition to this consideration, however. one must also observe the "selection rules" associated with each current. From the consideration of range. for example. the above single-pion exchange graphs are expected to be the most important. yet they contribute to lowest order only for the vector three-current and the axial-vector charge and only to isovector transitions. The other terms are higher order in $-i / m_{i}$ and or $k / m . x$.

In has become fairly common to include the effects of the one-meson-exchange currents ( with $\pi, \rho$. and $\rightarrow$ mesons) in modern treatments of electron scattering from nucies. For transitions that obey the MEC "selection rules" the MEC typically contribute as much as $10 \%$ in the region of maxima in transverse $\left(e, e^{\prime}\right)$ form factors. In the higher- $Q^{2}$ "tail" of nuclear form factor. .IEC usually fall off more gradually than the one-body contribution and hence are relatively more important. There are also special cases w. expected to play an important role or indeed dominate due to some "accidental" cancellation in the nucleon contributions to the currents (e.g., electrodisintegration of the deuteron near threshold). In such cases calculations of the one-meson exchange currents generally: provide a reliable description of the data and give confidence in our ability to treat the leading-order .IEC accurately.

In the context of semileptonic $\mathrm{NC}$ interactions, the MEC corrections to the isovector and non-strange isoscalar weak neutral vector currents are identical to those entering the $T=0$ and $T=1 \mathrm{E} . \mathrm{M}$ currents. This feature follows directly from Eq. (3.16). There exist. however, additional MEC considerations germane to $\mathrm{VC}$ matrix elements which do not enter the analysis of the E.M current. First, as noted above. the leading axial-vector .IEC's arise in the time component of $\left(\left\|J_{\mu s}^{N c}\right\|\right)$ rather than the spatial components. as in the case of $\left\langle\left\|J_{\mu}(T=1)\right\|\right\rangle$. Moreover, the two-body axial-vector charge operator. $\dot{\rho}_{3}^{2 !}$. enters at the same order in $v / c$ (i.e., $k_{F} / m_{N}$ or $\omega / m_{N}$ ) as the one-body axial-vector charge operä:nr In contrast, the leading MEC corrections to the isovector vector currents enter at relative order $v / c$ with regard to the one-body terms. This result carries a number of implicatıons for tite analysis of semileptonic NC observables. First, the PV asymmetry receives no axial-vector. MEC corrections from pion exchange to leading non-trivial order in $v / c$. The reason is that there exist no EM Coulomb multipoles having the same parity for a given $J$ as Coulomb and longitudinal projections of $\hat{\rho}_{5}$, so that the latter do not enter the interference response, $W^{(\mathrm{PV})}$ (see Eqs. (3.114) below). Only the axial-vector three-current enters this response via products of the matrix elements $\left\langle\left\|\hat{T}_{J}^{\text {els }}\right\|\right\rangle\left\langle\left\|\hat{T}_{J}^{\text {mag }}\right\|\right\rangle^{*}$ or $\left\langle\left\|\hat{T}_{J}^{\text {mags }}\right\|\right\rangle\left\langle\left\|\hat{T}_{J}^{e l}\right\|\right\rangle^{*}$. Consequently, axial-vector terms in the PV asymmetry, which involves the ratio of $W^{(\mathrm{PV})}$ and the $\mathrm{EM}$ response, receive $\mathrm{MEC}$ contributions primarily via the EM vector current matrix elements. In contrast, the neutrino scattering cross section. which is second order in the weak interaction, generally does contain products of axialvector charge matrix elements (see Eq. (3.150) below) and may, therefore, be sensitive to axial-vector MEC's at leading non-trivial order.

An additional set of MEC considerations involves the isospin and flavor dependence of the vector current operators. In general, the two-body isovector and isoscalar current 
operators possess different spin- and momentum-dependencies. so that two-body corrections to the one-body $T=1$ and $T=0$ matrix elements will differ. Since the weak neutral and E.M currents contain different linear combinations of the isovector and isoscalar currents see $\mathrm{E}_{\mathrm{q}} .(3.16)$, the corresponding matrix elements will also display different relative sensitivities to.$I E C$ 's. As a result, vector current terms in $A_{L R}$. which involves the ratio of weak neutral vector current and E.II current matrix elements. may retain a sensitivity to .IEC corrections. In the special case of pure isoscalar transitions, such as elastic scatter. ing form ${ }^{H} \mathrm{He}$. MEC contributions to the non-strange vector current terms in $t_{L A}$ cancel. since the isoscalar . $\mathrm{C}$ C and E.M nuclear operators are identical apart from the electroweak couplings. $\xi_{v}^{(T)}$.

The situation with the two-body corrections to the one-body strange quark currents is more subtle. In this case. one must distinguish among the so-called "pair". "mesonic". and "isobar" MEC's illustrated in Fig. 3.8. The pair currents (Fig. 3.3a) involve the creation of a virtual $X \bar{Y}$ pair by the exchanged; or $Z^{0}$. Conventionally, one derives an effective two-body operator for this process by retaining the negative energy part of the nucleon propagator and employing the same nucleon form factor (viz. $G_{E}$ or $\left.G_{M}\right)$ as appears in the one-body operator. Thus. for example. the leading $T=0$ and $T=1$ Coulomb operators have the form

$$
\dot{V}_{J}^{(1)}(T)+\dot{H}_{J}^{(2)}(T)^{\text {pair }}=G_{\varepsilon}^{\tau}\left[\hat{O}_{J}^{(1)}(T)+\dot{O}_{J}^{[2]}(T)^{\text {pair }}\right]
$$

where the $\hat{O}_{J}^{[1,2\}}(T)$ are nuclear operators. In contrast, the operators arising from the interaction of the $\gamma$ or $Z^{0}$ with the exchanged meson (Fig. 3.8b) or excitation of a nucleon resonance (Fig. 3.8c) involves different form factors. Again, in the case of the Coulomb operators. one has

$$
\begin{aligned}
\dot{H}_{J}^{[2]}(T)^{\text {mesonic }} & =F_{M} \hat{O}_{J}^{[2]}(T)^{\text {mesonic }} \\
\hat{M}_{J}^{(2]}(T)^{\text {isobar }} & =G_{E}^{(B, T)} \hat{O}_{J}^{[2]}(T)^{\text {isobar }},
\end{aligned}
$$

where $F_{M}$ and $G_{E}^{(B, T)}$ are form factors associated with the charge of the exchanged meson and with the isobar transition and $\hat{O}_{J}^{[2]}(T)^{\text {mesonic, isobar }}$ are the corresponding nuclear operators. To illustrate further the impact of these corrections. we consider PV elastic scattering from ${ }^{4} \mathrm{He}$, which is a pure isoscalar process. In this case, one has for the weak IC Coulomb matrix element

$$
\begin{aligned}
& \left\langle\text { g.s. }\left\|. \hat{H}_{0}^{[1]}(T=0)\right\| \text { g.s. }\right)^{\vee c}=\sqrt{3} \xi_{v}^{T=0}\left\{G_{E}^{T=0}\left\langle\text { g.s. }\left\|\hat{O}_{0}^{[1]}(T=0)+\hat{O}_{0}^{[2]}(T=0)^{\text {pair }}\right\| \text { g.s. }\right\rangle\right. \\
& +F_{M}\left\langle\text { g.s. }\left\|\dot{O}_{0}^{[2]}(T=0)^{\text {mesonic }}\right\| \text { g.s. }\right)+G_{\varepsilon}^{(B . T=0)}\left(\text { g.s. } \| \hat{O}_{0}^{[2]}(T=0)^{\text {isobar } \| \text { g.s. })}\right\} \\
& +\xi_{v}^{(0)}\left\{G_{\varepsilon}^{(s)}\left\langle\text { g.s. }\left\|\hat{O}_{0}^{[1]}(T=0)+\hat{O}_{0}^{[2]}(T=0)^{\text {pair }}\right\| \text { g.s. }\right\rangle\right.
\end{aligned}
$$

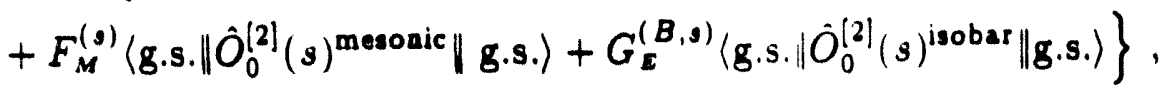


where " 3 " indicates a form factor or operator associated with $\overline{3} \gamma_{\mu} s$ and where we have assumed the exchange of only one type of meson and the excitation of only a single nucleon isobar for simplicity. The E.I C'oulomb matrix element has a similar structure

$$
\begin{aligned}
& \text { (g.s. } \left.\dot{M}_{0}^{[1}(T=0) \| \text { g.s. }\right)^{r . M}=\left\{G _ { x } ^ { r = 0 } \left(\text { g.s. }\left\|\hat{O}_{0}^{(1)}(T=0)+\dot{O}_{0}^{(2)}(T=0)^{\text {parr }}\right\|\right.\right. \text { g.s.) } \\
& +F_{M}\left(\text { g.s. } \mid \hat{O}_{0}^{(2)}(T=0)^{\text {mesonic }} \| \text { g.s. }\right)+G_{\boldsymbol{c}}^{(B . T=0)}\left(\text { g.s. } \| \hat{O}_{0}^{(2)}(T=0)^{\text {isobar } \| \text { g.s. })}\right\} \text {. }
\end{aligned}
$$

The one-body and pair operators multiplying $G_{\boldsymbol{r}}^{\mathrm{r}=0}$ and $G_{\boldsymbol{\varepsilon}}^{(0)}$ are identical since the corresponding diagrams have the same spin and isospin structure. Those arising from mesonic and isobar graphs. however. do not give identical operators for the isoscalar and strangequark currents. since the non-nucleonic degrees of freedom in the nucleus display a different flavor structure than the nucleons the nselves. The authors of Ref. [Sch90] have shown that for low-to-moderate momentum tri...fer, the one-body and pair currents dominate the E.I matrix element:

$$
\begin{gathered}
\mid F_{M}\left(\text { g.s. }\left\|\hat{O}_{0}^{[2]}(T=0)^{\text {mesonic }}\right\| \text { g.s. }\right) / G_{\varepsilon}^{T=0}\left\langle\text { g.s. }\left\|\dot{O}_{0}^{[1]}(T=0)+\partial_{0}^{(2)}(T=0)^{\text {pair }}\right\|\right. \text { g.s. } \\
\ll 1 .
\end{gathered}
$$

Were a similar result to hold for the mesonic and isobar contributions to the strange-quark matrix elements. then the MEC contributions to the strange-quark term in $A_{L R} \mid{ }^{4} \mathrm{He}$, would largely cancel, leaving a dependence primarily on the ratio of single-nucleon form factors, $G_{\varepsilon}^{(s)} / G_{\varepsilon}^{\mathrm{T}=0}$. A study of mesonic and isobar. MEC contributions to nuclear matrix elements of $\bar{s} \gamma_{\mu} s$ is in progress [Mus93b].

A final class of MEC corrections to PV electron scattering arises from nuclear PV. We postpone a discussion of these corrections until Sect. III.D.5 below.

\section{III.D.4. iSOSPIN-.MIXI.IG}

Another issue is the assumption of an exact isospin symmetry at the nuclear level. In the case of elastic PV electron scattering from $\left(J^{\pi} T\right)=\left(0^{+} 0\right)$ nuclei (Sect. IV.B), the assumption of exact isospin symmetry allows one to eliminate much of the dependence of the PV asymmetry on the details of the nuclear structure of the ground state (in the absence of s-quark contributions). The presence of charge-symmetry-breaking (CSB) nuclear forces, such as the Coulomb interaction between protons, reduces strong isospin to an approximate symmetry and the effects of this approximation must be understood and estimated.

Isospin-mixing has been the subject of study throughout much of the history of nuclear physics (see the discussion in (Don89]). While an exact description of nuclear structure remains elusive, enough phenomenological information is available to permit one to make reasonable estimates of the effects of isospin-mixing in PV $\left(\vec{e}, e^{\prime}\right)$ from a variety of nuclear targets. Such estimates are reported in Ref. [Don89] for a number of relevant nuclei and transitions. The authors conclude that the theoretical uncertainty 
associated with isospin-mixing is generally large enough to introduce non-negligible error, into Standard Model tests with PV electron scattering. However, they also find that ther" are also more favorable cases (including the critical elastic scattering from $\left(0^{+} 0\right.$ ) states in very ligh nuclei discussed in Sects. IV.B and $V$ ) where isospin mixing can be ignored at a level relesant to Standard Model tests.

The approach caken in Ref. Don89! is to consider mixing of ewo states $T_{1}$ and $T_{\text {: }}$ having exact isospin $T_{0}$ and $T_{1}$ respectively, to produce states of nominal isompin " $T_{11}$ and $" T_{1} "$ :

$$
\begin{aligned}
& \left.{ }^{\prime} T_{0}{ }^{\prime}\right\rangle=\cos \backslash T\left|T_{0}\right\rangle+\sin \backslash r \mid T_{1\rangle} \\
& \left.{ }^{\prime} T_{1}{ }^{\prime}\right\rangle=-\sin \backslash T T_{0\rangle}+\cos \backslash T\left(T_{1}\right\rangle .
\end{aligned}
$$

Since matrix elements of the charge-symmetry-breaking piece of the nuclear interaction in light nuclei are generally much smaller (up to a few hundred kel; than the "nery: splitting between states of identical spins but different isospins (typically a few Mel., ..." isospin mixing can be treated perturbatively. In this case. $I T$ is small and

$$
\begin{gathered}
\cos \backslash T \approx 1 \\
\sin \backslash T \approx{ }_{T T}=\left\langle T_{1}\left|\dot{H}_{C S B}\right| T_{0}\right\rangle / \Delta E,
\end{gathered}
$$

where $\dot{H}_{C S B}$ is a charge-symmetry-breaking interaction and $\Delta E$ is the energ: difference between the two states. giving

$$
\left|" T_{0} "\right\rangle \approx\left|T_{0}\right\rangle+\gamma T\left|T_{1}\right\rangle
$$

and

$$
\left|" T_{1} "\right\rangle \approx-\gamma T\left|T_{0}\right\rangle+\left|T_{1}\right\rangle
$$

For elastic scattering, the ground state will have the minimum allowed isospin $T_{0}={ }_{1} .{ }^{\circ}-$ $Z 1 / 2$. so only higher isospin $T_{1}>T_{0}$ need be considered. Furthermore, the transition operators we consider are either isoscalar or isovector, so states with isospin $T_{1}>T_{0}-1$ will not be connected to the dominant $\left|T_{0}\right\rangle$ terms in Eq. (3.78). Thus we limit discussion to mixing with states of $T_{1}=T_{0}+1$. One can also go beyond the simple two-state mixing of Eqs. (3.78) in first-order perturbation theory simply by adding additional states of isospin $T_{0}+1$.

In taking matrix elements between states of nominal isospin, the isospin selection rules are only approximately valid and we obtain additional terms of $O(X T)$ that would be missing for matrix elements between states of pure isospin. To illustrate, consider the case of electron scattering from a spin-0, (nominally) isospin-0 nucleus. To form the asymmetry one needs only matrix elements of the $\dot{M}_{0}$ projection of the electromagnetic and weak neutral currents. For pure isospin states, only the isoscalar operator contributes and we have (neglecting s-quark contributions)

$$
F_{C J}(q)=\left\langle 0^{+} 0\left\|\cdot \dot{M}_{0}(T=0)\right\| 0^{+} 0\right\rangle
$$


and

$$
\tilde{F}_{C J}(q)=\left(\frac{1}{2} \sqrt{3} \xi_{i}^{T=0}\right)\left(0^{+} 0\left\|\cdot \dot{K}_{0}(T=0)\right\| 0^{+} 0\right)
$$

so that in the PV asymmetry, which depends on the ratio of these form factors (see Sect. III.E , the single common matrix element cancels and we obtain for this ratio the (structure-independent) first factor in Eq. (3.82b). Including the isospin-mixed states with (small, $T=1$ components. however, allows for nonzero matrix elements of isovector operators as well

$$
\begin{aligned}
F_{C J}(q) & =\left(0^{+} \cdot 0^{*}\left\|\cdot \dot{H}_{0}(T=0)+. \dot{H}_{0}(T=1)\right\| 0^{+} \cdot 0^{*}\right\rangle \\
& \approx\left(0^{+} 0\left\|. \dot{H}_{0}(T=0)\right\| 0^{+} 0\right\rangle+2 \chi T\left(0^{+} 0\left\|. \dot{H}_{0}(T=1)\right\| 0^{+} 1\right)
\end{aligned}
$$

and

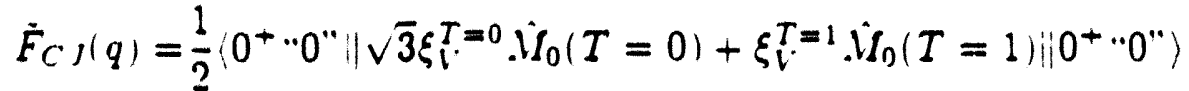

$$
\begin{aligned}
& \left.\approx \frac{1}{2} \sqrt{3} \xi_{1}^{T=0} 0^{+} 0\left\|\cdot \dot{U}_{0}(T=0)\right\| 0^{+} 0\right\rangle+x T \xi_{i}^{T=1}\left(0^{+} 0\left\|\cdot \dot{H}_{0}(T=1)\right\| 0^{-1}\right.
\end{aligned}
$$

Two different structure-dependent matrix elements now enter the form factors. Moreover. the numerator and denominator in their ratio. which governs the $\mathrm{PV}$ asymmetry. contain different linear combinations of these two matrix elements. Keeping only terms up to order $Y T$, one can write the ratio as

$$
A_{L R} \times \frac{1}{2} \sqrt{3} \xi_{V}^{T=0}+\Gamma(q)
$$

with

$$
\Gamma(q)=\gamma T\left(\xi_{V}^{T=1}-\sqrt{3} \xi_{V}^{T=0}\right) \frac{\left(0^{+} 0\left\|\hat{H}_{0}(T=1)\right\| 0^{+} 1\right\rangle}{\left\langle 0^{+} 0\left\|\hat{H}_{0}(T=0)\right\| 0^{+0}\right\rangle} .
$$

For transitions involving a single multipole, analogous expressions obtain. For other types of transitions where additional multipoles contribute, the effects of isospin impurities can be treated in a similar (though algebraically more complicated) manner, introducing small (of order $(T$ ) additional matrix elements for each multipole.

For elastic scattering from light $\left(0^{+} 0\right)$ nuclei, such as ${ }^{4} \mathrm{He},{ }^{12} \mathrm{C}$, and ${ }^{16} \mathrm{O}$. the authors ot Ket. [Donöyj estimate $\Gamma(q)<0.01$ over the entire momentum transfer range of practical interest for PV electron scattering experiments (see also Sect. IV.B). For heavier $\left(0^{+} 0\right)$ nuclei such as ${ }^{28} \mathrm{Si}$, the effect can be much larger with $\Gamma$ increasing from 0 at $q=0$ (a consequence of the orthogonality of the $T=0$ and $T=1$ states) to a few percent for 1 $\mathrm{fm}^{-1}<q<3 \mathrm{fm}^{-1}$. This significant difference between the light and heavier nuclei can be attributed to the relative inability of the former to support an isovector (monopole) breathing mode. For the $C 2$ (forward-angle) excitation of the $4.44 \mathrm{MeV}\left(2^{+} 0\right)$ first excited state in ${ }^{12} \mathrm{C}$, the isospin-mixing correction $\Gamma(q)$ may be as much as a few percent at intermediate $q$; this has important implications for the energy resolution require - for a elastic scattering experiment. Finally, for the $\mathrm{M} /$ excitation of the $12.71 \mathrm{MeV}\left(1^{+} 0\right)$ state (a transition frequently used to study the effects of isospin-mixing with the relatively strong, relatively close in energy, isovector $\mathrm{M} 1$ excitation of the $15.11 \mathrm{MeV}\left(1^{+} 1\right)$ state) the authors of Ref. [Don89] find effects as large as $\Gamma \approx 2$ at low $q$ and $\Gamma \approx 0.3$ at intermediate $q$ and conclude that, were the figure-of-merit not so low, $\left(\vec{e}, e^{\prime}\right)$ experiments would be excellent means for studying isospin-mixing in such cases. 


\section{III.D.j. PARITY-.IIXI.NG A.ND THE A.NAPOLE MOMENT}

In a manner analogous to the mixing of states with good isospin by charge-symmetry breaking forces. the weak...++ interaction induces small admixtures of opposite-parity states into states of a given. exact parity. One consequence of such parity-mixing is the existence of non-vanishing nuclear observables normally forbidden by parity-invariance. In the case of electron scattering. for example. a virtual photon emitted by the electron could couple to a nuclear multipole having the "wrong" parity for a given transition. I similar process involving an atomic electron interacting with the nucleus can give tise to a parity-riolating atomic Hamiltonian which. in turn. induces mixing between opposite parity atomic states. Such atomic parity-mixing would be manifested in the existenie of parity-forbidden atomic observables. These possibilities have motivated suggestions for studying the weak nuclear force with charged lepton probes. As noted in Sect. II. one hopen that such studies might complement other nuclear PV experiments and help to further ton the conventional picture of nuclear PV.

In this conventional picture. contributions from nuclear $\mathrm{PV}$ to semileptonic inter actions occur via the diagrams in Fig. 3.9. In Fig. 3.9a.b the exchange of the lightest pseudoscalar and vector mesons - with one vertex being parity-conserving and the ot ther parity-violating - mixes nuciear states of good parity which then couple to the virrual photon. The weak quark-quark interaction mediated by short range $(\sim 0.002 \mathrm{fm}) I^{\cdot \pm}$ and $Z^{0}$ exchanges occurs within the $\mathrm{PV}$ meson-nucleon vertex, while the resultant $\mathrm{PV} \times . .$. interaction occurs over a larger distance scale. In the conventional framework. this $\stackrel{-}{*}$ interaction takes the form

$$
\hat{H}_{P V}^{(2)}=\sum_{1} g_{N N M}^{(1)} h_{N N M}^{(1)} \hat{O}_{(1)}^{(2)}
$$

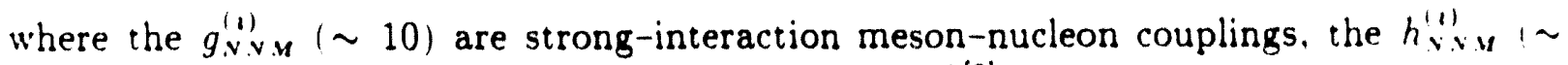
$10^{-i}$ ) are the weak PV meson-nucleon couplings, the $\hat{O}_{(1)}^{(2)}$ are two-body nuclear operators. and the sum over $i$ corresponds to the various exchanged mesons and isospin channels. Typically, one includes only $\pi-, \rho-$, and $\omega$-exchange. Under this truncation. the different isospin components of the weak interaction between light quarks $(T=0,1,2)$ give rise to seven terms in the sum of $\mathrm{Eq}$. (3.87) corresponding to the parameters. $h_{\pi}, h_{-}^{0.1}, h_{\mu}^{0.1 .2}$. and $h_{1,}^{\prime}$. Since the two-body operators $\hat{O}_{(1)}^{[2]}$ are generally momentum-dependent. one must also include PV meson-exchange current contributions (Fig. 3.9c) in matrix elements of the nuclear E.M current in order to satisfy the continuity equation: $Q^{\lambda}\left(f\left|J_{\lambda}^{E . M}\right| \imath\right)=0$.

In the simplest case of two-state parity-mixing, one has in analogy with Eq. (3.78)

$$
\begin{aligned}
\left|" \pi^{+"}\right\rangle & =\cos \chi_{P}\left|\pi^{+}\right\rangle+\sin \chi P\left|\pi^{-}\right\rangle \\
& \approx\left|\pi^{+}\right\rangle+\chi P\left|\pi^{-}\right\rangle .
\end{aligned}
$$

where $\left|\pi^{+}\right\rangle$and $\left|\pi^{-}\right\rangle$are states of exact, but opposite, parity and $\left|" \pi^{+} "\right\rangle$ is a state of nominal parity " $\pi^{+"}$. The mixing angle is simply

$$
\chi_{P}=\left\langle\pi^{-}\left|\hat{H}_{P V}^{(2)}\right| \pi^{+}\right\rangle / \Delta E,
$$


with $\triangle E$ being the difference in energy between $\left|\pi^{+}\right\rangle$and $\left|\pi^{-}\right\rangle$. Generically, one has $x_{P} \sim 10^{-i}$ (assuming typical nuclear level spacings) [Ade85], so use of the perturbative expression (3.88) is valid. For nuclei having nearly-degenerate opposite-parity states, the scale of $\backslash P$ may be enhanced by an order of magnitude or more [Ade85]. In a realistic nucleus. one must include mixing of the full spectrum of opposite-parity states into $\mid \pi^{+} ;$. although for nuclei possessing a pair of nearly degenerate opposite-parity levels. the use of two-state mixing may be a justifiable approximation.

To illustrate the impact of Eq. (3.88) on semileptonic processes, we consider an elastic process for which $\left\langle\pi^{+}\left|\hat{T}_{1 \lambda}^{\mathrm{el}}\right| \pi^{+}\right\rangle_{E M}=0=\left\langle\pi^{+}\left|\hat{T}_{1 \lambda}^{\mathrm{el}}\right| \pi^{+}\right\rangle_{E M}$, according to the selection rules discussed in Sect. III.D.I (the subscript E.M denotes multipole projections of the E.I current). Matrix elements of $\hat{T}_{1 \lambda}^{\mathrm{el}}$ in the parity-mixed state $\left|" \pi^{+} "\right\rangle$, however. need not vanish. Rather, one has

$$
\left\langle " \pi^{+} "\left|\hat{T}_{1 \lambda}^{\mathrm{el}}\right| " \pi^{+"}\right\rangle_{E M}=2 \operatorname{Re}\left\{\chi_{P}\left\langle\pi^{+}\left|\hat{T}_{1 \lambda}^{\mathrm{el}}\right| \pi^{-}\right\rangle_{E M}\right\} .
$$

The matrix element in Eq. (3.90) corresponds to the coupling of a virtual photon to the nucleus via the processes shown in Fig. 3.9a,b. The meson-exchange process of Fig. 3.9c induce a two-body E.M current operator having the spacetime transformation properties of an axial-vector:

$$
J_{\lambda S}^{E M}[2]=\sum_{i} g_{N N M}^{(i)} h_{N N M}^{(i)} \hat{j}_{\lambda S}^{[2]}
$$

where the $\hat{j}_{\lambda 5}^{[2]}$ are two-body axial-vector current operators. According to the selection rules discussed above, the current of Eq. (3.91) will also contribute to elastic matrix elements of $\hat{T}_{1 \lambda}^{\mathrm{el5}}$ as

$$
\left\langle " \pi^{+} "\left|\hat{T}_{1 \lambda}^{\mathrm{el},[2]}\right| " \pi^{+} "\right\rangle_{E M}=\left\langle\pi^{+}\left|\hat{T}_{1 \lambda}^{\mathrm{e} 15,[2]}\right| \pi^{+}\right\rangle+\mathcal{O}\left(\chi_{P}^{2}\right) .
$$

The full elastic transverse electric matrix element will be the sum of Eqs. (3.90) and (3.92). These two terms represent contributions of, respectively, matrix elements of the normal (polar vector) E.M current between states of opposite parity and matrix elements of the two-body axial-vector MEC between states of like parity.

The anaiysis for nuclear transitions is similar to that for elastic processes. In both cases, parity-mixing plus PV MEC's give rise to non-vanishing EM multipole matrix elements normally forbidden in the absence of nuclear PV. From the discussion of Sect. III.D.1, we observe that these parity-forbidden multipoles are precisely those which appear in matrix elements of the nuclear axial-vector weak neutral three-current. In effect, nuclear PV induces an axial-vector, conserved EM current which contributes in tandem with the nuclear axial-vector $\mathrm{NC}$ to semileptonic processes involving charged leptons:

$$
\left\langle f\left|\hat{O}_{J \lambda}^{5}\right| i\right\rangle_{N C} \rightarrow\left\langle f\left|\hat{O}_{J \lambda}^{5}\right| i\right\rangle_{N C}+\beta\left\langle f\left|\hat{O}_{J \lambda}^{5}\right| i\right\rangle_{E M}
$$

where

$$
\beta=-\frac{8 \sqrt{2} \pi \alpha}{G_{\mu} Q^{2}} \frac{Q_{e}}{g_{V}^{e}} .
$$

Although the nuclear PV contribution is generically suppressed by roughly $\alpha$ with respect to the tree-level, axial-vector NC interaction, nuclear structure effects and/or a fortuitous suppression of the axial-vector NC amplitude may enhance the relative importance of the nuclear PV contribution. 
The .iuclear Anapole . Moment

A special case of relevance to atomic PV and low-energy PV electron scattering is given by the low $-\left|Q^{2}\right|$ limit of the elastic $\hat{T}_{1 \lambda}^{\text {el }}$ matrix element discussed above. The requirements of current conservation, embodied in an "extended" version of Siegert 's Theorem [Sie37. Fri84. Fri85]. allows one to decompose this operator as [Hax89]

$$
\hat{T}_{1 \lambda}^{\mathrm{el}}=\hat{S}_{1 \lambda}^{\mathrm{el}}+\hat{R}_{1 \lambda}^{\mathrm{el}}
$$

where $\hat{S}_{1 \lambda}^{\text {el }}$ is a piece constrained by current conservation and $\hat{R}_{1 \lambda}^{e l}$ is an unconstrained remainder: For elastic processes, matrix elements of $\hat{S}_{1 \lambda}^{\mathrm{el}}$ vanish. For low $-q^{2}$. the remainder has the form

$$
\hat{R}_{1 \lambda}^{\mathrm{el}} \rightarrow-\frac{i}{\sqrt{6 \pi}} \frac{q^{2}}{m_{N}^{2}} \hat{a}_{\lambda}+\mathcal{O}\left(q^{4}\right)
$$

where

$$
\hat{a}_{\lambda}=\frac{m_{N}^{2}}{9} \int d^{3} r r^{2}\left\{\hat{J}_{\lambda}^{E \cdot M}(\vec{r})+\sqrt{2 \pi}\left[Y_{2}\left(\Omega_{r}\right) \gtrsim \hat{J}^{E M}(\vec{r})\right]_{1 \lambda}\right\}
$$

is the anapole moment operator. In Eq. (3.97). $\hat{J}^{E M}$ is the full E.M three-current operator. VIatrix elements of $\hat{a}_{\lambda}$ containing the polar vector component of $\hat{J}^{E M}$ are non-vanishing between opposite-parity components of a state of mixed-parity (as in Eq. (3.90)), while those containing the PV MEC operator are non-vanishing between same-parity components (Eq. (3.92)). The sum of these matrix elements defines the nuclear anapole moment (A.M).

While a detailed discussion of the anapole moment can be found elsewhere [Mus91. Hax89 and references therein], we make a few comments on the form of Eqs. (3.96) and (3.97). First, the matrix element of Eq. (3.96) vanishes for real photons $\left(q^{2}=0\right.$ in the Breit frame) and couples only to virtual photons entering semileptonic interactions with the nucleus. Moreover, when multiplied by the $1 / q^{2}$ appearing in the photon propagator in the semileptonic amplitude, the matrix element of $\mathrm{Eq}$. (3.96) gives rise to a contact interaction between leptonic and hadronic currents in co-ordinate space, like that engendered by low$q^{2} Z^{0}$-exchange. The result is that transverse electric projections of the axial-vector $\mathrm{NC}$ should be replaced by the following combination

$$
g_{V}^{e}\left\langle\left|\hat{T}_{1 \lambda}^{e \mid 5}\right|\right\rangle \rightarrow g_{V}^{e}\left\{\left\langle\left|\hat{T}_{1 \lambda}^{e \mid 5}\right|\right\rangle+\xi_{A M}\left\langle\left|\hat{a}_{\lambda}\right|\right\rangle\right\}
$$

where

$$
\xi_{A M}=\frac{Q_{e}}{g_{V}^{e}}\left[8 \sqrt{\frac{\pi}{3}}\right]\left(\frac{\alpha}{G_{\mu} m_{N}^{2}}\right) \approx 6.4 \times 10^{4}
$$

Third, the scale of $\langle\|\hat{a}\|\rangle$, typically $\sim 10^{-6} \rightarrow 10^{-7}$, grows as $A^{2 / 3}$. This result is suggested by the $r^{2}$ factor appearing in the integral (3.97) and has been shown rigorously by the authors of Refs. [Fla84, Hax89]. The scale of the anapole moment can also be strongly enhanced in nuclei having a pair of nearly degenerate opposite-parity states, where one of 
the states is the ground state. One finds. then. that for heavy and/or nearly-degenerate nuclei the scale of the axial-vector $\mathrm{IC}$ and nuclear anapole contributions to the low- $q^{2}$. elastic electron-nucleus interaction can be commensurate [Fla84, Hax89]. In addition. the relative importance of the anapole contribution is amplified in $T=0$ nuclei. whose axialvector $\mathrm{XC}$ matrix elements are suppressed. Consequently, were it possible to separate contributions from the axial-vector $\mathrm{VC}$ and anapole moment to semileptonic processes involving such nuclei. one could in principle impose new constraints on nuclear PV via a determination of $\langle\mid a \|\rangle$. This possibility is discussed more fully in Sect. IV.E.

Finally. from a formal standpoint. it is not possible to define the A.M of an elementary particle (lepton or quark) or nucleon unambiguously, since it depends on the choice of electroweak gauge parameter [Mus91]. The contributions made by the electron. quark. and nucleon A.I's to scattering amplitudes is more appropriately included in the full set of axial-vector radiative corrections, $R_{A}$. The many-body nuclear $A . M$ is, in contrast. gauge-parameter independent and. in principle. distinguishable from the $R_{A}$ due to its $A^{2 / 3}$ scaling behavior (Mus91). 


\section{III.E. Lepton Scattering from Nucleons and Nuclei}

In this section we discuss the basic formalism required in studying inclusive lepton scattering from nucleons and nuclei. In particular. we summarize in Sect. III.E.1 the expressions that will prove to be useful in the rest of the article for parity-conserving and -violating electron scattering, followed by discussions of the figure-of-merit for PV electron scattering in Sect. III.E.2. The formalism presented here is meant to provide a general framework in which the detailed treatment of specific cases om Sect. IV can be carried out. levertheless, to help in introducing the essential features of PC/PV electron scattering we begin the discussion of several important examples in this section. Specifically. we introduce the formalism for elastic scattering from spin-0, spin-1/2, and spin-1 nuclei as well as the essential features of quasielastic scattering from nuclei. . More detailed discussions of these cases are contained in Sects. IV.A-C and IV.F. We conclude this section by summarizing the formalism for treating inclusive neutrino scattering from nucleons and nuclei (Sect. III.E.3), again postponing more detailed discussions to a later section (Sect. IT.J)

For all cases of inclusive, semi-leptonic scattering from nucleons and nuclei. the cross section is proportional to the contraction of a leptonic tensor, $\eta_{\mu \nu}$, and hadronic tensor. $W^{\mu \nu}$. General covariance and symmetry considerations require this contraction to have the following form [Don85. Walioj]:

$$
\eta_{\mu \nu} W^{\mu \nu} \sim V_{C C} R^{C C}+2 V_{C L} R^{C L}+V_{L L} R^{L L}+V_{T} R^{T}-\lambda V_{T^{\prime}} R^{T^{\prime}} .
$$

where $\{C, L, T\}$ stand for (charge, longitudinal, transverse and involve the $\mu, \nu=0.3$. and 1 or 2 components of the electroweak currents, respectively (as usual, we choose the $z$-axis to be along $\vec{q}$ ). The $V$ 's are lepton kinematical factors and the $W$ 's are hadronic response functions; $\lambda= \pm 1$ is the incident lepton's helicity. In the extreme relativistic limit (ERL) where the lepton masses may be neglected with respect to their energies the lepton kinematical factors become [Don85, Wal75]:

$$
\begin{aligned}
& V_{C C} \longrightarrow 1 \times \cos ^{2} \frac{\theta}{2} \\
& V_{C L} \longrightarrow-(\omega / q) \times \cos ^{2} \frac{\theta}{2} \\
& V_{L L} \longrightarrow(\omega / q)^{2} \times \cos ^{2} \frac{\theta}{2} \\
& V_{T} \longrightarrow v_{T} \times \cos ^{2} \frac{\theta}{2} \\
& V_{T^{\prime}} \longrightarrow v_{T^{\prime}} \times \cos ^{2} \frac{\theta}{2},
\end{aligned}
$$

where $q=|\vec{q}|$ and $\omega$ are the magnitude of three-momentum and the energy transferred to the hadronic system. The four-momentum transf $r$ is then $Q^{\mu}=(\omega, \vec{q})$. Carrying the 
overall factor $\cos ^{2} \frac{\theta}{2}$, where $\theta$ is the lepton scattering angle, the ERL transverse lepton kinematical factors are given by

$$
\begin{aligned}
v_{T} & =\frac{1}{2}\left|\frac{Q^{2}}{q^{2}}\right|+\tan ^{2} \frac{\theta}{2} \\
v^{\prime} T^{\prime} & =\sqrt{\left|\frac{Q^{2}}{q^{2}}\right|+\tan ^{2} \frac{\theta}{2}} \tan \frac{\theta}{2} .
\end{aligned}
$$

The response functions in Eq. (3.100) in the general case contain products (with the appropriate sum over spin and isospin quantum numbers) of matrix elements of the nuclear currents. $R^{C C} . R^{C L} . R^{L L}$ involve products of matrix elements of nuclear vector currents with nuclear vector currents and of nuclear axial-vector currents with nuclear axial-vector currents while $R^{T}$ contains a product of nuclear vector with nuclear axial-vector current matrix elements. For conserved vector currents $(\mathrm{CVC})$ the purely vector $C C . C L$ and $L I$ responses are proportional to each other: then, defining another ERL lepton kinematical factor

$$
v_{L}=\left|\frac{Q^{2}}{q^{2}}\right|^{2}
$$

the first three terms in Eq. (3.100) can be combined into one:

$$
\left[V_{C C} R^{C C}+2 V_{C L} R^{C L}+V_{L L} R^{L L}\right]_{V V} \longrightarrow v_{L} R^{L} \times \cos ^{2} \frac{\theta}{2}
$$

where $R^{L} \equiv R^{C C}=(q / \omega) R^{C L}=(q / \omega)^{2} R^{L L}$ for purely vector-vector (VV) contributions.

\section{III.E.1. BASIC ELECTRON SCATTERING FORMALISM}

In electron scattering, both graphs of Fig. 3.1 enter and, in principle, $\left(e . e^{\prime}\right)$ experiments prohe the structure of both the electromagnetic and weak neutral currents. However. for $\left|Q^{2}\right| \ll M_{z}^{2}$, the photon-exchange amplitude of Fig. 3.1a is generally several orders of magnitude larger than the $Z^{0}$-exchange amplitude in Fig. $3.1 \mathrm{~b}$ and the latter can be safely neglected. In that case, the doubly-differential $\left(e, e^{\prime}\right)$ ERL cross section may be written [deF66]

$$
\frac{d^{2} \sigma}{d \Omega d \epsilon^{\prime}}=\sigma_{M}\left\{v_{L} R^{L}(q, \omega)+v_{T} R^{T}(q, \omega)\right\} \equiv \sigma_{M} W^{(\mathrm{EM})}(q, \omega, \theta)
$$

The incident electron is assumed to have four-momentum $K^{\mu}=(\epsilon, \vec{k})$ with three-momentum $k \equiv|\vec{k}|$ and energy $\epsilon=\sqrt{k^{2}+m_{e}^{2}}$ (where $\epsilon=k$ in the ERL); similarly, the scattered electron has four-momentum $K^{\prime \mu}=\left(\epsilon^{\prime}, \vec{k}^{\prime}\right)$. The four-momentum transfer is then given by $Q^{\mu} \equiv\left(K-K^{\prime}\right)^{\mu}=(\omega, \vec{q})$, so that $\omega=\epsilon-\epsilon^{\prime}$ and $q=|\vec{q}|=\left|\vec{k}-\vec{k}^{\prime}\right|$. The kinematics of electron scattering require that $Q^{2}=Q_{\mu} Q^{\mu}=\omega^{2}-q^{2} \leq 0$. In Eq. (3.104) 
the .Mott cross section for electron scattering from a point unit charge at angle $\theta$ is given by

$$
\sigma_{M}=\left[\frac{\alpha \cos \frac{\theta}{2}}{2 \epsilon \sin ^{2} \frac{\theta}{2}}\right]^{2}
$$

and the lepton kinematical factors are given in Eqs. (3.102). As discussed above. since the electromagnetic current is purely vector in nature only the longitudinal (really a combination of $\mu=0$ and 3 components, see above) and transverse response functions. $R^{L}$ and $R^{T}$. respectively, enter. These embody the hadronic matrix elements of the electromagnetic current operators discussed in Sects. III.C and III.D and depend only on $q$ and $\lrcorner$ (or. equivalently, only on $Q^{2}$ and $\nu \equiv \omega$ in the language more commonly used in high-energy physics). In writing the above expressions we have assumed the ERL for the electron $\left(\epsilon . \epsilon^{\prime} \gg m_{e}\right)$, which. while unnecessary, does simplify the formalism somewhat (see Refs. [deF66. Don85. Don86b] for discussions of the leptonic tensor when the ERL is not invoked). We have also restricted the treatment to the first-order plane-wave Born approximation: this is usually adequate for treating scattering from the low $Z$ targets that we will mostly be considering here. However, where necessary, distortion of the incoming and outgoing electron waves can and should be included.

For elastic and inelastic scattering to discrete nuclear states. $\omega$ is fixed by the excitation energy and momentum transfer $q$ and it is conventional to use the singly-differential cross section [deF66]

$$
\frac{d \sigma}{d \Omega}=4 \pi \sigma_{M} f_{\mathrm{rec}}^{-1} F^{2}(q, \theta)
$$

where the factor involving $f_{\text {rec }}=1+\left(2 \epsilon / M_{T}\right) \sin ^{2} \frac{\theta}{2}$ accounts for recoil of the target nucleus of mass $V_{T}$. The total form factor $F^{2}$ is the sum of longitudinal and transverse contributions

$$
F^{2}(q, \theta)=v_{L} F_{L}^{2}(q)+v_{T} F_{T}^{2}(q)
$$

where $F_{L}^{2}$ and $F_{T}^{2}$ are given by

$$
\begin{aligned}
& F_{L}^{2}(q)=\sum_{J \geq 0} F_{C J}^{2}(q) \\
& F_{T}^{2}(q)=\sum_{J \geq 1}\left[F_{E J}^{2}(q)+F_{M J}^{2}(q)\right],
\end{aligned}
$$

using the form factors introduced in Sect. III.D.1. The number of terms entering each of these sums depends on how many multipole projections are allowed by the angular momentum and parity quantum numbers of the initial and final nuclear states (see Sect. III.D.1).

In order to detect the presence of the very small weak neutral current contributions to electron scattering, one must search for a characteristic signature that only occurs when such contributions are present. Since electromagnetism obeys an exact parity symmetry while the neutral current amplitude contains a parity-violating piece, one such signature would be a difference between cross sections for scattering of electrons longitudinally polarized parallel ( + or $R=$ right-handed) and anti-parallel ( - or $L=$ left-handed) to their 
momenta. as this difference is parity-violating. Based on the same arguments about the relative size of the photon-exchange and $Z^{0}$-exchange amplitudes used above to neglect the latter in unpolarized scattering, we expect this parity-violating asymmetry to be dominated by the interference of the two amplitudes. That is, our focus will be on the PV asymmetry

$$
\begin{aligned}
A_{L R} & =\left\{\frac{d^{2} \sigma^{+}}{d \Omega d \epsilon^{\prime}}-\frac{d^{2} \sigma^{-}}{d \Omega d \epsilon^{\prime}}\right\} /\left\{\frac{d^{2} \sigma^{+}}{d \Omega d \epsilon^{\prime}}+\frac{d^{2} \sigma^{-}}{d \Omega d \epsilon^{\prime}}\right\} \\
& \equiv A_{L R}^{0} \times \frac{W^{(P V)}(q, \omega . \theta)}{W^{(\text {E.M) }}(q \cdot \omega, \theta)},
\end{aligned}
$$

where [Don88. Don89. Mus92a]

$$
W^{(\mathrm{PV})}(q \cdot \omega \cdot \theta)=v_{L} R_{A V}^{L} \cdot\left(q \cdot \omega^{\prime}\right)+v_{T} R_{A V}^{T} \cdot\left(q \cdot \omega^{\prime}\right)+v_{T}^{\prime} R_{V_{A}}^{T^{\prime}}\left(q \cdot \nu^{\prime}\right)
$$

and

$$
\mathcal{A}_{L R}^{0} \equiv \frac{G_{\mu}\left|Q^{2}\right|}{2 \pi \alpha \sqrt{2}} .
$$

The parity-violating helicity-difference cross section in Eq. (3.109) involves a product of the electromagnetic amplitude of Eq. (3.3) and the parity-violating $\mathrm{NC}$ amplitude of Eq. (3.5). The latter arises from product terms of leptonic vector currents $\times$ hadronic axialvector currents and vice versa. The subscripts on the response functions in Eq. (3.110) reflect this fact and identify which currents are involved: the subscript $A V$ denotes axialvector leptonic and vector hadronic currents, whereas $V A$ indicates the vector leptonic and axial-vector hadronic currents. Note the structure of the PV response: since one has a product of electromagnetic (purely vector) current hadronic matrix elements and weak neutral current hadronic matrix elements (with both vector and axial-vector contributions), the only terms in the g aral electroweak response (Eq. (3.100)) which can occur are those labeled " $L$ ", " $T$ " anc $\cdot T$ ". In particular, since no hadronic axial-vector $\delta$ hadronic axai "ector contributions are retained (they occur only in the square of the $Z^{0}$-exchange amplitude in Fig. 3.1b. which is ignored here), the $\mathrm{CVC}$ result in Eq. (3.103) can be invoked. An additional consequence of this is that the only place where hadronic axial-vector currents do occur is in the " $T$ " term which involves only transverse projections. In Sect. III.E. 3 we will draw comparisons of the different structure that occurs in discussing neutrino scattering.

For scattering to discrete states, the asymmetry is defined similarly:

$$
\begin{aligned}
A_{L R} & =\left\{\frac{d \sigma^{+}}{d \Omega}-\frac{d \sigma^{-}}{d \Omega}\right\} /\left\{\frac{d \sigma^{+}}{d \Omega}+\frac{d \sigma^{-}}{d \Omega}\right\} \\
& \equiv A_{L R}^{0} \times \frac{W^{(\mathrm{PV})}(q, \theta)}{F^{2}(q, \theta)},
\end{aligned}
$$

where

$$
W^{(\mathrm{PV})}(q, \theta)=v_{L} W_{A V}^{L}(q)+v_{T} W_{A V}^{T}(q)+v_{T}, W_{V A}^{T^{\prime}}(q)
$$


Here, in a form similar to Eqs. (3.108), we have [Don89]

$$
\begin{aligned}
& \amalg_{A \cdot}^{L} \cdot(q)=-g_{A}^{e} \sum_{J \geq 0} F_{C J}(q) \dot{F}_{C J}(q) \\
& \left.W_{A{ }^{\prime}}^{T} \cdot q\right)=-g_{A}^{e} \sum_{J \geq 1}\left[F_{E J}(q) \tilde{F}_{E J}(q)+F_{M J}(q) \dot{F}_{M J}(q)\right] \\
& \amalg_{i+1}^{\cdot T^{\prime}(q)}=-g_{i}^{e} \cdot \sum_{J \geq 1}\left(F_{E J}(q) \tilde{F}_{M J_{s}}(q)+F_{M J}(q) \dot{F}_{E J_{s}}(q) !\right.
\end{aligned}
$$

Again. the form factors which occur here have already been introduced in Sect. III.D.1: the tilde indicates which quantities arise from matrix elements of the weak neutral current. while the " 5 " indicates which are axial-vector form factors. The factors $g_{A}^{e}$ and $g_{i}^{e}$ in Eqs. (3.114) come from the coupling of the leptonic current to the exchanged $Z^{\prime}$ see Eqs. (3.t) and (3.5)]. Recall also that, as argued above. only transverse projections of the axial-vector current enter in $\mathrm{PV}$ electron scattering (to order $G_{\mu}$ ).

Let us rewrite these results in terms of the quantity

$$
\mathcal{E} \equiv\left[1+2\left|q^{2} / Q^{2}\right| \tan ^{2} \frac{\theta}{2}\right]^{-1}
$$

which characterizes the angle-dependences in the cross section and asymmetry: $\mathcal{E} \rightarrow 1$ for $\theta \rightarrow 0^{\circ}$ and $\mathcal{E} \rightarrow 0$ for $\theta \rightarrow 180^{\circ}$. Then $v_{L} / v_{T}=2\left|Q^{2} / q^{2}\right| \mathcal{E}$ and $v_{T^{\prime}} / v_{T}=\sqrt{1-\mathcal{E}^{2}}$. The (PC) electron scattering cross section in Eq. (3.106) may then be written

$$
\frac{d \sigma}{d \Omega}=\frac{4 \pi \alpha^{2}}{\left|Q^{2}\right|}\left(\frac{\epsilon^{\prime}}{\epsilon}\right) f_{\text {rec }}^{-1} \frac{1}{1-\mathcal{E}}\left[2\left|Q^{2} / q^{2}\right| \mathcal{E} F_{L}^{2}(q)+F_{T}^{2}(q)\right]
$$

and the PV asymmetry in Eq. (3.112) may be re-expressed as

$$
A_{L R}=A_{L R}^{0} \times\left[\frac{2\left|Q^{2} / q^{2}\right| \mathcal{E} W_{A V}^{L}(q)+W_{A V}^{T}(q)+\sqrt{1-\mathcal{E}^{2}} W_{V A}^{T^{\prime}}(q)}{2\left|Q^{2} / q^{2}\right| \mathcal{E} F_{L}^{2}(q)+F_{T}^{2}(q)}\right] .
$$

Elastic scattering from $\left(J^{\pi} T\right)=\left(0^{+} 0\right)$ nuclei

The simplest example of the formalism introduced above is that of elastic scattering from a spin-0, isospin-0 target, since then only monopole form factors can occur. From Eqs. (3.108) and (3.114) one has [Don89]

$$
\begin{aligned}
F_{L}^{2}(q) & =F_{C 0}^{2} \\
W_{A V}^{L}(q) & =-g_{A}^{e} F_{C 0}(q) \tilde{F}_{C 0}(q) \\
F_{T}^{2}(q) & =W_{A V}^{T}(q)=W_{V A}^{T^{\prime}}(q)=0,
\end{aligned}
$$


and hence the hadronic ratio in Eq. (3.112) simply involves the ratio of the two monopole form factors. $F_{C 0}$ (electromagnetic) and $\dot{F}_{C 0}$ (weak neutral current):

$$
\frac{A_{L R}}{A_{L R}^{0}}=-g_{A}^{e} \times \frac{\dot{F}_{C 0}(q)}{F_{C 0}(q)} \text {. }
$$

which depends on $q$. but is independent of $\theta$. Furthermore, in the limit that the ground state is an exact $T=0$ state. only the isoscalar pieces of these currents can contribute. since the isovector piece cannot connect $T=0$ initial and final states. In the absence of strange-quark contributions, as can be seen from Eqs. (3.30) and (3.39), the only surviving nuclear matrix elements are proportional to each other with the coupling $\sqrt{3} \xi_{1}^{T=1} / 2$ as the constant of proportionality. In this case the nuclear matrix elements then cancel in the ratio in Eq. (3.119), leaving a $q$-independent number: accordingly, the predicted asymmetry is independent of the details of nuclear structure [FeiTj. Walii].

The presence of strangeness and the consideration of the higher-order electroweak processes will introduce $q$-dependent. structure-dependent terms and yield the full form of the hadronic ratio (Mus92a):

$$
\left.\frac{A_{L R}}{A_{L R}^{0}}=-\frac{1}{2}\left\{\sqrt{3} \xi_{V}^{T=0} ! 1+\Gamma(q)\right]+\xi_{V}^{(0)} \frac{F_{C 0}(s)}{F_{C 0}(T=0)}\right\} .
$$

Here $\Gamma(q)$ is introduced to account for the effects of the breaking of isospin symmetry i see Sect. III.D.4). The final term in Eq. (3.120) isolates the strange-quark contribution to the (monopole) charge density. The leading-order approximation using one-body operators (see Sect. III.D.2) and working to the appropriate order in $Q^{2}$ (as discussed in more detail in Sect. IV.B) yields for the ratio of form factors in Eq. (3.120) simply the ratio of the corresponding single-nucleon form factors:

$$
\frac{F_{C 0}(s)}{F_{C 0}(T=0)}=\frac{G_{E}^{(s)}}{G_{E}^{T=0}} \text {. }
$$

The role of $\mathrm{PV}$ elastic electron sca'ering from a spin-0, isospin-0 nuclear target in a program of hadronc neutral current studies is discussed in detail in Sect. IV.B.

Elastic scattering from the proton

For elastic electron scattering from a spin-1/2 target the problem is somewhat more complicated than that described above. Using the results of Sect. III.D.1 for elastic scattering. We know in this case that the form factors to be considered are $F_{C 0}, F_{M_{1}}, \tilde{F}_{C 0}$, $\tilde{F}_{M_{1}}, \dot{F}_{E 1_{s}}$ and $\tilde{F}_{L 1_{s}}$ and fro $:$ : he general discussions above we need not consider the last, leaving five basic form factors that enter into the cross sections and asymmetry. From Eqs. (3.108) and (3.114) we have

$$
\begin{aligned}
F_{L}^{2}(q) & =F_{C 0}^{2} \\
F_{T}^{2}(q) & =F_{M 1}^{2} \\
W_{A V}^{L}(q) & =-g_{A}^{e} F_{C 0}(q) \tilde{F}_{C 0}(q) \\
W_{A V}^{T}(q) & =-g_{A}^{e} F_{M 1}(q) \tilde{F}_{M 1}(q) \\
W_{V A}^{T^{\prime}}(q) & =-g_{V}^{e} F_{M 1}(q) \tilde{F}_{E_{1 s}}(q) .
\end{aligned}
$$


When dealing with elastic scattering from the nucleon as a special case of a spin-1/2 target it is conventional to use the fully relativistic analogs of the formal...n summarized above and so to express $F^{2}$ directly in terms of the electric and magnetic Sachs form factors:

$$
F_{p}^{2}(\tau, \mathcal{E}) \equiv 4 \pi F^{2}(\tau, \mathcal{E})=\frac{1}{(1+\tau) \mathcal{E}}\left(\mathcal{E}\left[G_{\mathcal{E}}^{p}(\tau)\right]^{2}+\tau\left(G_{M}^{p}(\tau)\right]^{2}\right)
$$

where. instead of writing the total form factor as a function of $q$ and $\theta$ as in Eq. 13.10\% w. we write it equivalently as a function of $T=\left|Q^{2}\right| / 4 m_{N}^{2}$ and $\mathcal{E}$ (see Eq. 13.115 ). In a similar way. the $\mathrm{PV}$ response takes the relatively simple form [Don88. Mus92a]

$$
\begin{aligned}
& +\pi W^{(P V)}(\tau, \mathcal{E})=-\frac{1}{2(1+\tau) \mathcal{E}}\left(g_{A}^{e}\left[\mathcal{E} G_{E}^{p}(\tau) \tilde{G}_{E}^{p}(\tau)+\tau G_{M}^{p}(\tau) \tilde{G}_{M}^{p}(\tau)\right]\right. \\
& \left.+g_{i}^{e} \sqrt{1-\mathcal{E}^{2}} \sqrt{\tau(1+\tau)} G_{M}^{p}(\tau) \tilde{G}_{A}^{p}(\tau)\right) \text {. }
\end{aligned}
$$

The PV asymmetry is then proportional to the ratio of responses in the last two equations. Inserting the Standard Model results for the leptonic couplings (see Table 3.1). using Eqs. (3.31) for the proton $\left(\tau_{3}=1\right)$ and inserting these results into Eqs. (3.123) and (3.124) allows us to write the PV asymmetry as

$$
\begin{aligned}
A_{L R}(\vec{e} p)= & -\frac{1}{2} A_{L R}^{0} \\
\times & \left\{\xi_{V}^{p}+\left[\mathcal{E} G_{\varepsilon}^{p}\left\{\xi_{V}^{n} G_{\varepsilon}^{n}+\xi_{V}^{(0)} G_{\varepsilon}^{(0)}\right\}+\tau G_{M}^{p}\left\{\xi_{V}^{n} G_{M}^{n}+\xi_{V}^{(0)} G_{M}^{(0)}\right\}\right.\right. \\
& \left.-\left(1-4 \sin ^{2} \theta_{W}\right) \sqrt{1-\mathcal{E}^{2}} \sqrt{\tau(1+\tau)} G_{M}^{p} G_{A}^{p}\right] /\left[\mathcal{E}\left(G_{\varepsilon}^{p}\right)^{2}+\tau\left(G_{M}^{p}\right)^{2} !\right\} .
\end{aligned}
$$

where we have suppressed the $\tau$-dependences for clarity and used the weak .NC couplings labeled " $p$ " or " $n$ " defined in Eqs. (3.61). In obtaining this result, we have assumed the nucleon to be a state of pure isospin in order to rewrite the isoscalar and isovector neutral current form factors in terms of the proton and neutron form factors [Eqs. (3.31)]. We discuss several potentially interesting experiments for PV electron scattering from the proton and explore the various kinematic dependences and limits of Eq. (3.125) in detail in Sect. IV.A.

\section{Elastic scattering from the deuteron}

Next let us consider elastic scattering from spin-1 nuclei such as the deuteron. Using the results of Sect. III.D.1, we have as form factors in this case the following: $F_{C 0}, \tilde{F}_{C 0}$, $F_{C 2}, \tilde{F}_{C 2}, F_{M 1}, \tilde{F}_{M 1}$ and $\tilde{F}_{E 1}$, for a total of seven form factors (again, as above, $\tilde{F}_{L 1 \text { s }}$ does 
not euter in PV electron scattering). Equations (3.108) and (3.114) yield

$$
\begin{aligned}
F_{L}^{2}(q) & =F_{C 0}^{2}(q)+F_{C 2}^{2}(q) \\
F_{T}^{2}(q) & =F_{M_{1}}^{2}(q) \\
W_{. V}^{L}(q) & =-g_{A}^{e}\left[F_{C 0}(q) \dot{F}_{C 0}+F_{C 2}(q) \dot{F}_{C 2}\right] \\
W_{A V}^{T}(q) & =-g_{A}^{e} F_{M_{1}}(q) \dot{F}_{M_{1}}(q) \\
W_{V_{A}}^{T^{\prime}}(q) & =-g_{i}^{e} \cdot F_{M_{1}}(q) \dot{F}_{E_{1}}(q) .
\end{aligned}
$$

Using the Standard . Model values for the lepronic couplings (Table 3.1) we then obtain for the PV asymmetry

$$
A_{L R}=-A_{L R}^{0} \times\left\{\frac{v_{L}\left[F_{C O} \dot{F}_{C O}+F_{C 2} \dot{F}_{C 2}\right]+F_{M 1}\left[v_{T} \dot{F}_{M 1}+\left(1-t \sin ^{2} \theta_{w}\right) v_{T} \dot{F}_{E L_{S}}\right.}{v_{L}\left[F_{C O}^{2}+F_{C 2}^{2}+v_{T} F_{L 1}^{2}\right.}\right\} .
$$

where again we have suppressed the $q$-dependences for clarity. At the tree-level ir the absence of strangeness and assuming good isospin symmetry we again have that the weak

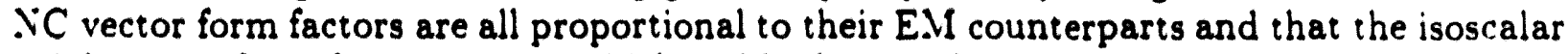
axial-vector form factor is zero, which yields the simple result

$$
-2 \frac{A_{L R}}{A_{L R}^{0}} \rightarrow \Delta_{(1)} \equiv \sqrt{3} \xi_{V}^{T=0}
$$

In Sect. IV.C we return to treat the case of elastic scattering from the deuteron in more detail. including there the complete expressions which incorporate radiative corrections and isospin-mixing together with discussions of how studies of this case this might serve to help in shedding light on the strangeness content of the nucleon.

Foliowing the review presented here of the basic formalism for elastic scattering from spin-0, spin-1/2 and spin-1 systems the next logical step is perhaps to consider inelastic excitations of discrete nuclear states. Since in general this subject is considerably more complicated than the formalism presented above (often having many more multipole form factors entering in the PC and PV cross sections), we choose not to continue along this path, but to postpone discussion of other discrete nuclear transitions until Sect. IV.D. Instead, we now consider excitations into the nuclear continuum, viz., quasielastic electron scattering.

\section{Quasielastic scattering}

While quasielastic scattering from a nuclear target is complicated by the details of the nuclear structure involved, the role of final-state interactions, etc., it is instructive to take as a starting point the "static" approximation in which one invokes the plane-wave impulse approximation [deF83]. In this case the QE cross section is given as an incoherent sum over the nucleons in the nucleus and involves integrals of the following general form

$$
d \sigma(\mathrm{QE})=\sum_{i} \int d E_{i} d k_{i} S_{i}\left(E_{i}, \vec{k}_{i}\right) d \sigma_{i}\left(E_{1}, \vec{k}_{i}\right) \delta\left(\omega-E_{\text {final }}+E_{\text {initial }}\right)
$$


where $S_{1}\left(E_{1}, k_{1}\right)$ is the spectral function. 2.e. the probability of finding the $\mathrm{i}^{\text {th }}$ nucleon (proton or neutron) moving with momentum $\vec{k}_{1}$ and energy $E_{1}$. The quantity $d \sigma_{1}, E_{1}, \vec{k}_{1}$, is the half-off-shell electroweak cross section for the $1^{\text {th }}$ nucleon and is also a function of $q .+$ and the electron helicity. The $\delta$-function enforces overall energy conservation and ontains the difference in nuclear energies. $E_{\text {final }}-E_{\text {initial. }}$ which in turn depends on q. $E_{1}$ and $\vec{k}_{1}$. liext. we assume that for the kinematics of interest in QE scattering. the $\sigma_{1}$ are each strongly peaked about some common $\vec{k}_{1} \equiv \vec{p}$ and $E=\sqrt{p^{2}-m_{k}^{2}}$. corresponding to having the struck nucleon on-shell and moving with momentum $\vec{p}$. In this case. one has

$$
\begin{aligned}
& \int d E_{1} d \vec{k}_{1} S_{1}\left(E_{1}, \vec{k}_{1}\right) d \sigma_{1}\left(E_{1}, \vec{k}_{1}\right) \dot{d}\left(\nu-E_{\mathrm{fnal}}+E_{\text {(nitial }}\right) \\
& \quad \approx d \sigma_{1}\left(E_{,} \vec{p}\right) \int d E_{1} d \vec{k}_{1} S_{1}\left(E_{1}, \vec{k}_{1}\right) \sigma\left(\omega-E_{\mathrm{fnal}}+E_{\text {(nitial }}\right) .
\end{aligned}
$$

In forming the parity-violating asymmetry we require the ratio of helicity difference and helicity-sum cross sections and so the integral in Eq. (3.130) will cancel. leaving only: the single-nucleon cross sections, $d \sigma_{1}(E, \vec{p})$ in the ratio. Finally, to obtain the crudest approximation (and so get some feeling for the form of the asymmetry) we take $\vec{p}=0$ and thus evaluate the single-nucleon cross sections in the rest frame of the struck nucleon.

In the static approximation we then find that $W^{\text {(E.M) }}$ is simply proportional to the sum of the response of $Z$ (on-shell) protons and.$\nu$ (on-shell) neutrons (Don92. .lus92a):

$$
W^{(E M)} \times \mathcal{E}\left[Z\left(G_{E}^{p}\right)^{2}+. V\left(G_{E}^{n}\right)^{2}\right]+\tau\left[Z\left(G_{M}^{p}\right)^{2}+. N\left(G_{M}^{n}\right)^{2}\right] .
$$

while the parity-violating response is

$$
\begin{aligned}
& -2 W^{(\mathrm{PV})} \times \mathcal{E}\left[Z G_{E}^{p} \dot{G}_{E}^{p}+. \nu G_{E}^{n} \tilde{G}_{E}^{n}\right]+\tau\left[Z G_{M}^{p} \tilde{G}_{M}^{p}+. V G_{M}^{n} \dot{G}_{M}^{n}\right] \\
& -\left(1-4 \sin ^{2} \theta_{w}\right) \sqrt{1-\mathcal{E}^{2}} \sqrt{\tau(1+\tau)}\left[Z G_{M}^{p} \dot{G}_{A}^{p}+. v G_{M}^{n} \tilde{G}_{A}^{n}\right] .
\end{aligned}
$$

where we have suppressed the $r$-dependences in the single-nucleon form factors and have used the Standard Model leptonic couplings (Table 3.1). The static model PV QE asymmetry is then given by

$$
\begin{aligned}
A_{L R}(Q E)_{\text {static }} & =-\frac{1}{2} A_{L R}^{0} \times\left\{\mathcal{E}\left[Z G_{E}^{p} \tilde{G}_{E}^{p}+. v G_{E}^{n} \dot{G}_{E}^{n}\right]+\tau\left[Z G_{M}^{p} \dot{G}_{M}^{p}+. v G_{. M}^{n} \dot{G}_{M}^{n}\right]\right. \\
& \left.-\left(1-\sin ^{2} \theta_{w}\right) \sqrt{1-\mathcal{E}^{2}} \sqrt{\tau(1+\tau)}\left[Z G_{M}^{p} \dot{G}_{A}^{p}+. N G_{M}^{n} \tilde{G}_{A}^{n}\right]\right\} \\
& \times\left\{\mathcal{E}\left[Z\left(G_{E}^{p}\right)^{2}+. V\left(G_{E}^{n}\right)^{2}\right]+\tau\left[Z\left(G_{M}^{p}\right)^{2}+N\left(G_{M}^{n}\right)^{2}\right]\right\}^{-1} .
\end{aligned}
$$

This result should be compared with the expression for the asymmetry for elastic scattering from the proton, Eq. (3.125), since the static model naturally reverts to the proton elastic asymmetry when $Z=1$ and $N=0$ (or to the elastic neutron asymmetry when $N=1$ and $Z=0$ ). The consequences of extending the treatment of PV quasielastic scattering beyond this simple static approximation are discussed in more detail in Sect. IV.F.

Similar considerations are applicable for excitation energies beyond the QE peak. In particular, the region dominated by quasi-free excitation of the $\Delta(1232)$ has received some attention; our discussion of this problem is postponed until Sect. IV.G. 


\section{III.E.2. FIGIRE-OF-IIERIT FOR PV ELECTRON SCATTERING}

While $A_{L R}$ depends directly on the matrix elements of the hadronic neutral current and presents a clear signature of the presence of these currents. it is a small quantity and is challenging to measure experimentally. The precision with which it may be determined depends on details of the experimental configuration (luminosity, detector resolution. beam polarization. etc.) as well as on the kinematic conditions under which a measurement is carried out. The latter also influence the degree to which various quantities of interest. such as $\xi_{v}^{p}$ or hadronic form factors. enter theoretical predictions of $A_{L R}$. Consequently: a non-trivial correlation exists between kinematical constraints imposed by considerations of experimental (statistical precision) issues and by considerations of interpretability. In many cases. these different sets of considerations conspire to constrain the optimal kinematical regime for performing a measurement. It is important. then, to understand the kinematicai conditions imposed by the requirement that the statistical uncertainty in.$_{L R}^{\text {exp }}$ be sufficiently small to make a given measurement theoretically meaningful.

To this end. we review the standard figure-of-merit (FOMI) for PV electron scattering which. when maximized. corresponds to a minimal $\left(\delta . A_{L R} / A_{L R}\right)_{s t a t i s t i c a l}$. To derive the FOM, we write the asymmetry as

$$
A_{L A} \equiv \Delta / \nu_{0}
$$

where $\Delta=\nu_{+}-\nu_{-}, v_{0}=\nu_{+}+\nu_{-}$and $\nu_{+(-)}$is the number of events with electrons polarized parallel (anti-parallel) to their incident momenta. The statistical error in $f_{L R}$ is, then

$$
\delta A_{L R}=\left|\left(\delta A_{L R}^{+}\right)^{2}+\left(\delta A_{L R}^{-}\right)^{2}\right|^{1 / 2}
$$

whore

$$
\delta A_{L R}^{+(-)} \equiv \frac{\partial A_{L R}}{\partial V_{+(-)}} \delta . V_{+(-)}
$$

lising $\delta . V_{+(-)} \equiv \sqrt{V_{+(-)}}$it is straightforward to obtain

$$
\delta A_{L R}=\frac{1}{\sqrt{V_{0}}}\left[1-A_{L R}^{2}\right]^{1 / 2}
$$

For the range of electron energy and momentum transfer of interest here, $\left|A_{L R}\right| \ll 1$ so that to an excellent approximation $\delta A_{L R} \cong 1 / \sqrt{\mathcal{V}_{0}}$. The relative error in $A_{L R}$, then, is*

$$
\frac{\delta A_{L R}}{A_{L R}}=\left[. V_{0} A_{L R}^{2}\right]^{-1 / 2}
$$

* Henceforth, we take $\delta A_{L R} / A_{L R}$ to be non-negative, that is, to denote the absolute value of the fractional error. 
Writing $._{0}=(d \sigma / d \Omega) \Delta \Omega \mathcal{L} T_{0}$, where $d \sigma / d \Omega$ is the helicity-independent differential cross section. $\Delta \Omega$ is the detector solid angle, $\mathcal{L}$ is the luminosity, and $T_{0}$ is the running time. we have

$$
\begin{aligned}
\frac{\delta A_{L R}}{A_{L R}} & =\left[\mathcal{F} X_{0}\right]^{-1 / 2} \\
\mathcal{F} & =\left(\frac{d \sigma}{d \Omega}\right) A_{L R}^{2} \\
X_{0} & =\mathcal{L} \Delta \Omega T_{0} .
\end{aligned}
$$

The quantity $\mathcal{F}$ is the figure-of-merit. It depends only on intrinsic properties of the target and lepton probe and on the relevant kinematic variables $\left(\left\{\epsilon, \epsilon^{\prime}\right.\right.$ and $\left.\theta\right\}$ or equivalently $\{q$. $\omega$ and $\theta\}$ ). In particular, the results given above for discrete states in Eqs. (3.116) and (3.11i) allow one to write (recall: $\omega$ is fixed by the excitation energy and $q$ in this case)

$$
\mathcal{F}(q, \theta)=\frac{\mathcal{F}_{0}(q, \theta)}{1-\mathcal{E}} \frac{\left[2\left|Q^{2} / q^{2}\right| \mathcal{E} W_{A V}^{L}(q)+W_{A V}^{T}(q)+\sqrt{1-\mathcal{E}^{2}} W_{V A}^{T_{A}^{\prime}}(q)\right]^{2}}{\left[2\left|Q^{2} / q^{2}\right| \mathcal{E} F_{L}^{2}(q)+F_{T}^{2}(q)\right]} .
$$

where for convenience the overall scale

$$
\mathcal{F}_{0}(q, \theta) \equiv \frac{G^{2}}{2 \pi} \mid Q^{2} i\left(\frac{\epsilon^{\prime}}{\epsilon}\right) f_{\mathrm{rec}}^{-1}
$$

has been introduced (see the discussions to follow in Sect. IV). Extrinsic experimental conditions are contained in the quantity $X_{0}$. For a given value of the latter, $\delta A_{L R} / A_{L R}$ is a decreasing function of $\mathcal{F}$. The values of $\mathcal{L}$ and $\Delta \Omega$ attainable also depend on properties of the target and kinematical conditions, as discussed in more detail in Sect. V. In analyzing the do-ability/interpretability correlation in Sect. IV, we take reasonable values of $X_{0}$ and work primarily with $\mathcal{F}$.

Before proceeding to specific examples we make several observations concerning the quantities given above for the typical conditions that apply when studying discrete nuclear states. First, since $F_{L, T}^{2}, W_{A V}^{L, T}$ and $W_{V A}^{T^{\prime}}$ all contain the square of a characteristic nuclear form factor $\left(\equiv F_{\text {nuc }}\right)$, the figure-of-merit $\mathcal{F}$, given in Eq. (3.140), is proportional to $F_{\text {nuc }}^{2}$. To a fair approximation, the $q$-dependence of this quantity can be parameterized as $F_{\text {nuc }}^{2} \approx$ $\exp \left[-\left(q / q_{0}\right)^{2}\right]$, where $q_{0} \approx 250 \mathrm{MeV} / \mathrm{c} \times A^{-1 / 6}$ gives the value of $q$ for which $F_{\text {nuc }}^{2}$ falls off by a factor of $e$ from its $q=0$ value. For instance, taking $A=12$ yields $q_{0}=165 \mathrm{MeV} / \mathrm{c}$ as the characteristic $1 / \mathrm{e}$ scale at which $\mathcal{F}$ falls off with $q$. Of course, there is usually other non-trivial momentum transfer dependence beyond this (see below); however, this parameterization sets the rough scale for the $q$-dependence, and one should expect that for momentum transfers significantly larger than $q_{0}$ the figure-of-merit will not be sufficient to render measurements practical. Secondly, when one applies these general expressions to situations involving discrete transitions the energy transfer $\omega$ is fixed once the threemomentum transfer $q$ and excitation energy $E_{x}$ are specified: $\omega=\sqrt{q^{2}+\left(M_{T}+E_{x}\right)^{2}}-$ $M_{T} \cong E_{x}+q^{2} / 2 M_{T}$. Since the three-momentum transfer must usually be kept rather low (say below $\approx 200-300 \mathrm{MeV} / \mathrm{c}$ except for the lightest nuclei, using the above estimates). 
the recoil energy $q^{2} / 2 . M_{T}$ is only a few $\mathrm{MeV}$ or less. The excitation energies of discrete nuclear states characteristically fall in the range $[0-10] \mathrm{MeV}$ and hence this range is also appropriate for $\omega$. As a consequence typically $\omega \ll q$ and the combination $\left|Q^{2} / q^{2}\right|=$ $1-(\omega / q)^{2}$ in the above equations may be taken to be approximately unity except when the three-momentum transfer is very small. Furthermore, $\epsilon^{\prime} / \epsilon$ and $f_{\text {rec }}$ satisfy the following inequalities

$$
\begin{aligned}
\frac{1-\omega / q}{1+\omega / q} & \leq \frac{\epsilon^{\prime}}{\epsilon} \leq 1 \\
1 & \leq f_{\mathrm{rec}} \leq 1+\frac{q+\omega}{M_{T}}
\end{aligned}
$$

and thus it can be argued that under typical conditions they are both also nearly unity. These arguments imply that in studying discrete-state transitions via PV electron scattering under typical conditions the scale factor $\mathcal{F}_{0}$ in Eq. (3.141) is nearly constant for fixed momentum transfer but varying sc tering angle. The angle-dependence in the figure-ofmerit is then isolated essentially in e factors $\mathcal{E}$ in Eq. (3.140). Importantly this expression contains the overall factor $(1-\mathcal{E})^{-1}$ which varies quite rapidly for small scattering angles. where $\mathcal{E}$ is only slightly less than unity, and leads us to expect that the figure-of-merit will usually be largest for small $\theta$.

In Figs. 3.10-3.12 the figure-of-merit calculated using Eq. (3.140) is shown as a function of incident electron energy at fixed scattering angle for several selected nuclear transitions. In particular, results are given for elastic scattering from ${ }^{12} \mathrm{C}$ and ${ }^{4} \mathrm{He}$, for inelastic scattering to the $J^{\pi} T=2^{+} 0(4.44 \mathrm{MeV}), 1^{+} 0(12.71 \mathrm{MeV})$ and $1^{+} 1(15.11$ $\mathrm{MeV}$ ) levels in ${ }^{12} \mathrm{C}$ and, to place the results in context, for elastic scattering from the proton. In each case the electromagnetic form factors have been determined directly from experimental cross sections [Car80, Fla78, Fla79, Fro67, Jan72, McC77, Reu82, Sic 70] ( simple parameterizations have been used and the parameters so introduced adjusted to produce fits to the data). We have set all strangeness form factors to zero in producing these results, although, when specific cases involving potential nonzero strangeness content are discussed below, the figures-of-merit are recomputed incorporating these additional contributions before arriving at numerical estimates of the fractional uncertainties in the quantities of intorest.

Three angles have been selected: a very forward angle $\left(10^{\circ}\right)$ which is characteristic of the limits that can be reached by the spectrometers being built at CEBAF, a typical forward angle $\left(30^{\circ}\right)$ and a typical backward angle $\left(150^{\circ}\right)$. In the figures $\mathcal{F}$ is given for the range in $\epsilon$ corresponding roughly to $0 \leq q \leq 500 \mathrm{MeV} / \mathrm{c}$. To interpret the results given here it is helpful to recall Eq. (3.139) where the fractional statistical precision obtainable for the asymmetry is given by $\left[\mathcal{F} X_{0}\right]^{-1 / 2}$, with $X_{0}=\mathcal{L} \Delta \Omega T_{0}$. As in our previous discussions, let we assume that the electron beam is $100 \%$ polarized, $\Delta \Omega=10 \mathrm{msr}, T_{0}=$ $1000 \mathrm{hr}=3.6 \times 10^{6} \mathrm{~s}$ and the luminosity is given by $\mathcal{L}\left[{ }^{12} \mathrm{C}\right]=1.25 \times 10^{38} \mathrm{~cm}^{-2} \mathrm{~s}^{-1}$ or $\mathcal{L}\left[{ }^{4} \mathrm{He},{ }^{1} \mathrm{H}\right]=5 \times 10^{38} \mathrm{~cm}^{-2} \mathrm{~s}^{-1}$. These assumptions yield: $X_{0}\left[{ }^{12} \mathrm{C}\right]=4.5 \times 10^{42} \mathrm{~cm}^{-2}$ and $X_{0}\left[{ }^{4} \mathrm{He},{ }^{1} \mathrm{H}\right]=1.8 \times 10^{43} \mathrm{~cm}^{-2}$. These values set the scale for the fractional precision in $A_{L R}$, namely

$$
\begin{array}{rlrl}
\mathcal{F}=10^{-39} \mathrm{~cm}^{2} \mathrm{sr}^{-1} & \longleftrightarrow\left|\delta A_{L R} / A_{L R}\right| \cong 1.5 \%\left({ }^{12} \mathrm{C}\right), & 0.75 \%\left({ }^{4} \mathrm{He},{ }^{1} \mathrm{H}\right) \\
10^{-41} \mathrm{~cm}^{2} \mathrm{sr}^{-1} & \longleftrightarrow & 15 \%, & 7.5 \% \\
10^{-43} \mathrm{~cm}^{2} \mathrm{sr}^{-1} & \longleftrightarrow & 150 \%, & 75 \% .
\end{array}
$$


It is then straightforward to determine the fractional precision with which specific transitions could be studied given the above experimental conditions. For elastic scattering from ${ }^{12} \mathrm{C}$ at the peak of the figure-of-merit in Figs. 3.:0-3.12 we find $\left|\delta A_{L R} / A_{L R}\right|=0.7 \%\left(10^{\circ}\right)$. $\left.2.2 \%, 30^{\circ}\right)$ and $31 \%\left(150^{\circ}\right)$. Essentially the same values are obtained for elastic scattering from ${ }^{4}$ He. since the smaller $Z$ is just compensated for by the slower fall-off of the nuclear form factor when comparing ${ }^{4} \mathrm{He}$ with ${ }^{12} \mathrm{C}$. For the excitation of the $2^{+} 0$ state in ${ }^{12} \mathrm{C}$. under conditions where its figure-of-merit peaks, we find $\left|\delta A_{L R} / A_{L R}\right|=2 \%\left(10^{\circ}\right) .6 \%\left(30^{\circ}\right)$ and $86 \%\left(150^{\circ}\right)$. Likewise for the excitation of the $1^{+}$states in ${ }^{12} \mathrm{C}$ where their respective figures-of-merit peak we have the following: for the $1^{+} 1$ state we find $\left|\delta A_{L R} / A_{L R}\right|=26 \%$ $\left(10^{\circ}\right), 6 \pi \%\left(30^{\circ}\right)$ and $163 \%\left(150^{\circ}\right)$, whereas for the $1^{+} 0$ state we find $\left|\delta A_{L R} / A_{L R}\right|=142 \%$ $\left(10^{\circ}\right) .413 \%\left(30^{\circ}\right)$ and $1197 \%\left(150^{\circ}\right)$. Of course. if a larger solid angle detector having sufficient resolution to permit the separation of the transition of interest were to be built. then the values of fractional precision in the PV asymmetry given here would be lower. For example, given a detector with $\Delta \Omega=0.16 \mathrm{sr}$, the above numbers would all be decreased by a factor of four. However. even were such a detector to be realized. some of the results presented above must still be regarded as rather uninteresting because of the large statistical uncertainties in the asymmetry that would be incurred. In context the results at forward angles and high energies (for the range of momentum transfers displayed in the figures) illustrate an observation that will be elaborated in Sect. IV.A: the proton asymmetry and figure-of-merit are atypically small for such kinematics. As noted in those discussions, this situation is due to the smallness of all of the terms which contribute. The asymmetry in Eq. (3.125) involves $1-4 \sin ^{2} \theta_{w}, G_{E}^{n}, G_{E}^{(s)}, \tau$ or $0.092 \sqrt{\tau(1+\tau)\left(1-\mathcal{E}^{2}\right)}$. all of which are suppressed for such kinematics. In contrast, for example, elastic scattering from $J=T=0$ nuclei instead involves $-4 \sin ^{2} \theta_{w}$ and therefore from this factor alone one expects the figure-of-merit in this case to exceed that of the proton by about two orders of magnitude. In addition. the many-body nuclear form factor diminishes the figure of merit for a nuclear target as $q$ increases. In the case of elastic scattering, however, the coherence factor $Z^{2}$ offsets this suppression for momentum transfers below the characteristic value $q_{0}$ introduced above.

\section{III.E.3. BASIC NEUTRINO SCATTERING FORMALISM}

Development of the expressions for neutrino and antineutrino $\mathrm{NC}$ scattering, $\left(\nu_{l}, \nu_{l}^{\prime}\right)$ and $\left(\bar{\nu}_{l}, \bar{\nu}_{l}^{\prime}\right)$. respectively, and for charge-changing neutrino and antineutrino reactions. $\left(\nu_{l}, \ell^{-}\right)$and $\left(\bar{\nu}_{l}, \ell^{+}\right)$, respectively, where $\ell$ labels the flavor of lepton, closely follows the derivation of the electron scattering cross sections above. In this case. however, the photon-exchange amplitude cannot contribute to leading order in electroweak couplings (cf. Fig. 3.1); only the diagrams in Fig. 3.13 involving $Z^{0}$ exchange (neutral current) or $W^{ \pm}$exchange (charge-changing current) between leptons and hadrons enter. The leadingorder contributions to the cross section are then $\mathcal{O}\left(G_{\mu}^{2}\right)$ and involve the weak interaction current matrix elements bilinearly. Consequently, the contributions to the general response functions in Eq. (3.100) are now somewhat more involved than in the case of PV response for electron scattering, which contain only a linear dependence on weak NC matrix elements. Moreover, the neutrino scattering $C C . C L, L L$ and $T$ responses all involve both products of nuclear vector currents and products of nuclear axial-vector currents 
whereas the $T^{\prime}$ response, as before, contains the interferences between the nuclear vector currents and the nuclear axial--vector currents. The development of the various cross sections for charge-changing and neutral current weak interaction processes involving neutrinos and antineutrinos has been discussed at length in previous review articles (see, e.g.. Ref. [Doni9a] where many of the conventions used are similar to those employed in the present work). Since the main focus of this work is PV electron scattering. we shall not discuss these other processes in as much detail. Rather our intent in this section is only to bring out the strong parallelism that exists in approaching the wider class of semi-leptonic electroweak interaction processes and to provide a basis for the discussions in Sect. IV.J where in context some of the main implications of neutrino scattering are summarized.

For the present discussions let us again assume the ERL, in which case Eqs. (3.101) apply in general and Eq. (3.103) may be used for the VV responses. The analog of Eq. (3.104) for neutrino and antineutrino scattering with exchange of a $Z^{0}$ may be written [Doni9a)

$$
\frac{d^{2} \sigma}{d \Omega d \epsilon^{\prime}}=\sigma_{0}\left\{S^{L}\left(q, \omega_{0}\right)_{0}+v_{T} R^{T}\left(q, \omega^{\prime}\right)_{0} \pm v_{T^{\prime}} R^{T^{\prime}}(q, \omega)_{0}\right\}
$$

where $\sigma_{0}$ is the elementary cross section (the neutrino scattering analog of the Mott cross section in Eq. (3.105))

$$
\sigma_{0}=\left[\frac{G_{\mu} \epsilon^{\prime} \cos \frac{\theta}{2}}{\pi \sqrt{2}}\right]^{2}
$$

and the $+/$ - sign on the third term corresponds to neutrino/antineutrino scattering (see also Ref. (Alb93b]). The leptons are labeled as in Sect. III.E.1. The responses in Eq. (3.144) are distinguished from their analogous electron scattering counterparts by the subscript. " 0 " (denoting neutral current weak interaction processes, see below). From our general discussions at the beginning of this section we can write

$$
\begin{aligned}
S^{L}(q, \omega)_{0}= & v_{L}\left[R^{L}(q, \omega)_{0}\right]_{V V} \\
& \quad+\left[R^{C C}(q, \omega)_{0}-\left(\frac{\omega}{q}\right) R^{C L}(q, \omega)_{0}+\left(\frac{\omega}{q}\right)^{2} R^{L L}(q, \omega)_{0}\right]_{A A} \\
R^{T}(q, \omega)_{0}= & {\left[R^{T}(q, \omega)_{0}\right]_{V V}+\left[R^{T}(q, \omega)_{0}\right]_{A A} } \\
R^{T^{\prime}}(q, \omega)_{0}= & {\left[R^{T^{\prime}}(q, \omega)_{0}\right]_{V A} . }
\end{aligned}
$$

It is straightforward to generalize these expressions to incorporate ERL charge-changing neutrino reactions. The structure is basically the same with the replacements

$$
\begin{aligned}
\sigma_{0} & \longrightarrow \sigma_{ \pm}=2 \sigma_{0} \\
(\text { Response })_{0} & \longrightarrow(\text { Response })_{ \pm}
\end{aligned}
$$

where the subscripts " \pm " correspond to $\left(\nu_{l}, \ell^{-}\right)$and $\left(\bar{\nu}_{l}, \ell^{+}\right)$reactions, respectively, and where the factor 2 is conventional [Wal75]. Furthermore, $\beta$-decay and charged-lepton capture can be added to the set of processes that can be inter-related, as can charge-changing 
neutrino reactions in circumstances where the ERL cannot be invoked (see Ref. Don $19 \mathrm{a}$ : for details).

For elastic and inelastic scattering to discrete states one has. in analogy with Eq. (3.106):

$$
\frac{d \sigma}{d \Omega}=4 \pi \sigma_{0} f_{\mathrm{rec}}^{-1} \tilde{F}^{2}(q . \theta)
$$

where $f_{\text {rec }}$ has been defined above and where. in analogy with the electron scattering formalism discussed above. we define a neutrino scattering form factor

$$
\tilde{F}^{2}(q, \theta)=\tilde{F}_{L}^{2}(q)+v_{T} \tilde{F}_{T}^{2}(q) \pm v^{\prime} T^{\prime} \tilde{H}_{T^{\prime}}(q) .
$$

In this case the $T^{\prime}$ contributions have been denoted $\tilde{H}_{T^{\prime}}$ rather than, say: $\tilde{F}_{T^{\prime}}^{2}$ since they can be positive or negative. As in the latter case. it is possible to perform a generalized Rosenbluth decomposition of the form factor into a sum of terms using the form factors defined in Sect. III.D:

$$
\begin{aligned}
\tilde{F}_{L}^{2}(q) & =v_{L} \sum_{J \geq 0} \tilde{F}_{C J}^{2}(q)+\sum_{J \geq 0}\left(\tilde{F}_{C J_{s}}(q)+\frac{\dot{\omega}}{q} \tilde{F}_{L J_{5}}(q)\right)^{2} \\
\tilde{F}_{T}^{2}(q) & =\sum_{J \geq 1}\left[\tilde{F}_{E J}^{2}(q)+\tilde{F}_{M J}^{2}(q)\right]+\sum_{J \geq 1}\left[\tilde{F}_{E J_{s}}^{2}(q)+\tilde{F}_{M J_{5}}^{2}(q)\right] \\
\tilde{H}_{T^{\prime}}(q) & =-2 \sum_{J \geq 1}\left[\tilde{F}_{E J}(q) \tilde{F}_{M J_{3}}(q)+\tilde{F}_{M J}(q) \tilde{F}_{E J_{s}}(q)\right],
\end{aligned}
$$

where. as before, the nuclear states are assumed to have good angular momentum and parity quantum numbers. In Eqs. (3.150) we have substituted the results for the neutrino couplings from Table 3.1: $g_{V}^{\nu}=-g_{A}^{\nu}=1$. Note the contrast with PV electron scattering (Eq. (3.114)): in the case of PV electron scattering the axial-vector contributions are suppressed by the ratio $\left|g_{V}^{e} / g_{A}^{e}\right|=1-4 \sin ^{2} \theta_{w} \cong 0.092$, whereas for neutrino scattering there is no suppression and the corresponding ratio is unity. Analogous expressions may be written for charge-changing neutrino reactions [Don $79 a$ ] by using the $F_{X J}^{ \pm}$and $F_{X J_{s}}^{ \pm}$ form factors introduced in Eqs. (3.56) and multiplying by 2, as in Eq. (3.147).

We conclude this section by considering two special cases of neutrino scattering: elastic scattering from $\left(J^{\pi} T\right)=\left(0^{+} 0\right)$ nuclei and elastic scattering from the nucleon. Treatment of all other cases is postponed until Sect. IV.J. Concerning the type of measurement which might be attempted, a few words are appropriate at this point. Since in the scattering of neutrinos or antineutrinos the outgoing lepton cannot be detected, some alternative signature must be found. For inelastic scattering, in certain cases the de-excitation of the final-state nuclear level by, say, emission of $\gamma$-rays can be used to study the neutral current neutrino excitation (see Ref. [Don79a] and Sect. IV.J.5). However, for elastic scattering all that happens is recoil of the target when it is struck by the neutrino or antineutrino. In specific circumstances (see Ref. [Don83] and the further discussion in Sects. IV.J.3 and IV.J.4) detection of the recoiling target may prove feasible for elastic scattering from nuclei; the present situation for elastic scattering from the proton is briefly reviewed in Sect. IV.J.2. 
For elastic scattering one has the following:

$$
\begin{aligned}
T_{R} & =2 . M_{T} \tau=\frac{\left|Q^{2}\right|}{2 . M_{T}}=\omega \\
q & =2 . M_{T} \sqrt{\tau(1+\tau)} \\
\epsilon & =. M_{T}\left[\cos \theta_{R} \sqrt{1+2 . M_{T} / T_{R}}-1\right]^{-1} \\
& =M_{T}\left[\cos \theta_{R} \sqrt{1+1 / \tau}-1\right]^{-1} \\
\sin ^{2} \theta / 2 & =\frac{\cos ^{2} \theta_{R}}{\cos ^{2} \theta_{R}+\left(1+\epsilon / . M_{T}\right)^{2} \sin ^{2} \theta_{R}}
\end{aligned}
$$

where $\epsilon$ is the energy of the incident neutrino (or antineutrino), $\theta$ is the neutrino scattering angle and $T_{R}$ and $\theta_{R}$ are the angle and kinetic energy of the final-state recoil. respectively: As usual..$V_{T}$ is the target mass. The lepton scattering angle $\theta$ can take on any value from $0^{\circ}$ to $180^{\circ}$ and accordingly the recoil angle goes between $90^{\circ}$ and $0^{\circ}$.

Elastic scattering from $\left(J^{\pi} T\right)=\left(0^{+} 0\right)$ nuclei

As in the discussions above of PV elastic electron scattering, the analysis of neutrino scattering from spin- 0 , isospin- 0 nuclei is simplified considerably since only isoscalar monopole form factors can occur. One finds that

$$
\begin{aligned}
\tilde{F}^{2}(q, \theta) & =\tilde{F}_{L}^{2}(q)=v_{L} \tilde{F}_{C 0}^{2}(q) \\
\tilde{F}_{T}^{2}(q) & =\tilde{H}_{T^{\prime}}(q)=0,
\end{aligned}
$$

where the neutral current form factor can be written in terms of the electromagnetic form factor (compare Eq. (3.120)):

$$
\tilde{F}_{C 0}(q)=\frac{1}{2}\left\{\sqrt{3} \xi_{V}^{T=0}[1+\Gamma(q)] F_{C 0}(q)+\xi_{V}^{(0)} F_{C 0}(s)\right\} .
$$

The Rosenbluth factor $v_{L}$ is given by

$$
v_{L}=(1+\tau)^{-2}=\frac{\left[1+2 \epsilon / M_{T}+\left(\epsilon / M_{T}\right)^{2} \sin ^{2} \theta_{R}\right]^{2}}{\left[1+\epsilon / M_{T}\right]^{4}} .
$$

Equation (3.148) may be used together with the form factor above to obtain the cross section for elastic scattering from $0^{+} 0$ targets: for fixed $\epsilon$ this may be expressed in the following form:

$$
\frac{d \sigma(\epsilon)}{d Q^{2}}=2 G_{\mu}^{2}\left(\frac{M_{T}}{\epsilon}\right)^{2}\left[\left(\epsilon / M_{\tau}-\tau\right)^{2}-\tau(1+\tau)\right] \tilde{F}^{2}(q),
$$


differential in the 4 -momentum transfer. or

$$
\frac{d \sigma(\epsilon)}{d \Omega_{R}}=\frac{8}{\pi} G_{\mu}^{2} \epsilon^{2}\left(1+\epsilon / . M_{T}\right)^{4} \frac{\sin ^{2} \theta_{R} \cos \theta_{R}}{\left(1+2 \epsilon / . M_{T}+\left(\epsilon / . M_{T}\right)^{2} \sin ^{2} \theta_{R}\right]^{3}} \dot{F}^{2}(q) .
$$

differential in the recoil solid angle.

One issue to which we return in Sect. IV.J involves the hadronic couplings occurring in Eqs. (3.120) and (3.153). These are the same at tree level, but have different radiative corrections, as discussed in Sect. III.A. In fact, if sufficiently high precision PV electron scattering and neutrino scattering experiments could be undertaken, then interesting information on the radiative corrections might emerge. The practical difficulties of achieving sufficient precision. however, make this goal a very difficult one to attain.

Elastic scattering from the nucleon

For elastic scattering from the nucleon we have expressions analogous to those in Eqs. (3.123) and (3.124) where the results are given as functions of $\tau$ and $\mathcal{E}$ :

$$
\begin{aligned}
& \tilde{F}_{. V}^{2}(\tau, \mathcal{E}) \equiv 16 \pi \tilde{F}^{2}(\tau, \mathcal{E})=\frac{1}{(1+\tau) \mathcal{E}}\left(\mathcal{E}\left[\tilde{G}_{E}^{v}(\tau)\right]^{2}+\tau\left[\tilde{G}_{. M}^{N}(\tau)\right]^{2}\right. \\
& \left.+(1+\tau)\left[\tilde{G}_{A}^{\mathrm{N}}(\tau)\right]^{2} \mp 2 \sqrt{1-\mathcal{E}^{2}} \sqrt{\tau(1+\tau)} \tilde{G}_{M}^{\mathrm{N}}(\tau) \tilde{G}_{A}^{\mathrm{N}}(\tau)\right)
\end{aligned}
$$

and where $V=p$ or $n$ with, as usual, the upper (lower) sign corresponding to neutrino (antineutrino) scattering. As before (compare Eq. (3.125)) the neutral current form factors may be re-expressed in terms of the electromagnetic and strangeness form factors using Eqs. (3.31).

For the charge-changing reactions $p\left(\bar{\nu}_{l}, \ell^{+}\right) n$ and $n\left(\nu_{l}, \ell^{-}\right) p$ we have similar expressions for the cross sections, although of course only isovector form factors enter:

$$
\begin{aligned}
F_{N \pm}^{2}(\tau, \mathcal{E}) \equiv 2 \pi & F_{ \pm}^{2}(\tau, \mathcal{E})=\frac{1}{(1+\tau) \mathcal{E}}\left(\mathcal{E}\left[G_{E}^{T=1}(\tau)\right]^{2}+\tau\left[G_{M}^{T=1}(\tau)\right]^{2}\right. \\
& \left.+(1+\tau)\left[G_{A}^{T=1}(\tau)\right]^{2} \mp 2 \sqrt{1-\mathcal{E}^{2}} \sqrt{\tau(1+\tau)} G_{M}^{T=1}(\tau) G_{A}^{T=1}(\tau)\right)
\end{aligned}
$$

where the upper (lower) sign corresponds to the reaction $\nu_{l}+n \rightarrow p+\ell^{-}\left(\bar{\nu}_{l}+p \rightarrow n+\ell^{+}\right)$.

Continuing with elastic scattering, the cross section may be obtained using the form factor given in Eq. (3.157) with the general formula in Eq. (3.155), where $M_{T} \rightarrow m_{N}$. $T_{R} \rightarrow T_{N}$ (the recoiling nucleon's kinetic energy) and $\theta_{R} \rightarrow \theta_{N}$ (the recoiling nucleon's angle with respect to the neutrino beam direction). In addition, it is straightforward to re-express the answer in terms of the familiar Mandelstam variables $s=(P+K)^{2}$, $t=\left(K^{-}-K^{\prime \prime}\right)^{2}=Q^{2}$ and $u=\left(P-K^{\prime}\right)^{2}$, where $P^{\mu}=\left(m_{N}, 0\right)$ is the struck nucleon's 4-momentum in the laboratory system (the other momenta have been defined above). It is straightforward to show that

$$
\left(\frac{s-u}{m_{N}^{2}}\right)=4\left(\frac{\epsilon}{m_{. v}}-\tau\right)
$$


and hence that the quantity $\mathcal{E}$ in Eq. (3.15i) (see Eq. (3.115)) may be written

$$
\mathcal{E}=\left[\left(\frac{s-u}{m_{. v}^{2}}\right)^{2}-16 \tau(1+\tau)\right] /\left[\left(\frac{s-u}{m_{. v}^{2}}\right)^{2}+16 \tau(1+\tau)\right] .
$$

The cross section may then be cast in the form frequently encountered in the literature:

$$
\frac{d \sigma(\epsilon)}{d Q^{2}}=\frac{G_{\mu}^{2} m_{v}^{2}}{8 \pi \epsilon^{2}}\left[A \pm\left(\frac{s-u}{m_{v}^{2}}\right) B+\left(\frac{s-u}{m_{s}^{2}}\right)^{2} C\right] .
$$

where the upper (lower) sign corresponds to the neutrino (anti-neutrino) cross section. ¿sing the above results the following identifications may be made:

$$
\begin{aligned}
& A(\tau)=\tau\left[(1+\tau)\left(\tilde{G}_{A}^{N}\right)^{2}-\left(\tilde{G}_{E}^{N}\right)^{2}+\tau\left(\tilde{G}_{M}^{N}\right)^{2}\right] \\
& B(\tau)=-\tau \tilde{G}_{A}^{N} \tilde{G}_{M}^{N} \\
& C(\tau)=\frac{1}{16}\left(\frac{1}{1+\tau}\right)\left[(1+\tau)\left(\tilde{G}_{A}^{N}\right)^{2}+\left(\tilde{G}_{E}^{N}\right)^{2}+\tau\left(\tilde{G}_{M}^{N}\right)^{2}\right] .
\end{aligned}
$$

In Sect. IV.J.2 we discuss the case of the nucleon in somewhat more detail. One situation discussed there involves elastic scattering from hydrogen. Another involves quasielastic scattering in nuclei where clearly the same approach followed in Sect. III.E.1 and developed in Sect. IV.F for QE PV electron scattering could also be pursued for neutrino scattering and neutrino reactions. In fact, the analog of Eq. (3.129) and the developments that follow can be used directly for the charge-changing neutrino reactions (merely replacing the PC or PV $p\left(e, e^{\prime}\right) p$ and $n\left(e, e^{\prime}\right) n$ cross sections with the $p\left(\bar{\nu}_{l}, \ell^{+}\right) n$ and $n\left(\nu_{l}, \ell^{-}\right) p$ cross sections). However, for the neutral current neutrino processes again some signature must be identified. For scattering in the quasielastic region one expects that a proton or neutron will be found in the final-state with energy-momentum corresponding roughly to elastic scattering from one of the nucleons in the nuclear ground state. Thus, one needs a different kind of inclusive cross section from those discussed heretofore, namely one where the final-state lepton kinematics are integrated over while the final-state ejected nucleon kinematics are not. Such $\left(\nu_{l}, N\right)$ and $\left(\bar{\nu}_{L}, N\right)$ cross sections are not "total cross sections" in the hadronic variables, in that such details as the propagation of the outgoing nucleon must be accounted for, placing more stringent demands on the nuclear model than is required for the rest of the inclusive cross sections discussed in this work (which are all "total cross sections" in the hadronic variables). As a consequence of this difference we have chosen to stop at this point and postpone further treatment of quasielastic neutrino scattering to Sect. IV.J.2. Instead, we now return to the main theme of this work and continue with more detailed discussions of $\mathrm{PV}$ electron scattering in the next section. 


\section{SPECIFIC CASES}

In Sect. IV we discuss a variety of specific cases where. using semileptonic electroweak interaction processes. the hadronic neutral current matrix elements may be explored. on the one hand. or where Standard Model tests may be performed. on the other. We begin in Sects. IV.A and IV.B with the important studies of PV elastic electron scattering from the proton and from $\left(J^{\pi} T\right)=\left(0^{+} 0\right)$ nuclei, respectively, and then continue in Sect. IT.C with a discussion of the role that $\mathrm{PV}$ elastic scattering from ${ }^{2} \mathrm{H}$ could play. Other discrete nuclear transitions initiated by inelastic PV electron scattering are treated in Sect. IV.D. while in Sect. IV.E we briefly discuss the axial-vector response and the anapole moment. also involving discrete nuclear states. Quasielastic PV electron scattering is treated in Sect. IV.F and PV electroexcitation of the $\Delta(1232)$ is discussed in Sect. IV.G. In the final three subsections of this major section we provide connections to scattering processes in other kinematic regimes (PV deep-inelastic electron scattering in Sect. IV. H) and to other electroweak processes (atomic PV in Sect. IV.I and $\nu$-scattering in Sect. IV.J) as these exhibit different sensitivities to hadronic content and to specifics of the underlying electroweak theory than does PV electron scattering and hence yield complementary information.

\section{IV.A. Elastic Scattering from the Proton}

At first glance, one would expect a measurement of the PV asymmetry for elastic scattering of polarized electrons from nucleons to provide the most direct and conceptually straightforward probe of the nucleon's weak neutral current. Several factors. however. conspire to render the interpretation of PV electron-nucleon scattering considerably more complicated than it might first appear. The lack of a free neutron target, for example. implies either restricting oneself to $\mathrm{PV}$ elastic $\vec{e} p$ scattering or turning to $A>1$ targets in order to access the $\mathrm{NC}$ of the neutron. The latter option, of course, introduces nuclear physics considerations into the interpretation of the PV asymmetry. Even in the case of $A_{L R}(\vec{e} p)$. however, one must account for the interplay between various hadronic form factors, hadronic uncertainties in radiative corrections, and the physics of the underlying electroweak gauge theory, as well as practical questions relating experimental doability to theoretical interpretability. The bottom line is that PV elastic $\vec{e} p$ is a fundamental component of any attempt to probe the nucleon's $\mathrm{NC}$, but that by itself, it is not sufficient for providing all the information one might want to acquire.

To illustrate the rationale for this conclusion, we return to the expression for the PV asymmetry given in Eq. (3.125)

$$
A_{L R}(\vec{e} p)=a_{0} \tau \frac{W^{(\mathrm{PV})}}{F^{2}}
$$


where $a_{0} \approx 3.1 \times 10^{-4}$ and where the hadronic ratio is given by

$$
\begin{aligned}
\frac{\left.I^{(P(P)}\right)}{F^{2}}= & \xi_{V}^{p} \\
& +\left[\mathcal{E} G_{E}^{p}\left\{\xi_{V}^{n} G_{E}^{n}+\xi_{V}^{(0)} G_{E}^{(s)}\right\}\right. \\
& +G_{M}^{p}\left\{\xi_{V}^{n} G_{M}^{n}+\xi_{V}^{(0)} G_{M}^{(s)}\right\} \\
& \left.-\sqrt{1-\mathcal{E}^{2}} \sqrt{\tau(1+\tau)}\left(1-4 \sin ^{2} n_{w}\right) G_{M}^{p} \tilde{G}_{A}^{p}\right] /\left[\mathcal{E}\left(G_{E}^{p}\right)^{2}+\tau\left(G_{M}^{p}\right)^{2}\right]
\end{aligned}
$$

The kinematic dependence of the various terms in Eq. (4.1) suggests a variety of possible $A_{L R}(\vec{e} p)$ measurements. A very low- $\left|Q^{2}\right|$ experiment (e.g., $q \lesssim$ few $100 \mathrm{Mel} / \mathrm{c}$. in which the contribution of the hadronic form factors is minimized. might allow one to extract $\xi_{v}^{p}$ and test the Standard .Model. The motivation for a moderate momentum transfer measurement (e.g., few $100 \mathrm{MeV} / c \rightarrow$ few $\mathrm{GeV} / c$ ) would be to determine the nucleon form factors. particularly those associated with the strange-quark currents. The $\theta$-dependence of the PV asymmetry suggests that one might consider a forward-angle measurement. which would constrain the electric form factors, $G_{E}^{(s)}$ and $G_{E}^{n}$ and a combination of intermediate- and backward-angle measurements at the same $Q^{2}$ with the intent of separating the axial-vector and weak magnetic responses. ideally allowing a determination of $G_{M}^{(s)}$. The experiments discuss ad in Refs. [.McK89, Bec91, Bei91b. Fin91] include two of these prospective measurements, and they would provide the first experimental bounds on the low- $\left|Q^{2}\right|$ behavior of $G_{M}^{(s)}$ and $G_{E}^{(s)}$.

As we illustrate below, however, completion of such a set of $\overrightarrow{e p}$ measurements . . ld not be sufficient to constrain the strangeness form factors to arbitrary precisinn. although it would provide valuable experimental limits. Specifically, the contribution from the axialvector response persists to sufficiently forward angles that a Rosenbluth-type separation of the weak magnetic and axial-vector form factors does not appear possible. Consequently. large theoretical uncertainties in the radiative corrections for the axial-vector current term impose an intrinsic theoretical bound on the precision to which $G_{M}^{\left({ }^{(s)}\right.}$ may be determined trom $A_{L R}(\vec{e} p)$. A similar limit on the achievable precision in $G_{M}^{(s)}$ is imposed by the experimental uncertainty in $G_{M}^{n}$, which also contributes to $A_{L R}(\vec{e} p)$ at backward angles. Moreover, this "intrinsic" uncertainty in $G_{M}^{(s)}$ enters the extraction of $G_{E}^{(s)}$ from forwardangle $A_{L R}(\vec{e} p)$ measurements. Assuming realistic experimental conditions, one finds that the resulting uncertainty in $G_{E}^{(s)}$ is somewhat larger than the theoretical error quoted for the model calculation of Ref. [Jaf89]. This conclusion also carries implications for prospective $A_{L R}(\vec{e} p)$ electroweak tests. Tighter limits on $G_{E}^{(s)}$ would be required before one could hope to extract $\xi_{V}^{p}$ from a low- $\left|Q^{2}\right| A_{L R}(\vec{e} p)$ measurement to $10 \%$ accuracy (as assumed in Fig. 2.4). Thus, a program of $\mathrm{NC}$ studies with polarized protons would require scattering from $A>1$ targets to complement elastic PV $\overrightarrow{e p}$ experiments.

We now proceed to discuss these results in more detail, drawing essentially from the treatment in Ref. [Mus92a].

\section{IV.A.1. BACKWARD-ANGLE SCATTERING}


In the $\theta \rightarrow 180^{\circ}$ limit. $\mathcal{E} \rightarrow 0$ and Eq. (4.1) simplifies to

$$
\frac{I^{\cdot(P V)}}{F^{2}} \longrightarrow \xi_{v}^{p}+\xi_{v}^{n} \frac{G_{M}^{n}}{G_{M}^{p}}+\xi_{v}^{(0)} \frac{G_{M}^{(s)}}{G_{M}^{p}}-\left(1-4 \sin ^{2} \theta_{w}\right) \sqrt{\frac{1}{\tau}+1} \frac{\dot{G}_{A}^{p}}{G_{M}^{p}} .
$$

Conservation of angular momentum and electron helicity (in the $\epsilon \gg m_{e}$ regime) require the proton to flip its spin. so that only the magnetic and axial-vector terms contribute. The first term in Eq. (4.2) comes from the piece of $\tilde{G}_{M}^{p}$ proportional to $G_{M}^{p}$ : hence the form factor dependence cancels with the $G_{M}^{p}{ }^{2}$ of the denominator. The second and third terms arise from the $G_{M}^{n}$ and $G_{M}^{(9)}$ terms in $\tilde{G}_{M}^{p}$. The S.A.UPLE experiment. presently underway at MIIT/Bates [Mck89], will measure the backward-angle $\vec{e} p$ asymmetry, thereby constraining, the strangeness magnetic moment. $\mu_{s} \equiv G_{M}^{(s)}(0)$. The anticipated experimental uncertainty: corresponds a limit on $\mu_{\mathrm{s}}$ of $\left|\delta \mu_{\mathrm{g}}\right|<0.22$. A second-generation SA.LPLE experiment having smaller experimental error might hope to tighten this limit. There are. however. other factors that must be considered. " $p$ to radiative corrections. $G_{M}^{n}$ and $G_{M}^{(s)}$ enter the asymmetry with equal weight $\left(\xi_{v}^{n}=\xi_{v}^{(0)}=-1\right.$ at tree level: see Table 3.2 and Eq. (3.24bi). so that an accurate extraction of $G_{M}^{(s)}$ requires very accurate knowledge of $G_{M}^{n}$. Of even greater concern are the complications introduced by the theoretical uncertainties in the axial-vector form factor appearing in Eq. (4.2). Uncertainties in $G_{A}^{p}$ arise from both $\eta_{\text {s }}$ and $G_{D}^{A}$ (see Sect. III.C) as well as from the radiative corrections, $R_{A}^{p}$. At the S.A.IPLE kinematics, uncertainties in the axial-vector dipole mass parameter induce roughly a one percent error in $\tilde{G}_{A}^{p}$; the corresponding induced error in $G_{M}^{(s)}$ is negligible for the present purposes. The impact of uncertainties in $\eta_{s}$ (see Table 2.2) is included in the projected SA.MPLE limits on $G_{M}^{(\mathbf{s})}$. In principle, the LSND neutrino oscillation experiment underway at LA.MPF [Lou89] could reduce the uncertainty in $\eta_{s}$, although with the expected $20 \%$ statistical uncertainty in the elastic $\nu-p$ cross section little improvement, if any, would be made over the uncertainty quoted in Table 2.2.* The error in the radiative correction. $R_{A}^{p}$. appears to be more problematic. As discussed in Sect. III, this quantity contains significant theoretical uncertainty in the case of PV $\overrightarrow{e p}$ scattering. Since this uncertainty is associated with low-energy hadronic contributions, one has little hope of calculating its magnitude with significantly better precision than given by the estimate of Ref. [Mus90]. For the present purposes, then, we consider this error tu be intrinsic. Together with uncertainty in $\mu_{s}$, it induces a fractional change in the backward-angle asymmetry of

$$
\frac{\delta A_{L R}}{A_{L R}} \approx \frac{\delta R_{A}^{p}}{5}-\frac{\delta \mu_{S}}{3}
$$

at the SA.MPLE kinematics. Thus, even if a "perfect" SAMPLE experiment (zero percent experimental uncertainty) were possible, one would not be able to constrain $\mu_{\text {s }}$ to better than

$$
\delta \mu_{\mathrm{s}} \approx 0.6 \delta R_{A}^{p} \approx \pm 0.12
$$

* The BNL error quoted in Table 2.2 does not include the impact of the $\eta_{\mathrm{s}}-M_{A}$ correlation. The LSND experiment would eliminate the latter source of uncertainty in $\eta_{s}$. 
according to the estimates of Ref. [Mus90). The relation between $\delta R_{A}^{p}$ and $\delta \mu$, for nonzero experimental uncertainty is indicated by the correlation plot of Fig. 4.1. As we illustrate below, this theoretical uncertainty may propagate through other experiments, introducing limitations on $G_{E}^{(\mathbf{s})}$ determinations as well as precision Standard . Nodel tests.

One might hope to reduce the uncertainties due to lack of knowledge of $\dot{G}_{A}^{p}$ by using the different $\theta$-dependences in Eq. (4.1) to separate the axial-vector term. Since $\mathcal{E} \rightarrow 1$ for $\theta \rightarrow 0^{\circ}$. the importance of the axial-vector term should be reduced for sufficiently small angles. As Fig. 4.2 illustrates, however, $\mathcal{F}_{A}$, the fraction of $A_{L R}$ due to the axial-vector term. does not begin to decrease significantly until $\theta \lesssim 40^{\circ}$ (depending on the energ! $)$. At this point the fraction $\mathcal{F}_{L}$ contributed by the longitudinal term increases and one can no longer reliably apply the backward-angle limit to isolate $G_{M}^{(\mathbf{*})}$.

A second possibility for separating the magnetic and axial-vector terms. a backwardangle measurement at higher energy. also depends on the different kinematical factors appearing with the second and third terms in Eq. (4.1). Since $\tau=\left(\epsilon \epsilon^{\prime} / m_{x}^{2}\right) \sin ^{2} \frac{\theta}{2}$ for elastic scattering with extremely relativistic electrons, the axial-vector term decreases in importance relative to the magnetic term for increasing energy at backward angles. Thus. one might hope to decrease the sensitivity of $A_{L R}(\vec{e} p)^{\theta-180^{\circ}}$ to the more uncertain axialvector term by performing an experiment at higher energy than envisioned for SA.MPLE. This gain, however. is soon offset by a decrease in the FOM for sufficiently high energies. resulting from a falloff in the proton magnetic form factor with increasing $\left|Q^{2}\right|$. In Fig. 4.3 we plot $\left(\delta A_{L R} / A_{L R}\right)_{s t a t}$ as a function of $\epsilon$ at $\theta=175^{\circ}$ for fixed experimental conditions a 1000-hour experiment, a luminosity of $5 \times 10^{38} \mathrm{~cm}^{-2} \mathrm{~s}^{-1}$, a beam polarization of $100 \%$. and a $1 \mathrm{sr}$ solid angle). Also shown is $\mathcal{F}_{A}$ as a function of $\epsilon$ at $\theta=175^{\circ}$. From these two curves we observe that although $\mathcal{F}_{A}$ decreases by a factor of two in going from $\epsilon=250$ to $1000 \mathrm{MeV},\left(\delta A_{L R} / A_{L R}\right)_{t a t}$ increases by nearly the same amount.

We conclude, then, that neither of these approaches will significantly improve the limits on $\mu_{s}$ attainable from a backward-angle $A_{L R}(\vec{e} p)$ measurement. A potentially more promising alternative is to measure either $R_{A}^{p}$ or $\mu$, with some other target for which they are not as strongly correlated as in the case of intermediate- or backward-angle $A_{L R}(\vec{e} p)$. Elastic scattering from the deuteron and quasielastic scatcering constitute two such possibilities, as discussed in Sects. IV.C and IV.F below.

\section{IV.A.2. FORWARD-ANGLE SCATTERING}

For $\theta \rightarrow 0^{\circ}$, one has $\mathcal{E} \approx 1$ and the axial-vector term in Eq. (4.1) becomes negligible. leading to

$$
\begin{aligned}
\frac{W^{(\mathrm{PV})}}{F^{2}} \longrightarrow & \xi_{V}^{p} \\
& +\left[G_{E}^{p}\left\{\xi_{V}^{n} G_{E}^{n}+\xi_{V}^{(0)} G_{E}^{(s)}\right\}\right. \\
& \left.+\tau G_{M}^{p}\left\{\xi_{V}^{n} G_{M}^{n}+\xi_{V}^{(0)} G_{M}^{(s)}\right\}\right] /\left[\left(G_{E}^{p}\right)^{2}+\tau\left(G_{M}^{p}\right)^{2}\right\}
\end{aligned}
$$


At moderately low momentum transfers ||$Q^{2} \mid \ll+(\mathrm{GeV} / \mathrm{c})^{2}$, so that $\tau \ll 1$, we can simplify the discussion by keeping only the terms through $O(\tau)$ :

$$
\frac{I^{\circ(P V)}}{F^{2}}-\xi_{v}^{p}-\tau\left[\mu_{n} \xi_{v}^{n}-\rho_{s} \xi_{v}^{(0)}-\mu_{p}\left\{\mu_{n} \xi_{v}^{n}+\mu_{s} \xi_{v}^{(0)}\right\}\right]+\mathrm{O}\left(\tau^{2}\right) \text {. }
$$

An extraction of the term of $O(\tau)$ in Eq. (4.6) would constrain a linear combination of $\rho$, and $\mu_{g}$. A series of $A_{L R}(\vec{e} p)$ measurements, carried out at different values of $\tau$ and designed to extract this term of $\mathrm{O}(\tau)$, has been discussed as a possibility for CEBAF (Bec91. Bei91b. Fin91]. As Eq. (4.6) demonstrates. however. the placement of constraints on $G_{E}^{(1)}$ from such an extraction would require one to account for correlations between uncertainties in all of the contributions of $\mathrm{O}(\tau)$.

To evaluate the impact of these various correlations on a $G_{\Sigma}^{(\text {s) }}$-determination. the authors of Ref. [Nus92a] have performed a sensitivity analysis for a prospective measurement carried out at the kinematics of Ref. [Nap91]: $\epsilon=2.6 \mathrm{GeV}$ and $0.1 \leq\left|Q^{2}\right| \leq 0.3\left(\mathrm{Gel} \mathrm{c}^{2}\right.$. In this regime, the strongest correlation occurs between $\rho_{\text {g }}$ and $\mu_{s}$. Assuming zero percent experimental uncertainty, for example, the uncertainties in these two parameters are related by

$$
\delta \rho_{s}=-\mu_{p}\left(1+\lambda_{\varepsilon}^{(g)} \tau\right) \delta \mu_{s} .
$$

For either $\tau=0$ or $\lambda_{\varepsilon}^{(g)}=0$, the uncertainty in $A_{L R}$ due to $\delta \mu_{\mathrm{s}}$ is weighted by a factor of $\mu_{p} \approx 2.79$ relative to the error induced by $\delta \rho_{s}$. For nonzero $\tau$ or $\lambda_{E}^{(s)}$, the impact of $\delta \mu$, on $\delta \rho_{s}$ may be enhanced. For example, taking $\lambda_{E}^{(s)}=\lambda_{n}=5.6$ and $\left|Q^{2}\right|=0.2(\mathrm{GeV} / \mathrm{c})^{2}$. corresponding to $\tau=0.06$, gives

$$
\delta \rho_{s} \approx-3.7 \delta \mu_{s}
$$

The $\rho_{s}-\mu_{s}$ correlation taken from Ref. [Mus92a] for a more realistic experiment $\left.\left(\delta A_{L R} / A_{L R}\right)_{\text {experiment }} \neq 0\right)$ is displayed in Fig. 4.4. The error bands correspond to the combined statistical and systematic errors projected for the measurement discussed in Ref. [Nap91]. From this analysis a limit of $\delta \mu_{\mathrm{s}} \approx 0.22$ from a backward-angle experiment will allow extraction of $G_{E}^{(s)}$ at a level of $\delta G_{E}^{(s)} \approx G_{E}^{n}$, while the limit of Eq. (4.4). $\delta \mu_{\mathrm{g}} \approx 0.12$ would give $\delta G_{\varepsilon}^{(\mathrm{s})} \approx 0.7 G_{\varepsilon}^{n}$. The latter uncertainty is somewhat larger than the theoretical uncertainty in the prediction of Ref. [Jaf89] and somewhat smaller than the total prediction of the broken SU(3) Skyrme model (see Table 2.3).

For comparison, the authors of Ref. [Mus92a] also examined the $G_{E}^{(\mathrm{s})}-G_{\Sigma}^{n}, G_{\Sigma}^{(\mathrm{s})}-G_{M}^{n}$ and $G_{E}^{(s)}-\sin ^{2} \theta_{w}$ correlations. Since $G_{E}^{(s)}$ and $G_{E}^{n}$ enter the terms of $O(\tau)$ with a relative weighting of $1: 1$ (up to radiative corrections), any uncertainty in $G_{\varepsilon}^{n}$ induces an identical uncertainty in $G_{\varepsilon}^{(0)}$. The former is presently about $\pm 0.5 G_{\varepsilon}^{n}$ and might he somewhat reduced by the completion of the current MIT/Bates experiments [Mil88]. One might ultimately hope for another factor of two or so improvement in $\delta G_{\varepsilon}^{n}$ within the next decade [Mad85, Are88, Mad89, Jon91, Chu88, Mil89, Ala91]. Lack of knowledge in $G_{M}^{n}$ may eventually be more problematic than uncertainty in $G_{E}^{n}$, due to the pre-multiplying factor of $\mu_{p}$ in 
Eq. (4.6). While the error in $G_{M}^{n}$ may be reduced to about $3 \%$ by the end of the decade 'Jou88). for example. it is roughly three times more important than the uncertainty in $G_{\varepsilon}^{n}$ as far as a $G_{\varepsilon}^{(\text {s) }}$-determination is concerned. Thus. a $3 \%$ uncertainty in $G_{M}^{n}$ will introduce more uncertainty in $G_{\varepsilon}^{(s)}$ than would a $10 \%$ uncertainty in $G_{\varepsilon}^{n}$. . Moreover. the error in $G_{M}^{n}$ would be roughly comparable with that corresponding to the $\left|\delta \mu_{g}\right|<0.12$ limit. Finally: for $Q^{2}=0.2(\mathrm{GeV} / c)^{2}$ and $\delta . A_{L R} / A_{L R}=0$, a one percent error in $\sin ^{2} \theta_{w}$ generates an uncertainty in $G_{\varepsilon}^{(\text {)" }}$ of $\delta G_{\varepsilon}^{\prime \prime \prime} \approx \pm 0.15 G_{\varepsilon}^{n}$. Thus. both the $G_{\varepsilon}^{n}$ and $\sin ^{2} \theta_{w}$ uncertainties in $G_{\varepsilon}^{(9)}$ are smaller than the error induced by $\mu$, when the latter is limited by the radiative correction uncertainty or by the $G_{M}^{n}$ uncertainty.

There is little doubt that a set of measurements of the asymmetry for $P V^{\circ}, \vec{\epsilon}, \epsilon^{\prime}$, from the proron will be a keystone in the determination of the contribution of $s$ quarks to the rector current of the nucleon. Given the large theoretical uncertainties associated with the axial-rector radiative corrections, $A_{L R}(\vec{e} p)$ is less suitable as a probe of strangeness axial-vector current of the nucleon. Indeed. the former uncertainty limits the extent to which a series of $A_{L R}(\vec{e} p)$ measurements could constraint $G_{\varepsilon}^{(s)}$ and $G_{M}^{(s)}$. Additional. complementary measurements will be needed to fully elucidate the role of the strange quarks in the nucleon.

\section{IV.A.3. STA.IDARD .MODEL TESTS}

In the limit that $\left|Q^{2}\right| \rightarrow 0$, the hadronic ratio $W^{(\mathrm{PV})} / F^{2}$ for forward-angle scattering is simply proportional to the proton neutral current coupling, $\xi_{V}^{p}=\left(1-4 \sin ^{2} \theta_{w}\right)\left(1-R_{t}^{p}\right)$. As indicated in Sect. II, a $10 \%$ determination of this quantity could nicely complement atomic $\mathrm{PV}$ or $\mathrm{PV}$ elastic ${ }^{12} \mathrm{C}(\vec{e}, e)$ scattering as a low-energy probe of physics beyond the Standard Model. At $Q^{2}=0$. this $10 \%$ figure translates directly into a $10 \%$ determinatirn of $A_{L R}(\vec{\epsilon} p)$. Any actual experiment must be carried out at $Q^{2} \neq 0$. since $A_{L R}$ ranishes with $Q^{2}$. In this case, one has, to lowest non-trivial order in $\tau$ one has

$$
\frac{\delta \xi_{V}^{p}}{\xi_{V}^{p}}=\left[1+\tau\left(\frac{B}{\xi_{V}^{p}}\right)\right]\left(\frac{\delta A_{L R}}{A_{L R}}\right)-\tau\left(\frac{\delta B}{\xi_{V}^{p}}\right)
$$

where

$$
B \equiv-\mu_{n} \xi_{v}^{n}+\rho_{s} \xi_{v}^{(0)}+\mu_{p}\left[\mu_{n} \xi_{v}^{n}+\mu_{s} \xi_{v}^{(0)}\right]
$$

The quantity $\delta B$ represents the error in $B$ from all sources, including $G_{\varepsilon}^{(s)}, G_{M}^{(s)}, G_{\varepsilon}^{n}, \xi_{v}^{n}$, and $\xi_{v}^{(0)}$. We now consider these sources of error.

First, we note that the errors induced in $\xi_{V}^{p}$ by $\delta B$ and $\delta A_{L R} / A_{L R}$ depend on the value of $\tau$. The impact of these errors is minimized as one decreases the value of $\tau$, as indicated by Eq. (4.9).* However, the FOM also decreases with $\tau$, so that the achievable statistical precision in $A_{L R}$ improves fo: increasing $\tau$. These two competing features of $A_{L R}(\vec{e} p)$ conspire to restrict severely the kinematical region in which one should attempt

* Note that both $\xi_{v}^{p}$ and $B$ are positive quantities. 
to carry out a $10 \%$ determination of $\xi_{v}^{p}$. In order to quantify these statements. We plot in Fig. 4.3 curves of $\left(\delta . A_{L R} / A_{L R}\right)_{\text {sot }}$ versus $T$ for constant values of the scattering angle. We have assumed a luminosity of $5 \times 10^{38} \mathrm{~cm}^{-2} \mathrm{~s}^{-1}$. a $100 \%$ beam polarization. a solid angle of 0.01 sr and a running time of 1000 hours. These conditions are within reasonable expectations for what might be achievable at CEBAF within the next decade (see Sect. $Y . C$ ). For each curve. increasing $\tau$ corresponds to increasing incident electron energy: On the same graph we plot the minimum $\left(\delta . A_{L R} / A_{L R}\right)_{\text {sat }}$ needed in order to keep the error in $\xi_{v}^{p}$ below ten percent (dashed line). The latter is derived from Eqs. 4.9 , and $(4.10)$ assuming tree-level Standard Model couplings and setting $\delta B=G_{M}^{\prime \prime}=G_{E}^{\prime \prime \prime}=0$.

From Fig. 4.4 one can see that an experiment should have $\epsilon \gtrsim 1100 \mathrm{Mel}$ and $\theta \leqslant 15^{\circ}$ to extract $\$ P$ at the $10 \%$ level. However. one cannot go to arbitrarily large values of $\epsilon$ without introducing problematic errors from $\delta B$. For example, in an experiment carried out at $\theta=10^{\circ}$. one needs $T \geq 0.011$. Inder these conditions. the uncertainty in $G_{E}^{\prime \prime \prime}$ must be $\delta G_{\varepsilon}^{(g)} \leq 0.4 i G_{\varepsilon}^{n}$ in order to keep the error induced in $\xi_{V}^{p}$ by $\delta B$ to less than ten percent. From our previous discussion. it does not appear possible to determine $G_{E}^{\prime \prime}$ " to this precision with a series of $\overrightarrow{e p}$ experiments alone. Further analysis of Ref. Mus92a. suggests that, anticipating a $10 \%$ uncertainty in $G_{\varepsilon}^{n}$ and a $5 \%$ uncertainty in $G_{M}^{n}$. it is the uncertainty from the strange-quark form factors that would most severely limit a determination of $\xi_{v}^{p}$. Thus, to extract $\xi_{v}^{p}$ to ten percent, one would need to improve the precision in $G_{E}^{\left({ }^{\prime)}\right.}$.

To realize this objective, one has a number of options: (i) a direct measurement of $G_{\varepsilon}^{(s)}$ with PV electron scattering from another target (see, e.g.. Sect. IV.B): ii । a more precise direct measurement of $G_{M}^{(s)}$ with PV electron scattering from another target. thereby reducing the $G_{M}^{(s)}$-induced error in an $A_{L R}(\vec{e} p)$ determination of $G_{E}^{(s)}$ (see. e.g.. Sect. IV.C): or (iii) a measurement of $\dot{G}_{A}^{p}$ using PV QE electron scattering (see Sect. IV.F). thereby reducing the $G_{M}^{(s)}$ and $G_{\Sigma}^{(s)}$ uncertainties associated with a series of $A_{L R}(\overrightarrow{e p})$ measurements. Alternatively, one could live with the larger $G_{\Sigma}^{(0)}$ uncertainty and perform an $A_{L R}(\vec{e} p)$ electroweak test at more forward angles than assumed in the foregoing analysis. For $\delta G_{E}^{(s)} \approx 0.7 G_{E}^{n}$, for example, a measurement at $\theta \lesssim 8^{\circ}$ would be needed. Such a measurement lies beyond the capabilities of presently envisioned facilities at CEBAF. and construction of a new detector would be required.

We conclude this section by pointing to one issue which arises in the extraction of constraints on new physics from a determination of $\xi_{V}^{p}$, namely, the appearance of theoretical hadronic uncertainties in the radiative correction, $R_{v}^{p}$. In order to arrive at the limits on $S$ and $T$ displayed in Fig. 2.4, one would necd to keep the error in this correction to $\mid \delta R_{V}^{p}$ (had) $\mid \lesssim 0.1$. This radiative correction arises primarily from hadronic loops in the $Z^{0}-$ $\gamma$ mixing tensor and from hadronic intermediate states in the $Z^{0}-\gamma$ "box" diagrams. The authors of Ref. [Mar84] have estimated the former using a dispersion analysis and find a corresponding uncertainty in the radiative correction of $\delta R_{v}^{p}(Z-\gamma$ mixing $) \approx 0.02$. Hadronic contributions to ihe box diagram correction have also been estimated in Ref. [Mar84] for the case of atomic PV. These authors considered proton intermediate states, whose dominant contribution to $R_{v}^{p}$ is suppressed by a factor of $\left(1-4 \sin ^{2} \theta_{w}\right)$. As a rough and. 


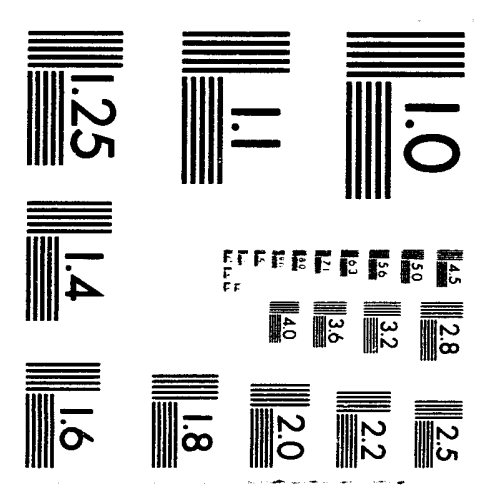



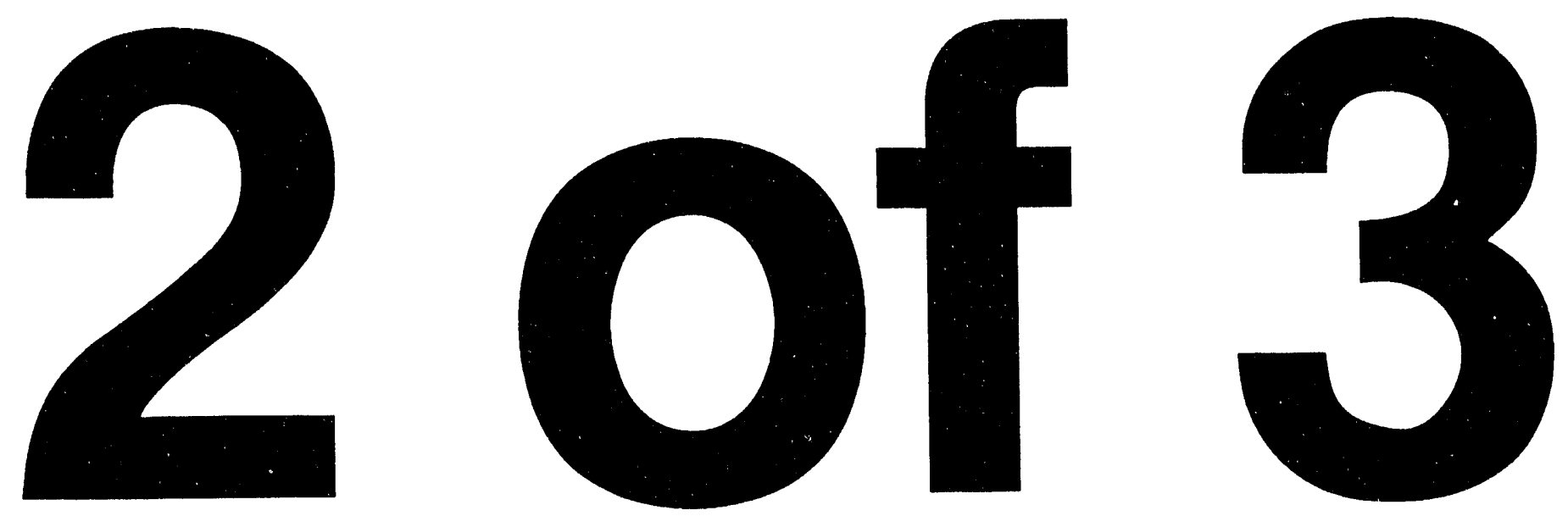
perhaps, liberal estimate, one might equate the hadronic error in $\delta R_{V}^{p}$ with their estimate of this "Born" contribution to $R_{V}^{p}$, resulting in an estimate of $\delta R_{V}^{p}= \pm 0.01$. well below the problematic limit. Contributions from higher-lying intermediate states, however, need not carry the $1-4 \sin ^{2} \theta_{w}$ factor and could introduce significantly larger uncertainty. Further study of these contributions is warranted for both PV electron scattering and atomic PV. 


\section{IV.B. Elastic Scattering from Spin-0 Nuclei}

In turning from the nucleon to nuclear targets one might imagine at least two sources of additional complications. The first would be the extra layer of strong-interaction physics manifested in details of nuclear structure: the second would be the variety of and interplay between the numerous multipole matrix elements that can contribute for arbitrary initialand/or final-state nuclear angular momenta. Indeed. as discussed later in Sect. IV.D. such complications coupled with practical considerations such as energy resolution probably. render most PV $\left(\vec{e}, e^{\prime}\right)$ experiments involving transitions between discrete nuclear states dubious at best for exploring the nucleonic current including its potential $s \bar{s}$ content. although such experiments might be of great value for studies of nuclear structure. However. there are also circumstances where specific features of nuclear structure can be used advantageously to provide simplification and/or to isolate or at least emphasize physics of interest. The best example of this is elastic PV electron scattering from a spin-0. isospin-0 nucleus. In general, elastic scattering has an advantage over inelastic scattering in that the FOM. being proportional to the E.M cross section, is enhanced in the forward direction and low $-\left|Q^{2}\right|$ by the $Z^{2}$ coherence factor in the monopole form factor. As Figs. 3.10-3.12 illustrate. this results in $\mathcal{F}$ being very large and consequently, the attainable statistical precision is improved by a factor of $1 / Z$ with respect to $\overrightarrow{e p}$ or inelastic scattering to a discrete state for a given luminosity and running time. Second, in the absence of strangeness and in the limit of exact isospin symmetry $\left(\left[\vec{T}, H_{\text {nuc }}\right]=0\right)$, elastic matrix elements of $j_{\mu}^{v} c$ and $J_{\mu}^{E M}$ are proportional for these targets, since only the $T=0$ components of the currents contribute. Accordingly, as discussed in Sect. III.E.1, in this limit the matrix elements cancel from the hadronic ratio $W^{(\mathrm{PV})} / F^{2}$, leaving only the particle physics coupling, $\sqrt{3} \xi_{\mathrm{T}}^{T=0}$. . Ioreover, $A_{L R}\left(0^{+} 0\right)$ is also free from magnetic and axial-vector contributions, since this target has $J=0$.

To begin our discussion of what happens when one proceeds beyond this simple limit. let us substitute the Standard Model couplings from Tables 3.1 and 3.2 into Eq. (3.120) to obtain

$$
-2 \frac{A_{L R}}{A_{L R}^{0}}=4 \sin ^{2} \theta_{W}\left[1+R_{V}^{T=0}+\Gamma(q)\right]+\left[1+R_{V}^{(0)}\right] \frac{F_{C 0}(s)}{F_{C 0}(T=0)}
$$

The $Q^{2}$-dependence here arises from three terms: $\Gamma$, the correction that represents the breaking of isospin symmetry discussed in Sect. III.D.4, the ratio $F_{C 0}(s) / F_{C 0}(T=0$ ) that comes from the presence of nonzero strangeness components in the hadronic neutral current and the radiative corrections $R_{V}^{T=0}$ and $R_{V}^{(0)}$ which were introduced in Sect. III.B. In the absence of the corrections, which will be discussed in more detail in the following subsections, the right-hand side of Eq. (4.11) yields the especially simple answer $4 \sin ^{2} \theta_{w}$ and accordingly elastic scattering from spin-0 nuclei was suggested as a place to test the Standard Model [Fei75, Wal77]. One experiment of this type has been attempted, the ${ }^{12} \mathrm{C}(\vec{e}, e)$ measurement at MIT/Bates, whose detailed results have been presented in Ref. [Sou90a] (see also the summary of these results in Sect. V.B.3). In this pioneering effort $A_{L R}\left(0^{+} 0\right) \approx 1.7 \mathrm{ppm}$ was measured with $23 \%$ statistical and $3 \%$ systematic errors. Future, higher-precision experiments of this type appear to afford the possibility of exploring 
in more detail all of the various facets of Eq. (4.11) and, accordingly, we shall follow the discussions in Refs. [Mus92a. Don89] and focus on these future issues. We begin by exploring the interpretation and implications of this basic equation for the extraction of the strange-quark contribution to the hadronic neutral current (Sect. IV.B.1) and then return to discuss such PV elastic scattering measurements as Standard Model tests (Sect. IV.B.2). Finally, in Sect. IV.B.3 we consider elastic scattering from spin-0 nuclei with $. V \neq Z$ i i.e. $T>0$ nuclei) as a new means to study their ground-state neutron distributions. Discussion of inelastic scattering to discrete nuclear states, including $J>0$ nuclei, will be defarred to Sect. IV.D.

Before entering into these more detailed discussions let us summarize some of the basic conclusions. As discussed in Ref. [Mus92a], high- $\left|Q^{2}\right|$ forward-angle measurements are potentially sensitive enough to $G_{E}^{(s)}$ to permit a determination of this form factor. For ${ }^{4} \mathrm{He}$. experiments carried out at moderately-forward angles $\left(\theta \sim 30^{\circ}\right)$ for energies in the regime $0.2 \lesssim \epsilon \lesssim 1.1 \mathrm{GeV}$ (below the first diffraction minimum) and $1.3 \lesssim \epsilon \lesssim 2.0 \mathrm{GeV}$ (abore the first diffraction minimum) could allow a $G_{E}^{(s)}$-determination with an uncertainty of roughly half what is attainable with $A_{L R}(\vec{e} p)$ measurements, up to presently unquantified theoretical uncertainties. Results for ${ }^{12} \mathrm{C}$ are similar, although the useful energy ranges are somewhat narrower. Experiments at more forward angles and higher energies would also achieve the same end. Viewed as a Standard Model test, the low- $\left|Q^{2}\right|$ forwardangle projections given in Ref. [Mus92a] show that $A_{L R}\left(0^{+} 0\right)$ is predominantly sensitive to $\sin ^{2} \theta_{W}$ and possible contributions from "non-standard," degenerate, heavy-fermion doublets [Mar90. Pes90], although potentially large and theoretically uncertain dispersion corrections could seriously cloud the interpretability of a $\lesssim 1 \%$ measurement, as discussed in Sect. II.A. Furthermore, that lack of knowledge in $G_{E}^{(s)}$ places tight requirements on the kinematic regime for which a $1 \%$ measurement of $A_{L R}\left(0^{+} 0\right)$ would be interpretable as a Standard Model test. For both ${ }^{4} \mathrm{He}$ ad ${ }^{12} \mathrm{C}$, a low-to-intermediate energy measurement would be required, whereas an exper: :ent performed at CEBAF energies (2-4 GoV) would introduce $G_{E}^{(s)}$-uncertainties at greater than the $1 \%$ level. Experiments at very-forward and moderately-forward angles both appear to be possible. Finally, it appears to be quite feasible to extract the rms radius of the ground-state neutron distribution for a wide range of nuclei to a precision of about $1 \%$ using PV elastic electron scattering.

\section{IV.B.1 SENSITIVITY TO THE STRANGENESS FORM FACTOR $G_{E}^{(s)}$}

$\mathrm{PV}$ elastic electron scattering from $0^{+} 0$ nuclei might ultimately allow a determination of $G_{E}^{(s)}$ to higher precision than is possible with $\vec{e} p$ scattering. By carrying out an $A_{L R}\left(0^{+} 0\right)$ experiment at higher values of $\left|Q^{2}\right|$, one enhances the importance of the strangenessdependent term of $\mathrm{Eq}$. (4.11) relative to the leading term [Bec89]. By going to sufficiently high- $\left|Q^{2}\right|$, one might also explore the non-leading $Q^{2}$-dependence of $G_{E}^{(s)}$. As discussed in Sect. III.D.4, for favorable $N=Z$ nuclei such as ${ }^{4} \mathrm{He}$ and ${ }^{12} \mathrm{C}$, the first of the corrections in Eq. (4.11), that relating to isospin-mixing $(\Gamma)$, has been conservatively estimated in Ref. [Don89] to remain less than 0.01 over the entire range of $Q^{2}$ of interest for extraction of strange-quark contributions (limited on the high- $\left|Q^{2}\right|$ side by the rapid falloff of the 
nuclear form factor and. correspondingly, the FOM - see Figs. 3.10-3.12). Moreover. the $Q^{2}$-dependence of $R_{V}^{T=0}$ and $R_{\downarrow}^{(0)}$ should be relatively weak. Thus. depending on the size of the strangeness form factor $F_{C 0}(s)$, measurement of the PV asymmetry for elastic scattering from such light $0^{+} 0$ nuclei may be expected to provide a relatively simple method for isolating the strangeness contribution to the nuclear vector current.

To understand how well elastic scattering from $0^{+}$nuclei can be used to learn about $G_{E}^{(s)}$. it is necessary to probe more deeply into the uncertainties that compete with the strangeness dependence. Let us begin with the nuclear many-body problem. If one treats the EMI and $\mathrm{XC}$ form factors as arising from matrix elements of one-body operators see Sect. III.D.2), then from Eq. (3.65) the relevant matrix elements can be written

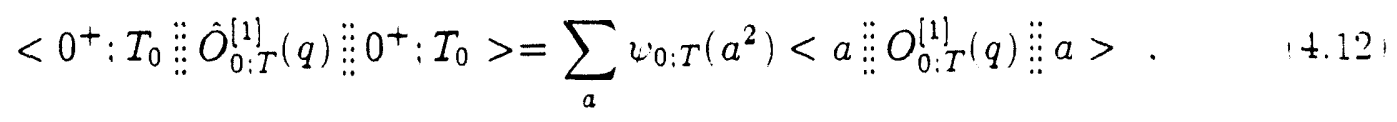

where we assume in general that the ground state has isospin $T_{0}$. The one-body densit? matrix elements can be shown to be [Don84]

$$
\begin{aligned}
& \psi_{0: 0}\left(a^{2}\right)=\frac{\sqrt{2 T_{0}+1}}{\sqrt{2\left(2 j_{a}+1\right)}}\left[V_{p}(a)+V_{n}(a)\right] \\
& \psi_{0: 1}\left(a^{2}\right)=-\frac{\sqrt{\left(T_{0}+1\right)\left(2 T_{0}+1\right)}}{\sqrt{6 T_{0}\left(2 j_{a}+1\right)}}\left[V_{p}(a)-V_{n}(a)\right] .
\end{aligned}
$$

where $V_{p, n}$ are the occupation numbers of the single-particle level labeled a see Sect. III.D.2). The single-particle matrix elements in Eq. (4.12) contain all of the momentum transfer dependence in the nuclear form factors. In particular, the coherence in the many-body matrix elements arises from the fact that the $T=0$ density matrix elements above involve a sum over protons and neutrons. Thus, at low momentum transfer the $T=0$ nuclear matrix elements are proportional to the sum over all of the nucleons in the nucleus, i.e., to $A$. In contrast, the $T=1$ density matrix elements involve proton-neutron differences and usually lead to smaller isovector effects. These arguments are independent of the nature of the electroweak probe - that aspect of the problem is contained entirely in the single-particle matrix elements. In particular, since in comparing the E.M and NC form factors the latter differ only by the hadronic weighting factors in Table 3.2 and by the inclusion of the appropriate single-nucleon form factors, $G_{E}^{T}$ with $T=0,1$ and $G_{E}^{(9)}$. one has the following proportionality relationship involving the nuclear many-body monopole form factors and the single-nucleon form factors:

$$
\begin{aligned}
\frac{F_{C 0}(s)}{F_{C 0}(T=0)} & =\frac{G_{E}^{(s)}}{G_{E}^{T=0}} \\
& =2 \rho_{s} \tau \xi_{E}^{(s)}
\end{aligned}
$$

using the parameterizations in Sect. III.C. Thus the ratio of nuclear form factors is simply a ratio of single-nucleon form factors in this one-body-operator approximation (see Sect. III.D.3 for a brief discussion of two-body meson exchange current corrections). 
Before proceeding further, let us make the following comment about the nuclear monopole form factors: in computing matrix elements of the one-body charge operator. $\hat{\rho}^{(1)}$. one faces an ambiguity as to whether to use Dirac and Pauli $\left(F_{1}, F_{2}\right)$ or $\operatorname{Sachs}\left(G_{E}, G_{\mathfrak{M}}\right)$ form factors (as above). Since the operator $\hat{\rho}^{(1)}$ arises as a non-relativistic reduction of the covariant nucleon currents, the nature of this ambiguity is most clearly characterized by expanding the operator in powers of $v / c$. We carry this expansion out to second order in $v / c$. since $F_{1}$ and $G_{E}$ differ by terms of $\mathcal{O}(\tau)$. For a spin-0 nucleus, the spin-dependent part of $\hat{\rho}^{(1)}$ may be neglected. so that the time component of the single-nucleon current involves

$$
F_{1}-\tau F_{2}-\frac{1}{2} \tau F_{1}=G_{E}\left(1-\frac{1}{2} \tau\right)+\mathcal{O}\left(\tau^{2}\right)
$$

up to terms of $\mathcal{O}(r / c)^{2}$ associated with spinor normalization. The factor $1-\frac{1}{2} \tau$ multiplying $G_{E}$ cancels from the hadronic ratio and yields the result in Eq. (4.14) to $\mathcal{O}\left(\tau^{2}\right)$. In short. the use of $G_{E}$ form factors effectively accounts for all relativistic corrections through order $\tau$. with truncation errors entering only at order $\tau^{2}$. Since $G_{E}^{(s)}$ is proportional to $T$. the error involved in making this approximation is only of $\mathcal{O}(r)$ relative to the leading term. Idditional corrections associated with relativistic many-body dynamics should enter at the same order in $\tau$ or $v / c$. For further discussion, see Ref. [Mus92a].

Within the context of the approximation in Eq. (4.14), one can analyze the errors in a potential determination of $G_{E}^{(s)}$ in terms of the "extended" Galster parameterization of $G_{E}^{(s)}$ (see Eqs. (3.41) and (3.42)) to obtain

$$
\begin{aligned}
\frac{\delta G_{E}^{(s)}}{G_{E}^{n}}=-\left(\frac{G_{D}^{V}}{G_{E}^{n}}\right)[ & \left\{2 \sin ^{2} \theta_{W}\left[1+R_{V}^{T=0}+\Gamma\right]+\left[1+R_{V}^{(0)}\right] \rho_{s} \tau \xi_{E}^{(s)}\right\}\left(\frac{\delta A_{L R}}{A_{L R}}\right) \\
& \left.-2 \sin ^{2} \theta_{W}\left\{\left(\frac{\delta \sin ^{2} \theta_{W}}{\sin ^{2} \theta_{W}}\right)\left[1+R_{V}^{T=0}+\Gamma\right]+\delta R_{V}^{T=0}+\delta \Gamma\right\}\right] .
\end{aligned}
$$

where we have used $G_{E}^{n}$ to set the size scale for $G_{E}^{(s)}$. From this result we observe that the sensituvitue of the form factor $G_{E}^{(s)}$ to $\delta R_{V}^{T=0}, \delta \Gamma$, and $\left(\delta \sin ^{2} \theta_{W} / \sin ^{2} \theta_{W}\right)$ are roughly the same. For $\left|\rho_{s} \tau \xi_{E}^{(s)}\right| \ll 1$, the sensitivity to $\left(\delta A_{L_{R}} / A_{L R}\right)$ is also similar to that of the other uncertainties.

Neglecting for the moment the uncertainties in $\sin ^{2} \theta_{w}, \Gamma$, and $R_{V}^{T=0}$, let us consider only the correlation between $\delta G_{E}^{(s)}$ and $\delta A_{L R}$. Fig. 4.6 shows the statistical uncertainties for elastic scattering from ${ }^{4} \mathrm{He}$ as a function of $\tau$ for different scattering angles under the experimental conditions of $\mathcal{L}=5 \times 10^{38} \mathrm{~cm}^{-2} \mathrm{~s}^{-1}, P_{e}=100 \%, T=1000$ hours, and $\Delta \Omega=0.01$ sr and $\Delta \Omega=0.16 \mathrm{sr}$. The curves in the two cases are similar, although the use of the larger solid angle for $\theta \approx 30^{\circ}$ would permit a slightly higher level of precision than that attainable in the small solid angle regime. The break at $\tau \approx 0.11$ corrnsponds to the diffraction minimum in the elastic form factor of ${ }^{4} \mathrm{He}$. Also shown are the .certainties in $A_{L R}$ required to reach limits on $G_{E}^{(s)}$ of $\left|\delta G_{E}^{(s)}\right| G_{E}^{n} \mid=1.4$ (dashed line $-c$.esponding to that expected from a forward-angle $\vec{e} p$ experiment with $\left.\delta \mu_{\mathrm{g}} \approx 0.22\right)$ and $\left|\delta G_{E}^{(s)} / G_{E}^{n}\right|=0.7$ (dotted line - roughly the best one could expect from $\vec{e} p$ under the circumstances discussed 
in Sect. IV.A). In both cases $\lambda_{\varepsilon}^{\prime}$ has been chosen to be zero (see Sect. III.C). Clearly. there are both low $-Q^{2}(\tau \approx 0.04)$ and somewhat higher $-Q^{2} \mid(\tau \approx 0.2)$ regions where $P V^{\circ}$ elastic scattering from ${ }^{4} \mathrm{He}$ holds the promise of a much better determination of $G_{E}^{(9)}$ than even the best conditions for $\overrightarrow{e p}$ (see below). Similar plots for elastic scattering from ${ }^{12} \mathrm{C}$ are also given in Ref. Mus92a! and similar conclusions can be drawn. In general however. the kinematic ranges over which elastic scattering is preferable to $\overrightarrow{e p}$ are much greater for ${ }^{4} \mathrm{He}$ than for ${ }^{12} \mathrm{C}$ and therefore. other conditions being equal. ${ }^{4} \mathrm{He}$ provides the greater flexibility. This results from the fact that, although the cross section appearing in the FOMI is larger at low- $Q^{2}$ for ${ }^{12} \mathrm{C}$ than for ${ }^{4} \mathrm{He}$ due to the $Z^{2}$ coherence factor in the charge form factor, the ${ }^{4} \mathrm{He}$ cross section falls off less rapidly with $\left|Q^{2}\right|$ (see Figs. 3.10-3.12). Moreover. a larger achi vable luminosity has been assumed for a helium target.

As can be seen from Fig. 4.6, the statistical error in $A_{L R}\left(0^{+} 0\right)$ that must be achieved to match the best $\overrightarrow{e p}$ determination of $G_{E}^{(9)}$ are approximately $5 \%$ at $\tau=0.04$ and $25 \%$ at $T=0.2$. These are to be compared with the other sources of possible uncertainty in $C_{E}^{\prime \prime}$ from Eq. (4.16). The isospin symmetry breaking correction $\Gamma$ is very model-depentint but. for the favorable cases such as ${ }^{4} \mathrm{He}$ and ${ }^{12} \mathrm{C}$. even if $\delta \Gamma \approx \Gamma$. this uncertainty is still unimportant. since $\Gamma \rightarrow 0$ rapidly as $Q^{2} \rightarrow 0$ and should be no larger than 0.01 at the higher values of $\tau$ considered. The error in $\sin ^{2} \theta_{w}$ is currently (see Sect. II.C.I, $\delta \sin ^{2} \theta_{w} / \sin ^{2} \theta_{w} \approx 1-2 \%$ and can be expected to be better than $1 \%$ in the next few years.

Sote that the maximum allowable statistical uncertainty grows with energy for constant $\delta G_{E}^{(s)} / G_{E}^{n}$. Since the weighting of other sources of uncertainty is roughly the same as that of $\delta A_{L R} / A_{L R}$ in their impact on $G_{E}^{(s)}$ in Eq. (4.16), the maximum allowable $\left(\delta A_{L R} / A_{L R}\right)_{s t a t}$ also sets the scale for the maximum allowable uncertainty from other sources. For example, consider an attempt to measure $\rho$, to roughly \pm 0.7 precision. For an experiment performed at $30^{\circ}$ and $\tau=0.04$ (near the first minimum in Fig. $4.6 \mathrm{~b}$. a $1 \%$ error in $\sin ^{2} \theta_{W}$ would be larger than $\left(\delta A_{L R} / A_{L R}\right)^{\text {stat }}$ and would. therefore. rule out a determination of $\rho$, to this precision. However, at $\tau=0.2$ (the second minimum in Fig. 4.6b). the same error in $\sin ^{2} \theta_{W}$ falls well below the maximum allowable statistical error and should not. therefore, be problematic in this case.

A second way to analyze the prospective value of such determinations of $G_{E}^{(s)}$ is to consider the constraints imposed on the $\rho_{\text {. }}$ and $\lambda_{E}^{(s)}$ parameters in the "Galster" parameterization of $G_{E}^{(s)}$ (see Sect. III.C). In Ref. [Mus92a] prospective ${ }^{4} \mathrm{He}$ measurements were analyzed for kinematics corresponding to $\tau=0.04$ and 0.2 at $\theta=30^{\circ}$. Since $G_{E}^{(s)}$ is as yet not known. the authors of Ref. [Mus92a] considered different parameterizations for $G_{E}^{(s)}$ : here we employ two of those, viz., $(A)\left(\lambda_{E}^{(s)}, \rho_{s}\right)=\left(\lambda_{n}, 0\right)$ and $(B)\left(\lambda_{E}^{(s)}, \rho_{s}\right)=\left(\lambda_{n},-2\right)$. where the value of $\rho_{\mathbf{s}}=-2$ corresponds roughly to the average prediction of Ref. [Jaf89]. The results of this analysis are shown in Fig. 4.7. For comparison, the constraints from the $\overrightarrow{e p}$ determination of $G_{E}^{(s)}$ discussed previously are also included. The bands in the figure correspond to the uncertainty in $\rho$, associated with the uncertainty in $A_{L R}$ for given values of $\lambda_{E}^{(3)}$.

As Fig. 4.7 illustrates, it does not appear to be possible to constrain the value of $\lambda_{E}^{(g)}$ in the event that $\rho$, is zero (parameterization $A$ above). Moreover, in this case, the 
lower-energy measurement gives the tightest constraints in the $\left(\lambda_{E}^{(s)}, \rho_{s}\right)$ plane. Fo new information is added by a higher-energy experiment. In contrast. if $\rho_{g} \neq 0$. it is in principle possible to restrict the uncertainty in both $\rho_{s}$ and $\lambda_{E}^{(s)}$ with a combination of ${ }^{4}$ He measurements. In none of these cases. however, would the $\overrightarrow{e p}$ results add significant information to the series of helium measurements. We consider in Sect. IV.F.3 the possibility that a high- $\mid Q^{2}$ QE measurement would contribute additional constraints to those of Fig. $4 . \bar{T}$.

Several other features of these results are worth noting. First, the statistical precision in $A_{L R}$ associated with the lower-energy constraints is roughly one percent isee Ref. [Mus92a]). Since other sources of uncertainty enter into a determination of $\delta G_{E}^{(3)}$ at roughly the same level as $\delta A_{L R} / A_{L R}$, as mentioned above in discussion Eq. $(4.16)$. one might worry about the impact of these as yet unquantified uncertainties - particularly. those associated with dispersion corrections - on the low-energy bands in Fig. 4.7. In contrast. the higher-energy measurement corresponds to $\delta A_{L R} / A_{L R}$ on the order of $10 \%$. so that theoretical uncertainties would only become problematic when they reach this scale.

\section{IV.B.2 STANDARD MODEL TESTS}

In the absence of $s$-quark contributions, radiative corrections, and isospin-mixing. the PV asymmetry of Eq. (4.11) is simply proportional to $\sin ^{2} \theta_{w}$ and historically $\mathrm{PV}$ electron scattering from $0^{+} \mathrm{O}$ nuclei was viewed as a test of the Weinberg-Salam model and a measure of $\sin ^{2} \theta_{w}$ (see, e.g., Refs. [Fei75, Wal77]). Use of such an experiment for a contemporary test of the Standard Model would require a measurement accurate enough to allow determination of $R_{V}^{T=0}$ to within \pm 0.01 . Such a requirement demands a statistical uncertainty in $A_{L R}$ of $\delta A_{L R} / A_{L R} \approx 1 \%$, as well as knowledge of the other terms in Eq. (4.11), $\Gamma(q)$ and $G_{E}^{(s)} / G_{E}^{T=0}$, to the same level of accuracy - we now proceed to consider the latter.

As discissed in Sect. III.D.4, drawing upon the work of Ref. [Don89] we expect $|\Gamma(q)|<0.01$ at least for the lightest $0^{+} 0$ nuclei such as ${ }^{4} \mathrm{He}$ and ${ }^{12} \mathrm{C}$. The correction $\Gamma(q) \rightarrow 0$ as $q \rightarrow 0$, since the isovector charge operator $\hat{\rho}^{T=1} \rightarrow \hat{T}_{3}^{\text {tot }}$ in this limit and since the states are eigenstates of this operator. At moderate values of momentum transfer. however, $\Gamma(q)$ need not be small. In particular, for nuclei heavier than ${ }^{16} \mathrm{O}$ the correction can be several percent; however, for nuclei in the $1 s$ - and $1 p$-shells the correction is somewhat smaller because of the difficulty of sustaining an isovector breathing mode in the relevant nuclear shell model space (see Ref. [Don89]). The most favorable cases for PV electron scattering studies appear to be ${ }^{4} \mathrm{He}$ and ${ }^{12} \mathrm{C}$. Of the two targets. ${ }^{4} \mathrm{He}$ is advantageous from the standpoint of interpretability. The first excited state in ${ }^{4} \mathrm{He}$ lies at $20.1 \mathrm{MeV}$ as compared to $4.44 \mathrm{MeV}$ in ${ }^{12} \mathrm{C}$, thereby introducing less likelihood of contamination from inelastic events. Furthermore, luminosity loss over the length of the target is less problematic for ${ }^{4} \mathrm{He}$ than for ${ }^{12} \mathrm{C}$ (see Sect. V.C). On the other hand, the ${ }^{12} \mathrm{C}$ FOM is larger than that of ${ }^{4} \mathrm{He}$ at very low- $\left|Q^{2}\right|$ due to the larger value of $Z$, thereby increasing the range of energies over which a $1 \%$ Standard. Model test could be performed.

As shown in Fig. 4.5, uncertainty in the strange-quark contribution, even when limited by the "best" determination of $G_{E}^{(s)}$ possible from a $\overrightarrow{e p}$ experiment as discussion in 
Sect. IV.A. renders a determination of $R_{V}^{T=0}$ at the level of $\delta R_{V}^{T=0} \approx \pm 0.01$ impossible. Only in a very limited kinematic range for small $\tau$ can one expect to reduce the uncertainties to the necessary level. As $T \rightarrow 0 . G_{E}^{(s)} \rightarrow 0$ and the significance of the strange-quark term. even if $\delta G_{E}^{(s)} \approx G_{E}^{(s)}$. is reduced. On the other hand, the asymmetry $A_{L R}$ also $\rightarrow 0$ as $Q^{2} \rightarrow 0$ and the statistical uncertainty attainable for given experimental conditions begins to increase at very small $\tau$. For fixed $\lambda_{E}^{(s)}$. it is possible with either the high-or low$Q^{2}{ }^{4} \mathrm{He} G_{E}^{(3)}$ determination to reduce the uncertainty in $\rho_{s}$ to well below what is needed to make a $1 \% \sin ^{2} \theta_{w}$ determination for the kinematic ranges discussed above. Once $\lambda_{E}^{\prime \prime \prime}$ is allowed to vary, however. this statement no longer holds, as discussed in Ref. Mus92a: However. from that work it was concluded that a low- $\left|Q^{2}\right| G_{E}^{(s)}$ determination using ${ }^{4} \mathrm{He}$ could be sufficient to keep the $G_{E}^{(s)}$-induced error below a problematic level for a ${ }^{12} \mathrm{C}$ Standard Model test.

To evaluate the prospect of running a low- $\left|Q^{2}\right|$ experiment. the $(\delta A / A)_{\text {stat }}-$ - relationship for potential ${ }^{12} \mathrm{C}(\vec{e}, e)$ and ${ }^{4} \mathrm{He}(\vec{e}, e)$ experiments under conditions possible at MIT/Bates. Mainz. or CEBAF was analyzed in Ref. [Mus92a]. The resultant curves are shown in Fig. 4.8. In arriving at these plots, luminosities of $\left.\mathcal{L}^{12} \mathrm{C}\right]=1.25 \times 10^{38} \mathrm{~cm}^{-2}, s^{-1}$ and $\mathcal{L}\left[{ }^{4} \mathrm{He}\right]=5 \times 10^{38} \mathrm{~cm}^{-2} \mathrm{~s}^{-1} .100 \%$ beam polarization. and a running time of 1000 hours were assumed, together with two choices of solid angle, $\Delta \Omega=0.01$ and 0.16 sr. The solid curves give the statistical uncertainty $\delta A_{L R} / A_{L R}$ as a function of $\tau$, for different values of $\theta$. The two sets of dashed curves give the $G_{E}^{(s)}$-induced error in $A_{L R}$ for two different values of $\rho_{s} .1 .4$ and 0.7 (where the former corresponds to the "ideal" $\vec{e} p$ limits discussed in Sect. IV.A). In order to perform a precision $(\lesssim 1 \%)$ Standard Model test, both the statistical and $G_{E}^{(s)}$-induced uncertainties must fall below $1 \%$. These requirements thus determine the appropriate kinematics.

From these results, we observe first that although the low $-\left|Q^{2}\right|$ FOM is larger for ${ }^{12} \mathrm{C}$ than for ${ }^{4} \mathrm{He}$, owing to the difference in $Z$, the larger luminosity achievable for ${ }^{4} \mathrm{He}$ compensates for most of this difference, leading to roughly comparable $\left(\delta A_{L R} / A_{L R}\right)_{s t a t}$ in most cases. In the very-forward-angle regime, experiments on these targets would need to be carried out at $\theta \lesssim 15^{\circ}$ and at energies in the 400 to $800 \mathrm{MeV}$ range. The lower bound is set by the requirement that $\left(\delta A_{L_{R}} / A_{L_{R}}\right)_{\mathbf{s t a t}} \leq 1 \%$, while the upper limit is determined by the uncertainty in $\rho_{\mathbf{s}}$. The range of allowable energies, for a given angle, is slightly larger for ${ }^{12} \mathrm{C}$ than for ${ }^{4} \mathrm{He}$. The general characteristics for the intermediate-angle case are similar. Here, the decrease in FOM, relative to very forward angles, is compensated by the larger solid angle. However, lower energies are needed in order to keep the statistical and $\delta A_{L R} / A_{L R}$ error below $1 \%$.

We emphasize that the limits corresponding to $O \rho_{\mathrm{s}}= \pm 1.4$ apply only in the event that a sequence of high precision, forward-and backward-angle $A_{L_{R}}(\vec{e} p)$ measurements are performed. In particular, the $\overrightarrow{e p}$ experiments proposed thus far would not be sufficient to reach this level.

Even if an experimental determination of $R_{V}^{T=0}$ can be accomplished at the required level, one must be able to interpret the experimental value. As discussed in Sect. III.B, 
$K_{V}^{T=0}$ receives contributions from at least three possible sources: one-quark radiative corrections in the Standard Model, non-Standard-.Model physics, and hadronic processes beyond the one-quark terms. The first of these is in principle calculable to arbitrary accuracy given values for the $t$-quark and Higgs masses with a much stronger dependence on the former than the latter. Physics beyond the minimal Standard . Model may be expressed in terms of the $S$ and $T$ parameters described in Sects. II.C and III.B. The relevant contribution here enters roughly as $0.016 S$ so that a determination of $R_{V}^{T=0}$ to $\delta R_{V}^{T=0} \approx \pm 0.01$ would limit the uncertainty in $S$ to $\delta S<0.6$ assuming the other contributions to $R_{V}^{T=0}$ were precisely known. Atomic PV experiments using, e.g., ${ }^{133} \mathrm{Cs}$, show a similar sensitivity to $S$ : current results from atomic $P V$ give $S=-2.7 \pm 2.3$.

Unfortunately, it is not presently clear that one can calculate the hadronic contribution to $R_{V}^{T=0}$ with sufficient accuracy to permit a competitive determination of $S$. The largest such contribution probably arises from the dispersion corrections discussed in Sect. III.B. In particular. since $A_{L R}$ is a ratio of the $Z^{0}$-exchange to the photon-exchange term in the one-boson exchange approximation. it is the difference between two-photon dispersion correction and the photon $-Z^{0}$ dispersion correction that enters $R_{l}^{T=0}$. As discussed in Sect. III.B these two corrections have significantly different analytic structures al.d $Q^{2}$-dependences. Furthermore, analysis of a recent $(e, e)$ experiment on ${ }^{12} \mathrm{C}$ and a comparison of $e^{+}$and $e^{-}$scattering from both ${ }^{12} \mathrm{C}$ and ${ }^{208} \mathrm{~Pb}$ suggest that the two-photon dispersion corrections may enter at the level of a few percent, a significantly larger contribution than previously estimated theoretically. Therefore one clearly needs to develop a greater confidence in the reliability of theoretical calculations of such dispersion corrections before one can expect to study physics beyond the minimal Standard Model.

\section{IV.B.3 DETERIINING THE GROUND-STATE NEUTRON DISTRIBLTION}

In the previous discussion we have relied upon the isospin symmetry of $T=0$ nuclei to select only the isoscalar piece the hadronic current. In the more general case of $. V \neq Z$ (but still spin-0) nuclei, $T \neq$ and consequently both the isoscalar and isovector currents enter. In this case using Eq. (3.59a) one has

$$
-\frac{A_{L R}}{A_{L R}^{0}}=\frac{\tilde{F}_{C 0}}{F_{C 0}}=\frac{\sqrt{3} \xi_{V}^{T=0} F_{C 0}(T=0)+\xi_{V}^{T=1} F_{C 0}(T=1)+\xi_{V}^{(0)} F_{C 0}(s)}{2\left[F_{C 0}(T=0)+F_{C 0}(T=1)\right]} .
$$

For convenience let us define the following sum and difference of the $T=0,1$ monopole form factors:

$$
F_{C 0}( \pm) \equiv F_{C 0}(T=0) \pm F_{C 0}(T=1)
$$

and then Eq. (4.17) may be re-written using Eqs. (3.61)

$$
\begin{aligned}
-2 \frac{A_{L R}}{A_{L R}^{0}} & =\xi_{V}^{p}+\left(\frac{\xi_{V}^{n} F_{C 0}(-)+\xi_{V}^{(0)} F_{C 0}(s)}{F_{C 0}(+)}\right) \\
& \equiv\left(\xi_{V}^{p}+\frac{N}{Z} \xi_{V}^{n}\right)\{1+\tilde{\Gamma}(q)\},
\end{aligned}
$$


where following Ref. [Don89] we have introduced the $q$-dependent quantity

$$
\tilde{\Gamma}(q)=\xi_{i}^{\prime} \cdot\left\{\left[1-\frac{F_{C 0}(-) / N}{F_{C 0}(+) / Z}\right]-\left[\frac{\xi_{v}^{(0)}}{\xi_{V}^{n}} \frac{Z F_{C 0}(s)}{N F_{C 0}(+)}\right]\right\} .
$$

Here

$$
\xi_{V}^{\prime} \equiv-\left[1+\frac{Z \xi_{V}^{p}}{V \xi_{V}^{n}}\right]^{-1}
$$

which only varies slightly with the choice of target $\left(\xi_{V}^{\prime} \approx-1.1\right.$ at tree-level for all $0^{-}$ nuclei). . Lote also that $\xi_{V^{*}}^{(0)} / \xi_{V}^{n}=1$ at tree-level.

If initially we ignore the strangeness form factor and use the definitions of the form factors written in terms of Fourier transforms of ground-state matrix elements of the appropriate density operators (see, for example, Refs. [deF66. Don75. Don79a]), we have (for one-body operators)

$$
\begin{aligned}
& F_{C 0}(+)=\frac{1}{\sqrt{4 \pi}} \int d \vec{x} j_{0}(q x) \rho_{p}(\vec{x}) \\
& F_{C \cdot 0}(-)=\frac{1}{\sqrt{4 \pi}} \int d \vec{x} j_{0}(q x) \rho_{n}(\vec{x}),
\end{aligned}
$$

where $\rho_{p}\left(\rho_{n}\right)$ is the proton (neutron) density normalized to $Z(N)$. Then $\tilde{\Gamma}(q)$ in Eq. $(4.20$ ) becomes

$$
\tilde{\Gamma}(q)=\xi_{V}^{\prime}\left\{1-\frac{\int d \vec{x} j_{0}(q x) \rho_{n}(\vec{x}) / N}{\int d \vec{x} j_{0}(q x) \rho_{p}(\vec{x}) / Z}\right\}
$$

or, equivalently, the PV asymmetry involves

$$
-2 \frac{A_{L R}}{A_{L R}^{0}}=\xi_{V}^{p}+\xi_{V}^{n} \frac{\int d \vec{x} j_{0}(q x) \rho_{n}(\vec{x})}{\int d \vec{x} j_{0}(q x) \rho_{p}(\vec{x})} .
$$

At tree-level. the first term in Eq. (4.24) becomes $\xi_{V}^{p}=\left(1-4 \sin ^{2} \theta_{w}\right) \approx 0.092$, while the coefficient of the second term $\xi_{V}^{n}=-1$ and accordingly the second term is dominant. The Fourier transform of the proton density occurring in the denominator in the second term of Eq. (4.24) is determined by unpolarized electron scattering. Thus, measurement of the $P V$ asymmetry in this case is nearly a direct measurement of the (Fourier transform of the) neutron density.

The ratio of the Fourier transforms of the neutron and proton densities occuring in the dominant term in Eq. (4.24) has some interesting properties. For pure $T=0$ nuclei, $\rho_{n}=\rho_{p}$, this ratio is 1 and Eq. (4.24) yields the simple result $\sqrt{3} \xi_{V}^{T=0}$ discussed above (as expected). For $T=0$ nuclei with small isospin impurities introduced e.g., by Coulomb interactions between protons, $\rho_{n} \approx \rho_{p}$ and deviations of this ratio from unity are related to a nonzero $\Gamma$ term in Eq. (4.11). For $T>0$ (usually $N>Z$ ) nuclei, the ratio deviates from 1 even at $q=0$. In addition, if $\rho_{n}$ and $\rho_{p}$ have different radial dependences, the Fourier transforms of these densities will have different diffraction structure with diffraction 
minima occurring at different $q$. In this case the $\mathrm{PV}$ asymmetry will show rather dramatic deviations from the simple monotonic increase with momentum transfer that obtains for $\rho_{n} / \stackrel{V}{ }=\rho_{p} / Z$. This "beat" phenomenon thus leads to a very sensitive dependence of $A_{L R}$ to differences in the proton and neutron densities.

Including the strangeness contribution in Eq. (4.20) does not significantly alter the conclusions reached here. Instead of $\rho_{n}$ in Eqs. (4.23) and (4.24) it is only necessary to make the replacement

$$
\rho_{n} \longrightarrow\left[\rho_{n}\right]_{\text {eff }} \equiv \rho_{n}+\frac{1}{2}\left\{\rho_{p}+\rho_{n}\right\}\left(\frac{G_{E}^{(s)}}{G_{E}^{T=0}}\right) .
$$

again treating the density operators as one-body operators. For the very small values of momentum transfer where one might attempt to extract the neutron distribution isee below) the second term in Eq. (4.25) is only about a percent or so of the first term. the one that involves the neutron distribution. Of course, were extremely high-precision called for. then the second term could be treated as a correction with $G_{E}^{(s)}$ as a quantity that would have been determined from the other measurements described elsewhere in the present work.

Examples of how sensitive a probe of the ground-state neutron distribution PV elastic electron scattering can be are shown in Fig. 4.9 which is taken from Ref. [Don89]. Despite many years of effort using a variety of experimental probes, detailed knowledge of neutron densities in nuclei remains extremely limited. The authors of Ref. [Don89] have concluded that a measurement of the PV asymmetry for ${ }^{208} \mathrm{~Pb}$ at $q \approx 0.5 \mathrm{fm}^{-1}$ could provide a determination of the radius of the neutron density to an accuracy of $1 \%$; they also conclude that such an experiment is eminently feasible given reasonable beam and detector parameters. Because such experiments are both difficult and costly it is unlikely that $(\vec{e}, \epsilon)$ will ie used in an extensive program to map out neutron densities in a variety of nuclei. Hnwever, it should be possible to use it to provide critical "benchmark" measurements against which to compare both theoretical predictions and other experimental probes of neutron densities. 


\section{IV.C. Elastic Scattering from the Deuteron}

As with elastic scattering from $\left(0^{+} 0\right)$ targets, elastic ${ }^{2} \mathrm{H}(\vec{e}, e)$ scattering serves as an isospin "filter". since the ground state is nominally $T=0$. Thus, as with the $\left(0^{+} 0\right)$ cases discussed in the previous section, one might hope to eliminate some of the uncertainties associated with the multitude of form factors entering $\overrightarrow{e p}$ elastic scattering through the use of a deuterium target. On the other hand. since this nucleus has spin-1. the elastic asymmetry depends on isoscalar magnetic dipole. axial-vector dipole and Coulomb quadrupole form factors as well as the Coulomb monopole form factors entering $A_{L R}\left(0^{+} 0\right)$. For this reason, one or more measurements of $A_{L R}\left({ }^{2} \mathrm{H}\right)$ could nicely complement the experiments discussed in the foregoing sections as a means for further constraining the strangeness form factors. In fact. the isoscalar character of this nucleus enhances the sensitivity of $A_{L R}\left({ }^{2} \mathrm{H}\right)$ to $G_{M}^{(s)}$ at backward angles by roughly $\mu_{p} / \mu^{T=0}$ over the corresponding sensiti. ity of $A_{L R}\left({ }^{1} \mathrm{H}\right)$, while reducing the impact of theoretical uncertainties in the axial-vector contribution. Moreover, uncertainties associated with the nuclear wave function appear to be tolerably small at low $-\left|Q^{2}\right|$. Consequently, one might hope to improve the constraints on $\mu_{\mathrm{s}}$ by a factor of two over the "ideal" constraints with $\overrightarrow{e p}$ elastic (see Eq. (4.4). Elastic scattering from deuterium does present a challenge to experimental resolution. given the small $(2.22 \mathrm{MeV})$ binding energy of the ground state. It appears, however, that an experiment which admits a non-negligible amount of inelastic contribution would not seriously impair the extraction of interesting constraints on $G_{M}^{(s)}$. Furthermore, while no analogous enhancement factor arises in the longitudinal contribution to $A_{L R}\left({ }^{2} \mathrm{H}\right)$, it appears that a moderate-energy, moderately-forward-angle measurement of this asymmetry could permit tighter constraints on $G_{E}^{(s)}$ than are possible with $\overrightarrow{e p}$ elastic scattering alone.

To illustrate the rationale for these conclusions, we return to the expressions for the deuteron asymmetry given in Sect. III.E (see Eq. (3.127)). Let us extend the leading-order result in Eq. (3.128) to the following:

$$
-2 \frac{A_{L R}}{A_{L R}^{0}} \equiv \Delta_{(1)}+\Delta_{(2)}+\Delta_{(3)}
$$

where $\Delta_{(1)}$ gives the tree-level Standard Model contribution in the absence of strangeness, $\Delta_{(2)}$ contains the strangeness contribution to deuteron vector current form factors, and $\Delta_{(3)}$ contains the axial-vector terms. The latter two terms vanish in the absence of strangeness and electroweak corrections. In terms of the quantities introduced in Sect. III. we have

$$
\begin{aligned}
& \Delta_{(1)}=\sqrt{3} \xi_{V}^{T=0} g_{A}^{e}=-4 \sin ^{2} \theta_{w}\left[1+R_{V}^{T=0}+\Gamma\right] \\
& \Delta_{(2)}=\xi_{V}^{(0)} g_{A}^{e}\left[v_{L}(\right.\left.\left.F_{C 0}(T=0) F_{C 0}(s)+F_{C 2}(T=0) F_{C 2}(s)\right)+v_{T} F_{M 1}(T=0) F_{M 1}(s)\right] \\
& \times\left[v_{L}\left(F_{C 0}^{2}(T=0)+F_{C 2}^{2}(T=0)\right)+v_{T} F_{M 1}^{2}(T=0)\right]^{-1} \\
& \Delta_{(3)}=-v_{T^{\prime}} g_{V}^{e} F_{M 1}(T=0)\left[\xi_{A}^{T=0} F_{E 1}^{5}(8)+\xi_{A}^{(0)} F_{E 1}^{5}(s)+\beta F_{E 1}^{5}(A M)\right] \\
& \times\left[v_{L}\left(F_{C 0}^{2}(T=0)+F_{C 2}^{2}(T=0)\right)+v_{T} F_{M 1}^{2}(T=0)\right]^{-1}
\end{aligned}
$$


We have neglected in $\Delta_{(3)}$ a term that is second-order in the nominally small quantities $\xi_{A}^{T=0}$ and $F_{. M 1}(s)$. The term containing $3 F_{E 1_{s}}(A, M)$ is generated by the many-body nuclear anapole moment (see Sect. III.D.j), where $F_{E 1_{s}}(A M)=\left\langle\right.$ g.s. $\left\|\hat{T}_{1}^{\text {el5 }}\right\|$ g.s ${ }^{E . M}$ and 3 is defined in Eq. (3.94). The A.M is one of a number of many-body effects which do not enter in scattering from single nucleons. Nor does it arise in scattering from $\left(0^{+} 0\right)$ targets. since it requires a target having nonzero spin.

The term $\Delta_{(1)}$ is analogous to the leading term in the $\left(0^{+} 0\right)$ asymmetry. It arises from the piece of the isoscalar neutral vector current proportional to $J_{\mu}^{E M}(T=0)$ and is. therefore, nominally independent of nuclear physics. Nuclear corrections enter via both $R_{v}^{T=0}$ (e.g., dispersion corrections) and $\Gamma$, which represents the mixing of $T \neq 0$ continuum states into the deuteron ground state. For purposes of this discussion. we will take the nucleus-independent parts of $\Delta_{(1)}$ to be sufficiently well determined from experiments in uner sectors and focus on $\Delta_{(2)}$ and $\Delta_{(3)}$. In what follows, we let $\Delta_{(2)}^{E}$ and $\Delta_{(2)}^{M}$ denote the contributions to $\Delta_{(2)}$ from $G_{E}^{(s)}$ and $G_{M}^{(9)}$. respectively.

After casting the results of Ref. [Pol90] in the formalism outlined in Sect. III. we obtain the following impulse approximation (IA) expressions for the deuteron form factors:

$$
\begin{aligned}
\sqrt{4 \pi} F_{C 0}(T=0) & =\sqrt{3\left(1+\tau_{\mathrm{d}}\right)}\left\{G_{E}^{T=0}\left[\left(1-\frac{2}{3} \tau_{\mathrm{d}}\right) D_{C}+\frac{2}{3} \tau_{\mathrm{d}} D_{M}^{E}+\frac{4}{3} \tau_{\mathrm{d}}^{2} D_{Q}\right]+\frac{2}{3} \tau_{\mathrm{d}} G_{M}^{T=0} D_{M}^{M}\right\} \\
\sqrt{4 \pi} F_{C 2}(T=0) & =\sqrt{2\left(1+\tau_{\mathrm{d}}\right)} \tau_{\mathrm{d}}\left\{G_{E}^{T=0}\left[\left(1+\frac{2}{3} \tau_{\mathrm{d}}\right) D_{Q}-D_{C}+D_{M}^{E}\right]+G_{M}^{T=0} D_{M}^{M}\right\} \\
\sqrt{4 \pi} F_{M 1}(T=0) & =-\frac{q}{M_{D}} \sqrt{1+\tau_{\mathrm{d}}}\left(G_{E}^{T=0} D_{M}^{E}+G_{M}^{T=0} D_{M}^{M}\right) \\
\sqrt{4 \pi} F_{E_{15}} & =-\tilde{G}_{A}^{T=0} D_{M}^{M},
\end{aligned}
$$

where $\tau_{\mathrm{d}} \equiv\left|Q^{2}\right| / 4 M_{D}^{2}$ and where $D_{C}, D_{Q}, D_{M}^{M}, D_{M}^{E}$ and $D_{A}$ are integrals dependent on the $\mathrm{S}$ - and $\mathrm{D}$-state components of the deuteron wave function defined in Ref. [Pol90]. The vector $\mathcal{X}$ form factors $\tilde{F}_{C 0}, \tilde{F}_{C_{2}}$, and $\tilde{F}_{M_{1}}$ have identical forms to the E.M form factors in Eq. (4.28) with the replacements $G_{E}^{T=0} \rightarrow \tilde{G}_{E}^{T=0}$ and $G_{M}^{T=0} \rightarrow \tilde{G}_{M}^{T=0}$. Making use of these formulae, we now consider $\Delta_{(2,3)}$ at forward and backward angles. Results for other kinematic regimes may be found in Ref. [Pol90].

\section{C.: D.ACKWARD-ANGLE SCATTERING}

Let us begin by considering backward-angle PV electron scattering. Using Eqs. (4.27) and $(4.28)$ and the fact that $v_{L} / v_{T} \ll 1$ as $\theta \rightarrow 180^{\circ}$ we have

$$
\Delta_{(2)} \rightarrow-\left\{\frac{G_{M}^{(s)}}{G_{M}^{T=0}}+\lambda \frac{G_{E}^{(s)}}{G_{M}^{T=0}}\right\}\left[1-\delta_{N U C}+R_{V}^{(0)}\right]
$$

where $\lambda \equiv D_{M}^{E} / D_{M}^{M}$, and

$$
\delta_{N U C} \equiv \frac{\lambda\left(G_{E}^{T=0} / G_{M}^{T=0}\right)}{1+\lambda\left(G_{E}^{T=0} / G_{M}^{T=0}\right)} \approx \lambda\left(G_{E}^{T=0} / G_{M}^{T=0}\right)
$$


The terms in Eqs. (4.29) and (4.30) arise purely from the ratio of magnetic deuteron form factors. $F_{M_{1}}(s) / F_{M_{1}}(T=0)$. Contributions from the Coulomb form factors are highly suppressed by $v_{L} / v_{T}$ at backward angles. The presence of the nucleon form factors $G_{E}^{T=0}$ and $G_{E}^{(s)}$ in the deuteron magnetic form factors arises from magnetic projections of the convection part of the one-body vector current:

$$
\vec{J}(q)_{\text {conv }}=G_{E}\left(q^{2}\right) \frac{\left(\vec{p}+\vec{p}^{\prime}\right)}{2 m_{N}}
$$

and similarly for the strange-quark vector current. Since the contribution of Eq. 14.31 , to the magnetization, $\vec{m}(\vec{r})=\frac{1}{2}(\vec{r} \times \vec{J})$, is proportional to the orbital angular momentum operator. contributions containing $G_{E}^{T=0}$ and $G_{E}^{(s)}$ to $F_{M_{1}}(T=0)$ and $F_{M_{1}}(s)$ vanish for a pure S-wave. Hence, the contributions involving $G_{E}$ are weighted by the integrai $D_{M}^{E}$, which depends only on the $\mathrm{D}$-state component of the deuteron wave function. The correction factors $\delta_{\mathrm{N} v \mathrm{C}}$ and $\lambda$ thus vanish in the limit that $\mathrm{D}$-wave components of the deuteron are neglected.

Simple estimates for the scale of terms in Eqs. (4.29) and (4.30) may be obtained by considering sufficiently small values of $q$ such that terms of order $(q r / 2)^{2}$ and higher in the Bessel functions appearing in the $D$-integrals are negligible. Since the percent of $D$-wave in the deuteron is generally considered to be $\leq 10 \%$ of the total, we have that the ratio $D_{M}^{E} / D_{M}^{M}$ is approximately

$$
\lambda=\frac{D_{M}^{E}}{D_{M}^{M}} \approx\left(\frac{3}{4}\right) \frac{\% \mathrm{D} \text {-wave }}{\% \text { S-wave }} \lessgtr 0.08 .
$$

. Woreover, at $\left|Q^{2}\right|=0$, one has $G_{E}^{T=0}(0)=\frac{1}{2}$ and $G_{M}^{T=0}(0)=\frac{1}{2}\left(\mu_{p}+\mu_{n}\right) \approx 0.44$. Hence. at $\left|Q^{2}\right|=0$,

$$
\delta_{N U C} \approx\left(\frac{G_{E}^{T=0}}{G_{M}^{T=0}}\right)\left(\frac{D_{M}^{E}}{D_{M}^{M}}\right) \lessgtr 0.1
$$

The scale of $\Delta_{(2)}^{M}\left(\theta \rightarrow 180^{\circ}\right)_{I A}$ is thus given by $\mu_{s} / \mu^{T=0}$ with a small $(\leq 10 \%)$ nuclear physics correction. The model-dependence of this correction has been analyzed in Ref. [Pol90], using nonrelativistic deuteron wave functions. For example, the quantity $\delta_{N V C}$ does depend on momentum transfer, running from $\approx 0.05$ at $\left|Q^{2}\right|=0$ to $\approx 0.2$ at $\left|Q^{2}\right|=0.5(\mathrm{GeV} / \mathrm{c})^{2}$. The latter number is quite dependent on the details of the deuteron wave function. The Bonn potential yields a value of 0.13 , while the Paris potential gives 0.23 , and Reid soft core 0.29 . (None of these nonrelativistic models should be considered to be totally reliable at momentum transfers much above this scale, in any case.) At low$\left|Q^{2}\right|$, however, $\delta_{N U C}$ is constrained to the value in Eq. (4.33) by static properties of the deuteron, primarily the ratio of $\mathrm{D}$ - to $\mathrm{S}$-wave content.

The result of Eq. (4.29) has two notable features. First, the presence of $\mu^{T=0}$ rather than $\mu_{p}$ in the denominator of $\Delta_{(2)}^{M}$ enhances the backward-angle $\mu_{s}$ signal by roughly a factor of six over the corresponding signal in $A_{L R}(\vec{e} p)$. Second, the small magnitude of the nuclear physics correction to $A_{L R}\left(\theta \rightarrow 180^{\circ}\right)_{I A}$ implies that theoretical uncertainties 
in the deuteron wave function should not introduce serious theoretical ambiguities into the interpretation of a backward-angle measurement. As discussed above. for $\left|Q^{2}\right|<0.1$ $(\mathrm{GeV} / \mathrm{c})^{2} . \delta_{\mathrm{v} \cup \mathrm{C}} \leqslant 0.1 \mathrm{in}$ all deuteron models considered, with an uncertainty of less than +0.03 .

From Eqs. (4.29) and (4.30) we also observe that the backward-angle strangeness radius signal is suppressed with respect to the $\mu$, term by the same factor which minimizes the nuclear physics correction in $\Delta_{(2)}^{M}$. Specifically.

$$
\left.\frac{\Delta_{(2)}^{E}}{\Delta_{(2)}^{M}}\right|_{\theta-180^{\circ}} ^{t A} \approx \frac{\tau \rho_{s}}{\mu_{s}} \lambda \approx 0.56 \tau
$$

for small $\mid Q^{2}$ and using the values for $\mu_{\text {s }}$ and $\rho$, from Ref. [Jaf89]. If these estimates are realistic, one would need to go to extremely large values of $\left|Q^{2}\right|$ in order for $\Delta_{(2)}^{E}$ to compete with $\Delta_{(2)}^{M}$ at backward angles. Consequently. unless the ratio $\left|\rho_{\mathbf{g}} / \mu_{\mathbf{g}}\right|$ is much larger than the prediction of Ref. [Jaf89], a nonzero value of the strangeness radius should pose no serious difficulty for the extraction of a value of $\mu$, from low $-\left|Q^{2}\right|, \theta \rightarrow 180^{\circ}$ measurements.

Following a similar line of reasoning as above, we arrive at the following low- $\left|Q^{2}\right|$, backward-angle signal from the axial-vector term of $\Delta_{(3)}$ :

$$
\begin{aligned}
\Delta_{(3)}\left(\theta \rightarrow 180^{\circ}\right)_{I A} \approx & g_{v}^{e}\left(\frac{v_{T^{\prime}}}{v_{T}}\right) \frac{M_{D}}{q} \sqrt{1+\tau_{\mathrm{d}}} \\
& \times\left\{\frac{\tilde{G}_{A}^{T=0}}{G_{M}^{T=0}}-\frac{Q_{e}}{g_{V}^{e}}\left[\frac{16 \pi \alpha}{\sqrt{3} G_{\mu} m_{V}^{2}}\right] \frac{\langle D\|\hat{a}\| D\rangle}{G_{M}^{T=0} D_{. M}^{M}}\right\}\left[1-\delta_{N v^{\prime} C}\right] .
\end{aligned}
$$

where $\langle D\|\hat{a}\| D\rangle$ is the elastic matrix element of the anapole operator defined in Eq. (3.9T)

It is interesting to compare the relative sensitivities of $A_{L R}\left({ }^{1} \mathrm{H}\right)$ and $A_{L R}\left({ }^{2} \mathrm{H}\right)$ to the dominant sources of uncertainty. In the latter case, one has

$$
\frac{\delta A_{L R}}{A_{L R}} \approx 2 \delta \mu_{s}+\frac{1}{5} \frac{M_{D}}{q} \delta \tilde{G}_{A}^{T=0},
$$

which. for an experiment performed at the SA.MPLE kinematics $(q \approx 300 \mathrm{MeV} / \mathrm{c})$ leads to

$$
\frac{\delta A_{L R}}{A_{L R}} \approx 2 \delta \mu_{s}+\frac{6}{5} \delta \tilde{G}_{A}^{r=0} .
$$

Comparing this result with Eq. (4.3), one observes that the elastic deuteron asymmetry is much more sensitive to $\mu_{\mathrm{s}}$ than is $A_{L R}\left({ }^{1} \mathrm{H}\right)$. Moreover, although $\mu_{\mathrm{g}}$ and uncertainties in the axial-vector form factors are correlated at the same level in both cases, one has that $\delta \tilde{G}_{A}^{T=0} \ll \delta \tilde{G}_{A}^{T=1}$ from the effects of radiative corrections. Thus, in contrast to the situation with $\vec{e} p$ scattering, the impact of axial-vector radiative correction uncertainties on a determination of $\mu_{\text {s }}$ with $\vec{e} D$ scattering appears to be less problematic. 
To ascertain the potential constraints on $\mu$, one might achieve with a measurement of $A_{L R}\left({ }^{2} \mathrm{H}\right)$, we show in Fig. 4.10 the fractional statistical uncertainty in the asymmetry as a function of energy for fixed scattering angle, assuming reasonable experimental conditions isee figure caption). On the same plot we give the induced uncertainty from $\mu$, associated with the "ideal" SA.MPLE determination of this parameter. From these curves it is apparent that one might hope to improve upon the best case S.A.MPLE results by a factor of two by performing a large--solid-angle elastic $\vec{e} D$ experiment at $\epsilon \approx 200 \mathrm{Mel}^{*}$.

Such an experiment. of course. would have to confront the issue of resolution. For a detector like S.A.MPLE. it is not possible to resolve the ground state and consequently the asymmetry would receive contributions from excitation of continuum states isee Ref. [Hwa86] for a discussion of $A_{L R}\left({ }^{2} \mathrm{H}\right)$ near threshold). In Ref. [Mus92a] the impact of contributions from excitation of the first continuum state $\left({ }^{1} \mathrm{~S}_{0}\right)$ on a determination of $\mu$. was analyzed. A simple estimate for $q=300 \mathrm{MeV} / \mathrm{c}$ leads to

$$
\delta \mu_{9} \approx \frac{3}{20} \frac{f^{\text {inel }}}{f^{\mathrm{el}}}\left[\delta R_{A}^{T=1}+\delta \Delta_{M E C}\right] .
$$

where $f^{e l}\left(f^{\text {inel }}\right)$ is the fraction of the total cross section arising from elastic (inelastic) scattering, $\delta R_{A}^{T=1}$ is the theoretical uncertainty in the isovector axial-vector radiative corrections, and $\delta \Delta_{M E C}$ is a correction due to meson-exchange currents. Using the estimate of $\delta R_{A}^{T=1}= \pm 0.2$ from Ref. [Mus90] one has $\delta \mu_{\mathrm{s}} \approx 0.03\left(f^{\text {inel }} / f^{\text {el }}\right)$ from radiative correction uncertainties. One expects uncertainties in the meson-exchange correction to contribute at a similar, if not smaller, level. To leading order in $v / c$, the latter contribute only to the magnetic transition matrix element appearing in the denominator of the asymmetry. This contribution. dominated by $\pi$-exchange at low momentum transfer, is well constrained by experimental knowledge of thresiold deuteron electrodisintegration [Are82, Auf85. Beri1. Gan 72 , Hoc 73 , van91]. Hence, to the extent that one can experimentally restrict inelastic contributions to those arising from the isovector transition to the first continuum state. the inclusion of inelasticities should not seriously weaken the $\mu$, constraints attainable from purely elastic $\bar{e} D$ scattering.

\section{IV.C.2 FORWARD-A.NGLE SCATTERING}

In the extreme forward direction, the axial-vector term $\Delta_{(3)}$ vanishes since $v^{\prime} T^{\prime} \propto$ $\tan \theta / 2$. Thus, we need only consider $\Delta_{(2)}^{M}$ and $\Delta_{(2)}^{E}$ in this kinematical regime. In contrast to the backward-angle limit, where $v_{T} \gg v_{L}$, the $\theta \rightarrow 0^{\circ}$ vector current terms receive contributions from both the charge and magnetic form factors. In this limit. using similar arguments as employed previously to estimate the scale of the wave function integrals, one has from Eqs. (4.27) and (4.28) the following IA expressions for $\Delta_{(2)}^{M}$ and $\Delta_{(2)}^{E}$ :

$$
\begin{aligned}
& \Delta_{(2)}^{M}\left(\theta \rightarrow 0^{\circ}\right)^{I A} \approx-2 \frac{G_{M}^{(s)} G_{M}^{T=0}}{\left(G_{E}^{T=0}\right)^{2}} \tau_{\mathrm{d}} \\
& \Delta_{(2)}^{E}\left(\theta \rightarrow 0^{\circ}\right)^{I \wedge} \approx-\frac{G_{E}^{(s)}}{G_{E}^{T=0}} .
\end{aligned}
$$


In arriving at Eqs. (4.39), we have omitted terms of $\mathcal{O}\left(\tau_{\mathrm{d}}\right)$ as well as those involving the $D_{Q}$ integral. Since this integral, which depends only on the $D$-state component of the wave function. vanishes as $Q^{2}$, it should be negligible in comparison with $D_{C} . D_{M}^{M}$, and $D_{M}^{E}$ at small values of momentum transfer. We have also neglected small additional nuclear physics corrections $\sim \delta_{\mathrm{v} u^{\mathrm{C}} \mathrm{C}}$.

Comparing Eqs. (4.39) and (4.29), we observe that $\Delta_{(2)}^{M}\left(\theta \rightarrow 0^{0}\right)^{t A}$ is suppressed with respect to the corresponding backward-angle limit by roughly $0.4\left(q / . I_{D}\right)^{2}$. On the other hand. $\Delta_{(2)}^{E}$, which is suppressed with respect to the ratio $G_{E}^{(s)} / G_{E}^{T=0}$ by $\lambda \approx 0.1$ at backward angles, now differs from $G_{E}^{(s)} / G_{E}^{r=0}$ by only a small. $Q^{2}$-dependent nuclear physics correction. Thus, one expects the $\rho$, signal as $\theta \rightarrow 0^{\circ}$ to be relatively much stronger

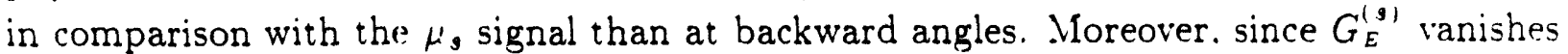
as $Q^{2}$ for small momentum transfer. the ratio $\Delta_{(2)}^{E} / \Delta_{(2)}^{K}$ should be largely independent of momentum transfer in the low- $\left|Q^{2}\right|$ limit. In particular, neglecting the small nuclear physics corrections and taking $M_{D} \approx 2 m_{N}$, we have

$$
\frac{\Delta_{(2)}^{E}\left(\theta \rightarrow 0^{\circ}\right)^{I A}}{\Delta_{(2)}^{M}\left(\theta \rightarrow 0^{\circ}\right)^{I A}} \approx 2 \frac{\rho_{s}}{\mu_{s}} \frac{G_{E}^{T=0}}{G_{M}^{\tau=0}} \approx 15
$$

using the estimates of Ref. [Jaf89]. Thus, the strangeness radius signal at forward angles could be relatively significant.

To estimate the constraints that a forward-angle $A_{L R}\left({ }^{2} \mathrm{H}\right)$ measurement could place on $\rho_{\text {s, }}$, we have considered a 1000 hour measurement at $\theta=30^{\circ}$, assuming a luminosity of $\mathcal{L}=5 \times 10^{38} \mathrm{~cm}^{-2} \mathrm{~s}^{-1}$, solid angle $\Delta \Omega=0.16 \mathrm{sr}$, and beam polarization $P_{\mathrm{e}}=100 \%$. Inder these conditions, the most stringent constraints on $\rho_{\text {s }}$ would be obtained at energy $\epsilon \approx 800$ $\mathrm{MeV}$. for which the statistical uncertainty would be roughly $2 \%$. The resulting uncertainty on $\rho$, would be about a factor of five smaller than is achievable with an "ideal" series of $f_{i n}(\vec{e} p)$ measurements and comparable to the constraints attainable from a measurement of $\left.f_{z: .}{ }^{4} \mathrm{He}\right)$ at $\mathrm{i}\left(\mathrm{v}-\left|Q^{2}\right|\right.$. The technical feasibility and impact of inelastic contributions remain to be analyzed.

In summary, the use of $\vec{e} D$ scattering as an additional constraint on the nucleon strangeness form factors has several advantageous features: enhanced sensitivity due to the nucleon's small isoscalar magnetic moment and its small axial-vector isoscalar form factor. weaker sensitivity to axial-vector radiative corrections than in the free proton case, and the possibility of a kinematic separation of $\mu$, effects from $\rho_{\text {s }}$ effects. Moreover, the impact of nuclear wave function uncertainties appears to be negligible at low $-\left|Q^{2}\right|$. Finally, in the case of backward-angle scattering, it appears that an experiment could integrate into some of the inelastic region without seriously affecting a determination of $\mu_{s}$. These features suggest that further theoretical study of contributions from the anapole moment. dispersion corrections, and relativistic effects as well as experimental analysis of technical feasibility appear warranted. 


\section{IV.D. Other Discrete Nuclear Transitions}

In this section we consider a few examples of $\mathrm{PV}$ electron scattering involving discrete nuclear transitions other than those discussed above. The treatment presented here is not meant to be comprehensive: while the procedures followed are straightforward extensions of the formalism applied above to elastic scattering from spin-0 systems (see Sect. IV.B). it is generally necessary to deal with several multipole matrix elements when considering transitions between nuclear states which have non-trivial angular momenta and this significantly complicates the analysis. On the one hand. the atility to select specific transitions with good quantum numbers for the initial and final nuclear states can. in principle. be an advantage for then the focus can be placed on particular pieces of the electroweak current. On the other hand, given the proliferation of different electroweak matrix elements that can occur in general, the description of the asymmetry now usually becomes more dependent on details of the nuclear modeling than is the case for the very special monopole situation that occurs for elastic scattering from spin-0 nuclei. Both of these aspects of the problem will be illustrated in the discussions to follow using specific nuclear transitions.

It is clear that under certain circumstances the PV asymmetry may be quite sensitive to the single-nucleon content and therefore it would appear that choosing the transition and kinematics judiciously might provide a means to explore, for instance. the nucleon's strangeness form factors (given that the nuclear many-body uncertainties are not too large). However. as discussed in detail below, this is usually not the case. The reason is not one of principle, but of practice: for most discrete nuclear transitions of interest other than elastic scattering, which is coherent and so has a large cross section, the figure-ofmerit introduced in Sect. III.E is very small. As a consequence, unfortunately it is rather unlikely that high-precision determinations of the PV asymmetry can be contemplated for most discrete nuclear transitions in the foreseeable future.

\section{IV.D.1 ILLLSTRATIVE EXAMPLES OF TRANSITIONS IN $. \mathrm{N}=Z$ NCCLEI}

To make the treatment reasonably tractable in this subsection let us restrict our attention to $. V=Z$ nuclei with ground states having angular momentum and isospin 0 with positive parity. The discrete excited states will be assumed to have good quantum numbers $J^{\pi} T$ and accordingly specific transitions may be classified in the following ways: first, the transitions are either isoscalar $(T=0)$ or isovector $(T=1)$. Secondly, they fall into two classes, natural parity transitions where $\pi=(-)^{J}$ :

$$
\begin{aligned}
F_{L}^{2} & =F_{C J}^{2} \\
F_{T}^{2} & =F_{E J}^{2} \\
W_{A V}^{L} & =-F_{C J} \tilde{F}_{C J} \\
W_{A V}^{T} & =-F_{E J} \tilde{F}_{E J} \\
W_{V A}^{T^{\prime}} & =\left(1-4 \sin ^{2} \theta_{W}\right) F_{E J} \tilde{F}_{M J_{3}}
\end{aligned}
$$


and non-natural parity transitions where $\pi=(-)^{J+1}$ and one has

$$
\begin{aligned}
F_{L}^{2} & =0 \\
F_{T}^{2} & =F_{M J}^{2} \\
W_{A V}^{L} & =0 \\
W_{A V}^{T} & =-F_{M J} \tilde{F}_{. M J} \\
W_{V_{A}^{\prime}}^{T^{\prime}} & =\left(1-4 \sin ^{2} \theta_{W}\right) F_{M J} \tilde{F}_{E J_{3}} .
\end{aligned}
$$

using Eqs. (3.108) and (3.114) in Sect. III.E.1. Clearly the special case of elastic scattering from spin-0 systems discussed in Sect. IV.B is contained in Eq. (4.41a), i.e.. when only Coulomb monopole form factors are retained: $F_{L}^{2}=F_{C 0}^{2} . W_{A \mathrm{~L}}^{-L}=-F_{C 0} \dot{F}_{C 0}$ and $F_{\bar{T}}^{2}=$ $W_{A V}^{T}=W_{V A}^{T^{\prime}}=0$. In the discussions to follow we shall compare the figures-of-merit for a few selected transitions with that for elastic scattering from spin-0 nuclei and hence it is appropriate to re-state the result for the latter (see also Sect. IV.B):

$$
\mathcal{F}=\mathcal{F}_{0} \frac{\mathcal{E}}{1-\mathcal{E}} 2\left|Q^{2} / q^{2}\right| \tilde{F}_{C 0}^{2}
$$

The general behavior of the FOM has been discussed in Sect. III.E.2. There elastic scattering from the proton. ${ }^{4} \mathrm{He}$ and ${ }^{12} \mathrm{C}$ was compared with three particular representative inelastic excitations in ${ }^{12} \mathrm{C}$. Let us now examine the asymmetries and figures-of-merit for these illustrative examples in somewhat more detail to bring out the reasons why the specific results shown in Figs. 3.10-3.12 are obtained.

\section{.on-..Yatural Parity Transitions}

Let us start with non-natural parity transitions, since this case is somewhat simpler than the natural parity case as no Coulomb matrix elements occur. The cross section is proportional to the square of the magnetic form factor $\left(\propto F_{M J}^{2}\right)$, whereas from Eq. (3.11i) the asymmetry is

$$
\frac{A_{L R}}{A_{L R}^{0}}=-\left[\tilde{F}_{M J}-\sqrt{1-\mathcal{E}^{2}}\left(1-4 \sin ^{2} \theta_{W}\right) \tilde{F}_{E J_{B}}\right] / F_{M J} .
$$

Since in general $F_{M J}$ and $\tilde{F}_{M J}$ have different $q$-dependences and hence different locations of their diffraction minima, it is clear that the asymmetry can become quite large near a minimum in $F_{M J}$. While that may seem at first sight to be advantageous for studying PV electron scattering, it should be remembered that the FOM characterizes the precision with which the asymmetry can be determined. Equation (3.140) yields

$$
\mathcal{F}=\frac{\mathcal{F}_{0}}{1-\overline{\mathcal{E}}}\left[\check{F}_{M J}-\sqrt{1-\mathcal{E}^{2}}\left(1-4 \sin ^{2} \theta_{W}\right) \check{F}_{E J_{B}}\right]^{2},
$$

which does nothing special as one passes through the diffraction minima of $F_{M J}$. Said in other words: it does no good to have a large asymmetry but a small cross section if 
they conspire as they do here to yield only a typical small figure-of-merit ( see below 1 , for then using Eq. (3.139) the fractional uncertainty in the asymmetry is large. As we shall see below, under typical conditions the asymmetry itself is not sufficiently sensitive to. for example. details of the strangeness or axial-vector single-nucleon form factors to overcome this large fractional uncertainty.

As particular examples. let us consider further the $J=1 . T=0.1$ cases shown in Figs. 3.10-3.12 and use the following to characterize the form factors that enter: $\left.F_{M 1^{\prime}} T\right) \equiv$ $-(q / M) G_{M}^{T} K_{M 1}, \dot{F}_{M 1}(T) \equiv-(q / M) \tilde{G}_{M}^{T} \tilde{K}_{M 1}$ and $\dot{F}_{E_{1 s}}(T) \equiv-2 \tilde{G}_{A}^{T} \tilde{K}_{E 1_{3}}$. where we have isolated the overall dependences on $q$ which must occur. as well as specific choices for the dependences on the single-nucleon form factors (see below). Isospin-mixing is ignorer in this section. $K_{M_{1}}, \tilde{K}_{M_{1}}$ and $\tilde{K}_{E_{1}}$, contain all of the remaining $q$-dependences: all may be nonzero at low momentum transfer. The normalizations are chosen here in order that for specific spin-flip-dominated cases. where the convection current contributions are unimportant, one has $K_{M 1}=\tilde{K}_{M 1}=\check{K}_{E_{1}}$. Specifically, for the excitation of the $1^{-1}$ state at tree level we have from Eq. (4.44)

$$
\mathcal{F}=\frac{\mathcal{F}_{0}}{1-\mathcal{E}}\left[0.546 F_{M_{1}}\right]^{2} \times\left(1+\sqrt{1-\mathcal{E}^{2}}\left\{\frac{85 . \mathrm{MeV} / \mathrm{c}}{q}\right\}\left[\frac{G_{D}^{A}}{G_{D}^{+}}\right]\left[\frac{\check{K}_{E 1_{s}}}{K_{M 1}}\right)^{2}\right.
$$

Taking $\check{K}_{E l_{s}}=K_{M_{1}}$ for this spin-flip-dominated transition (which is borne out by more detailed analysis: see Ref. (Don 79 a) ) together with the minimum fractional uncertainties deduced from Figs. 3.10-3.12 we obtain the following for the fractional uncertainties of the axial-vector contributions to the asymmetry: $300 \%\left(10^{\circ}\right), 320 \%\left(30^{\circ}\right)$ and $465 \%, 150^{\circ}$ Clearly some dramatic (unanticipated) improvement in experimental do-ability would have to occur before interesting information concerning the isovector axial-vector form factor could be extracted.

Similar conclusions are reached for the excitation of the $1^{+} 0$ state in ${ }^{12} \mathrm{C}$. Treating this as an eigenstate of isospin (which is known not to be the case: see Refs. [Doni9a. Flai9] where isospin-mixing is discussed), we have from Eq. (4.44)

$$
\mathcal{F}=\frac{\mathcal{F}_{0}}{1-\mathcal{E}}\left[F_{M 1}\right]^{2} \times \frac{1}{2}\left(\left[\frac{\tilde{G}_{M}^{T=0}}{G_{M}^{T=0}}\right]\left[\frac{\tilde{K}_{M 1}}{K_{M 1}}\right]-\sqrt{1-\mathcal{E}^{2}}\left\{\frac{173 \mathrm{MeV} / \mathrm{c}}{q}\right\}\left[\frac{\dot{G}_{A}^{T=0}}{G_{M}^{T=01}}\right]\left[\frac{\check{K}_{E 1 s}}{K_{M 1}}\right]\right)^{2}
$$

For simplicity let us assume that this transition is also spin-flip-dominated (this is not as good an approximation as it is for the excitation of the $15.11 \mathrm{MeV}$ level in ${ }^{12} \mathrm{C}$. as discussed in Ref. [Don79a]; however it is adequate for the present purposes where we merely wish to set the general scale of the problem), in which case the ratios of $K$ 's are both unity. Furthermore, if we for example set the vector strangeness form factor of the nucleon to zero and take the EMC value for the axial-vector strangeness form factor (see Eq. (3.47)), we also have $\tilde{G}_{M}^{T=0} / G_{M}^{T=0}=-0.918$ and $\tilde{G}_{A}^{T=0}(0) / G_{M}^{T=0}(0)=-0.441 \pm 0.070 \pm$ 0.105 . We then obtain the following fractional uncertainties in the latter when the $1^{+0} 0$ figures-of-merit at their first (second) maxima are employed: $3070 \%(3245 \%)$ for $10^{\circ}$. $3240 \%(3365 \%)$ for $30^{\circ}$ and $4705 \%(4734 \%)$ for $150^{\circ}$. If instead we ignore the axial-vector contribution (which can be made small by working in the forward direction) and allow for a 
nonzero magnetic strangeness contribution, then we have $\tilde{G}_{. M}^{T=0} / G_{. M}^{T=0}=-0.918(1+2.5 \mu$, using our parameterization of $G_{M}^{(g)}$. The fractional uncertainty in $\mu_{\mathrm{s}}$ is $\left|\delta A_{L R} / A_{L R}\right| / \mu_{\mathrm{s}}$. implying from the numbers given above that the magnetic strangeness could only be defined to several $\times 100 \%$ using the $1^{+} 0$ state in ${ }^{12} \mathrm{C}$ which is not competitive with other cases discussed in Sects. IV.A-C. Clearly no useful information is likely to be forthcoming from studies of this transition.

\section{Natural Parity Transitions}

The natural parity class of transitions is somewhat more complicated. In this case the PC cross section (Eq. (3.116)) involves both Coulomb and electric form factors i $x$ $\left.2\left|Q^{2} / q^{2}\right| \mathcal{E} F_{C J}^{2}+F_{E J}^{2}\right)$ and the hadronic ratio is given by

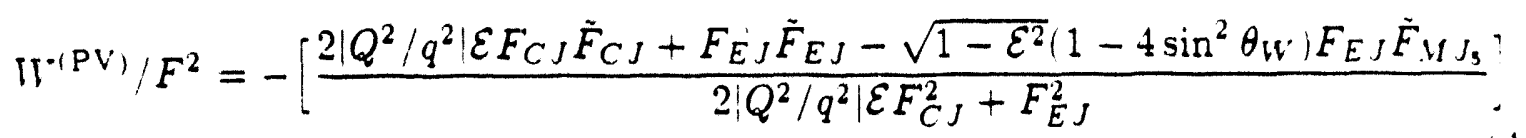

Using Eq. (3.140) the FOM can be written in the following way:

$$
\mathcal{F}=\frac{\mathcal{F}_{0}}{1-\mathcal{E}} \frac{\left[2\left|Q^{2} / q^{2}\right| \mathcal{E} \tilde{F}_{C I}+\gamma_{J}\left[\tilde{F}_{E J}-\sqrt{1-\mathcal{E}^{2}}\left(1-4 \sin ^{2} \theta_{W}\right) \tilde{F}_{M J_{s}}\right]\right]^{2}}{2\left|Q^{2} / q^{2}\right| \mathcal{E}+\gamma_{J}^{2}} .
$$

where for convenience we have defined $\gamma_{J} \equiv F_{E J} / F_{C J}$. In the long wavelength limit $(q \rightarrow 0)$ the Coulomb and electric multipole matrix elements are related by the continuity equation [deF66], which implies that $\gamma_{J} \rightarrow-\sqrt{(J+1) / J}(\omega / q)$ in this limit. For example. in Figs. 3.10-3.12 the figure-of-merit is given for the $2^{+} 0$ state at $4.44 . \mathrm{MeV}^{*}$ in ${ }^{12} \mathrm{C}$. The peak value of $\mathcal{F}$ occurs at $q \approx 265 \mathrm{MeV} / \mathrm{c}$ which yields $\left|\gamma_{2}\right| \approx 2.0 \times 10^{-2}$ using the above approximation. Experiment [Fla78] gives $3.9 \times 10^{-2}$, implying that the long wavelength limit, while not precisely correct at such values of momentum transfer, is in fact a reasonable measure of the relative importance of Coulomb and transverse multipole contributions. In either case, at least for this specific transition. $\left|\gamma_{2}\right|$ is rather small and Eq. (4.48) can roughly be approximated by

$$
\mathcal{F}=\frac{\mathcal{F}_{0}}{1-\mathcal{E}} 2\left|Q^{2} / q^{2}\right| \mathcal{E} \tilde{F}_{C J}^{2}
$$

except when $\mathcal{E}$ becomes very small (i.e., at rather backward angles) and consequently when the transverse multipoles must be retained. As seen in Figs. 3.10-3.12 at large $\theta$ the FOM is diminished and consequently we shall focus on forward-angle scattering. There the FOM has the same form as that found for elastic scattering ( $\mathrm{Eq} .(4.42)$ ) up to corrections of order $\gamma_{J}$ for natural-parity transitions that behave as the $4.44 \mathrm{MeV}$ transition in ${ }^{12} \mathrm{C}$ does. $T$..e requirement that must be met is that the Coulomb/convection current currents must dominate. Examples with this character include the rather collective low-lying $2^{+}$states such as the $4.44 \mathrm{MeV}$ level in ${ }^{12} \mathrm{C}$ and the usual (convective) giant dipole resonance. In contrast, certain cases exist which are spin-flip dominated, such as the spin-flip giant dipole resonance [Don68, Don70]; for these more detailed analyses would need to be performed. 
The general observation to be drawn from studies of natural-parity transitions is that they apparently offer no special advantage over elastic scattering and. in fact. have the detriment that the FOM in the latter case is generally more favorable due to the coherence of elastic scattering. A possible exception to this statement could come from using highenergy forward-angle scattering to probe the weak neutral current in nuclei at somewhat higher values of $q$ than may be permitted with elastic scattering. To be specific. in ${ }^{12} \mathrm{C}$ the elastic form factor falls off with increasing momentum transfer sufficiently rapidly that the $2^{+} 0$ competes successfully with it at $q$ greater than about $265 \mathrm{MeV} / \mathrm{c}$ and then. for instance. using the results for $10^{\circ}$ (Fig. 3.10) for the $2^{+} 0$ transition we find $c A_{L R} / A_{L R}=$ $5.1 \%$ at $\epsilon=2.5 \mathrm{GeV}(q=435 \mathrm{MeV} / \mathrm{c})$. The forward-angle asymmetry involves the ratio $\tilde{G}_{E}^{T=0} / G_{E}^{T=0}=-0.918\left(1+2.2\left(G_{E}^{(s)} / G_{E}^{T=0}\right)\right)$ and, in contrast to the magnetizationdominated $1^{+} 0$ case discussed above. has only very small strangeness sensitivity because of the suppression of $G_{E}^{(9)}$ at low momentum transfer. Using the abore conditions thiwould result in a $45 \%$ determination of $G_{E}^{(9)}$ (taking model (B) discussed in Sect. IV.B.1 for the electric strangeness form factor of the nucleon). While this might constitute a good alternative to elastic scattering. it should be remembered that the transverse multipole contributes at some level (quantified above for the $2^{+} 0$ transition in ${ }^{12} \mathrm{C}$. which is cne of the few that have been adequately studied using PC electron scattering) and consequently that additional nuclear model dependence enters the problem for any case other than $0^{ \pm} \rightarrow 0^{ \pm}$transitions where only the monopole can contribute.

\section{IV.D.2 NCCLEAR TRA.NSITIONS: DISCLSSION AND SLMIMARY}

A few additional comments concerning discrete nuclear transitions are in order. One pertains to the question of experimental resolution in studies of $\mathrm{PV}$ and $\mathrm{PC}$ electron scattering. As discussed in Sect. $V$, when extreme values of luminosity are demanded (implying thick targets) and large solid angles are required to make high-precision measurements of $A_{L R}$ it may not be possible to resolve individual discrete states. Instead. under some conditions. it may be necessary to sum over a few transitions to obtain a total asymmetry

$$
A_{L R}^{\mathrm{log}}=\sum_{1} p_{\mathrm{i}} A_{L R, 2},
$$

where $A_{L R .}$ is the asymmetry for the $\mathrm{i}^{\text {th }}$ transition and $p_{\mathrm{i}} \equiv(d \sigma / d \Omega)_{1} /\left[\sum,(d \sigma / d \Omega)_{3}\right]$ with $(d \sigma / d \Omega)$, the $\mathrm{PC}$ cross section for the $\mathrm{i}^{\text {th }}$ transition. An example where this might occur is when the $2^{+} 0$ transition in ${ }^{12} \mathrm{C}$ is summed together with elastic scattering. Again using the results for scattering at forward angles shown in Fig. 3.10, we see that only about $6 \%$ of the total asymmetry comes from the inelastic transition. Of course. for very high precision studies of the PV asymmetry this may be significant. For instance, as discussed in Sect. IV.B, one issue that arises is that of isospin-mixing, which, being theoretically uncertain (see the treatment in Ref. (Don89]), could lead to an intolerable uncertainty in the total asymmetry. Clearly the problem is only worse with poorer resolution where more transitions must be summed and accordingly where a larger fraction of the total asymmetry comes from inelastic excitations. 
A related comment also involves summing over several transitions. If the range of excitation involves only $T=0$ states in $. V=Z$ nuclei (e.g., takes into account only states below $15.11 . \mathrm{MeV}$ in ${ }^{12} \mathrm{C}$. ignoring isospin-mixing problems), then the tree-level asymmetry is proportional to

$$
\left(W^{-1 \mathrm{PV})} / F^{2}\right)^{\mathrm{tot}}=0.454+\sum_{i} p_{i}\left(W^{(\mathrm{PV})} / F^{2}\right)_{1}^{(s)} .
$$

where " $(s)$ " indicates contributions coming only from terms with nonzero strangeness. Insofar as complications from isospin-mixing can be ignored (or modeled successfully) the total asymmetry could be used to obtain some indication about the presence or absence of strangeness: if experiment yielded the result 0.454 for Eq. (4.51). then it would at least be suggestive that the strangeness contributions were small. Of course. accidentally they could cancel in the sum on the right-hand side of the equation and no conclusion could be drawn. Such a measurement might serve to get a first glimpse at the importance of strangeness in elec'ron scattering in that. being summed over several levels. it would have the maximal FOMI atcinable for a given nucleus.

In this section we have focused on discrete transitions in $V=Z$ nuclei. since the analysis is reasonably tractable and serves to set the scale for nuclei in general. OddA nuclei can, of course, also be investigated, although the proliferation of multipoles that occurs for non-trivial values of $J_{i}^{\pi_{i}}$ and $J_{f}^{\pi_{f}}$ takes us beyond the intent of the present work. Only one case will be mentioned - that of elastic scattering from spin-1/2 targets (see also Sect. IV.E). Focusing first on forward-angle scattering where the FOM is expected to be large. we have for this case that the PV asymmetry is proportional to

$$
W^{(\mathrm{PV})} / F^{2}=\frac{\rho_{C}+\delta^{2} \rho_{M}}{1+\delta^{2}},
$$

where $\rho_{C} \equiv-\tilde{F}_{i v} / F_{C n} . \rho_{M} \equiv-\tilde{F}_{M 1} / F_{M 1}$ and $\delta \equiv-\left|q^{2} / Q^{2}\right| F_{M 1} / \sqrt{2} F_{C 0}$. In the long wavelength limit we have tine following: $\rho_{C} \rightarrow 0.5(\mathrm{~N} / Z)-0.046$ neglecting strangeness. $\rho_{. M}$ is of order unity and $\delta \rightarrow(q / 2 M) \times(\mu / Z)$, where $\mu$ is the magnetic moment of the nuclear ground state in nuclear magnetons. (Recall also from the general arguments above that $\left|Q^{2} / q^{2}\right| \approx 1$.) Consider a case such as ${ }^{207} \mathrm{~Pb}$ where $\mu=0.584$. Using a momentum transfer of about $100 \mathrm{MeV} / \mathrm{c}$ (i.e., employing the estimate $q_{0}$ given above) yields $\delta \cong 3.8 \times 10^{-4}$ and therefore for such high- $Z$ cases the transition is completely dominated by the $\mathrm{C} 0$ multipole. Even for a case as light as ${ }^{13} \mathrm{C}$ (with $\mu=0.7022, q_{0} \cong 163 \mathrm{MeV} / \mathrm{c}$ ) one finds $\delta \cong 1.0 \times 10^{-2}$ and the magnetic effects are typically only a percent or so at low momentum transfer. For backward-angle scattering the axial-vector dipole form factor also plays a role. Defining $\rho_{E} \equiv \tilde{F}_{E 1_{s}} / F_{M 1}$ the complete hadronic ratio may be written

$$
W^{(\mathrm{PV})} / F^{2}=\frac{\rho_{C}+\left[\rho_{M}+\left(1-4 \sin ^{2} \theta_{w}\right) \sqrt{1-\mathcal{E}^{2}} \rho_{E}\right] \delta^{2} / \mathcal{E}}{1+\delta^{2} / \mathcal{E}} .
$$

Backward-angle scattering corresponds to $\mathcal{E} \ll 1$ and thus the factor $\sqrt{1-\mathcal{E}^{2}}$ may safely be taken to be unity. For the remaining $\mathcal{E}$-dependence two regimes exist: one is at extreme 
angles where $\mathcal{E} \ll \delta^{2}$, in which case the hadronic ratio goes to $\rho_{. M}+\left(1-4 \sin ^{2} \theta_{w} ! \rho_{E}\right.$. yielding a relatively large sensitivity to the axial-vector form factor. However. this regime is very hard to study. For example, even for a nucleus as light as ${ }^{13} \mathrm{C}$ one has $\delta \sim 10^{-2}$ and therefore the angle at which $\mathcal{E}=\delta^{2}$ is so far backward $\left(178^{\circ}\right)$ as to prove impractical. The other regime is where $\delta^{2} \ll \mathcal{E} \ll 1$. This corresponds to angles between about $90^{\circ}$ and $170^{\circ}$. using the fact that $\mathcal{E} \approx 1 / 3$ for $\theta=90^{\circ}$. In this regime the axial-vector term is not suppressed appreciably because of the factor $\sqrt{1-\mathcal{E}^{2}}$ : however. the factor $\delta^{2} / \mathcal{E}$ is unfortunately rather small (e.g., about $1 \%$ at $\theta \approx 165^{\circ}$ and even smaller at more forward scattering angles). Consequently. it would appear that it will be rather difficult to learn anything about the axial-vector contributions to elastic :attering if they enter with typical single-particle strengths. On the other hand. if these contributions are enhanced by collective effects ( see the discussions of the anapole moment in Sect. IV.E), then it may be possible to study them in this way.

The arguments here can be generalized to include elastic scattering from auclei with spins greater than 1/2. The basic conclusion will be unchanged: at low $-q$ and forwart angles where the FOM is large, the elastic scattering PV asymmetry will come almost entirely from the $\mathrm{C} 0$ multipole in all but the lightest nuclei. This implies that elastic scattering can be used to determine the radius of the ground-state neutron distribution even in odd-A nuclei (see the discussions in Sect. IV.B). Of special interest in this regard is the case of ${ }^{133} \mathrm{Cs}$ because of its importance in studies of atomic PV (see Sect. IV.I).

Our conclusions for the prospects of studying $A_{L R}$ for discrete nuclear transitions are mixed: on the one hand, the case of elastic scattering (from spin-0 nuclei especially. but also as mentioned above from nuclei with spins greater than zero) is likely to be quite important as it provides a way to isolate the effects of $G_{E}^{(s)}$ from the other nucleon form factors and consequently will complement studies of the proton (see Sects. IV.A and B). A few transitions such as the excitation of the $2^{+} 0$ state at $4.44 \mathrm{MeV}$ in ${ }^{12} \mathrm{C}$ might add to the elastic scattering studies and perhaps early, poor resolution experiments could yield some information about the rough level at which strangeness enters; however. in most cases we have found that the figure-of-merit for excitation of discrete states is too low for there to be much hope that useful information on the electroweak currents will be forthcorning from $\mathrm{PV}$ electron scattering in the foreseeable future. 


\section{IV.E Axial Hadronic Response}

Thus far. we have focused primarily on ways in which nucleon structure physics and the underlying lepton-quark electroweak interactions manifest themselves in the hadronic response functions. As alluded to earlier, however, these response functions are also sensitive to the many-body physics of the target nucleus. In this respect, one case of particular interest involves the piece of the PV hadronic response which involves nuclear matrix elements of the axial current [Don86b]:

$$
W_{i, A}^{*} \times \operatorname{Re}\left\{\left\langle f\left|J_{+1}^{E, M}\right| i\right\rangle\left\langle f\left|J_{+1,3}^{v c}\right| i\right\rangle^{*}-\left\langle f\left|J_{-1}^{E M}\right| i\right\rangle\left\langle f\left|J_{-1,3}^{N C}\right| i\right\rangle^{*}\right\}
$$

where " \pm 1 " refer to the transverse components of the currents and the subscript $" . "$

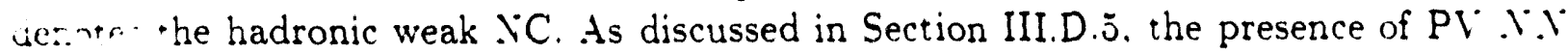
interactions in the nucleus requires one to replace multipole matrix elements of $J_{\lambda_{5}}^{*} \mathrm{c}$ with the $\operatorname{sum}\left\langle f\left|\hat{O}_{J}^{5}\right| i\right\rangle^{v C}+3\left\langle f\left|\hat{O}_{J}^{5}\right| i\right\rangle^{E M}$, where 3 is given in Eq. (3.94). The many-body physics of interest - in this case the PV $N . V$ force - is contained in $\left\langle f\left|\hat{O}_{J}^{3}\right| i\right)^{E M}$. Although we postpone a more detailed discussion of atomic PV until Section IV.I. we note that a special case of $\left\langle f\left|\hat{O}_{J}^{3}\right| i\right\rangle$ - the nuclear anapole moment $\left(f=i\right.$ and $\left.\hat{O}_{J}^{s} \rightarrow \hat{T}_{1}^{\text {els }}\right)$ - is also accessible with atomic $\mathrm{PV}$ observables. In tandem with the hadronic axial. $\mathrm{NC}$. the anapole moment (A.M) induces a nuclear spin-dependent (.VSD) PV atomic Hamiltonian

$$
\mathcal{H}_{\mathrm{atom}}^{P V}(\mathrm{NSD})=\frac{G_{\mu}}{2 \sqrt{2}} \tilde{\kappa} \vec{I} \cdot \int d \vec{x} \hat{\psi}_{\mathrm{e}}^{\dagger}(\vec{x}) \vec{\alpha} \psi_{\mathrm{e}}(\vec{x}) \delta(\vec{r})
$$

where $\vec{\alpha}$ is the vector of Dirac matrices acting in the space of lepton spinors, $\vec{I}$ is the nuclear spin. $\vec{r}$ is the electron coordinate, and the strength $\tilde{\kappa}=\tilde{\kappa}_{N C}+\tilde{\kappa}_{A M}$ is determined by nuclear PV $\left(\tilde{\kappa}_{A M}\right)$ and the weak NC interaction $\left(\tilde{\kappa}_{N C}\right)$ [Fla80]. The interaction in Eq. (4.j4) mixes atomic states of good parity, thereby giving rise to $\mathrm{PV}$ atomic observables, such as optical $z^{*}$ ation or circular polarization of incident radiation [Nov75]. Since $\tilde{\kappa}_{v C} \sim \tilde{\kappa}_{A M}$ for heavy and/or neariy degenerate nuclei, a measurement of an appropriate atomic PV observable can provide access to nuclear PV.

In seeking to study nuclear PV with charged leptons, one faces two challenges: (a) finding a nucleus in which the contribution from nuclear $\mathrm{PV}$ (e.g., the A.M or $\left\langle f\left|\hat{O}_{J}^{S}\right| i\right\rangle^{E . M}$ ) is at least commensurate with that of the axial $\mathrm{NC}$, and (b) to find an observable in which the total axial contribution is large enough to be observed experimentally. For both atomic PV and PV electron scattering, meeting the latter requirement is a non-trivial exercise. Fortunately, in order to place interesting new constraints on nuclear PV. one need not measure the axial response with the same degree of high-precision as required by electroweak tests or probes of nucleon strangeness. With these comments in mind. we discuss three cases which have received some attention as possible means for studying nuclear PV.

Atomic PV in heavy atoms 
The experimental difficulty in achieving sufficient precision to observe atomic $\mathrm{PV}$ can be mitigated by performing experiments with heavy atoms. To illustrate the reasons why: consider the mixing matrix element between $S_{1 / 2}$ and $P_{1 / 2}$ states in an atom such as Cs. In the limit that only single-electron states enter this mixing, one has [Novi5]

$$
\begin{aligned}
& \left\langle S_{1 / 2}\right| \mathcal{H}_{\text {atom }}^{P V}(\text { NSID })+\mathcal{H}_{\text {atom }}^{P V}(\text { NSD })\left|P_{1 / 2}\right\rangle \\
& \quad=\frac{i G_{\mu}}{2 \sqrt{2}} Z^{2} \cdot \mathcal{I}\left\{Q_{w}-\tilde{\kappa}\left[\frac{2 \gamma+1}{3}\right] g_{\imath}\left[F(F+1)-I(I+1)-\frac{3}{4}\right]\right\}
\end{aligned}
$$

where NSID and NSD refer to the nuclear spin-independent (Eq. (4.96)) and spindependent (Eq. (4.54)) components, respectively, of the atomic Hamiltonian: $Q_{w}$ is the weak charge defined in Eq. (4.97); $\mathrm{N}$ is a structure-dependent overall normalization factor: $\gamma=\sqrt{1-\alpha^{2} Z^{2}}: g_{i}\left\langle\right.$ g.s. $\left|\vec{\sigma}_{n}\right|$ g.s. $\rangle=\langle$ g.s. $|\vec{I}|$ g.s. $\rangle$, with $\left\langle\right.$ g.s. $\left|\vec{\sigma}_{n}\right|$ g.s. $\rangle$ being the spin of an unpaired nucleon and $\langle$ g.s. $|\vec{I}|$ g.s. $\rangle$ being the total nuclear spin in the ground state: and $\vec{F}=\vec{I}+\vec{J}$ is the total atomic angular momentum. The NSD part of the electron-nucleus interaction is extracted from PV observables which depend on $F$, such as transitions between different hyperfine levels. The overall $Z^{2}$ factor appearing in Eq. (4.55). in tandem with the coherence enhancement of $Q_{w}(\approx N)$ raises the scale of the NSID contribution to the mixing matrix element to an observable level for heavy atoms. In contrast. the $\mathrm{CSD}$ contribution receives no such coherence enhancement and is thus suppressed by roughly a factor of $N$ with respect to the NSID part. Moreover, in the absence of nuclear PV. one has for atomic Cs $\tilde{\kappa}=\tilde{\kappa}_{N C} \approx \frac{2}{9}\left(1-4 \sin ^{2} \theta_{w}\right) \approx 0.022$. Thus, the NSD part can be down by almost two orders of magnitude relative to the NSD contribution. Given this difference in scale between the two terms, it is significant that a value of $\tilde{\kappa}=0.42(23)$ has been extracted from the recent ${ }^{133} \mathrm{Cs}$ atomic $\mathrm{PV}$ experiment carried out by the Boulder group [Noe88, Blu90]. In that experiment, the NSD contribution was determined from measurements of the rates for transitions between different hyperfine levels involving parity-mixed $6 S_{1 / 2}$ and $7 S_{1 / 2}$ atomic levels. In the same experiment, a value for $Q_{w}$ with $\approx 2 \%$ experimental error was obtained. The experimenters also report that their data implies a $97 \%$ probability that $\tilde{\kappa}$ is larger than zero. The experimental result has the same sign and order of magnitude as the prediction of Ref. [Fla84], in which a single-particle effective PV nuclear Hamiltonian and Woods-Saxon potential were used. The large scale predicted for $\tilde{\kappa}_{A M}$, relative to that of $\tilde{\kappa}_{N C}$, results from two factors: (i) the nuclear PV contribution contains no $g_{V}^{e}=-1+4 \sin ^{2} \theta_{w}$ suppression factor, and (ii) an nuclear enhancement resulting from the $A^{2 / 3}$ scaling behavior of the nuclear AM. A new calculation of $\tilde{\kappa}_{A M}$ using the full two-body PV nuclear Hamiltonian and including PV MEC's (see Section III.D.5), is in progress [Hax93].

On the experimental side, the prospects for better limits on $\tilde{\kappa}_{A M}$ appear encouraging. From a new atomic Cs measurement in progress at Boulder, one expects to reduce the experimental uncertainty by a factor of four or five over the previous Cs determination [Wie93]. It is hoped that future Cs experiments will bring about another factor of five improvement in the anapole limit. In principle, one might hope to extract a limit on $\tilde{\kappa}_{A M}$ from the atomic $\mathrm{Pb} \mathrm{PV}$ experiment nearing completion in Seattle. However, the natural 
$\mathrm{Pb}$ used in that experiment mostly consists of ${ }^{208} \mathrm{~Pb}$, whose ground state is spin-0 and cannot support an anapole moment: the natural abundance of ${ }^{207} \mathrm{~Pb}$ ( $\operatorname{spin}-\frac{1}{2}$ ) is only about $20 \%$. Consequently, the expected scale of the contribution from the ${ }^{207} \mathrm{~Pb}$ A.M to the PV observable in this experiment is an order of magnitude smaller than the level of statistical precision. A future atomic PV measurement by the same group using thallium. however. is likely to be sensitive to the Th A.M at the level expected from theoretical predictions [Lam93].

\section{Elastic PV electron scattering}

The possibility of studying nuclear PV with elastic PV electron scattering has been explored in some detail by Serot for the case of ${ }^{13} \mathrm{C}$ [Ser 79 ]. Since this nucleus has spin $J=\frac{1}{2}$. the elastic asymmetry receives contributions from Coulomb. magnetic dipole. and the $J=1$ axial transverse electric multipole (see Eq. (3.50) of Section III.E). Contributions from nuclear $\mathrm{PV}$ arise only in the last of these multipoles. which can be decomposed according to Eq. (3.93) as

$$
\tilde{F}_{E 1_{s}} \longrightarrow \tilde{F}_{E 1_{s}}^{N C}+3 F_{E 1_{s}}^{E M}
$$

For elastic scattering, conservation of the E.M current implies that the E.M contribution to $F_{E 1 \text { s }}$ is finite as $Q^{2} \rightarrow 0$. In this limit, the E.M term in Eq. (4.56) behaves as

$$
F_{E 1_{s}}^{E M} \propto\left\langle\text { g.s. }\left\|i \hat{R}_{1}^{\text {el }}\right\| \text { g.s. }\right\rangle \rightarrow \frac{1}{\sqrt{6 \pi}} \frac{q^{2}}{m_{N}^{2}}\langle\text { g.s. }\|\hat{a}\| \text { g.s. }\rangle .
$$

The leading $q^{2}$-dependence on the right side of Eq. (4.57) cancels the $1 / q^{2}$ (in the Breit frame) contained in $\beta$, rendering a finite contribution to Eq. (4.56) at zero $q^{2}$. One consequence of this result is that even in the presence of nuclear PV, the $L, T$, and $T^{\prime}$ contributions to the ${ }^{13} \mathrm{C}$ PV response vary with $\theta$ for fixed $\epsilon$ in a manner entirely analogous to the case of a proton target, for which the axial contribution is largest at backward angles and lower energies.

Vuclear PV contributions to $A_{L R}\left({ }^{13} \mathrm{C}\right)$ at back angles were calculated in Ref. [Ser 79] . using a one-body average of $\hat{H}_{P V}^{[2]}$ and the [Des80] value for $h_{N N \pi} \sim 5 \times 10^{-7}$. Only the $\pi$-exchange part of the PV Hamiltonian was included, and contributions to $\tilde{F}_{E 1_{5}}^{E M}$ were not explicitly accounted for. Nevertheless, the estimates of Ref. [Ser79] should provide a rough guide as to the scale and kinematic dependences of various contributions to $A_{L R}\left({ }^{13} \mathrm{C}\right)$. The results of this work indicate that the nuclear PV and total NC (vector and axial vector) contributions to $A_{L R}\left({ }^{13} \mathrm{C}\right.$ ) at backward angles are comparable for low incident electron energies $(\epsilon \lesssim 100 \mathrm{MeV})$, while the total NC contribution dominates by one or two orders of magnitude for larger energies $(\epsilon \gtrsim 200 \mathrm{MeV})$. Thus, the low-energy regime appears to be the most appropriate for elastic scattering studies of nuclear PV. To estimate the possible precision with which such a measurement could be made, we consider a 1000 hour experiment at $\epsilon=50 \mathrm{MeV}$ and backward angles, a luminosity $\mathcal{L}\left[{ }^{13} \mathrm{C}\right]=1.25 \times 10^{38} \mathrm{~cm}^{-2} \mathrm{~s}^{-1}$, solid angle $\Delta \Omega=1 \mathrm{sr}$, and $100 \%$ beam polarization as an illustrative case. Under these conditions, one expects a statistical uncertainty in the asymmetry of $40 \%$. Were all other contributions to the asymmetry known to infinite precision, and were $\pi$-exchange the 
dominant contribution. one could then determine $h_{\mathrm{v} v \mathrm{v} \pi}$ with an uncertainty of $\pm 2 \times 10^{-i}$. Although the latter figure is somewhat larger than the range on $h_{v v \pi}$ obtained from the ${ }^{18} \mathrm{~F}$ experiment. it is sufficiently small to provide one with a test of the ${ }^{18} \mathrm{~F}$ result.

A more detailed analysis of this case would require a calculation using the full. twobody PV Hamiltonian and including PV .MEC's. In addition. one would require an analysis of the $\theta$-dependence of the different contributions to the asymmetry, given the large solid angle required to obtain the above-mentioned statistical precision. We also note that if the low-energy nuclear PV contribution to elastic scattering is dominated by the leading $q^{2}$-dependence of matrix elements of $\hat{R}_{1 \lambda}^{\text {el }}$, then the $A^{2 / 3}$ scaling behavior of this operator suggests that a heavier target could be more favorable than ${ }^{13} \mathrm{C}$.

\section{Inelastic PV electron scattering}

Two cases of inelastic PV electron scattering have been analyzed as possible means of studying nuclear $\mathrm{PV}$ : the excitation of the $15.11 \mathrm{MeV}\left(1^{+}, 1\right)$ state in ${ }^{12} \mathrm{C}[$ Ser 79$]$ and electrodisintegration of the deuteron [Hwa81]. In the former case, which is a pure isovector transition. parity and angular momentum selection rules restrict the set of allowed multipole form factors to the following: (a) $F_{M 1}, \tilde{F}_{M 1}, \tilde{F}_{E_{15}}, \tilde{F}_{C_{15}}$, and $\tilde{F}_{L_{1} \text { s }}$ for transition matrix elements between components of the ground state and $15.11 \mathrm{MeV}$ state having positive parity; (b) $F_{C 1}\left(F_{L_{1}}\right)$ and $F_{E 1}$ for E.M transitions involving a state of negative parity mixed into either the ground state or excited state; (c) $F_{C 1_{3}}\left(F_{L_{1} 1_{3}}\right)$ and $F_{E_{1}}$ arising from PV E.M MEC's; and (d) $\tilde{F}_{C 1}\left(\tilde{F}_{L 1}\right), \tilde{F}_{E 1}$, and $\tilde{F}_{M 1_{3}}$ for. $\mathrm{V}$ transition matrix elements involving a negative-parity component of either the ground state or excited state. The latter set of form factors (d), as well as all other Coulomb and longitudinal multipoles in (b) and (c), appear in $A_{L R}$ only at second order in the weak interaction. so one may neglect them for present purposes. Hence, only the form factors $F_{M 1}, \tilde{F}_{M 1}, \tilde{F}_{E_{15}}, F_{E 1}$, and $F_{E_{1}}$ s appear at leading non-trivial order in the weak interaction. Of these form factors. only the last two contain information on nuclear PV. In the analysis of Ref. [Ser 79 ]. which did not include PV .MEC contributions, no contribution from $F_{E 1_{s}}$ appears.

The remaining form factor sensitive to nuclear PV, $F_{E 1}$, which arises from paritymixing in the ground state and $15.11 \mathrm{MeV}$ state, receives contributions from matrix elements of both $\hat{S}_{1 \lambda}^{\text {el }}$ and $\hat{R}_{1 \lambda}^{\mathrm{el}}$. This situation contrasts with the elastic case, for which matrix elements of $\hat{S}_{1 \lambda}^{\text {el }}$ vanish. Consequently, $F_{E 1}$ need not vanish as $Q^{2}$ for small $Q^{2}$, as it must for elastic scattering. An important consequence of this fact is that the parity-mixing contribution to $A_{L R}$ does not vanish as $\theta \rightarrow 0^{\circ}$, whereas the weak NC contributions do vanish in this limit. In fact, the results of Ref. [Ser79] indicate that the parity-mixing term dominates the asymmetry at forward angles. For sufficiently low incident energies $(\epsilon \lesssim 50 \mathrm{MeV})$, this dominance of the nuclear PV component persists to backward angles. To illustrate, consider scattering at $\epsilon=30 \mathrm{MeV}$ and $\theta=30^{\circ}$. At these kinematics, the parity-mixing part of the asymmetry is roughly an order of magnitude larger than the weak NC terms. Moreover, under the same experimental conditions as assumed above for ${ }^{13} \mathrm{C}$, but with a solid angle of $0.16 \mathrm{sr}$, one has $\left(\delta A_{L R} / A_{L R}\right)_{\text {stat }} \approx 0.13$. Hence, assuming all other contributions are known with sufficient precision, a measurement of $A_{L R}$ under 
these conditions could allow for a significant improvement in the present constraints on $h_{\mathrm{v}, \mathrm{r}}$ and possibly help in resolving the current discrepancy.

In their analysis of $\mathrm{PV}$ electrodisintegration of the deuteron. the authors of Ref. [Hwa81] find that the weak NC and nuclear PV contributions to the asymmetry have comparable magnitude for low incident electron energies $(\epsilon \lesssim j 0) \mathrm{MeV}$ and low relative final state $n p$ energies $\left(\dot{E}_{\mathrm{rel}} \lesssim 1 \mathrm{MeV}\right)$. assuming the [Des80] values for the $h_{, v, v}$. In this analysis, a Reid soft-core potential [Rei68] was used to describe the strong.$V$ interaction. Gauge invariance was maintained through the use of Siegert's Theorem [Sie3i] and explicit inclusion of MEC's. The authors also suggest that by measuring the asymmetry at a variety of kinematic conditions, one could in principle constrain different linear combinations of the $h_{N, M_{M}}$. The experimental doability (i.e., $\left(\delta A_{L R} / A_{L R}\right)_{s t a t}$ ) of such measurements. however, was not analyzed. and it is not presently know what statistical precision would be achievable.

\section{Summary}

From the foregoing discussion, it appears that a combination of atomic PV and $f_{L R}$ measurements may produce useful new constraints on the conventional model of the PV weak V.V force. From both a theoretical and experimental perspective, progress in this direction is more advanced for atomic PV than for PV electron scattering. In the former case, new experiments which should improve on the present atomic cesium anapole limits are planned by the Boulder and Seattle groups [Wie93, Lam93]. In the latter case. lowenergy, moderate-to-forward angle measurements of $A_{L R}$ for nuclear transitions appear most promising, based on initial studies. Further analysis, including a search for the most favorable cases, more sophisticated nuclear calculations, and a detailed study of experimental doability, appears to be warranted. 


\section{IV.F. Quasielastic Scattering}

While PV elastic scattering from $\left(0^{+} 0\right)$ targets and from the deuteron hold out the possibility of determining $G_{E}^{(\mathbf{s})}$ and $G_{.1}^{(s)}$ more precisely than is possible with $\vec{e} p$ scattering alone. as discussed in Sects. IV.B and IV.C. a precision $A_{L R}(\mathrm{QE})$ measurement might allow one to measure $R_{A}^{T=1}$. which is responsible for a large portion of the theoretical hadronic uncertainty in the S.A.MPLE determination of $\mu_{\mathrm{g}}$ (see Sect. IV.A). More generally. QE PV scattering has the attraction that if the kinematics are chosen carefully, the resultant figures-of-merit can be rather large. Ideally, one would carry out an experiment at :ecisely quasi-free kinematics, in which case cross sections and helicity-differences are predominantly given by the corresponding quantities for individual nucleons. To the extent that the process is sufficiently "quasi-free" and so has controllably small nuclear model uncertainties from final-state interactions, meson-exchange currents, etc.. then $Q E$ scattering may provide valuable information about the single-nucleon form factors themselves.

$A$ few general comments about PV QE scattering are in order. Let us re-write the PV asymmetry in Eq. (3.109) in the following form involving ratios of the relevant response functions:

$$
A_{L R}(\mathrm{QE})=A_{L R}^{0} \times\left\{\frac{2\left|Q^{2} / q^{2}\right| \mathcal{E}\left[\frac{R^{L}}{R^{T}}\right]\left[\frac{R_{\Lambda \Psi}^{L}}{R^{L}}\right]+\left[\frac{R_{\Lambda \psi}^{T}}{R^{\Psi}}\right]+\sqrt{1-\mathcal{E}^{2}}\left[\frac{R_{\Lambda Y}^{T^{\prime}}}{R^{\Psi}}\right]}{1+2\left|Q^{2} / q^{2}\right| \mathcal{E}\left[\frac{R^{L}}{R^{T}}\right]}\right\} .
$$

where as usual $\mathcal{E}$ is given by Eq. (3.115). Each of the responses here may be decomposed into $T=0$ and $T=1$ contributions (see below). First, we note from the results presented in Ref. [Don92] (see also Ref. [Bei91a]) that $A_{L R}(\mathrm{QE})$ is dominated by the isovector transverse response and is. therefore, rather insensitive to $G_{E}^{(\mathbf{s})}, G_{M}^{(\mathbf{s})}$ and $G_{A}^{(\mathbf{s})}$, which contribute to the isoscalar longitudinal and transverse responses, respectively. The isovector part of the $R_{l \cdot A}^{T^{\prime}}$ response, however, depends on $\tilde{G}_{A}^{T=1}$, so that a measurement of backward-angle PV QE scattering should serve in constraining the problematic $R_{A}^{T=1}$. Second, as discussed in more detail below, the longitudinal responses are expected to be suppressed with respect to the dominant isovector transverse responses and to be highly sensitive to isospin-dependent nuclear correlations at moderate momentum transfers [Don92]. Consequently, forwardangle PV QE scattering may offer a new window on nuclear dynamics. Third, in the event that the electric strangeness form factor is unsuppressed at $\left|Q^{2}\right| \sim$ few $(\mathrm{GeV} / \mathrm{c})^{2}$. it may overwhelm the correlation effects in the $\mathrm{PV}$ longitudinal response and hence could be determined by measuring $A_{L R}(\mathrm{QE})$ at high momentum transfer and forward angles.

Recent work [Don92] within the context of the relativistic Fermi gas model bears out these expectations and suggests that experiments may be feasible in which the QE PV asymmetry could be determined to a fractional precision of about $1-2 \%$. To reach such high precision will likely require integrating over some region around the $\mathrm{QE}$ peak. While this integration may increase the do-ability of the experiment, it may also introduce contaminations from physics beyond the quasi-free approximation, including significant final-state interaction effects at low excitation energies, differences in reaction mechanism as in the case of pion production and the effects of two-body meson exchange currents. One must 
ask. then. whether theoretical uncertainties associated with these contaminations would cloud the interpretation of $A_{L R}(Q E)$ measurements at a prohlematic level. Although extensions for complex nuclei beyond the initial relativistic Fermi gas modeling undertaken thus far will have to be pursued* before definitive answers can be obtained. it is nevertheless encouraging that these initial studies indicate that the asymmetry can be relatively insensitive to the above uncertainties, at least for specific, well-chosen kinematics. In particular, the fact that the asymmetry involves ratios of responses as in Eq. 14.58 i and is therefore not as critically dependent on details of the nuclear model as are the individual responses suggests that the $\mathrm{QE}$ region might be a relatively good one for high-precision $\mathrm{PV}$ studies. Also supporting these expectations is the case of QE PV scattering from ${ }^{-} \mathrm{H}$ which has recently been studied in detail [Had92]. It provides a special situation in which the nuclear modeling can be undertaken at a more sophisticated level than is generally the case for many-body systems. In Ref. [Had92] realistic $. N . \%$ potentials were employed in obtaining bound ${ }^{2} \mathrm{H}$ and continuum $n p$ wave functions for use in calculating ilectroweak current mitrix elements. By compa:ing with models that contain only plane-wave final states (the plane-wave impulse approximation. PWIA. and the plane-wave Born approximation, PWBA), it was found in that work that $A_{L R}\left({ }^{2} \mathrm{H}, \mathrm{QE}\right)$ is rathe: insensitive to final-state interaction effects for intermediate-to-high values of momentum transfer ( say, above $\sim 400 \mathrm{MeV} / \mathrm{c}$ ). We shall return to discuss some of these results below.

To obtain some feeling for the issues involved in studies of PV QE scattering. let us continue with the static approximation introduced in Sect. III.E. Writing each form factor in Eqs. (3.131)-(3.133) in terms of the decomposition given in Eqs. (3.31) we have

$$
\begin{aligned}
Z G_{E}^{p} \tilde{G}_{E}^{p} & +. V G_{E}^{n} \tilde{G}_{E}^{n} \\
& =(Z+. \nu)\left\{G_{E}^{T=0}\left[\sqrt{3} \xi_{V}^{T=0} G_{E}^{T=0}+\xi_{V}^{(0)} G_{E}^{(s)}\right]+G_{E}^{T=1}\left[\xi_{V}^{T=1} G_{E}^{T=1}\right]\right\} \\
& +(Z-. V)\left\{G_{E}^{T=1}\left[\sqrt{3} \xi_{V}^{T=0} G_{E}^{T=0}+\xi_{V}^{(0)} G_{E}^{(s)}\right]+G_{E}^{T=0}\left[\xi_{V}^{T=1} G_{E}^{T=1}\right]\right\}
\end{aligned}
$$

(and likewise with $E \rightarrow M$ ) for the combinations involving only vector form factors. together with

$$
\begin{aligned}
Z G_{M}^{p} \tilde{G}_{A}^{p} & +N G_{M}^{n} \tilde{G}_{A}^{n} \\
& =(Z+N)\left\{G_{M}^{T=0}\left[\xi_{A}^{T=0} G_{A}^{(8)}+\xi_{A}^{(0)} G_{A}^{(s)}\right]+G_{M}^{T=1}\left[\xi_{A}^{T=1} G_{A}^{(3)}\right]\right\} \\
& +(Z-N)\left\{G_{M}^{T=1}\left[\xi_{A}^{T=0} G_{A}^{(8)}+\xi_{A}^{(0)} G_{A}^{(s)}\right]+G_{M}^{T=0}\left[\xi_{A}^{T=1} G_{A}^{(3)}\right]\right\}
\end{aligned}
$$

for the combinations involving both vector and axial-vector form factors. The denominator in the asymmetry involves the EM form factors:

$$
Z\left(G_{E}^{p}\right)^{2}+N\left(G_{E}^{n}\right)^{2}=(Z+N)\left\{\left(G_{E}^{T=0}\right)^{2}+\left(G_{E}^{T=1}\right)^{2}\right\}+(Z-N)\left\{2 G_{E}^{T=0} G_{E}^{T=1}\right\}
$$

* Initial steps in this direction have been taken in Refs. [Hor93a, Hor93b] and [Alb93a]. 
and likewise with $E \rightarrow . M$. First. we note that the mixed isovector-isoscalar components in Eqs, 4.59 ) and 14.60 ) are suppressed with respect to the pure isovector or isoscalar pieces by $(Z-. P) /(Z+. \dot{V})$. Hence, in making the following arguments (but not in the results taken from Ref. (Don92/) we neglect these terms. Second. we note that in the transverse responses in Eqs. 14.59 ) involving $G_{M}^{T}$ and $\dot{G}_{M}^{T}$ or $\dot{G}_{A}^{T}$. the isovector component is enhanced with respect to the isoscalar by a factor of $G_{M}^{T=1} / G_{M}^{T}=0 \approx 5$ at low- $Q^{2}$ Hence, the effect of $\mu$, appearing in $G_{M}^{T}=0{ }^{0}{ }_{M}^{T}=0$ will be less important. relative to the leading magnetic term, than it is in the elastic $\vec{e} p \mathrm{PV}$ response (see Eq. $(3.124,1$. A similar remark applies to $G_{A}^{(s)}$. which appears in the combination $G_{.1}^{T=0} \tilde{G}_{A}^{(s)}$ in Eq. 14.53 , and is suppressed with respect to the leading $G_{M}^{T}=1{ }^{T}{ }_{A}^{T}=1$ axial-vector response. Finally. we note that in the simple static approximation where incoherent sums over protons and neutror, occur. the $\mathrm{Pl}$ longitudinal response is highly suppressed with respect to the trans:er:e responses (however, see the further discussion in Sect. IV.F.2). This feature is most ensf: seen by writing the PV longitudinal response in the form

$$
\begin{aligned}
\frac{1}{2}\left(Z G_{E}^{p} \tilde{G}_{E}^{p}+. \dot{V} G_{E}^{n} \tilde{G}_{E}^{n}\right) & =Z G_{E}^{p}\left[\left(1-4 \sin ^{2} \theta_{W}\right) G_{E}^{p}-G_{E}^{n}-G_{E}^{\prime \prime}\right) \\
& +. \nu G_{E}^{n}\left[\left(1-4 \sin ^{2} \theta_{w}\right) G_{E}^{n}-G_{E}^{p}-G_{E}^{\prime \prime \prime}\right]
\end{aligned}
$$

where radiative corrections have been omitted for simplicity. What would be the largest component - the term containing $\left(G_{E}^{p}\right)^{2}-$ is suppressed by the $\left(1-4 \sin ^{2} \theta_{W}\right) \approx 0.092$ premultiplying factor. The next largest component. given by $(Z+. \nu) G_{E}^{p} G_{E}^{n}$, is small at low- $Q^{2}$ due to the smallness of $G_{E}^{n}$. In the case of the magnetic response $E-. M$ in Eq. (4.61)), on the other hand, no such form factor suppression arises. Hence, one expects the transverse isovector response to dominate $A_{L R}(\mathrm{QE})$, especially given the large scale of $G_{.1}^{T=1}$ appearing in this term.

\section{IV.F.1. B.ACKWARD-A.NGLE QE SCATTERI.NG: $\tilde{G}_{A}^{T=1}$-SE.NSITIVITY}

Let us begin by discussing the possibility that a measurement of $A_{L R}(Q E)$ might eliminate one of the sources of uncertainty in the interpretation of $A_{L R}(\vec{e} p)$. namely. the radiative correction $R_{A}^{T=1}$ contained in $R_{A}^{p}$. According to the estimates of Ref. Nus90). one has $R_{A}^{T=1} / R_{A}^{T=0} \cong-3$, with uncertainties $\delta R_{A}^{T=0} \cong{ }_{-0.03}^{+0.04}$ and $\delta R_{A}^{T=1}={ }_{-0.20}^{+0}$. Hence. a sufficiently precise measurement of $\tilde{G}_{A}^{T=1}=-g_{A}^{(1)} G_{D}^{A}\left(1+R_{A}^{T=1}\right)$ could eliminate most of the theoretical uncertainties associated with the axial-vector contribution to a SA.MPLEtype experiment [Bei91a]. For scattering at backward angles $A_{L R}(Q E)$ depends primarily on the transverse PV response functions, $R_{A V}^{T}, R_{V A}^{T^{\prime}}$ (see Eq. (3.110)) and the transverse PC electromagnetic response function, $R^{T}$ (see Eq. (3.104)), with only small effects from longitudinal contributions. As discussed above, all of these transverse contributions are dominated by isovector spin-flip currents. As $\theta \rightarrow 180^{\circ}$ the leptonic kinematic factors attain the limit $v_{T^{\prime}} / v_{T} \rightarrow 1$ (equivalently, $\mathcal{E} \rightarrow 0$ in Eq. (3.115)) and consequently one obtains from Eq. (4.58) only two terms,

$$
\frac{A_{L R}(Q E)}{A_{L R}^{0}} \rightarrow \frac{R_{A V}^{T}}{R^{T}}+\frac{R_{V A}^{T^{\prime}}}{R^{T}}
$$


The first (second) involves the $\dot{G}_{M}\left(\dot{G}_{A}\right)$ dependence in Eq. (3.132). Typically the second term is about one quarter of the first. and thus the backward-angle asymmetry derives about $20 \%$ of its strength from the response which contains the axial-vector currents. Giver that the PV QE scattering asymmetry can be measured to $\sim 1-2 \%$ ( see Ref. 'Don32! . this implies that the axial-vector contributions would be determined to $\sim 5-10 \%$ if the first. purely vector pieces were perfectly known. Of course, these vector contributions are uncertain at some level. since they contain form factors which are only known with finite precision. Additionally, the nuclear modeling itself entails some uncertainty i see also Sect. IV.F.2 and Ref. [Don92] for some discussion of the level of confidence that might be expected when specific nuclear models are employed). To the extent that one does not incur too much uncertainty from the nuclear modeling. the backward-angle asymmetry can then be used to shed light on the interplay of the $G_{M i}^{p, n}, G_{\| l}^{\prime \prime}$ and $G_{A}$ form factors. As noted above. the primary reason for using PV QE scattering (i.e.. from a nucleus toget her with elastic scattering from the proton is that the interplay of the form factors is different in the two cases and may permit separations of the effects from the various form factorto he diser:-angled. Clearly. using only elastic $\vec{e} p$ scattering alone involves too many form factors and too few observables to permit such separations to be made.

Let us expand a little further on these ideas. Writing the transverse ratios in Eq. 4.62) in terms of the single-nucleon form factors (i.e., taking $\mathcal{E} \rightarrow 0$ in Eq. (3.133) and substituting for the $\dot{G}$ form factors - again we invoke the static approximation in making these arguments), we note the following: (1) the denominators in Eq. (4.62) are proportional to $Z\left(G_{M}^{p}\right)^{2}+N\left(G_{M}^{n}\right)^{2}:(2)$ the non-strange part of the first (vector) ratio involves the combination $A G_{M}^{p} G_{M}^{n}$ in its numerator, whereas the magnetic strangeness content occurs there in the form $\left(Z G_{M}^{p}+\nu G_{M}^{n}\right) G_{M}^{(s)}$; and (3) the axial-vector content in the second term appears in the combinations $\left(Z G_{M}^{p}-. \nu G_{M}^{n}\right) \tilde{G}_{A}^{T=1}$ and $\left(Z G_{M}^{p}+.{ }_{M} G_{M}^{n}\right) \dot{G}_{A}^{T=0}$. where any strangeness axial-vector form factor occurs in the latter. We may then make at least two important observations. First, for elastic scattering from the proton, obtained from these expressions by setting $Z=1$ and $. V=0$ (compare Eqs. (3.123) and $(3.124)$. the only dependence on $G_{M}^{n}$ occurs in the numerator of the hadronic ratio. In contrast. for PV $O E$ scattering where typically.$V \sim Z$, such dependence occurs both in the numerator and the denominator of the hadronic ratio. As a consequence, the effect of having limited precision from PC electron scattering on $G_{M}^{n}$ is diminished somewhat in the nuclear case. This fact is illustrated in panel (a) of Fig. 4.11, which is taken from Ref. [Don92] inote that these results were obtained using the full relativistic Fermi gas model, not just the static approximation). The correlation of $\bar{G}_{A}^{T=1}(0)$ with $\rho_{M_{n}}$ is shown for ${ }^{1} \mathrm{H},{ }^{12} \mathrm{C}$ and ${ }^{184} \mathrm{~W}$ (see also below). Clearly the correlation is weaker for the nuclear cases than for the proton. Second, any occurrence of $G_{M}^{(0)}$ or $\dot{G}_{A}^{T=0}$ enters multiplied by the combination $Z G_{M}^{P}+. V G_{M}^{n}$, whereas the isovector axial-vector form factor, $G_{A}^{T=1}$, enters multiplied by $Z G_{M}^{p}-v G_{M}^{n}$. Thus, the relative importance of the two classes of contributions is governed by

$$
\frac{Z G_{M}^{p}+. \nu G_{M}^{n}}{Z G_{M}^{p}-. \nu G_{M}^{n}} \sim \frac{Z \mu_{p}+. V \mu_{n}}{Z \mu_{p}-. V \mu_{n}}
$$

This yields 1 for elastic scattering from the proton and, for example, in $. V=Z$ nuclei such as ${ }^{2} \mathrm{H}$ or ${ }^{12} \mathrm{C}$ a much smaller value, 0.187 . Thus, effects from $G_{M}^{(0)}$ and $\dot{G}_{A}^{T=0}$ are 
suppressed in PV QE scattering from nuclei. possibly permitting the focus to be placed on the unsuppressed form factor. $\dot{G}_{A}^{T=1}$. One may take these observations to their natural extreme and choose a nucleus which has $. \dot{Z}: Z$ very close to $\mu_{p}: \mu_{n}$ and consequently. yields nearly zero for the ratio in Eq. (4.63). For example, in Ref. (Don92) the target ${ }^{184} 11$. was chosen for discussion: then the ratio becomes -0.009 and the suppression is virtually. complete. The correlations of $\dot{G}_{A}^{T=1}(0)$ with $\mu_{s}$ and $g_{A}^{(s)} \equiv G_{A}^{(0)}(0)$ are shown in panels (b) and (c) of Fig. 4.11 for the three nuclear targets discussed. Clearly these are rather strong correlations for the case of the proton, as noted previously in Sect. IV.A where we have discussed the problem of making an unambiguous determination of $G_{M}^{\prime \prime}$ in a S.A.MPLE-type experiment, i.e., without incurring some uncertainty from the axial-vector form factors. For nuclei. where only very weak correlations are seen to occur. it should be possible to focus on the isovector axial-vector form factor and accordingly remove at least this source of uncertainty. Of course. $\dot{G}_{A}^{T=1}$ is interesting in its own right and PV QE scattering from appropriately chosen nuclei may help to shed light on it.

The special case of QE scattering from deuterium may be used to illustrate these ilea. in a different way. Let us concentrate on the form factors $G_{A}^{T=1} . G_{.11}^{\prime \prime \prime}$ and $G_{E}^{\prime \prime}$. ('sing the parameterizations discussed in Sect. III.C (specifically, taking $\lambda_{E}^{(s)}=0$ ) we may write the $\mathrm{Pl}$ asymmetry in the following form:

$$
A_{L R} \equiv a_{L R}\left[1-b_{A} \dot{G}_{A}^{T=1}\left(\left|Q^{2}\right|=0\right)+b_{M} \mu_{S}+b_{E} \rho_{S}\right] .
$$

where the numbers $\left(a_{L_{R}}: b_{A}, b_{M}, b_{E}\right)$ reflect the way the asymmetry depends on these three particular form factors. Ling the results from Ref. [Had92] for $q=300 . \mathrm{MeV} \mathrm{c}$ and $\theta=150^{\circ}$ (corresponding to an incident electron energy of $321 \mathrm{MeV} / \mathrm{c}$ at the $\mathrm{QE}$ peak t the form factor dependences are the following (see Eq. (4.64)):

$$
\begin{aligned}
\left(a_{L R} ; b_{A}, b_{M}, b_{E}\right) & =\left(-1.54 \times 10^{-3} ; 0.197,-0.461,-0.005\right) \text { proton elastic } \\
& =\left(-2.07 \times 10^{-5} ; 0.166,-0.070,-0.003\right) \text { deuteron QE - FSI (SdT) } \\
& =\left(-2.07 \times 10^{-5} ; 0.166,-0.068,-0.003\right) \text { deuteron QE - FSI }(Y) \\
& =\left(-2.06 \times 10^{-3} ; 0.168,-0.073,-0.002\right) \text { deuteron QE - PIVIA }
\end{aligned}
$$

As expected, the effects due to $G_{E}^{(0)}$ are very small at backward angles. All of the models for deuterium represented here (see the discussions above) give answers which are very similar and again show the rather weak model dependence for these (favorable) kinematics. Our expectations concerning the differences between the proton and deuteron cases are borne out in detail. Importantly, the relative dependence on the magnetic strangeness form factor (embodied in the parameter $\mu_{0}$ ) is more than six times stronger in the proton case than for the deuteron. Clearly the latter case is relatively more sensitive to the isovector axial-vector form factor (represented by the parameter $\dot{G}_{A}^{T=1}(0)$ ). A high enough precision measurement of the QE PV asymmetry in deuterium should help in defining $\dot{G}_{A}^{T=1}$ and so. used in concert with elastic PV scattering from the proton, permit $G_{M}^{(0)}$ to be determined with better precision than is possible with the proton alone. 


\section{IV.F.2. ISOSPIN-DEPENDE.IT CORRELATIONS}

One source of nuclear physics uncertainties is the impact of nuclear correlations i see. e.g. Ref. Alb90|). Such correlations may be responsible to some degree for the well-known failure of the Coulomb sum rule for PC QE scattering. Since the various nuclear response functions display different sensitivities to inclusion of final-state interaction and mesonexchange current effects. and since these have different isospin-dependences. we expect the individual nuclear ingredients in the PV asymmetry to be isospin-dependent as weil. In particular. the transverse responses (the $P C{ }^{2}$-response and $P V^{\prime} T$ - and $T^{\prime}$ - responses in Eqs. $(4.62)$ are dominated by nuclear matrix elements of isovector spin-flip operators. $\sim \sigma T$ ( see. e.g.. Ref. (Don92]). This can be made clear by writing the ratios of response functions in Eq. $(4.38)$ as follows (here we take $. V=Z$ to simplify the expressions:

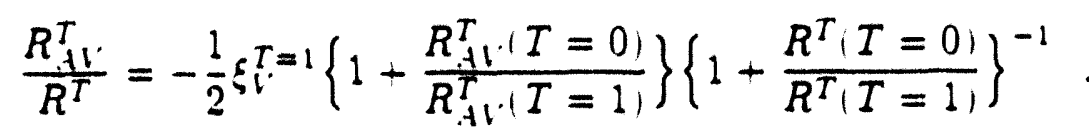

where the $\mathrm{PV}$ ratio in the static model is given by

$$
\frac{R_{A V}^{T} \cdot(T=0)}{R_{A V}^{T} \cdot(T=1)}=\left(\frac{G_{. I}^{T=0}}{G_{. V}^{T=1}}\right)^{2} \frac{1}{\xi_{V}^{T=1}}\left(\sqrt{3} \xi_{V}^{T=0}+\xi_{V}^{(0)} \frac{G_{E}^{(0)}}{G_{E}^{T=0}}\right)
$$

and where the corresponding E.M ratio is

$$
\frac{R^{T}(T=0)}{R^{T}(T=1)}=\left(\frac{G_{11}^{T=0}}{G_{M}^{T=1}}\right)^{2} \cong 0.035
$$

Thus. the isoscalar effects can be expected to contribute to these ratios only at about the $3 \%$ '.el. Isospin correlations will modify the relative amounts of the responses that are isosralar and isovector and hence change the ratios $R_{A V}^{T} \cdot(T=0) / R_{.+1}^{T}+T=1$ ) and $\therefore$ I $I=0), R^{T}(T=1)$ in Eqs. (4.6i) from their static model values. However. even a reasonably large change of these ratios is still a minor effect on $R_{A V}^{T} / R^{T}$ because of the isovector dominance. Consequently. isospin correlations are not expected to have much effect on the asymmetry as long as the transverse responses are dominant, viz. at backward scattering angles. Similar expressions may be written for $R_{V_{A}}^{T^{\prime}} / R^{T}$ which is likewise isovector dominated.

The situation for the longitudinal PC and PV nuclear responses is quite different there the balance of isoscalar-to-isovector content is such that changes from the static model predictions for the relative amounts of each are expected to be quite large [Don92]. For instance, in the PC QE longitudinal response the balance is approximately $1: 1$ and so if isoscalar correlations effectively provide a reduction from the najve answer (say by moving strength to low excitation energies), then roughly half of this response will be affected proportionately, whereas the transverse responses will be almost unchanged. The PV QE longitudinal response is especially sensitive to isospin correlations and consequently might provide an interesting new window on such many-body effects. To see how this arises. let 
us again assume that $. P=Z$ for simplicity and write the isoscalar/isovector parts of $R_{41}$ in the following way:

$$
R_{+\cdot A}^{L}(T)=. \ell\left(1+\Delta_{T}\right) G_{E}^{(T)} \dot{G}_{E}^{(T)}
$$

where $l^{\circ}$ is an overall normalization and $\Delta_{T}$ with $T=0.1$ represent the amounts that the correlations cause the actual responses to deviate from their static model values. One then has

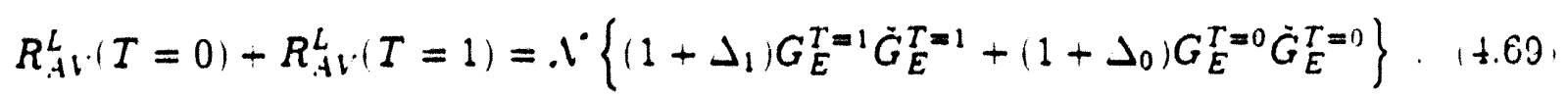

Writing the $G_{E}^{(T)}$ and $\dot{G}_{E}^{(T)}$ in terms of $G_{E}^{n, p}$ and $\dot{G}_{E}^{n, p}$ using the inverse of Eqs. (4.61, gives

$$
\begin{aligned}
R_{.4 V}^{L}=\frac{. l}{2}\{[1 & \left.+\frac{1}{2}\left(\Delta_{0}+\Delta_{1}\right)\right]\left[G_{E}^{p} \dot{G}_{E}^{p}+G_{E}^{n} \dot{G}_{E}^{n}\right\} \\
& \left.+\frac{1}{2}\left(\Delta_{0}-\Delta_{1}\right)\left[G_{E}^{p} \dot{G}_{E}^{n}+G_{E}^{n} \dot{G}_{E}^{p}\right]\right\}
\end{aligned}
$$

and inserting the tree-level Standard model results (Eq. (3.31)) we have that the first term in Eq. $(4.70)$ is proportional to

$$
\left(-1+4 \sin ^{2} \theta_{W}\right)\left[\left(G_{E}^{p}\right)^{2}+\left(G_{E}^{n}\right)^{2}\right]+2 G_{E}^{p} G_{E}^{n}+\left(G_{E}^{p}+G_{E}^{n}\right) G_{E}^{(s)}
$$

which is suppressed with respect to order unity by factors of $\left(-1+4 \sin ^{2} \theta_{W}\right), G_{E}^{n}$. or $G_{E}^{s}$ (compare $\mathrm{Eq}$. (4.61)). The second term, which is sensitive to the difference in isospindependent correlation effects, is proportional to

$$
\left(G_{E}^{p}\right)^{2}+\left(G_{E}^{n}\right)^{2}+2\left(-1+4 \sin ^{2} \theta_{W}\right) G_{E}^{p} G_{E}^{n}+\left(G_{E}^{p}+G_{E}^{n}\right) G_{E}^{(s)}
$$

Due to the presence of the first term. Eq. (4.72) can be a factor of 10 or more larger than Eq. (4.71) for low- $\left|Q^{2}\right|$. Hence, one sees that the effect of differences in isospin-dependent correlations can be significant in $R_{A V}^{L}$. A measurement of $A_{L A}(Q E)$ sufficiently sensitive to $R_{A l}^{L}$. might, therefore, provide an effective probe of these correlations. Recent work [Alb93a] has been aimed in part at exploiting this sensitivity to nuclear dynamics.

\section{IV.F.3. FORWARD-A.NGLE QE SCATTERING: $G_{E}^{(s)}$-SENSITIVITY}

An exception to the strong nuclear model dependence found in forward-angle scattering can occur. As noted in Ref. [Don92], it may turn out that the strangeness electric form factor is unsuppressed at high momentum transfer $\left(\lambda_{E}^{(s)} \approx 0\right)$ and consequently dominates in the longitudinal response (see Eq. (4.61)). A special (calculable) case where such high$\left|Q^{2}\right|$, forward-angle studies have recently been explored (Had92] is that of $Q E$ scattering 
from deuterium. At $q=1 \mathrm{GeV} / \mathrm{c}$ and $\theta=12.5^{\circ}$ the following results were obtained in that work:

$$
\begin{aligned}
\left(a_{L R}: b_{A}, b_{M}, b_{E}\right) & =\left(-3.43 \times 10^{-3}: 0.035,-0.495,-0.172\right) \text { proton elastic } \\
& =\left(-5.25 \times 10^{-3}: 0.028,-0.080,-0.085\right) \text { deuteron QE - FSI SdT) } \\
& =\left(-5.40 \times 10^{-3}: 0.027,-0.073,-0.078\right) \text { deuteron QE - FSI }\left({ }^{\prime}\right) \\
& =\left(-4.87 \times 10^{-3}: 0.032,-0.082,-0.108\right) \text { deuteron QE - PIVIA }
\end{aligned}
$$

The proton results are for elastic scattering (see Sect. IV.A), whereas the deuteron results are for kinematics corresponding to the $Q E$ peak: in both cases the incident electron energy is $4.36 \mathrm{GeV}$. The scattering angle was taken to be the minimum possible with the spectrometers that are being built in Hall $A$ at CEBAF. For the deuteron. three sets of results are given, viz. two with different.$\vee$. potentials labelled FSI(SdT) and FSI $(Y$, and the last for the PVIA. Details of the two potentials can be fcund in [deTi3. deTiJ. Cot 6 . (SdT) and [Bre6i. Sea68] ( $Y$ ). Here the two FSI models differ in $a_{L R}$ by less than $3 \%$ and yet the relative effect of $G_{E}^{(s)}$ could be as large as $16-17 \%$ in the FSI models 1 or $22 \%$ in the PWIA) if $\rho_{s}=-2$ as in the model of Ref. [Jaf89]. The differences between the FSI and PWIA results are due to the non-relativistic expansion procedure used in the former: for the purpose of predicting the asymmetry at high momentum transfer the relativistic PWIA should be more reliable. Comparing the proton and deuteron results. we see rather striking differences. In elastic scattering from the proton at forward scattering angles the effects from $G_{.1}^{(s)}$ are quite important and any significant uncertainty in this form factor from backward-angle determinations will propagate into connected uncertainty in $G_{E}^{\prime \prime \prime}$. no matter how precisely the small angle proton asymmetry is measured. However. taken together with the QE PV asymmetry on the deuteron where the magnetic strangeness dependence is much weaker, new information about the form factor dependences could be extracted. As the results of Ref. [Had92] suggest, it should be possible to undertake such comparisons with rather high confidence in the deuterium $\mathrm{QE}$ predictions.

\section{IV.F.4. SCWMIARY}

Thus far only one quasielastic PV electron scattering experiment has been performed. the pioneering measurement on ${ }^{9} \mathrm{Be}$ at Mainz [Hei89]. For future studies at extreme luminosities there appear to be several attractive features of high-precision PV QE scattering to explore. Foremost is likely the possibility of extracting information on $R_{A}^{T=1}$ from backward-angle scattering at modest momentum transfers and consequently relatively low electron energies $(\sim 300-500 \mathrm{MeV} / \mathrm{c})$. As a choice of target, the deuteron has the merit of being "calculable" to the degree that at moderate energies, where relativistic effects are believed to be relatively unimportant, the nuclear physics uncertainties in the modeling of the asymmetry are likely to be under control. Even for heavier nuclei, the expectation is that the nuclear model uncertainties are rather small for the purely transverse responses and thus for the asymmetry at backward scattering angles. More theoretical work will be required before one can be certain about the scale at which nuclear dynamics (from final-state interactions, meson-exchange currents and relativistic corrections) effect the 
asymmetry: more importantly, it will be necessary to quantify the level of uncertainty in modeling the nuclear dynamics if or when high-precision measurements become feasible.

Forward-angle PV QE electron scattering may also prove to be interesting. There the possibility of learning about isospin correlations exists. since. as discussed in the previous subsections. the various contributions to the $\mathrm{PV}$ longitudinal response at the level of the static approximation are all very small. whereas the terms which arise when isospin correlations are present are about one order of magnitude larger. Consequently. even relatively small amounts of isospin correlation will be significantly magnified and appear as large changes in the forward-angle PV asymmetry. Aside from studies of such nuclear many-body effects, another potential circumstance where forward-angle scattering might prove interesting is that of adding information on $G_{E}^{(\text {s) }}$ at high momentum transfer isee also Sects. IV.A and IV.B). In the event that the electric strangeness form factor is unsuppressed at large- $\left|Q^{2}\right|$ it could become the dominant contribution to the longitudinal response (since all other terms are relatively small, as mentioned abovei. having only the isospin correlation effects with which to compete.

Cltimately, it may be necessary to study a given nucleus over a range of kinematics and/or to study several d:ferent nuclei to disentangle the nuclear many-body effects from those that relate directly to the properties of the nucleon. By comparing the $\mathrm{PV}$ asymmetry measured at low- and high- $\left|Q^{2}\right|$, for forward- and backward-angle scattering. in each case as a function of $\omega$ should help in quantifying the level at which nuclear modeling uncertainties enter. For example, from recent work [Alb93a] it appears that the nuclear modeling could be tested by choosing the kinematics wisely (in particular. to emphasize the aspects such as isospin correlations which need to be understood better) and then used with increased confidence for other kinematics where the nuclear many-body effects are less important and where the single-nucleon form factor dependences are best revealed.

In Sect. $V$ we return to discuss the future experimental program for PV QE electron scattering as it is presently perceived. 


\section{IV.G. The Nucleon-to-Delta Transition}

In this section. we consider the excitation of the $\Delta(1232)$ resonance. $\vec{e}+. V \rightarrow \epsilon+\Delta$. Since this is an isovector transition, measurements of the PV asymmetry could allow di. rect extraction of both the vector and axial-vector isovector couplings. As discussed in Sect. III.B. the radiative corrections due to heavy-quark contributions are quite different for an isovector transition than for both isoscalar and elastic nucleon scattering. involving the $S$ and $I$ parameters with almost equal weight. In analogy to the elastic cases considered above. such PV electroproduction measurements might provide a possible test of the Standard Model that is essentially independent of details of the underlying hadronic physics. This is only true to the extent that (i) kinematics (or neutrino data) can be used to eliminate axial-vector terms: (ii) the corresponding electromagnetic transition matrix elements which contribute to the non-resonant background are well measured: and iii . hadronic (target-dependent) contributions to electroweak radiative corrections are understood. One advantage afforded by $\left.A_{L R}(.) \rightarrow \Delta\right)$ is that uncertainties from isoscalar contributions (e.g. nucleonic s-quark content) are suppressed. in contrast to the elastic cases considered above. Alternatively, if electroweak couplings are ' sen as given input. PV $\Delta$-production may be useful as a means to measure the weak transition matrix elements. giving information on difficult-to-measure isovector amplitudes and on the currently uncertain axial-vector transition strength.

The.$V \rightarrow \Delta$ asymmetry has previously been calculated assuming elastic $\lambda$ production in the high-energy [Cah 78 ] and intermediate-energy [Jon80, Vat82] regimes. "Elastic" here means treating the $\Delta$ as a stable ( $\operatorname{spin}-\frac{3}{2}$ ) particle. Existing data on photoand electro-production of pions indicate, however, that there is a non-negligible bacl:ground to the $\Delta$-production (see Ref. [Moo 8 8]), and since this contains both isovector and isoscalar pieces, one needs an estimate of the size and uncertainty of these contributions. Such estimates have been performed in the context of specific nuclear models [Li82. Rei8i]. Here. we focus on what hadronic model-independent statements can be made. To set the scale, we note the limits this asymmetry could place on the $S$ and $T$ parameters mentioned ai "e. From Fig. 2.4 $A_{L R}(N \rightarrow \Delta)$ would need to be measured at the several percent leve, or better. in order to be effective in complementing atomic PV and the other PV electron scattering experiments discussed earlier.

Since axistine data on the $N \rightarrow \Delta$ transition have been obtained from $. N\left(e, e^{\prime} \pi\right) . N$ and .$V\left(e . e^{\prime} . V\right) \pi$ experiments, we consider pion electroproduction below the 2-pion threshold. ignoring electroweak radiative corrections. One can write a general expression for the weak and electromagnetic cross sections in a multipole expansion [Ad168, Pol87, Ras89]. . Vear the $\Delta(1232)$-resonance. the vector magnetic dipole multipole $\left(\equiv M_{1+}\right)$ dominates, leading to a very simple expression when neglecting backgrounds (cf., Eqs. (4.79) and (4.81) below). In addition. all multipoles can be expanded in terms of their isospin structure: namely. we can decompose a generic electromagnetic multipole, $T$, (suppressing all spin/parity labels) as [Adl68]

$$
\begin{aligned}
T_{n \pi^{+}} & \equiv \frac{1}{\sqrt{2}} T^{\frac{3}{2}}+T^{\frac{1}{2}}-\sqrt{2} T^{0} \\
T_{p \pi^{0}} & \equiv T^{\frac{3}{2}}-\frac{1}{\sqrt{2}} T^{\frac{1}{2}}+T^{0} \\
T_{n \pi^{0}} & \equiv T^{\frac{3}{2}}-\frac{1}{\sqrt{2}} T^{\frac{1}{2}}-T^{0} \\
T_{p \pi^{-}} & \equiv \frac{1}{\sqrt{2}} T^{\frac{3}{2}}+T^{\frac{1}{2}}+\sqrt{2} T^{0},
\end{aligned}
$$


where $T^{0}$ is isoscalar, and $T^{\frac{1}{2}}$ and $T^{\frac{3}{2}}$ are linearly independent isovector multipoles. going to final states with isospin $\frac{1}{2}$ and $\frac{3}{2}$ respectively. For pure $\Delta$-production. of course, $T^{0}=$ $T^{\frac{1}{2}}=0$. Relating the vector $\mathrm{NC}$ multipoles to the EM multipoles via Eqs. (3.16), one has

$$
\begin{aligned}
\tilde{T}^{0} & =\sqrt{3} \xi_{V}^{T=0} T^{0} \\
\tilde{T}^{\frac{1}{2}}, \frac{3}{2} & =\xi_{V}^{T=1} T^{\frac{1}{2}, \frac{3}{2}} .
\end{aligned}
$$

If one performed a coincidence pion-electroproduction experiment and measured final charge states, the total cross section for a proton target would be obtained by adding $n \pi^{-}$ and $p \pi^{0}$ cross sections incoherently. Such an asymmetry measurement would be misleading for purposes of studying PV effects, however, since PC helicity-differences can also occur (specifically, the so-called $5^{\text {th }}$ response function will be nonzero and generally much larger than the PV observables: see Ref. [Ras89]). For inclusive electron scattering the helicitydifferences are only PV and hence unambiguous. The latter, however, are less selective in that all final states must be considered; this lack of specificity means that background effects are less easily controlled (see below). Since the theoretical analysis of the coincidence measurement is somewhat simpler and will permit us to bring out the main features of potential PV studies in the $\Delta$ region, we focus on this case in what follows. knowing full well that extensive analyses of the inclusive reactions will be required as well. The asymmetry involves the interference of weak and electromagnetic amplitudes, and each vector term in the multipole expansion will then contribute as

$$
\left[\tilde{T}^{*} T\right]_{p \pi^{0}}+\left[\tilde{T}^{*} T\right]_{n \pi^{+}}=\xi_{V}^{T=1}\left(\left|T_{p \pi^{0}}\right|^{2}+\left|T_{n \pi^{+}}\right|^{2}\right)+2 \xi_{V}^{n}\left(T_{p \pi^{0}}-\sqrt{2} T_{n \pi^{+}}\right)^{*} T^{0}, \quad \text {, 4.i } 6
$$

using Eq. (3.24a). Here Eq. (4.76) is derived using Eqs. (4.74) and (4.75) for the $\mathrm{NC}$ multipoles, and then adding and subtracting the isoscalar multipole, $T^{0}$, to the $\mathrm{NC}$ isovector terms, in order to pull out the overall isovector factor of $\xi_{V}^{T=1}$ in the first term. This term is then exactly proportional to the (unpolarized) electromagnetic cross section. The remainder should be quite small. Since from Eq. (4.74) one has $T_{p \pi^{0}}-\sqrt{2} T_{n \pi^{+}} \propto T^{0}-T^{\frac{1}{2}} / \sqrt{2}$. the final term in Eq. (4.76) is a product of two background amplitudes with final-state isospin $\frac{1}{2}$.

The resulting electron asymmetry is given by a sum of three terms (we use a superscript $\pi$ to indicate single-pion production),

$$
A_{L R}(N \rightarrow \Delta)=-\frac{1}{2} A_{L R}^{0} \times\left\{\Delta_{(1)}^{\pi}+\Delta_{(2)}^{\pi}+\Delta_{(3)}^{\pi}\right\}
$$

where $\Delta_{(1)}^{\pi}$ gives the Lorentz vector, isovector contributions (both resonant and nonresonant), corresponding to the first term of Eq. (4.76) above; $\Delta_{(2)}^{\pi}$ gives the remaining isospin- $\frac{1}{2}$ channel, Lorentz vector, non-s sonant background piece; $\Delta_{(3)}^{\pi}$ gives the axialvector contributions, both resonant and non-resonant; and $A_{L_{R}}^{0}$ has been introduced in Sect. III.E. The terms are given explicitly by

$$
\Delta_{(1)}^{\pi}=g_{A}^{e} \xi_{V}^{T=1}
$$




$$
\begin{aligned}
F^{2} \Delta_{(2)}^{\pi} & =-2 g_{A}^{e} \xi_{V}^{n} \sum_{l} \operatorname{Re} \\
& \times\left\{v _ { T } \left[l(l+1)^{2}\left(\frac{3}{\sqrt{2}} \cdot M_{l+}^{0 *} \cdot M_{l+}^{\frac{1}{2}}-3\left|\cdot M_{l+}^{0}\right|^{2}\right)+l^{2}(l+1)\left(\frac{3}{\sqrt{2}} \cdot M_{l-}^{0 *} \cdot M_{l-}^{\frac{1}{2}}-3\left|\cdot M_{l-}^{0}\right|^{2}\right)\right.\right. \\
& \left.+(l+2)(l+1)^{2}\left(\frac{3}{\sqrt{2}} E_{l+}^{0 *} E_{l+}^{\frac{1}{2}}-3\left|E_{l+}^{0}\right|^{2}\right)+l^{2}(l-1)\left(\frac{3}{\sqrt{2}} E_{l-}^{0 *} E_{l-}^{\frac{1}{2}}-3\left|E_{l-}^{0}\right|^{2}\right)\right] \\
& \left.+v_{L}\left[(l+1)^{3}\left(\frac{3}{\sqrt{2}} S_{l+}^{0 *} S_{l+}^{\frac{1}{2}}-3\left|S_{l+}^{0}\right|^{2}\right)+l^{3}\left(\frac{3}{\sqrt{2}} S_{l-}^{0 *} S_{l-}^{\frac{1}{2}}-3\left|S_{l-}^{0}\right|^{2}\right)\right]\right\} \\
& \cdot F^{2} \Delta_{l 3)}^{\pi}=2 g_{V}^{e} v_{T^{\prime}} \sum_{l} \operatorname{Re}\left[l(l+1)^{2} \tilde{E}_{l+}^{5 *} \cdot M_{l+}-(l+1)^{2}(l+2) \cdot \tilde{K}_{l+}^{5 *} E_{l+}\right. \\
& \left.\quad-l^{2}(l+1) \tilde{E}_{l-}^{5 *} \cdot M_{l-}+l^{2}(l-1) \tilde{M}_{l-}^{5 *} E_{l-}\right]
\end{aligned}
$$

where the superscripts indicate the isospin decomposition of Eq. (4.74), the subscripts indicate the angular momentum and parity of the multipoles, and we have used Eq. (4.76) to separate out the first and second terms. The $E$ 's, $M$ 's, and $S$ 's are transverse electric. transverse magnetic, and longitudinal multipoles, respectively [Ad168, Pol87, Ras89]. The isospin structure of the axial-vector term $\Delta_{(3)}^{\pi}$ is not explicitly decomposed in Eq. (4.78). as there is no electromagnetic analog from which to extract information.

At the $\Delta(1232)$-resonance, and in the high-energy limit, $\Delta_{(1)}^{\pi}$ dominates the nonresonant backgrounds. In this case, the asymmetry takes on the the particularly simple form

$$
A_{L R}(N \rightarrow \Delta)=-\frac{1}{2} A_{L R}^{0} \times \xi_{V}^{T=1}
$$

....: $:$ h is the isovector analog of the form for purely isoscalar transitions, Eq. (3.128). At lower energies. there does exist one significant difference, however. At forward angles, the axial-vector contribution to $A_{L R}(N \rightarrow \Delta)$ does not vanish, as is the case for elastic scattering. This arises because of the different kinematics: since $2 M_{p} \omega=M_{\Delta}^{2}-M_{p}^{2}+\left|Q^{2}\right|$, in the limit $\theta \rightarrow 0, Q^{2} \rightarrow 0$, we have

$$
\begin{gathered}
q \rightarrow \omega \rightarrow \frac{M_{\Delta}^{2}-M_{p}^{2}}{2 M_{p}} \neq 0 \\
v_{T^{\prime}} / v_{T} \rightarrow \frac{\epsilon^{2}-\epsilon^{\prime 2}}{\epsilon^{2}+\epsilon^{\prime 2}} \neq 0 .
\end{gathered}
$$

In this limit, at large $\epsilon$, the relative contribution of the axial-vector term is indeed suppressed by $1 / \epsilon$, but at threshold $\left(\epsilon^{\prime} \rightarrow 0\right)$ the ratio $v_{T^{\prime}} / v_{T}$ is unity. Consequently, the axial-vector transition matrix element contributes at forward angles, suppressed at low energies only by the coefficient $g_{V}^{e}$. 
Ignoring the isoscalar background term $\Delta_{(2)}^{\pi}$, but retaining the resonant axial-vector term results in the formula of Ref. [Jon80]. Because the axial-vector terms are all suppressed by $g_{v}^{e}$, one can reasonably hope to estimate their contribution by including just the $1^{+}$resonant amplitude. Doing so gives

$$
F^{2} \Delta_{(3)}^{\pi}=8 g_{V}^{e} v_{T}^{\prime} \operatorname{Re} \dot{E}_{1+}^{5 *} \cdot M_{1+}
$$

which requires only the one axial-vector multipole, $\tilde{E}_{1+}^{5}$. The latter has been studied using charge-changing neutrino cross sections, but is still not very well known (see Refs. [Adl68. Zuci1. Llei2. Sch 73. Rei87, Kit90]). Lsing several different possible parameterizations of this axial-vector term gives variations in the small-angle asymmetry of $1.5-2.5 \%$ at $4 \mathrm{GeT}$ incident electron energy. The corresponding uncertainty in $\xi_{v}^{T=1}$ is also $1.5-2.5 \%$. While the relative contribution from the axial-vector term does not vanish at small angles. higher beam energies decrease the strength of this term. Dropping $\epsilon$ from 4 to $1 \mathrm{Gel}$ increases the above mentioned uncertainties to closer to $10 \%$. However, raising $\epsilon$ to $20 \mathrm{GeV}$, while keeping $Q^{2}$ fixed) reduces the sensitivity to the axial-vector term by roughly a factor of five, bringing the uncertainties due to this term below the $1 \%$ level.

One practical limitation to a model-independent approach, where the multipoles appearing in Eq. (4.78) are to be taken from electromagnetic and charge-changing neutrino scattering, is that the inelastic electromagnetic transition multipoles appearing, for example, in $\Delta_{(2)}^{\pi}$, must be broken down into isoscalar and isovector pieces separately. Such a separation is difficult for the $\Delta$-resonance, requiring detailed neutron data to accomplish a full separation of the three independent isospin pieces. Because of the complete cancellation of nucleon structure from the $\Delta_{(1)}^{\pi}$ term, however, this isospin decomposition is only relevant for the non-resonant background terms.

Part of the background piece $\Delta_{(2)}^{\pi}$ can be re-written in terms of electromagnetic cross sections; namely, that part which contains terms of the form $T^{0} T^{\frac{1}{2}}$. Multiplying by the appropriate kinematic coefficients yields exactly $\frac{1}{2}\left(\sigma_{n}^{\gamma}-\sigma_{p}^{\gamma}\right)$, with $\sigma^{\gamma}$ the full electromagnetic differential cross section. The remaining piece of this background is purely isoscalar. going as $\left|T^{0}\right|^{2}$. For a PV coincidence measurement, then, one in principle requires only electromagnetic cross section data, rather than the detailed multipole decomposition. plus information on the explicitly isoscalar background amplitudes, to evaluate $\Delta_{(2)}^{\pi}{ }^{*}$

In order to set the scales involved one can use photoproduction data which have been broken down into isospin components to get an estimate of the effect of non-resonant background on the asymmetry at $Q^{2}=0$. The background is approximately $3-4 \%$ of the total photoproduction cross section, implying a similar amount for the asymmetry. This result, however, depends sensitively on the poorly known neutron cross section: a $10 \%$ shift in $\sigma_{n}^{\gamma}$ would raise the background contribution to almost $7 \%$ of the asymmetry. Thus, the uncertainty from background contributions appears to be non-negligible even at $Q^{2}=0$. At large- $\left|Q^{2}\right|$, the uncertainties become larger. This feature is due in part

* This separation is completely arbitrary, however, as there is no a priori reason to assume the $T^{0}$ background multipoles should be significantly smaller than the $T^{\frac{1}{2}}$ multipoles. 
to the difficulty in extracting detailed information from $\nu$-scattering, which means that the $Q^{2}$-dependence of the axial-vector form factors in the term $\Delta_{(3)}^{\pi}$ is uncertain. Also. the $\Delta$-resonance drops in strength relative to the backgrounds with growing momentum transfer. making the separation of non-resonant contributions, $\Delta_{(2)}^{\pi}$, more problematic. It seems likely that one would want to use theoretical models to predict and correct for this effect.

This discussion has focused on the particular case of incoherent summation of final charge states to illustrate the general methods of calculation. To reiterate. false asymmetries in coincidence measurements mean that one should probably consider inclusive $\vec{e}$-scattering, in which case the background piece analogous to $\Delta_{(2)}^{\pi}$ will also include interference terms between the background and resonance multipoles. and thus may not be as small as the pure background considered here. An analysis of this case will appear in forthcoming work [Pol92b].

On the experimental side, a proposal was recently made to measure $A_{L R}(N \rightarrow \Delta)$ at SLAC [Lou92a]. The asymmetry in that case would be determined at kinematics corresponding to $\epsilon=12.9 \mathrm{GeV},\left|Q^{2}\right|=2(\mathrm{GeV} / \mathrm{c})^{2}$, and $\theta=6.5^{\circ}$ with a precision of $15 \%$ in the asymmetry. From Fig. 2.4, we observe that roughly an order-of-magnitude improvement in precision would be needed to make the use of such a measurement as a probe of new physics competitive with others. The scale of the axial-vector contribution at these kinematics is well below the experimental uncertainty. A less definitive statement can be made about the non-resonant background contributions, although at lower energies their contribution is less than $10 \%$. While the existing proposal appears to reach the limits of precision attainable at SLAC, the higher beam current and larger solid angle attainable in CEBAF Hall A could make a high-precision $A_{L R}(N \rightarrow \Delta)$ measurement feasible there. For example, a 1000 hour Hall A experiment for $\epsilon \sim 4 \mathrm{GeV}\left(\left|Q^{2}\right| \gtrsim 0.6(\mathrm{GeV} / c)^{2}\right)$ could yield a $1 \%$ asymmetry measurement if an $80 \%$ beam polarization were achievable [Lou92b]. A combination of forward-angle measurements might then afford a separation of the axialvector, background, and/or new physics contributions. The prospects for such a scenario at backward angles appears somewhat less promising. The maximum achievable precision for the asymmetry in this regime is roughly $5 \%$.

We conclude that a high-accuracy PV measurement in the $\Delta$-regime, as a means to measure $\xi_{v}^{T=1}$ in a model-independent way, is currently limited by uncertainties in $\nu$ - and electro-production data. A more thorough multipole and isospin decomposition is needed, as well as better data on neutrino pion-production. Higher beam energies provide an advantage in eliminating the uncertain axial-vector contributions, provided $\left|Q^{2}\right|$ is not so large that the resonance peak gets washed out. With current experimental electroproduction uncertainties, one will have to rely on model-dependent analyses to approximate the background. Since the total background and axial-vector contributions appear to be small at moderate momentum transfers, the resulting model dependence of the extraction should be fairly weak. Turning the argument around, however, the axialvector transition strength is itself of theoretical interest, and lower energy and/or large angle $\Delta$-production may provide useful information on this resonant multipole. 


\section{IV.H. Parity-Violating Deep Inelastic Scattering}

Thus far. we have concentrated on semileptonic . $\mathrm{NC}$ studies at low- and intermediateenergies. A considerable degree of insight into the structure of the lepton-quark electroweak interaction has also been derived from experiments carried out in the deep inelastic regime. Although a detailed discussion of these experiments (see e.g.. Ref. (Ama87)! lies beyond the scope of the present article. we briefly treat one case of historical interest. Indeed. the first PV electron scattering experiment, performed with a deuterium target at SLAC [Prei8. Prei9], illustrates the different set of physics issues encountered in higherenergy semileptonic $\mathrm{NC}$ scattering as compared with the. $\mathrm{NC}$ processes treated elsewhere in this article. In what follows, we make no pretense of providing a complete discussion: our intent, rather, is to illustrate the aforementioned comparison with lower-energy experiments.

At the energy and momentum transfers associated with the SLAC experiment inz.. $\left\langle\omega^{\prime}\right\rangle \approx 5 \mathrm{GeV} .\left|\left\langle Q^{2}\right\rangle\right| \approx 1.3(\mathrm{GeV} / \mathrm{c})^{2}$ [Prei9]), the nuclear target is unlikely to retain its identity in the final state. Moreover, the wavelength of the virtual vector-boson probe $\left(\hat{*}^{*}, Z^{0 *}\right)$ is much smaller than the size of the nucleon. and the timescale for its interaction with a given quark is much shorter than the timescale associated with the strong quarkquark interaction [Bjo69]. Thus, to a good approximation, the scattering may be treated as an incoherent process involving the nucleon's constituents. In the parton picture. these constituents are point-like valence and sea quark-partons, characterized by momentum distribution functions $f_{q}(x)$, where

$$
x \equiv \frac{-Q^{2}}{2 m_{N} \omega}
$$

is the Bjorken scaling variable (note that the quantity $\omega$ used in this work is usually called $\nu$ in high-energy physics). The SLAC data were centered about a value of $x=0.165$. In the infinite momentum frame, $x$ is the fraction of longitudinal momentum carried by a given parton. The distribution functions satisiy the sum rule

$$
\int_{0}^{1} d x \sum_{q} x f_{q}(x)=1
$$

The differential cross section for scattering from a nuclear target is then given by

$$
\frac{d \sigma(e N \rightarrow e X)}{d x d y}=\sum_{q} x \hat{x}_{q}(x)\left(\frac{d \sigma}{d x d y}\right)_{e-q}
$$

where

$$
y \equiv \frac{p \cdot Q}{p \cdot K} \stackrel{\text { lab }}{\longrightarrow} 1-\frac{\epsilon^{\prime}}{\epsilon}
$$

and where the differential cross section appearing on the right-hand side of Eq. (4.84) is for scattering of the electron from a given quark-parton $q$. The helicity independent and 
dependent $e-q$ cross sections are proportional to the contraction of the lepton tensors (in the ERL)

$$
\begin{aligned}
L_{\mu \nu}^{E M)} & =2 Q_{e}^{2}\left(K_{\mu}^{-} K_{\nu}^{-1}+K_{\nu}^{-} K_{\mu}^{-1}-g_{\mu \nu} K^{-} \cdot K^{-1}\right) \\
L_{\mu \nu}^{(E M-V C}(h)= & 2 h Q_{e} g_{A}^{e}\left(K_{\mu} K_{\nu}^{-1}+K_{\nu} K_{\mu}^{-1}-g_{\mu \nu} K \cdot K^{-1}\right) \\
& -2 i h Q_{e} g_{\nu}^{e} \epsilon_{\mu \nu \alpha 3} K^{-\alpha} K^{-3 \prime} .
\end{aligned}
$$

where $h$ is the electron helicity, with the corresponding helicity independent tensors $I_{\mu \nu}$ EM and $W_{\mu \nu}$ EM-NC associated with the quark-parton. The resulting $P C$ and $P V$ cross sections depend on the variable $y$. It is straightforward to work out the asymmetry as a function of $x$ and $y$

$$
A_{L R}(x, y)=A_{L R}^{0} \times \frac{W^{(P V)}(x, y)}{W^{(E M)}(x)} .
$$

where the deep inelastic hadronic ratio is given by [Cahi8]

$$
\frac{W^{(\mathrm{PV})}}{W^{\left(E_{\mathrm{M}}\right)}}=\frac{\sum_{q} f_{q}(x) Q_{q}\left\{g_{A}^{e} g_{V}^{q}+g_{A}^{q} g_{V}^{e}\left[\frac{1-(1-y)^{2}}{1+(1-y)^{2}}\right]\right\}}{2 \sum_{q} f_{q}(x) Q_{q}^{2}}
$$

- Vote how, in contrast to the situation that occurs in low-and intermediate-energy scattering to discrete states, where the hadron structure physics enters via one- and many-body form factors (see Sects. III.D and III.E), the deep inelastic hadronic ratio depends on target structure via the distribution functions $f_{q}(x)$. The reason for this difference is essentially contained in the incoherent approximation embodied in Eq. (4.84) (summing probabilities rather than amplitudes - in this regard, it is more akin to the quasielastic scattering discussed in Sects. III.E and IV.F). Note also that, in general, the structure of the $e-q$ electroweak interaction enters through the $g_{V}^{q}, g_{A}^{q}$ etc., rather than the through the isospin couplings $\xi_{v}^{(a)}$.

As with lower-energy scattering, it is possible to eliminate much of the hadronic physics dependence of Eq. (4.88) through a judicious choice of target. In the case of the deuteron, which is symmetric in valence $u$ and $d$ quarks, one has $f_{u}^{v}(x)=f_{d}^{v}(x)$. In this case, the hadronic ratio may be written in the simple form

$$
\frac{W^{(\mathbf{P V})}}{W^{(\mathbf{E M})}}=\frac{9}{10}\left\{\tilde{a}_{1}+\tilde{a}_{2}\left[\frac{1-(1-y)^{2}}{1+(1-y)^{2}}\right]\right\},
$$

where

$$
\begin{aligned}
& \tilde{a}_{1}=\frac{1}{3} g_{A}^{e}\left(2 g_{V}^{u}-g_{V}^{d}\right)=-\frac{1}{3}(3 \tilde{\alpha}+\tilde{\gamma}) \\
& \tilde{a}_{2}=\frac{1}{\xi} g_{V}^{e}\left(2 g_{A}^{u}-g_{A}^{d}\right)=-\frac{1}{3}(3 \tilde{\beta}+\tilde{\delta})
\end{aligned}
$$

at tree-level and neglecting sea-quark contributions. One may account for the latter as well as for the presence of "non-standard" physics by through correction factors as

$$
\begin{aligned}
& \left.\tilde{a}_{1}=\left(1-\frac{20}{j} \sin ^{2} \theta_{w}\right)\left[1+R_{1}\left(\text { st }^{\prime} \mathrm{d}\right)+R_{1} \text { (new }\right)+R_{1} \text { (had) }\right] \\
& \left.\tilde{a}_{2}=\left(1-4 \sin ^{2} \theta_{w}\right)\left[1+R_{2}\left(\text { st'd }^{\prime} \mathrm{d}\right)+R_{2} \text { (new }\right)+R_{2} \text { (had) }\right],
\end{aligned}
$$


where the $R_{(1)}$ (st'd) are radiative corrections in the Standard. Model and the $R_{(1)}$ (new) represent contributions from physics beyond the Standard. Model. In the $S$ and $T$ framework one has approximately

$$
\begin{aligned}
& R_{1}(\text { new })=0.02 T-0.017 S \\
& R_{2} \text { (new) }=0.155 T-0.206 S
\end{aligned}
$$

The hadronic structure corrections, obtained by keeping the sea-quark contributions in Eq. $(4.88)$, have the form

$$
\begin{aligned}
& R_{1}(\text { had }) \approx \frac{\sum_{q} Q_{q} g_{v}^{q} F_{q}^{s}(x)}{f_{q}^{v}(x)\left(Q_{u} g_{v}^{u}+Q_{d} g_{v}^{d}\right)}-\frac{\sum_{q} Q_{q}^{2} F_{q}^{s}(x)}{f_{q}^{v}(x)\left(Q_{u}^{2}+Q_{d}^{2}\right)} \\
& R_{2}(\text { had }) \approx \frac{\sum_{q} Q_{q} g_{A}^{q} \delta f_{q}^{s}(x)}{f_{q}^{v}(x)\left(Q_{u} g_{A}^{u}+Q_{d} g_{A}^{d}\right)}-\frac{\sum_{q} Q_{q}^{2} F_{q}^{s}(x)}{f_{q}^{v}(x)\left(Q_{u}^{2}+Q_{d}^{2}\right)} .
\end{aligned}
$$

where $F_{q}^{s} \equiv f_{q}^{s}+f_{q}^{s}$ and $\delta f_{q}^{s} \equiv f_{q}^{s}-f_{q}^{s}$ are the sum and difference. respectively. of the sea quark $(q)$ and anti-quark $(\bar{q})$ distributions. The reason that the difference $\delta f_{q}^{s}$ appears in $R_{2}$ (had) rather than the sum as in $R_{1}$ (had) is that both $Q_{q}$ and $g_{v}^{q}$ change sign on going from quark to anti-quark, whereas the sign of $g_{A}^{q}$ remains the same [Cahis]. In the case of $u$ and $d$ quarks, the valence distribution is defined as $f_{q}^{v}(x)=f_{q}(x)-f_{q}(x)$. so that the $\delta f_{q}^{s}(x) \equiv 0$ for $q=u, d$.

The foregoing results merit several observations. First, from Eq. (4.89) one observes that a measurement of $A_{L R}(x, y)$ as a function of $y$ allows a separate determination of $\tilde{a}_{1}$ and $\tilde{a}_{2}$. At the simplest level, these two quantities are hadronic physics independent (neglecting the $R_{(1)}\left(\right.$ had)). Thus, a separation of the $\tilde{a}_{(i)}$ allows one either to test various electroweak models at tree-level (by specifying the $g_{v}^{q}, g_{A}^{q}$, etc.) or to place constraints on the two linear combinations of the "model-independent" $e-q$ couplings appearing in Eq. (4.90). Such a separation was performed using the SLAC data. leading to the constraints (see, e.g., Refs. [Pre78, Pre79, Com83])

$$
\begin{aligned}
& \tilde{\alpha}+\downarrow \tilde{\gamma}=-0.60 \pm 0.16 \\
& \tilde{\beta}+\downarrow \tilde{\delta}=0.31 \pm 0.51 .
\end{aligned}
$$

Alternately, one may assume the Standard Model forms for the $\tilde{a}_{(1)}$ and extract a value of $\sin ^{2} \theta_{w}$. The SLAC results were found to be consistent with the Standard Model with $\sin ^{2} \theta_{w}=0.224 \pm 0.020$.

While the $9 \%$ determination of $\sin ^{2} \theta_{w}$ from the SLAC experiment represented a triumph for this first PV electron scattering experiment, present and future electroweak tests in other sectors are approaching precision of $1 \%$ or better. A deep inelastic $\vec{e} D$ measurement at this level of precision could be sensitive to physics beyond the Standard Model, such as would be characterized, for example, by $S$ and $T$ appearing in the $R_{(3)}$ (new). To extract meaningful constraints on these parameters, however, one must also make a reliable determination of the structure corsections. $R_{(\mathrm{t})}($ had). 
An indication of the scale of these corrections may be obtained by employing a parameterization of the distribution functions fit to E.MC data [Slo88. Sch88]. In this param. eterization. one neglects contributions from heavy quarks $(c, b, t)$ in the sea and assumes equality of sea quark and anti-quark distributions for the three lightest quarks:

$$
f_{u}^{s}=f_{u}^{s}=f_{d}^{s}=f_{d}^{s}=f_{s}^{s}=f_{u}^{s} \equiv f^{s} .
$$

Hence. one has $c f_{q}^{s}=0$ and $F_{q}^{s}=2 f^{s}$ in $E q .(4.93)$. In order to obtain the valence distributions for the deuteron. we assume good isospin symmetry for the nucleon. so that $f_{u}^{\prime}($ proton $)=f_{d}^{\prime}$ (neutron) and $f_{d}^{\prime}$ (proton) $=f_{u}^{v}$ (neutron). Thus. one has $f_{j}^{\prime}$ (deuteron $=$ if $f_{u}^{v}$ (proton) $-f_{d}^{v}$ (proton)). From the fits of Ref. [Slo88] we then obtain the $R_{1,}$, had as functions of $x$. as shown in Fig. 4.12.

As expected. the $R_{(1)}\left(\right.$ had) become negligible for $x \gtrsim 0.2$. Even though $x f_{1}^{\prime}(x)$ and $x f_{q}^{s}(x)$ both vanish as $x \rightarrow 1$, the sea distribution falls of more rapidly with $x$, becoming negligible for $x \gtrsim 0.2$. while the valence distributions persist at an appreciable level to somewhat larger values of $x$. Cnder the assumptions employed in parameterizing the $f_{q}^{v s}$. it is the ratio $f^{s} / f_{q}^{v}$ which governs the $x$-dependence of the $R_{(1)}$ (had). so that the latter become vanishingly small for $x \gtrsim 0.2$. Ideally, then, one would perform a future measurement of the deep-inelastic $A_{L A}\left({ }^{2} \mathrm{H}\right)$ in a kinematic region for which $x \geq 0.2$ if one were interested in constraining $S$ and $T$ or other possible extensions of the Standard Model.

The mean value of $x$ for SLAC $A_{L R}\left({ }^{2} \mathrm{H}\right)$ measurements was somewhat below 0.2 . To illustrate the impact of hadronic uncertainties at the SLAC kinematics. we consider a measurement at $x=0.15$. At this kinematic point, the values of the $R_{(1)}$ (new) would be the same as the $R_{(i)}($ had) if one had $S=-1.4$ and/or $T=1.18(i=1)$ and $S=0.31$ and/or $T=-0.68(i=2)$. Of course, it is the uncertainty in the $R_{(1)}($ had) rather than their overall scale that is potentially problematic for the extraction of limits on $S$ and $T$. At $x=0.15$, for example, a non-negligible uncertainty is introduced by the sea-quark distribution functions, parameterized in Ref. [Slo88] by the form $x f^{s}(x)=C_{3}(1+q)(1-x)^{*}$. We take the quoted uncertainty.$\therefore$ y as a rough indication of the overall uncertainty in the $f^{s}(\alpha)$. The corresponding uncertainties induced in $S$ and $T$ via the $\delta R_{(1)}($ had) are $105 . \delta T) \approx(0.46 .0 .39)(i=1)$ and $(\delta S, \delta T) \approx(0.17,0.23)(i=2)$, where the values for $\delta S$ are obtained assuming $\delta T=0$ and vice-versa. These values do not change appreciably at smaller $x$ and fall off by a factor of ten for $x \geq 0.4$. Moreover, they are significantly smaller than the prospective low-energy constraints of Fig. 2.4. A much larger degree of uncertainty in the constraints on $S$ and $T$ is introduced by the experimental error. For the SL.AC data, the experimental uncertainty in the asymmetry $(\sim 10 \%)$ is generally more than an order of magnitude larger than the uncertainty introduced by the quark distribution functions. Consequently, the constraints on $S$ and $T$ from the SLAC experiment are somewhat loose.

A future, more precise measurement of the deep-inelastic deuterium asymmetry could tighten these constraints without requiring substantial improvements in one's knowledge of the quark distribution functions. Such a measurement has recently been proposed for SLAC [Bos92]. The proposed experiment would measure the deep inelastic deuterium asymmetry at several kinematic points having $x \gtrsim 0.2$ with overall uncertainties in the 
asymmetry ranging from one to two percent. In this kinematic range, the impact of $i_{1} x$ uncertainties is negligible. but uncertainties associated with higher-twist contributions could be worrisome Bos92!. The proposal aiso called for measurements of the as:mmet: at several points with $x \leqslant 0.2$, where the sea-quark contributions become impor:ant. The goal of such measurements would be to obtain two new observables which. when combined with other DIS data. could permit a separation of light-quark distribution tunctions. Such a separation m:ght shed light on the violation of the Gottiried Sum Rule 'Got6i' reported by I.IIC Ama91: Although the proposal Bos92| was not approved by the 1392 SLAC PAC a revised version will be submitted to the upcoming PAC [Bos93]. 


\section{IV.I. Atomic Parity Violation}

Atomic PV provides a class of experiments which is sensitive to a rather different set of correction factors and uncertainties than the $P V$.ctron scattering observable. $f_{L R}$. considered so far Bouit. For80. For84. Bou86]. Because of the very small momentum transfers involved in atomic transition matrix elements. atomic $\mathrm{PV}$ is generally less sen. sitive to unknown (or poorly known) hadronic form factors and certain types of radiati:e correction uncertainties than is PV electron scattering. On the other hand. new difficul. ties do arise. including atomic wave function uncertainties :Blu90! and nuclear structure uncertainties. In general, atomic $\mathrm{PV}$ appears to be nicely complementary to $\mathrm{PV}$ electron scattering. and may continue to prove extremely useful in yielding high-precision Standard Model tests.

To illustrate. consider the PV atomic Hamiltonian which mixes opposite-pari? atonic states. and leads to the presence of atomic $\mathrm{PV}^{\circ}$ observables:

$$
\dot{\mathcal{H}}_{\mathrm{atom}}^{P V}=\frac{G_{\mu}}{2 \sqrt{2}} \int d \vec{x} \dot{U}_{\mathrm{e}}(\vec{x}) \cdot \operatorname{s} \dot{C}_{\mathrm{e}}(\vec{x}) \rho \cdot N C_{(\vec{x})}+\cdots .
$$

where $i_{e}(\vec{x})$ is the electron field and $\rho^{v C_{(}}(\vec{x})$ is the Fourier transform of the matrix element of the charge (0) component of the weak neutral current operator (see Eqs. (3.43a) . (3.jia). and $(3.59)$ ). We omit the contribution of the three-vector part of the nuclear current in Eq. (4.96), since it is highly suppressed due to the small effective momentum transfer involved in atomic transitions. The leading term given in Eq. (4.96) is also enhanced relative to omitted nuclear axial-vector terms, at least in heavy atoms. due to the coherent behavior of the nuclear charge operator.

Following Refs. [For90] and [Mus92a], we write the matrix element of $\dot{\mathcal{H}}_{\mathrm{atom}}^{P l}$ between

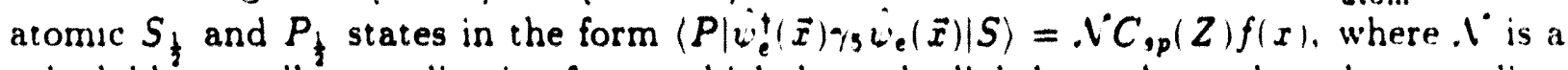
calculable overall normalization factor which depends slightly on the nuclear charge radius. $C_{\text {sp }}(Z)$ is an atomic structure-dependent function, and $f(x)=1-\frac{1}{2}\left(x / x_{0}\right)^{2}+\cdots$ gives the spatial dependence of the electron axial-vector charge density. In a simplified model where a charge- $Z$ nucleus is taken as a sphere of constant electric charge density out to radius $R$, one has $x_{0}=R / Z \alpha$, neglecting small corrections involving the electron mass. In this case, the atomic matrix elements of Eq. $(4.96)$ become

$$
\left\langle P\left|\hat{\mathcal{H}}_{\text {atom }}^{P V}\right| S\right\rangle=\frac{G_{\mu}}{2 \sqrt{2}} \mathcal{N} C_{\text {sp }}(Z)\left[Q_{W}^{(0)}+\Delta Q_{W}^{(n, p)}+\Delta Q_{W}^{(s)}+\Delta Q_{W}^{(T)}\right]+\cdots,
$$

where

$$
\begin{aligned}
Q_{W}^{(0)} & =\frac{1}{2}(Z-. V) \xi_{V}^{T=1}+\frac{1}{2} \sqrt{3}(Z+. V) \xi_{V}^{T=0}, \\
\Delta Q_{W}^{(n, p)} & =\frac{1}{2}\left[\sqrt{3} \xi_{V}^{T=0}+\xi_{V}^{T=1}\right]\left\langle T_{0} \| \sum_{k=1}^{A} \frac{1}{2}\left(1+T_{3}(k)\left|h\left(x_{k}\right) \| T_{0}\right\rangle,\right.\right.
\end{aligned}
$$




$$
\begin{aligned}
& -\frac{1}{2} i \sqrt{3} \xi_{l}^{T=0}-\xi_{i}^{T} T \|\left(\mid T_{0} \| \sum_{k=1}^{t} \frac{1}{2}\left(1-T_{3}(k) \mid h\left(x_{k}\right) \| T_{0}\right), \quad 14.98 \mathrm{~b}\right. \\
& \Delta Q_{\| !}^{(1)}=-\xi_{l}^{(0)}\left(\frac{\rho_{0}}{4 m_{?}^{2}}\right)\left\langle T_{0}\left\|\sum_{k=1}^{t} \Gamma_{k}^{2} h\left(x_{k}\right)\right\| T_{0}\right\rangle \\
& \Delta Q_{W}^{(T)}=\lambda \xi_{i}^{T=1}\left[\left\langle T_{0}\left\|\sum_{k=1}^{t} h\left(x_{k}\right) T_{3}(k)\right\| T_{1}\right\rangle+\left(T_{1}-T_{0}\right)\right]+\cdots
\end{aligned}
$$

are contributions to the so-called "weak charge", with $h(x) \equiv f(x)-1$. and where $\left.T_{0}\|\dot{\mathcal{O}}\| T_{0}\right\rangle$ denotes reduced matrix elements of a nuclear operator $\dot{\mathcal{O}}$ in a nuclear ground state having nominal isospin $T_{0}$.

The term in Eq. (4.98a) is the leading contribution to the weak charge usually dis. cussed in treatments of atomic PV. Including Standard Model radiative corrections in the IIS renormalization scheme, as well as some possible (new) heavy-quark phyisics correc. tions. leads to

$$
\begin{aligned}
Q_{W}^{(0)}= & Z(1-4 \bar{x})\left[1+R_{v}^{p}\left(\text { st }^{\prime} \mathrm{d}\right)+R_{v}^{p}(\text { new })\right) \\
& -N\left[1+R_{v}^{n}\left(\text { st }^{\prime} \mathrm{d}\right)+R_{v}^{n}(\text { new })\right]+\Delta Q_{\text {tsee }}^{\text {new }}(. ., Z)
\end{aligned}
$$

From Ref. (Mar90) one has that

$$
\bar{x} \equiv \sin ^{2} \hat{\theta}_{w}\left(. M_{Z}\right)=0.2323 \pm 0.0007
$$

gives the weak mixing angle and

$$
\begin{aligned}
& R_{v}^{p}\left(\text { st'd }^{\prime}\right) \approx-0.054 \pm 0.033 \\
& R_{v}^{n}(\text { st'd })^{\prime} \approx-0.0143 \pm 0.0004
\end{aligned}
$$

are the Standard Model one-loop corrections in the $\overline{M S}$ scheme. The errors shown in Eqs. (4.100) and (4.101) result from uncertainties in experimental input parameters. and also in evaluations of one-loop diagrams. We note that since the effective momentum transfer for atomic transitions is so small, uncertainties associated with two-photon dispersion corrections should be negligible. This situation contrasts with that of $\mathrm{PV}$ electron scattering from nuclei, where these corrections introduce a non-negligible source of theoretical ambiguity. Contributions from $S$ and $T$, which signal the presence of non-Standard Model physics entering loops, are contained in the $R_{v}^{p, n}$ (new) as indicated in Eq. (3.26). In contrast, the term $\Delta Q_{\text {tree }}^{\text {new }}$ accounts for new physics contributions arising at tree-level, e.g. coming from extra $Z^{0}$ bosons. For example, in $\mathrm{SO}(10)$ models with the exchange of an additional " $Z_{x}$ " boson with no $Z-Z_{x}$ mixing, one has

$$
\Delta Q_{\text {iree }}^{Z_{x}} \approx 0.4(2 . V+Z) \cdot M_{w}^{2} / . M_{Z_{\gamma}}^{2}
$$


as given in Ref. $\mathrm{Mar} 90 \mid$. The value of $د Q_{\text {troe }}^{2}$ one would extract from existing $C$ 's atomic Pl measurements. if all other radiative and heavy physics were ignored. is $2.2=1.6=0.9$. This value corresponds in the $S O(10)$ model to $M_{z_{1}} \approx 300 \mathrm{GeV}$.

It has recently been suggested [Dzu86. .Ion90. Mar90] that one perform measurements of the weak rharge for atoms along an isotopic chain rather than for a single isotope. In the ratio $\left.Q_{W}, Z \ldots I^{*}, Q_{W}, Z, V^{*}\right)$, the coefficient $C_{, p}(Z)$ exactly cancels. thereby eliminat ing much of the atomic physics uncertainties. Moreover. this ratio will carry a different sensitivity to new physics than does $Q_{W}$ for a single isotope. Due to the largeness of.$Z$ in heavy atoms, the weak charge of Eqs. $(4.98)$ and (4.99) is almost completely indepen. dent of $T$. The isotope ratio. however. carries the same relative dependence on $S$ and $T$ as do various $Z^{0}$-pole observables. Isotope ratios also provide a different linear combination of $S . T$. and $J Q_{\text {tree }}^{\text {new }}$ than does $Q_{W}$ for a single isotope. Thus, with a combination of isotope ratio measurements and measurements of other $\mathrm{XC}$ observables 1 e.g... $f_{L R}$. onte might hope to disentangle contributions from various types of physics beyond the Standari Model.

In order for such a scenario to be realized. theoretical uncertainties associated with additional contributions to the weak charge must be resolved. In particular. the term $د Q_{11}^{n} \cdot{ }^{\prime \prime}$ in $E_{q .}$ ( 4.98 ) carries a dependence on the ground-state neutron radius. $R_{n}$. The impact of uncertainties in $R_{n}$, and indeed in the full spatial neutron distribution. on the use of atomic PV for high--precision electroweak tests has been discussed in some detail in Refs. For90, Poi92a]. To illustrate, we use here the simplifying assumptions of Eq. (4.98) for the case of atomic cesium, on which the most recent high-precision experimental measurements have been made. and for which the atomic physics calculations can be done to quite high accuracy. One then needs to know $R_{n}$ to roughly $10 \%$, to reduce the uncertainty induced in $S$ to $\approx \pm 0.6$ (the equivalent to a $1 \% A_{L n}\left(0^{+} 0\right)$ measurement . For ${ }^{209} \mathrm{~Pb}$. another promising future experimental possibility, one needs to know $R_{n}$ to roughly $4 \%$. This requirement is fairly stringent, and the reliability of existing nuclear model predictions for $R_{n}$ may begin to be questioned at such a level. In Ref. Don89?. the idea of using PV elastic electron scattering to determine $R_{n}$ was explored isee also Sect. IV.B. For the case of lead, the conclusion was that a $1 \%$ determination of $R_{n}$ is possible, requ ing under 1000 hours of beam time with experimental conditions which could in principle be provided at CEBAF. It seems likely that a similar determination of $R_{n}$ could be made for cesium. In the case of isotope ratio measurements, one requires knowledge of the change in neutron radii between isotopes, which presents additional challenges to theory and/or electron scattering experiment.

Eq. (4.98) gives the leading contribution to $Q_{W}$ from $G_{E}^{(0)}$. For the case of ${ }^{133} \mathrm{Cs}$, one has under the assumptions leading to Eqs. (4.98d) [.Mus92a]

$$
\Delta Q_{w}^{(g)}=-\left(\frac{3}{4}\right) Z^{2} \cdot A^{1 / 3} \alpha^{2}\left(\frac{1}{m_{, v} r_{0}}\right)^{2} \delta \rho_{s},
$$

* In the $Z_{x}$ model, and indeed in all such models with an extra $U$ (1) symmetry arising from $E_{b}$, the vector coupling of the extra neutral boson to $u$ quarks is zero. Consequently, the ratio of neutron to proton couplings in Eq. (4.102) is exactly $2: 1$, since only $d$ quarks contribute. 
where $r_{0} \approx 1.1 \mathrm{fm}$ and where only the leading term in $h(x)$ has been retained. For a value of $\rho_{s}$ on the order of the prediction of Ref. [Jaf89]. Eq. (4.103) would lead to an error in $\sin ^{2} \theta_{w}$ from $Q_{W}^{(0)}$ of roughly $0.1 \%$. about an order-of-magnitude smaller than the dominant theoretical errors associated with atomic and nuclear structure. Given the constraints on $G_{E}^{(s)}$ likely to be achieved with $A_{L R}$ measurements. nucleon strangeness uncertainties should remain well below a problematic level for electroweak tests with atomic $\mathrm{PV}$. We note further that given the simple additive nature of Eq. (4.98), $G_{\varepsilon}^{(9)}$ contributions to isotope ratios are further suppressed from their already small impact on $Q_{W}$. We also point out that additional contributions to $Q_{w}$ arising from the single-nucleon E.I charge radii are discussed elsewhere [Pol92a], but as an example. the net effect of the nucleon": known internal electromagnetic structure results in a correction of $Q_{W}$ for ${ }^{133} \mathrm{C}$ s of only. approximately $0.1 \%$.

Eq. (4.98d) gives the leading contributions to $Q_{w}$ from isospin impurities in the nuclear ground state. We have shown explicitly only the contribution to $\triangle Q_{(V)}^{(T)}$ arising from the mixing of a single state of isospin $T_{1}$ into the ground state of nominal isospin $T_{n}$ with strength $\lambda$. Further discussion can be found in Ref. [Mus92a], but this term appears likely to be very small in most cases of interest.

There exists another class of possible atomic PV experiments, namely those with muonic atoms. Such experiments may be achievable at the $1-10 \%$ level in the future at PSI [Lan91]. A recent discussion of the effects of radiative corrections and non-Standard Model physics on light muonic atoms may also be found in Ref. [Lan91]. The possibility of performing $\mathrm{PV}$ experiments with heavy muonic atoms has interest from a somewhat different perspective. Because the ratio of Bohr radii for muonic and electron atoms goes as $a_{0}^{e} / a_{0}^{\mu}=m_{\mu} / m_{e} \approx 20 \tau$. the muon is much more tightly bound than the correspond. electron for a given set of orbital quantum numbers. Consequently, one might expect an enhanced sensitivity of $Q_{W}(\mu)$ to short-range effects, such as the ground-state neutron distribution and the nucleon strangeness radius. In the case of the latter, one may estimate the scale of this effect by solving the Dirac equation for a single charged lepton moving in the field of a nuclear sphere of uniform charge and retaining the lepton mass (Mus92c). The resul is to make the replacement $x_{0}=R / Z \alpha \rightarrow\left[3 R / 4 m_{\mu} Z \alpha\right]^{1 / 2}$ in $h(x)$, which yields an enhancement of $Q_{W}^{(s)}(\mu)$ over $Q_{W}^{(s)}(e)$ by roughly $4 m_{\mu} R / 3 Z \alpha$. For atomic cesium. this enhancement factor is $\approx 8$, making $Q_{W}(\mu C s)$ nearly as sensitive to $\rho_{s}$ as is $A_{L R}(\vec{e} p)$. For light muonic atoms, on the other hand, the sensitivity to $\rho_{s}$ is too weak to be observable. In the case of heavy muonic atoms, where nucleon strangeness may be observable, nuclear structure uncertainties also become correspondingly more important. A combination of atomic $\mathrm{PV}$ and electron scattering experiments ( $\mathrm{PV}$ and $\mathrm{PC}$ ) would then appear necessary in order to separate nuclear structure and strangeness contributions. A more thorough investigation of this possibility is in progress [Mus93d]. From a theoretical standpoint. muonic atoms have the additional advantage of being essentially a one-lepton problem. making a determination of the muon wave function much more straightforward than for electron atoms.

In summary, apart from questions of atomic theory and important uncertainties in nuclear structure, the unique dependencies on new physics means that atomic PV experiments can yield additional high-precision Standard .lodel tests, unattainable from 
direct high-energy experiments at the $Z^{0}$-pole. In addition, the relative insensitivity to certain classes of radiative corrections, and hadronic form factors. makes atomic PV nicely complementary to PV electron scattering. 


\section{IV.J. Neutrinos}

The subject of neutrino scattering from nuclei is sufficiently broad and detailed that one could devote an entire review article to it alone. Indeed, when considering the full range of neutrino beam energies, one encounters a variety of physics issues which may be addressed in this way. including charge-changing deep inelastic scattering measurements aimed at testing the Standard Model or probing quark distributions at high-energies and low-to-intermediate energy inelastic excitations of nuclear levels as a means of studying the nuclear weak current. Given the existence in the literature of other reviews of semileptonic neutrino reactions (see, for example, [Don 79 a]), we limit the present discussion to those aspects of low- and intermediate-energy neutrino-hadron . $\mathrm{IC}$ processes not considered in previous studies. In particular, we focus on the use of neutrinos to probe nucleon strangeness content. noting the relative advantages or disadvantages this offers when compared with PV electron scattering. We also pay particular attention to three low- and intermediate-energy neutrino . $\mathrm{NC}$ experiments: the recently completed Brookhaven (B.NL, $\nu-\mathrm{p} / \bar{\nu}-\mathrm{p}$ experiment [Ahr87], the KARMEN experiment involving inelastic neutrino excitation of a discrete state in ${ }^{12} \mathrm{C}$ [KAR92] and the LS.ND experiment at LA.MPF [Lou89] currently in progress.

As with PV electron scattering, it is important when reviewing neutrino scattering prospects to keep considerations of doability firmly in mind. For the energies and momentum transfers of interest here, typical cross sections $d \sigma / d Q^{2}$ range from $\sim 10^{-38} \rightarrow$ $10^{-40} \mathrm{~cm}^{2}(\mathrm{GeV} / \mathrm{c})^{-2}$. We shall return in Sect. V.A.2 to discuss the existing or planned neutrino facilities (see Table 5.3 for characteristics of these facilities). Typically the fluxes of neutrinos or antineutrinos obtained are about $10^{7} \mathrm{~cm}^{-2} \mathrm{~s}^{-1}$, occasionally somewhat more or somewhat less. (We shall not discuss the subject of reactor antineutrino physics where considerably larger fluxes and much smaller cross sections than those encountered below are relevant - see Ref. [Don79a] for further treatment of this subject.) To set the scale of neutrino-nuclear physics let us assume a target with $10^{31}$ protons (typically a target+detector will have about $10 \%$ hydrogenic protons) and consequently would weigh about 200 tons - for example, as does the LSND detector discussed in Sect. V.C.J. This yields an effective neutrino-proton luminosity of $10^{38} \mathrm{~cm}^{-2} \mathrm{~s}^{-1}$. For a typical $\nu-p$ total cross section of $10^{-40} \mathrm{~cm}^{2}$ [Don83] this yields an event rate of $10^{-2} \mathrm{~s}^{-1}$. To accumulate $10^{4}$ events and thus reach the level of $1 \%$ statistical error needed to make such measurements relevant as probes of strangeness or non-standard physics would take $278 \mathrm{hr}$. Naturally the running time will scale with the flux and consequently will vary with the nature of the neutrino-producing facility (see Table 5.3 ). Additionally, the $\nu-p$ total cross section varies with energy from about $0.2 \times$ the above number for beam stop neutrino sources to a few $\times$ the above number for DIF facilities [Don83]. Accordingly, the typical running time for $1 \%$ statistics goes from a few hundred to a few thousand hours. The level of precision realistically achievable depends on kinematics and neutrino species $(\nu$ or $\bar{\nu})$. Similarly, the sensitivity of cross sections to different form factors or electroweak couplings is also highly dependent on the same factors. Consequently, any discussion of the interpretation of neutrino cross sections must take into account considerations of experimental doability. 


\section{IV.J.1. GE.VERAL FEATLRES}

The summary of accessible kinematic regimes for neutrino beams given in Table 5.3 points to an important difference between neutrino and electron scattering experiments. In the latter instance. one may tune $\epsilon$ and $\theta$ to define a rather narrow kinematic window and thereby perform Rosenbluth-type separations and. in so-doing, highlight contributions from different pieces of the hadronic $\mathrm{NC}$. Neutrino scattering experiments. on the other hand. integrate over a range of energies about some peak energy as determined by the neutrino spectrum $O(\epsilon)$. The corresponding inclusive observable is the energy-integrated differential cross section

$$
\overline{\frac{d \sigma}{d Q^{2}}}=\int_{\epsilon_{1}}^{\epsilon_{2}} \phi(\epsilon) \frac{d \sigma(\epsilon)}{d Q^{2}},
$$

where $d \sigma(\epsilon) / d Q^{2}$ is the energy-dependent $\nu$ or $\bar{\nu}$ differential cross section and $\left(\epsilon_{1}, \epsilon_{2}\right)$ define the range of available beam energy for a given facility (see Table 5.3). For a given value of $Q^{2}$. then. the integral in Eq. (4.104) effectively integrates over the lepton scattering angle. thereby precluding the possibility of making a Rosenbluth-type separation of the inclusive cross section. Consequently, the strategy appropriate to neutrino scattering experiments is to consider measurements at a variety of facilities, thereby accessing different $\epsilon$ and $Q^{2}$ regimes, in order to emphasize in Eq. (4.104) the dependence on different form factors or electroweak couplings. Lq. (4.104) also points to the importance of one's knowledge of the neutrino spectrum $\phi(\epsilon)$ in the interpretation of the measured cross section.

When studying electron scattering or charge-changing neutrino/antineutrino reactions it is possible to detect the final-state charged lepton and therefore inclusive cross sections can be measured. In contrast, for neutrino/antineutrino scattering it is not practical to detect the scattered lepton and hence some hadronic signature must be found to know that a scattering event has occurred. This means that a different kind of inclusive cross section must be studied: the scattered neutrino is not detected (and thus the cross section is in general inclusive in the leptonic sector), whereas some hadronic final-state particle detection is involved (and thus the cross section is not inc.usive in the hadronic sector). In other words, the measurement is more like a coincidence (semi-inclusive) electron scattering experiment where the srattered electron is not detected, viz., more like nuclear photoreactions.

In the case of elastic neutrino scattering, for example, one typically measures tile nuclear recoil having kinetic energy $T_{R}$ and angle $\theta_{R}$ relative to the incident beam direction. as discussed in Sect. III.E.3 (see Eqs. (3.151)). To set the scale for the subject of the next subsection, in Table 4.1 we give the accessible ranges in $Q^{2}, \tau$ and $T_{N}$ and their equivalents in $\theta_{N}$ and $\theta$ for a range of values of $\epsilon$ available at different facilities ranging from low-energies (beamstop facilities) to medium-energies (decay-in-flight (DIF) facilities) for neutrino-nucleon elastic scattering. Following the discussion of $\nu-\mathrm{N}$ elastic scattering in Sect. IV.J.2, in Sects. IV.J.3 and IV.J.4 we return to consider elastic scattering from the deuteron and from spin-0 nuclei such as ${ }^{4} \mathrm{He}$, respectively.

In the case of inelastic neutrino or antineutrino scattering alternatives to detecting the recoiling target nucleus can be sought. For example, as discussed in more detail in 


\begin{tabular}{|c|c|c|c|c|c|}
\hline \multicolumn{6}{|c|}{ TABLE 4.1} \\
\hline $\begin{array}{l}\text { Seutrino energy } \\
\qquad(\mathrm{MeV})\end{array}$ & $\theta$ range & $\theta_{. v}$ range & $\begin{array}{l}\left|Q^{2}\right| \text { range } \\
(\mathrm{GeV} / \mathrm{c})^{2}\end{array}$ & $\tau$ & $\begin{array}{c}\text { Recoil energy } \\
\text { MeV }\end{array}$ \\
\hline 35 & $0^{\circ} \rightarrow 180^{\circ}$ & $90^{\circ} \rightarrow 0^{\circ}$ & $0 \rightarrow 0.00046$ & $0 \rightarrow 0.0013$ & $0 \rightarrow 2.43$ \\
\hline 50 & $0^{\circ} \rightarrow 180^{\circ}$ & $90^{\circ} \rightarrow 0^{\circ}$ & $0 \rightarrow 0.0090$ & $0 \rightarrow 0.0026$ & $0 \rightarrow 4.81$ \\
\hline 100 & $0^{\circ} \rightarrow 180^{\circ}$ & $90^{\circ} \rightarrow 0^{\circ}$ & $0 \rightarrow 0.033$ & $0 \rightarrow 0.0093$ & $0 \rightarrow 17.6$ \\
\hline 200 & $0^{\circ} \rightarrow 180^{\circ}$ & $90^{\circ} \rightarrow 0^{\circ}$ & $0 \rightarrow 0.112$ & $0 \rightarrow 0.032$ & $0 \rightarrow 59 . \bar{\imath}$ \\
\hline 500 & $0^{\circ} \rightarrow 180^{\circ}$ & $90^{\circ} \rightarrow 0^{\circ}$ & $0 \rightarrow 0.484$ & $0 \rightarrow 0.137$ & $0 \rightarrow 257.9$ \\
\hline 1000 & $0^{\circ} \rightarrow 180^{\circ}$ & $90^{\circ} \rightarrow 0^{\circ}$ & $0 \rightarrow 1.278$ & $0 \rightarrow 0.362$ & $0 \rightarrow 680 . j$ \\
\hline
\end{tabular}

Table 4.1 Achievable $\left|Q^{2}\right|, \tau$, recoil energy $\left(T_{N}\right)$, together with equivalent recoil nucleon scattering angle $\left(\theta_{N}\right)$ and (undetected) neutrino scattering angle $(\theta)$ for various neutrino energies, $\epsilon$ (see also Table 5.3). The target is assumed to be a nucleon at rest.

Sect. IV.J.J, it is possible to excite a discrete state in a nucleus via inelastic neutrino scattering and then detect the decay of that state, say by the emission of a photon. In a sense such measurements involve "nothing in" and "nothing out" except the emission (when the neutrino-producing beam is on) of a photon. Of course, other final-state hadronic signals may be sought (emission of an $\alpha$-particle, ejection of a proton or neutron, etc.). Some of these are discussed in Ref. [Don79a] and the references contained therein. We shall return in Sect. IV.J.5 to consider only a few cases to illustrate the basic nature of such neutrino-nuclear studies.

Let us note for the present that even the above classes of measurements are not always so cleanly separated when real experiments are contemplated. For instance, for purposes of interpreting elastic $\nu-\mathrm{N}$ and $\bar{\nu}-\mathrm{N}$ cross section measurements, one faces a third level of complication not encountered in elastic PV $\overrightarrow{e p}$ scattering. Typically, the elastic neutrinonucleon cross section must be extracted from a measurement of the quasielastic $A(\nu, N) A^{\prime}$ cross section, where $A$ and $A^{\prime}$ denote target and daughter nuclei. In the BNL $\nu-p / \bar{\nu}-\mathrm{p}$ experiment, for example, $80 \%$ of the "elastic" events actually involved scattering from protons bound in ${ }^{12} \mathrm{C}$ nuclei. The remaining $20 \%$ resulted from scattering from ${ }^{1} \mathrm{H}$ nuclei in the $\mathrm{CH}_{2}$ target. Extraction of the elastic $\nu-.$. cross section from the $A(\nu, N) A^{\prime}$ reaction generally introduces more theoretical uncertainty than would enter the extraction from the quasielastic inclusive $A\left(\nu, \nu^{\prime}\right) X$ reaction, were a measurement of the latter possible. 
Interpretation of the former process, which is semi-inclusive. requires knowledge of the final-state interactions between the outgoing nucleon and residual nucleus. In contrast the inclusive quasielastic measurement involves detection of the outgoing lepton (assuming such were possible - as it is for the charge-changing neutrino and antineutrino reactions where a charged lepton is produced). and no knowledge of the hadronic final-state interactions is needed in the interpretation. An indication of the scale of theoretical uncertainties in the interpretation of $\left.A(\nu, .)^{\prime}\right) A^{\prime}$ reactions might be obtained from analyses of quasielastic $A\left(e, e^{\prime} \vec{p}\right) \because^{\prime \prime}$ coincidence cross sections. Typically the final-state interactions play a very importan. role in interpreting such electromagnetic coincidence cross sections. but play a much less significant part in determining the inclusive $A\left(e . e^{\prime}\right)$ cross section. Quantifying this statement requires a case-by-case analysis and lies beyond the context of the present review. However. one can get some sense of the importance of final-state interactions by noting that for coincidence electron scattering the DWIA (where final-state interactions are included) differs from the PWIA (where they are not) typically by factors of about 0.6-0.7 (see. e.g.. [Fru84]). while for inclusive scattering much smaller modifications from final-state interaction effects are usually found. We shall return briefly to this point in the next section when discussing the ideas put forward by the authors of Ref. [Gar32].

\section{IV.J.2. ELASTIC NELTRINO-.VTCLEON SCATTERING}

The aforementioned theoretical challenges notwithstanding, measurements of elastic and low-lying inelastic neutrino cross sections offer a number of potential advantages. We focus first on elastic neutrino-nucleon scattering, for which the primary attraction is its sensitivity to $\tilde{G}_{A}^{\mathrm{v}}$. Unlike its contribution to $A_{L R}(\vec{e} N)$, which is suppressed by $g_{v}^{e}=$ $-1+4 \sin ^{2} \theta_{w} \approx-0.092$. the $\tilde{G}_{A}^{v}$ contribution to $d \sigma^{\nu(\tilde{\nu})} / d Q^{2}$ receives no such suppression. . Woreover, the interpretation $\tilde{G}_{A}^{N}$, as determined by neutrino scattering, is theoretically less ambiguous than in the case of PV electron scattering. Recall from Eq. (3.31) that one has $\tilde{G}_{A}^{N}=\xi_{A}^{T=1} G_{A}^{(3)} \tau_{3}+\xi_{A}^{T=0} G_{A}^{(8)}+\xi_{A}^{(0)} G_{A}^{(s)}$, where $G_{A}^{(3)}$ and $G_{A}^{(8)}$ may be determined from neutron and hyperon semileptonic decays, respectively, and where the $\xi_{A}^{(a)}$ are renormalized electroweak axial-vector $\mathrm{NC}$ couplings determined by the underlying gauge theory. As noted earlier, $\xi_{A}^{T=0}$ vanishes at tree-level in the Standard Model, but becomes nonzero once electroweak radiative corrections are included. For PV electron scattering, these corrections, which enter all three of the $\xi_{A}^{(a)}$, are enhanced over the generic $\alpha / 4 \pi$ scale and contain theoretical hadronic uncertainties on the same scale. For neutrino scattering, on the other hand, the corrections are significantly smaller and more reliably calculable. Consequently, an extraction of $\tilde{G}_{A}^{N}$ from the elastic neutrino-nucleon cross section offers a theoretically "cleaner" probe of, e.g., axial-vector nucleon strangeness, than does the corresponding determination from $A_{L R}(\vec{e} p)$.

In addition to its dependence on $G_{A}^{(s)}$, the energy-integrated elastic $\nu-p(\nu-n)$ cross section displays a non-negligible dependence on other quantities of interest, such as the electroweak couplings $\xi_{v}^{p}\left(\xi_{v}^{n}\right)$, the axial-vector dipole mass parameter (see Eq. (3.45)), and the vector current strangeness form factors (see Sect. III.C). For low-energy, low 
momentum transfer scattering. one may expand the cross section in Eq. (3.161) to leading order in $\epsilon / m_{v}$ and $\tau$ and obtain [Gar92]

$$
\begin{aligned}
\frac{d \sigma}{d Q^{2}}(\epsilon)=\frac{G_{\mu}^{2}}{8 \pi} & {\left[\left(1+\rho^{2}\right)\left(\tilde{G}_{A}^{\mathrm{N}}\right)^{2}+\left(1-\rho^{2}\right)\left(\tilde{G}_{E}^{\mathrm{v}}\right)^{2}\right.} \\
& \left.-4 \rho \sqrt{\tau}\left\{\frac{1}{2}\left(\left(\tilde{G}_{A}^{\mathrm{N}}\right)^{2}+\left(\tilde{G}_{E}^{\mathrm{N}}\right)^{2}\right) \pm \tilde{G}_{A}^{\mathrm{N}} \tilde{G}_{M}^{\mathrm{N}}\right\}\right] .
\end{aligned}
$$

where

$$
\rho \equiv \sqrt{\tau}\left(\frac{m_{v}}{\epsilon}\right)=\sqrt{\left|Q^{2}\right| / 4 \epsilon^{2}}=\sqrt{1+\tau} \cos \theta_{v}-\sqrt{\tau}
$$

so that $0 \leq \rho \leq\left[1+2 \epsilon / m_{\mathrm{v}}\right]^{-1 / 2}$. From Eq. (4.105) one may observe the relative importance of the axial-vector form factor in comparison with contributions from others, especially for target protons. In this case, the. $\mathrm{XC}$ electric form factor of Eq. (3.31) is suppressed due to the $\xi_{V}^{p}=\left(1-4 \sin ^{2} \theta_{w}\right)$ coefficient of $G_{E}^{p}$ and the leading $Q^{2}$-dependence of $G_{E}^{n}$ and $G_{E}^{\prime}$ as they enter $\tilde{G}_{E}^{p}$. The contribution from $\tilde{G}_{M}^{\mathrm{N}}$ to Eq. $(4.105)$ is suppressed by $\sqrt{\tau}$ relative to the leading $\tilde{G}_{A}^{N}$ contribution. Hence, one expects $\tilde{G}_{A}^{N}$ to dominate the cross section at low-energy and low momentum transfer [Don74]. The situation for target neutrons is somewhat different, since the leading contribution to $\tilde{G}_{\varepsilon}^{n}$ is $\xi_{v}^{n} G_{E}^{p}$, which is not suppressed.

To illustrate the sensitivities of the elastic $\nu-p$ and $\bar{\nu}-\mathrm{p}$ differential cross sections in various kinematic regimes, we give in Table 4.2 the fractional shift in $d \sigma^{\nu(\nu)}(\epsilon) / d Q^{2}$ due to variations in several parameters of interest. The higher energy and momentum transfer results correspond to the BNL kinematics, while those for lower energies and $\left|Q^{2}\right|$ are appropriate to the LS.ND measurement. Although the BNL experiment integrated over beam energy from 0.2 to $5.0 \mathrm{GeV}$, the spectrum $\phi(\epsilon)$ was peaked in the neighborhood of $1 \mathrm{GeV}$. One expects the sensitivities at this energy, then, to represent rather fairly the sensitivity of the energy-integrated cross section.

From the entries in Table 4.2, one may observe immediately the level of precision required for a meaningful electroweak test. In all but a few cases, measurements of $d \sigma(\epsilon) / d Q^{2}$ to better than $1 \%$ precision are needed to determine $\xi_{v}^{p}$ to $10 \%$ (the precision assumed in deriving the corresponding bands in Fig. 2.4). Since the cross sections were determined to $\sim 10 \%$ precision in the BNL experiment, while the LSND expectation is for a $10 \%$ determination of the cross section, significantly higher precision experiments would be required at existing or future facilities to make a meaningful extraction of $\xi_{v}^{p}$. For the form factors, on the other hand, significant constraints could be achieved under realistic experimental conditions. In the case of $G_{M}^{(s)}$, for example, a modest improvement in statistics over the BNL experiment could lead to a determination of $\mu_{\mathrm{s}}$ at a level comparable to the projected SA.MPLE constraints. In contrast, the sensitivity to $G_{E}^{(0)}$ appears to be well below the observable level. Given this insensitivity, we have not included in Table 4.2 the shifts associated with variations in $\lambda_{E}^{(0)}$. The cross sections display the greatest sensitivity to variations in $G_{A}^{(s)}$, assuming its current level of uncertainty. As the sensitivity to $\lambda_{D} \hat{b}$ makes clear, it is desirable to attempt a determination of $\eta_{s}$ at lower energy and momentum transfer. At the BNL kinematics, for example, the variation associated with the 


\begin{tabular}{|c|c|c|c|c|}
\hline \multicolumn{5}{|c|}{ TABLE 4.2} \\
\hline$\left(\epsilon .: Q^{2} \mid\right)$ & Parameter & range & $\begin{array}{c}\text { variation in } \\
d \sigma^{\nu}(\epsilon) / d Q^{2}(\%)\end{array}$ & $\begin{array}{l}\text { variation in } \\
d \sigma^{\nu}(\epsilon) / d Q^{2}\left(\psi_{1}\right)\end{array}$ \\
\hline$(1.0,0.5)$ & $\xi_{v}^{p}$ & $0.1003 \rightarrow 0.0821$ & $0.7 \rightarrow-0.6$ & $-0.6 \rightarrow 0 . \bar{j}$ \\
\hline$(1.0,0 . \overline{0})$ & $\mu_{s}$ & $-0.2 \rightarrow 0.2$ & $6 \rightarrow-5$ & $-4-5$ \\
\hline$(1.0 .0 .5)$ & $\rho_{s}$ & $-1.4 \rightarrow 1.4$ & $0.3 \rightarrow-0.7$ & $0.6 \rightarrow-2$ \\
\hline$(1.0,0.5)$ & $\eta_{\mathbf{s}}$ & $-0.12 \pm 0.07$ & $14 \mp 8$ & $25 \mp 15$ \\
\hline$\therefore .0,0.5)$ & $\lambda_{D}^{A}$ & $3.12 \rightarrow 3.52$ & $5.7 \rightarrow-5.3$ & $10.1 \rightarrow-9.0$ \\
\hline$(1.0,1.0)$ & $\xi_{V}^{p}$ & $0.1003 \rightarrow 0.0821$ & $0.8 \rightarrow-0.7$ & $-2.1 \rightarrow 2.2$ \\
\hline$(1.0,1.0)$ & $\mu_{s}$ & $-0.2 \rightarrow 0.2$ & $6 \rightarrow-6$ & $-1 \bar{u} \rightarrow 18$ \\
\hline$(1.0,1.0)$ & $\rho_{\mathbf{s}}$ & $-1.4 \rightarrow 1.4$ & $0.2 \rightarrow 0.6$ & $1.3 \rightarrow 5.1$ \\
\hline$(1.0 .1 .0)$ & $\eta_{\mathbf{s}}$ & $-0.12 \pm 0.07$ & $13 \mp 7$ & $40 \mp 25$ \\
\hline$(1.0 .1 .0)$ & $\lambda_{D}$ & $3.12 \rightarrow 3.52$ & $8.2 \rightarrow-7.2$ & $24.5 \rightarrow-19.9$ \\
\hline$(0.15,0.05)$ & $\xi_{v}^{p}$ & $0.1003 \rightarrow 0.0821$ & $0.4 \rightarrow-0.3$ & $-0 . \overline{5} \rightarrow 0 . \overline{5}$ \\
\hline$(0.15 .0 .05)$ & $\mu_{s}$ & $-0.2 \rightarrow 0.2$ & $3 \rightarrow-3$ & $-4 \rightarrow 4$ \\
\hline$(0.15 .0 .05)$ & $\rho_{s}$ & $-1.4 \rightarrow 1.4$ & $0.07 \rightarrow 0$ & $0.05 \rightarrow-0.03$ \\
\hline$(0.15,0.05)$ & $\eta_{0}$ & $-0.12 \pm 0.07$ & $17 \mp 10$ & $25 \mp 15$ \\
\hline$(0.15,0.05)$ & $\lambda_{D}$ & $3.12 \rightarrow 3.52$ & $0.9 \rightarrow-0.9$ & $1.3 \rightarrow-1.3$ \\
\hline
\end{tabular}

Table 4.2 Variation in elastic $\nu-p$ and $\bar{\nu}-p$ differential cross section associated with uncertainiies in electroweak couplings and form factor parameters (units: $\epsilon[\mathrm{GeV}],\left|Q^{2}\right|$ $\left.\left[(\mathrm{GeV} / \mathrm{c})^{2}\right]\right)$. The final two columns give the percent deviation from nominal Standard Model, no-strange predictions for the cross sections. The central value of the axialvector dipole parameter $\lambda_{D}=3.32$ corresponds to $M_{A}=1.032 \mathrm{GeV}$. Variations due to $G_{\Sigma}^{(s)}$ uncertainty were computed assuming $\lambda_{E}^{(0)}=0$. 
present uncertainty in $\lambda_{D}^{A}$ is comparable to that associated with the error in $G_{A}^{\text {" ' }}$ ( using the B.NI results 1. whereas at the LS.ND kinematics, the $\lambda_{D}^{A}$-variation is an order-of-magnitude smaller than that associated with $\eta_{\mathbf{s}}$. To improve upon the B.VL $G_{A}^{(\mathbf{s})}$ constraints. however. a measurement of $d \sigma^{\nu(\hat{\nu})}(\epsilon) / d Q^{2}$ at LS.ND kinematics would need to be carried out with better precision than presently projected. We note in passing that an analysis similar to the above was carried out in Ref. [Bei9la]. In the latter work. the shift in $c \sigma^{\nu(\nu)}(\epsilon) / d Q^{2}$ from the zero-strangeness predictions are given. assuming the predictions of Ref. .Jaf 89 ;: for the vector current form factors and the E.MC value for $\eta_{\text {s }}$ (see Table 2.3).

Any extraction of constraints on form factors and couplings must. of course. account for simultaneous uncertainties in the parameters of interest. These currelations are somewhat more complicated than in the case of $\mathrm{PV}$ electron scattering. where the $\mathrm{IC}^{\circ} \mathrm{C}$ form factors and electroweak couplings enter $A_{L R}$ linearly. In contrast, the differential $v-$. and $\bar{\nu}-. \dot{ }$ cross sections are quadratic in these quantities. so that the correlations define generalized conic sections in a multi-dimensional parameter space. To illustrate. we consider elastic $\nu-p$ and $\bar{\nu}-p$ measurements at the B.NL kinematics. The B.IL experiment was originally analyzed assuming that all vector strangeness form factors vanish identically. A reanalysis of the data allowing for nonzero $G_{E}^{(0)}$ and $G_{M}^{\left({ }^{\prime}\right)}$ would require detailed knowledge of the neutrino energy spectra. normalizations, systematic error correlations. as well as other systematics including charge-changing and quasielastic cross sections for calibration, etc. Such a reanalysis has in fact recently been carried out by the authors of Ref. [Gar93]. but we wish to examine here. in a more general way, the sensitivity of these data to the strangeness form factors and electroweak couplings.

To this end, we generate "fictitious" data points by assuming the Standard Nodel with no strangeness in the nucleon and add arbitrary random statistical errors of $\mathcal{O}(10 \%$, (roughly the same magnitude as the actual BNL errors) to $d \sigma / d Q^{2}$ for the same kinematic points as in the B.NL experiment. Performing a $\chi^{2}$-minimization on this fictitious data set to fit different input parameters then yields an effective uncertainty for the parameters considered. This procedure affords at least an estimate of the scale of the uncertainties and correlations that a more complete reanalysis would provide. At the same time. this procedure neglects systematic errors, so that the uncertainty estimates provided below should be considered underestimates.

We chose as parameters to be varied $\xi_{V}^{p}, \eta_{s}, \mu_{s}, \rho_{s}$, and $M_{A}$. For simplicity, we did not consider additional uncertainties associated with the $\lambda_{E . M}^{(s)}$. Setting all but one of the parameters to their nominal Standard. Model values and allowing the last to vary. we take as the statistical uncertainty for a one-parameter fit the range in this parameter which keeps $x^{2}$ within one unit of its minimum. Allowing a second parameter to vary leads to a $67 \%$ confidence contour whose extrema define the correlated uncertainties. For example, allowing only $\xi_{v}^{p}$ to vary gives about a $44 \%$ uncertainty in this parameter*. Allowing $\eta$, to vary as well gives $\delta \xi_{V}^{P} / \xi_{V}^{p} \approx 0.66$ at $67 \%$ confidence (see Fig. 4.13). A three-parameter fit in which $M_{A}$ is also allowed to vary leads to an $88 \%$ uncertainty in $\xi_{v}^{p}$. From these results

* Recall that a $10 \%$ uncertainty in $\xi_{v}^{p}$ corresponds roughly to a $1 \%$ uncertainty in $\sin ^{2} \theta_{w}$ 
one would conclude that a future medium-energy $\nu-p / \bar{\nu}-p$ elastic scattering experiment would have to be performed with significantly higher precision than obtained in the B.IL experiment in order to constrain new physics at a level competitive with atomic PV or prospective PV electron scattering experiments.

The situation with regard to the strangeness form factor constraints is somewhat more hopeful. In Table $\downarrow .3$ we summarize these prospective constraints. allowing for correlated uncertainties among the various form factors assuming $s_{t}^{p}$ takes on its Standard Model value.

\begin{tabular}{|c|c|c|c|}
\hline \multicolumn{3}{|c|}{ TABLE 4.3 } \\
\hline Case & Varied Parameters & Experimental Uncertainty & Constraints \\
\hline B.iL & $M_{A}, \eta_{\mathbf{s}}$ & $\pm 10 \%$ in $\nu, \bar{\nu}$ cross sections & $\delta \eta_{\mathbf{s}}= \pm 0.12$ \\
& & & $\delta . M_{A}=0.1$ \\
\hline B.IL & $M_{A}, \eta_{\mathbf{s}}, \mu_{\mathbf{s}}$ & $\pm 10 \%$ in $\nu, \bar{\nu}$ cross sections & $\delta \eta_{\mathbf{s}}= \pm 0.16$ \\
& & & $\delta . M_{A}=0.1$ \\
& & & $\delta \mu_{\mathbf{s}}=0.2$ \\
\hline B.NL & $\rho_{\mathbf{s}}, \mu_{\mathbf{s}}, \eta_{\mathbf{s}}$ & $\pm 10 \%$ in $\nu, \bar{\nu}$ cross sections & $\delta \rho_{\mathbf{s}}= \pm \overline{0} .0$ \\
& & & $\delta \mu_{\mathbf{s}}= \pm 0.3$ \\
& & & $\delta \eta_{\mathbf{s}}= \pm 0.06$ \\
\hline LS.ND & $\eta_{\mathbf{s}}$ & $\pm 18 \%$ in $\nu$ cross section & $\delta \eta_{\mathbf{s}}= \pm 0.1$ \\
\hline
\end{tabular}

Table 4.3 Multi-parameter fits to "fictitious" BNL and LSND data, assuming $\xi_{v}^{p}$ at its Standard Model value.

From the three-parameter fit in Table 4.3, we observe that the constraint $\delta \mu_{\mathrm{s}}= \pm 0.3$ is nearly as stringent as that expected from the SAMPLE experiment $\left(\delta \mu_{s} \approx \pm 0.2\right)$. The recent reanalysis of the actual BNL data by (Gar93) gives error estimates quite compatible with the above numbers, if somewhat larger due to their inclusion of the (systematic) normalization uncertainties. In contrast, the uncertainty in $\rho_{\mathbf{s}}$ is nearly three times less 
stringent than would be achievable from a series of $A_{L R}(\vec{e} p)$ measurements alone. The constraint on $\eta_{*}$. achieved for fixed $V_{A}$. could not be approached by PV electron scattering. given the large axial-vector radiative correction uncertainties arising in the latter. experiment, where the impact of Since the $\eta$, uncertainty increases in a two-parameter $\|_{t}-\eta$, fit. it is desirable to carry out a determination of $G_{A}^{(3)}$ at the lower-energy and lower- $Q^{2}$ of the LS.ID experiment, where the impact of $V_{A}$ uncertainties on the differential cross sertion is negligible ( see Table 4.2 ). However, the projected $20 \%$ statistical error for the LS.'D determination of the cross section corresponds to an uncertainty in $\eta$, only slightly better than the uncertainty taken from the fictitious B.IL data allowing for the correlation with . $H_{A}$ [Bei9la].

In order to achieve or surpass the aforementioned form factor constraints. improve. ments in both experimental precision and the reliability of theoretical modeling would be required. Prospects for experimental progress are discussed in Sect. V. As far as thet) retical analysis is concerned. contributions from final-state hadronic interactions render the extraction of $d \sigma(\nu . \dot{V}) / d Q^{2}$ from the $\left.A(\nu . .)^{\prime}\right) A^{\prime}$ cross section theoretically problem. atic, as discussed above. It may prove advantageous to make use of existing analyses of $A\left(e, e^{\prime} . V\right) . f^{\prime}$ reactions to "calibrate" the hadronic physics and thus. at least to some extent, to remove the final-state interaction effects as uncertainties. Given the possibility of high-precision measurements in the future, more theoretical work on these issues will have to be undertaken.

In order to minimize the impact of some of these ambiguities. the authors of Ref. [Gar92] have proposed measuring the ratio $R(\epsilon)$ of proton to neutron vields in quasielastic nucleon knockout. Assuming the static approximation (initial nucleon at rest and neglecting final-state interactions, one has

$$
R(\epsilon)=\frac{\int_{\left|Q_{1}^{2}\right|}^{\left|Q_{2}^{2}\right|}\left\{d \sigma^{\nu P}(\epsilon) / d Q^{2}\right\} d Q^{2}}{\int_{\left|Q_{1}^{2}\right|}^{\left|Q_{1}^{2}\right|}\left\{d \sigma^{\nu n}(\epsilon) / d Q^{2}\right\} d Q^{2}} .
$$

These authors compute $R$ for $\epsilon=200 \mathrm{MeV}$ and detection of outgoing nucleons in the energy range $50 \leq T_{N} \leq 59.7 \mathrm{MeV}$. Assuming quasifree kinematics and taking the initial nucleon to be at rest, this spread corresponds to a range in $\left|Q^{2}\right|$ of $0.094 \leq\left|Q^{2}\right| \leq 0.112$ $(\mathrm{GeV} / \mathrm{c})^{2}$ or, from Eq. $(3.151)$, a range in forward angles of $23.3^{\circ} \geq \theta_{N} \geq 0^{\circ}$. The resultant value of $R$ depends on the strangeness parameters $\mu_{\text {s }}$ and $\eta_{\text {, as }}$

$$
R \approx 1-3.16 \eta_{\mathrm{\rho}}-0.362 \mu_{\mathrm{s}}+0.073 \eta_{\mathrm{s}}^{2}+0.024 \eta_{\bullet} \mu_{\mathrm{\rho}},
$$

where in accordance with Table 4.2 the dependence on uncertainties in $M_{A}$ has been neglected. The authors also point out that $R$ is insensitive to uncertainties in neutrino flux, since the same flux is used in extracting both proton and neutron knockout cross sections and then forming the ratio. Furthermore, $R(\epsilon)$ carries only a gentle dependence on $\epsilon$ for $160 \leq \epsilon \leq 240 \mathrm{MeV}$, so that one need not possess precise knowledge of $\phi(\epsilon)$ in order to interpret this ratio. One might also hope that at these kinematics, the final-state interactions for outgoing protons and neutrons behave similarly, so that their impact on $R$ 
would be much smaller than on the individual cross sections. Perhaps the most important feature of Eq. $(+.108)$ is its large sensitivity to $\eta_{s}$. Indeed. a $10 \%$ determination of $R$ could constraint $\eta_{\text {s }}$ to $i \eta_{\mathrm{s}}= \pm 0.03$, while the error induced in $\eta_{\text {, from }} \delta \mu$, assuming the S.A.IPLE projections would be roughly $\delta \eta_{\mathrm{\rho}}= \pm 0.02$. Hence. further analysis of the impact of final-state interactions on the interpretation of $R$, as well as exploration of experimental feasibility, appears to be warranted.

\section{IV.J.3. EL.ASTIC SCATTERING FROII $\mathrm{He}$}

The basic formalism for elastic neutrino and antineutrino scattering from spin-0 nuclei has been introduced in Sect. III.E.3. In Ref. [Don83] a selection of targets all with spin-0 except ${ }^{2} \mathrm{H}$. which was also studied in that work - see the following discussions in Sect. [V.J.4) was investigated with an eye to the problem of elastic scattering. The genera! characteristics of coherent scattering involve a recoil kinetic energy which falls with mass number as $t^{-1}$. but a cross section which increases with the square of a combination of the proton (atomic) and neutron numbers. $\left(Z \xi_{V}^{p}+. v \xi_{V}^{p}\right)^{2}$. as discussed in Sect. III.D.1. Thus. the challenge for such studies is to measure small cross sections (albeit. much larger than single-particle transition cross sections due to the coherence) by detecting very low energy recoils. In the present subsection we shall only briefly touch upon a single example of this class of reactions. viz., elastic scattering from ${ }^{4} \mathrm{He}$. . Vo evaluation of the do-ability of using such a target will be attempted here, although it should be remarked that the fact that ${ }^{4} \mathrm{He}$ is a scintillator could make such a target/detector promising.

Typical conditions for use with DIF neutrino beams are represented in Table 4.4 (taken from Ref. (Don83]).

\begin{tabular}{|c|c|c|c|}
\hline \multicolumn{4}{|c|}{ TABLE 4.4 } \\
\hline Quantity & $\epsilon=100 \mathrm{MeV}$ & $\epsilon=150 \mathrm{MeV}$ & $\epsilon=200 . \mathrm{MeV}$ \\
\hline$\theta_{R}^{\text {peak }}$ & $62^{\circ}$ & $64^{\circ}$ & $69^{\circ}$ \\
$\left|Q^{2}\right|(\mathrm{GeV} / \mathrm{c})^{2}$ & 0.0084 & 0.016 & 0.018 \\
$T_{N}$ & 0.0024 & 0.0045 & 0.0052 \\
$q(\mathrm{MeV} / \mathrm{c})$ & 92 & 126 & 136 \\
$T_{R}(\mathrm{MeV})$ & 1.13 & 2.13 & 2.48 \\
$d \sigma / d \Omega_{R}\left(\times 10^{-41} \mathrm{~cm}^{2} \mathrm{sr}^{-1}\right)$ & 2.6 & 4.6 & 6.6 \\
\hline
\end{tabular}


Table 4.4 Elastic neutrino scattering from ${ }^{4}$ He at in ermediate energies. For given values of the neutrino energy. $e$. the quantities in the table are given for recoil angles corresponding to the peak in the differential cross section. The dimensionless momen. tum transfer $\tau_{N}$ is calculated using the nucleon mass $\left(\equiv\left|Q^{2}\right| / 4 m_{V}^{2}\right.$ ) rather than the mass of the.

Clearly, even for a target as light as the, the recoil energies are rather small for detec. tion in a large-active-volume detector. The quantity of interest is that which multiplies the electromagnetic form factor in Eq. (3.153), namely

$$
X \equiv \sqrt{3} \xi_{i}^{T=0}[1+\Gamma(q)]+\xi_{V}^{(0)} F_{C 0}(s) / F_{C 0}(T=0)
$$

Estimating the form factor ratio in last term by

$$
\frac{F_{C 0}(s)}{F_{C 0}(T=0)}=\frac{G_{E}^{(s)}}{G_{E}^{T=0}} \cong 2 \rho_{s} T
$$

setting $\xi_{E}^{(s)}$ to unity, since the momentum transfer is very small, and for the isospin-mixing factor writing approximately

$$
\Gamma(q) \cong \Gamma_{0} \tau+\mathcal{O}\left(\tau^{2}\right)
$$

where from Ref. [Don89] one has that $\Gamma_{0} \approx 0.04$. At tree-level, the entire multiplying factor above then has the form

$$
X=-4 \sin ^{2} \theta_{w}\left[1+\gamma_{0} \tau+\mathcal{O}\left(\tau^{2}\right)\right]
$$

where tree-level coupling have been employed and where $\gamma_{0}=\Gamma_{0}+\rho_{0} / 2 \sin ^{2} \theta_{w}$. For $|\rho$, set to the value 2 [Jaf89] one finds that $\left|\gamma_{0}\right| \cong 4.4$ with only a $1 \%$ correction from $\Gamma_{0}$. The term $\gamma_{0} \tau$ then contributes about $1-2 \%$ compared to the leading term in $X$ for the conditions in Table 4.4 and it thus provides only a small modification of the basic $4 \sin ^{2} \theta_{w}$ dependence in Eq. (4.112). For the conditions studied here the problem is similar to that of attempting a Standard Model test with PV elastic electron scattering at low-energies (see Sect. IV.B.2). Naturally, as in the discussions in Sect. IV.B, at higher-energies the influence of both the isospin-mixing term and the electric strangeness term is larger; the possibility exists that the clarity of the nuclear recoil as a signature for elastic scattering might make neutrino scattering advantageous for studies of hadronic structure in much the same way that is expected for PV electron scattering. Given sufficiently high-quality determinations from both types of lepton scattering measurements, it would then be possible to address the issue of how the radiative corrections differ for the various flavors of leptons. 


\section{IV.J.ł. ELASTIC NELTRINO-DELTERON SCATTERING}

Experimental considerations make it less likely that elastic $v-D$ scattering will prove useful as a probe of nucleon strangeness or non-standard physics than will either elastic neutrino-nucleon or elastic $\mathrm{PV}{ }^{2} \mathrm{H}\left(\vec{e}, e^{\prime}\right)$ scattering. Tevertheless. elastic $\nu-D$ scattering illustrates the special issues one encounters when seeking to study nucleon structure or electroweak physics with elastic neutrno scattering from nuclei with nonzero spin.

Apart from experimental considerations, the interpretation of elastic $\nu-D$ measure. ments involves complications not present in $\nu-.$. or PV electron scattering. In particular. both the nuclear and nucleon form factors contribute to $d \sigma(\nu D) / d Q^{2}$. In contrast. by a suitable choice of target and kinematics. much of the nuclear physics may be eliminated from $A_{L A}$. which involves a ratio of hadronic response functions. In the case of neutrino scattering. one might similarly attempt to minimize one s sensitivity to nuclear physics by studying the $\nu-D / \bar{\nu}-D$ asymmetry [Hen91. Fre92]

$$
\Delta_{\nu \nu}=\frac{d \sigma /\left.d Q^{2}\right|_{\nu D}-d \sigma /\left.d Q^{2}\right|_{\nu D}}{d \sigma /\left.d Q^{2}\right|_{\nu D}+d \sigma /\left.d Q^{2}\right|_{\nu D}}
$$

With these considerations in mind. we consider briefly the interpretation of the elastic $\nu-D$ cross section. paying particular attention to the similarities and differences with elastic $\nu-.{ }^{\prime}$ and $\mathrm{PV}$ elastic ${ }^{2} \mathrm{H}\left(\vec{e}, e^{\prime}\right)$ scattering. To that end. we convert from a discussion of $d \sigma / d Q^{2}$ to $d \sigma / d \Omega$ (see Sect. III.E.3) and consider different regimes in $\nu$ scattering angle. even though a recoil deuteron rather than outgoing neutrino would be detected. Following the formalism outlined in Sect. III.E.3 (see Eqs. (3.148) and (3.149)), we write the qua:tity appearing in $\frac{d \sigma}{d \Omega}(\nu D)$ as

$$
\dot{F}_{D}^{2}(q, \theta) \equiv 4 \dot{F}^{2}(q, \theta) \equiv R_{(1)}+R_{(2)}+R_{(3)}+R_{(4)} .
$$

where the response functions have the forms

$$
\begin{aligned}
& R_{(1)}=3\left(\xi_{V}^{T=0}\right)^{2}\left\{v_{L}\left(F_{C 0}^{2}(T=0)+F_{C 2}^{2}(T=0)\right)+v_{T} F_{M 1}^{2}(T=0)\right\} \\
& R_{(2)}=2 \sqrt{3} \xi_{V}^{T=0} \xi_{V}^{(0)}\left\{v_{L}\left(F_{C 0}(T=0) F_{C 0}(s)+F_{C 2}(T=0) F_{C 2}(s)\right)\right. \\
& \left.+v_{T} F_{M_{1}}(T=0) F_{M_{1}}(s)\right\} \\
& R_{(3)}= \pm 2 v_{T^{\prime}} \sqrt{3} \xi_{V}^{T=0} F_{M_{1}}(T=0)\left\{\xi_{A}^{T=0} F_{E 1_{s}}(8)+\xi_{A}^{(0)} F_{E 1_{0}}(s)\right\} \\
& R_{(4)}=v_{T}\left\{\xi_{A}^{T=0} F_{E 1_{s}}(8)+\xi_{A}^{(0)} F_{E_{1}}(s)\right\}^{2} \\
& +\left(\xi_{V}^{(0)}\right)^{2}\left\{v_{L}\left(F_{C 0}^{2}(s)+F_{C 2}^{2}(s)\right)+v_{T} F_{M 1}^{2}(s)\right\} \\
& \pm 2 v_{T^{\prime}} \xi_{V}^{(0)} F_{M_{1}}(s)\left\{\xi_{A}^{T=0} F_{E_{1}}(8)+\xi_{A}^{(0)} F_{E_{1}}(s)\right\}
\end{aligned}
$$


and where the form factors are defined in Sect. III.D.1.

The response functions $R_{(1)}(i=1 \ldots t)$ are the $\nu-D$ analogs of the $\Delta_{11}(i=1 \ldots 3)$ in $A_{L R} \mid \bar{E} D$. $R_{(1)}$ contains the leading vector current contribution in the absence of strangeness: $R_{(2)}$ depends linearly on the strangeness vector current form factors: and $R_{i 3}$ contains the product of vector and axial-vector form factors. Although the combinations of form factors entering the $R_{(1)}$ are, for the most part. identical to those appearing in the numerators of the corresponding $\lambda_{(1)}$, a few differences exist: (i) the combination $v_{L}\left(F_{C O}{ }^{2}+F_{C_{2}}{ }^{2}\right)+v_{T} F_{M_{1}}{ }^{2}$ appearing in $R_{11}$ is canceled from $\Delta_{(1)}$ by an identical quantity appearing in the denominator of $W^{-P V} / F^{2}$ : no such cancellation occurs for $\nu-D$ scatter. ing: (ii) the A.M form factor $F_{E 1},(A . M)$ contained in $J_{(3)}$ does not enter $R_{13}$ since the neutrino has no E.I charge: and (iii) the $R_{(1)}$ depend on products of weak neutral current amplitudes rather than on the weak-electromagnetic interference product which governs the $\vec{\epsilon} D$ asymmetry. Consequently, the $R_{(1)}$ are bilinear in weak neutral current couplings. whereas the $J_{(1)}$ depend on them linearly.

The terms in $R_{(4)}$ have no analog in $A_{L R}(\vec{e} D)$. They arise because $\frac{d \sigma}{d \Omega}(\nu D)$ is proportional to a product of weak. neutral current amplitudes. Since $R_{(t)}$ is second-order in the small strangeness form factors and $\xi_{A}{ }^{(8)}+\frac{3}{\sqrt{3}} \xi_{A}{ }^{(0)}$ axial-vector coupling, one might expect it to be negligible in comparison with the other $R_{(i)}$.

In principle. $\frac{d o}{d \Omega}(\nu D)$ can be quite sensitive to vector strangeness nucleon form factors. as is $A_{L R}(\vec{e} D)$. In practice, however, kinematics conspire to make the neutrino experiments more difficult. To see why, consider first the magnetic contribution, $F_{M_{1}}(s)$. Its contribution becomes most significant at backward angles, where momentum transfer is largest. However. the deuteron body form factors defined in Eq. (4.28) fall off rapidly with momentum transfer. thereby suppressing the backward-angle cross section. For example, the magnetic term $D_{M}^{M}\left(Q^{2}\right)$, whose contribution dominates $F_{M_{1}}(s)$, is down by two ordersof-magnitude from its static value $D_{M}^{M}(0)$, for backward-angle scattering with incident energies of only $500 \mathrm{MeV}$. For the single-nucleon form factor, on the other hand, on the other hand, the suppression is only 0.25 at this energy. By going to lower-energy neutrino beams, one may mitigate this suppression to some extent. At low- $\left|Q^{2}\right|$, however. $F_{M 1}(s)$ also vanishes linearly with momentum transfer. As a result, optimal neutrino energies. balancing these two constraints, are roughly several hundred $\mathrm{MeV}$. For these kinematics. one then encounters experimental difficulties due to the extremely low resulting recoil deuteron energy. In contrast, no such difficulties arise in a measurement of $A_{L R}\left({ }^{2} \mathrm{H}\right)$, which involves detection of the outgoing lepton. Moreover, at backward angles, the deuteron body magnetic form factor essentially cancels from the PV asymmetry (see Sect. IV.C.1), so that the large two-body suppression does not appear in the observable of interest.

At forward angles and low momentum transfer, the $\nu-D$ cross section goes like

$$
\begin{aligned}
d \sigma / d \Omega\left(\theta \rightarrow 0^{\circ}, q \text { small }\right) \times 3\left(\xi_{V}^{T=0}\right)^{2}\left(F_{C 0}^{2}(T=0)+F_{C 2}^{2}(T=0)\right) \\
+\frac{1}{2}\left(\xi_{A}^{T=0} F_{E 1_{s}}(8)+\xi_{A}^{(0)} F_{E 1_{s}}(s)\right)^{2}+\mathcal{O}\left(q^{2}\right),
\end{aligned}
$$

where the $\mathrm{O}\left(q^{2}\right)$ terms include contributions from the magnetic $\left(F_{M_{1}}(T=0)\right)$ and strangeness charge $\left(F_{C 0, C 2}(s)\right)$ multipoles. In comparison, the $\mathrm{PV}$ asymmetry goes like 
$\vee \overline{3} \xi_{i}^{T}=0-O\left(q^{2}\right)$. In both cases, the strange-quark vector current contributions are sup. pressed by factors of $Q^{2} / m^{2}$ " At forward angles, however the electromagnetic cross section gets very large, which keeps the FOM for PV electron scattering fairly high "Pol90". Thus. ignoring systematic errors, one could hope to measure $\left.A_{L R}\right|^{2} \mathrm{H}$ ) with sufficient precision to be sensitive to $Q^{2}$-suppressed terms. in analogy with the single nucleon case. For the $\nu-D$ cross section. however, there is no. Mott divergence. and thus nothing to improve the statistics at forward angles. Considering high-energy forward-angle $v-D$ scattering leads to only a marginal improvement in sensitivity. When kinematic coefficients of order - are large enough to allow significant strange quark contributions. the deuteron body form factor is again very small.

The strangeness axial-vector current contributes to the differential neutrino cros; section via $R_{13}$, and $R_{14}$. In comparing the sensitivities of $d \sigma(\nu D) / d Q^{2}$ and $A_{L R^{2}}{ }^{2} \mathrm{H}$, is the axial-vector form factor. we reiterate that the interpretation of a neutrino meastrs. ment in terms of ax.al-vector strangeness is much less ambiguous than in the case of $\mathrm{Pl}$ electron scattering. Both the interference term $R_{(3)}$ and its analog $\Delta_{(3)}$, in $A_{L R}$ ?i suppressed at forward angles. The axial-vector contribution to $R_{13}$. however. carries no $g_{v}^{e}$ suppression factor so that its backward-angle contribution can be more significant for $\nu-D$ scattering than for $\mathrm{PV}{ }^{2} \mathrm{H}\left(\vec{e}, e^{\prime}\right)$ scattering. The axial-vector contribution via $R_{(4)}$ has no analog in $A_{L A}\left({ }^{2} \mathrm{H}\right)$. This term has no explicit forward-angle kinematic suppression. but is still quadratic in the presumably small axial-vector isoscalar form factor. To use this term to limit the axial-vector isoscalar form factor to less than or about $=0.15$ would require absolute forward $v-D$ cross section measurements at below the $10 \%$ level. In the backward-angle limit, the axial-vector contributions from interference and quadratic terms can be formally combined by

$$
\begin{aligned}
& \frac{d \sigma}{d \Omega}\left(\begin{array}{c}
\nu \\
i
\end{array}\right)\left(\theta \rightarrow 180^{\circ}\right) \times\left(\left[\sqrt{3} \xi_{V}^{T=0} F_{M 1}(T=0)+\xi_{V}^{(0)} F_{M 1}(s)\right]\right. \\
&\left. \pm\left[\xi_{A}^{T=0} F_{E_{1 s}}(8)+\xi_{A}^{(0)} F_{E_{1 s}}(s)\right]\right)^{2} .
\end{aligned}
$$

At moderate energies. (where the cross sections are extremely small. as discussed above the axial-vector contributions contribute comparably to the strangeness magnetic form factors. Since $F_{M 1} \times\left(q / m_{N}\right)$ for low momentum transfer, however. the backward-angle cross section is much more sensitive to the axial-vector terms at low-energies, becoming essentially directly proportional to the square of the axial-vector form factor. There is also a range of energies where the'vector and axial-vector terms become comparable. In this kinematic regime, the difference between the $\nu-D$ and $\bar{\nu}-D$ cross sections can be large. thereby enhancing the sensitivity of $\Delta_{\nu \nu}$ to nucleon strangeness.

Finally, we observe that the use of elastic $\nu-D$ scattering to probe physics beyond the Standard Model through an extraction of the $\xi_{v, A}$ from the differential cross section is more problematic than with other processes discussed in this review. The reason is the

* At small momentum transfers, $F_{M_{1}}^{2}(T=0)$ is explicitly proportional to $q^{2}: F_{C 0}(s)$. being proportional to $\tau$ because the deuteron has no net strangeness, is likewise small. 
additional level of nuclear physics uncertainties not encountered in the interpretation of the elastic $\nu-. . / \bar{\nu}-. . \bar{l}$ cross sections. The impact of these uncertainties on $A_{L R}$ can be mitigated by a suitable choice of target since the asymmetry involves a ratio of nuclear response functions. The extent to which an observable such as $\Delta_{\nu \bar{\nu}}$ can reduce the sensitivity of an electroweak test to nuclear physics uncertainties remains to be analyzed. At present. elastic $v$-D scattering seems most suited to probing the structure of the nucleon.

\section{IV.J.J. INELASTIC .NELTRINO SCATTERING}

We end our discussion of special cases with brief remarks about a few selected inelastic neutrino scattering transitions where knowledge of the cross sections is expected to help in determining the single-nucleon weak-interaction form factors. Of particular interest in this regard is the $\mathrm{NC}$ transition between the ground state of ${ }^{12} \mathrm{C}$ and the $1+1,15.11 \mathrm{MeV}$ excited state in the same nucleus, together with the charge-changing $(\mathrm{CC})$ transitions to the analog ground states of ${ }^{12} \mathrm{~B}$ and ${ }^{12} \mathrm{~N}$. From our summary of the formalism in Sect. III.E.3 we see that the NC multipole matrix elements that enter include $\tilde{F}_{M 1}, \tilde{F}_{C_{1}}, \tilde{F}_{L_{13}}$ and $\tilde{F}_{E 1_{1}}$. together with their $C C$ analogs. For the states involved only isovector currents occur. This system is much-studied theoretically (see, for example. Refs. [OCo72. Doni3. Donit. Don76. Don 79b]) and recently has been the focus of several ${ }^{12} \mathrm{C}\left(\nu_{e}, e^{-}\right)^{12} \mathrm{~N}(\mathrm{~g} . \mathrm{s}$.$) , charge-$ changing [Al190, KAR92] and ${ }^{12} \mathrm{C}\left(\nu, \nu^{\prime}\right)^{12} \mathrm{C}(15.11)$, neutral-current [KAR91] experimental investigations. As emphasized in Refs. [Don74. Don76, Don79b], at low-energies the allowed multipole is of the Gamow-Teller form and consequently the nuclear matrix element involved in 3-decay is closely related to the dominant contribution entering in chargechanging neutrino reactions and neutral-current neutrino scattering. For instance, to the extent that the long wavelength limit (LWL) can be taken one may write the $\mathrm{NC}$ cross section in terms of the ${ }^{12} \mathrm{~N}$ and ${ }^{12} \mathrm{~B} \beta$-decay rates, $\omega_{\beta}^{ \pm}$, respectively (see Eq. (4.13) in [Doni9b]):

$$
\sigma_{\nu, \nu^{\prime}}^{L W L}(J=T=1)=\frac{3}{4} \pi^{2} G_{\mu}^{2} m_{N}^{2}\left[\frac{\nu-\omega}{m_{N}}\right]^{2}\left(\xi_{A}^{T=1}\right)^{2} \times \frac{\omega_{3}^{ \pm}(J=1)}{G_{\mu}^{2} \cos ^{2} \theta_{c} f^{ \pm}\left(W_{0}^{ \pm}\right)^{5}},
$$

where $W_{0}^{ \pm}$is the energy of the decay, $\theta_{c}$ is the Cabibbo angle and $f^{ \pm}$is a dimensionless phase-space integral [Don79b]. Using the average of the ${ }^{12} \mathrm{~B}$ and ${ }^{12} \mathrm{~N} \beta$-decay rates, this yields (Eq. (4.53) in [Don79b])

$$
\sigma_{\nu, \nu^{\prime}}^{L W L}(J=T=1)=2.7 \times 10^{-39} \mathrm{~cm}^{2}\left[\frac{\nu-\omega}{m_{N}}\right]^{2}\left(\xi_{A}^{T=1}\right)^{2} .
$$

Of course, these simple relationships are not exact, since as noted above there are more nuclear matrix elements than one to consider (and these enter differently in the various electroweak processes); in addition, the momentum transfer dependence of the nuclear form factors must at some level be taken into account. For this well-studied $A=12$ system, as discussed in depth in Ref. [Don79b], it is possible to constrain the nuclear one-body density matrix elements (see Sect. III.D.2) to a very high degree using electron scattering, $\gamma$-decay, 
$3^{ \pm}$-decay and $\mu^{-}$-capture information. Consequently, it is not necessary to resort to the strict LWL model. It is reassuring, however, that the simple model does yield an excellent approximation to the more sophisticated result (typically being in error by only about $10-15 \%$ at low-energies. although somewhat worse at intermediate-energies) and. on the basis of the refinements that go into the latter. it is expected that for this special case the confidence level of the theoretical predictions for the neutrino-induced reactions should be better than $10 \%$.

Indeed. the recent $C C$ results [All90. KAR92] strengthen this expectation. since rather good agreement is found with the predictions: from early modeling [Doni3] the integrated cross section for a beamstop facility was found to be $0.94 \times 10^{-41} \mathrm{~cm}^{2}$ (see also Refs. [Fuk8S. Min89]) while experiments have yielded $1.05 \pm 0.10$ (stat) \pm 0.10 (syst) $\times 10^{-41} \mathrm{~cm}^{2} \quad A 1190$ and $0.81 \pm 0.09$ (stat) \pm 0.075 (syst) $\times 10^{-41} \mathrm{~cm}^{2}$ [KAR92]. With further running it might prove possible to lower the total experimental uncertainty to below $10 \%$ ! K.AR91. K.AR9? . commensurate with the estimated level of the model uncertainty.

The situation for $\mathrm{NC}$ neutrino-excitation of the $15.11 \mathrm{MeV}$ level in ${ }^{12} \mathrm{C}$ followed by 7 -decay back to the ground state is similarly quite satisfactory. The theoretical modeling [Don74, Don76. Don79a] updated to a modern value for $\sin ^{2} \theta_{w}$ yields $0.97 \times 10^{-41}$ $\mathrm{cm}^{2}$ for the integrated beamstop $\nu_{e}+\bar{\nu}_{\mu}$ cross section (see also Refs. [Beri9. Fuk88. Kol92] which predict very similar numbers), to be compared with the experimental value $1.08 \pm 0.51$ (stat) \pm 0.11 (syst) $\times 10^{-41} \mathrm{~cm}^{2}$ [KAR91]. With extended running it is expected here as well that the total experimental uncertainty may be reduced to below $10 \%$ [KAR91. KAR92]. In fact, a theory-to-experiment comparison of the ratio of NC to CC integrated cross sections might ultimately provide the best-determined quantity, both from the experimental point of view where flux normalization uncertainties may be minimized. as well as from the theoretical point of view where the ratio is expected to have only a very weak dependence on the nuclear modeling.

Of particular ir--arest for the $\mathrm{NC}$ measurements in which it is the sum of the $\nu_{e}$ and $\bar{\nu}_{\mu}$ scattering cross sections that is determined is the fact that the VA-interference term changes in going from particle- to antiparticle-scattering (see Eq. (3.149)) and consequently largely cancels in taking the sum (it does not exactly cancel, since the beamstop spectra for $\nu_{e}$ and $\bar{\nu}_{\mu}$, while rather similar, are not exactly the same - see Fig. $5.1 \mathrm{a}$ ). In fact the predictions for the quantity $\left.\delta<\sigma_{\nu_{e}}+\sigma_{\nu_{\mu}}\right\rangle / \delta \sin ^{2} \theta_{w}$ yield the very small value 0.06 , reflecting the weak dependence on the vector currents. Since the summed result is almost completely determined by the isovector axial-vector form factor and is quadratic in this quantity (see the above discussions on neutrino reactions), this suggests that extended experimental measurements in the $A=12$ system could lead to a determination of $\tilde{G}_{A}^{T=1}(0)$ with about $5 \%$ uncertainty. Coupling such a result with a high-precision determination of the equivalent quantity from PV electron scattering where the radiative corrections are expected to be rather different would provide a very interesting test of the underlying weak-interaction model.

Let us end this section with a brief discussion of another interesting case drawn from Ref. [Don79a]. The same motivation of studying M1/Gamow-Teller NC excitations can be extended to include isoscalar transitions, as emphasized by the authors of Refs. [Doni4, 
Doni6). However, in this case there are no 3-decay rates to use to (largely) determine the nuclear matrix element: instead one may use the corresponding $\gamma$-decay rate $\Gamma_{\gamma}(J=$ 1. $T=0$ ) to write (see Eq. $(4.17$ ) in $[$ Doni9a]):

$$
\begin{aligned}
\sigma_{\nu, \nu^{\prime}}^{L W L}(J=1 . T=0)=\frac{3}{4 \alpha} \pi^{2} G_{\mu}^{2} m_{N}{ }^{4} & \left(\frac{2 J_{0}+1}{2 J_{1}+1}\right)\left[\frac{\nu-\omega^{\prime}}{m_{N}}\right]^{2}\left(\frac{\xi_{A}^{T=0} G_{A}^{(8)}+\xi_{A}^{(0)} G_{A}^{(s)}}{F_{2}^{T=0}}\right)_{L W L}^{2} \\
& \times \frac{1}{\lrcorner^{3}} \Gamma_{\gamma}(J=1, T=0),
\end{aligned}
$$

where the ground state has angular momentum $J_{0}$ while the excited state has $J_{1}$ and $\left|J_{1}-J_{0}\right| \leq 1$. Specifically, let us consider the $1^{+} 0$ ground and $2^{+} 0,7.028 \mathrm{Mel}$ excited states in ${ }^{14} . \mathrm{N}$ (see Eq. (4.51) in [Don79a] and associated discussion). Keeping only the axial-vector strangeness form factor and using Eq. (3.47) one obtains

$$
\sigma_{\nu, \nu^{\prime}}^{L W L}(J=1, T=0)=2.5 \times 10^{-38} \mathrm{~cm}^{2}\left[\frac{\nu-\omega^{\prime}}{m_{v}}\right]^{2}\left(\eta_{\bullet}\right)^{2} .
$$

Given the value $\eta_{\mathbf{s}} \approx-0.12$ in Table 4.2 this yields roughly $1 / 4$ the number of photons for this isoscalar ${ }^{14} \mathrm{~N}$ case as obtained for the above isovector ${ }^{12} \mathrm{C}$ case (per neutrino per unit time for equal masses of carbon and nitrogen) and consequently may prove feasible for future experiments.

Other nuclear transitions are discussed in Ref. [Don79a] for NC neutrino scattering of reactor anti-neutrinos and beamstop and DIF intermediate-energy neutrinos $\left(\nu_{e}, \nu_{\mu}\right.$ and $\bar{\nu}_{\mu}-$ see Sect. V.A). The results given there can usually be extended quite easily to the incorporate strangeness content that was ignored when that previous review was written. 


\section{EXPERIMENTAL CONSIDERATIONS}

In this section we discuss some of the experimental considerations that enter into treatments of parity-violating electron scattering and neutrino scattering from nucleons and nuclei. Our aims are several: we wish to summarize the general considerations that occur in experimental investigations of these classes of reactions to provide the reader with an assessment of what issues are likely to determine the future course of the field (Sect. V.A): we then turn in Sect. V.B to overviews of past studies of electron and neutrino scattering for the purpose of learning about the hadronic neutral current - here sufficient detail is given in several cases to permit an appreciation of the level of difficulty involved in both classes of reactions: finally, in Sect. V.C we summarize the present and proposed worldwide experimental program as we are aware of it and attempt to indicate where future initiatives in this general area of research may lie.

\section{V.A General Considerations}

We begin with a discussion of the general experimental considerations that pertain when studying PV electron scattering (Sect. V.A.1) or neutrino scattering (Sect. V.A.2).

\section{V.A.1. ELECTRON SCATTERING FACILITIES}

Electron accelerators which have been used for PV electron scattering studies span the energy range from a few hundred $\mathrm{MeV}$ to tens of $\mathrm{GeV}$. Modern facilities are designed to produce intense beams of polarized electrons and the newest machines will also provide $\mathrm{CW}$ beams. High-duty-factor beams, with greatly reduced instantaneous counting rates (more than two orders-of-magnitude), would make possible for the first time the capability of using particle-counting techniques. Both particle discrimination and background rejection would be : :znificantly "nhanced.

There are many experimental challenges associated with carrying out PV experiments at the $1 \%$ level using high-energy electrons. These involve limitations on luminosity, beam polarization, detector acceptance, resolution and the ability to control systematic errors. The relative importance of the different factors is a strongly physics-dependent issue. The luminosity is given by

$$
\begin{aligned}
\mathcal{L} & =\frac{I \rho N_{0}}{A} \\
& \cong 4 \times 10^{38} \frac{I \rho}{A},
\end{aligned}
$$

where the luminosity is in units of $\mathrm{cm}^{-2} \mathrm{~s}^{-1}$, the average current $I$ is in units of $100 \mu \mathrm{A}$, the target density $\rho$ is in $\mathrm{g} \mathrm{cm}^{-2}$ and $A$ is the target mass number. Essentially all PV experiments are designed to run at maximum luminosity in the interest of running time and cost. For accelerators in the one to few $\mathrm{GeV}$ range (see Table 5.1), polarized-beam intensities of $100 \mu \mathrm{A}$ are reasonable. For a CW accelerator the laser requirements are modest, while for pulsed machines very high-powered lasers are required to produce the 
polarized beams and such accelerators are usually not designed to provide much higher beam intensities. Some lower-energy $(<100 \mathrm{MeV})$ electron machines have capabilities in the ampere range. although these do not have polarized beam capability ar present. Table 5.1 summarizes the beam characteristics of electron accelerators which are designed to deliver polarized beams. Both the MIIT/Bates and .VIKHEF* facilities have recently been upgraded with the addition of stretcher/storage rings whirh make possible essentially: $\mathrm{CW}$ extracted beams, as well as very high current internal stored beams. Polarized beams will be available in both operating modes.

\begin{tabular}{|c|c|c|c|}
\hline \multicolumn{4}{|c|}{ TABLE 5.1} \\
\hline LABORATORY & $\begin{array}{c}\text { E.EERGY RA.IGE } \\
(\mathrm{GeV})\end{array}$ & $\begin{array}{c}\text { BE.A.II } \\
\text { CLRRE.NT }\end{array}$ & DLTY FACTOR \\
\hline \multicolumn{4}{|l|}{.MIT/Bates } \\
\hline Pulsed & $0.1-1.0$ & $100 \mu \mathrm{A}$ & 0.01 \\
\hline CW Extracted & $0.3-1.0$ & $50 \mu \mathrm{A}$ & $\mathrm{CW}$ \\
\hline CW Internal & $0.3-1.0$ & $80 \mathrm{~mA}$ & $\mathrm{CW}$ \\
\hline CEBAF(design) & $0.5-6.0$ & $200 \mu \mathrm{A}$ & $\mathrm{CW}$ \\
\hline Mainz & $0.2-0.9$ & $100 \mu \mathrm{A}$ & $\mathrm{CW}$ \\
\hline \multicolumn{4}{|l|}{ NIKHEF } \\
\hline Pulsed & $0.1-0.9$ & $65 \mu \mathrm{A}$ & 0.001 \\
\hline CW Extracted & $0.3-0.9$ & $65 \mu \mathrm{A}$ & $\mathrm{CW}$ \\
\hline CW Internal & $0.3-0.9$ & $200 \mathrm{~mA}$ & $\mathrm{CW}$ \\
\hline \multicolumn{4}{|l|}{ SLAC } \\
\hline End Station $A($ Present $)$ & $1-23$ & $10 \mu \mathrm{A}$ & $2 \times 10^{-4}$ \\
\hline End Station A (Ľpgrade) & $1-50$ & $100 \mathrm{nA}$ & $10^{-3}$ \\
\hline
\end{tabular}

Table 5.1. Properties of existing and planned electron accelerators.

A very practical luminosity-related consideration is the power-handling capability of the scattering target. It is possible to construct liquid hydrogen, deuterium and helium targets which can dissipate $\sim 1 \mathrm{~kW}$ of beam power. Certain solid targets, such as carbon and tungsten, have somewhat higher power-handling capabilities, although the target thickness is in general limited to approximately $(5-10) \mathrm{g} / \mathrm{cm}^{2}$ of material. Other considerations that limit target thickness involve radiative effects which can limit the resolution in the scattered

* NIKHEF does not currently have a polarized injector. although plans exist to install one. 
electron energy and affect the accuracy of the results. In particular. bremsstrahlung effects set limits which are comparable to the target's maximum power-handling capabilities.

\section{Polarized Electron Sources}

Polarized beams are a relatively new capability for electron accelerators. They now provide many important physics opportunities connected with electroweak studies and with experiments designed to exploit spin degrees of freedom in electron scattering. In addition to the $\mathrm{PV}$ experiments discussed here. major efforts are underway to measure $G_{E}^{n}$ and various observables involving nucleon and nuclear structure.

The measurement of small asymmetries, in the range of $10^{-4}-10^{-b}$. places severe demands on the minimal requirements that a polarized source must achieve to make such experiments practical. The most important of these are high intensity ( up to hundreds of mA peak c!rrsnt fur pulsed accelerators) and high polarization. Other factors being equal. the figure-of-merit in such experiments is proportional to $\mathrm{Pe}_{\mathrm{e}}^{2} A_{L R}^{2} \sigma$. where $\mathrm{P}_{\mathrm{e}}$ is the beam polarization. $A_{L R}$ is the asymmetry to be measured and $\sigma$ is the differential cross section (see Sect. III.E.2). In order to control systematic effects, rapid and precise polarization reversal is essential and it must leave all other beam parameters unchanged.

Although various techniques have been used to construct polarized electron sources. only the $\mathrm{Li}$ atomic beam and photoemission sources have been used as injectors in accelerators to date and essentially all sources now in use on electron machines are based on photoemission from GaAs. Photoemission from semiconductors as a source of polarized electrons was first proposed by Garwin, Pierce and Siegmann [Gar74] and by Lampel and Weisbuch [Lami5]. These crystals can provide the very high peak currents required by the older low-duty-factor machines and allow for rapid polarization reversal and stability demanded by PV experiments. A source was constructed by Sinclair and co-workers for the SLAC PV experiment of Prescott et al. [Pre79], and most electron accelerator sources now in use are variants of this early SLAC design.

The upcration of a GaAs source is relatively simple. Polarized electrons are produced in the conduction band of the semiconductor crystal by shining circularly-polarized laser light of an appropriate wavelength on the material. To allow the electrons to escape from t..e crystal, the surface is covered with a very thin layer of alkali metal and oxidants. This lowers the work-function of the surface so that the energy of an electron in the vacuum is lower than it is in the bulk material (a condition known as negative electron affinity).

The theoretical maximum polarization possible with a bulk GaAs crystal is limited by level degeneracies to $50 \%$. Polarizations achieved in practice are in the range of 25 to $43 \%$. Since the FOM is proportional to the square of the polarization, almost an orderof-magnitude improvement is still possible. The sign of the electron polarization is simply changed by reversal of the circular polarization of the light and devices are available for achieving this on time scales shorter than a microsecond.

During the past decade, much research activity has been directed at understanding why working sources have a polarization less than the theoretical maximum of $50 \%$ and 
finding approaches for achieving much higher polarizations. Significant progress has occurred. A recent review by Cardman summarizes these latest efforts [Car92]. The most promising research is directed towards approaches which would remove the degeneracy in GaAs and allow for much higher polarizations. These approaches depend on altering the nature of the crystal structure by: (1) applying stress to the crystal: (2) constructing artificial structures with varying band gap energies: and (3) finding new crystals where the degeneracy is absent due to lack of symmetry.

Experiments with strained crystals have been carried out by . Vakanishi et al. Yak91. In their approach a layer of GaAs is grown on a surface of $G_{a} P_{x} A s_{1-x}$ (with $x \sim 0.17$ ) which has a lattice mismatch of $0.6 \%$. This stressed crystal has shown electron polarizations as high as $\$ 3 \%$ [Mar92]. The quantum efficiency has however been reduced by an orderof-magnitude in comparison with using bulk GaAs. This may not be a problem for $\mathrm{CII}^{\circ}$ accelerators. but could be a serious limitation for the older pulsed machines.

Multi-layered heterostructures, such as alternating $\mathrm{GaAs}$ and $\mathrm{Al}_{x} \mathrm{Ga}_{1-x}$.ts. have also been constructed in another approach in which the band degeneracy is broken by the introduction of a periodic variation in the band energy. A $K E K / \triangle$ iagoya/ $\triangle E C$ group [Omo91] has investigated this technique and achieved a polarization of $71 \%$. The quantum efficiency is. however, almost two orders-of-magnitude below bulk GaAs.

The third approach to higher polarization involves the use of direct band gap materials which do not have the valence band degeneracy of GaAs; one class of such materials is the chalcopyrites. These materials could in principle provide both high polarization and high quantum efficiency and several groups are now investigating these possibilities.

An important consideration in the operation of polarized sources, in addition to high polarization, is source lifetime. At present the lifetimes of typical accelerator photocathode sources are in the range of a few hundred hours, after which time the crystal surface must be cleaned and reactivated. Most evidence indicates that lifetime is for the most part related to vacuum quality. Since the vacuum requirements are extremely high, efforts are also under way to find less sensitive materials and/or techniques for continuously cleaning the crystal surface.

There has been much progress in our ability to generate and use intense polarized electron beams since the first PV experiment at SLAC. The recent developments in source technology show possibilities for substantial improvement in polarization for the future.

\section{$\underline{\text { Detectors }}$}

Detectors that have been used and/or proposed for PV experiments have acceptances from a few millisteradians to more than a steradian (see Table 5.2). The small-acceptance devices are usually magnetic spectrometers with relatively good momentum resolution $(<$ $0.1 \%$ ), whereas the large-acceptance devices involve the use of Cerenkov counters optimized for the detection of minimum-ionizing particles. The latter have essentially no momentum resolution, since the thresholds are usually set very low. There have also been some novel ideas for systems in the range of $(50-200) \mathrm{msr}$ which have reasonable momentum 
resolution. One such system uses a multi-sector toroidal magnet configuration as proposed for an experiment in Hall $C$ at CEBAF. As discussed in Sect. V.C.J. the possibility of using a large solenoid as a detector has also been studied and looks quite promising. particularly. at relatively low energies $(<1 \mathrm{GeV})$. All experiments until now have used detection of the scattered electrons. The toroidal system. mentioned above, is designed to look at the flux of recoil protons instead. which potentially has some advantages for experiments where the scattered electron is at an unfavorably far forward angle.

Basic physics considerations set the criteria for the required momentum resolution. Experiments on hydrogen have the least restrictions: in this case inelasticities in excess of $m_{\pi}$ must be accounted for in precise measurements. For nuclear scattering the issue involves contributions due to inelastic levels or electrodisintegration. Elastic scattering on deuterium requires a resolution of better than $2.2 \mathrm{MeV}$. for ${ }^{12} \mathrm{C}$ less than 4.4 . Me. and in the case of ${ }^{t} \mathrm{He}$ this is relaxed somewhat to $20.1 \mathrm{Mel}$. Depending on the inciden: electron energy: this corresponds to momentum resolutions of a few percent to less than $0.1 \%$. Magnetic devices provide the only practical means for achieving such resolutions. In addition. for experiments at the highest luminosity. where event-by-eve. l $^{*}$ tracking is not possible, the detector must have intrinsic momentum and angular focusing properties as well.

A monoenergetic beam of electrons passing through a target loses energy at the rate of $\sim 2.2 \mathrm{MeV} \mathrm{cm} / \mathrm{gm}$ and develops an energy-spread at approximately $1 / 4$ this rate. For example. a target of $5 \mathrm{gm} / \mathrm{cm}^{2}$ will develop an energy-spread of approximately 2.5 . $\mathrm{Hel}^{\circ}$ due to jonization straggling. This sets an obvious limit on maximum target thickness due to these considerations alone. This limit is, however, comparable to that due to the maximum level that is practical in handling the dissipated power.

Each experiment has to identify and solve a number of problems connected with systematic uncertainties. Most important are those associated with beam-related helicitycorrelated differences. Beam parameters such as energy, intensity, position and direction can have major effects. The MIT/Bates ${ }^{12} \mathrm{C}$ results [Sou90a] show that asymmetry uncer ainties at the level of $2 \times 10^{-8}$ are achievable in an electron scattering measurement and in polarized proton scattering at SIN [Kis87] the systematic errors were of comparable size. It is expected that it should be possible to improve on these results with the modern CW electron accelerators.

There have been suggestions, at different times, that PV studies might be feasible using polarized targets or recoil polarimetry. Practical considerations, however, make this highly unlikely given present technology. High-density polarized targets are available as cryogenic gas cells $\left({ }^{3} \mathrm{He}\right)$ and solids $\left({ }^{1} \mathrm{H},{ }^{2} \mathrm{H}\right)$. In the case of polarized ${ }^{3} \mathrm{He}$ a density of $10^{22} \mathrm{~cm}^{-2}$ can be contemplated. At a beam current of $100 \mu \mathrm{A}$, which could present some difficulty for maintaining target polarization, the maximum luminosity is only $10^{37} \mathrm{~cm}^{-2} \mathrm{~s}^{-1}$. This is nearly two orders-of-magnitude less than that achieved in the MIT/Bates ${ }^{12} \mathrm{C}$ experiment. In the case of polarized solid targets the situation is even worse. State-of-the-art solid cryogenic polarized ${ }^{2} \mathrm{H}$ targets can reach densities of $10^{23}$ $\mathrm{cm}^{-2}$ at high polarization [Bur93, Day93]. The beam current must, however, be limited to less than $100 \mathrm{nA}$ in order to maintain target polarization. The resulting luminosity 
TABLE 3.2

\begin{tabular}{|c|c|c|c|c|}
\hline & Type & $\begin{array}{l}\text { Acceptance } \\
\text { (msr) }\end{array}$ & $\begin{array}{c}\text { Momentum } \\
\text { Resolution } \\
(\%)\end{array}$ & $\begin{array}{l}\text { Scattering } \\
\text { Angle }\end{array}$ \\
\hline \multicolumn{5}{|l|}{ SLAC } \\
\hline $197 i$ & Magnetic & 0.5 & 20 & $t^{\circ}$ \\
\hline \multicolumn{5}{|l|}{ End Station A } \\
\hline \multirow[t]{2}{*}{$\therefore \quad$ (Elastic) } & Magnetic (2) & 0.6 & 0.5 & $6.5^{\circ}$ \\
\hline & & 3 & 0.1 & $67.9^{\circ}(p)$ \\
\hline (DIS) & .lagnetic (2) & 1 & 3 & $3.70^{\circ}$ \\
\hline \multicolumn{5}{|l|}{ Mainz } \\
\hline & Cerenkov & 2500 & - & $115^{\circ}-145^{\circ}$ \\
\hline & Calorimeter & 2200 & 4 & $35^{\circ}-50^{\circ}$ \\
\hline \multicolumn{5}{|l|}{.MIT/Bates } \\
\hline${ }^{12} \mathrm{C}$ & Magnetic (2) & 20 & 6 & $35^{\circ}$ \\
\hline SA.MPLE & Čerenkov & 2000 & - & $135^{\circ}-160^{\circ}$ \\
\hline \multirow[t]{2}{*}{ Solenoid } & Magnetic & 600 & 1 & Forward \\
\hline & & 2000 & 1 & Backward \\
\hline \multicolumn{5}{|l|}{ CEBAF } \\
\hline Hall $A$ & Magnetic (2) & 16 & .01 & $12.5^{\circ}$ \\
\hline \multirow[t]{2}{*}{$G^{0}$} & $\begin{array}{c}\text { Toroidal } \\
\text { Spectrometer }\end{array}$ & 870 & 10 & $60^{\circ}-77^{\circ}(\mathrm{p})$ \\
\hline & (reversed) & 500 & 10 & $108^{\circ}$ \\
\hline
\end{tabular}

Table 5.2. Properties of parity-violating electron scattering detector systems (existing and proposed).

is only a few times $10^{34} \mathrm{~cm}^{-2} \mathrm{~s}^{-1}$. These targets, in addition, usually have associated impurities which could affect the interpretation of the results. Polarization reversal is also more difficult and the frequency would be much reduced in comparison to reversing beam electron polarization. These considerations are of great importance in the control of systematic errors. Furthermore, while we have not evaluated in any detail the case of recoil electron polarimetry, it would appear that involving a second scattering with its attendant 
cross section. analyzing power and efficiency would not be favorable.

Three Pl electron scattering experiments of the type described in this section have been completed to date. These will be discussed in more detail in Sect. V.B. followed in Sect. V.C by a description of the several new experiments which have been proposed or are currently in progress.

\section{V.A.2. IELTRINO SCATTERING FACILITIES}

Veutrino beams for medium-energy physics research are in use now at the Los liamos .leson Physics Facility (L.A.IIPF) and at the Rutherford Appleton Laboratory ISIS . Future facilities are planned. for the KAON project in Vancouver and for a Pulsed Lepton Source (PLS) at L.A.IPF. These neutrino beams can be put into two generic classes. In the beamstop source. neutrinos result predominantly from the production and subsequent decay at rest of single positive pions. The decay sequence $\pi^{+} \rightarrow \mu^{+} \nu_{\mu}$ is followed by $\mu^{+} \rightarrow e^{+} \nu_{e} \bar{\nu}_{\mu}$, which produces equal numbers of $\nu_{\mu} \cdot \nu_{e}$. and $\bar{\nu}_{\mu}$ neutrinos with up to 52.8 $\mathrm{Mel}$ kinetic energy. In the decay-in-flight (DIF) source. a decay region following a pion-production target allows energetic $\nu_{\mu}\left(\bar{\nu}_{\mu}\right)$ neutrinos to be produced from the decay of high-energy $\pi^{+}\left(\pi^{-}\right)$.

A brief survey of the characteristics of present and future medium-energy neutrino beams is displayed in Table 3.3 . The spectrum of a beamstop source, as shown in Fig. j.1a. consists of a monochromatic $\nu_{\mu}$ line at $29.8 \mathrm{MeV}$ from the two-body decay at rest of the $\pi^{+}$and the well-determined $\nu_{e}, \bar{\nu}_{\mu}$.Michel spectrum, with average energy about $35 . \mathrm{Mel}$. from the three-body decay at rest of the $\mu^{+}$. . Vuclear absorption of negative pions and muons typically reduces $\bar{\nu}_{\mathrm{e}}$ contamination from $\mu^{-}$decays at rest to less than $j \times 10^{-4}$ of the $\mu^{+}$decay flux. Neutrinos from a beamstop source can be separated in time for suitably short proton pulses. As shown in Table 5.3 , both the ISIS ( $100 \mathrm{~ns}$ ) and the proposed PLSL.A.MPF (270 ns) time structures are short compared to the muon lifetime, and so allow separation of $\nu_{\mu}$ from $\nu_{e}$ and $\bar{\nu}_{\mu}$ neutrinos. This is a particularly useful feature in neutral current measurements.

The more energetic $\nu_{\mu}$ spectra from DIF sources are shown in Figs. $5.1 \mathrm{~b}$ and $5.1 \mathrm{c}$ for a modified LA.MPF beamstop and for the future KAON facility. The LA.MPF neutrino flux. produced by an $800 \mathrm{MeV}$ proton beam, extends from 60 to $250 \mathrm{MeV}$, while the K.AON neutrino flux. produced irom $30 \mathrm{GeV}$ protons, would peak at about $1 \mathrm{GeV}$ and extend to $6 \mathrm{GeV}$. Charge selectivity in the pion focusing elements of the future KAON and PLSLAMPF beams will enhance the $\nu_{\mu}\left(\bar{\nu}_{\mu}\right)$ beam while decreasing the $\bar{\nu}_{\mu}\left(\nu_{\mu}\right)$ contamination.

Knowledge of the absolute neutrino flux is very difficult to obtain experimentally and is thus usually determined through Monte Carlo simulations and calibrations. For the rather complicated geometries of the LA.MPF and ISIS beamstops, absolute values to $\pm 7 \%$ accuracy were obtained through Monte Carlo calculations [Bur90] of the stopping $\pi^{+}$/proton ratio, as calibrated by an experimental measurement of that ratio in a simplified. instrumented beamstop [All89]. As an example of the accuracy of DIF sources, the BNL 


\section{TABLE 3.3}

\begin{tabular}{|c|c|c|c|c|c|}
\hline Laboratory & $\begin{array}{l}\text { Deutrino } \\
\text { Source }\end{array}$ & $\begin{array}{c}\text { Deutrino } \\
\text { Type }\end{array}$ & $\begin{array}{c}\text { Energy Range } \\
(\mathrm{MeV})\end{array}$ & $\begin{array}{c}\text { Time } \\
\text { Structure }\end{array}$ & $\begin{array}{c}\text { Flux } 0 \\
\nu \mathrm{cm}^{-2} \mathrm{~s}^{-1}\end{array}$ \\
\hline L.A.MPF & $\begin{array}{c}\text { beamstop } \\
\text { DIF }\end{array}$ & $\nu_{e}, \nu_{\mu} \cdot \bar{\nu}_{\mu}$ & $\begin{array}{c}0-33 \\
30-250\end{array}$ & $\begin{array}{c}500 \mu \mathrm{s} \\
\quad 1\end{array}$ & $\begin{array}{l}4.2 \times 10^{\circ} \\
1.1 \times 10^{5}\end{array}$ \\
\hline ISIS & beamstop & $\nu_{e}, \nu_{\mu}, \bar{\nu}_{\mu}$ & $0-53$ & $100 \mathrm{~ns}$ & $1.3 \times 10^{5}$ \\
\hline KAON & DIF & $\mu_{\nu}$ & $100-6000$ & $3.6 \mu \mathrm{s}$ & $2.3 \times 10^{8}$ \\
\hline PLS-LA.MPF & $\begin{array}{l}\text { beamstop } \\
\text { DIF }\end{array}$ & $\nu_{e}, \nu_{\mu} \cdot \bar{\nu}_{\mu}$ & $\begin{array}{c}0-33 \\
60-300\end{array}$ & $\begin{array}{c}270 \mathrm{~ns} \\
" \prime\end{array}$ & $\begin{array}{l}1.2 \times 10^{\circ} \\
7.5 \times 10^{5}\end{array}$ \\
\hline
\end{tabular}

Table 5.3. Characteristics of current and future neutrino sources. The decay-inflight sources (DIF) have a decay region following the pion production target. The time structure is that of the incident proton beam. Neutrino fluxes are calculated for representative geometries for the different facilities.

neutrino beam intensity has been quoted [Ahr85] as $\pm 12 \%$ by calibration with measured charged-current $\nu_{\mu}$ events.

Investigation of hadronic neutral currents is in progress at ISIS and at LA.MPF. The KAR.MEN collaboration has observed $[\operatorname{Bod} 91]$ the reaction ${ }^{12} \mathrm{C}\left(\nu, \nu^{\prime}\right)^{12} \mathrm{C}^{*}(10.11 \mathrm{MeV}$ ). for combined $\nu_{e}, \bar{\nu}_{\mu}$ neutrinos from the ISIS beamstop source (see also Sect. IV.J.j). The IS.ND collaboration at LAMPF is proceeding to measure both this reaction and the $p\left(\nu_{\mu}, \nu_{\mu}\right) p$ elastic scattering cross section at low momentum transfer [Lu90!. Elastic scattering from a nucleus, $A\left(\nu_{\mu}, \nu_{\mu}\right)$, has been considered for the prcposed LA.MPF-PLS facility [Don83]. 


\section{V.B. Past Studies of Electron and Neutrino Scattering}

\section{Y.B.1. DEEP-INELASTIC SCATTERING FROM DELTERITMISLAC)}

The first scattering experiment to investigate PV effects due to neutral currents in electromagnetic interactions was carried out at SLAC in the late 1970 's 'Prei9!. It involved the measurement of an asymmetry in deep-inelastic scattering (incoherent scattering trum individual quarks I of longitudinally-polarized electrons from a deuterium target at energiein the range $(16-22) \mathrm{Gel}$. These results provided one of the important early verifications for the predictions of the gauge theories and for the Weinberg-Salam-Glashow $\mathrm{SL}_{1} \mathbf{2}_{\mathrm{L}}$. I I I $_{1}$ theory Standard Model $i$ of the weak and electromagnetic interactions in particular.

The scale of the predicted PV asymmetry for the SLAC kinematics was small in $10^{-4} Q^{2} \mid\left(\mathrm{GeV}^{-} / \mathrm{c}^{2}\right.$ and as a consequence presented some very difficult technical problem. It required the development of an intense polarized electron source. beam control and monitoring. together with counting techniques capable of achieving the required sensitivy and precision. These developments provided a technical base and model for the experimentat intermediate-energies which were to be carried out later at . Nainz and .IIT Bates.

\section{Polarized Electron Source}

The polarized electron sources used as injectors in linear accelerators, to date. operate on the principle of photoemission from a GaAs crystal. At SLAC the longitudinallypolarized electrons were extracted following photoexcitation from the $J=3 / 2$ valence band to the $\mathrm{J}=1 / 2$ conduction band using circularly-polarized $710 \mathrm{~nm}$ light from a flashlamppumped dye laser. The theoretical maximum polarization achievable using this technique is $P_{\mathrm{e}}=0.50$. although in actual use the beam polarization as measured is more typically: $P_{e}=0.40$. A schematic of the original SLAC source is shown in Fig. 5.2. The Mainz and .MIT / Bates polarized sources as well as that planned for CEBAF are modern versions of very similar design. As described in Sect. $V A$, research is currently underway on tovaloping crystal structures which could yield polarizations as high as $P_{e}=0.80$.

The electron helicity was reversed rapidly by reversing the circular polarization of the laser light. A typical arrangement of optical elements used to achieve this is shown in Fig. 3.3. The light is linearly-polarized in a Glan-Thomson calcite prism and then circular polarization is achieved by means of a Pockels cell operating as a quarter-wave plate. The cell has a birefringence which is linear with the applied electric field allowing the polarization to be controlled by the application of a voltage prior to each beam pulse. Switching the polarity of the voltage reverses the helicity of the photons and the helicity of the electrons, allowing reversal rates up to $1 \mathrm{kHz}$ to be achieved.

In order to isolate asymmetry contributions due to systematic effects (for example. such as those connected with changes in beam parameters) from the PV asymmetries. it is .nportant to have an independent control of the electron helicity. At SLAC, a slow helicity-reversal technique was implemented by rotating the Glan-Thomson prism through $90^{\circ}$ using a movable holder. 


\section{Beam and Instrumentation}

The electron accelerator was operated at $120 \mathrm{~Hz}$ with beam pulse lengths of $1 . \overline{\mathrm{s}} \mathrm{s}$. Currents in the range $(1-4) \times 10^{11} e^{-} /$pulse were accelerated and delivered to the target. A major objective of the experiment was to eliminate and control the sources of helicitycorrelated systematic errors. Beam parameters were measured and controlled to keep the corrections to the measured asymmetry small. The measured beam parameters included average polarizat on. energy, current, position and angle at the target.

Beam polarization was measured using . Moller scattering in which the longitudinally-polarized electrons were elastically scattered from polarized target electrons. The target was a thin $\mathrm{Fe}$ foil located in a magnetizing external field. The scattering cross section is large and for relativistic energies the analyzing power is a maximum at $90^{\circ}$ in the electron-electron center-of-mass. Since the scattering process is precisely described by: QED. the beam polarization could be measured to an accuracy of $\pm 5 \%$. limited mainly by: the uncertainty in background subtraction.

The beam-monitoring system was crucial for controlling beam position and direction at the target. Resonant microwave cavities. with a node in the response which could be located on the beam axis, were used to measure the beam position. Pairs of monitors. sensitive to horizontal and vertical displacements. were located at $2 \mathrm{~m}$ and $50 \mathrm{~m}$ upstream of the target. It was possible to achieve pulse-to-pulse measurements of beam position. averaged over the pulse, with resolutions better than $10 \mu \mathrm{m}$ and corresponding angle resolutions better than $\pm 0.3 \mu$ radians.

Beam currents were measured ( $0.02 \%$ resolution) on a pulse-to-pulse basis by means of non-intercepting toroid monitors. The charge per pulse was used to normalize the detector flux signals as well as the beam position monitors. Beam energy was measured pulse-to-pulse by means of a microwave cavity position monitor at a location in the beamline with nonzero dispersion. The relative energy sensitivity was $\pm 0.01 \%$.

The beamline optics were typically nulled in order to minimize sensitivity to helicitycorrelated systematics. Beam energy, position. and angle were continuously monitored and stabilized using computer-controlled feedback loops.

\section{Target and Spectrometer}

The incident electrons were scattered in a $30 \mathrm{~cm}$ long liquid deuterium target and momentum-analyzed in a magnetic spectrometer. The spectrometer was operated at a scattering angle of $4^{\circ}$ and had a very large momentum acceptance. Angular acceptances were $\Delta \theta= \pm 7.5 \mathrm{mrad}$ and $\Delta \phi= \pm 16.6 \mathrm{mrad}$. Two counters were used to detect the scattered electrons: the first was a gaseous $\nu_{2}$ Cerenkov counter operated at atmospheric pressure in which a spherical mirror collected and focused the Cerenkov light to an offaxis phototube. This Cerenkov counter was backed by a shower counter constructed from nine radiation lengths of lead glass in which Cerenkov light produced in the lead glass was collected by an array of phototubes. A third counter was located behind a thick shielding wall which absorbed the electron shower. This counter was designed to measure 
the background due to $\pi$ 's, $\mu$ 's and $K$ 's and the associated asymmetry. The experimental arrangement was very clean and the measured contribution of the background to the final asymmetries was less than $1 \%$.

A statistical precision of $10^{-3}$ requires $10^{i}$ beam pulses or approximately one day at $120 \mathrm{~Hz}$ and therefore a flux-counting technique was developed to deal with the very high rates of scattered particles. At the SLAC kinematics each $1.5 \mu$ s beam pulse yielded $10^{3}$ scattered electrons. The resulting anode currents for each detector were integrated. digitized and stored to tape. Cormalizing the flux to the incident charge vields a quantity proportional to the acceptance averaged cross section. Since background discrimination is not possible in such a flux counting measurement, the kinematics and experiment were designed to minimize the contribution of unwanted processes.

\section{Results}

The data were collected in runs of a few hours (1-3) duration. During each run there was random pulse-to-pulse reversal of beam helicity using the Pockels cell. Between runs. two other methods were employed to achieve independent helicity reversal. A rotation of the calcite polarizing prism. in front of the Pockels cell, resulted in a rotation of the plane of linear polarization and beam polarization reversal. Some data were also taken with the prism at the $45^{\circ}$ (unpolarized beam) position.

The second method of spin reversal was based on the precession of the electron spin relative to its direction. due to the electron's anomalous magnetic moment $(g-2$ precession). The electron beam was transported with a net deflection of $24.5^{\circ}$ between accelerator and target. A beam energy change of $3.237 \mathrm{GeV}$ results in a helicity reversal. The experiment was run at four energies in the range $(16.2-22.2) \mathrm{GeV}$ resulting in approximately two spin reversals due to precession. Figure 5.4 shows the results for the measured asymmetries for each of the different energies. The kinematics for the scattering process were adjusted by correcting the asymmetry for the varying $Q^{2}$.

The above methods for spin reversal were designed to identify and measure false asymmetries due to systematic effects. These independent spin-reversal techniques allow for the experimental cancellation of helicity-correlated effects that the source may have on the beam parameters. It is important however, that the level of such cancellations or corrections be kept small relative to the measured physics asymmetry.

The SLAC data for the two highest-energy points yielded an experimental asymmetry $A_{L R} /\left|Q^{2}\right|=(-9.5 \pm 1.6) \times 10^{-5}(\mathrm{GeV} / \mathrm{c})^{-2}$ (deuterium). The data point is at the average value of $y=1-\epsilon^{\prime} / \epsilon=0.21$ and the average value of $\left|Q^{2}\right|=1.6(\mathrm{GeV} / \mathrm{c})^{2}[\operatorname{Pre} 78]$. The quoted error is a linear combination of equal statistical and systematic contributions. The main systematic uncertainties come from the measurement of beam polarization, $P_{e}(\sim 5 \%)$ and helicity-correlated differences in beam parameters $(\sim 3 \%)$. The beam parameter errors are summarized in Table 5.4. As expected. the energy differences were the most important. 


\begin{tabular}{|l|c|}
\hline \multicolumn{2}{|c|}{ TABLE 5.4 } \\
\hline Parameter & $\Delta A_{L R} /\left|Q^{2}\right|$ \\
\hline$E_{0}$ & $-0.3 i \times 10^{-5}$ \\
$Q$ & $-0.03 \times 10^{-5}$ \\
$X_{t}$ & $+0.04 \times 10^{-5}$ \\
$Y_{t}$ & $-0.02 \times 10^{-5}$ \\
$\theta_{x}$ & 0.00 \\
$\theta_{y}$ & $+0.01 \times 10^{-5}$ \\
& \\
\multicolumn{1}{|c|}{ Total } & $-0.37 \times 10^{-5}$ \\
\hline
\end{tabular}

Table 5.4. Systematic errors for the SLAC experiment [Pre79].

. Oormalization corrections have also been applied to the data for $\pi^{-}$background ( $\left.2 \%\right)$ and radiative effects $(3 \%)$.

In Fig. 5.5 the measured asymmetry is shown as a function of $y$ (see Ref. [Prei9]). The results are clearly consistent with the Standard Model and were used to extract a best fit for the weak mixing angle: $\sin ^{2} \theta_{W}=0.224 \pm 0.020$. The best fit for the modelindependent parameters is $a_{1}=-(9.7 \pm 2.6) \times 10^{-5}$ and $a_{2}=(4.9 \pm 8.1) \times 10^{-3}$. where $a_{i}=\left(9 G_{\mu} / 20 \sqrt{2} \pi \alpha\right) \tilde{a}_{i}$ with $\tilde{a}_{i}$ defined in Eq. (4.89). A less accurate measurement was also done using a liquid hydrogen target at $19.4 \mathrm{GeV}$. The experimental asymmetry was $A_{L R} /\left|Q^{2}\right|=(-9.7 \pm 2.7) \times 10^{-5}(\mathrm{GeV} / \mathrm{c})^{-2}$ (hydrogen). The quoted error includes both the statistical and systematic uncertainties. One expects a slightly smaller asymmetry for the proton due to the different mix of quarks.

\section{V.B.2. QUASIELASTIC SCATTERING FROM BERYLLIUM (MAINZ)}

The PV asymmetries observed in the scattering of high-energy longitudinallypolarized electrons from deuterium at SLAC and in atomic physics measurements at lowenergies were sensitive mainly to the hadronic vector current. These two measurements are essentially orthogonally dependent on the linear couplings $\tilde{\alpha}$ and $\tilde{\gamma}$ of Table 3.2. Contributions due to the hadronic axial-vector currents were too small to provide a measure of the corresponding coupling constants $\tilde{\beta}$ and $\tilde{\delta}$.

A recent PV experiment completed at Mainz [Hei89], involved the scattering of medium-energy electrons at backward angles. The measurement involved the inclusive scattering of polarized electrons of energy $\epsilon=300 \mathrm{MeV}$ from ${ }^{9} \mathrm{Be}$ at an average scattering angle of $\bar{\theta}=130^{\circ}$. At these kinematics, the cross section is dominated by quasielastic scattering and the sensitivity to the hadronic axial-vetor current is enhanced, as discussed in 
Sect. IV.F. The expected asymmetry at this momentum transfer is approximately $10^{-3}$. which is about 10 times smaller than for the SLAC experiment. The problems associated with achieving the required statistical accuracy and the control of systematic errors were severe.

\section{Technical Considerations}

The polarized source that was built for this experiment followed closely the SLAC design. For injection into the linac the source produced current pulses of $150 \mathrm{mAt}$ peak and $3 . \overline{\mathrm{s}} \mathrm{s}$ duration at a repetition rate of $50 \mathrm{~Hz}$. An average current of about $i \mu \mathrm{A}$ was delivered to the target. Proper accelerator operation required injected beam pulses with a high degree of amplitude stability as well as flatness. An optical device. consisting of a transverse Pockels cell mounted between a pair of Glan-Thomson polarizers. was incorporated in the laser beamline to provide automatic control of the pulse-shape. A short-termi amplitude stability of $\sim 0.1 \%$ and flatness within $3 \times 10^{-3}$ were achieved. Helicity-correlated beam intensity differences had to be maintained at a level $<10^{-5}$ - this was achieved with an active feedback system incorporated into the laser beamline optics. The source lifetime during operation was as long as 200 hours. A short interruption was required approximately once per day to change the flashlamps and the laser dye. As in the SLAC experiment, rapid polarization reversal was achieved by reversing the voltage applied to the Pockels cell. A slow reversal cycle, approximately every 15 min. was accomplished by rotating the half-wave plate between the Glan prism and the Pockels cell.

The beam transport line to the target consisted of two successive oppositely deflecting $90^{\circ}$ achromatic bends. As a consequence, the parallel displacement of the electron beam leaves the longitudinal polarization unchanged. The relatively low beam energy precluded the use of spin precession for independent spin reversal.

Beam polarization was measured using three different techniques. Prior to injection intu ine linac. a Wien filter was used to produce transverse polarization. This was followed by spin analysis of the Mott-scattered $44 \mathrm{keV}$ electrons. The polarization of the $300 \mathrm{MeV}$ beam could also be measured directly using a Møller polarimeter downstream of the first $90^{\circ}$ achromat.

An on-line Compton polarimeter was used to provide relative polarization measurements during the experiment. It consisted of a magnetized iron absorber between two ionization chambers. The ratios of the ionization currents in the two chambers for positive and segative electron helicity measures the circular polarization of the bremsstrahlung. This in turn is proportional to the longitudinal polarization of the electron beam. The Compton polarimeter was calibrated relative … rhe Møller polarimeter. The beam polarization during the experiment averaged between $44 \%$ and $50 \%$.

The beamline was fully instrumented to control and minimize helicity-correlated differences in beam parameters such as energy, intensity, position and angle at the target. The monitoring system included toroid intensity monitors, microwave cavity position monitors and steering correctors. 


\section{Detector System}

Given the small PV asymmetry and the cross section for quasielastic scattering it was necessary to have a detector system of very large solid angle to achieve the desired statistical accuracy. Conventional magnetic spectrometers were inadequate. The detector system (Fig. 5.6) consisted of 12 ellipsoidal mirror gas Cerenkov counters mounted symmetrically about the beam axis. Electrons scattered in the angular range $110^{\circ}<\theta<145^{\circ}$ were detected. The Cerenkov light emitted by the electrons travelling through air between target and mirror is focused onto the photomultiplier cathode. The mirrors subtended a solid angle of $20 \%$ of $4 \pi$.

The detector has essentially no momentum resolution. With atmospheric pressure air as the Cerenkov medium the threshold energy for detecting electrons was about 21 $\mathrm{MeV}$. This provided a low-energy cut-off for the electron spectrum and some discrimina. tion of backgrounds due to heavier particles. Each of the mirrors could be tilted remotely. in order to scan the target image over the face of the photomultiplier. This allowed the mirror orientation to be optimized and the signal-to-background ratio measured. Four additional photomultipliers positioned out of the mirror focus and mounted symmetrically about the beam axis provided continuous on-line background information for each counter. One important background source in this experiment was beam halo which could be directly scattered in the direction of the photomultipliers. Under optimal accelerator tuning conditions the contribution due to beam halo was kept below $1 \%$.

\section{Measurements}

The scattered rate into each detector during a beam burst $\left(\sim 3 \times 10^{4} /\right.$ pulse $)$ was too high for individual event counting. Instead, the resulting flux (anode current) for each detector was integrated, digitized and stored to tape. Beam information, charge and position. was similarly averaged and recorded for each beam burst. The electron helicity was randomly chosen for a beam burst. The following beam pulse was set to have the opposite helicity. This process was repeated for succeeding pulse pairs. In analyzing the data, asymmetries were formed from such adjacent pulse pairs. This approach compensated for unavoidable slow drifts in the accelerator and detector hardware.

Figure 5.7 shows a histogram of pulse-pair asymmetries $(\sim 22,500)$ for a $15 \mathrm{~min}$ run at a fixed orientation of the half-wave plate. The result shows the expected normal distribution. In addition, the width of the distribution is consistent with the expected number of scattered electrons. One concludes that contributions due to fluctuations of the beam parameters have been suppressed to a level below the statistical errors. 
$\underline{\text { Results }}$

There were several sources of systematic false asymmetries which had to be understood and controlled to a very high level. One of these was electronic cross-talk correlated with the high voltage switching of the Pockels cell. Careful wiring and grounding of the electronics followed by detector tests with light diodes showed that the electronic asymmetry was less than $8 \times 10^{-8}$, which is insignificant. Møller and Mott scattering depends on the polarization of the electron beam and can give rise to false asymmetries. The axially symmetric detector system reduced possible .lott effects to less than $4 \times 10^{-8}$ and . Holler scattering was estimated to contribute at a level of less than $8 \times 10^{-8}$. The most troublesome systematic errors were the helicity-dependent differences in the beam parameters such as position, angle. energy, and intensity. The sensitivity to each of these parameters was determined experimentally from the normal jitter of the beam itself. This jitter was typically 100 times larger than the measured correlated differences in any of the parameters.

After applying various corrections, the measured corrected asymmetry was found to be $A_{L R}^{c o r r}=(-3.5 \pm 0.7 \pm 0.2) \times 10^{-6}$. where the first error is the statistical uncertainty and the second is the systematic. The experimental asymmetry was extracted from $A_{L R}^{c o r r}$ by normalizing for the dilution due to the background ( $B / S$, assumed to produce no asymmetry $)$ and the dependence on the electron polarization $\left(P_{t}\right.$ using the relation

$$
A_{L R}^{e x p}=A_{L R}^{c o r r} \frac{(1+B / S)}{P_{e}} .
$$

Csing averaged values for $B / S=0.19$ and $P_{e}=0.443$ the final result is

$$
A_{L R}^{e x p}=(-9.4 \pm 1.8 \pm 0.5) \times 10^{-6} .
$$

In interpreting this result one should note that at least four distinct scattering processes contributed significantly to the asymmetry. These include: quasielastic scattering ( $59 \%)$, evcitatinn of the $\Delta$-rosonance $(6.5 \%)$, contributions from the "dip" region $(12.5 \%)$ and contributions from the radiative tail for low final electron energies $(22 \%)$. The spectrum was assumed to be cut off by the $21 \mathrm{MeV}$ detector threshold.

This measured result can be combined with previous model-independent determinations of $\tilde{\alpha}$ and $\tilde{\gamma}$ [Pre79, Kim81] to obtain a relation between the two axial-vector coupling constants

$$
\tilde{\beta}+0.04 \tilde{\delta}=0.005 \pm 0.17 .
$$

The errors refiect a combination of the errors in the measurement of $\ddot{\alpha}$ and $\tilde{\gamma}$ and the total relative error of this experiment. The results for $\tilde{3}$ and $\tilde{\delta}$ are shown plotted in Fig. $\tilde{j} .8$ in the standard form involving the quark coupling constants $C_{2 d}=(-\tilde{\beta}+\tilde{\delta})$ and $C_{2 u}=(\tilde{\beta}+\tilde{\delta})$ together with the results of the SLAC experiment. The results of this experiment are consistent with a vanishing axial-vector interaction $(\tilde{3}=i=0)$. The resulting error band has been reduced by a factor of three in comparison with earlier work. 


\section{V.B.3. ELASTIC SCATTERING FROM CARBON (MIT/BATES)}

An experiment measuring the $\mathrm{PV}$ asymmetry in the elastic scattering of longitudinally-polarized electrons from ${ }^{12} \mathrm{C}$ was successfully completed at . VIT/Bates [Sou90a). The measurement was motivated by the theoretical work of Feinberg [Feiij] and Walecka [WaliT], as discussed in Sect. IV.B: since ${ }^{12} \mathrm{C}$ is a spinless and isoscalar nucleus. the electromagnetic amplitude is described by a single form factor. At low-energies where a phenomenological four-fermion interaction is a good approximation. the asymmetry may be written as in Eq. (3.119). The expression for the asymmetry is independent of the form factors if the effects from strangeness. isospin-mixing and dispersion corrections (all of which are expected to be small, see Sect. IV.B) are ignored. The goal of the .IIT/Bates ${ }^{12} \mathrm{C}$ experiment was to measure $\sin ^{2} \theta_{w}$ precisely using a technique which involved very little theoretical ambiguity in the interpretation of its result. The choice of optimal kinematics for elastic scattering from ${ }^{12} \mathrm{C}$ was influenced by several important considerations. To first-order it was important to maximize the FOMI. Signal-to-background considerations, contamination due to Mott asymmetry and contributions due to inelastic levels in ${ }^{12} \mathrm{C}$ also influenced the choice. The experiment was designed for a beam energy: of $250 \mathrm{MeV}$, a scattering angle of about $35^{\circ}$ giving a momentum transfer $q=150 \mathrm{MeV}, \mathrm{c}$. With a beam polarization $P_{e}=37 \%$ the Standard . Model predicts an experimental asymmetry $A_{L R}^{e x p}=A_{L R} P_{e}=0.70 \times 10^{-6}$. Such a small asymmetry places severe demands on the apparatus, in terms of both achieving sufficient statistical precision and maintaining adequate control of systematic errors.

\section{Beam and Instrumentation}

The apparatus for this measurement consisted of a polarized electron source, a pair of single-quadrupole spectrometers, a Møller polarimeter, precision beam monitors. and a high-capacity data acquisition system. A schematic layout of the experimental apparatus in shown in Fig. 5.9.

The source, which provided an intense beam of longitudinally-polarized electrons. was built especially for this experiment. Similar to the SLAC source, it was based on photoemission from a GaAs crystal using circularly-polarized light, but in addition involved a unique three-chamber design which allowed for greater flexibility to select crystals with high quantum efficiency, lifetime and polarization. The main components of the optical system were a $2 \mathrm{~W} \mathrm{CW}$ krypton-ion laser and a system of optical shutters and circular polarizers. An electro-optical shutter, consisting of two crossed polarizers, half-wave plate and a Pockels cell modulated the laser beam to match the $1 \%$ duty factor of the accelerator. A "flipper" system consisting of a half-wave plate, crossed polarizers and a Pockels cell produced the circularly-polarized light and controlled helicity reversal. The half-wave plate served as a "slow" helicity reversal device. The two different orientations of the device reversed the relationship between the polarity of the potential difference applied to the Pockels cell and the handedness of the emerging laser light. This made it possible to change the overall sign of all downstream helicity-correlated differences including the electroweak PV asymmetry. A converging lens provided point-to-point focusing between the Pockels cell and the GaAs crystal and made the position of the laser beam on the crystal 
insensitive to the angular fluctuations that could be induced in the beam by misalignments or instability in the operation of the Pockels cell.

The beam delivery system was designed and carefully instrumented to ensure that the expected small asymmetries could be accurately measured. A set of monitors measured the undesirable effects of helicity reversal. These included seven current monitors to measure the intensity. four microwave cavity position monitors in front of the target to measure the position and angle of the beam and a position monitor to measure the beam energy at a location in the chicane where the beam was dispersed in momentum. All of these measurements were performed on a pulse-by-pulse basis together with the data-taking.

A steering coil pulsing scheme allowed steering correctors along the beam transport line to be synchronously adjusted (ramped) during data acquisition. The coil current changes were triggered at a $47 \mathrm{~Hz}$ rate. which was chosen to be fast enough so that the system calibration would be unaffected by drifts and slow enough to add negligibly to the statistical fluctuations in the cross section measurements. The coil-pulsing system was used for approximately one-third of the data collection time.

The combination of precision monitors and the coil pulsing scheme allowed for the measurement and correction of systematic errors. It was also possible to calibrate the position monitors and to measure the sensitivity of the detector system to known displacements of beam energy, position and angle at the target. As a consequence the beam position and direction could be located and maintained in a way that minimized the sensitivity of the experiment to beam parameters.

The detector system consisted of a pair of single-quadrupole spectrometers. $\mathrm{CV} T$ lucite Cerenkov counters, analog and control electronics and a high-capacity data acquisition system. The two spectrometers were fixed on either side of the beamline at a nominal scattering angle of $35^{\circ}$. Each had an acceptance of $\sim 10 \mathrm{msr}$ and a momentum resolution of $\sim 12 \mathrm{MeV}$. Lead collimators at the front, rear, and midplane of the quadrupoles defined the acceptance and momentum discrimination of the system. The focusing was in the vertical plane. A bank of 12 Cerenkov detectors directly coupled to photomultipliers formed the detector $\dot{a}_{\text {. }}: \mathrm{ay}$.

\section{Measurements}

The electron beam impinged on a $5 \mathrm{~g} / \mathrm{cm}^{2}$ segmented carbon target. About $10^{5}$ electrons were detected during each $17 \mu \mathrm{s}$ beam burst and the integrated responses over the beam pulse were recorded by 16-bit ADC's. A Møller polarimeter, similar to the SLAC design, was used to measure the beam polarization during the course of the experiment.

A major consideration in implementing a data-taking scheme was the issue of noise: it was important to use a technique where the noise contributions would be less than the statistical error per pulse. To achieve this the accelerator was operated at a pulse rate of $600 \mathrm{~Hz}$ locked to the $60 \mathrm{~Hz}$ line frequency. The noise associated with the $60 \mathrm{~Hz}$ frequency was minimized by dividing the data into ten separate "timeslots" corresponding to the 60 $\mathrm{Hz}$ harmonics and then analyzing the data for each timeslot independently. The beam 
helicity was set quasi-randomly for each pulse. Ten random helicities were chosen. one for each timeslot. The pattern was complemented for the next ten beam bursts. and ten asymmetries were computed. each based on a complementary pulse pair. This process was repeated every ten pulse pairs, i.e., at a $30 \mathrm{~Hz}$ rate. Typical beam currents on target averaged 30-60 $\mu \mathrm{A}$. The accumulated data amounted to $30 \mathrm{~T}$ half-hour runs. With each timeslot treated independently, this corresponds to 3070 individual "mini-runs". The statistical error for each mini-run was computed using the variance of the asymmetries. Approximately $1 \%$ of the data was rejected by loose cuts which were associated with accelerator malfunctions. A histogram of the measured asymmetry for each mini-run normalized to its statistical error is shown in Fig. 5.10. The shape. as demonstrated by the solid curve. is Gaussian with the expected width over more than two decades.

The main objective of the experiment was to measure a cross section which. apart from the electroweak physics. is independent of the sign of the helicity. The goal was to maintain the level of systematic errors below the statistical precision. There were many sources of systematic error. They included: beam parameters (intensity. energy: position. direction. enittance, transverse polarization); hardware (drifts and noise. crosstalk/pickup, linearity): nuclear physics (isospin admixtures, inelastic contributions. target purity. strangeness). Correlations of beam parameters with helicity. such as energy, position and intensity were the most important class of systematic errors. These were controlled by minimizing helicity correlations during data acquisition and corrections were made to the raw asymmetries using position monitor data during analysis. A major source of helicity-dependent correlations was the intensity of the laser light striking the GaAs photocathode. The resultant helicity-correlated current changes are coupled by accelerator beam loading to energy changes. Since the cross section is a very strong function of the energy it was necessary to maintain the helicity-dependent current fluctuations to within $\Delta I / I<7 \times 10^{-7}$. The principal cause of the intensity correlation was a coupling of a slightly elliptically polarized laser beam to an asymmetric optical transport system in which the transport efficiency depends on the orientation of the ellipse axes. In practice this effect was easily controlled. It was possible to adjust the appropriate phase electro-optically by small changes in the voltages applied to the Pockels cell. A slow feedback loop was implemented to minimize the intensity asymmetry. The result was that the helicity-correlated intensity asymmetry averaged over the experiment was reduced to about $1 \mathrm{ppm}$.

\section{$\underline{\text { Results }}$}

During data analysis, it was necessary to make small correction for systematic asymmetries as measured by the beam monitors. The corrected asymmetries were related to the raw asymmetries by the algorithm

$$
A_{L R}^{\text {corr }}=A_{L R}^{r a w}-\Sigma a_{i}\left(\delta M_{i}\right),
$$

where $A_{L R}^{r a w}$ is the measured uncorrected asymmetry, $\delta M_{i}$ are the helicity-correlated differences measured by the beam monitors, and the $a_{i}$ are correction coefficients, which are a measure of the sensitivity of the asymmetry to fluctuations in the beam parameters. The 
last were determined simultaneously with data-taking by using the coil-pulsing system. Correlation analysis of the energy measurement in the beamline chicane was used to extract the energy-sensitive coefficient. An energy vernier (fast phase shifter) on one of the klystron amplifiers provided a controlled and independent measure of the sensitivity. Typical values for the $a_{1}$ were about $10 \mathrm{ppm} / \mu \mathrm{m}$ and the averaged helicity-correlated position differences were typically a fraction of $1 \mu \mathrm{m}$.

Table $5 . j$ lists all of the corrections (ppm) that were applied to the experimental asymmetry together with their estimated uncertainty. The root-mean-square value of the corrections due to the position and energy monitor differences for individual runs was 0.3 $\mathrm{ppm}$. The average correction for the entire data sample was only $0.04 \mathrm{ppm}$. An important check on the systematics is a measurement of a null asymmetry. If the half-wave-plate reversal is neglected in the data analysis. the measured asymmetry is $0.04 \pm 0.14 \mathrm{ppm}$. Similarly the difference in the measured asymmetry between the two spectrometers is $0.14 \pm 0.14 \mathrm{ppm}$. This latter result is important in determining that contributions due to transverse polarizations are not significant in this experiment.

\begin{tabular}{|l|c|c|}
\hline \multicolumn{3}{|c|}{ TABLE 5.5 } \\
\hline Correction & Value & Error \\
\hline Energy and Position . Monitors & 0.04 & \pm 0.006 \\
Electronic Cross-Talk & - & \pm 0.001 \\
Transverse Polarization & - & \pm 0.005 \\
Nonlinearities & - & \pm 0.007 \\
Phase Space & - & \pm 0.006 \\
Magnetized Iron Background & - & \pm 0.010 \\
$\quad$ Total & & \pm 0.016 \\
\hline
\end{tabular}

Table 5.5. Systematic errors (in units of $\mathrm{ppm}$ ) for the Bates ${ }^{12} \mathrm{C}$ experiment [Sou90a].

The result for the measured raw asymmetry is $A_{L R}^{\text {raw }}=0.56 \pm 0.14 \mathrm{ppm}$ and the corrected PV asymmetry is $A_{L R}^{\text {corr }}=0.60 \pm 0.14 \pm 0.02 \mathrm{ppm}$, where the first error is statistical and the second is systematic.

It was necessary to account for various normalization factors in extracting a value for $\sin ^{2} \theta_{w}$. These included the average effective $\left|Q^{2}\right|$, the beam polarization $P_{e}$, and the backgrounds due to inelastic nuclear levels and scattered neutrons. These factors are summarized in Table 5.6. Applying these normalization factors the final result for the 
experimental asymmetry is $A_{L R}^{e x p}=(1.65 \pm 0.39 \pm 0.06) \times 10^{-6}$. The resulting extracted value for $\bar{z}$ ( see Table 3.2 ) is

$$
\tilde{y}=0.136 \pm 0.032 \pm 0.009 \text {, }
$$

which is consistent with the prediction of the Standard Model. where

$$
\tilde{i}_{S . M}=0.155 \pm 0.002 \text {. }
$$

using a value of $\sin ^{2} \theta_{w}=0.233 \pm 0.002$.

\begin{tabular}{|l|l|}
\hline \multicolumn{2}{|c|}{ TABLE 5.6 } \\
\hline Beam Polarization. $P_{e}$ & $0.37 \pm 0.02$ \\
.uclear Structure & $1.00 \pm 0.01$ \\
Background & $0.98 \pm 0.02$ \\
$\left\langle\left|Q^{2}\right|\right\rangle /\left\langle\left|Q_{\diamond}^{2}\right|\right\rangle$ & $1.00 \pm 0.02$ \\
\hline
\end{tabular}

Table 5.6. Normalization factors for the Bates ${ }^{12} \mathrm{C}$ experiment [Sou90a].

This experiment achieved a statistical precision which is about five times smaller than that for the most sensitive previous electron scattering measurement. Based on these very promising results, especially the very small systematic errors, it is clear that significant improvements in a future ${ }^{12} \mathrm{C}$ measurement are feasible. It is reasonable to imagine large acceptance spectrometers with factors of 10-30 increased solid angle which together with substantially longer running time would result in a statistical error of a few percent. At this level of precision theoretical uncertainties may become important. We will need to understand that the effects of hadronic contributions to the radiative corrections and isospin-mixing are tractable at this level before committing to such a major effort. A significantly larger contribution, about which there is currently much speculation and a lot of interest, could come from a large charge radius of strange quarks in the nucleon (see Sects. IV.B and V.C).

\section{V.B.4. NEUTRINO-PROTON SCATTERING (BNL)}

Another class of experiments involves those where neutrino-proton elastic scattering cross sections are measured. As discussed in Sect. IV.J, these experiments are the best choice for accessing the axial-vector form factors, both in terms of sensitivity and because the axial-vector radiative corrections are much smaller than in electron scattering. The relevant differential cross section is given in Eqs. (4.105).

The most recently completed experiment of this type is that of Ahrens et al. [Ahr87], performed at the Brookhaven AGS facility. The $28 \mathrm{GeV}$ proton beam, incident on a 
production target. produced pions and kaons, which decay in-flight into primarily muon neutrinos. ¿sing a magnetic horn the secondary beams were focused into a decay tunnel. producing a wide-band beam of either $\nu$ or $\bar{\nu}$. depending on the polarity of the horn. The neutrino beams covered a spectrum of 0.2 to $j \mathrm{GeV}$. peaked at $\sim 1 \mathrm{Gel}$. The contamination from electron neutrinos was typically $1 \%$ and from wrong-helicity neutrinos was $\sim 3 \%$ for the $\nu$ beam and $\sim 9 \%$ for the $\bar{\nu}$ beam. In general, the neutrino flux cannot be measured in the neutral current scattering process, so secondary measurements of quasielastic chargedcurrent scattering are performed and the data are normalized to the reactions $\nu_{\mu} n-\mu^{-} p$ and $\bar{\nu}_{\mu} p \rightarrow \mu^{+} n$.

As can be seen in Eq. (4.105), the neutrino cross sections are proportional to $G_{\mu}^{2}$. and $=0$ are very small. Thus, it is important to have as high a luminosity and as large a detector as possible. Typically one uses an active target. In the case at hand. the target/detector was comprised of 170 metric tons of liquid scintillator cells. In addition to the liquid scintillator, each of the 112 cells contained crossed planes of proportional drift tubes to measure particle tracks. Particle identification was performed by measuring the energy deposited in each liquid scintillator plane, and the $\nu-p$ signature was ideritified by the scattered proton. A shower counter directly downstream of the main detector was used to contain the electromagnetic shower from neutrino interactions in the downstream half of the detector. A muon spectrometer was located behind the shower counter and was used to measure the neutrino flux via charged-current scattering. An important consequence of having to use an active target such as liquid scintillator is that only $20 \%$ of the protons in the target are actually free protons; the other $80 \%$ are bound in ${ }^{12} \mathrm{C}$. This introduces a systematic error associated with how well the neutrino scattering process is described by quasifree scattering.

Much of the background was reduced by using the time structure of the beam to eliminate events which arrived out of time with respect to the AGS beam extraction. In this way the background from inelastic pion production was removed. Neutron-induced events ware also removed with this cut, and also by reducing the active volume of the detector to only the central region (an $80 \%$ reduction). The remainder of the backgrounds were modeled by a Monte Carlo calculation. The overall normalization error in the experiment was $10 \%$, and came predominantly from the Monte Carlo calculation.

The differential cross sections for both neutrinos and antineutrinos are shown in Fig. 5.11. The data were fit to Eq. (4.105), averaged over the neutrino energy spectrum. The vector form factors $F_{1}$ and $F_{2}$ were assumed to have a dipole $\left|Q^{2}\right|$-dependence with $. M_{V}=0.843 \mathrm{GeV}$, and both vector strangeness form factors were taken to be zero. The axial-vector form factor was also assumed to have a dipole dependence, and although there was an allowance for a strange axial-vector contribution through the parameter $\eta_{s}$, it was assumed that the $\left|Q^{2}\right|$-dependence of the strange contribution is the same as the nonstrang. part. The adjustable parameters of the fit were $\sin ^{2} \theta_{W}$, the axial-vector

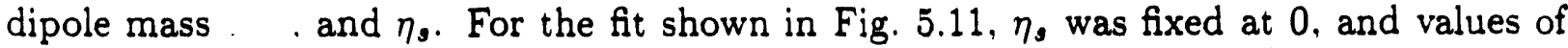
$\sin ^{2} \theta_{W}=0 .: \quad \pm 0.016$ (stat) \pm 0.03 (syst) and $M_{A}=1.06 \pm 0.05 \mathrm{GeV} / \mathrm{c}^{2}$ were simultaneously extracted. Overall normalization adjustments of $s_{\nu}=1.05$ for $\nu_{\mu} p$ and $s_{\bar{\nu}}=1.09$ for $\bar{\nu}_{\mu} p$ was made due to the $10 \%$ normalization uncertainty in the data. In a second fit. $M_{A}$ 
and $\eta$, were simultaneously fit while $\sin ^{2} \theta_{W}$ was held at the then world average of $0.220 . A$ contour plot of $\gamma^{2}$ in $\eta$, and $\Psi_{A}$ space is shown in Fig. j.12. and, as stated by the authors. it is seen that they are highly correlated. Nonetheless, by fixing $M_{A}$ at the then world average of 1.03 a value of $\eta_{s}=0.12 \pm 0.07$ was extracted from the data. corresponding to $G_{A}^{\prime \prime \prime} \mid 01=-0.15 \pm 0.08$. This result is. in fact. consistent with the published result of the E.IC collaboration iAsh89] (assuming the momentum scale dependence of the latter is negligible). It is. however. also consistent with zero at the $2 \sigma$ level.

In assessing the results of this experiment. several questions come to mind which one might hope to address in future experiments. Firstly, the majority of the protons detected were actually quasielastically knocked out of ${ }^{12} \mathrm{C}$ nuclei. Is it reasonable to assume that the both $M_{A}$ and $G_{A}\left(Q^{2}\right)$ are the same for nucleons bound in nuclei as for free nucleons? At least one author has suggested (Bro88] that medium modifications might lead to a change in the value of $W_{A}$ on the order of $5 \%$ [Whi90) and. since $K_{A}$ and $\eta_{\text {, }}$ are so highly correlated, this could lead to a large change in $\eta_{\mathbf{s}}$. Secondly, is the assumption that $G_{A}^{\prime \prime}$ 'has the same $\left|Q^{2}\right|$-dependence as $G_{A}^{T=1}$ a good one? It has been suggested [Ber90! that. within the context of soliton models. the isoscalar axial-vector dipole mass should be somewhat larger than $M_{A}$. Linfortunately, there is no other experimental information available at this time. Both of these assumptions could be avoided if an experiment were to be performed at a lower average value of $\left|Q^{2}\right|$. A third concern is the assumption that both $F_{1}^{\left({ }^{\prime}\right.} Q^{2}$ ) and $F_{2}^{(s)}\left(Q^{2}\right)$ are zero. If one assumes the Jaffe parameterizations, the contributions from the vector strange form factors at these kinematics are each about $10-15 \%$ Bei9lai. At lower- $\left|Q^{2}\right|$ the contribution from $F_{1}^{(s)}$ will certainly be smaller; however, $F_{2}^{(s)}$ is as yet undetermined.

A re-analysis of the Brookhaven data has recently been performed [Gar93], taking into account several of the above considerations. The authors use more recent world averages of $\sin ^{2} \hat{\theta}_{W}=0.2325$ and $._{A}=1.061 \pm 0.026$ in the new fit. They also use a nonzero value for the neutron electric form factor. The vector form factors $F_{1}^{(s)}$ and $F_{2}^{(s)}$ were included in the fit and a dipole dependence with dipole mass. $M_{V}$ was assumed. The fit parameters were $M_{A}, \eta_{s}, \mu_{s},\left\langle r_{s}^{2}\right\rangle$ (effectively) and the overall normalization parameters $s_{\nu}$ and $s_{\bar{\nu}}$. Five different fits are performed, in which $M_{A}, \eta_{s}, \mu_{s}$, and $\left\langle r_{s}^{2}\right\rangle$ were either held constant or extracted from the fit. Two of the five fits attempt to account for the fact that the majority of the target protons were bound in carbon by reducing the effective number of protons from 6 to $5.4 \pm 0.6$. Reducing the number of target protons generally has the effect of increasing the fitted value of $\eta$, without having much effect on the extracted values of the vector form factors. The authors point out that because of the observed $Q^{2}$-dependence of the data, a positive slope of $F_{1}^{(s)}\left(Q^{2}\right)$ is preferred. Their combined fit of vector and axial-vector parameters yields uncertainties of roughly order \pm 0.5 for the strangeness magnetic moment, and $\pm 0.1 \mathrm{fm}^{2}$ for the square of the strangeness radius. The errors in the fitted vector form factors (as well as those between the axial-vector form factor and the axial-vector dipole mass) are highly correlated.

* In Ref. [Gar93] the authors define the parameter $F_{1}^{9}=-\frac{1}{6}\left\langle r_{9}^{2}\right\rangle$. In the present notation, this corresponds to $-\left.\frac{d F^{(\bullet)}}{d Q^{2}}\right|_{Q^{2}}=0$. 
The implication is that the B.IL data do potentially provide some rough constraints on the vector. as well as axial-vector, nucleon form factors. This comes largely from the fact that $\nu$ and $\bar{\nu}$ cross sections have nicely orthogonal dependences on the vector form factors. Unfortunately, the large values of $\left|Q^{2}\right|$ in this experiment introduce a strong dependence on the poorly known $\left|Q^{2}\right|$-variation of the form factors (in his case. parameterized by only two dipole masses. $V_{V}$, and $M_{A}$.) Thus. the constraints are necessarily rather weak. But coupled with future data. including electron scattering and lower- $Q^{2}$ neutrino-proton scattering, the B.VL results should continue to play a role in extracting the desired strangeness parameters. 


\section{V.C. Present and Proposed Experiments - Future Prospectives}

The ${ }^{12} \mathrm{C}$ experiment described in the last section is an example of the level of systematic error that can be achieved at present-day electron accelerators. Since the completion of the ${ }^{12} \mathrm{C}$ experiment. accurate measurements of the mass of the Z-boson in high-energ: experiments at CER.i. Fermilab and SLAC have caused a change in focus of PV experiments at medium-energies. The next generation of experiments are centered around measurements of the strange matrix elements. relying on the fact that $\sin ^{2} \theta_{w}$ is now known with suff 'nt accuracy that uncertainties due to $\sin ^{2} \theta_{w}$ are much smaller than uncertainties due to strange-quark contributions. .IIT/Bates. CEBAF. Mainz and SLAC all have proposed extensive programs to measure the strange-quark contributions to vector matrix elements. In addition. a new experiment at L.A.IPF is currently underway to further investigate the axial-vector strange form factor. This section will describe and contrast each of the proposals, as well as provide some discussion of where one might go in the future.

\section{V.C.1. S.A.MPLE (MIIT/Bates)}

The next PV experiment currently being prepared at MIT /Bates [McK89] is intended to build on much of the work accomplished in the ${ }^{12} \mathrm{C}$ experiment. This experiment will focus on measurement of the strange magnetic contribution to the proton. specifically $\mu$, introduced in Sect. III.C. Performing an experiment at backward angles allows one to focus on the second and third terms of Eq. (3.125). The axial-vector term is further suppressed by the factor $\left(1-4 \sin ^{2} \theta_{w}\right)$, so to a first approximation one is left with an asymmetry proportional to $\tilde{G}_{M}^{p}$. In the limit of zero momentum transfer, the only unknown parameter is $\mu$. The experiment will make a measurement at a beam energy of $200 \mathrm{Mlel}^{\circ}$ and scattering angles of $135<\theta<160^{\circ}$, which is an average momentum transfer of $Q^{2} !=0.1$ $(\mathrm{GeV} / \mathrm{c})^{2}$. At these kinematics the Standard Model asymmetry is $8 \times 10^{-6}$. which is about five times larger than that measured in the ${ }^{12} \mathrm{C}$ experiment, and comparable to the Mainz experiment. The present goal is to achieve a statistical accuracy of $5 \%$ with a systematic error of $5 \%$. This can be achieved in approximately 500 hours of running with $40 \mu \mathrm{A}$ of beam polarized to $40 \%$. The resulting overall error on $\mu_{\mathrm{s}}$ will be $\Delta \mu_{\mathrm{s}}=0.22$.

The polarized injector, many of the beamline components and most of the detector electronics will be the same as those used in the MIT/Bates ${ }^{12} \mathrm{C}$ experiment. One added complication that the ${ }^{12} \mathrm{C}$ experiment did not have is that the beamline at the target is not at $0^{\circ}$ with respect to the accelerator, so the electron spin will precess away from longitudinal by $16.5^{\circ}$ by the time it reaches the scattering target. This effect can be removed by purposely aligning the electron spin away from longitudinal just after the source but before acceleration. With the appropriate initial alignment angle, the electron spin will precess into the longitudinal direction at the target. This will be accomplished using a Wien filter, a device with crossed electric and magnetic fields. The beam polarization will again be measured with a Møller polarimeter, and in this case it is important that the polarimeter be accurate enough to detect not only the large longitudinal Møller asymmetry, but also the transverse asymmetry which is seven times smaller. 
With the small $\mathrm{PV}$ asymmetries anticipated in experiments of this type. it is desirable to obtain the maximum possible luminosity from the accelerator. The polarized electron beam will be incident on a $40 \mathrm{~cm}$ long circulating liquid hydrogen target. The total power deposited in the target by a $40 \mu \mathrm{A}$ beam is $500 \mathrm{~W}$. so sufficient cooling power must be available in order to keep the target from boiling. A $700 \mathrm{~W}$ He gas refrigerator will be used to cool the target. In addition, any volume of liquid hydrogen seen by a $15 \mu \mathrm{sec}$ beam pulse must be out of the path of the beam by the next beam pulse or density fluctuations in the liquid will occur due to boiling. This is achieved by flowing the aydrogen parallel to the beam at a rate of approximately $10 \mathrm{~m} / \mathrm{s}$. At such a high velocity, the liquid is highly turbulent and a significant transverse component is also present.

Figure 3.13 shows a layout of the S.A.MPLE detector and target system. The target will be viewed by a large array of Cerenkov detectors which cover $\sim 2$ sr of solid angle The detector consists of a set of ten mirrors. each $0 . i \times 0.7 \mathrm{~m}^{2}$. which focus Cerenkor light produced in air onto en 8 inch diameter photomultiplier tubes. This detector system is quite similar to that used in the Mainz experiment. with the modification that the phototubes must be able to view the entire $40 \mathrm{~cm}$ of target length. The scattered electron rate into each phototube is $\sim 4000 /$ pulse. so the yield in each tube is integrated orer the 1.5 $\mu$ sec pulse length. It is therefore important that background processes which might cause a signal in the detector be kept to a minimum. With a beam energy of $200 \mathrm{Mel}$. it is not possible to produce pions in the target directly, and the inelastic scattering rate into the detector is kept reasonably low. The largest source of background is expected to come from low-energy inelastic scattering from $\mathrm{Al}$ in the target plumbing downstream of the target cell. Because these are mainly low momentum transfer processes. the main effect on the asymmetry is an overall dilution factor.

As mentioned above. it is the latter two terms in the PV asymmetry which are the dominant contributions. The axial-vector term is suppressed because of the $\left(1-4 \sin ^{2} \theta_{w}\right)$ term. but it still contributes approximately 20\% (see Sect. IV.A). The weak radiative corrections to the vector term are quite small $(\sim 3 \%)$, but the corrections to the axialvector term are about $20 \%$ and rather uncertain (see Sect. III.B). These uncertainties put a natural limitation on the level of precision one might currently expect to achieve in extracting $\mu_{\mathrm{g}}$. It is then interesting to ask whether it is possible to make some kind of calibration measirement in order to reduce the theoretical uncertainties of these effects. In the case of SA.MPLE, an ideal calibration experiment is quasielastic scattering from deuterium at the same kinematics. As in the case of the Mainz experiment on ${ }^{9} \mathrm{Be}$ and as discussed in Sect. IV.F, the magnetic strangeness effects in deuterium are an incoherent sum of the neutron and the proton and nearly cancel. The axial-vector term contributes approximately the same fraction to the asymmetry as in the case of the proton. Both the asymmetry and the cross section are about 1.5 times larger than in the proton, which means that only $1 / 3$ as much running time is needed to achieve the same level of accuracy. If a deviation fron the Standard Model asymmetry is seen in the proton, a measurement on deuterium would provide an important check on the results. A complication arising in the case of deuterium is the fact that one will detect not only electrons from quasielastic scattering, but also from ilastic and threshold inelastic processes. These processes each coniribute about $10 \%$ to the scattering rate. The elastic rate is particularly troublesome because it is highly sensitive to strange-quark effects. Fortunately, the elastic and inelastic asymmetries contribute with opposite sign and roughly cancel each other, leading to an overall correction factor of $\sim 2 \pm 1 \%$ at $200 \mathrm{Mel}$. 


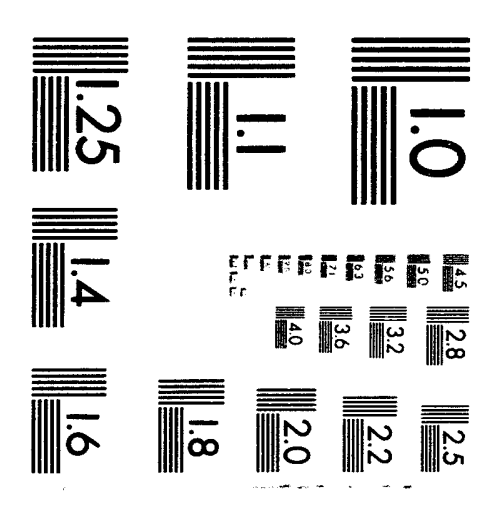



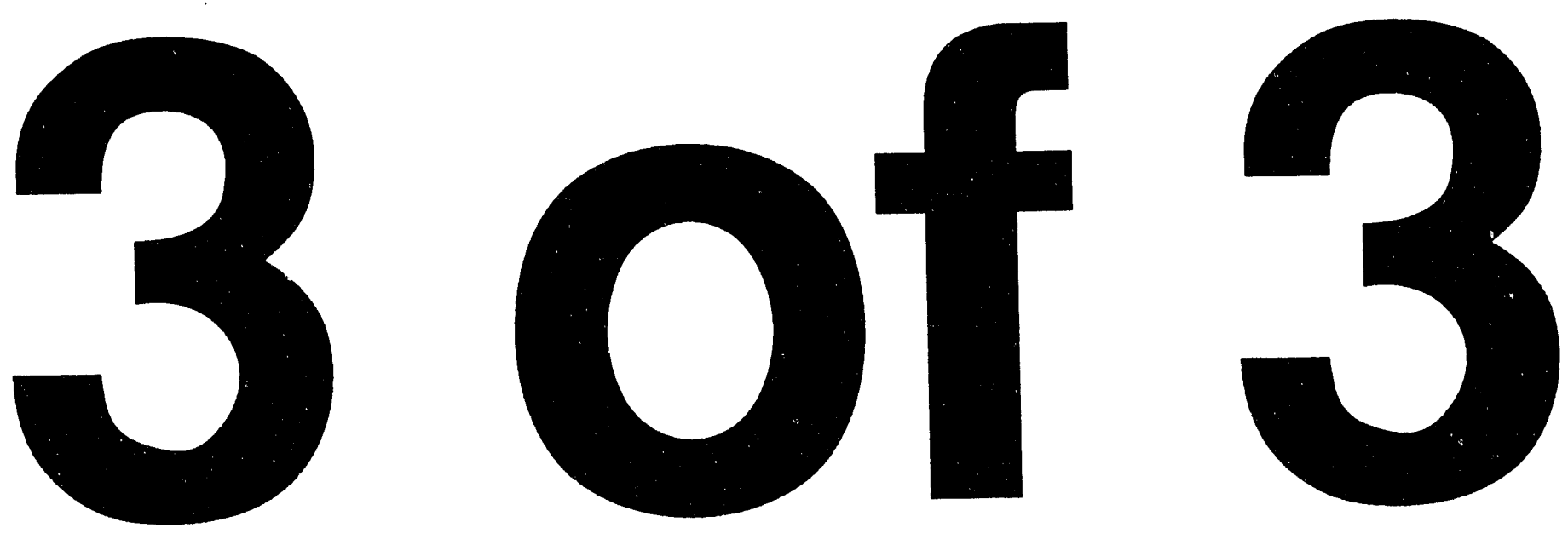


\section{V.C.2. MAINZ}

A proposal has recently been submitted to the Mainz . Microtron for a new PV measurement at forward electron angles [Hei93] whose goal is to measure the contribution of the strange-quark electric form factor $G_{E}^{(s)}$ to the asymmetry for elastic scattering from the proton. The proposed kinematics would be a beam energy of $850 \mathrm{MeV}$ and an average scattering angle of $35^{\circ}$. corresponding to $\left|Q^{2}\right|=0.23(\mathrm{GeV} / \mathrm{c})^{2}$. At these kinematics the $\mathrm{PV}$ asymmetry is sensitive to $\left(G_{E}^{(s)}+G_{E}^{n}+0.2 G_{M}^{(s)}\right)$, and with no strange quarks is $\sim i \times 10^{-6}$. Preliminary plans are to build a calorimeter of six lavers of liquid Xenon modules $5.4 \times 5.4 \times 39 \mathrm{~cm}$. covering about $2 \mathrm{sr}$ of solid angle. With the assumed $35 \mu \mathrm{A}$ polarized beam current and a $10 \mathrm{~cm} \mathrm{LH}_{2}$ target. the data-rate into each detector module would be approximately $2 \mathrm{MHz}$. The choice of liquid Xenon is driven by the desire for a fast detector which could count individually scattered electrons at this rate. On average. a scattered electron would generate an electromagnetic shower covering an area of $3 \times 3$ modules. Individual detectors would be triggered at a signal size corresponding to onethird of the total elastically scattered electron energy, and signals over the $3 \times 3$ cluster would be summed together. This would make it possible to separate elastic scattering from events below the pion production threshold. A $20 \mathrm{~cm}$ polyethylene absorber would be placed in front of the Xenon modules to reduce background coming from the target. The PV asymmetry would be measured with a statistical error of $\sim 4 \%$, which could be done in 400 hours assuming a beam polarization of $80 \%$. To achieve this level of systematic accuracy, the beam polarization must be measured to $3 \%$ or better. This level of precision has not currently been achieved and will require additional development.

\section{V.C.3. CEBAF}

At higher beam energies and forward angles one has the possibility of obtaining information on the first of the three terms in Eq. (3.125). An explicit extraction of $G_{E}^{(9)}$ is complicated both by the neutron electric form factor and by $G_{M}^{(s)}$, as discussed in Sect. IV. In addition, the uncertainty in the neutron charge form factor enters directly into the uncertainty in $G_{E}^{(s)}$. This may not be such a serious limitation. however, since several experiments are currently proposed or underway [Mil88, Day89, Mad89. McK91, Ott90. Pla90, Lun93] to improve the limits on $G_{E}^{n}$ to $\sim \pm 10 \%$. Two proposals for PV $\vec{e} p$ measurements have already been submitted to $\mathrm{CEBAF}$ and are conditiunally approved.

Hall A experiment $91-010$

The first CEBAF proposal [Sou91] is a measurement at $\left|Q^{2}\right|=0.5(\mathrm{GeV} / \mathrm{c})^{2}$ using the two high-resolution spectrometers in Hall $\mathrm{A}$. The pair of spectrometers would be positioned symmetrically on either side of the beamline at their most forward scattering angle of $12.5^{\circ}$, yielding a total acceptance of $16 \mathrm{msr}$. At these kinematics, the beam energy is 3.5 $\mathrm{GeV}$, and the calculated asymmetry with no strange-quark effects is $-2 \times 10^{-5}$. With $100 \mu \mathrm{A}$ of $50 \%$ polarized electrons and a $15 \mathrm{~cm}$ long $\mathrm{LH}_{2}$ target, a statistical accuracy 
of $5 \%$ can be achieved in about 400 hours. The elastic proton cross section at these kinematics is approximately $700 \mathrm{nb} / \mathrm{sr}$. leading to a total rate into each spectrometer of $\sim$ $2 \mathrm{MHz}$. In order to handle these high rates, the standard detector package of the Hall $A$ spectrometers will be replaced with a lead glass shower counter and integrating electronics. The focusing properties of the spectrometer are such that it is possible to separate elastic scattering events kinematically from other possible backgrounds by using a reduced area of the spectrometer focal plane.

A single measurement alone would not allow separation of $G_{E}^{(s)}$ and $G_{M}^{(s)}$. but it would determine if sirange quarks play a significant role in the structure of the proton. modulo the uncertainty in $G_{E}^{n}$. If large effects are seen, a more extended program of measurements at lower $-\left|Q^{2}\right|$ on ${ }^{1} \mathrm{H}$ and ${ }^{4} \mathrm{He}$ targets is anticipated. A measurement on ${ }^{4} \mathrm{He}$ has the advantage of being sensitive only to the electric contribution. but at low momentum transfer it represents a significantly greater challenge due to the smaller asymmetries and lower sensitivity to strange quark effects.

\section{Hall C experiment $91-017$}

A second proposal [Bec91] advocates the construction of a dedicated apparatus with an open geometry and a large solid angle. which could be used for both a forward-angle experiment as well as backward-angle measurements at slightly lower momentum transfer. This apparatus, to be placed in Hall $\mathrm{C}$ at CEBAF, would consist of a segmented ironfree toroidal magnet and an array of scintillators placed along the focal planes of the magnet. A layout of the apparatus is shown in Fig. 5.14. The goal of this experiment is to perform several sets of measurements which would allow a complete separation of the three terms in Eq. (3.125). At forward electron kinematics, the detector would be sensitive to recoil protons $\left(60^{\circ}<\theta_{p}<77^{\circ}\right)$. corresponding to momentum transfers of $0.1<\left|Q^{2}\right|<0.5(\mathrm{GeV} / \mathrm{c})^{2}$ and a Standard Model asymmetry of $\sim-5 \times 10^{-0}$. The entire range of momentum transfer would be covered in a single measurement, with a solid angle acceptance of $0.87 \mathrm{sr}$ and a target length acceptance of $20 \mathrm{~cm}$. The proposed measurements would use $40 \mu \mathrm{A}$ of polarized beam. corresponding to very high counting rates $(\sim 10 \mathrm{MHz})$ in the detector. The recoil protons would be counted with scalers. Additional time-of-flight information would be provided by modifying the CEBAF beam microstructure to be $32 \mathrm{nsec}$ between beam bunches with increased peak current (the accelerator design value is $2 \mathrm{nsec}$ between bunches). This measurement is sensitive to the combination $G_{E}^{(s)}+G_{E}^{n}+0.2 G_{M}^{(s)}$, and a $5 \%$ measurement of the asymmetry could be achieved in approximately 700 hours.

A second set of measurements would involve reversing the orientation of the spectrometer to detect electrons in the backward direction, $\theta_{e} \sim 108^{\circ}$. Four measurements, 700 hours each, are proposed. The beam energies in this configuration would be 335.428 , 512 , and $590 \mathrm{MeV}$ corresponding to momentum transfers of $0.2,0.3,0.4$, and $0.5(\mathrm{GeV} / \mathrm{c})^{2}$. This set of measurements would be sensitive to a different linear combination of the strange 
form factors. Finally, a 500 hour QE measurement on deuterium would provide some constraints on the uncertainties associated with the term proportional to the axial-vector form factor. as discussed in Sect. IV.F. Combining the proton detection. electron detection and deuterium quasielastic measurements would allow the separation of $G_{E}^{(s)}$ and $G_{M}^{(s)}$ over a relatively large range of momentum transfer. Although a model-independent extraction of $G_{E}^{(s)}$ would still be limited by the uncertainty in $G_{E}^{n}$, the $\operatorname{SL}(3)$ singlet combination $G_{E}^{(0)}$ would be determined with good accuracy.

Hall A experiment $91-004$

As discussed in Sect. IV.B. another approach to learning something about strangequark effects in a hadronic system is to use PV elastic scattering from a $J=0 . T=0$ nucleus. Only the ground-state vector monopole matrix elements of the electromagnetic and weak neutral currents occur in this case and the asymmetry is given by Eq. (3.120). As discussed in Sect. IV.B, in a light nucleus such as ${ }^{4} \mathrm{He}$, isospin violations are expected to be quite small and accordingly this case may be the best to use in looking for effects due to the presence of strange quarks. Musolf and Donnelly [Mus92a] have argued that two measurements on a $\left(0^{+} 0\right)$ nucleus, at low- and moderate- $\left|Q^{2}\right|$. might ultimately constrain $G_{E}^{(s)}$ to a higher level of precision than that achievable with a measurement on a proton target. since uncertainties associated with $G_{M}^{(\mathbf{s})}, G_{E}^{n}$ and $\tilde{G}_{A}^{p}$ are no longer presen:.

This third CEBAF proposal [Bei91b] is for a measurernent of ${ }^{4} \mathrm{He}(e, e)$ at $\left|Q^{2}\right|=0.6$ $(\mathrm{GeV} / \mathrm{c})^{2}$. again using the Hall $\mathrm{A}$ spectrometers. The target will be ${ }^{4} \mathrm{He}$ gas operating at $10 \mathrm{~K}$ and $70 \mathrm{~atm} .15 \mathrm{~cm}$ long. The standard detector package of the spectrometers need not be modified in this case, since at the highest CEBAF design luminosity $\left(\mathcal{L} \sim 3 \times 10^{38}\right.$ $\mathrm{cm}^{-2} \mathrm{~s}^{-1}$ ) the counting rate into each spectrometer is $\sim 2 \mathrm{kHz}$. The asymmetry with no strange quarks is $5 \times 10^{-3}$, large on the scale of PV experiments, thus reducing the demand on the helicity-correlated properties of the CEBAF beam. This allows for removal of target wall background since elastically scattered electrons are uniquely identified with the tracking capabilities of the detector package. Small trigger scintillators will be installed in the detector package to limit the focal-plane acceptance of the spectrometers to the region of the elastic peak.

Because of the lower counting rates a good statistical measure of the asymmetry will take somewhat more running time than the previous two experiments. However, even a $30 \%$ measure of the asymmetry may be able to constrain significantly the current limits of the strange electric contribution to hadronic matter. This level of precision could be reached with 1000 hours of CEBAF beam, assuming $40 \%$ beam polarization. 


\section{V.C.4. SLAC}

Two proposals were recently developed for measuring PV asymmetries at very high energies at SLAC. One proposal suggests the measurement of an asymmetry in elastic and inelastic electron scattering from the proto at both high- and low- $\left|Q^{2}\right|<2.0$ and 0.5 $\left(\mathrm{GeV} / \mathrm{C}^{2}\right.$, Che92]. The other proposes to measure the deep-inelastic scattering (DIS) asymmetry on both hydrogen and deuterium at high- $Q^{2} \mid\left(2-9(\mathrm{GeV} / \mathrm{c})^{2}\right)$, Bos92]. Both experiments would take advantage of high beam energies. significant $\left|Q^{2}\right|$ and very forward scattering angles with large cross sections and asymmetries. resulting in good statistical accuracy. The DIS experiment is perhaps the most ambitious of all proposed electron scattering PV measurements, in terms of the expected accuracy. Both experiments are an example of future directions in PV electron scattering. Rather than presenting a discussion of each proposal in detail. a general discussion is given of both the possibilities and the constraints they might present.

$\underline{\text { Proton Elastic and Inelastic Scattering }}$

At beam energies in the $30-50 \mathrm{GeV}$ region it would be possible through $\epsilon-p$ elastic scattering to explore the high- $\left|Q^{2}\right|$ behavior of the strange form factors. Because of the increasing value of $\tau$, the PV asymmetry becomes more sensitive to $G_{M}^{(s)}\left(Q^{2}\right)$ than to $G_{E}^{(s)}\left(Q^{2}\right)$. Unlike the lower-energy experiments, possible contamination due to the poorly known axial-vector form factor and its radiative correction are negligible (see Sect. IV.A.

Data could also be acquired simultaneously for excitation of the inelastic nucleon resonances. Of great interest is a measurement of the electron axial-vector/quark vector current isovector coupling for the $V \rightarrow \Delta$ transition discussed in Sect. IV.G. If the non-resonant background can be neglected. the isovector couplings can be isolated. The excitation is also expected to be sensitive to higher-order electroweak processes and possible contributions from strange quarks.

The biggest difficulty that higher-energy experiments face is the rapidly falling $e-p$ cross section. Although the asymmetries are large, even modest levels of precision require long periods of running with a conventional magnetic spectrometer. Nonetheless, these experiments would be exploring completely unknown territory for which there are currently few theoretical predictions.

\section{$\underline{\text { Deep-Inelastic Scattering }}$}

The recently submitted letter of intent [Bos93] for deep-inelastic scattering is perhaps the most ambitious of all proposed PV experiments in terms of expected accuracy. The primary objective of this experiment would be to provide a precision test of the Standard Model and to determine the flavor composition of the sea quarks. The proposed measurement would improve upon the results of the original SLAC PV experiment by almost an order-of-magnitude. At the level of $1.5 \%$ uncertainty it would be comparable to many of the current Standard Model tests. A planned 1\% atomic PV experiment would achieve 
approximately the same uncertainty in $\sin ^{2} \theta_{w}$. However, the SLAC and atomic PV experiments are complementary. since the atomic PV experiment measures $C_{1 u}+C_{1 d}$ and the DIS experiment measures $2 C_{1 u}-C_{1 d}$. Combining the results would determine $C_{1 u}$ and $C_{1 d}$ independenty to \pm 0.005 (see also Sects. IV.H and IV.I).

It would also be possible to measure the quark distribution functions. The overall $x$-dependence of the quark sea is reasonably well understood, but the flavor composition is not. At present, the available data from muon, neutrino, and antineutrino scattering, as well as from the Drell-Yan process, are insufficient to disentangle the five unknown quantities $u_{\text {val }}, d_{\text {val }}, u_{\text {sea }}, d_{\text {sea }}$ and $s_{\text {sea }}$. An experiment at SLAC would provide results for two new observables. By taking ratios of asymmetries from hydrogen and deuterium targets. light sea-quark distribution functions would be much more unambiguously determined.

At beam energies of $30-50 \mathrm{GeV}$ and very forward angles, the PV asymetry could be as large as $10^{-3}$. Techniques similar to those used in previous experiments to control helicity-correlated beam properties could be used. Count rates would also be high due to the relatively large deep-inelastic cross sections, so modest beam currents and target lengths would be sufficient. For the hydrogen/deuterium ratio measurements. accurate knowledge of the relative target densities would be required. Absolute densities on the order of $1 \%$, as well as $1 \%$ knowledge of the relative beam-related density changes, are feasible. As was demonstrated in the previous SLAC PV experiment, the use of magnetic spectrometers and segmented shower counters makes it possible to maintain high count rates while keeping background to a minimum.

The primary technical challenge associated with a precision PV experiment would be knowledge of the beam polarization at the level of $1 \%$. The standard technique used by most experiments is Møller scattering from polarized atomic electrons in thin foils. Analyzing power is a maximum for scattering at $90^{\circ}$ in the center-of-mass. There are several effects which limit the accuracy of this method: uncertainty in the effective foil polarization, background asymmetries under the .Møller peak and atomic effects connected with our knowledge of the atomic electron polarization. Significant efforts will be required to understand the various contributions and reduce the overall systematic errors to the level of the statistical uncertainties in the experiment itself. An alternative approach for determining beam polarization would be to use Compton scattering which involves the backscattering of circularly-polarized laser photons from the beam of polarized electrons. The technique is complicated and expensive, but can probably achieve a systematic error of $<1 \%$ in measuring the beam polarization.

The two proposals submitted to SLAC are examples of the future directions one might pursue at a higher-energy electron machine such as that currently under consideration by the European community [Pro91, EEF93]. 


\section{V.C.J. SOLENOIDAL SPECTROMETFR}

Recent PV experiments have demonstrated that the present limitation for such measurements is counting statistics. The next generation of such studies. with its very ambitious physics goals. will require the development of large acceptance devices with appropriate momentum resolution. A novel approach is to use a large solenoid spectrometer: at energies up to $1 \mathrm{Gel}^{*}$ this requires a device of up to $4 \mathrm{~m}$ diameter. $4 \mathrm{~m}$ long and a central field of $\sim 2$ T. A recent analysis by Souder et al. [Sou90b] has shown that there are many advantages with such an approach. The device is suitable for both forward-and backward-angle measurements. In the forward direction. for an experiment such as elastic scattering on ${ }^{12} \mathrm{C}$. acceptances of up to $600 \mathrm{msr}$ are possible with adequate resolution to exclude inelastic scattering. This is more than an order-of-magnitude larger acceptance than has typically been available up to now. At backward angles. solid angles in excess of $2 \mathrm{sr}$ are possible. In both cases the spectrometer can accommodate extended taroets such as $\mathrm{LH}_{2}$ and $\mathrm{LD}_{2}$. An important advantage of a solenoid is that it makes use of all of the azimuthal acceptance possible within a given angular range. It also allows for the ability to reverse the direction of the magnetic field without any change in the beam optics. This could potentially be very important for understanding systematic effects arising from Moller scattering backgrounds.

\section{V.C.6. LS.ND}

The LSND collaboration at LA.MPF is currently undertaking a new elastic neutrino scattering experiment using the A-6 beamline. The high current $(800 \mu \mathrm{A}) 780 \mathrm{MeV}$ proton beam is stopped in a water-cooled $\mathrm{C}_{11}$ beam dump, from which charged pions below 600 $\mathrm{MeV}$ are produced. Roughly equal numbers of $\nu_{e}, \nu_{\mu}$ and $\bar{\nu}_{\mu}$ are produced from pion decay mechanisms at rest. The muon neutrinos result from the process $\pi^{+} \rightarrow \mu^{+} \nu_{\mu}$ and are monoenergetic at $27.8 \mathrm{MeV}$. The other two species result from $\mu^{+} \rightarrow \epsilon^{+} \nu_{e} \bar{\nu}_{\mu}$ and have a distribution up to a maximum energy of $52.8 \mathrm{MeV}$. A small percentage of pions will deray in flight and produce neutrinos with energies in the range of 100-200 $\mathrm{Mfel}$. The ratio of $\bar{\nu}_{\mu}$ from $\pi^{-}$decay-in-flight to $\nu_{\mu}$ from $\pi^{+}$decay is $\sim 0.2$, and thus the majority of the higher-energy beam is $\nu_{\mu}$. An additional decay path at the beginning of the beamline will enhance the yield of decay-in-flight neutrinos. The primary goal of the experiment is a continuing study of neutrino oscillations, for which the decay-at-rest beams are used.

The experiment once again involves an active detector: a cylindrical tank filled with 200 tons of liquid scintillator, $8.5 \mathrm{~m}$ long by $5 \mathrm{~m}$ diameter. High-quality eight inch diameter photomultiplier tubes cover approximately $25 \%$ of the surface area of the detector. The detector is surrounded by a veto shield [Nap89] constructed of planes of $\mathrm{Pb}$ and liquid scintillator to veto events coming from cosmic ray muons and neutrons. The entire detector is covered with $\sim 2000 \mathrm{~g} / \mathrm{cm}^{2}$ of steel and earth to provide additional cosmic ray shielding. A water tank downstream of the detector shields the open end of the detector.

Neutrinos in the $150 \mathrm{MeV}$ range elastically scatter from the protons in the scintillator (again, $80 \%$ of which are bound in carbon) with an average momentum transfer of $\left|Q^{2}\right|=$ $0.07(\mathrm{GeV} / \mathrm{c})^{2}$. The recoil protons $\left(20<E_{p}<40 \mathrm{MeV}\right)$ deposit energy in the scintillator and are detected. Cosmic ray background events are rejected with the veto shield and 
with the good timing resolution of the photomultiplier tubes. Vertex reconstruction and a $50 \mathrm{~cm}$ fiducial cut on the central volume of the detector provides additional background rejection together with energy and angle resolution. It is expected that the $\nu_{\mu}$ elastic scattering from free protons can be determined to $\sim 20 \%$ and the $\nu_{\mu}$ cross section from free and bound protons can be measured to $\sim 10 \%$. Because of the very low momentum transfer. the contribution from $G_{E}^{(s)}$ is virtually negligible. The remaining ambiguities are from the strange magnetic moment (which also should be reduced at lower- $Q^{2}$ ), and from the nuclear physics uncertainties associated with quasielastic scattering from ${ }^{12} \mathrm{C}$. Ling the results from the SA.MPLE experiment discussed above will resolve the former issue. To address the latter problem the collaboration has proposed two possible solutions. The first is to use two different types of liquid scintillator and perform a subtraction to isolate quasielastic scattering from true elastic neutrino-proton events. A second proposal is to make use of the fact that in addition to recoil protons. the LS.ND detector will be sensitive to recoiling neutrons. It has been suggested [Gar32] that the ratio of quasielastic $(\nu, p) /(\nu, n)$ from the ${ }^{12} \mathrm{C}$ in the detector may provide additional sensitivity to $G_{A}^{(s)}$ (see also Sect. IV.J). Performing such a ratio measurement should reduce some of the systematic uncertainties associated with nuclear binding. This technique is also less sensitive to the uncertainties in the neutrino flux than $(\nu, p)$ elastic cross section measurements. The nuclear physics uncertainties must still be understood, and are the subject of future work by the same authors. The LSND experiment is expected to start production running late in 1993. It is anticipated that in several years of running $G_{A}^{(\mathbf{s})}(0)$ will be determined to about a factor of two better than was determined from the B.NL experiment. 


\section{SUMMARY AND CONCLUSIONS}

As a result of the foregoing discussion. we hope to have convinced the reader that intermediate-energy: semileptonic neutral current scattering offers a promising means for studying a wide array of physics issues. At the same time. we have endeavored to illustrate the necessity for a coordinated program of experimental and theoretical work in this field. Indeed. it should be apparent that a combination of several different measurements. together with careful and detailed theoretical analysis. is necessary if one wishes to extract useful new information on physics beyond the Standard Model. hadronic structure or nuclear dynamics. To this end. several challenges remain. On the experimental side. one must push the limits of luminosity ( viz.. beam current) and electron polarization or neutrino flux in order to achieve statistical uncertainties on the order of one percent. Moreover. systematic errors, such as false asymmetries arising from electron helicity-correlated differences. must be held to the same level. These considerations impose rather tight restrictions on the choice of target and kinematics if one is to make meaningful measurements. From a theoretical perspective, one would like a better grasp of nuclear dispersion corrections. nuclear medium effects in elastic and quasielastic scattering, and hadronic contributions to electroweak radiative corrections.

With these general remarks in mind. we summarize the salient features of the present and future semileptonic, weak neutral current scattering program as we view it:

(1) For the immediate future, intermediate-energy neutrino and PV electron scattering seem best suited as tools for studying strange-quark vector and axial-vector form factors of the nucleon. Higher-energy processes, such as deep inelastic scattering, and lowerenergy experiments, such as atomic PV measurements, cannot access these form factors. In this respect. intermediate-energy semileptonic scattering is unique.

(2) Atomic PV" experiments are presently more suited to the study of "non-standard" electroweak physics, provided that theoretical nuclear structure uncertainties can be reduced. To this end. a measurement of the ground-state neutron distribution in cesium or lead. through a combination of PV and PC electron scattering experiments, would provide a useful calibration point for theoretical calculations. Looking somewhat further down the road. once the nucleon's strange-quark form factors are sufficiently constrained. PV electron scattering could provide for a set of low- and intermediate-energy electroweak tests complementary to those performed with atomic PV.

(3) For either type of study (hadron structure or electroweak physics), neutral current scattering from the proton would not be sufficient, due to the presence of several unknown or poorly constrained form factors in the PV asymmetry and neutrino cross section. A program which includes a combination of scattering from pr ton and nuclear targets therefore appears warranted. The components of such a program could include the following:

(a) A combination of backward-angle $A_{L_{R}}(\vec{e} p)$ measurements, as in the present S.A.MPLE and conditionally approved " $G^{0}$ " experiments, with a backward-angle $A_{L_{R}}(\mathrm{QE})$ measurement, could allow one to constrain separately $\mu_{s}$ and the large and theoretically uncertain radiative correction, $R_{A}^{T=1}(\vec{e} p)$. Alternatively, a series of backward-angle $A_{L R}(\vec{e} p)$ and elastic $A_{L R}\left({ }^{2} \mathrm{H}\right)$ measurements would accomplish the same purpose. In 
either case. the resultant limits on $\mu_{\text {s }}$ could be sufficiently tight to test some of the model predictions discussed in Sect. II. In the case of quasielastic scattering. a deu. terium target appears to be the most favorable. since one stands the greatest chance of performing reliable nuclear calculations with this nucleus.

(b) A combination of low- and moderate- $\left|Q^{2}\right|$ elastic $A_{L R}\left(0^{+} 0\right)$ measurements has the potential to determine $G_{E}^{(s)}$ at a level sensitive to most of the model predictions appearing in Table 2.3. Moreover, the resultant constraints could reduce the $G_{E}^{\prime \text { "1 }}$ uncertainty to a level needed to make an interesting electroweak test possible with a subsequent $A_{L R}\left(0^{+} 0\right)$ measurement. The $G_{E}^{(s)}$ constraints obtained from a series of forward-and backward-angle $A_{L R}(\vec{e} p)$ alone would be much less stringent. Vevertheless. measurements made with a proton target would be an important check on $A_{L R}\left(0^{+} 0\right)$ results.

(c) For purposes of constraining $G_{A}^{(s)}$. low- $\left|Q^{2}\right|$ neutrino-nucleon elastic scattering. as in the LS.ND experiment, seems better suited than does PV electron scattering. owing to the large theoretical uncertainties appearing in the radiative correction $R_{A}^{T=1}(\vec{\epsilon} p$, and the possible large degree of $\mathrm{SU}(3)$-breaking in the octet axial-vector current matrix elements. The degree of theoretical uncertainty is much smaller in the case of $R_{A}^{T=0}(\nu p)$. Moreover, since $\left|R_{A}^{T=0}(\nu p)\right| \ll\left|R_{A}^{T=0}(\vec{e} p)\right|$, neutrino scattering determinations of $G_{A}^{(s)}(0)$ are much less sensitive to axial-vector $\mathrm{SU}(3)$ breaking. In addition. a determination of the ratio of $\nu p$ and $\nu n$ yields in $\mathrm{QE}$ neutrino scattering could provide 'r enhanced sensitivity to $G_{A}^{(s)}$ while reducing the experimental and theoretical systematic uncertainties, and continued studies of selected nuclear transitions could yield, for example. high-precision determinations of axial-vector nucleonic couplings.

(d) Once the strange form factors are constrained with the foregoing program of measurements, one could consider performing Standard Model tests with PV electron scattering. In this respect, the most promising targets are the proton and $\left(0^{+} 0\right)$ nuclei. Elastic $A_{L R}$ measurements on these targets could complement atomic $\mathrm{PV}$ measurements as low-energy probes of physics beyond the Standard Model. An additional possibility is a measurement of the $N \rightarrow \Delta P V$ asymmetry, although for such a measurement to be competitive, better data on the axial-vector and background contributions to this asymmetry would be needed.

(4) Going beyond strangeness form factors and electroweak tests, one encounters a host of other possibilities for studying hadron-structure and nuclear dynamics. These possibilities include the following:

(a) A determination of the axial-vector.$V \rightarrow \Delta$ matrix element, which at present is only loosely constrained by neutrino scattering data. Obtaining an improved determination with $\mathrm{PV}$ electron scattering would require better knowledge of non-resonant background contributions than is presently available.

(b) Obtaining a new set of constraints on the conventional picture of nuclear PV appears within the realm of possibility. Based on some initial studies, it seems that inelastic $A_{L R}$ measurements at low-energies $(\epsilon \lesssim 100 \mathrm{MeV})$ would be most ideal. Moreover. 
given the relatively broad constraints which presently exist on the PV meson-nucleon couplings. one can live with nearly an order-of-magnitude less precision to make meaningful measurements than is required for strangeness and electroweak studies. In this regard. $\mathrm{PV}$ electron scattering could complement atomic PV determinations of the nuclear anapole moment as a probe of nuclear PV.

(c) A determination of the ground-state neutron distribution. A relatively short ( $\sim$ few hundred hours) PV electron scattering experiment on a heavy nucleus such as lead or cesium could determine $\rho_{n}(\vec{r})$ at a level significantly beyond the typical present degree of knowledge. Such an improved determination would provide an important calibration point for theoretical calculations of $\rho_{n}(\vec{r})$ needed in the analysis of Standard . Model tests using atomic PV measurements with a series of isotopes.

In comparison with the situation of just a few years ago. it seems that considerable progress has been made in achieving a comprehensive understanding of the issues germane to intermediate-energy semi-leptonic scattering - especially with regard to the interplay between different types of physics issues which enter and the complementarity of different prospective measurements. Whereas once the future direction of the field appeared rather murky, one may now envision a rather well-defined program of experiments and theoretical work. If successfully carried out, such a program could provide a rich payoff of new insight into the structure of hadrons, physics beyond the Standard. Model and nuclear dynamics. 


\section{ACKNOWLEDGEMENTS}

It is a pleasure to thank R. Burman. C. Williamson. J. Napolitano. G. Dodson. D. Beck. C. Wieman. S. Lamoreaux. P. Bosted. R. Lourie, and A. Molinari for useful discussions. 


\section{REFERENCES}

Abe8T K. Abe et al.. Phys. Rev. Lett. 58 (1987) 636.

Abe89 K. Abe et al.. Phys. Rev. Lett. 62 (1989) 1709.

Abe91 F. Abe et al.. Phys. Rev. D43 (1991) 2070.

Abr\$6 H. Abramowicz et al.. Phys. Rev. Lett. 57 (1986) 298.

Ade85 E. G. Adelberger and W. C. Haxton. Ann. Rev. Nucl. Part. Sci. 35 (1985) 501.

Adl68 S. L. Adler, Ann. Phys. 50 (1968) 189.

Ahr85 L. A. Ahrens et al. Phys. Rev. Lett. 54. 18 (1985).

Ahr87 L. A. Ahrens et al.. Phys. Rev. D35 (1987) 785.

Ala91 MIIT/Bates Experiment 91-09. R. Alarcon and J. van den Brand. spokespersons.

Alb88 W. M. Alberico. A. Molinari. T. W. Donnelly. E. L. Kronenberg and J. W. Van Orden. Phys. Rev. C38 (1988) 1801.

Alb90 W. M. Alberico. T. W. Donnelly, and A. Molinari. Nucl. Phys. A512 (1990) 541.

Alb93a W. M. Alberico et al., Nucl. Phys. A (1993) to be published.

Alb93b W. M. Alberico, M. B. Barbaro, A. De Pace, and M. Molinari. Phys. Lett. B303 (1993) 5.

Ali92 J. Alitti et al., Phys. Lett. B276 (1992) 354.

All87 J. V. Allaby et al., Zeit. für Phys. C36 (1987) 611.

All89 R. C. Allen, H. H. Chen, M. E. Potter, R. L. Burman. J. B. Donahue. D. A. Krakauer.

R. L. Talaga, E. S. Smith and A. C. Dodd, Nucl. Inst. Meth. A284 (1989) 347.

Al190 R. C. Allen et al., Phys. Rev. Lett. 64 (1990) 1330.

Alt91 G. Altarelli, Helv. Phys. Acta 64 (1991) 761.

Ama87 C. Amaldi, et al., Phys. Rev. D36 (1987) 1385.

Ama91 P. Amandruz et al, Phys. Rev. Lett. 66 (1991) 2712.

Aok82 K. Aoki, Z. Hioki, R. Kawabe, M. Konuma, and T. Muta, Suppl. Prog. Theo. Phys. 73 (1982) 1.

Are82 H. Arenhövel, Nucl. Phys. A384 (1982) 287.

Are88 H. Arenhövel, W. Leidemann and E. L. Tomusiak, Z. für Phys. A331 (1988) 123.

Ash89 J. Ashman et al., Nucl. Phys. B328 (1989) 1.

Auf85 S. Auffret et al., Phys. Rev. Lett. 55 (1985) 1362.

Bec89 D. H. Beck, Phys. Rev. D39 (1989) 3248. 
Bec91 CEBAF proposal PR-91-017. D.H. Beck, spokesperson.

Bei91a E. J. Beise and R. D. Mckeown, Comments in Nucl. Part. Phys. 20 (1991) 105.

Bei91b CEBAF proposal PR-91-004. E. J. Beise, spokesperson.

Bei92 E. J. Beise. in Proceedings of the International Conference on the Structure of Baryons and Related Mesons. .I. Gai. Ed., World Scientific (1992) 392.

Beri1 .M. Bernheim. Phys. Rev. Lett. 46 (1971) 402.

Berī9 J. Bernabeu and P. Pascual, Nucl. Phys. A324 (1979) 365.

Ber90 V. Bernard. ‥ Kaiser. and U.-G. Meissner. Phys. Lett. B237, 545 (1990).

Ber91 W. Bertozzi. R. W. Lourie, and E. J. Moniz, in Modern Topics in Electron Scattering. B. Frois and I. Sick. Eds., World Scientific (1991) 419.

Bjo69 J. D. Bjorken and E. i. Paschos, Phys. Rev. 185 (1969) 1975.

Blo89 C. Blondell et al., CERN report CER.N/EP 89-101 (1989).

Blu90 S. A. Blundell, W. R. Johnson, and J. Sapirstein, Phys. Rev. Lett. 65 (1990) 1411.

Bod91 B. Bodmann et al. Phys. Lett. B267, 321 (1991).

Bos92 SL.AC proposal E149, P. Bosted, contact person.

Bos93 SLAC proposal E149. P. Bosted, contact person. Note proposal status "deferred" by EPAC, June 1993.

Bour4 .I. A. Bouchiat, C. C. Bouchiat, Phys. Lett. B48 (1974) 111; J. Phys. (Paris) 35 (19i4) 899; J. Phys. (Paris) 36 (1975) 493.

Bou83 M. Bourquin et al., Z. für Phys. C21 (1983) 27.

Bou86 .. A. Bouchiat and L. Pottier, Science 234 (1986) 1203.

Bou91 C. Bouchiat and C. A. Piketty, Zeit. für Phys. C49 (1991) 91.

Bre67 G. Breit. Rev. Mod. Phys. 39 (1967) 560.

Bre90 V. Breton et al., Phys. Rev. Lett. 66 (1991) 572.

Bro88 G. E. Brown, C. B. Dover, P. B. Siegel, and W. Weise Phys. Rev. Lett. 60, 2723 (1988).

Bur89 CEBAF Proposal, "Electroproduction of the $P_{33}(1232)$ Resonance", (1989) V. Burkert and R. Minehart, spokespersons.

Bur90 R. L. Burman, M. E. Potter and E. S. Smith, Nucl. Inst. Meth. A291, 621 (1990).

Bur93 V. Burkert, private communication.

Cah78 R. N. Cahn and F. J. Gilman, Phys. Rev. D17 (1978) 1313.

Car80 L. S. Cardman et al., Phys. Lett. B91 (1980) 203. 
Car92 L. S. Cardman. Nucl. Phys. A546, 31īc (1992).

Cheila T. P. Cheng and R. Dashen. Phys. Rev. Lett. 26 (19i1) 594.

Cheilb .I. Chemtob and .I. Rho. Nucl. Phys. A163 (1971) 1.

Chei6 I. P. Cheng. Phys. Rev. D13 (1976) 2161.

Che84 T. P. Cher. and Ling-Fong Li. Gauge Theory of Elementary Particle Physics. Oxford L. Press (1984).

Che92 SL.AC proposal E148. J. P. Chen, contact person.

Chu88 .IIT/Bates Experiment \#88-10. T. Chupp and A. Bernstein. spokespersons.

Colis J. Collins. F. Wilczek, and A. Zee. Phys. Rev. D18 (1978) 242.

Com83 E. D. Commins and P. H. Bucksbaum. Weak Interactions of Leptons and Quarks. Cambridge $L^{-}$. Press (1983) Chapter 9 and references therein.

Con89 .M. Consoli. W. Hollik, and F. Jegerlehner in Z Physics at LEP. G. Altarelli. R. Kleiss. and C. Verzegnassi. CER.N report \# 89-08, Geneva, Switzerland (1989) Vol. 1. p. T.

Cot76 J. Coté. B. Rouben, R. de Tourreil, and D. W. L. Sprung, Nucl. Phys. A273 (1976) 269.

Dai91 J. Dai. M. J. Savage, J. Liu, and R. Springer, Phys. Lett. B271 (1991) 403.

Day89 CEBAF proposal 89-018, D. Day, contact person.

Day93 D. Day, private communication.

deF66 T. de Forest, Jr. and J. D. Walecka, Adv. in Phys. 15 (1966) 1.

deF83 T. de Forest, Jr., Nucl. Phys. A392 (1983) 232.

Deg89 G. Degrassi. A. Sirlin, and W. J. Marciano, Phys. Rev. D39 (1989) 287.

Des80 B. Desplanques, J. F. Donoghue, and B. R. Holstein, Ann. Phys. (N. Y.) 124 (1980) 449.

deT73 R. de Tourreil and D. W. L. Sprung, Nucl. Phys. A201 (1973) 193.

deT75 R. de Tourreil, B. Rouben and D. W. L. Sprung, Nucl. Phys. A242 (1975) 445.

Don68 T. W. Donnelly, J. D. Walecka, I. Sick, and E. B. Hughes, Phys. Rev. Lett. 21 (1968) 1196.

Don70 T. W. Donnelly, Phys. Rev. C1 (1970) 833.

Doni3 T. W. : nelly, Phys. Lett. 43B (1973) 93.

Don74 T. W nnelly, D. Hitlin, M. Schwartz, J. D. Walecka, and S. J. Wiesner Phys. L 49B (1974) 8.

Don75 T. W. Donnelly and J. D. Walecka, Ann. Rev. Nucl. Sci. 25, (1975) 329. 
Doni6 T. W. Donnelly and R. D. Peccei. Phys. Lett. 65B (1976) 196.

Doni9a T. W. Donnelly and R. D. Peccei. Phys. Rep. 50 (1979) 1.

Doni9b T. W. Donnelly and W. C. Haxton. At. Data and Nucl. Data Tables 23 (1979) 103

Don80 T. IV. Donnelly and W. C. Haxton. At. Data and Nucl. Data Tables 25 (1980) 1

Don83 T. WV. Donnelly in Proceedings of the Los Alamos Neutrino Workshop (1983).

Don84 T. W. Donnelly and I. Sick. Rev. Mod. Phys. 56 (1984) 461.

Don85 T. W. Donnelly. Prog. in Part. and Nucl. Phys. 13 (1975) 183.

Don86a J. F. Donoghue and C. R. Nappi, Phys. Lett. B168 (1986) 105.

Don86b T. W. Donnelly and A. S. Raskin. Ann. Phys. 169 (1986) 247.

Don88 T. W. Donnelly. J. Dubach. and I. Sick. Phys. Rev. C37 (1988) 2320.

Don89 T. W. Donnelly, J. Dubach and I. Sick, Nucl. Phys. A503 (1989) 589.

Don92 T. W. Donnelly, .I. J. Musolf. W. M. Alberico, .I. B. Barbaro, A. De Pace and A. . Nolinari. Nucl. Phys. A541 (1992) 525.

Dor89 J. Dorenbosch et al., Zeit. für Phys. C41 (1989) 567.

Dre59 S. D. Drell and S. Fubini, Phys. Rev. 113 (1959) 741.

Dubi5 J. Dubach, Ph.D. thesis, Stanford University (1975) unpublished.

Dub76 J. Dubach, J. H. Koch, and T. W. Donnelly, Nucl. Phys. A271 (1976) 279.

Dub80 J. Dubach, Nucl. Phys. A340 (1980) 271.

Dub90 D. Dubbers. W. Mampe, and J. Döhner, Europhys. Lett. 11 (1990) 195.

Dum83 O. Dumbrajs et al., Nucl. Phys. B216 (1983) 277.

Dzu86 V. A. Dzuba. V. V. Flambaum, and I. B. Khriplovich, Zeit. für Phys. D1 (1986) 243.

EEF93 "The EEF Project: Report of the Mainz Workshop", J. Arvieux, E. de Sanctis. T. Walcher, and P. K. A. de Witt Huberts, Eds., Nuovo Cim. (1993) to be published.

Ell90 J. Ellis and G. L. Fogli, Phys. Lett. B249 (1990) 543.

Fei75 G. Feinberg, Phys. Rev. D12 (1975) 3575.

Fet71 A. L. Fetter and J. D. Walecka, Quantum Theory of Many-Particle Systems, McGrawHill, N.Y., (1971).

Fin91 CEBAF proposal PR-91-010, J. M. Finn and P. A. Souder, spokespersons.

Fla78 J. B. Flanz, et al., Phys. Rev. Lett. 41 (1978) 1642.

Fla79 J. B. Flanz, et al., Phys. Rev. Lett. 43 (1979) 1922.

Fla80 V. V. Flambaum and I. B. Khriplovich, Zh. Eksp. Teor. Fiz. 79 (1980) 1656 [Sov. Phys. JETP 52 (1980) 835. 
Fla84 V. V. Flambaum. I. B. Khriplovich, and O. P. Sushkov. Phys. Lett. 146B (1984) $36 \mathrm{~T}$.

For80 E. A. Fortson and L. Wilets, Adv. in Atomic and Molec. Phys. 16 (1980) 319.

For34 E. ‥ Fortson and L. L. Lewis, Phys. Rep. 113 (1984) 289.

For90 E. ‥ Fortson. Y. Pang, and L. Wilets. Phys. Rev. Lett. 65 (1990) 285 T.

Fre90 S. Freedman. Comments in Nucl. Part. Phys. 19 (1990) 209.

Fre92 T. Frederico. E. M. Henley, S. J. Pollock, and S. Ying, Phys. Rev C46 (1992) 34t

Frit4 J. L. Friar and .I. Rosen. Ann. Phys. 87 (1974) 289.

Fri84 J. L. Friar and S. Fallieros. Phys. Rev. C29 (1984) 1645.

Fri8j J. L. Friar and W. C. Haxton, Phys. Rev. C31 (1985) 2027.

Fro6T R. F. Frosch. J. F. McCarthy, R. E. Rand. and M. R. Yearian. Phys. Rev. 160 (196T) 874 .

Fru84 S. Frullani and J. Mougey, Adv. in Nucl. Phys. 14 (1984).

Fuk88 M. Fukugita, Y. Kohyama, and K. Kubodera. Phys. Lett. B212 (1988) 139.

Gai84 J. M. Gaillard and G. Sauvage, Ann. Rev. Nuc. Part. Sci. 34 (1984) 351.

Gal71 S. Galster et al., Nucl. Phys. B32 (1971) 221.

Gani2 D. Ganichot. B. Grossetete, and D. B. Isabelle, Nucl. Phys. A178 (1972) 545.

Gari4 E. L. Garwin. D. T. Pierce, and H. C. Siegmann, Helv. Phys. Acta 47393 (1974).

Gar92 G. T. Garvey, S. Krewald, E. Kolbe, a..d K. Langacke, Phys. Lett. B289 (1992) 249.

Gar93 G. T. Garvey. W. C. Louis, and D. H. White, Los Alamos preprint LA-LR-93-0037.

Gas91 J. Gasser, H. Leutwyler, and M. E. Sainio, Phys. Lett. B253 (1991) 252.

Gei89 D. Geiregat et al., Phys. Lett. B232 (1989) 539.

Glai0 S. L. Glashow, J. Iliopoulos and L. Maiani, Phys. Rev. D2 (1970) 1285.

Gol90 .M. Golden and L. Randall, Nucl. Phys. B361 (1991) 3.

Got67 K. Gottfried, Phys. Rev. Lett. 18 (1967) 1174.

Gre69 G. K. Greenhut, Phys. Rev. 184 (1969) 1860.

Had92 E. Hadjimichael, G. I. Poulis, and T. W. Donnelly, Phys. Rev. C45 (1992) 2666.

Hax89 W. C. Haxton, E. M. Henley, and M. J. Musolf, Phys. Rev. Lett. 63 (1989) 949.

Hax93 W. C. Haxton, E.M. Henley, and M.J. Musolf, to be published.

Hei89 W. Heil et al., Nucl. Phys. B327 (1989) 1. 
Hei93 . Iainz proposal \# A4/1-93. E. Heinen-Konschak et al. collaborators. D. von Harrach. spokesperson.

Heni3 E. M. Henley. A. H. Huffman, and D. L. L. Yu. Phys. Rev. D7 (1973) 943.

Heni9 E. .I. Henley, W.-Y. P. Hwang, and G. X. Epstein, Phys. Lett. B88 (1979) 349.

Hen82 E. M. Henley and W.-Y. P. Hwang. Phys. Rev. C26 (1982) 2376.

Hen91 E. .M. Henley, G. Krein. S. J. Pollock, and A. G. Williams. Phys. Leti. B269 (1991, 31.

Hocỉ3 J. Hockert, D. O. Riska. M. Gari, and A. Huffman. Nucl. Phys. A217 (1973) 14.

Hohi6 G. Höhler et al, Nucl. Phys. Bi14 (1976) 505.

Hol89 B. R. Holstein. Weak Interactions in Nuclei. Princeton Lniversity Press. Princeton. ‥ J.. (1989) ch. 5 .

Hol90 B. R. Holstein in Proceedings of the Caltech Workshop on Parity Violation in Electron Scattering. E. J. Beise and R. D. McKeown, Eds.. World Scientific (1990) 27.

Hor93a C. J. Horowitz, Phys. Rev. C47 (1993) 826.

Hor93b C. J. Horowitz and J. Piekarewicz, Phys. Rev.. to be published (1993).

Hun76 P. Q. Hung and J. J. Sakurai, Phys. Lett. B63 (1976) 295; see also Table 9.1 of Ref. [Com83].

Hwa81 W.-Y. P. Hwang, E. M. Henley, and G. A. Miller, Ann. Phys. 137 (1981) 378.

Hwa86 W.-Y. P. Hwang and T. W. Donnelly, Phys. Rev. C33 (1986) 1381.

Jaf89 R. L. Jaffe, Phys. Lett. B229 (1989) 275.

Jani2 J. A. Jansen. R. Th. Peerdeman, and C. De Vries, Nucl. Phys. A188 (1972) 337.

Jeg89 F. Jegerlehner. PSI Preprint \# PR-89-23 (1989).

Jen91 E. Jenkins and A. V. Manohar, Phys. Lett. B255 (1991) 558.

Jon80 D. R. T. Jones and S. T. Petcov, Phys. Lett. 91B (1980) 137.

Jon89 G. T. Jones et al., Zeit. für Phys. C43 (1989) 527.

Jon91 C. E. Jones-Woodward et al., Phys. Rev. C44 (1991) R571.

Kal89 N. Kalantar-Nayestanaki et al., Phys. Rev. Lett. 63 (1989) 2032.

Kap88 D. B. Kaplan and A. Manohar, Nucl. Phys. B310 (1988) 527.

Kap92 D. B. Kaplan and M. J. Savage, UCSD Prinprint \#PTH 92-04 (1992)

KAR91 KARMEN, Phys. Lett. B267 (1991) 321.

KAR92 KARMEN, Phys. Lett. B280 (1992) 198. 
Ken89 D. C. Kennedy, B. W. Lynn. C. J.-C. Im, and R. G. Stuart, Nucl. Phys. B321 (1989) $\$ 3$.

Ken90 D.C. Kennedy and P. Langacker. Phys. Rev. Lett. 65 (1990) 296i: Phys. Rev. Lett. 66 (1991) 395: Phys. Rev. D44 (1991) 1591.

Kim81 J. E. Kim. P. Langacker. M. Levine, and H. H. Williams. Rev. Mod. Phys. 53. 211 (1981).

Kin62 T. Kinoshita. J. Math. Phys. 3 (1962) 6 jั0.

Kis87 S. Kistryn et al., Phys. Rev. Lett. 58 (1987) 1616.

Kit90 T. Kitagaki et al.. Phys. Rev. D42 (1990) 1331. and refs therein.

Kioe92 W. Koepf. E. .II. Henley, and S. J. Pollock. Phys. Lett. B288 (1992) 11.

Kol92 E. Kolbe. K. Langacke. S. Krewald. and F.-K. Thielemann. Nucl. Phys. A540 1992 j99.

Lamij G. Lampel and C. Weisbuch. Solid State Comm. 16 (19ij) $87 i$.

Lam93 S. Lamoreaux. private communication.

Lan90 P. Langacker, Č. Penn. pre-print \# LPR-0435T (1990) (PASCOS-90).

Lan91 P. Langacker. Phys. Lett. B256 (1991) 277.

Lee64 T. D. Lee and M. Nauenberg, Phys. Rev. 133 (1964) B1549.

Li82 S.-P. Li. E. M. Henley, and W.-Y. P. Hwang, Ann. Phys. 143 (1982) 372.

Llei2 C. H. Llewellyn-Smith. Phys. Rep. 3 (1972) 261.

Lou89 LSND collaboration, LA.MPF proposal \#1173, Los Alamos National Laboratory report LA-L'R-89-3764 (1989), W.C. Louis, contact person.

Lou92a SLAC proposal E148 (1992) R. Lourie, spokesperson.

Lou92b R. Lourie. private communication.

Lu90 X. Q. Lu et al. Los Alamos Report LA-11842-P, Los Alamos National Laboratory (1990).

Lun93 A. Lung et al., Phys. Rev. Lett. 70 (1993) 718.

.Mad89 CEBAF proposal 89-005, R. Madey, contact person.

Mad92 R. Madey et al., private communication.

Mar80 W. J. Marciano and A. Sirlin, Phys. Rev. D22 (1980) 2695.

Mar81 W. J. Marciano and A. Sirlin, Phys. Rev. Lett. 46 (1981) 163.

Mar83 W. J. Marciano and A. Sirlin, Phys. Rev. D27 (1983) 552.

Mar84 W. J. Marciano and A. Sirlin, Phys. Rev. D29 (1984) 75. 
.Mar90 W. J. Marciano and J. L. Rosner. Phys. Rev. Lett. 65 (1990) 2963.

.Mar93 P. Markowitz et al., accepted for publication in Phys. Rev. C (June 1993).

McCiT J. S. McCarthy. I. Sick. and R. R. Whitney, Phys. Rev. C15 (19ii) 1396.

McK89 .IIT Bates proposal 89-06. R. Mickeown and D. H. Beck. contact people.

IIch91 CEB.AF proposal 91-020, R. .Ickeown. contact person.

Mil88 MIIT/Bates proposal 88-25, R. Milner. R. Mckeown. T. Chupp. and A. Bernstein. contact people.

Mil89 .IIT/Bates Experiment 89-12. R. Milner and J. van den Brand, spokespersons.

.Min89 S. L. .Mintz and .I. Pourkaviani. Phys. Rev. C40 (1989) 2458.

Mo69 L. W. Mo and Y. S. Tsai. Rev. Mod. Phys. 41 (1969) 205.

Mon90 C. Monroe. W. Swann. H. Robinson. and C. E. Wieman. Phys. Rev. Lett. 65 !1990, 1571.

Moois R. G. Moorhouse. Electromagnetic Interactions of Hadrons, Vol. 1. A. Donnachie and G. Shaw. Eds., Plenum Press (1978) 83.

Mus90 M. J. Musolf and B. R. Holstein, Phys. Lett. 242B (1990) 461.

Mus91 M. J. Musolf and B. R. Holstein. Phys. Rev. D43 (1991) 2956.

Mus92a MI. J. Musolf and T. W. Donnelly. Nucl. Phys. A546 (1992) 509.

Mus92b .I. J. Musolf in Proceedings of the CEBAF Summer Workshop, F. Gross and R. Holt. Eds.. . lewport . News, Va. (1992), 321.

Mus93a ..I. J. Musolf and .M. Burkardt, CEBAF Theory Pre-print \# TH-93-01.

Mus93b .M. J. Musolf. T. W. Donnelly, and T. S. H. Lee, work in progress.

Mus93c .M. J. Musolf and T. W. Donnelly, Zeit. fur Phys. C57 (1993) 559.

Mus93d M. J. Musolf, S. J. Pollock, and M. D. Havey, work in progress.

.ak91 T. Nakanishi et al., Phys. Lett. A158, 345 (1991).

Nap89 J. Napolitano et al., Nucl. Instr. Meth. A274 (1989) 152.

Sap90 J. Napolitano in Proceedings of the Caltech Workshop on Parity Violation in Electron Scuttering, E.J. Beise and R.D. McKeown, Eds., World Scientific (1990) 90.

. Vap91 J. Napolitano, Phys. Rev. C43 (1991) 1473.

Nat82 L. M. Nath, K. Schilcher, and M. Kretzschmar, Phys. Rev. D25 (1982) 2300.

Noe88 M. C. Noecker, B. P. Masterson, and C. E. Wieman, Phys. Rev. Lett. 61 (1988) 310.

Nov75 V. N. Novikov, and I. B. Khriplovich, JETP Lett. 22 (1975) 74.

OCoi2 J. S. O'Connell, T. W. Donnelly, and J. D. Walecka, Phys. Rev. C6 (1972) 719. 
Omo91 T. Omori et al., Phys. Rev. Lett. 67. 3294 (1991).

Ott90 . Mainz proposal A1/3-90. E. Otten, contact person.

Par91 I. IV. Park. J. Schechter and H. Weigel. Phys. Rev. D43. 869 (1991).

Pasi3 E. Paschos and L. Wolfenstein. Phys. Rev. D7 (1973) 91.

Pes90 .I. E. Peskin and T. Takeuchi, Phys. Rev. Lett. 65 (1990) 964.

Pla90 S. Platchkov et al., Nucl. Phys. A510 (1990) 740.

Pol8i S. J. Pollock. Ph. D. Thesis, Stanford University (1987) unpublished.

Pol90 S. J. Pollock. Phys. Rev. D42 (1990) 3010.

Pol92a S. J. Pollock. E. N. Fortson. and L. Wilets. Phys. Rev. C46 (1992) 258 i.

Pol92b S. J. Pollock and M. J. Musolf. to be published.

Pol93 S. J. Pollock and .I. J. Musolf, to be published.

Preis C. Y. Prescott et al.. Phys. Lett. B77 (19i8) $34 i$.

Prei9 C. Y. Prescott et al.. Phys. Lett. B84 (1979) 524.

Pro91 Proceedings of the European Workshop on Hadron Physics with Electrons Beyond 10 GeV. B. Frois and J.-F. Mathiot, Eds., Nucl. Phys. A532 (1991).

Ras89 A. S. Raskin and T. W. Donnelly, Ann. Phys. 191 (1989) 78.

Rei68 R. V. Reid, Jr.. Ann. Phys. 50 (1968) 411.

Rei8i D. Rein. Zeit. für Phys. C35 (1987) 43.

Reu82 WV. Reuter, G. Fricke, K. Merle, and H. Miska, Phys. Rev. C26 (1982) 806.

Ros90 J. L. Rosner. Phys. Rev. D42 (1990) 3107.

RPP92 Review of Particle Properties, Phys. Rev. D45 (1992) Part II.

Sac62 R. G. Sachs, Phys. Rev. 126 (1962) 2256.

Sal68 A. Salam, in Elementary Particle Physics, ed. N. Svartholm, Stockholm (1968) 367.

Schi3 P. A. Schreiner and F. Von Hippel, Nucl. Phys. B58 (1973) 333.

Sch88 Andreas Schäfer, Phys. Lett. B208 (1988) 175.

Sch90 R. Schiavilla, V. R. Pandharipande, D. O. Riska, Phys. Rev. C41 (1990) 309.

Sea68 R. E. Seamon, K. A. Friedman, G. Breit. R. D. Haracz, J. M. Holt and A. Prakash, Phys. Rev. 165 (1968) 1579.

Ser79 B. D. Serot, Nucl. Phys. A322 (1979) 408.

Sici0 I. Sick and J. S. McCarthy, Nucl. Phys. A 150 (1970) 631.

Sic91 I. Sick, personal communication (1991). 
Sie37 A. J. F. Siegert. Phys. Rev. 52 (1937) i8i.

Sims0 G. G. Simon. F. Borkowski, Ch. Schmitt. and V. H. Walther. Z. Naturforsch. 35A $11980,1$.

Sir30 A. Sirlin. Phys. Rev. D22 (1980) 971.

Slo88 T. Sloan. G. Smadja, and R. Voss, Phys. Rep. 162 (1988) 45.

Sou90a P.A. Souder. et al. Phys. Rev. Lett. 65 (1990) 694.

Sou90b P. Souder. in Proceedings of the Caltech Workshop on Parity-Violating Electron Scat. tering. E. J. Beise and R. D. McKeown. Eds.. World Scientific (1990).

Sou91 CEBAF proposal PR-91-00T. P. Souder and J.MI. Finn, contact people.

van91 .I. van der Schaar. Phys. Rev. Lett. 66 (1991) 2855.

Vap88 O. Vapenikova. Zeit. für Phys. C37 (1988) 251.

Walis J. D. Walecka in Muon Physics, Vol. II. V. W. Hughes and C. S. Wu. Eds.. Academic Press (1975) 113.

Walii J. D. Walecka. Nucl. Phys. A285 (1977) 349.

Wei67 S. Weinberg, Phys. Rev. Lett. 19 (1967) 1264.

Whi90 D. H. White. in Proceedings of the Caltech Workshop on Parity Violation in Electron Scattering, E. J. Beise and R. D. McKeown, Eds., (1990) 224.

Wie93 C. Wieman. private communication.

Woli9 L. Wolfenstein, Phys. Rev. D19 (1979) 3450.

Zel5i Ia. B. Zeldovich. Zh. Eksp. Teor. Fiz. 33 (1957) 1531 [Sov. Phys. JETP 6 (1958) 1184].

Zel60 Ia. B. Zeldovich and A. M. Perelomov, Zh. Eksp. Teor. Fiz. 39 (1960) 1115 [Sov. Phys. JETP 12 (1961) 777).

Zuci1 P. Zucker. Phys. Rev. D4 (1971) 3350. 


\section{FIGURE CAPTIONS}

Fig. 2.1 Perturbative contributions to non-valence quark axial-vector and vector current matrix elements of the nucleon. Loops involving heavy quarks renormalize light quark current operators via gluon exchange (curly lines).

Fig. 2.2 . Von-perturbative contributions to strange-quark vector and axial-vector matrix elements of the nucleon. Virtual $s \bar{s}$ pair with low-momenta $\left(p \leqq . \perp_{Q C D}\right)$ interacts with valence quarks to form strange hadronic intermediate states (i.e. $K$. 1 . o....

Fig. 2.3 Experimental constraints on parity-violating meson-nucleon couplings entering meson-exchange model for the PV .X. interaction [Hol89]. In the text. $f_{\pi}$ is denoted by $h_{x, v \pi}$ to avoid confusion with the pion decay constant. The black circle refers to theoretical "best value" of Ref. (Des80). For a detailed description of experimental bands, see Ref. Ade85].

Fig. 2.4 Present and prospective low-energy constraints on $S$ and $T$ parameterization of physics beyond the Standard Model. Short-dashed lines give present constraints from atomic PV [Noe88. Mar90]. Long-dashed lines give limits attainable with a $10 \%$ determination of $\xi_{V}^{p}$ from a prospective forward-angle $A_{L_{R}}(\vec{e} p)$ experiment. Solid lines correspond to potential constraints from a one percent measurement of $A_{L R}\left(0^{+} 0\right)$. Dot-dashed lines indicate possible constraints from a $10 \%$ determination of $\xi_{i}^{T=1}$ obtainable from an $A_{L R}(V \rightarrow \Delta)$ measurement [.Mus92b. Mus93c]. In all cases. common central values for $S$ and $T$ are assumed, and only ranges permitted by experimental and theoretical uncertainties are indicated.

Fig. 3.1 Leading-order parity-conserving (a) and parity-violating (b) amplitudes for scattering of polarized electrons from a nuclear target.

Fig. 3.2 Higher-order contributions to amplitude for neutral current (E.I and weak) scattering involving two elementary fermions (lepton or quark), $f$ and $f^{\prime}$. Shaded circle represents radiative processes (loops). (a.c) indicate vertex corrections. (b.d) represent corrections to propagators, and (e) indicates exchange of two vector bosons $\left(V . V^{\prime \prime}=\gamma, Z^{0}, W^{ \pm}\right)$.

Fig. 3.3 "One-quark" electroweak radiative corrections to lepton-hadron scattering. Onequark processes refer to scattering involving only one quark at a time and result in renormalization of the electroweak couplings $\xi_{v}^{(a)}$ from their tree-level values (see Table 3.2).

Fig. 3.4. Strong interaction corrections to vector boson propagators. (a) indicates gluon exchange (curly line) between virtual quark and anti-quark, while in (b) virtual $q \bar{q}$ pair interacts strongly with target quark.

Fig. 3.5. Representative Feynman diagrams for "many-quark" electroweak radiative corrections to lepton-nucleon scattering. In contrast to processes of Fig. 3.3, many-quark 
processes involve electroweak interactions between target quarks. leading to the formation of virtual hadronic intermediate states $(.0 \%, \rho$, etc. $)$.

Fig. 3.6. Feynman diagrams used in modelling many-quark electroweak contributions to $1 \cdot \epsilon) \mathrm{s}$ Al $N$ ) scattering amplitude. Pion-loop (a) and vector meson pole (b) diagrams contain a weak. $\mathrm{PV}$ meson nucleon vertex (crossed circle) and parity-conserving strong and electromagnetic vertices open circles), respectively.

Fig. 3.T. Dispersion corrections to lepton-hadron (nucleon or nucleus) scattering. Exchange of vector bosons $\left(V . V^{\prime}=\gamma, Z^{0} . W^{ \pm}\right)$may excite and de-excite virtual hadronic intermediate states (denoted by double line).

Fig. 3.8. Two-body meson-exchange contributions to nuclear electromagnetic and weak neutral currents. In (a), an off-shell photon or $Z^{0}$ couples to a virtual . $V . \bar{r}$ pair. which interacts with remaining nucleons via meson exchange. (b) and (c) give current. associated with exchanged meson and virtual isobar intermediate states. respectively:

Fig. 3.9. Meson-exchange model for nuclear PV contributions to PV lepton-nucleon scattering. Exchanged mesons interact strongly (open circle) with one nucleon and weakly (crossed circle) with second nucleon. PV component of the latter generates PV... interaction. which mixes nuclear states. (a.b) illustrate parity-mixing of initial and final nuclear states, which couple to the final and initial states via the one-body. E.I current operator. (c) shows meson-exchange current contributions, which are required by current conservation.

Fig. 3.10. Figure-of-merit for PV electron scattering (see Eq. (3.139)) at $\theta=10^{\circ}$ versus incident electron energy $\epsilon$. The various cases presented are discussed in the text.

Fig. 3.11. As for Fig. 3.10, except now at $\theta=30^{\circ}$.

Fig. 3.12. As for Fig. 3.10, except now at $\theta=150^{\circ}$.

Fig. 3.13. Leading-order neutrino and anti-neutrino charged-current $(a, b)$ and weak neutral current (c,d) scattering from a nuclear target.

Fig. 4.1. Correlation between $\mu_{\mathrm{g}}$ and $g_{A}^{(1)}=-\dot{G}_{A}^{T=1}(0)$ for a backward-angle $A_{\llcorner R}(\vec{e} p)$ measurement. Solid bands correspond to different assumptions for experimental uncertainty in $A_{L R}$. Dashed lines give error induced in $\mu_{\mathrm{g}}$ due to the $20 \%$ uncertainty in $g_{A}^{(1)}$ resulting from the theoretical uncertainty in $R_{A}^{T=1}$. Arrows indicate constraints on $\mu$, anticipated from SAMPLE measurement [Bei92].

Fig. 4.2. Fraction of total $\overrightarrow{e p}$ asymmetry resulting from longitudinal $\left(F_{L}\right)$ and axial-vector $\left(F_{A}\right)$ responses as a function of scattering angle for different incident energies (given in $\mathrm{MeV}$ ). 
Fig. 4.3. Fractional statistical uncertainty (a) and axial-vector contribution to $A_{L R}(\vec{\epsilon} p)$ (b) at backward-angles as a function of incident energy. Luminosity $\mathcal{L}=5 \times 10^{38} \mathrm{~cm}^{-2} \mathrm{~s}^{-1}$. $100 \%$ beam polarization. and 1000 hours of running time assumed in (a).

Fig. 4.4. Correlation between $\mu_{\mathbf{s}}$ and dimensionless strangeness radius. $\rho_{\mathbf{s}}$, in a measurement of the forward-angle $\vec{e} p$ PV asymmetry. Solid lines correspond to different assumptions for experimental uncertainty in $A_{L R}$. Dashed lines indicate uncertainty in $\rho$, assuming theoretical uncertainty in $\mu$, determined from an "ideal" backward-angle $A_{L R} \mid \vec{E} p$, measurement. Arrows indicate expected S.A.MPLE limits on $\mu_{s}$ [Bei92].

Fig. 4.j. Doability curves for Standard Model test with PV $\overrightarrow{e p}$ scattering. Solid curves give statistical uncertainty in $A_{L R}(\vec{e} p)$ under same conditions as in Fig. 4.3 a a but with solid angle $د \Omega=0.01 \mathrm{sr}$. Dashed curve indicates maximum statistical uncertainty allowed for a $\leqslant 10 \%$ determination of $\xi^{p}$.

Fig. 4.6. Doability curves for $G_{E}^{(s)}$ determination from $A_{L R}\left({ }^{4} \mathrm{He}\right)$ measurements under two different sets of kinematic conditions and assumptions about detector. In each case. a luminosity of $\mathcal{L}\left[{ }^{4} \mathrm{He}\right]=5 \times 10^{38} \mathrm{~cm}^{-2} \mathrm{~s}^{-1} .100 \%$ beam polarization, and 1000 hours of running time are assumed. Error associated with $G_{E}^{(0)}$-uncertainty indicated by dashed and dotted curves for $\delta \rho_{s}=1.4$ and 0.7 , respectively. In both cases. a value of $\lambda_{\boldsymbol{E}}^{\boldsymbol{s}}=0$ is assumed.

Fig. 4.7. Constraints on $G_{E}^{(9)}$ obtainable from prospective PV elastic electron scattering experiments. Dashed lines give constraints from a series of $A_{L R}(\vec{e} p)$ measurements. including an "ideal" backward-angle measurement and a forward-angle measurement carried out under the conditions of Ref. [Nap91]. Dashed-dot and solid bands give constraints from series of low- and moderate- $\left|Q^{2}\right|$ measurements of the ${ }^{4} \mathrm{He}$ asymmetry: Conditions of Fig. 4.6(b), with $\theta=30^{\circ}$, and $\tau$ corresponding to successive minima in the statistical uncertainty in $A_{L R}$, are assumed. Black dot gives central values for ( $\rho_{\mathbf{s}}$. $\left.\lambda_{\boldsymbol{E}}^{\boldsymbol{s}}\right)$ under models $(\mathrm{A})$ and $(\mathrm{B})$ discussed in section IV.B.

Fig. 4.8 Doability curves for Standard Model tests with $A_{L R}\left(0^{+} 0\right)$ measurements. Solid curves indicate statistical uncertainty in $A_{L R}$ assuming $\mathcal{L}\left[{ }^{4} \mathrm{He}\right]=5 \times 10^{38} \mathrm{~cm}^{-2} \mathrm{~s}^{-1}, \mathcal{L}\left[{ }^{12} \mathrm{C}\right]=$ $1.25 \times 10^{38} \mathrm{~cm}^{-2} \mathrm{~s}^{-1}, 100 \%$ beam polarization, 1000 hours of running time, and solid angles as indicated in the figure. Dashed and dotted curves have same meaning as in Fig. 4.6. Arrows indicate maximum allowable error (either experimental or $G_{\varepsilon}^{(s)}$ induced) for a $\lesssim 1 \%$ determination of $\xi_{v}^{T=0}$.

Fig. 4.9. The PV asymmetry (upper panels) and structure-dependent part of the asymmetry defined in Eq. (4.23) (lower panels) for elastic scattering from ${ }^{40} \mathrm{Ca},{ }^{48} \mathrm{Ca}$ and ${ }^{208} \mathrm{~Pb}$. Shown are calculated results for $\rho_{n}(\vec{x}) / \rho_{p}(\vec{x})=. V / Z$ where $\tilde{\Gamma}(q)=0$ (solid lines) and for $\rho_{n}(\vec{x}) / \rho_{p}(\vec{x}) \neq N / Z$ (dashed lines) using density parameterizations taken from Ref. [Don89]. 
Fig. 4.10. Doability curves for $G_{M}^{(9)}$ determination with backward-angle $A_{L R}\left({ }^{2} \mathrm{H}\right)$ measurements. Solid curves give fractional statistical uncertainty in the asymmetry assuming $\dot{L}=$ $j \times 10^{38} \mathrm{~cm}^{-2} \mathrm{~s}^{-1}, 100 \%$ beam polarization, $\Delta \Omega=1 \mathrm{sr}$, and 1000 hours of running time. Dashed curve gives error induced in $A_{L R}\left({ }^{2} \mathrm{H}\right)$ at $\theta=170^{\circ} \mathrm{by} G_{M}^{\left({ }^{\prime}\right)}$ assuming $\delta \mu_{s}= \pm 0.12$ (corresponding to "ideal" backward angle $\vec{e} p$ determination).

Fig. 4.11. Correlation fiuts for backward-angle. PV elastic electron scattering from hydrogen (labelled $\mathrm{H}$ ) and quasielastic scattering from carbon $(\mathrm{C})$ and tungsten (IV). In the latter two cases, conditions $q=500 \mathrm{Mev} / c, \theta=150^{\circ}$, and $\omega=\frac{1}{2} Q^{2} \mid / m_{, v}$ are assumed. As in Fig. $4.1, g_{A}^{(1)}=-\dot{G}_{A}^{T=1}(0)$. Bands correspond to $\pm 1 \%$ experimental uncertainty. in measured asymmetry.

Fig. 4.12. Hadronic (sea-quark) corrections to Standard Model values for the $\tilde{a}_{i}$ parameters appearing in the deep inelastic $\mathrm{PV}{ }^{2} \mathrm{H}$ asymmetry. Dashed line gives $R_{1}$ (had) while solid line gives $-R_{2}$ (had) as a function of the Bjorken scaling variable $x$.

Fig. 4.13. $\chi^{2}$ contour showing $6 \% \%$ confidence region in extraction of $\xi_{v}^{p}$ and $\eta$, only. from our fictitious data set. Systematic errors are ignored, and data is generated as described in the text (integrated over an incident neutrino spectrum similar to that of the B.IL experiment.) The $\gamma^{2}$ minimum here is about 13.(/14 d.o.f)

Fig. 5.1. Neutrino spectrum from a $\pi-\mu-e$ beamstop neutrino facility: this arises from the decay $\pi^{+} \rightarrow \mu^{+} \nu_{\mu}(0.026 \mu \mathrm{s})$ followed by $\mu^{+} \rightarrow e^{+} \nu_{e} \bar{\nu}_{\mu}(2.20 \mu \mathrm{s})$.

Fig. 5.2. The SLAC polarized electron source of 1979.

Fig. 5.3. Optical elements of the laser beamline for a SLAC style polarized electron source.

Fig. 5.4. Measured asymmetry vs. beam energy in the SLAC experiment. The beam helicity reverses every $3.237 \mathrm{GeV}$ due to $g-2$ precession.

Fig. 5.j. Measured asymmetry vs. $y=1-\epsilon^{\prime} / \epsilon$ in the SLAC experiment.

Fig. 5.6. The detector system for the Mainz quasielastic ${ }^{9} \mathrm{Be}$ experiment (see ref. [Hei89]).

Fig. 5.7. Histogram of pulse-pair asymmetries from the Mainz experiment.

Fig. 5.8. Limits on $\tilde{\beta}$ and $\tilde{\delta}$ for the Mainz experiment (dotted lines) compared with data from other sources. Hashed lines are from the SLAC data [Pre79], and broken lines represent data from $\nu$-hadron scattering [Kim81].

Fig. 5.9. Experimental apparatus for the Bates ${ }^{12} \mathrm{C} \mathrm{PV}$ experiment (see ref. [Sou90a]).

Fig. 5.10. Histogram of the measured asymmetry in each mini-run normalized to its statistical error in the ${ }^{12} \mathrm{C}$ experiment. 
Fig. j.11. Cross section vs. $\left|Q^{2}\right|$ for $\left(\nu_{\mu}, p\right)$ and $\left(\bar{\nu}_{\mu}, p\right)$ in the Brookhaven experiment [Ahr8 $\bar{i}_{\text {]. }}$.

Fig. 5.12. Contour plot of $\eta$ vs. $M_{A}$ taken from Ref. [Ahr87]. ( $\eta$ in Ref. [Ahr87] has the same meaning as $\eta_{s}$ in the notation of the text.)

Fig. 5.13. Detector and target system for the Bates SA.MPLE experiment (see ref. [MIcK89]).

Fig. 5.14. Apparatus for the proposed CEBAF $G^{0}$ experiment (see ref. [Bec91]). 

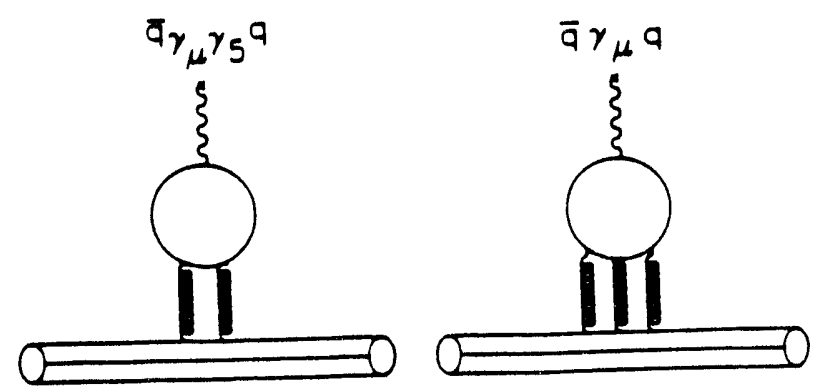

Fig. 2.1

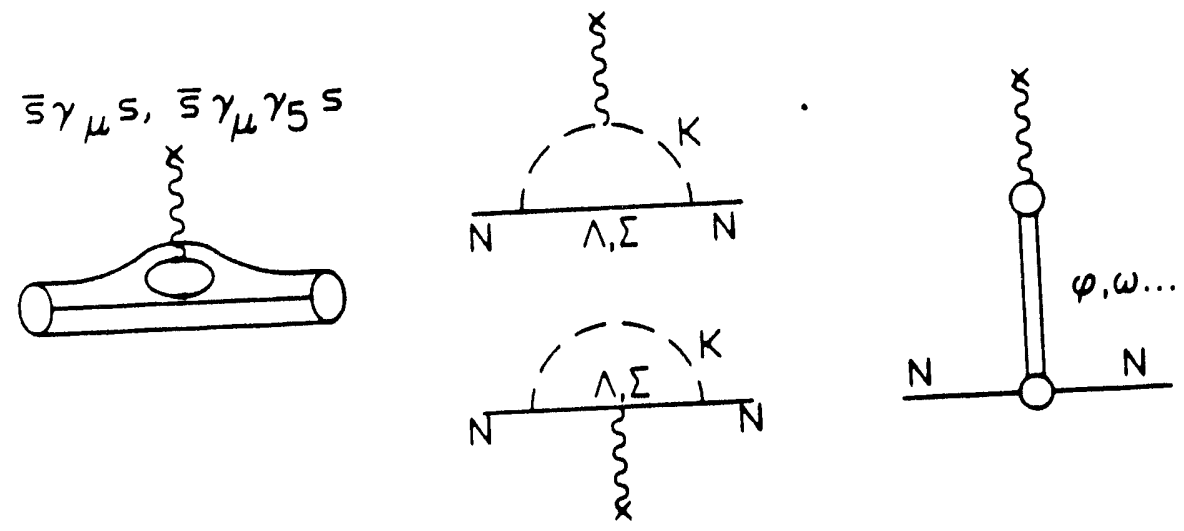

Fig. 2.2 


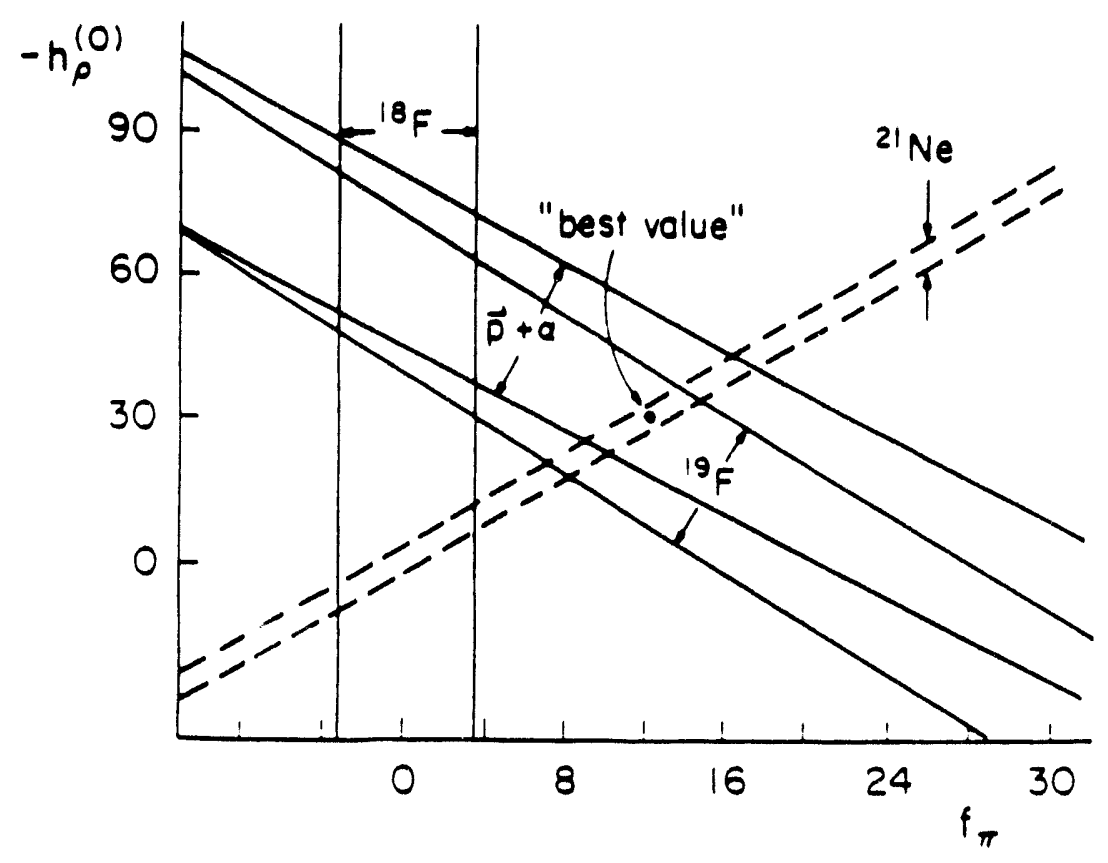

Fig. 2.3

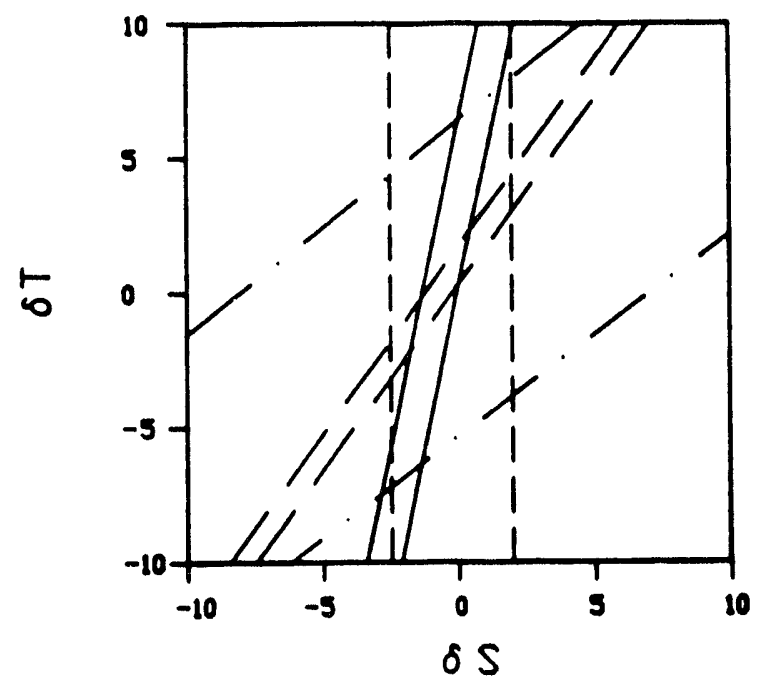

Fig. 2.4 

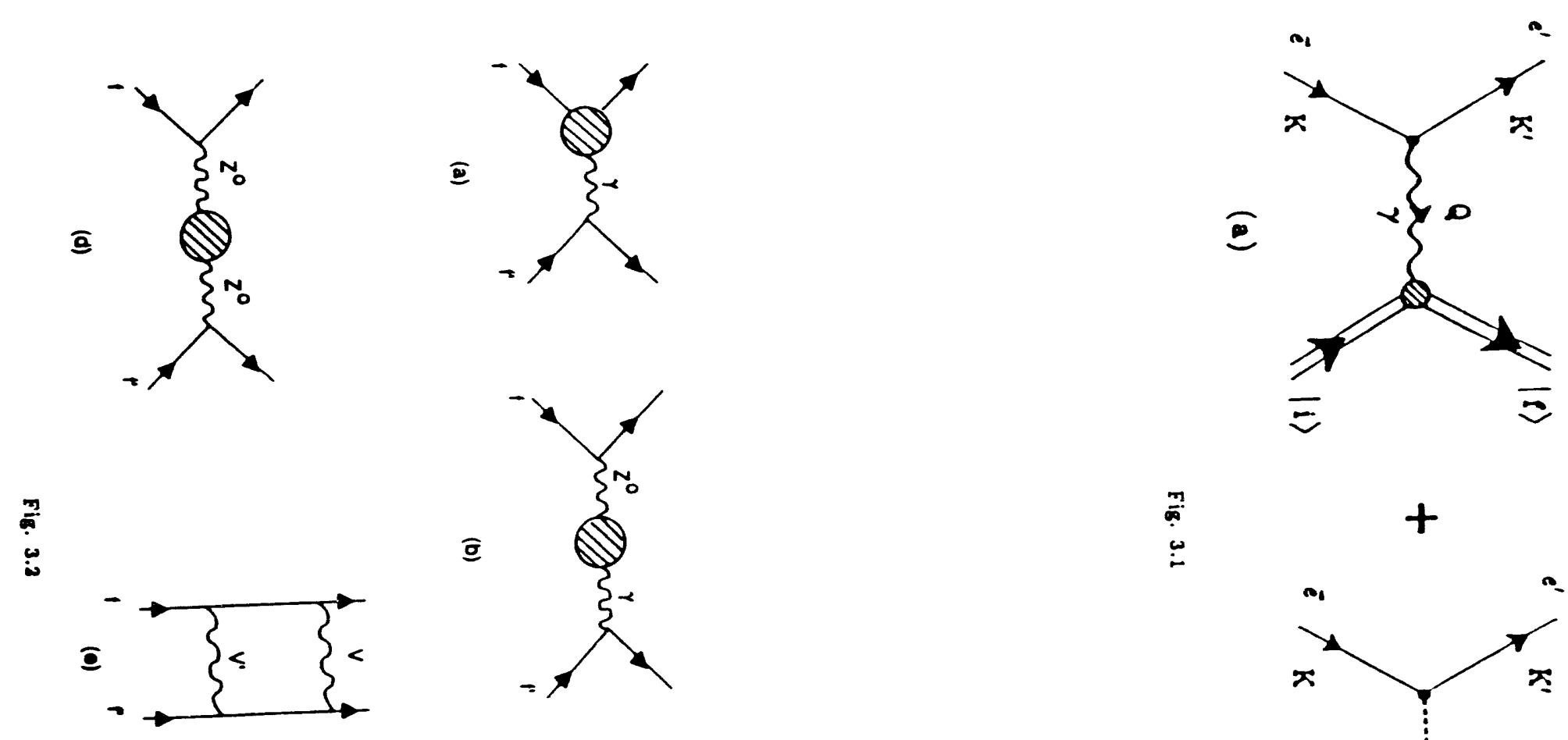

ㅎ

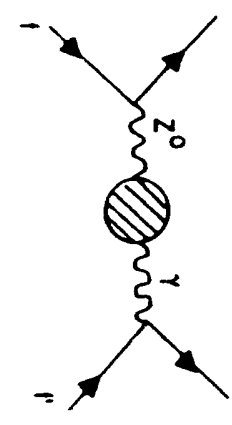

3
$\vdots$
$\vdots$

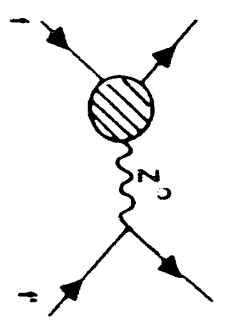




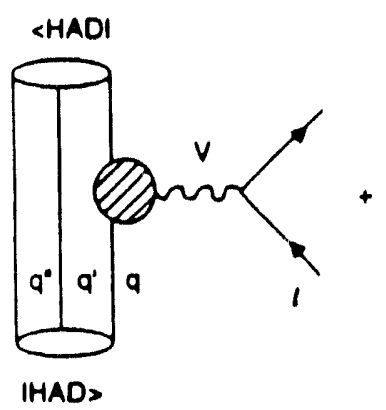

(a)

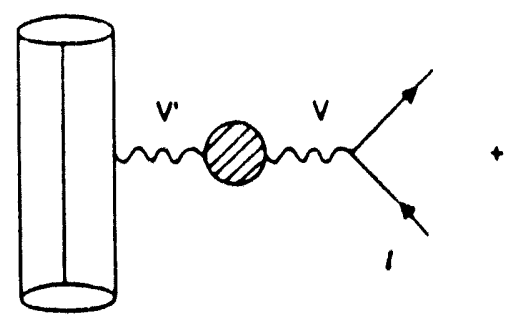

(b)

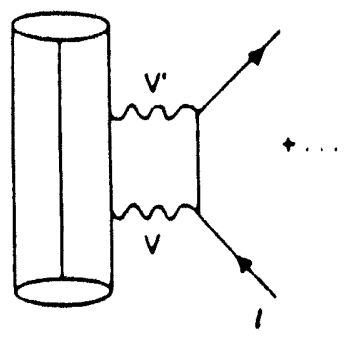

(c)

Fig. 3.3

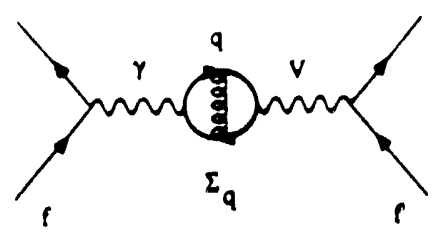

(a)

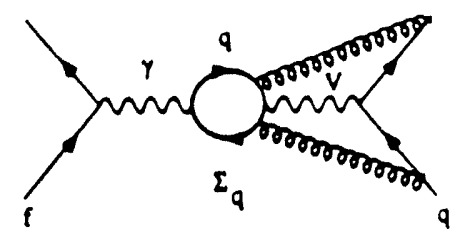

(b)

Fig. 3.4

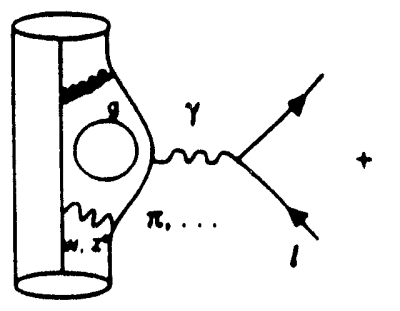

(a)

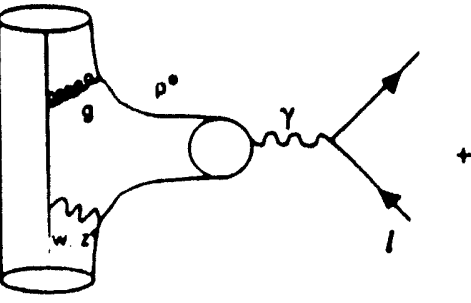

(b)

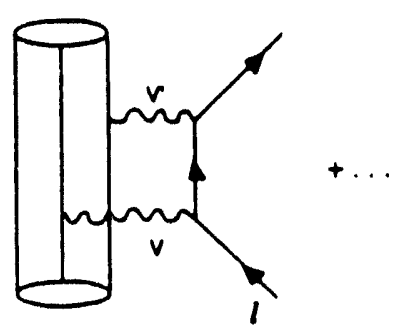

(c)

Eig. 3.3 


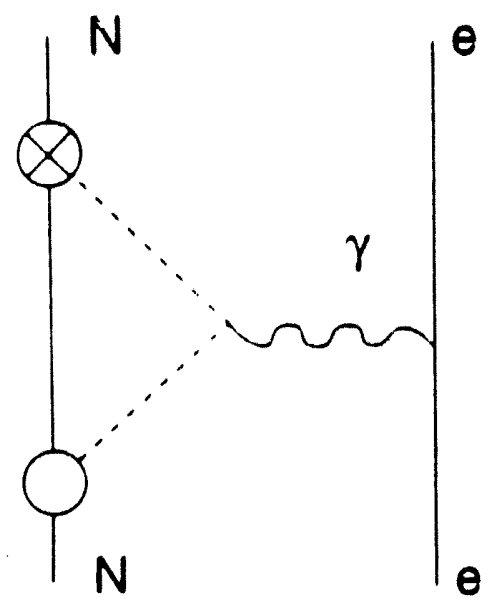

(a)

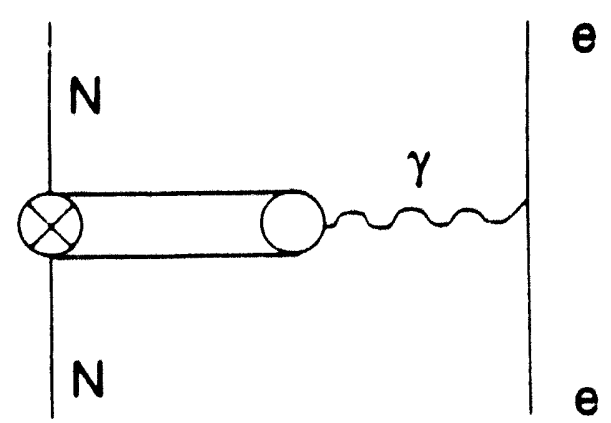

(b)

Fig. 3.6

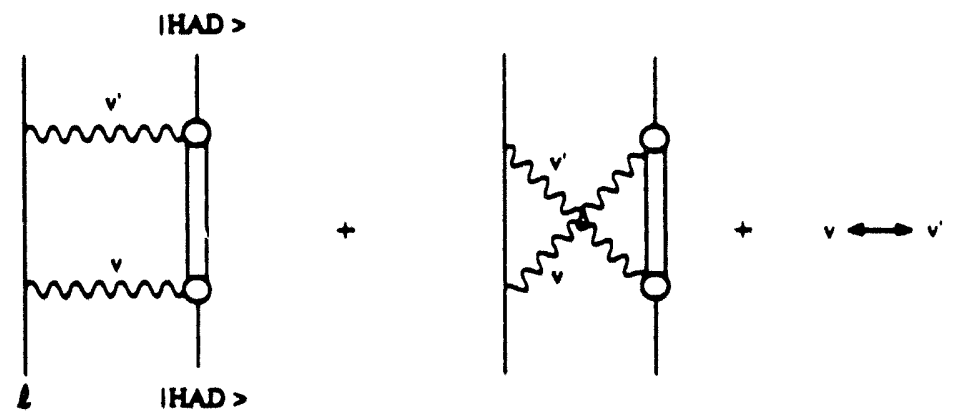

Fig. 3.7 

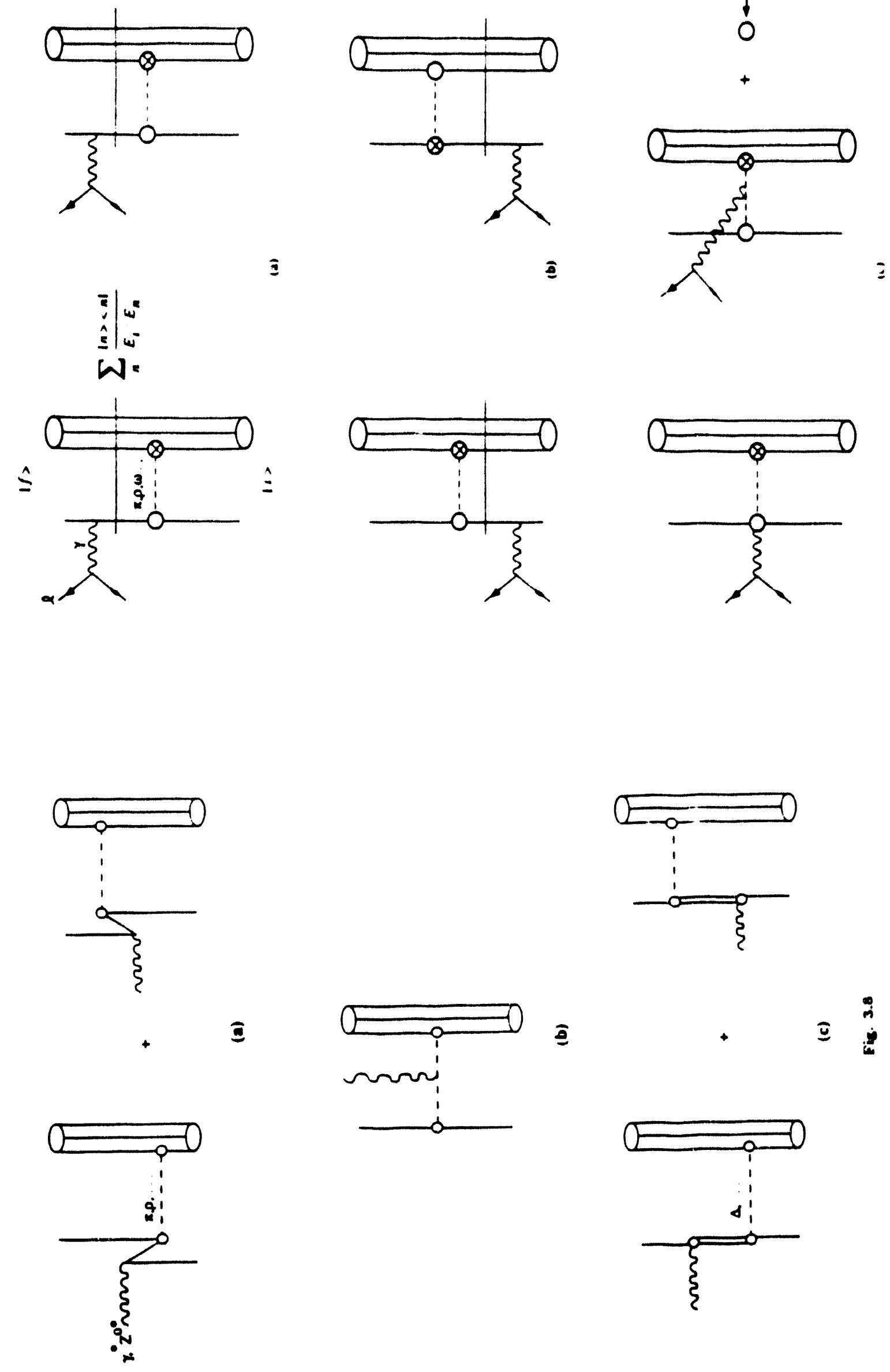

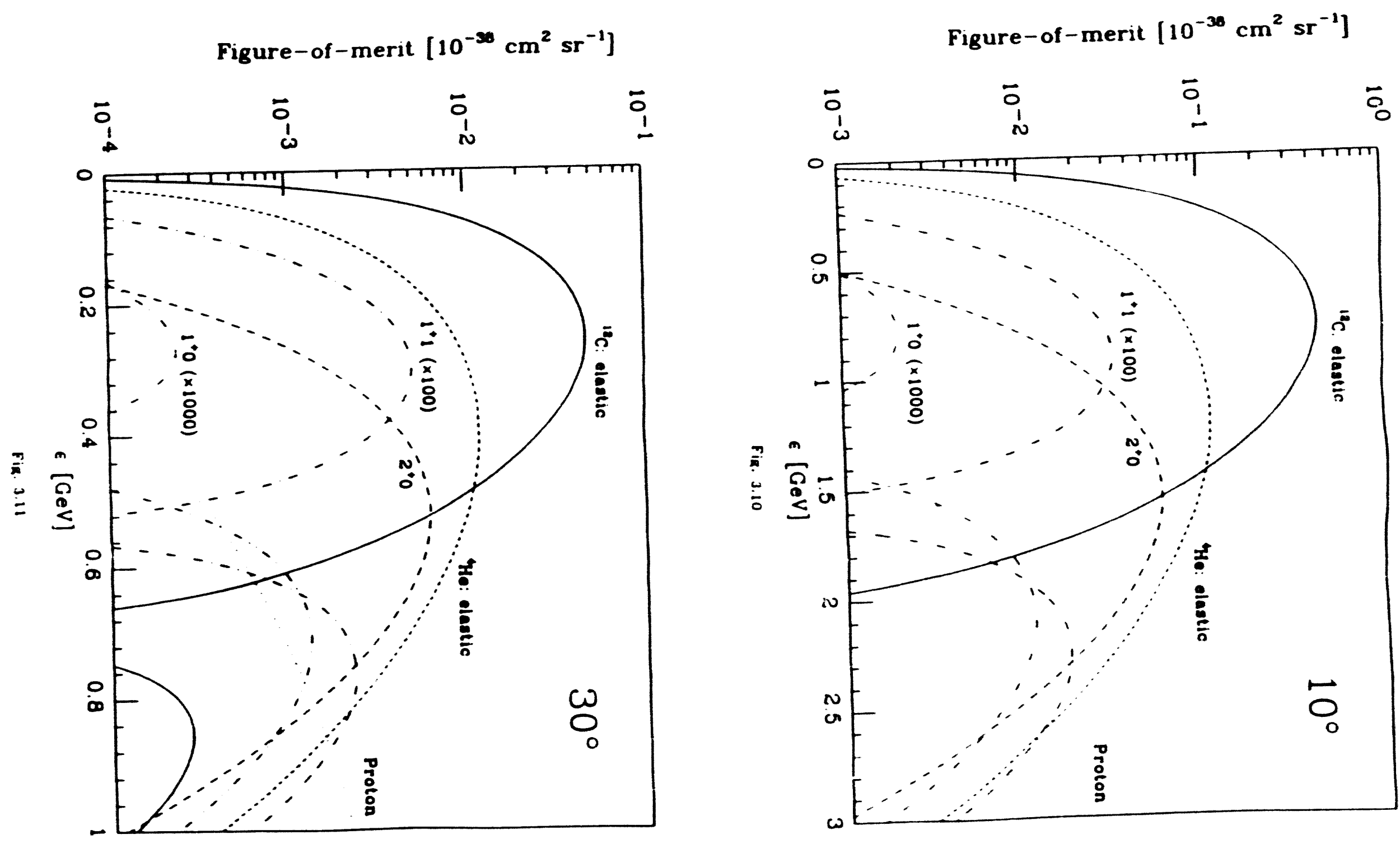

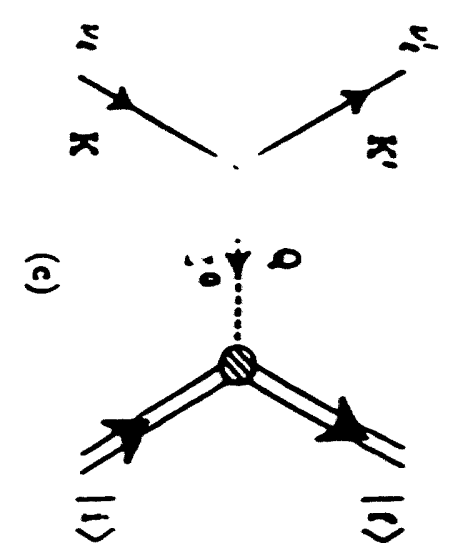

$\underset{2}{2}$

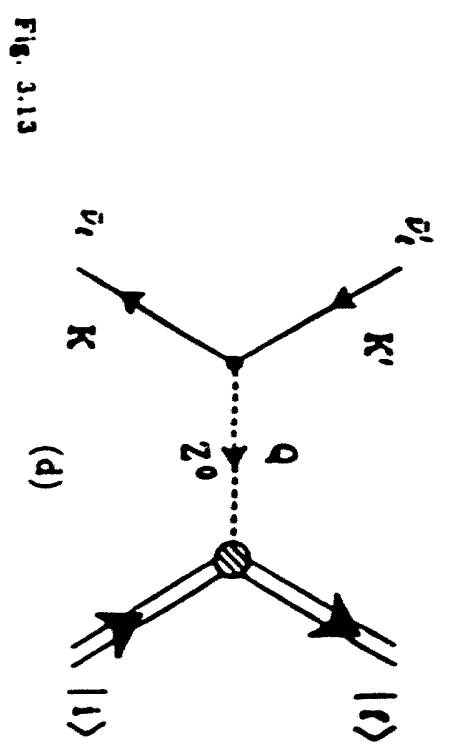

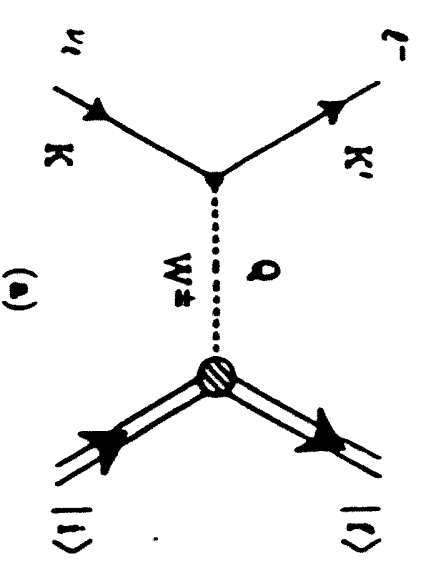

Figure-of-meril $\left[10^{-30} \mathrm{~cm}^{2} \mathrm{sr}^{-1}\right]$

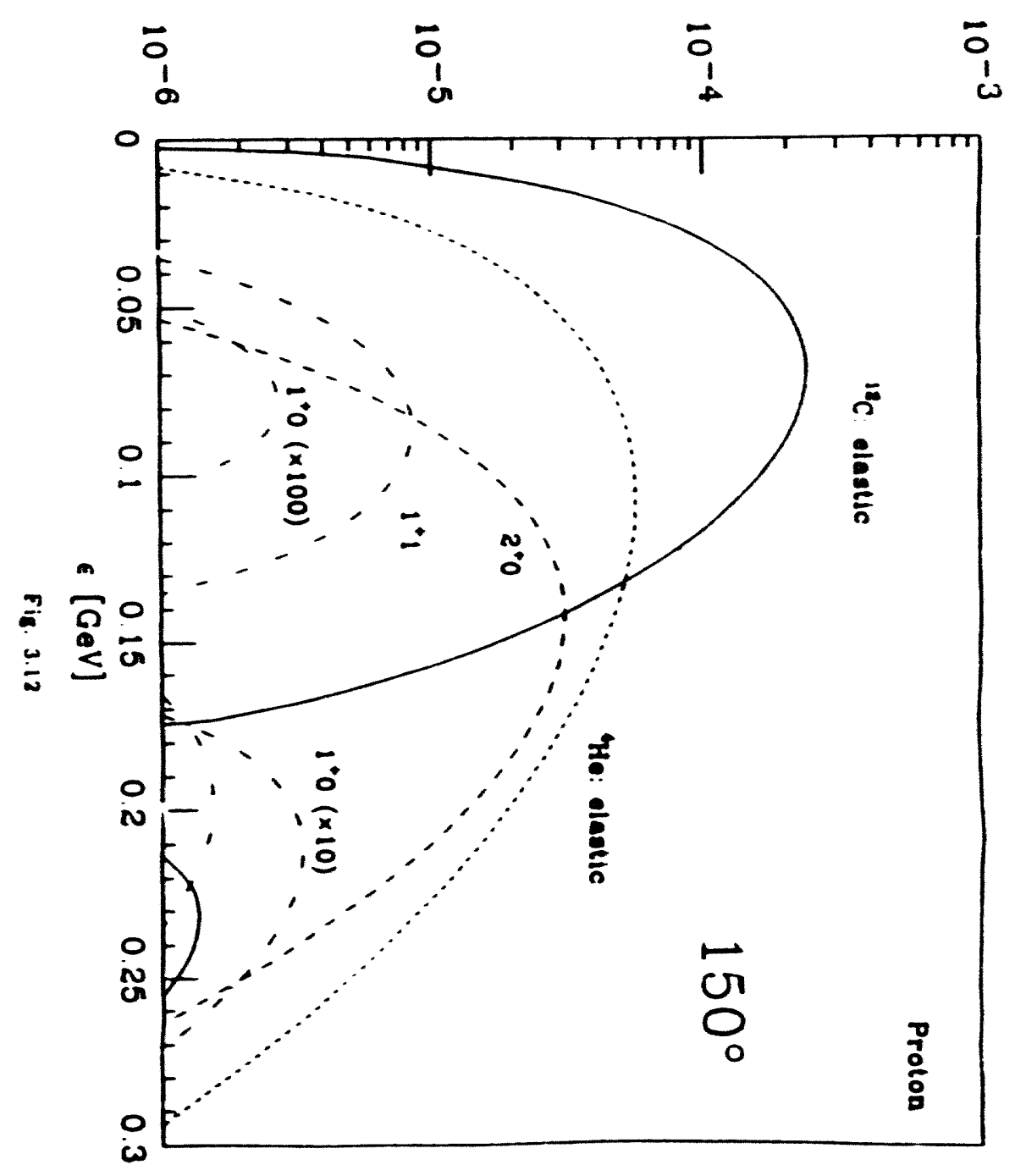



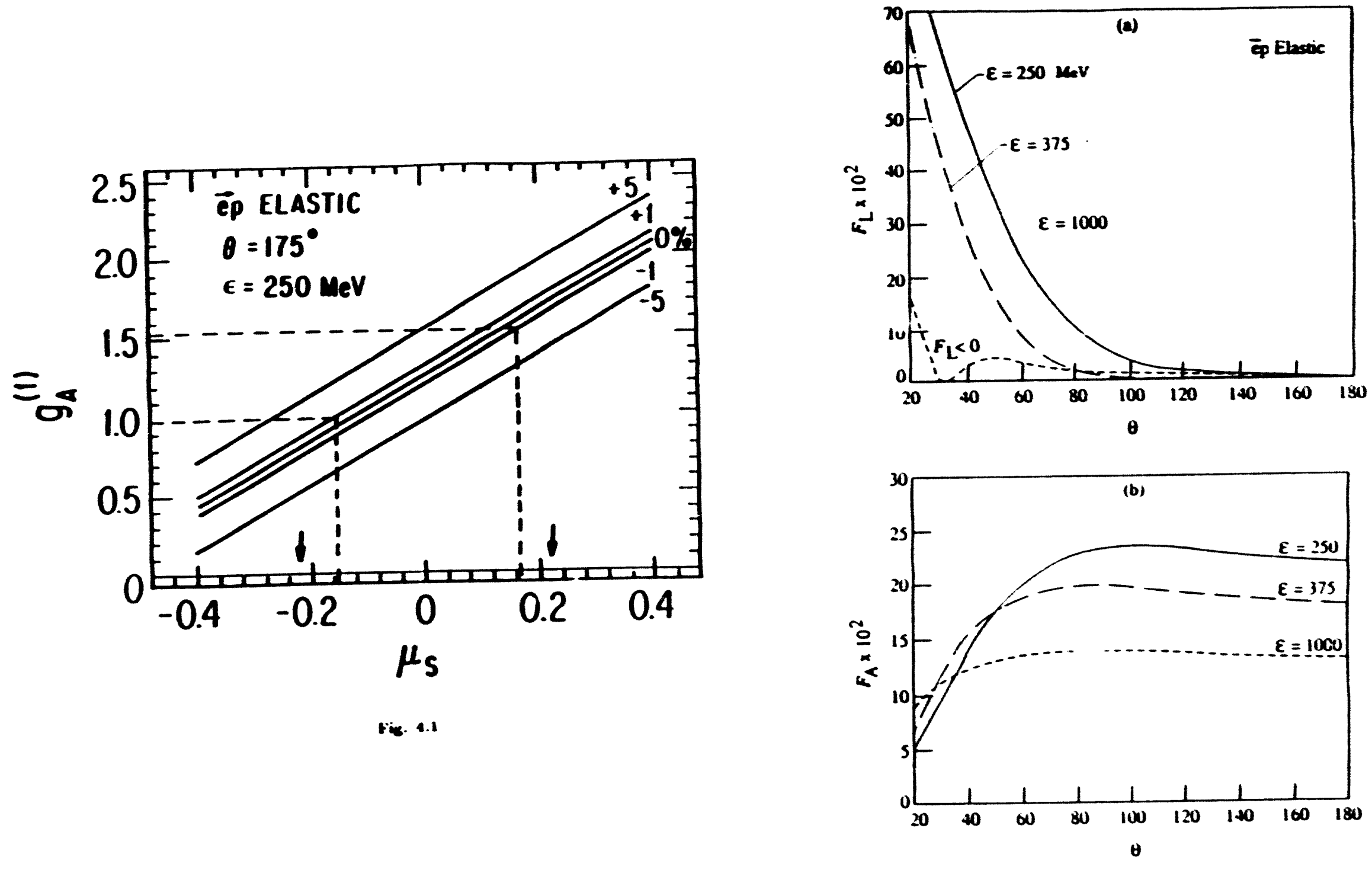

1... 12 

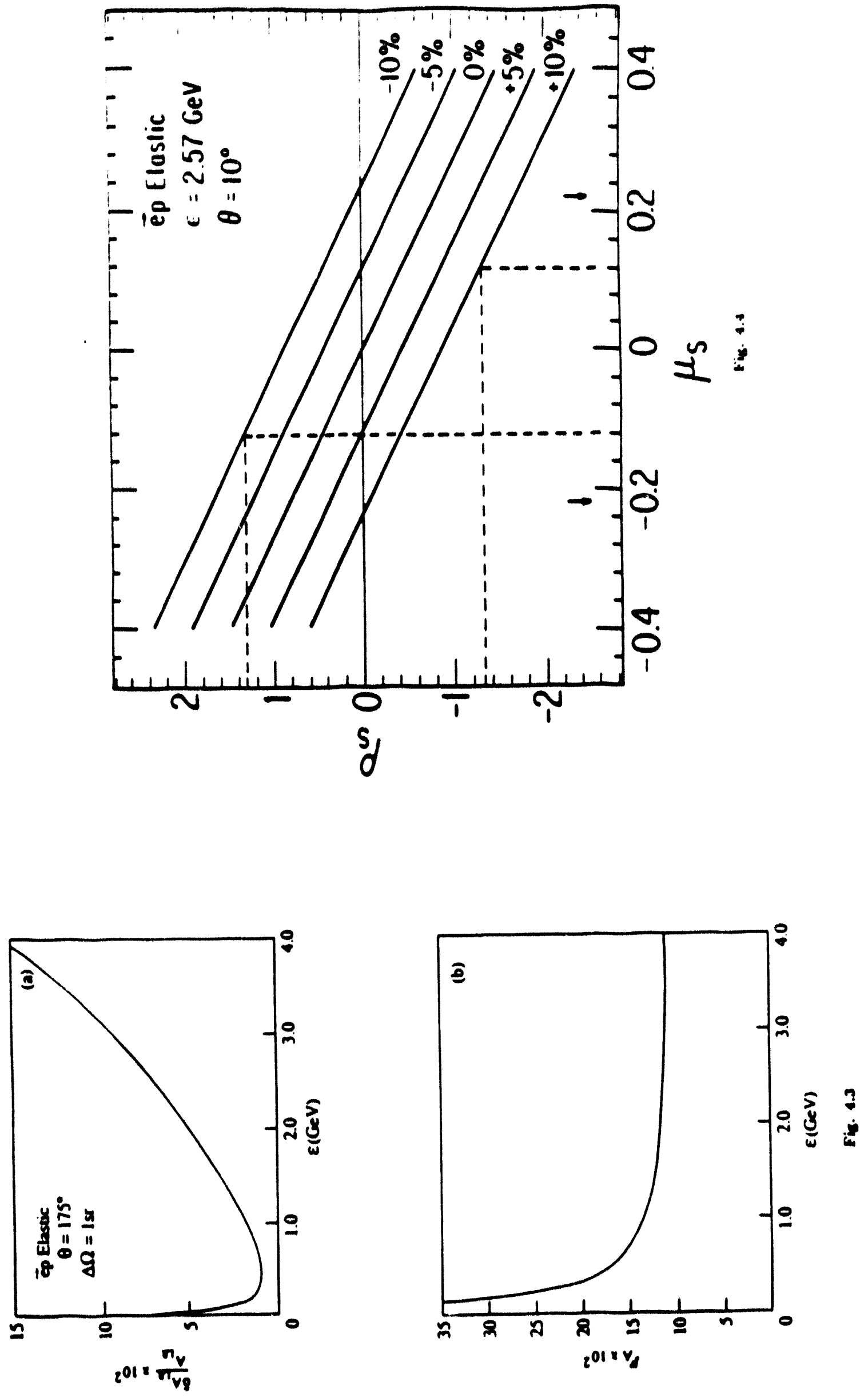

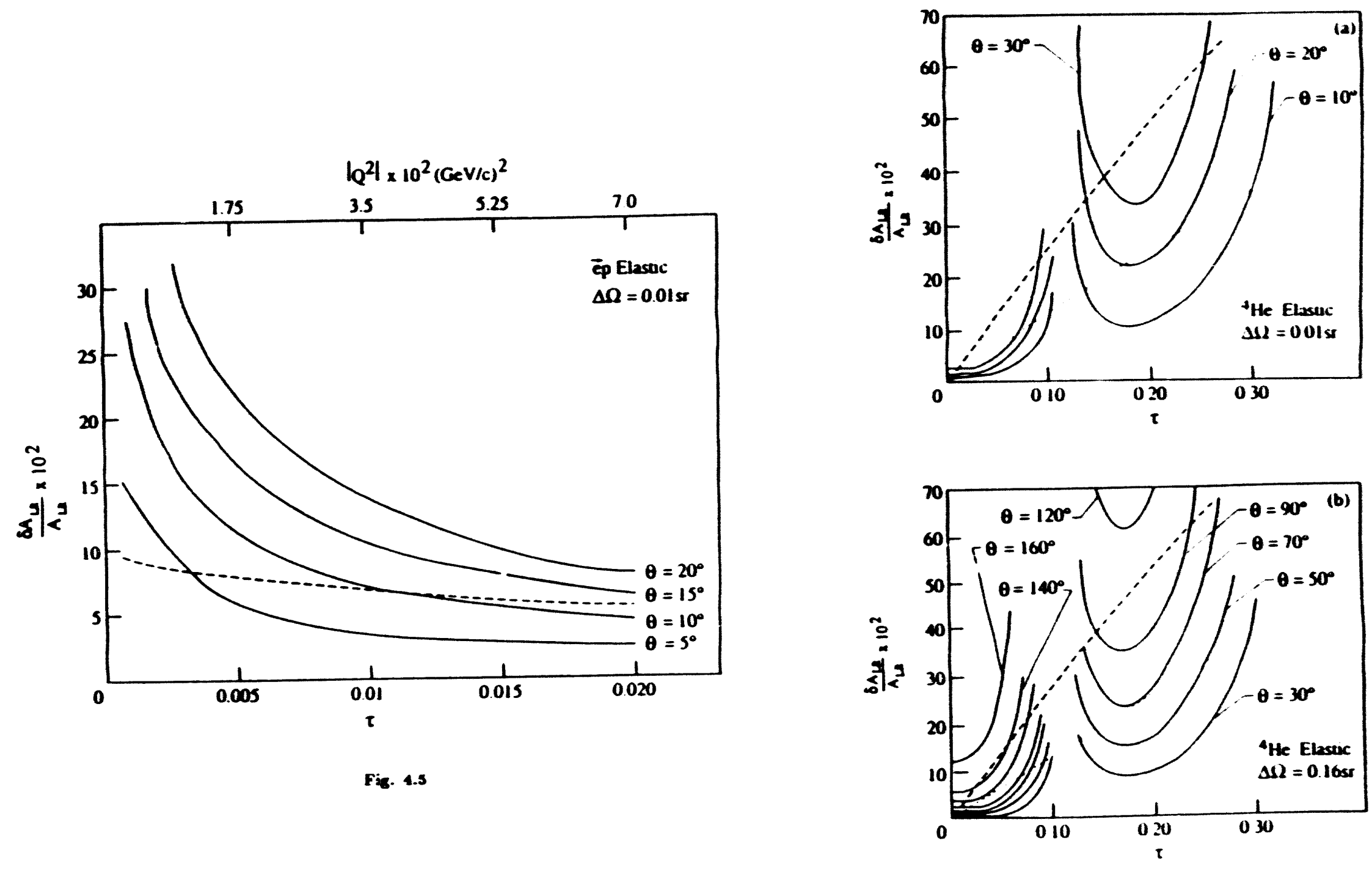

t.8. 1.6 

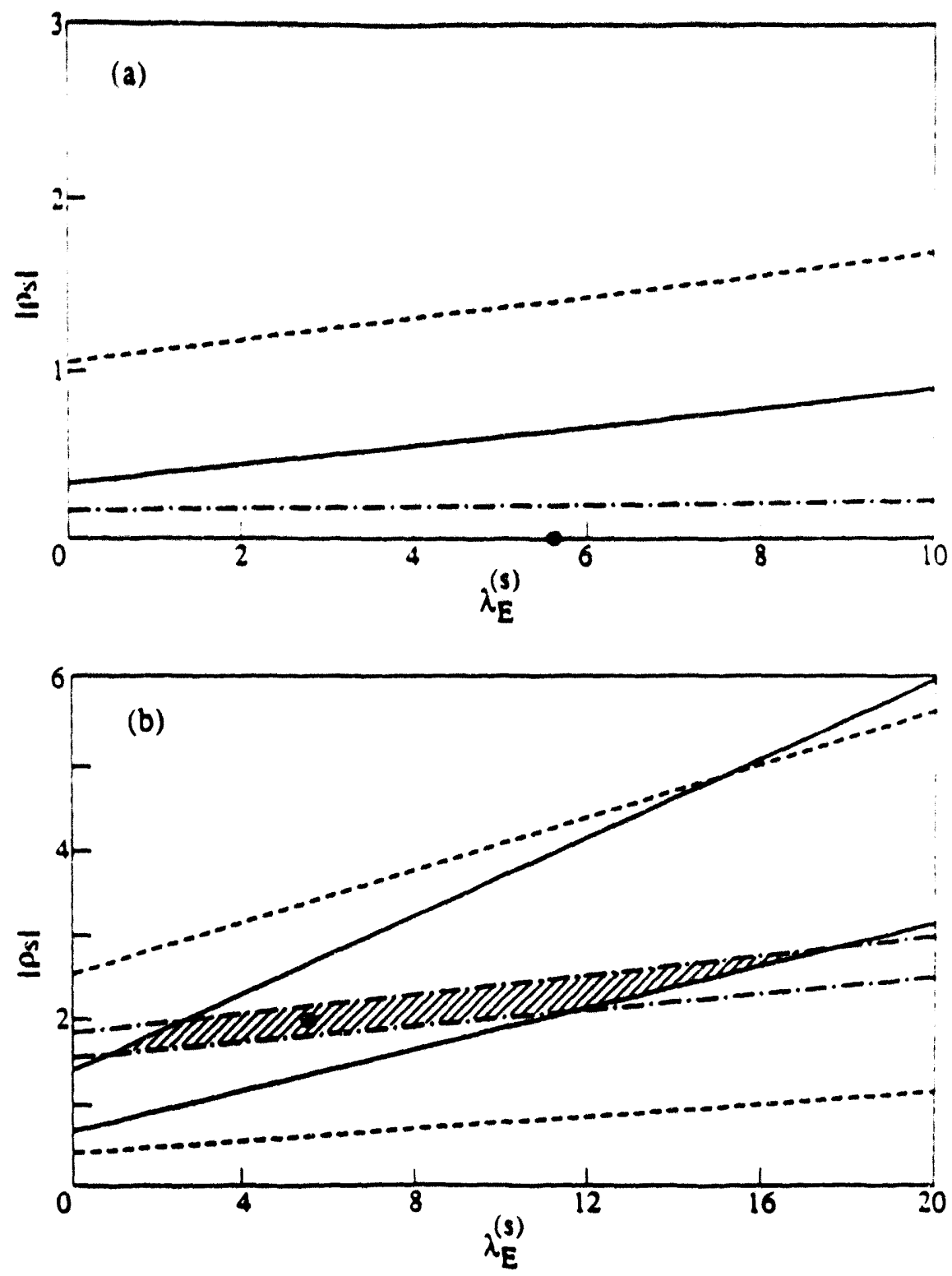

Fig. 4.7 

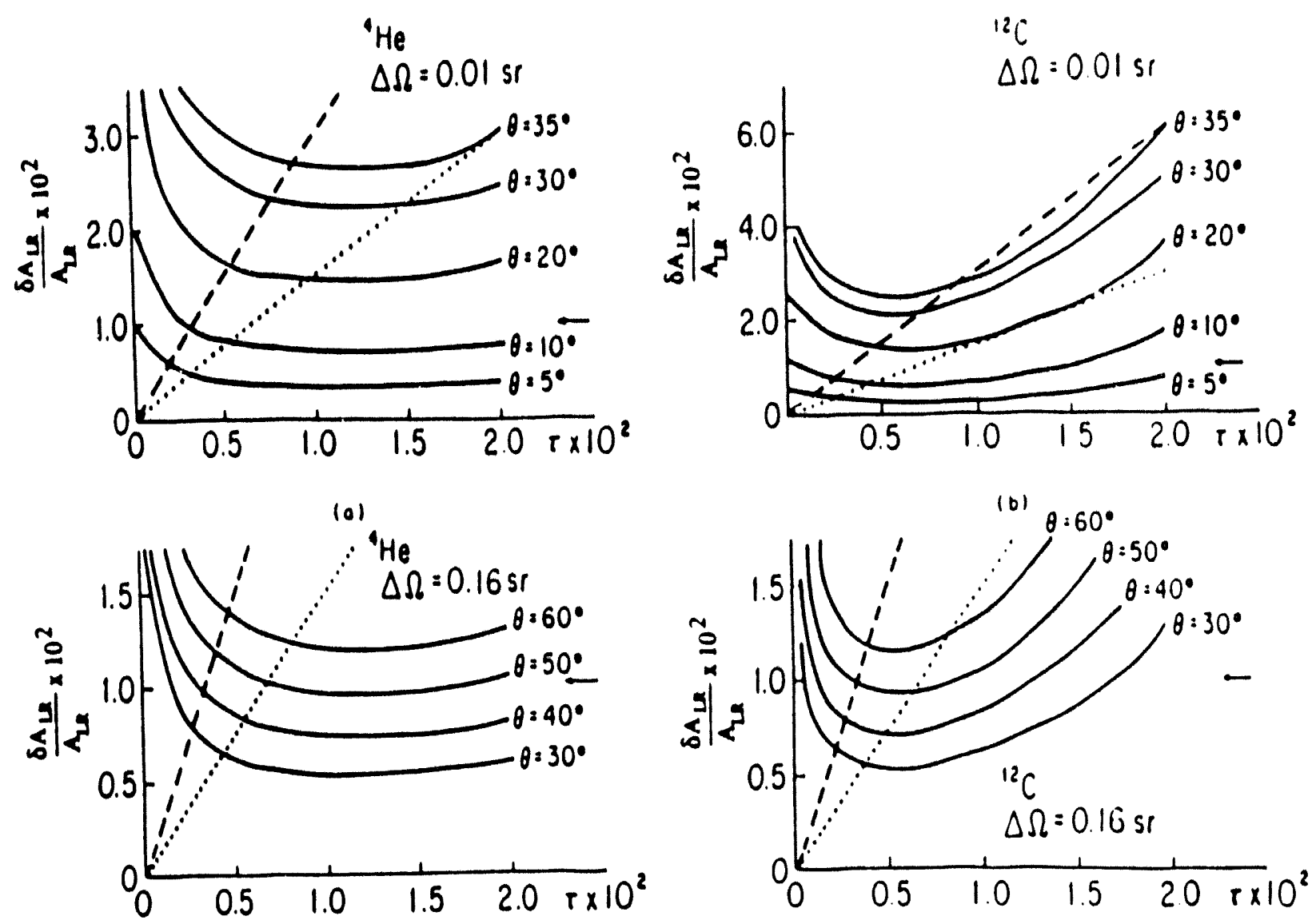

(c)

Fig. 4.8

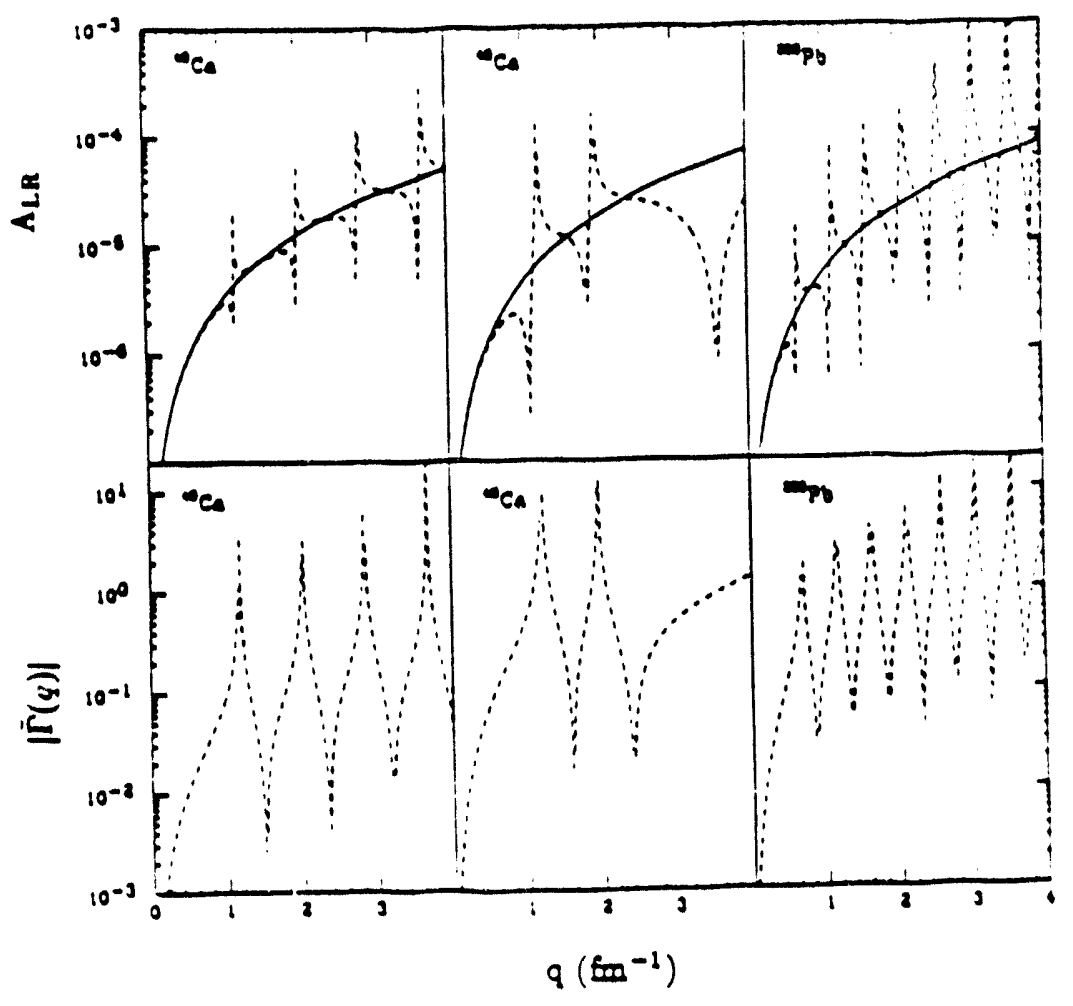

Fis. 4.8 


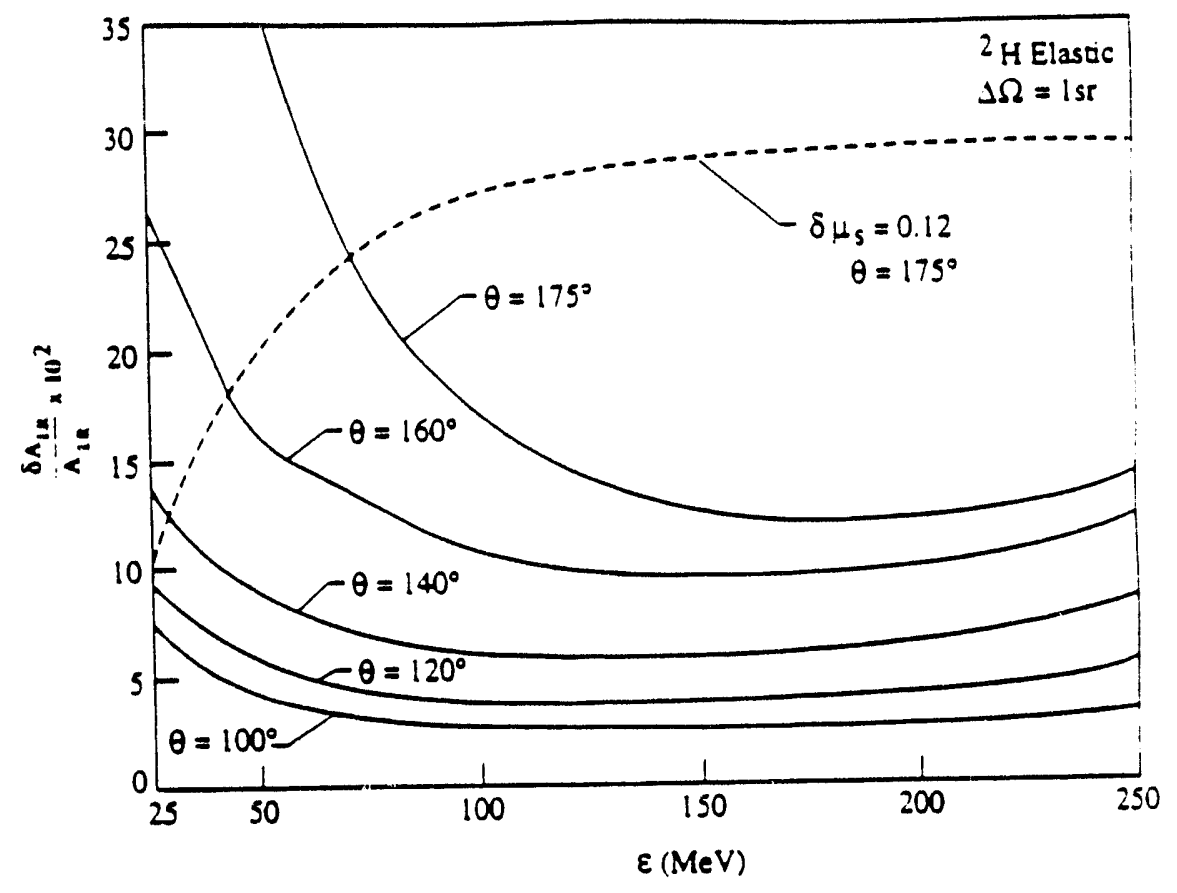

Fig. 4.10
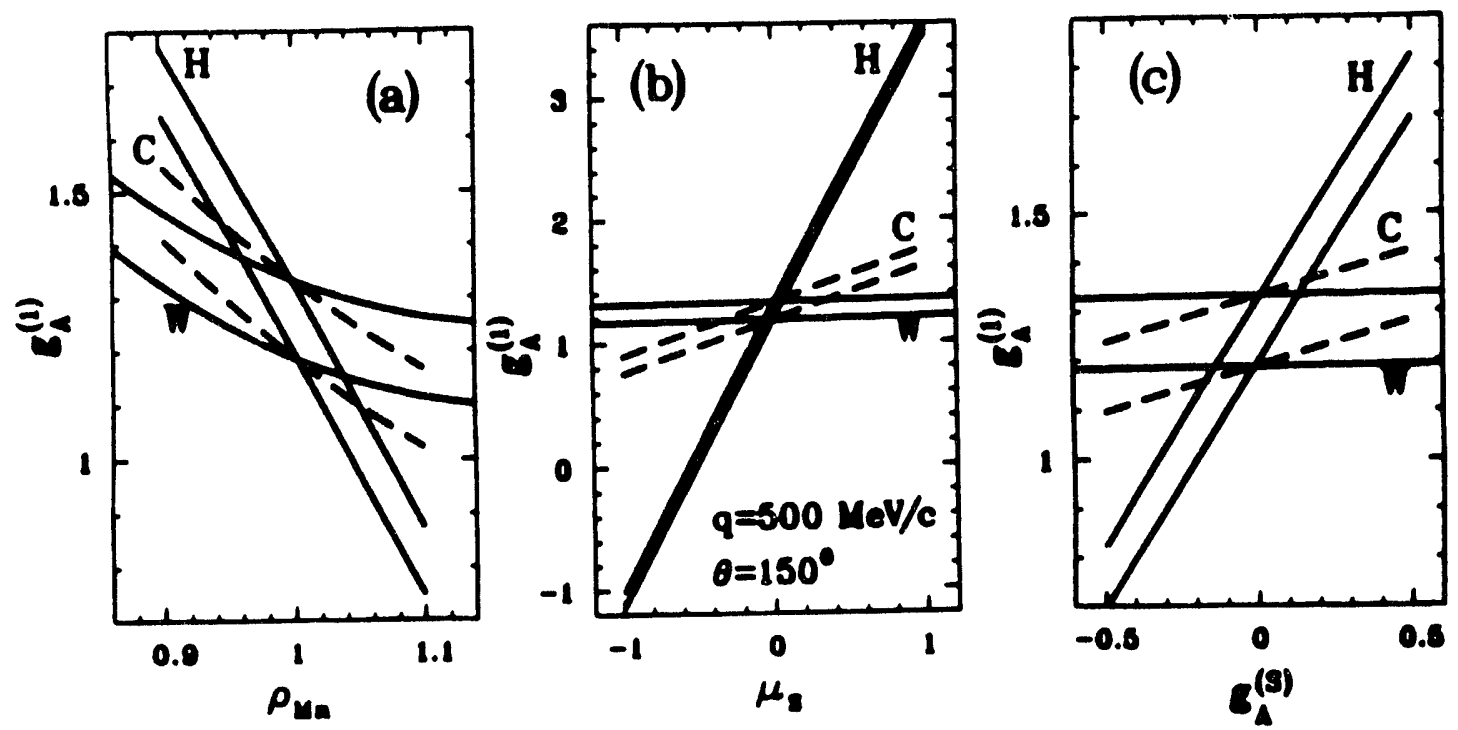

Fig. 4.11 


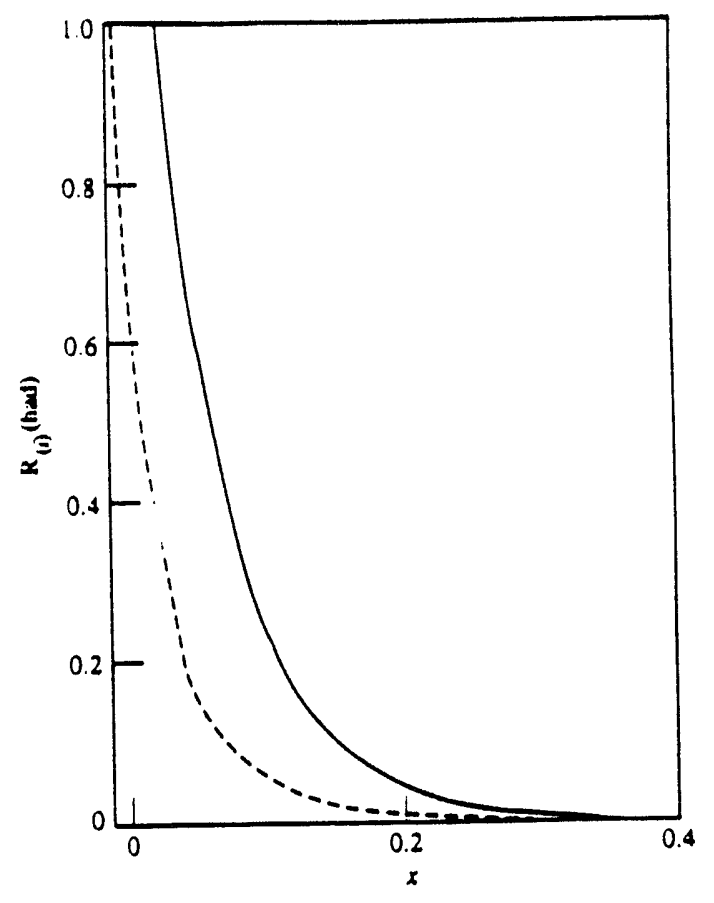

Fig. 4.12

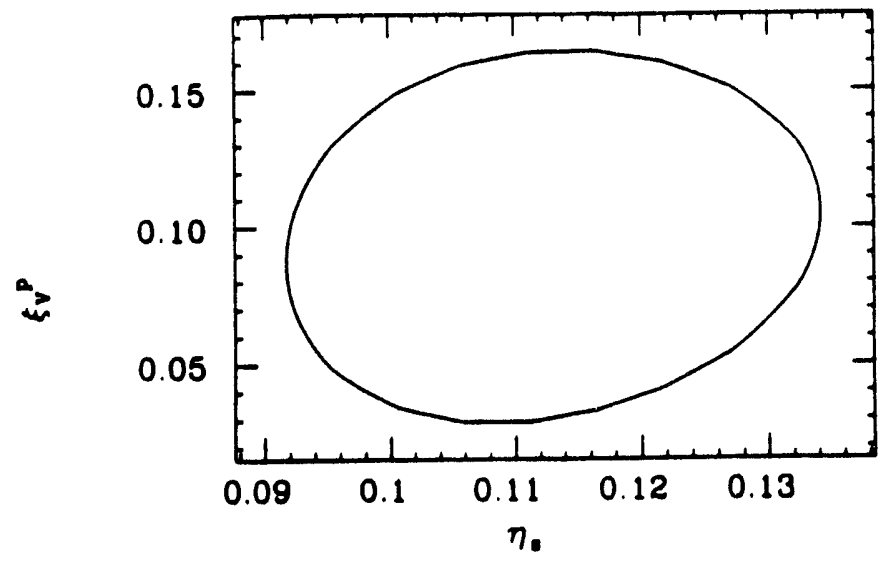

Fig. 4.13 


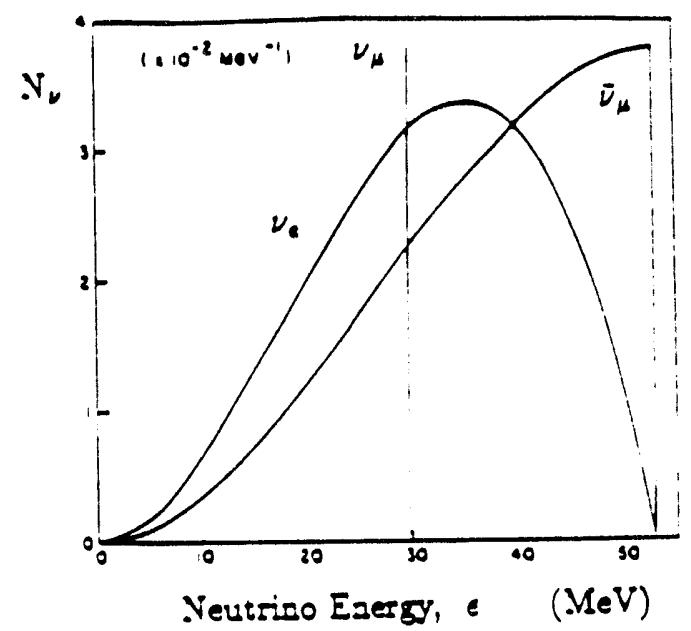

Fig. 3.1

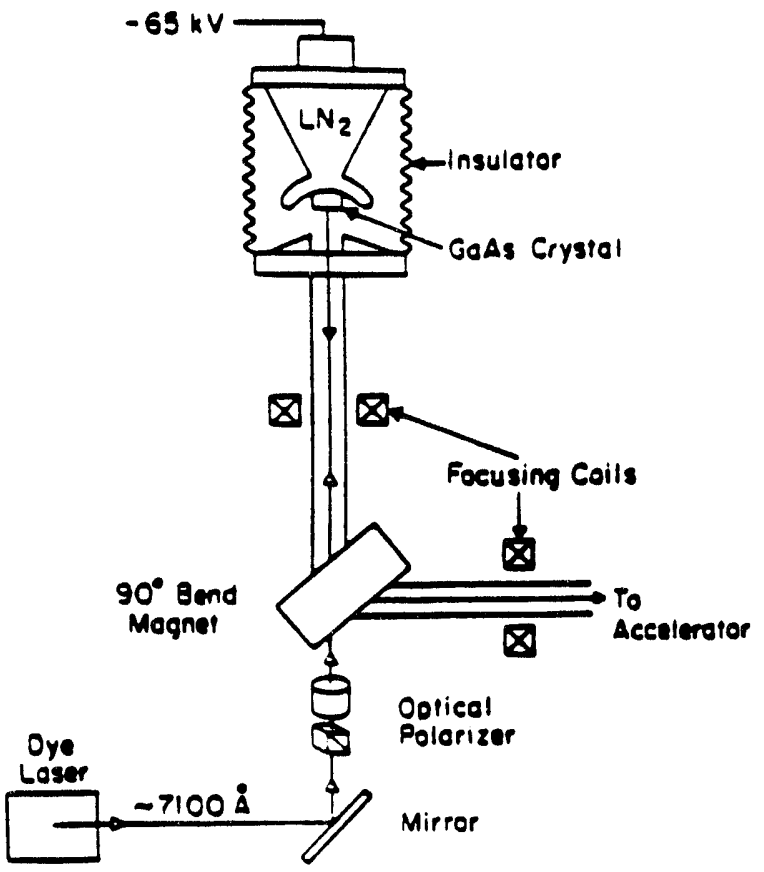

Fig. 3.2 


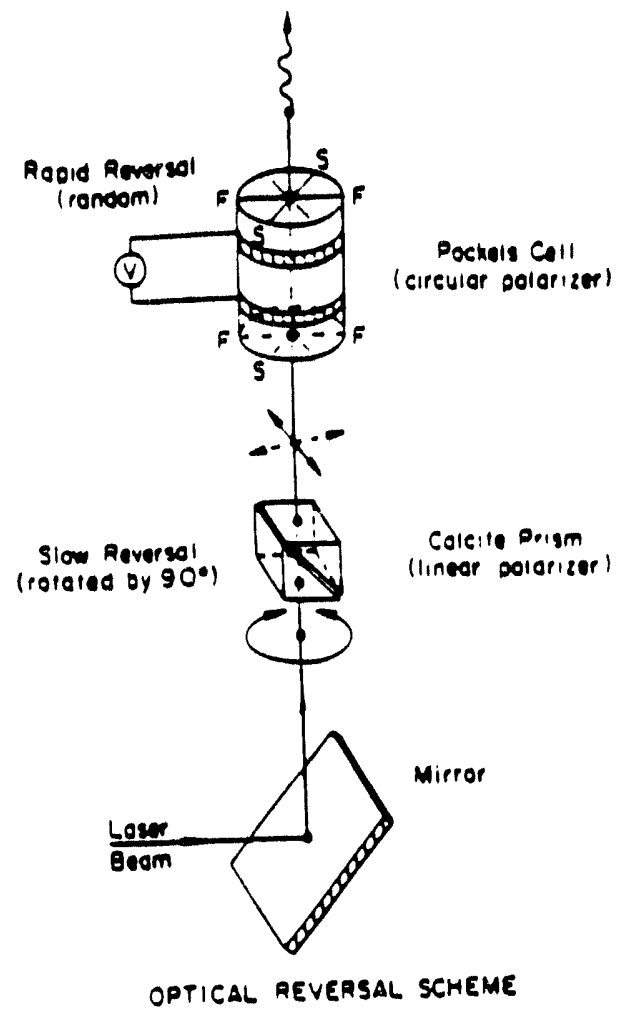

Fig. 5.3

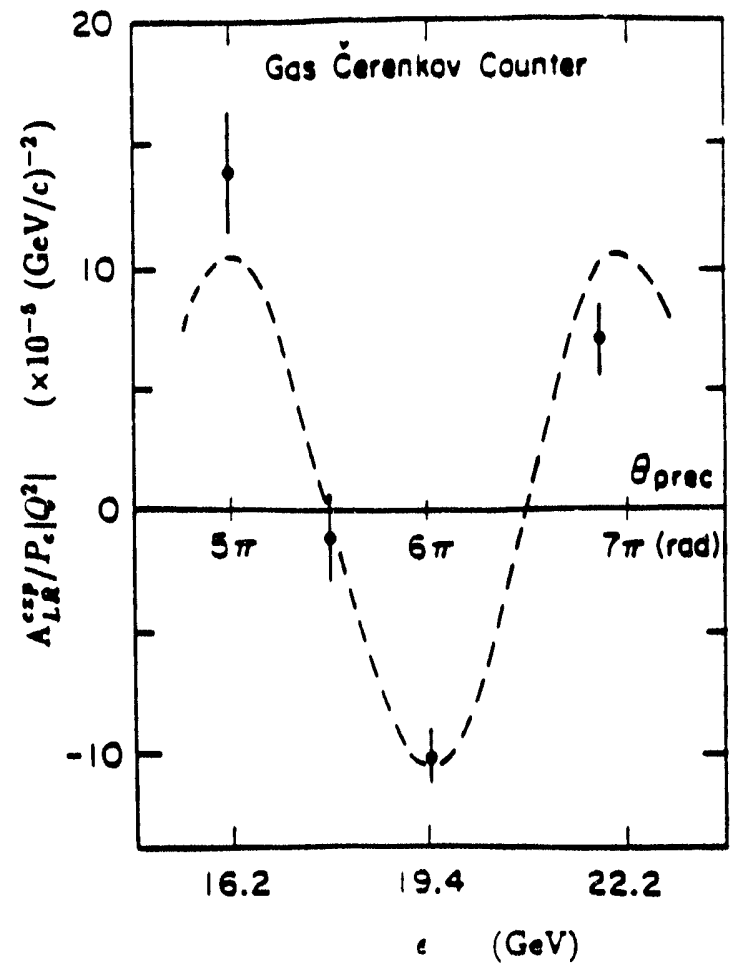

Fig. 3.4 


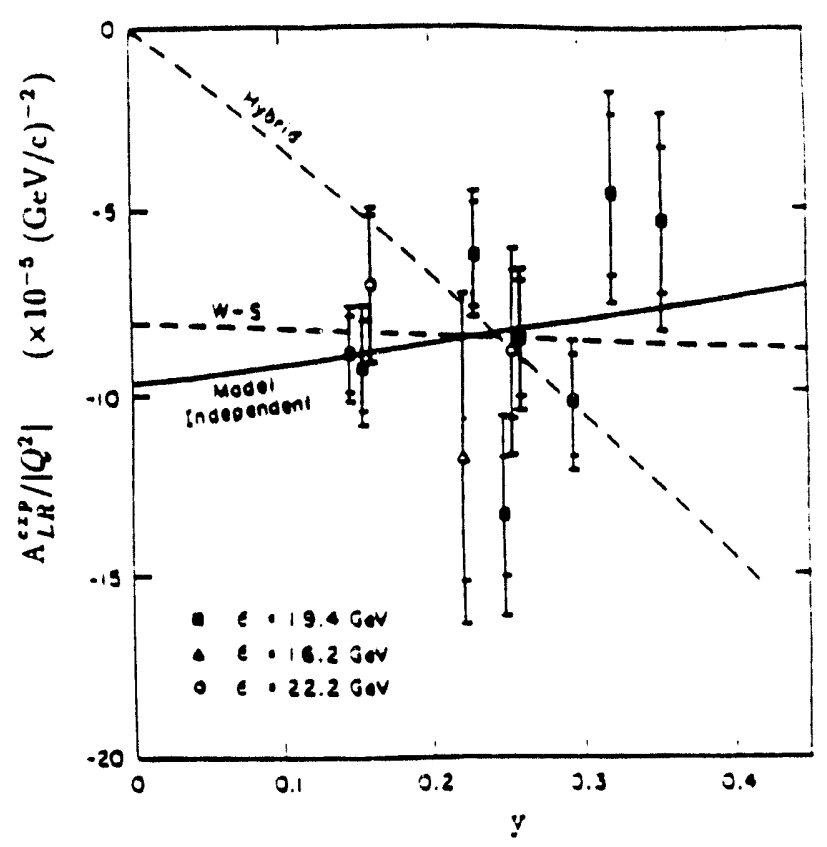

Fig. 3.5

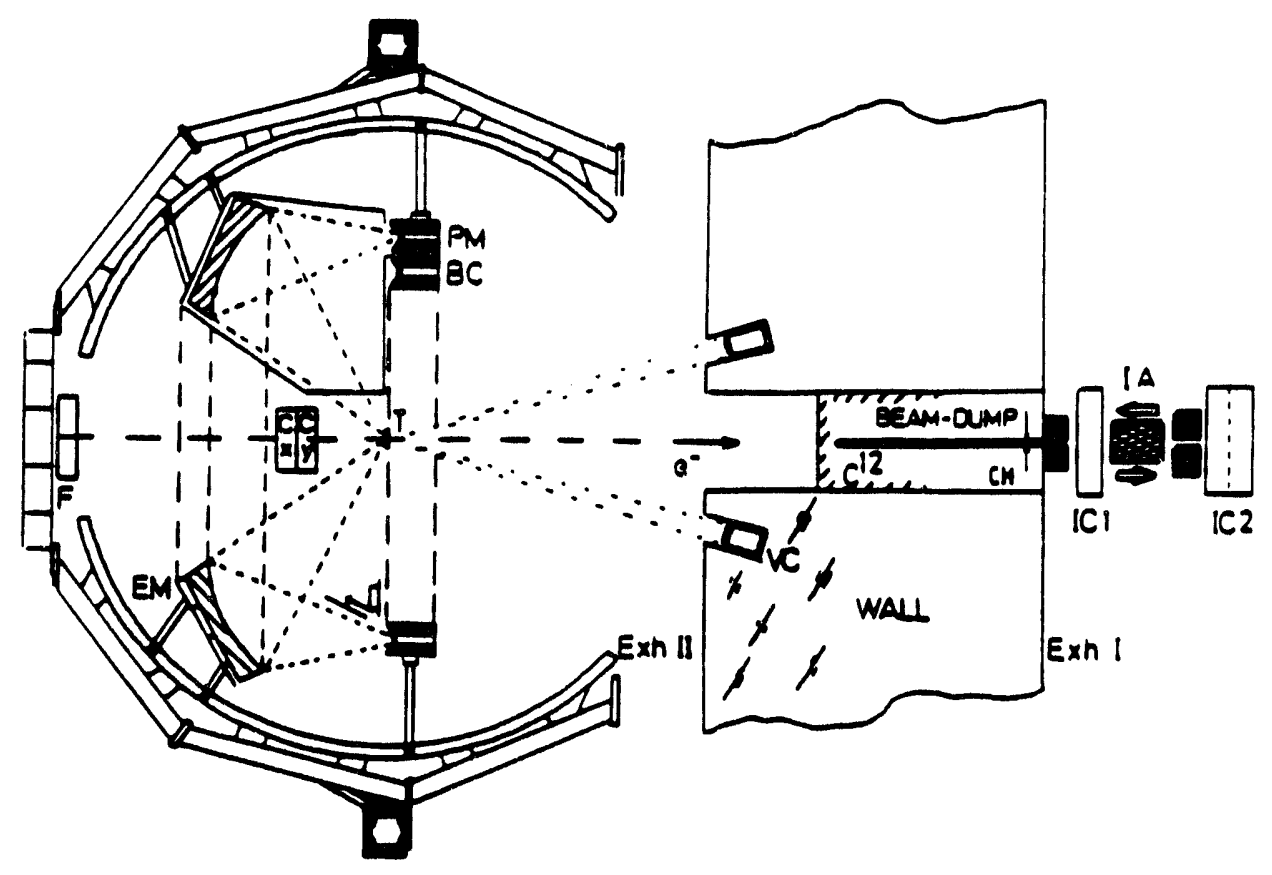

Fig. 5.6 

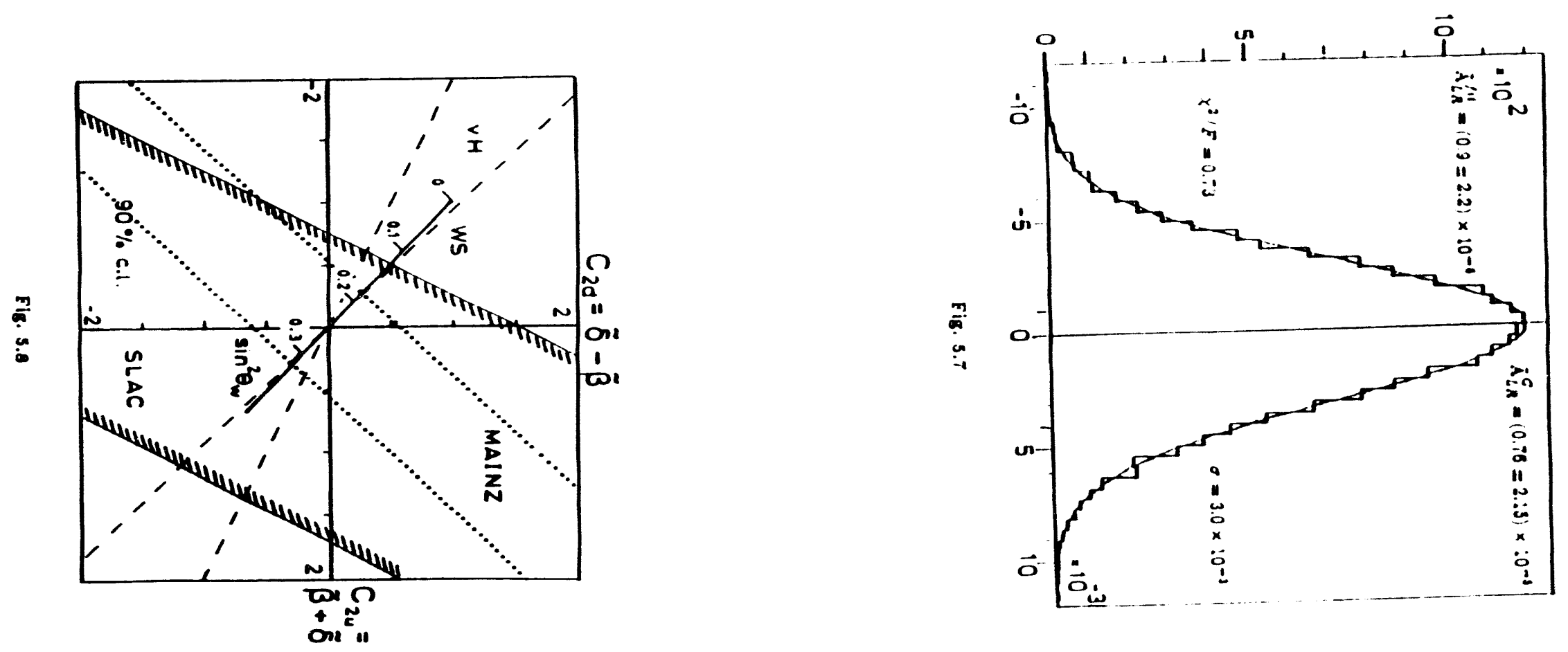


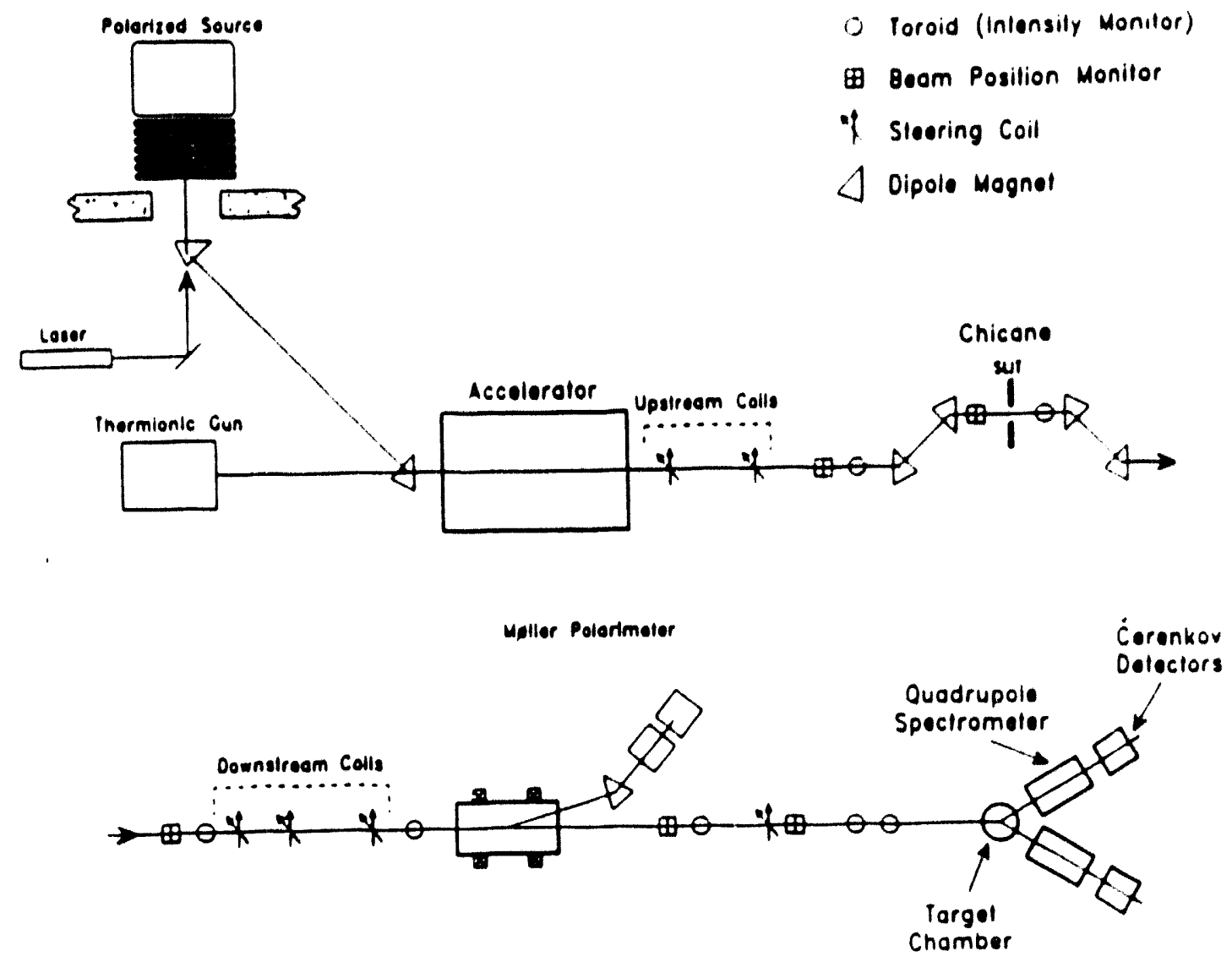

Fig. 5.9
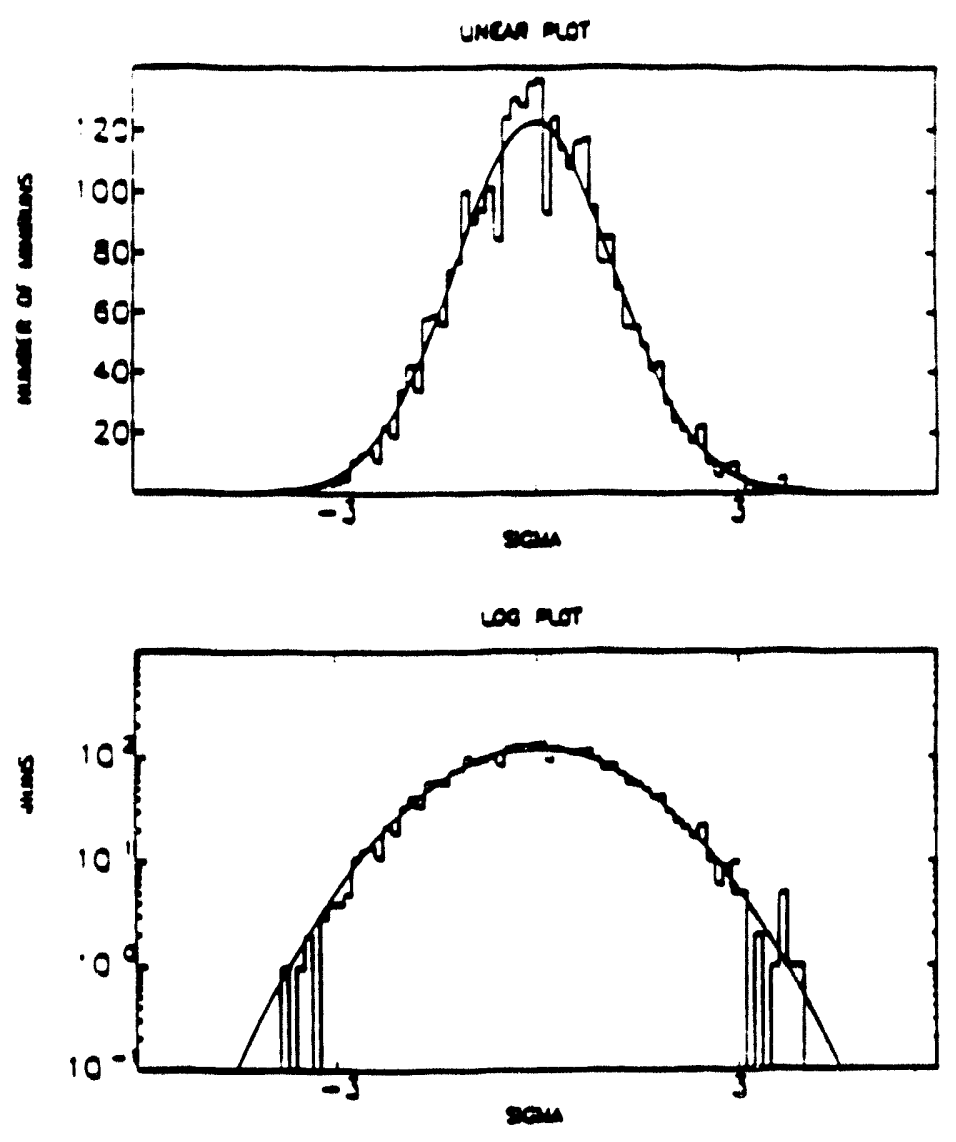

Fis. 5.10 

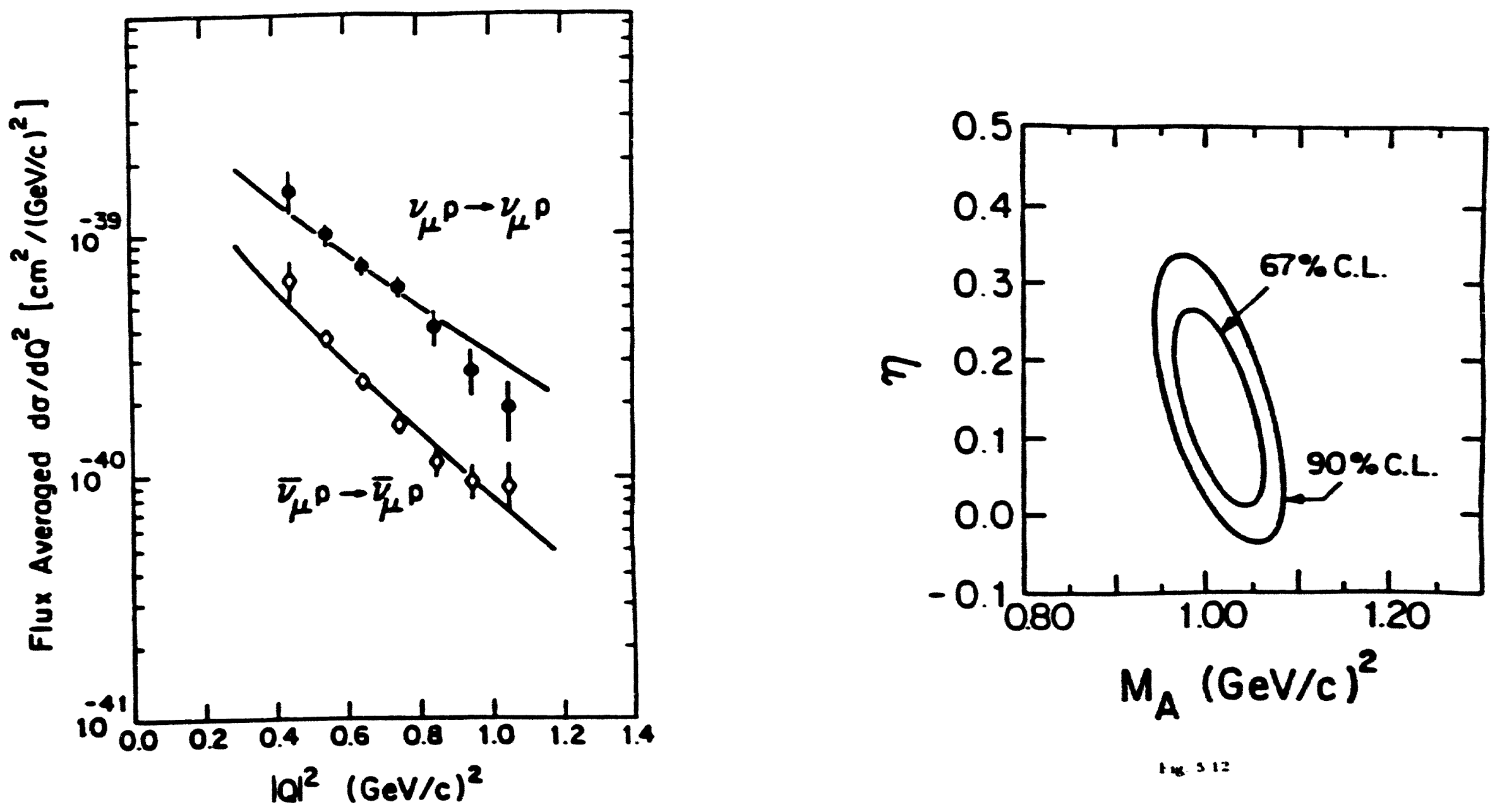

Fie. 5.11 


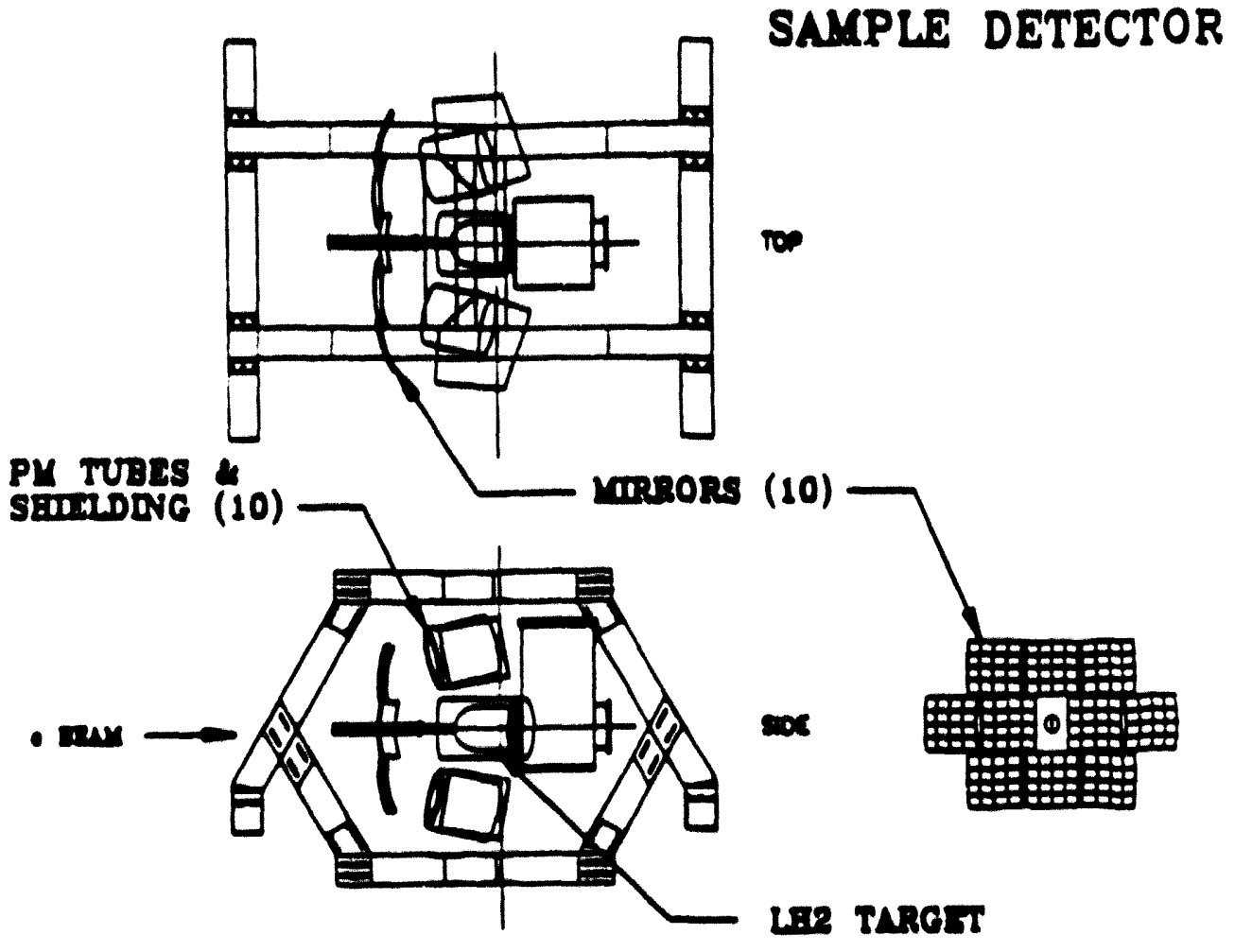

Fig. 5.13

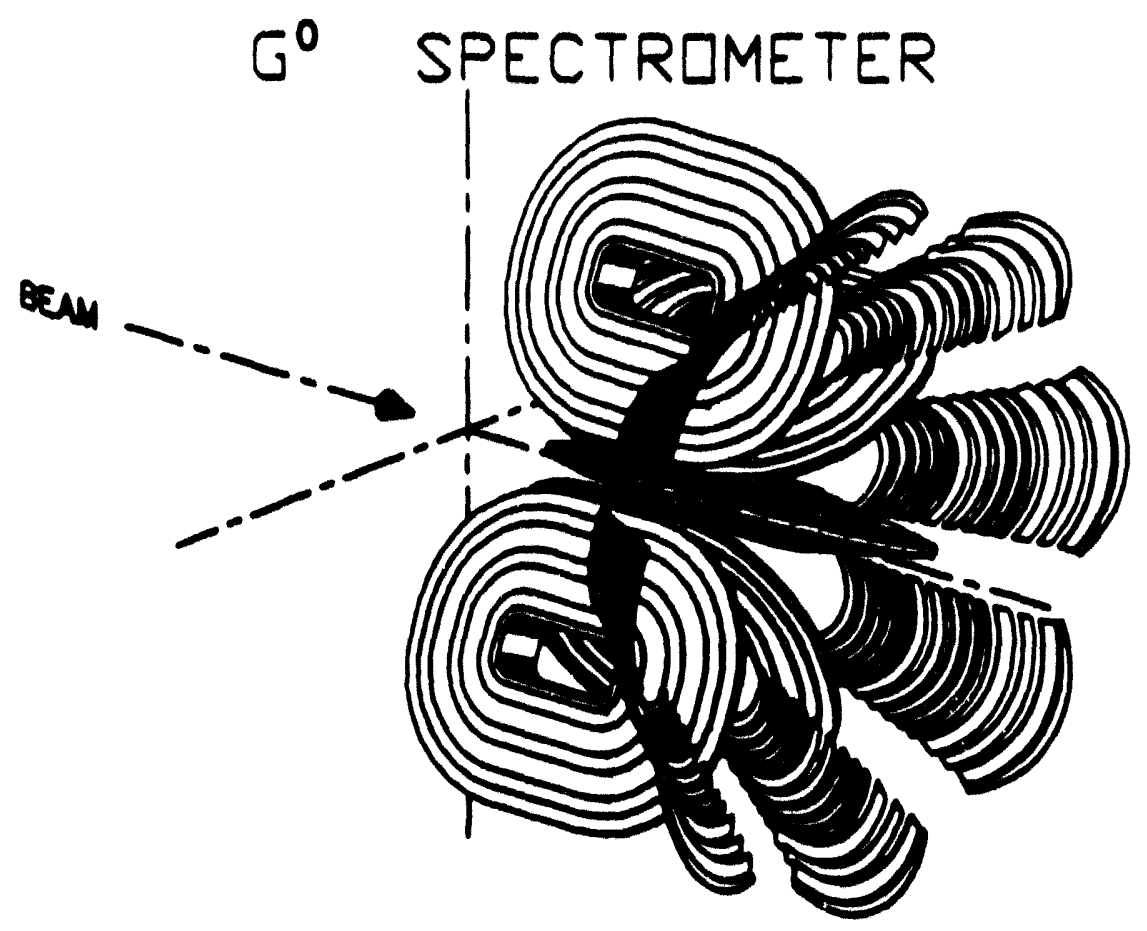

Fk. 3.14 


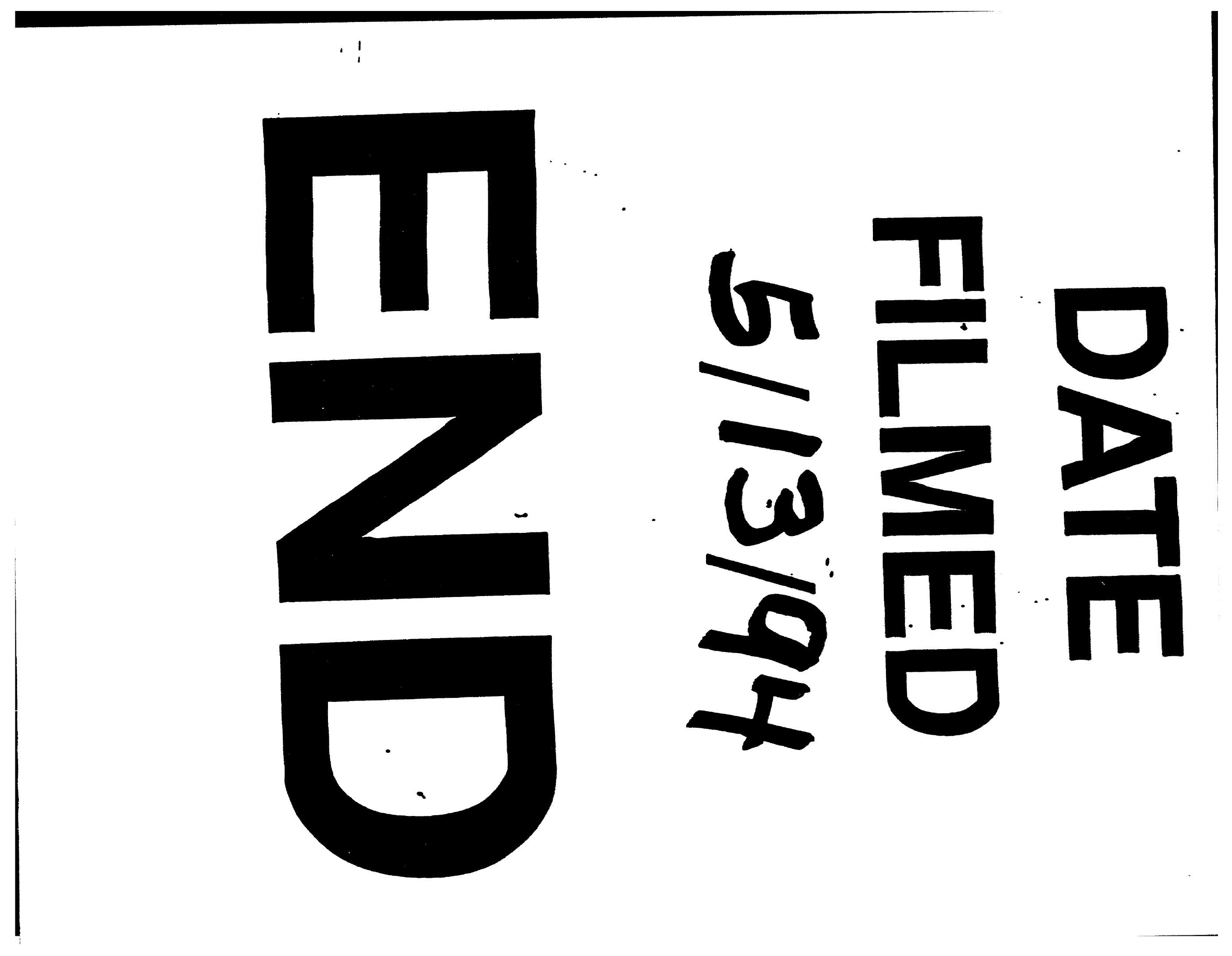




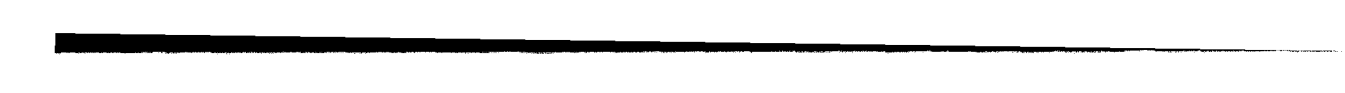

